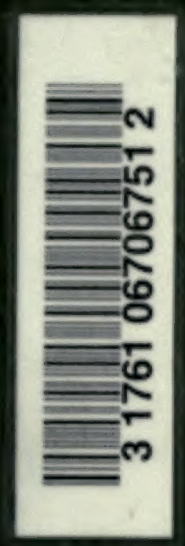

UNIVDF

IORONTO

LBBRARY 


Digitized by the Internet Archive in 2007 with funding from Microsoft Corporation 

$B$.

\section{CHECK LIST}

OP THE

\section{LE P I D O P T E R A \\ OF}

\section{BOREAL AMERICA}

BY

WM. BARNES, S. B., M. D.

AND

J. McDUNNOUGH, PH. D.

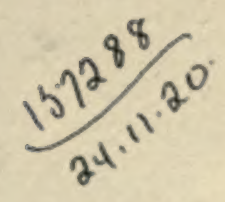

DECATUR, ILL.

HERÁL PRESS

FEB. 1917 


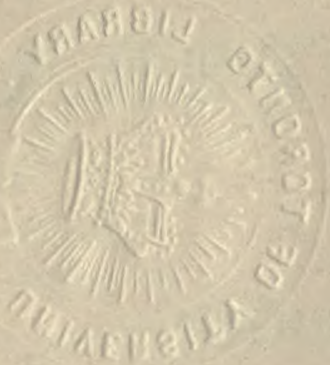

PUBLISHED UNDER

THE

PATRONAGE

OF

MISS JESSIE D. GILLETT

ELKHART, ILL. 
14 


\section{INTRODUCTION}

Thirteen years have elapsed since the publication of the last complete list of North American Lepidoptera by Dr. J. B. Smith. In the meantime many new species have been described and-what is still more important-revisions of various groups have been made by specialists, resulting in many instances in very radical changes, so that Smith's list, whilst excellent when published, has now become entirely antiquated and of little use as a basis on which to arrange a collection.

It has seemed to us therefore that the publication on our part of this present list may be of some value to students of Lepidoptera who wish not only to arrange their collections but to have before them in a concise form the latest views on the classification of this order of insects.

In general this list is based on the latest revision of each group, combined in many instances, more especially in the so-called Macrolepidoptera, with work which has been carried on for several years in the Barnes Collection in connection with the study of the existing types of North American species and the comparison of specimens with these same types; the synonymy of each species is given as fully as possible in so far as it relates to species described from North American material, and we have incorporated a number of changes in the list which have been commented on in several papers by ourselves which recently appeared in print.

In the DIURNals we have made the arrangement cor- 
respond as far as possible with that advocated by Dr. Seitz in his Macrolepidoptera of the World; we have revised the Lycænid and Hesperid genera to make them conform with the rules of nomenclature as adopted by the International congress, and in this connection would express our appreciation of the kind assistance rendered us by Mr. Bethune Paker of Edgbaston, England, in determining the Lycænid genera which it is advisable to employ.

In the SPHINGIDE we have followed Rothschild and Jordan's masterly revision, making only a few generic changes which we considered advisable.

The general scheme of the ARCTIIDE and Noctuide has been that of Sir George Hampson's Catalogue of the Lepidoptera Phalænæ of the British Museum, but in the latter family we have been obliged to change the generic conceptions considerably as the method employed by Hampson in determining generic types is not permissable according to the International Rules; these changes have been made the subject of a special paper published recently.

In the GEOMETRID $\&$ we have followed Mr. L. B. Prout's careful revisions in the Genera Insectorum and in Seitz's Macrolepidoptera as far as published, making only such changes as our more extended acquaintance with the North American species and their types has rendered necessary; our most hearty thanks are due Mr. Prout for the invaluable aid he has so cheerfully given us in connection with the difficult genera of this group.

The PYRALID E remain more or less as listed by Dr. Smith in so far as the genera are concerned, but numerous shifting of species has been necessitated, especially in the Phycitinae, as our knowledge of the same has been increased by long series of both sexes. 
Finally with regard to the Microlepidoptera Mr. August Busck of the U. S. National Museum has had the kindness to read over our whole manuscript relating to this group, suggesting many important changes in connection with the sequence of the families and genera, a fact which has rendered this portion of the list much more up-to-date than we could ever have hoped for without Mr. Busck's generous assistance.

With regard to certain generic terms we might note that, following the majority of the European authorities, we have disregarded Hubner's 'Tentamen' as being unpublished; this has resulted in several rather unfortunate substitutions, notably the displacement of the well-known genera Olethreutes and Coleophora in favor of 'Verzeichniss' names which are not so generally known; we feel, however, that such changes, while regrettable, should be made for the sake of uniformity in the list and in accordance with a strict adherence to the rules of nomenclature.

In the text we have as far as possible used the subdivisions $a, b, c$, etc., to denote geographical races which have often been listed, especially in Europe, as var. The term form we have applied to a variety which exists alongside the nimotypical form or which may be a seasonal one and we have confined the term $a b$. to so-called freak specimens or sports. In many instances, of course, our grouping is more or less tentative.

Certain European and foreign species which have been erroneously recorded from North America as well as a few species in which the type material, although stated to be North American, has proved foreign we have omitted and append a list of these at the conclusion of the work. 
Names preoccupied or cited in error we have marked with an $\ddagger$.

Finally we have thought it advisable to include a full index of all the species as well as the genera in order that the numerous shiftings which have been necessary should give no trouble to the average collector in locating any given species.

A few additions and corrections will be found at the end of the list proper.

Decatur, Febry., 1917. 


\section{Additions and Corrections}

Read gen. vern. immaculata Ckll.

immaculata Skin. \& Aar.

47 Add $a b . \&$ pallida Ckll.

60 Add $a b$. nigrofasciata Reiff.

250 Add ab. tristra Clill.

ab. rohweri Ckill.

263b Read $a b$. rufescens $E d w$.

$a b$. nigrescens $E d w$.

448 Add lorquini Behr.

1052 Read Heliolonche Grt.

2061 Read piffardi WLE.

atrifasciata Morr.

2880,1 olivacea Grossb.

2882,1

3051

numa Druce

3070

3097

Add form normani Bartsch.

3120

Add form nigra Eastman.

Add $a b$. chiquita Bartseh.

Add form melanotica Reiff.

a. novangliæe Reiff.

3409 Add $a b$. Puscostigma Clell.

$a b$. albostigma Ckll.

5805,1

naidaria Swett.

3850,1

davisi Grossb.

3977,1

populata Linn.

$\$ 025$

Add as olivata $W g t$.

b. pallidata $W g t$.

4031,1 switzeraria $W g t$. form swetti Wgt.

4500, 1 Read melanocarpa Swett. tremularia B. \& McD.

4577,1 cypressaria Grossb.

4600,1 4798,1

5760 unipunctaria Wgt. pallidovenata Grossb.

5761

Add planatella Grossb.

5762,1

Add verecuntella Grossb.

Wunderia Grossb. neæriatella Grossb.

5835,1 albicostella Grossb.

5917,1 5925,1 simplicius Grossb. perplexus Grossb.

Read Vespamima Beut. 



\section{LIST OF LEPIDOPTERA}

\section{Superfamily PAPILIONOIDEA}

\section{PAPILIONIDE}

Papilio L.

1 philenor $L$.

astinons Dru.

ab. wasmuthi Weeles.

$a b$. obsoleta $E h r$.

a acauda Oberth. nezahualcoyotl Stkr. corbis G. S.

b hirsuta Skin.

2 devilliers Godt. villiersi Bd.-Lec.

3 polydamas $L$.

4 polyxenes Fabr. a americus Koll.

$b$ asterius Cram. form curvifascia Skin. form ampliata Men. materioide Reak. ab. alunata Skin \& Aar. ub. semialba $E h r$. ab. calverleyi Grt.

c brevicauda Saund. enticostiensis Stkr.

5 bairdi Edu.

utahensis Stkr.

form oregonia $E d w$.

form brucei $E d w$.

form hollandi Edw.

6 zelicaon Lue.

zolicron Bdv.

culifornica Men.

coloro Wright.

7 indra Reak.

$a$ pergamus $H$ y. Edw.
8 nitra Edu.

9 machaon $L$.

a aliaska Seudd. joannisi Verity.

10 thoas $L$. a autocles $R$. \& J.

11 cresphontes Cram. oxitus $\mathrm{Hbn}$.

12 aristodemus Esp. daphnis Gray. cresphontinus Martyn a ponceana Schaus.

13 glaucus $L$.
ajax L.
antilochus L.
alcidamas Cram.

ab. of fletcheri Kemp.

form \& turnus $L$.

$a$ canadensis $R$. \& $J$. areticus Skinner.

$b$ australis Mayn.

14 rutulus Luc.

$a$ arizonensis $E d w$.

$b$ ammoni Behrens.

15 daunus $B d v$.

16 eurymedon Luc. form albanus Feld.

17 pilumnus $B d v$.

18 troilus $L$. iloneus A.\& S.

a texanus Ehr. ab. radiatus Sikr.

19 palamedes Dru. chaleas Feld. 
20 marcellus Cram.

ajaxt Auct.

walshi Edw.

form ménétriesi $H y . E d w$. lusca Stich.

abboti Edw.

$a b$. lorquini Oberth.

form floridensis Holl.

$a b$. immaculata Skin.

form telamonides Feld.

f. aest. lecontei $R . \& J$.

c gallatinus Stich. marcellus $\ddagger \mathbf{B d v}$.

21 celadon $L$ Luc.

sinont Auet.

Parnassius Latr.

22 eversmanni Men.

a thor $\boldsymbol{H} y, \boldsymbol{E} d w$.

23 nomion Fisch.

24 clodius Men.

$a b$. altaurus D D yar.

a claudianus Stich.

$b$ baldur Ediv.

25 smintheus Dbldy \& Hew.

$a b$. nanus Neum. mendica Stich. minor Verity. form alt. hermodur $\boldsymbol{H} y$.

$E d w$.

$a b$. nigerrima Verity.

$a$ apricatus Stichel.

b magnus Wright. pseudoeorybas Verity.

$c$ sayi $E d w$.

$d$ behri $E d w$.

ab. niger Wright.

\section{PIERID正}

Neophasia Behr.

26 menapia Feld. tau Scudder. ninonia Bdv. suffusa Stretch.

27 terlooti Behr.

epyaxa Stkr.

form \& princetonia Poling

\section{Appias Hbn.}

28 ilaire Godt.

molpodia $\mathrm{Hbn}$.

margarita $\mathrm{Hbn}$.

mysia Godt.

poeyi Butl.

a neumoegeni Skin. hollandi Rob.

Pieris Schrank.

29 amaryllis $\mathrm{Fabr}$.

a josepha $G$. \& S.

30 monuste $L$.

cleomes Bdv. \& Lec.

form \& phileta Fabr.
31 beckeri $E d w$.

32 sisymbrii $B d v$.

33 occidentalis Reak.

$a$ calyce $E d w$.

b nelsoni $E d w$.

34 protodice $B d v$. \& Lec. gen vern. vernalis $E d w$.

35 napi $L$.

$a$ arctica Verity.

$b$ pseudobryonir Verity.

c hulda $E d w$.

$d$ borealis Grt.

e frigida Scud. pseudoleracea Verity. gen. aest. acadica Edw.

$f$ gen vern. oleracea Harris. gen. aest. cruciferarum

$B$ iv。

casta Kirby. aestiva Edw.

$g$ gen. vern. venosa Scud. nasturtii Bdv. gen. aest. castoria Reak. resedae Bdv. 
h. gen. vern. marginalis Seud. gen. aest. pallida scued.

$a b$. flava $E d w$.

$i$ virginiensis $E$ du.

k. pseudonapi B. \& $\mathrm{MCD}$. gen. aest. pallidissima B. \& MeD. -36 ochsenheimeri Staud.

87 rapae $L$.

yreka Reak.

gen. vern. immaculata

Skin. \& Aar. ab. \& novanglize Scud.

Nathalis Rdv.

38 iole $B d v$.

irene Fitch.

luteolus Reak.

Euchloe Ithn.

39 creusa Dbldy. elsa Beut.

a hyantis $E d w$. pseudoausonides Verity.

$b$ orientalides Verity.

c lotta Beut. belioides Verity.

40 ausonides $B d v$.

a coloradensis $\mathrm{H} y . \mathrm{Ed} w$. montana Verity.

Zegris Rmb.

41 olympia $E d w$. a rose $E d w$.

Anthocharis Bdr.

42 lanceolata $B d v$. edwardsi Behr.

a australis Grinnell

43 genutia Fabr. midea $\mathrm{Hbn}$.

44 cethura Feld. cooperi Behr. angelina Bdv. a morrisoni Edue.

$b$ deserti Wright

45 pima $E d w$.

a caliente Wright

46 sara Bde. mollis Wright. gen. vern. reakirti Edu.

a flora Wright

b julia Edw. thoosa Seud.

c stella Edw.

d browningi Skin.

Catopsilia IIhn.

47 eubule $L$.

$$
\begin{aligned}
& \text { form senna } L \text {. } \\
& \text { marcellina Cram. } \\
& \text { yamana Reak. }
\end{aligned}
$$

48 philea $L$.

aricye Cram. melanippe Cram.

*49 argante Fabr. larra Fabr. cridie Godt.

50 agarithe $B d v$. a maxims Neum. floridensis Rob.

51 statira Cram. a floridensis Nerm.

Amynthia Swains.

52 elorinde Godt. swainsonia Swain. maerula Hbn. godarti Perty.

Kricogonia Reak.

53 lyside Godt.

of lanice Lint.

form \& terissa Lue.

form \& unicolor G. \& S. xanthophila Rob.

form \& fantasia Butl. \& lanice Lint. 


\section{Zerene IIlon.}

54 eurydice $B d v$.

wosnesenski Men.

lorquini Bdv.

helena Reak.

gen. aest. amorphæ Hy.

a bernardino $E d w$.

$E d w$.

55 cresonia Stoll.

caroliniana Pet.

gen. autumn. rosa McNeill

Eurymus Swains.

56 meadi $E d w$.

$a$ elis Stkr.

57 hecla Lef.

a glacialis McLach. chrysothemoides Verity.

$b$ hela Stkr.

$c$ pallida Skin. \& Men.

58 boothi Curt.

form chione Curt.

59 eurytheme $\boldsymbol{B} d v$.

ariadne Edw.

form amphidusa $B d v$.

keewaydin Edw.

californiana Men.

$a b$. \& fumosa Stler.

form eriphyle $\boldsymbol{E} d w$.

hageni Edw.

gen. vern. autumnalis Clell.

$a b$. intermedia Ckll.

ab. \& pallida Clcll.

gen. vern. kootenai Cockle.

60 philodice Godt.

palaeno Cram.

europome Steph.

$a b$. pallidice Scud.

$a b$. nigridice Scud.

nigrina Stkr.

ab. miscidice Scud.

$a b$. luteitincta Wollc.

$a b$. virida Stkr.

gen. vern. anthyale $\mathrm{Hbn}$.
61 occidentalis Scud.

a chrysomelas $H y . E d w$.

b barbara $H y$. $E d w$.

62 harfordi $H y . E d w$.

63 interior Scud. laurentina Scud.

64 christina $E d w$. form astræa $E d w$. $a b$. \& pallida Ckll. form gigantea Stkr. pelidneides Staud.

65 alexandra $E d w$. alba Stkr.

$a$ edwardsi Behr.

$b$ emilia $E d w$.

66 scudderi Reak. $a b$. flavotincta Ckll.

67 pelidne $B d v$.

a labradorensis Scud. ab. of mira Verity $a b$. \& moeschleri $G r$. Gr.

$b$ skinneri Barnes.

c minisni Bean menisme Verity

68 palæno $L$. philomene Hbn. lapponica Staud. werdandi $\mathrm{H}$. S.

$a$ chippewa $E d w$. helenał Edw.

69 nastes $B d v$.

a rossi $G n$.

$b$ streckeri $G r . G r$.

c cocandicides Verity $a b$. obscurata Verity.

70 moina Stkr.

71 behri $E d w$.

Eurema Hbn.

72 gundlachia Poey.

73 proterpia Fabr.

74 mexicana $B d v$.

\& damaris Feld.

depuiseti Bdv. 
i5 boisduvaliana Feld. ingrata Feld.

76 nicippe Cram. ab. Aava Stkr.

77 westwoodi $\mathrm{Bdv}$. dina Geyer.

78 euterpe $\mathbf{M}$ en.

lise Bdv.

form clappi Mayn.

form $\&$ alba Stkr.
80 delia Crum. demoditas Hbn. daire Godt.

81 jucunda Bdv. ebriola Poey. albine Poey.

82 blakei Mayn.

Dismorphia Hlhu.

83 melite $L$.

79 linda $E d w$.

\section{DANAIDFE}

Danaus I.inn.

84 archippus Fabr.

form atergatis Dbldy. \&

plexippus $\ddagger$ Auct. (nec. L.) Mechanitis Falor.

megulippe $\mathrm{Hbn}$.

*87 californica Reak.

ab. fumosus $\mathrm{Hlst}$. ab. pulchra Stkr.

85 berenice Cram.

Dircenna Dhhly.

form strigosa Betes.

Lycorea Dhldy.

86 eleobses Godt.

88 klugi $H b n$.

Dynothea Reak.

89 lycaste Fabr. a negreta Reak.

\section{SATYRIDAE}

Enodia Hbn.

90 portlandia Fabr. andromacha $\mathrm{Hbn}$.

91 creola Skin.

Neonympha Hbn.

92 gemma $\mathrm{Hbn}$. cornelius Godt.

93 henshawi $E d w$.

94 phocion Fabr. areolatus A. \& S. helcita Hbn.
Cissia Dbldy.

95 mitchelli Freh.

96 sosybiug Fabr. camerta Cram.

97 eurytus $\mathrm{Fabr}$. cymela Cram. eurytris Fabr. viola Mayn.

98 rubricata Edu.

Paramecera Butl.

99 xicaque Reak.

- Doubifulty North Amerlcan. 
Satyrodes Seud.

100 canthus $L$. eurydice Joh. boisduvali Harris.

a fumosus Leuss.

Cœnonympha Hbn.

101 california West \& Hew. ceres Butl.

form galactinus $B d v$. ab. palla $H y$. Edw.

$a$ eryngii $H y . E d w$.

102 kodiak $E d w$.

a yukonensis Holl.

:03 inornata $E d w$.

104 ampelos $E d w$.

$a$ elko $E d w$.

105 ochracea $E d w$. brenda Edw.

*106 pamphilus Linn. pamphiloides Reak.

107 haydeni $E d w$.

Neominois Scud.

108 ridingsi $E d w$. stretchi Edw.

109 dionysius Scud. ashtaroth Stkr.

Cercyonis Speyer.

110 pegala Fabr.

111 alope Fabr.

a maritima $E d w$.

$b$ texana $\boldsymbol{E} d w$.

c nephele Kirby.

d olympus $E d w$.

$e$ ariane $B d v$. incana Edw. form boopis Behr. form baroni Edw.

112 stephensi Wright
113 wheeleri $E d w$. hoffmani Stkr.

114 gabbi $E d w$.

115 meadi $E d w$.

116 behri Grinnell

117 sthenele $B d v$.

118 silvestris $E d w$. okius Oberth.

a paulus Edw.

119 oetus $B d v$. charon Edw.

a phocus $E d w$.

Oeneis Hbn.

120 macouni Edw.

121 nevadensis Feld. gigas Butl. californica Bdv. form iduna $E d w$.

122 chryxus Dbldy. \& Hew.

a calais Scud.

$b$ ivallda Mead.

123 jutta $\mathrm{Hbn}$. balder Bdv.

$a$ alaskensis $\boldsymbol{H o l l}$.

124 uhleri Reak.

$a$ varuna $E d w$.

125 nahanni Dyar

126 alberta Elwes

a oslari Skin.

127 norna Thun.

a caryi Dyar.

128 katahdin Newc.

129 taygete $\mathrm{Hbn}$. bootes Bdv.

130 dauria Stkr.

131 semidea Say form nigra $E d w$.

132 peartix $E d w$.

133 subhyalina Curt. crambis Frey also Moesch.

- Doubifully North American. 
134 oeno Bdv.

a assimilis Butl.

135 brucei Edu*

136 beani Elwes.

Erebia Dal.

137 discoidalis Kirby

138 fasciata Butl.

139 rossi Curt.

140 disa Thun.

a mancinus $\mathrm{Dbl}$. Hew.

141 vidleri Elwes.

142 epipsodea Butl. rhodia Edw. a brucei Elwes. sineocellata Skin.

143 youngi Holl.

144 magdalena Stkr.

145 tyndarus Esp. a callias Edw.

146 sofia Stkr. ethela Edw. a alaskensis $\mathrm{Holl}$.

Gyrocheilus Butl.

147 patrobas Hew. a tritonia $E d w$.

\section{NYMPHALIDFE}

Heliconiina

Heliconius Latr.

148 charithonia $L$.

Colænis IIhn.

149 julia $H b n$.

aleionea Cram.

a delia Fabr.

Dione IIbn.

150 vanills $L$. pessifloras Fabr. $a$ insularis Mayn.

Nymphalinze

Euptoieta Dbldy.

151 claudia Cran. columbine Godt. dnunus Hbst.

152 hegesia Cram.

Argynnis Fabr.

153 idalia Dru.

ab. astarte Fish.

ab. ashtaroth Fish. infumata Oberth.
154 diana Cram.

155 nokomis Edw.

156 nitocris $E d w$. nigrocaerulea Ckll. a crerulescens Holl.

157 leto Behr. $a b$. letis Wright. a charlotti Barnes.

158 cybele Fabr. daphnis Cram. ab. baal Stkr. ab. bartschi Reiff. a carpenteri $E d w$.

159 aphrodite Fabr. daphnis Martyn. $a$ alcestis $E d u$. $a b$. suffusa Wole.

$b$ cypris Edu.

c columbia $\boldsymbol{H} y$. Edu.

160 lais $E d w$.

161 nausicaa $E d w$.

162 atlantis Edw.

163 hesperis $E d v$.

164 electa Edu. cornelia Edw.

165 chitone Edu'. 
166 bremneri Edw.

a hippolyta $E d w$.

167 behrensi $E d w$.

168 zerene $B d v$.

monticola Behr.

169 hydaspe $B d v$.

a purpurascens $\boldsymbol{H} y$. Edw.

$b$ xhodope $E d w$.

ab. sordida Wright.

c sakuntala Skin.

170 irene $B d v$.

171 nevadensis $E d w$.

a meadi $E d w$.

172 edwardsi Reak.

173 platina Skin.

174 halcyone $E d w$.

175 snyderi Skin.

176 semiramis $E d w$.

a atossa $E d w$.

177 adiaste $B e h r$. adianthe $\mathrm{Bdv}$.

178 callippe $B d v$.

179 coronis Behr.

liliana Hy. Edw.

$a b$. baroni $E d w$.

180 rupestris Behr.

181 juba $B d v$.

laura Edw.

$\boldsymbol{a}$ inornata $E d w$.

182 macaria $E d w$.

a laurina Wright.

183 montivaga Behr. egleis Bdv.

a oweni $E d w$.

184 mormonia $B d v$.

arge Stkr.

a erinna $E d w$.

$a b$. cunninghami Owen.

185 bischoffi $E d w$.

$a$ opis $E d w$.

$b$ washingtonia $B, \& M C D$.
186 euxynome $E d w$.

$a$ clio $E d w$. artonis Edw.

$b$ luski $B$. \& $M c D$.

Brenthis Hbn.

187 myrina Cram. myrissa Godt.

188 euphrosyne $L$. $a$ andersoni Dyar.

189 aphirape $\mathrm{Hbn}$.

$a$ triclaris $H b n$.

$b$ alticola $B$. \& $M c D$.

$c$ dawsoni $B$. \& $M C D$.

190 kriemhild Stkr. laurenti Skin.

191 helena $E d w$.

192 montinus Scud.

193 chariclea Schneid.

a boisduvali Dup.

$b$ rainieri $B$. \& $M c D$.

c grandis $B$. \& $M c D$.

$d$ arctica $Z$ ett.

$e$ obscurata McLach.

$f$ butleri $E d w$.

194 pales $D$. \& $S$.

a alaskensis $\mathrm{Holl}$.

195 freija Thun.

a tarquinius Curt.

196 polaris $B d v$.

$a$ americana Strand.

197 frigga Thun.

a saga Stgr.

b improba Butl.

c alaskensis Lehm.

198 youngi $\mathrm{Holl}$.

199 bellona Fabr. myrina Martyn.

200 epithore $B d v$.

201 alberta $E d w$.

202 astarte Dbldy \& Hew. victoria Edw. 
Euphydryas Seud.

208 phaeton Dru.

pheetana $\mathbf{H b n}$.

phactoneta Godt.

phaedon H. S.

ab. superba Stkr.

ab. phaethusa Hlat.

ab. streckeri Ells.

204 chalcedona Dbldy. \& Hew.

ab. fusimacula Barres.

grundeli Cool.

lorquini Oberth.

ab. mariana Barnes.

$a$ dwinelli $\boldsymbol{H} y$. Edw. macglashani Rivers.

b olanchs Wright.

205 colon $E d w$.

206 cooperi Behr.

207 perdiceas $E d w$.

208 baroni $E d u$.

209 quino Behr.

ab. mirabilis Wright.

angusta Edw.

ab. augustina Wright.

210 rabicunda $\boldsymbol{H} y$. Edw.

211 nubigena Behr.

a colonia Wright.

$b$ beani Skin.

212 editha $B d v$.

213 taylori $\boldsymbol{E} d w$.

214 helvia Scud.

215 sierra Wright.

216 wheeleri $H_{y}$. Edw.

217 hermosa Wright.

218 maria Skinner.

219 anicia Dbldy. \& Hev.

brueei Edw.

a capella Barnes.

220 gilletti Barnes.

Melitsea Fabr.

221 sterope Edw.

222 neumoegeni Skin.
223 gabbi Behr.

sonorne Bdv.

a abina Wright.

224 acastus $E d w$.

225 damoetas Skin.

226 palla $B d v$.

\& eremita Wright.

ab. wardi Oberth.

a whitneyi Behr.

227 hoffmanni Behr. helcita Bdv. ab. abnorma Wright.

228 harrisi Scud.

229 callins $B d v$. ulriea Edw. imitata Stkr.

230 perse $E d w$.

231 dymas Edw. larunda Stkr.

o senrabii Barnes.

232 chara $E d w$.

233 leanira $B d v$. form leona Wright.

form obsoleta $\boldsymbol{H}$. Edv.

234 wrighti Edw.

235 cyneas $G$. \& S.

236 alma Stkr.

cerrita Wright.

237 fulvia $E d w$.

$a b$. sinefascis $W i l l$.

238 theona Men.

a thekla Edu.

$b$ bolli $E d w$.

239 definits Aaron.

240 pols $B d v$. ab. albiplaga Aaron.

a arachne $E d w$. minuta $\ddagger$ Auct.

241 minuta $E d w$.

approximata Stlar. arachnet Auct.

a nympha Edw. 
Phyciodes Dbldy.

212 gorgone $\mathrm{Hbn}$.

ismeria Bdv. \& Lec.

carlota Reak.

$a b$. nigra Cary.

243 nycteis $\mathrm{Dbl}$. \& Hew.

oenone Scud.

$a$ drusius $E d w$.

244 hanhami Fletch:

245 vesta $E d w$.

hiemalis Edw.

form aestiva $E d w$.

246 phaon $E d w$.

q gorgone Hbn.

form hiemalis $E d w$.

247 tharos Dru.

cocyta Cram.

morpheus Fabr.

form vern. marcia $E d w$.

$a b$. packardi Saund.

$a b$. reaghi Reiff.

a pascoensis Wright.

248 batesi Reak.

249 campestris Behr.

pratensis Behr.

pulchella Bdv.

250 camillus $E d w$. emissa Edw.

251 montana Behr.

orsa Bdv.

pratensis Scud.

252 orseis $E d w$.

253 picta $E d w$.

canace Edw.

ab. jemezensis Brehme.

254 mylitta Edw.

colline Behr.

epula Bdv.

a pallida Edw.

matn Reak.

255 barnesi Skin.
Microtia Bates.

256 elva Bates.

Anthanassa Seud.

257 frisia Poey. gyges Hew.

258 texana $E d w$. smerdis Hew. a seminole Skin.

259 tulcis Bates. punctata Edw. archesilea Feld.

*260 cincta Edw.

Chlosyne Butl.

261 erodyle Bates.

262 janais Dru.

263 lacinia Geyer.

$a$ adjutrix Scud.

$b$ crocale $\boldsymbol{E} d w$. rufescens Wright. nigrescens Wright.

264 californica Wright.

265 endeis $G$. \& $S$.

Mestra Hbn.

266 amymone Men. a floridana Stkr.

Hypolimnas $\mathrm{Hbn}$.

267 misippus $L$.

Polygonia Hbn.

268 interrogationis $F a b r$.

fabricii Edw.

form umbrosa Lint.

269 comma Harris.

harrisi Edw.

form dryas $E d w$.

270 satyrus $E d w$.

form chrysoptera Wright. $a$ marsyas $E d w$.

- ronatufully North Ameriean. 
271 faunus Edw.

a rusticus $E d w$. 8 silviu Edw.

272 hylas $E$ du.

273 zephyrus $k d w$.

thiodamas Scud.

274 silvius Edw.

275 gracilis G. \& $R$.

c-argenteum Scud.

276 oreas $E d w$.

a silenus $E d w$.

277 progne Cram.

c. argenteum Kirby.

form l-argenteum Scud.

\section{Aglais Dal.}

278 j-album Bdv. \& Le Con. aureomarginate Ckll. 279 californica $B d v$. 280 milberti Godt. fureillata Say. form subpallida Ckll. 281 antiopa $L$.

ab. grandis Ehrm.

ab. obscura Ckll.

ab. hippolits Lym.

$a b$. hygiaea Heyd.

lintneri Fitch.

Vanessa Fubr.

282 atlanta $L$.

admiralis Retz.

283 virginiensis $D$ ru.

huntera Fabr.

iole Cram.

hunteri $\mathrm{Hbn}$.

$a b$. fulvia Dodge.

284 cardui $L$.

ab. minor Ckll.

$a b$. elymi $R a m b$.

inb. ate Stkr.

285 carye $\mathrm{Hbn}$. charie Blanch.

ab. muelleri Leteh.

Junonia IIbn.

286 cenia Hbn。

orythia A. S.

form nigrosuffusa B. \&

ICD.

negra $\ddagger$ Auct.

287 genoveva Cram.

Anartia IIbn.

288 jatrophe $L$.

289 fatima Fabr.

Victorina Blanch.

290 steneles $L$. lavinia Fabr.

Hypanartia Hbn.

291 lethe Fabr. demonica $\mathrm{Hbn}$.

Eunica Hbn.

292 monima Cram. myrta Godt. modesta Bates.

293 tatila H. S. cnerulea G. \& S.

Myscelia Dbldy.

294 ethusa $B d v$.

295 skinneri Mengel.

Dynamine Hbn.

296 dyonis $\mathrm{Hbn}$.

Callicore Hibn.

297 elymena Cram.

Ageronis Hbn.

298 fornax $H b n$.

299 feronia $L$. 
Timetes Bdv.

300 coresia Godt. zerynthia $\mathrm{Hbn}$. sylla Perty.

301 chiron Fabr. marius Cram. chironias Hbn.

Athena Hbn.

302 peleus Sulz.

petreus Cram. tethys Fabr.

303 eleuchea $\mathrm{Hbn}$. pellenis Godt.

\section{Basilarchia Scud.}

304 astyanax Fabr.

ephestion Stoll. ursula Godt.

$a b$. cerulea $E h r$.

form albofasciata Newc. $\boldsymbol{a}$ arizonensis $\boldsymbol{E} d w$.

305 arthemis Dru.

lamina Fabr.

form proserpina $E d w$. hyb. arthechippus Scud. a rubrofasciata $B . \& M c D$. 306 weidemeyeri $\boldsymbol{E} d w$.

$a$ angustifascia $B . \& M c D$. $a b$. sinefascia $E d w$.

307 lorquini $B d v$. $a b$. eavesi $H y . E d w$.

a burrisoni Mayn.

.308 archippus Cram. disippe Godt.

$a b$. psuedodorippus Stkr. $a b$. lanthanis Cook \& Wat.

a floridensis Stkr. eros Edw. ab. nig Stkr.
309 obsoleta Edw. hulsti Edw.

Heterochroa Bdv.

310 bredowi $\mathrm{Hbn}$. eulatia Dbldy. \& Hew. $a$ californica Butl.

Chlorippe Bdv.

311 celtis $B d v$. \& Lec. $a b$. inornata Wolc.

$a$ alicia $E d w$.

$b$ antonia $E d w$.

c montis $E d w$.

312 leilia $E d w$. cocles Lint.

313 clyton $B d v$. \& Lec. ocellata Edw. form proserpina Scud. $a$ flora $\mathbf{E} d w$. $b$ texana Skin. $c$ subpallida $B, \& M c D$.

Smyrna Hbn.

"314 karwinski $\mathrm{Hbn}$.

Historis Hbn.

*315 odius Fabr. orion Fabr. danae Cram.

*316 acheronta Fabr. eodamus Cram. pherecydes Cram.

Anæa Hbn.

317 andria Scud. ops Druce.

318 portia Fabr.

319 morrisoni $\boldsymbol{E} d w$.

- Doubtfully North American. 


\section{LIBYTHEID $\approx$}

Libythea 11 bn.

320 bachmani $K$ irt.
321 carinentn Cram. ab. \& larvata Stkr.

\section{RHIODINIDE}

Apodemia Feld.

322 mormo Feld. dumeti Behr. mormonia Bdv.

a virgulti Behr. sonorensis Feld. cythera Edw.

8 duryi $E d w$. 8 mejicanus Behr.

323 palmeri $E d w$. a hepburni $G$. \& $S$. 324 nais $E d w$.

Emesis Fabr.

325 zela Butl.

a ares $E d w$.

form cleis $E d w$.
Lasaia Bates.

326 narses Staud.

Caria Hbn.

327 domitianus Fabr. $a$ ino $G$. \& $S$. melicerta Schans.

Calephelis G. \& R.

328 virginiensis Gray. pumila Bdv. \& Lec. caenius $\ddagger$ Auet.

329 borealis $G$. \& $R$.

330 nemesis $E d w$. australis Edw. guadeloupe Stkr.

\section{LYCANIDE}

Theclinx

Eumæus IIbn.

> 331 atala Poey. toxea Gray. 332 minyas $\mathrm{Hbn}$. godarti Bdv. toxana Bdv.

Habrodais Seud. > 333 grunus $B d v$.

\section{Atlides Hbn.}

- 334 halesus Hbn. dolichus $\mathrm{Hbn}$. juanita Seud.
Hypaurotis Send.

> 335 crysalus $E d w$. $a$ citima $H y . E d w$.

Strymon Hbn.

336 hugon Godt. hrgo Dbldy. \& Hew.

337 jada Hew.

338 telea Hew.

339 critola Hew.

340 martialis $\boldsymbol{H}$. S.

341 acis' Dru.

mars Fabr.

342 simæthis Dru. sarita Skin. lyeus $\mathrm{Hbn}$. 
343 pastor Butl. \& Dru.

ᄀ 344 cecrops Fabr. poeas $\mathrm{Hbn}$.

345 columella Fabr. eurytulus $\mathrm{Hbn}$. modesta Mayn. ocellifera Grt. istapa Reak. salona Hew.

346 laceyi $B$. \& $M c D$.

347 clytie $E d w$.

348 azia Hew.

¿49 leda $E d w$.

$a$ ines $E d w$.

¿50 avalona Wright.

$351 \mathrm{~m}$. album $B d v$. \& Lec. psyche Bdv. \& Lec.

- 352 melinus $\boldsymbol{H b n}$. hyperici Bdv. \& Lec. favonius Bdv. \& Lec. humuli Harr. pan Harr. a pudica $\boldsymbol{H} y$. Edw.

353 favonius $A$. \& $S$.

354 autolycus Edw. $a$ ilavia Beut. mirabelle Barnes.

955 ontario Edw.

356 alcestis $E d w$.

357 osiari Dyar.

358 titus Fabr. mopsus $\mathrm{Hbn}$.

a immaculosus Comst.

$3 \pi 9$ acadica $E d w$. souhegan Whit.

360 californica $\boldsymbol{E} d w$. borus Bdv. cygnus Edw.

361 dryope $E d w$.

.362 sylvinus $\boldsymbol{B} d v$.

$a$ itys $E d w$.

b putnami Hy. Edw.

-36.) edwardsi Saund. fabricii Kirby.
364 wittfeldi $E d w$.

- 365 calanus $H b n$. falacer Godt. inorata Grt. lorata G. \& R.

366 heathi Fletch.

ᄀ 367 liparops $B d v$. \& Lec. a strigosa Harris.

, 368 auretorum $B d v$. tacita Hy. Edw. tetra Behr.

a spadix $H y$. Edw.

> 369 adenostomatis $H y$. Edw.

, 370 sæpium $B d v$.

a fulvescens $\boldsymbol{H} y . E d w$.

371 chalcis Behr.

Mitoura Seud.

- 372 spinetorum $B d v$. ninus Edw.

373 johnsoni Skin.

374 nelsoni $B d v$. $a$ exoleta $H y . E d w$. b muiri $H y$. Edw.

$>375$ siva $E d w$.

> 376 xami Reak. blenina Hew.

377 damon Cram. gryneus $\mathrm{Hbn}$. damastus Godt. smilacis Bdv. \& Lec. auburniana Harris form patersonia Brehme.

$a$ castalis $E d w$. discoidalis Skin.

378 loki Skin.

Incisalia Scud.

> 379 augustus Kirby. augustinus West.

" 380 iroides $B d v$. $a b$. immaculata Cock.

$>381$ irus Godt. arsace Bdv. \& Lec. 
382 hadros cook of Wut.

> 383 henrici $\boldsymbol{G}$. \& $\boldsymbol{R}$. a solatus $C$. C $W$.

384 mossi Hy. Edw.

385 polios $C$. \& $W$.

386 fotis Stkr.

387 eryphon Bdr.

388 niphon $H b n$.

Callophrys [Billh.

- 389 dumetorum Bill. viridis $\mathrm{Edw}$.

390 affinis Edw.

? 391 sheridani Edw.

> 392 apama $\boldsymbol{E} d w$.

Erora Seud.

393 leta Edw.

$\%$ elothilde Edw.

Callipsyche s'end.

> 394 behri Edu. kali Stkr.

Satyrium Seud.

395 fuliginosa Erlu'. suasa Bdv.

Feniseca Grt.

396 tarquinius Fabr. crataegi Bdv. \& Lec. poraenna Scud.

Chrysophaninze

\section{Tharsalea Scud.}

- 397 arota Bdu.

398 virginiensis Erlu.

399 hermes Edue,

del-sud Wright.

Heodes Dalm.

400 gorgon Bdv.

401 xanthoides Brlv.

? 402 dione Scud.
403 editha Mead.

404 thoe Bdv.

40.) mariposa Reuk. seroe Bdv.

406 nivalis Balv. ianthe Edw.

- 407 helloides Bdy. castro Reak.

a florus Edw.

408 dorcas Kirhy.

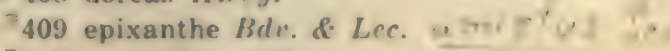

$>410$ hypophlseas $B d v$.

phlaeas Bdv. \& Iec. americana D'U'rban.

ab. fulliolus Hlst.

ab. fasciata Stkr.

ab. obsoleta Send.

caeca Reiff.

$a$ arethusa Dod.

$b$ feildeni McLach.

$>411$ cupreus Edu.

.412 snowi Edu. + 6. :-

7413 rubidus Behr.

, a girius $E d w$.

$>414$ heterones $B d i$. a clara $H y$. Edu.

Lycrenina

Leptotes Sirurl.

$>\mathbf{4 1 5}$ theonus Lre.

a floridensis Morr. striata Edw.

.416 marina Reak.

Brephidium S.'ull.

-417 exilis $B d \%$. fea Edw.

418 isophthalma H.-S. pauedofea Morr.

Hemiargus Hbn.

419 catalina Fabr. ammont Auct. 
3

420 hanno Stoll.

antibubastus $\mathrm{Hbn}$.

pseudoptiletes Bdv. \& Lec. filenus Poey.

421 gyas $E d w$.

astragalus Wright. florencia Clem.

a zachæina Butl. \& Dru.

422 cyna $E d w$. mela Stkr.

$>423$ isola Reate. \& zachaeina Butl. \& Dru. alce Edw.

Everes Hbn.

424 comyntas Godt. sissona Wright. $a$ herri Grinnell. 425 amyntula $B d v$.

Plebeius Linn.

Polyommatus Fabr.

$>426$ scudderi $E d w$.

> $a$ aster $E d w$.

$b$ kodiak $E d w$.

, c annetta Mcad.

-427 melissa $E d w$.

a lotis Lint.

428 anna $E d w$.

cajona Reak.

argyrotoxus Behr. philemon Bdv.

$>429$ aquilo $B d v$. franklini Curt. $a$ rustica $E d u$.

$b$ podarce Feld. tehama Reak. cilla Behr. nestos Bdv.

$>430$ sæpiolus $B d v$. form rufescens $B d v$. $a$ æhaja Behr. hilda Grinnell.

431 amica $E d w$.

- 7432 yukona Holl. $\supset 433$ icarioides $B d v$.

ㅇ maricopa Reak. phileros $B d v$. fulla Edw. ? mintha Edw. $a b$. dædalus Behr.

$a$ helios $E d w$.

$b$ evius $B d v$.

$c$ ardea $E d w$.

$d$ lycea $E d w$. rapahoe Reak. e pembina $E d w$.

434 pheres $B d v$.

$a b$ orcus $E d w$.

¿435 shasta Edw. zelmira Feld. calchas Behr. nivium Bdv. $a$ minnehaha Scud.

¡436 acmon Dbldy. \& Hew. antaegon Bdv. a cottlei Grin.

$>437$ lupini $B d v$.

$>438$ monticola Clem.

439 chlorina Skin.

- 440 emigdionis Grin. melimona Wright.

441 neurona Skin.

Philotes Scud.

(1) 442 battoides Behr.

$a$ bérnardino $B . \& M c D$.

443 glaucon $E d w$.

- 444 enoptes $B d v$. 445 rita $B$. \& $M c D$.

$>446$ speciosa $H y . E d w$.

, 447 sonorensis Feld. regia Bdv.

Phædrotes Scud.

>448 piasus $B d v$. sagittigera Feld. viaca Edw.

a catalina Reak. rhaea Bdv.

$b$ daunia $E d w$. 
Glaucopsyche Scud..

$\$ 49$ lygdamus Dbldy.

$>$ a couperi Grt.

\& afre Edw.

$b$ oro Seud.

- behri Edw.

-450 xerces $B d v$.

form antiacis $B d v$.

form mertila $E d u$.

form polyphemus $B d v$.

Lycrna L.

4.)1 pardalis Behr. erymus Bdv.
Lycrnopsis Feld.

452 pseudargiolus $B d v$. L Lee. violacea Edw.

form \& nigra $E d u$. form \& intermedia Stkr. form Jucia Kirby. form marginata $E d w$.

$>$ gen. aeat. neglects Edw. form argentata Fletch.

b gen aest. neglecta-major

e nigrescens Fleteh. form quesnelli Cockle.

$d$ cinerea $E d u$. gen. aest. arizonensis Edw.

e echo Edw.

$f$ gozora $B d v$.

\section{HESPERIID}

Pyrrhopygina

Pyrrhopyge IIton.

453 araxes Hew.

$a$ arizonæ $G . \& S$.

\section{Hesperiinae}

Phocides IIbn.

$\$ 54$ lilea Reak. ulbicilla H. S. socius Butl. \& Dru. decolor Mab. denuba Ploetz. 455 urania Weat. \& Hew. texana Scud.

456 batabano Lef. mancinus H. S. okeechobee Worth.

Acolastus Seud.

457 amyntas Fubr. lividus $\mathrm{Hbn}$. savignyi Latr. a arizonensis Skin.
Goniurus Hbn.

458 proteus $I$.

459 dorantes Stoll. amisus Hew. protillus H. S. a rauterbergi Skin. 460 simplicius Stoll.

*461 eurycles Latr.

462 alcæus Hew.

463 zilpa Butl.

464 albofasciatus Hew.

Plestia Mab.

465 dorus $E d w$.

Proteides Illon.

466 idas Cram. mercurius Fabr.

Epargyreus Hbn. 467 zestos Hbn. oberon Worth.

468 tityrus $F a b r$. clarus Cram. 
469 exadeus Cram. socus $\mathrm{Hbn}$.

Nascus Wats.

470 hesus West. \& Hew.

Hetoropia Mab.

471 melon G. \& S. $a$ arizonensis Skin.

Achalarus Scud.

$\triangle 72$ lycidas $A . \& S$. lycides $\mathrm{Hbn}$.

Rhabdoides Scud.

473 cellus $B d v$. \& Lec. festus $\mathrm{Hbn}$.

474 pseudocellus Cool. \& Clem.

Murgaria Wats.

475 albociliata $M a b$. coyote Skin.

Cogia Butl.

476 calchas $H$. S. terranea Butl.

477 outis Shin.

478 hippalus $E d w$. gila Ploetz.

Phaedinus G. \& S.

479 mysie Dyar.

480 caicus $H$. S. schaefferi Ploetz. noschus Edw.

481 epigona $H . S$. eprigena Butl. orestes Lint.

Cocceius G. \& S.

482 drusius $E d w$. 483 pylades Scud. $a b$. immaculata Skin.
Thorybes Seud.

484 mexicanus $H$. S. ananius Ploetz.

a nevada Scud. aemilea Skin.

485 daunus Cram. bathyllü A. \& S.

Telegonus Hbn. 486 hahneli Staud.

Cabares G. \& S. 487 potrillo Lef.

Hesperia Fabr.

488 centaureæ Ramb. wyandot Edw.

489 ruralis $B d v$. caespitalis Bdv. ricara $\mathrm{Edw}$. petreius Edw.

490 xanthus $E d w$. 491 macdunnoughi Oberth 492 seriptura $B d v$.

Pyrgus Hbn.

493 syrichtus Fabr. montivagus Reak.

494 philetas Edw.

495 tessellata Scud. communis Grt. $a$ occidentalis Skin.

Celotes G. \& S. 496 nessus $E d w$. notabilis Stkr.

Chiomara G. \& S. 497 asychis Cram. 498 gesta $H . S$. llano Dodge. 
Heliopetes Billb.

499 domicella Erich. nearchus Edw.

500 macaira Reak. oceanus Edw. locutia Hew.

501 nivella Mab. nivea Scud.

502 laviana Hew. pastor Feld. leca Butl.

503 ericetorum Bdv. alba Edw.

Systasea Butl.

504 pulverulenta Feld. zampa Edw.

Eantis Bdv.

505 thraso $\mathrm{Hbn}$. tamenund Edw.

Xonophanes G. \& S.

506 tryxus Cram.

Grais G. \& S.

507 stigmaticus Mab.

Timochares G. \& S. 508 ruptifasciatus Ploetz.

\section{Hesperopsis Dyar.}

509 alpheus $E d w$. oricus Edw.

510 libya Send. 511 lena $E d w$.

\section{Pholisora Send.}

512 catullus Fabr. a mejicanus Reak.

Staphylus G. \& S. 513 hayhursti Edw.
Bolla Mab.

514 ceos Edw。

515 brennus G. \& S.

Melanthus Mab.

516 brunnea $H$. S.

Thanaos Bdv.

517 icelus Lint.

518 brizo Bdv. \& Lec.

a somnus Lint.

$b$ lacustra Wright.

519 burgessi Skin.

520 lucilius Lint.

521 persius Scud.

$a$ afranius Lint.

b pernigra Grin.

522 lilius Dyar.

523 callidus Grin.

524 martialis Scud.

$a b$. susonius Lint.

525 juvenalis $\mathrm{Fabr}$. juvenis Hbn. costalis West \& Hew. ennius Scud. \& Burg.

526 propertius Seud. \& Burg. ? tibullus Scud. \& Burg.

a borealis Cary.

527 horatius Seud. \& Burg. virgilius Scud. \& Burg. petronins Lint.

528 plautus Scud. \& Burg.

529 terentius Seud. \& Burg. ovidine Scud. \& Burg. naevius Lint.

530 pacuvius Lint.

531 scudderi Skin.

532 tatius Edw.

533 clitus $E d w$. maestus G. \& S.

534 tristis Bdv.

535 funeralis Seud. \& Burg. 


\section{Pamphilina}

Carterocephalus Led.

536 palæmon Pall. mandan Edw. mesapano Scud. skada Edw.

Dalla Mab.

537 pirus $E d w$.

538 microsticta $G . \&$ S.

539 polingi Barnes.

Adopæa Billb.

540 eunus $E d w$.

541 wrighti $E d w$.

Copæodes Speyer.

542 aurantiaca Hew.

waco $\mathrm{Edw}$.

minima Edw.

procris Edw.

candida Wright.

543 rayata $B . \& M c D$.

Ancyloxypha Feld.

544 numitor $\mathrm{Fabr}$ : puer $\mathbf{H b n}$. marginatus Harris.

545 longleyi Frch.

546 arene $E d w$. myrtis Edw.

Oarsima Scud.

547 garita Reak. hylax Edw.

548 edwardsi Barnes.

549 powescheik Parker.

Chærephon G. \& S.

550 rhesus $\boldsymbol{E} d w$.

551 carus $\boldsymbol{E} d w$.

552 simius $E d w$.
Pamphila Fabr.

553 uncas $E d w$.

ㅇ axius Ploetz.

554 lasus $E d w$.

555 licinus $E d w$.

556 metea Scud. ridingsi Reak.

557 manitoboides Fletch.

558 morrisoni $E d w$. morissoni Ploetz.

559 columbia Scud. california Wright. erynnioides Dyar.

560 cabelus $E d w$.

561 harpalus $E d w$. 562 comma $L$.

a nevada Scud. form colorado Scud.

$b$ manitoba Scud. laurentina Lym.

$c$ idaho $\boldsymbol{E} d w$.

$d$ assiniboia Lym. $e$ oregonia $E d w$.

563 juba Scud.

$a$ viridis $E d w$.

$b$ woodgatei Will.

564. ruricola $B d v$.

565 attalus $E d w$.

$a$ seminole Scud. slossonae Skin.

566 leonardus Harris.

567 meskei $E d w$. straton Edw.

568 ottoe $E d w$.

569 pawnee Dodge.

a montana Skin.

570 sassacus Harris.

$a$ dacotæ Skin.

Hylephila Billb.

571 phylæus $D r u$. augias $\mathrm{Hbn}$. bucephalus Steph. 
Ochlodes Seud.

572 sylvanoides $B d v$. agricolat Auct. frenciara Ploetz.

a napa Edw.

573 nemorum Bdv. verua Edw.

a pratincola $B d v$.

574 agricola $B d v$. yreka Edw.

575 milo Edu.

\section{Polites Scud.}

576 cernes Bdv. \& Lec.

? taumas Fabr. ahaton Harris.

577 manataaqua Seud. cernes Harris.

578 rhena $E d w$. aleina Skin.

579 baracos Luc. amadis $\mathrm{H}$. S. myus French.

580 sonora Seud. sylvanoides: Auct. siris Edw.

a utahensis Skin.

581 mystic Seud.

a dacotah $E d w$. pallida Skin.

582 mardon $E d w$.

583 peckius Kirby. ? coras Cram. wamsutta Harris.

584 sabuleti $B d v$. genon Ploetz.

$a$ tecumseh Grin. chispa Wright.

585 chuska Edu.

586 draco Edu.

587 brettus Bdv. wingina Scud. unna Ploetz. a brettoides Edw.
588 stigma Skin.

Atalopedes Scud.

589 campestris $B d v$. huron Edw.

Catia G. \& S.

590 otho A. \& S. drury Latx. pustula Geyer. aetwa Scud. ursa Worth. form egeremet Scud. cinna Ploetz.

Poanes Send.

591 massasoit Scud. form suffusa Laur.

592 hobomok Harris. form \& pocohontes Scud. quadraquina Scud.

593 zabulon Bdv. \& Lee.

594 taxiles $E d w$.

595 melane $\boldsymbol{E} d w$.

Atrytone Scud.

596 byssus $E d w$.

? bulenta Bdv. \& Lec.

597 logan Edw.

o delaware Edw.

598 lagus $E d w$.

599 arogos $B d v$. \& Lee.

vitellius A. \& $\mathrm{S}$. iowa Scud. mutius Ploetz.

Phycanassa Seud. 600 viator Edw.

Paratrytone G. \& S.

601 howardi Skin. 602 aaroni Skin. 603 yehl Skin. 
Euphyes Scud.

604 vestris $B d v$.

metacomet Harris. rurea Edw.

kiowah Reak.

osyka Edw.

osceola Lint.

$a b$. ㅇ immaculatu

605 bimacula $G . \& R$.

acanootus Scud.

illinois Dodge.

606 arpa $B d v . \&$ Lec.

607 palatka $E d w$.

floridensis Ploetz.

608 streckeri Skin.

609 dion $E d w$.

610 conspicua $E d w$.

ô pontiac Edw. orono Scud.

Atrytonopsis G. \& S.

611 yuma $E d w$.

scudderi Skin.

612 snowi $E d w$.

613 verna $E d w$. vetulina Ploetz. pottawattomie Worth.

614 loammi Whitney. regulus Edw.

615 hianna Scud. grotei Ploetz.

616 lunus $E d w$.

617 deva Edw.

618 vierecki Skin.

619 pittacus $E d w$.

620 python $E d w$.

a margarita Skin.

621 cestus $E d w$.

622 edwardsi $B$. \& $M c D$.

Thespieus G. \& $\mathbf{S}$.

623 macareus $H . S$.
Amblycirtes Scud.

624 nanno $E d w$. 625 elissa $G$. \& S.

626 vialis $E d w$.

627 meridionalis Dyar.

628 nysa $E d w$. similis Stkr.

629 ænus $E d w$.

630 cassus $E d w$.

631 eos $E d w$.

632 celia Skin.

633 comus $E d w$. nilus Edw. quinquemacula Skin.

634 textor $\mathrm{Hbn}$. oneko Scud. wakulla Edw.

635 hegon Scud. samoset Scud. nemoris Edw. alternata G. \& R. 636 nereus $E d w$.

Mastor G. \& S.

637 oslari Skin.

638 bellus $E d w$.

639 phylace $E d w$.

Epiphyes Dyar. 640 carolina Skin.

Megistias G. \& S. 641 fusca $G$. \& $R$.

Lerema Scud.

642 accius $A . \& S$. monoco Scud. nortoni Edw. punctella G. \& R.

643 horus $E d w$.

Lerodea Scud.

644 arabus $E d w$. 645 eufala $E d w$.

floridae Mab. 
646 maculata Edw. norue Ploetz.

Calpodes IIbn.

647 ethlius Cram. chemnis Fabr.

olynthus Bdv. \& Lec.

648 coscinis $H$. S.

? ares Feld.

Prenes Scud.

649 nero $\mathrm{Fabr}$.

650 panoquin Scud. ophis Edw.

651 panoquinoides Skin.

652 errans Skin.
653 ocola Edw.

Megathymina

Megathymux Seud.

654 yucea Bdv. \& Lee. a coloradensis Riley.

b navajo Skin.

655 ursus Poling.

656 cofaqui Stkr.

657 streckeri Skin.

$a$ texana $B . \& M c D$.

658 smithi Druce.

659 neumoegeni $E d w$. a stephensi Skin.

660 aryxna Dyar.

661 polingi Skin.

\section{Superfamily SPHINGOIDEA}

\section{SPHINGIDFE}

Herse Oken.

662 cingulata Fabr.

afinis Goeze.

pungens Eschsch.

druraei Don.

ab. decolora $\boldsymbol{H} y$. Edw.

Cocytius IIbn.

663 antreus Dru.

a medor Merian.

Protoparce Burm.

664 sexta Johan.

curolina L.

lyeopersici Bdv.

665 quinquemaculata Haw. celeus $\mathrm{Hbn}$.

666 rustica Fabr. chionanthi A. \&

667 brontes Dru.

a cubensis Grt.

668 muscosa $R$. \& J.
669 florestan Stoll. brevimargo Butl.

Chlænogramma Sm. 670 jasminearum $B d v$. rotundate Roths.

Dolba Wik.

671 hylæus Dru. prini A. \& S.

Dolbogene R. \& J. 672 hartwegi Butl.

Isogramma R. \& J. 673 hageni Grt.

Ceratomia Harris.

674 amyntor $\mathrm{Hbn}$. quadricornie Harr.

675 undulosa Wlk. repentinus Clem. 676 catalpx Bdv. 
Isoparce R. \& J.

677 cupressi $B d v$.

Dictyosoma R. \& J.

678 elsa Stkr.

Atreus Grt.

679 plebeja Fabr.

Sphinx I.

680 lugens $W l k$.

681 eremitus $\mathrm{Hbn}$. sordida Harr.

682 geminus $R$. \& $J$.

683 eremitoides Stkr.

684 separatus Neum.

685 istar $R . \& J$.

686 leucophratus Clem.

687 chersis Hbn. cinerea Harris.

$a$ pallescens $R$. $\& J$.

$b$ oreodaphne $H y$. Edw.

688 vancouverensis $H y$. Edvo. vashti Stkr.

$a b$. albescens Tepper.

689 libocedrus $H y . E d w$.

a insolita Lint.

690 perelegans $H y . E d w$.

form asellus $R$. \& J.

691 canadensis $B d v$. plota Stkr.

692 francki Neum.

693 kalmiæ $A$. \& S.

694 gordius Cram.

a oslari $R . \& J$.

695 luscitiosa Clem. $a b$. \& una Skin.

696 drupiferarum A. \& S. a utahensis $H y . E d w$.

697 dolli Neum.

$a$ coloradus $\mathrm{S} m$.

698 sequoix $B d v$.

699 pinastri $L$.

saniptri Stkr.
Lapara Wlk.

700 coniferarum $A . \& S$. cana Martyn.

701 bombycoides Wlk. harrisi Clem. $a b$. pineum Lint.

Exydrium Grt.

702 halicarniæ Stkr.

Protambulyx R. \& J.

703 strigilis $L$.

704 carteri $R$. \& J

Smerinthus Latr.

705 jamaicensis Dru.

f. norm. geminatus Say. clarkiae Franck.

$a b$. tripartitus Grt.

$a b$. flavitincta Nixon.

706 cerisyi Kirby.

$a$ astarte Strk.

$b$ ophthalmicus $B d v$. vancouverensis Butl. form pallidulus $\boldsymbol{E} d w$.

$c$ saliceti $B d v$ :

\section{Paonias Hbn.}

707 excæcata $A . \& S$. pavonina Geyer.

708 myops $A . \& S$. rosacearum Bdv.

709 astylus Dru. io Gray. integerrima Harris. hyb. interfaunus Neum.

Cressonia G. \& R.

710 juglandis $A . \& S$. pallens Stkr. robinsonii Butl. $a b$. hyperbola Slosson. 
Pachysphinx R. \& J.

711 modesta Hurris. princeps Wik. populicula Bdv. cablei Reiz. occidentalis Hy. Edw.

a imperator Stlor. form kunzei $\boldsymbol{R}$. \& J.

Pseudosphinx Burm.

712 tetrio $L$. hasdrubal Cram. rustica Sepp.

Erinnyis $\mathrm{Hbn}$.

713 alope Dre.

Ravicans Goeze.

fasciata Swain. edwardsi But].

714 lassauxi $B d v$. a merianæ Grt. janiphae Bdv.

715 ello $L$.

716 renotrus Stoll. picta Sepp. melancholica Grt. piperis G. \& R.

717 crameri Schaus. 718 obscura Fabr. stheno $\mathrm{Hbn}$. pallida Grt. cinerosa G. \& R. rhaebus Bdv.

719 domingonis Butl. festa Hy. Edw.

720 guttularis Wlk. pallida Bdv.

Grammodia R. \& J. 721 caicus Cram.

Pachylia Wาk.

722 ficus $L$.

crameri Men. lyncea Clem.
723 resumens Wik. inconspicus Wik. verauta Clem. tristis Men.

Madoryx Bdv.

724 pseudothyreus Grt.

Hemeroplanes Hbn.

725 parce Fabr. licastus Stoll. galianna Burm.

Enyo Hbn.

726 lugubris $L$. fegens Cram. luctuosus Bdv.

727 ocypete $L$. camertus Cram. danum Cram.

Cautethia Grt. 728 grotei $H y . E d w$.

Aellopos IIhn.

729 tantalus $L$. form zonata Dru. form clavipes $R . \& J$. 730 titan Cram. 731 fadus Cram. annulosum Swains. balteata Kirtland.

Hæmorrhagia Grt.

732 thysbe Fabr. pelasgus Cram. ruficaudis Kirby. etolus Bdv.

form cimbiciformis Steph. foridensis G. \& R. uniformis G. \& R. buj̃aloensis G. \& R. pyramus Bdv.

form fuscicaudis Wlk. 733 gracilis $G$. \& $\boldsymbol{R}$. 
734 senta Stkr.

brucei French.

735 diffinis $B d v$.

marginalis Grt.

f. vern. tenuis Grt.

fumosa Stkr. metathetis Butl.

f. aest. axillaris $G . \& R .749$ nessus Crant. grotei Butl.

$a$ æthra Stkr.

$b$ ariadne $B . \& M c D$.

$c$ thetis $B d v$. palpalis Grt. form cynoglossum $\mathrm{H} y$.

$E d w .752$ clarkiæ $B d v$.

$d$ rubens $H y$. Edw.

\section{Pholus Hbn.}

736 anchemolus Cram.

737 satellitia Dru.

$a$ pandorus $\mathrm{Hbn}$.

738 achemon Dru.

739 typhon Klug.

740 vitis $\operatorname{Linn}$.

hornbeckiana Harr.

linnei G. \& R.

741 fasciatus Sulz. jussieuae $\mathrm{Hbn}$.

742 labruscæ $L$. clothro Fabr.

Ampeløca R. \& J.

743 versicolor Harr.

744 myron Cram.

pampinatrix A. \& $\mathbf{S}$.

form cnotus $\mathrm{Hbn}$.

Darapsa Wlk.

745 pholus Cram. choerilus Cram. azalene A. \& S. chlorinda Martyn.

Sphecodina Blanch.

746 abbotti Swains.
Deidamia Clem.

747 inscriptum Harris.

Arctonotus $\mathrm{Bdv}$.

748 lucidus $B d v$.

Amphion $\mathrm{Hbn}$.

Proserpinus Hhn.

750 gauræ $\mathrm{Hbn}$. $a$ circæ $\boldsymbol{H} y, E d w$.

751 juanita $S t k r$. $a$ oslari $R$. \& $J$. victoria Grt.

753 flavofasciata $W l k$.

a ulalume Stkr.

$b$ rachel Bruce.

754 vega $D$ yar.

Euproserpinus G. \& R.

755 phaeton $G$. \& $R$. erato Bdv.

756 euterpe $H y . E d w$.

Xylophanes Hbn.

757 pluto Fabr. croesus Dal. thorates $\mathrm{Hbn}$.

758 porcus $\mathrm{Hbn}$.

759 falco $W l k$. fugax Bdv. mexicana Ersch.

760 tersa $L$.

Celerio Oken.

761 gallii Rott.

$a$ intermedia Kirby. canadensis $\mathrm{Gn}$. chamaenerii Harris. oxybaphi Clem.

762 lineata $F a b r$. daucus Cram. melancholica Grt. piperis G. \& R. 


\section{Superfamily SATURNIOIDEA}

\section{SATURNIIDE}

Philosamia Grt.

763 cynthia Dru.

a walkeri Feld. form advena Paele.

Rothschildia Frt.

764 orizaba Went.

765 jorulla Weat.

splendida $\uparrow$ Clem. cinctus Tepp.

Samia Itbn.

766 cecropia $L$.

767 gloveri Stkr.

a reducta Neum.

768 columbia $S m$.

a nokomis Brodie.

$b$ winonah Brodie.

769 rubra Behr.

euryalus Bdv. califorrica Grt. ceanothi Behr. $a b$. parvimacula Grt.

a kasloensis Ckll.

Eupackardia Ckll.

570 calleta West. polyommata Tepper.

Callosamia Pack.

771 promethea Dru. ab. \& creca Ckll.

772 angulifers $W l k$.

a carolina Jones.

Tropæa IIbn.

773 luna $L$.

form dictynna Walk. form rubromarginata

ab. rossi Ross.

Davis.

ab. rubrosuffusa Ckll.
Telea Hbn.

774 polyphenus Cram. paphia L. fenestra Perry. ab. flava Grt.

a oculea Neum. aurelia Druce.

$b$ olivacea Ckll.

Agapema N. \& D.

775 galbina Clem.

a anona Ottol.

776 homogena Dyar.

Calosaturnia Sm.

777 mendocino Behrens.

Automeris IIbn.

778 pamina Neum.

$a$ aurosea Neum.

boncardi Druce.

779 zephyria Grt.

780 io $\mathrm{Fabr}$.

corollaria Perry. varia WIk.

fabricii Bdv.

$a b$. argus $N . \& D$.

$a$ lilith Stkr.

b fuscus Luther.

lutheri Ckll.

- coloradensis Chll.

Thauma Hy. Edw.

781 socialis Feist.

angulifera Wik.

ribesii Hy. Edw.

Coloradia Blake.

782 pandora Blake.

loiperda Dyar.

a doris Barnes.

lois Dyar. 
Hemileuca Wlk.

783 electra Wright. ab. rickseckeri Wats.

784 maia Dru. proserpina Fabr.

785 lucina $\boldsymbol{H} y$. Edw. $a b$. obsoleta Reiff. a latifascia $B . \& M c D$. 786 nevadensis Stretch. californica Wright. artemis Pack.

787 juno Pack. yavapai Neum.

788 grotei $G$. \& $\boldsymbol{R}$. diana Pack.

789 neumoegeni $H y$. Edw.

790 burnsi Wats.

$a b$. ilmæ Wats.

$a b$. nigrovenosa Wats. $a b$. conjuncta Wats. ab. paradoxa Wats.

791 tricolor Pack.
792 sororius $\mathrm{Hy}$. Edw. a hualapai Neum. 793 olivia Ckll. $a b, \delta$ grisea Ckll. $a b$. ㅇ sufiusa Ckll.

Pseudohazis G. \& R.

794 eglanterina $B d v$. normalis Dyar. form shastaensis Behr. form denudata Neum. boisduvali Oberth. harrisi Oberth.

a nuttalli Stkr. uniformis Ckll. form arizonensis Stkr.

795 hera Harris. pica Wlk.

a marcata Neum.

Hylesia Hbn.

796 alinda Druce.

\section{CERATOCAMPIDE}

Anisota $\mathrm{Hbn}$.

797 stigma $\mathrm{Fabr}$.

798 senatoria $A$. \& S.

799 consularis Dyar.

800 virginiensis $\mathrm{Dru}$.

astymone Oliv. pellucida H. S.

801 oslari Roths. skinneri Bied. neomexicana Brehme.

802 rubicunda Fabr.

$a$ alba $G r t$. pallida Bowles.

Adelocephala H. S.

803 bicolor Harris. distigma Walsh. form suprema Neum. form immaculata Jewett.

806 quadrilineata $G$. \& $R$.
807 bisecta Lint. nebulosa Neum. 808 albolineata $G$. \& $R$. raspa $\mathrm{Bdv}$.

809 heiligbrodti Harv. a hubbardi Dyar.

Citheronia Hbn.

810 regalis $\mathrm{Fabr}$. regia A. \& S. $a$ infernalis Stkr. $a b$. saengeri Neum. 811 sepulchralis $G . \& R$. 812 mexicana $G$. \& $R$.

\section{Basilona Bdv.}

813 imperialis Dru. imperatoria A. \& S. $a b$. punctatissima Neum. a didyma Beauv.

$b$ nobilis Neum.

c oslari Roths. 


\section{Superfamily BOMBYCOIDEA}

\section{SYNTOMIDE}

Cosmosoma Hbn.

814 myrodora Dyar.

815 teuthras Wlk. rubrigutta Skin.

Syntomeida Harris.

816 impomese Harr. feros: Wlk. euterpe H. S.

817 epilais $W I k$.

a jucundissima Dyar.

818 hampsoni Barnes. befana Skin.

Pseudomya Hbn.

819 minima Grt.

Didasys Grt.

820 belæ Grt.

Horama Hbn.

821 texana Grt.

Eucereon IIbn.

822 carolina $\boldsymbol{H}$ y. $E d w$.

Lymire Wlk.

823 edwardsi Grt.

Scepsis Wlk.

824 fulvicollis $H b n$. semidiaphana Harr. a pallens $H y . E d w$.
825 packardi Grt. mathewi Hy. Edw.

a cocklel Dyar.

826 wrighti Stretch. a gravis $H$ y. Edw.

Lycomorpha IIarr.

827 grotei Pack. palmeri Pack.

a pulchra Dyar.

828 regulus Grinnell.

829 fulgens $H y$. Edw. tenuimargo Holl.

830 splendens $\boldsymbol{B}$. \& $\boldsymbol{M} \boldsymbol{c D}$.

831 pholus Dru.

a miniata Pack.

832 desertus $\boldsymbol{H} y$. $\boldsymbol{E} d w$.

Ctenucha Kirby.

833 venosa $W l k$.

834 cressonana Grt. form sanguinaria Stkr. form lutea Grt.

835 brunnea Stretch.

836 multifaria Wlk. form luteoscapus $N$. \& $D$. 837 rubroseapus $M$ en. walsinghami Hy. Edw. form ochroseapus $G$. \& $R$. corvina Bdv.

838 virginica Charp. latreillana Kirby.

Dahana Grt.

839 atripennis Grt.

\section{ARCTIIDF}

Nolin

Celama WIk.

840 aphyla Hamp.
841 pustulata Wlk. nigrofasciata Zell. obaurata Morr.

842 cilicoides Grt. 
843 sorghiella Riley.

844 triquetrana Fitch. trinotata Wlk. sexmaculata Grt.

845 minna Butl. hyemalis Stretch.

846 ovilla Grt.

847 clethræ Dyar.

Nola Leach.

848 apera Druce. involuta Dyar. $a$ exposita $D$ yar.

849 lagunculariæ Dyar. obliquata B. \& McD.

Nigetia Wlk.

850 formosalis Wlk. melanopa Zell.

Rœselia Hbn.

851 minuscula Zell. a phylla Dyar.

852 minor Dyar.

853 dentata Dyar.

854 fuscula $G r t$. conspicua Dyar.

\section{Lithosinx}

Lexis Wallgr.

855 bicolor Grt. argillacea Pack.

Crambidia Peck.

856 pallida Pack.

857 lithosioides Dyar.

858 uniformis Dyar.

859 dusca B. MCD.

860 casta Sanb.

candida Hy. Edw.

861 pura $B$. \& $M c D$.

862 cephalica $G$. \& $R$.

863 suffusa $B$. \& $M c D$.

864 impura $B$. \& $M C D$.

865 allegheniensis $\mathrm{Holl}$.
Agylla Wlk.

866 septentrionalis $B . \& M c D$.

Comacla Wlk.

867 simplex Wlk. murina Wlk. clarus G. \& R. texana Frch.

868 fuscipes Grt.

Neoplynes Grt.

869 eudora Dyar.

Ptychoglene Feld.

870 coccinea $H y . E d w$.

871 phrada Druce.

flammans Dyar.

872 sanguineola $B d v$.

Cisthene Wlk.

873 arida Skin.

Inopsis Feld.

874 modulata $H y$. Edw.

Pygoctenucha Grt.

875 terminalis Wlk. harrisi Bdv. pyrrhoura Hulst. votiva $\mathrm{Hy}$. Edw.

876 funerea Grt.

Afrida Moesch.

Aresia B. \& McD.

877 minuta Druce.

878 ydatodes Dyar. parva B. \& $\mathrm{McD}$.

Ozodania Dyar.

879 schwarziorum Dyar.

880 subrufa $B$. \& $M c D$.

881 tenuifascia Harv.

Illice Wlk.

882 unifascia $G$. \& $R$. 
a kentuckiensis Dyar.

883 perrosen Dyar.

a barnesi Dyar.

b angelus $D$ yar.

884 injecta $D$ yar.

a gamma Dyer.

885 subjecta $W / k$.

packandi Grt.

886 striata Otlol.

887 plumbea Stretch.

888 conjuncta $B$. \& $M C D$.

889 dorsimacula Dyar.

form liberomacula Dyar.

890 nexa $B d v$.

grisen Pack.

deserta Feld.

891 faustinula $B d v$. form fusca Stretch.

Clemensia Pack.

892 albata Puck.

albida Wik.

cana W]k.

irrorata $\mathbf{H y}$. Edw.

form umbrata Pack.

893 lactea Stretch.

Palpidia Dyar.

894 pallidior Duar.

Lerina Wlk.

895 incarnata $B d v$. robinsoni Bdv.

Bruceia Neum.

896 pulverina Neum.

897 hubbardi Dyar.

Hrematomonis Hump.

898 mexicana Druce.

Hypoprepia Hbn.

899 miniata Kirby. vittate Harr.
900 Pucosa $H b n$.

tricolor Fitch.

form plumben $\boldsymbol{H}$. Edw.

a subornata $N$. \& $D$.

form dolli Dyar.

901 cadaverosa Stkr.

902 inculta $H y . E d w$.

\section{Arctiina}

Eupseudosoma Grt.

903 involutum Sepp.

a floridum Grt.

Bertholdia Schaus.

904 trigona Grt.

Ammalo Wik.

905 tenera $H b n$. collaris Fitch. antica Wlk.

a sciurus $B d v$. yosemite $\mathrm{Hy}$. Edw.

906 insulata Wlk. cadaverose Grt. affiris Grt. aurata Butl.

907 inopinatus $H y . E d w$. nivalis Stretch.

Opharus Wik.

908 astur Cram.

$a$ arizonensis Roths.

fumatn B. \& McD.

Hemihyalea Hamp.

909 splendens $\boldsymbol{B}$. \& $M$ M D.

910 argillacea Roths.

911 labecula Grt.

912 edwardsi Pack.

translucida Wlk.

quereus Bdv. 


\section{Halisidota Hbn.}

913 argentata Pack.

a subalpina French.

b sobrina Stretch.

914 ingens $\boldsymbol{H} . \boldsymbol{E} d w$.

scapularis Stretch.

915 caryæ Harris. annulifascia Wlk. porphyrea H. S.

916 mixta Neum. psuedocarye Roths.

917 pura Neum. flavescens Roths.

918 maculata Harr. fulvoflava Wlk. guttifera $\mathrm{H}$. S. $a$ angulifera $W l k$. alni Hy. Edw.

$b$ agassizi Pack. californica Wlk. salicis Bdv.

$c$ texana Roths.

$d$ eureka Dyar.

919 indistincta $B . \& M c D$.

920 cinctipes $H y$. $E d w$. davisi Hy. Edw.

921 tessellaris $A . \& S$. antiphola Walsh.

922 harrisi Walsh.

923 oslari Roths.

924 longa Grt.

925 annulosa Wlk.

926 lurida $H y$. Edw. otho Barnes.

Neritos Wlk.

927 prophæa Schaus.

Aemilia Kirby.

928 ambigua Stkr. bolteri Hy. Edw. syracosia Druce.

929 roseata Wlk. cinnamomea Bdv. sanguivenosa Neum. $a$ significans $H y . E d w$.
930 occidentalis Frch.

Eubaphe Hbn.

931 læta Guer. treati Grt.

a rubropicta Pack.

932 intermedia Graef.

$a$ parvula $N . \& D$.

$b$ cocciniceps Schaus.

933 ostenta $H y$. $E d w$. + calera Barnes.

934 aurantiaca $\mathrm{Hbn}$.

a rubicundaria $\mathrm{Hbn}$. rosa Frch. diminutiva Græf.

$b$ ferruginosa Wlk.

c brevicornis Wlk. belfragei Stretch.

$d$ quinaria Grt. choriona Reak. bimaculata Saund.

935 fragilis Stkr.

936 costata Stretch. oppelloides Graef.

937 opella Grt. obscura Stretch. rubricosta Ehrm.

a nigricans Reak. nigrifera Wlk.

$b$ belmaria $\mathrm{Ehrm}$.

938 immaculata Reak. a trimaculosa Reak.

Dodia Dyar.

939 albertæ Dyar.

Hypocrisias Hamp.

940 minima Neum. armillata Hy. Edw.

Leptarctia Stretch.

941 californiæ Wlk. lena Bdv. adnata Bdv. fulvofasciata Butl. wrighti French. form decia $B d v$. 
borisduvali Butl.

latifasciata Butl.

abifascie Freh.

occidentali Freh.

furm dimidiata siretch. strefchi Butl.

\section{Hyphoraia Hbn.}

\section{2 parthenos Harr. borealis Moesch. \\ 943 lapponice Thun. alpina Quens. thulea Dal. nvia $\mathrm{Hbn}$. hypoborea Curt.}

944 subnebulosa Dyar.

Neoarctia $\mathrm{N}$. \& D.

945 brucei $H y . E d w$.

946 beani Neum. form fuscosa Neum.

947 yarrowi Stretch. form remissa $\boldsymbol{H y} . \mathbf{E d w}$.

Phragmatobia Steph.

948 fuliginosa Linn. rubriensa Harr.

a borealis Staud.

949 assimilans Wlk. a franconica Sloss.

Mænas Hihn.

950 vestalis Pack. a amelaina Dyar.

Euerythra Harv.

951 phasma Harv. 952 trimaculata Sm.

Diacrisia IIhn.

953 latipennis Stretch. 954 virginiea Fabr. form fumosa Stlor.

955 vagans $B d v$. pteridis Hy. Edw. bicolor Wlk. a rufula $B d v$. punctata Pack. proba Hy. Edw.

b kasloa Dyar.

956 rubra . Veum.

a walsinghami Butl.

$b$ danbyi Neum.

Isia Wlk.

957 isabella $A$. \& $S$. californica Pack.

Hyphantria Harris.

958 textor Harr. candida WIk.

959 cunea Dru. punctatissime A. \& S. budea Hbn. punctata Fitch. pallida Pack. sufusa Stkr. brunnea Stkr. 959, 1 sspera Grt.

Estigmene Hbn. 960 acræa Dru. caprotina Dru. menthrustrina Martyn. pseuderminea Peck. californica Pack. packardi Schaupp. klagesi Ehrman. a dubis Wlk. rickseckeri Behr. $b$ arizonensis Roths. 961 albida Stretch. 962 prima Slosson. 963 congrus Wlk. entigone Stkr. $a$ athene Sthr.

Ecpantheria Hbn. 964 deflorata Fabr. seribonia Stoll. oculatissima A. \& S. chryseis Oliv. 
form confluens Oberth.

a denudata Sloss.

965 muzina Oberth.

albicollis Oberth.

abscondens Oberth.

depauperata Oberth.

sennetti Lint.

966 oslari Roths.

\section{Arachnis Geyer.}

967 aulæa Geyer. incarnata. Wlk.

968 picta Pack.

a maia Ottol.

$b$ citra $N . \& D$.

c hampsoni Dyar.

969 zuni Neum.

970 semiclara Stretch.

Apantesis Wlk.

971 virgo $L$.

ab. simplex Stretch.

$a b$. citrinaria $N . \& D$.

972 intermedia Stretch.

form stretchi Grt.

973 parthenice Kirby. saundersi Grt. circa Stretch. $a b$. approximata Stretch.

974 rectilinea Frch.

form conspicua Stretch.

975 oithona Stkr.

976 michabo Grt.

a minea Sloss.

977 arge Dru.

dione Fabr.

incarnatorubra Goeze.

coelebs Martyn.

nerea Bdv.

doris Bdv.

$a b$. nervosa $N$. \& $D$.

978 ornata Pack.

shastaensis French.

simplicior Butl. perpicta Dyar.

form achaia $G$. \& $R$. maculosa Stretch.

barda Hy. Edw. rivulosa Stretch. form edwardsi Stretch. $a b$. obliterata Stretch. form ochracea Stretch.

a complicata $W l k$.

979 anna Grt.

form persephone Grt. 980 quenseli Payk.

strigosa Fabr.

liturata Men.

a gelida Moesch.

b turbans Christ.

981 cervinoides Stkr.

982 virguncula Kirby. speciosa Moesch. $a b$. otiosa $N$. \& $D$.

983 proxima Guer. docta Wlk. mexicana G. \& R. arizoniensis Stretch.

a mormonica Neum.

$b$ autholea $B d v$.

984 favorita Neum.

985 nevadensis $G$. \& $R$. behri Stretch.

a geneura Stkr. incorrupta $\mathrm{Hy}$. Edw.

b sulphurica Neum. ochracea Neum.

986 bolanderi Stretch.

987 blakei Grt.

a superba Stretch.

$b$ elongata Stretch.

c diecki Neum.

988 williamsi Dodge. form determinata Neum.

a tooele $B$. \& $M C D$. form ophir $B$. \& $M C D$.

989 phyllira Dru. b-ata Goeze. dodgei Butl. 
990 figurata Dru.

coramica Hbn.

form snowi Grt.

form excelsa Neum.

ab. lugubris Hlst.
1006 oslari Roths.

1007 fusca Roths.

1008 elegans Streteh.

a roseicapitis $N$. \& $D$.

b Aavidorsalis B. \& MCD.

form franconia $H$ y. Edw. 1009 lorula $D$ yar.

991 celia saund. preciosa Nixon.

992 \&. pallida Stkr.

998 quadranotata Sikr. moierra Dyar.

a sociata B. \& MeD.

994 placentia A.\& S.

form flammea Neum.

995 nais Dru.

cunerata Goeze.

defloriana Martyn.

ochreata Butl.

996 vittats Fabr.

decorata Saund.

$a$ radians $W l k$.

colorata Wik. incompleta Butl.

$b$ phalerata Harris.

pulcherrima Stretch.

rhoda Butl.

ab. incarnata Stretch.

Kodiosoma Stretch.

997 fulva Stretch.

form nigra Stretch.

form eavesi Stretch.

form tricolor Streteh.

998 otero Barnes.

Pygarctia Grt.

999 abdominalis Grt.

1000 grossbecki Davis.

1001 eglenensis Clem.

1002 vivida Grt.

1003 spraguei Grt.

conspicue Neum.

1004 neomexicana Barnes.

1005 murina Stretch.

a albistrigats $B$. \& $M C D$.
Euchætias Lyman.

1010 antica W/k.

zonalis Grt.

1011 albicosta WIk.

fumidus Hy. Edw.

1012 bolteri Stretch.

of seepsiformis Graef.

1013 perlevis Grt.

1014 gigantea $B$. \& $M c D$.

1015 egle Dru.

form cyclica $H y . E d w$.

1016 pudens $H y . E d w$.

1017 castalla B. \& McD.

$a b$. griseopunctata $B$. \&

$M c D$.

1018 oregonensis Stretch.

1019 zella Dyar.

Seirarctia Pack.

1020 echo A. \& S.

niobe Stkr.

Turuptiana Wlk.

1021 permaculata Pack. reducta Grt. caeca Stkr.

Calidota Dyar.

1022 strigosa $W l k$. laqueata Hy. Edw. cubensis Grt.

1023 muricolor Dyar.

Parasemia Hbn.

1024 plantaginis $L$.

petrose Wlk.

chicorii G. \& R.

caespitio G. R. 
form \& hospita $D$. \& S. form modesta Pack. alascensis Stretch. form scudderi Pack. selwyni Hy. Edw. form geometrica Grt. form geddesi Neum.

\section{Arctia Schrank.}

1025 caja $L$.

a phreosoma Butl. $a b$. opulenta $\boldsymbol{H} y . E d w$.

$b$ americana Harr.

c utahensis $\boldsymbol{H} y . \mathbf{E} d w$. $a b$. transmontana $N$. \& $D$. d parva Roths.

\section{Ectypia Clem.}

Euverna N. \& D.

1026 bivittata Clem. nigroflava Graef.

1027 clio Pack. $a b$. thona Stkr.

a jessica Barnes.

Platyprepia Dyar. 1028 guttata $B d v$.

form virginalis $B d v$. form ochracea Stretch.

Utetheisa Hbn.

1029 bella $L$.

form hybrida Butl. intermedia Butl. form terminalis $N$. \& $D$. form nova $\mathrm{Sm}$.

$a$ venusta $D a l$. speciosa Wlk.

1030 ornatrix $L$.

form stretchi Butl. pura Butl.

Haploa Hbn.

1031 clymene Brown. interruptomarginata Beau. comma Wlk.

1032 colona $\mathrm{Hbn}$.

carolina Harris.

form conscita Wlk. lactata Sm. form fulvicosta Clem. duplicata N. \& D. form reversa Stretch. suffusa Sm.

1033 lecontei $B d v$. leucomelas H. S. form militaris Harris. confinis WIk. harrisi Dyar. form smithi Dyar. form dyari Merrick. form vestalis Pack.

1034 confusa Lyman. form lymani Dyar. form triangularis $\mathrm{Sm}$. 1035 contigua Wlk.

form lumbonigera Dyar.

AGARISTIDFE

Copidryas Grt.

1036 gloveri $G . \& R$.

Misa Karseh.

1037 sabulosa $\boldsymbol{B} d v$. noctuiformis Moesch.

1038 cosyra Druce.
Androloma Grt.

1039 mac-cullochi Kirby.

a lorquini $G$. \& $R$.

$b$ similis Stretch. edwardsi Bdv. $a b$. conjuncta $H y$. $E d w$. 1040 disparata $H y . E d w$. 
gracilenta Grae?.

1041 brannani Strelch.

Alypis IIbn.

1042 octomaculata $\mathrm{Fabr}$.

bimaculata Gmel. quadriguttalis Hbn.

ab. \& albomaculata Stoll.

1043 matuta $\boldsymbol{H y}$. Edw.

1044 wittfeldi Hy. Edw.

1045 dipsaci $G$. \& $R$.

1046 langtoni Couper. seramenti G. \& R.

hudsonica Hy. Edw.

1047 ridingsi Grt.
1048 mariposa $G$. \& $R$.

form lunata Strefeh.

Alypiodes Grt.

1049 bimaculatn $H$. S. trimaculata Bdv. form crescens $W \mid k$. grotei Bdv. Ravilinguis Grt. form dugesi Ckll.

1050 geronimo Barnes.

Pseudalypia $\mathrm{Hy}$. Eilw.

1051 crotchi $\boldsymbol{H} y$. Edw.

form atrata $H y$. Edw.

\section{NOCTUIDE}

\section{Agrotina}

Heliophana Grt.

1052 modicella Grt. 1053 indiana $\mathrm{Sm}$.

Heliophana Grt.

1054 bina $G n$.

1055 amaryllis $S m$.

1056 mitis Grt. obliquata Sm.

Heliosea Grt.

1057 pictipennis Grt.

Melicleptria Hbn.

1058 celeris $\mathrm{Grt}$.

1059 scissa Grt.

1060 graefiana Tepper.

1061 pulchripennis Grt.

form languida $\boldsymbol{H} y$. Edro

1062 antonio $S m$.

1063 sabulosa $S m$.

1064 cresina Sm.

1065 dobla Sm.

1066 edwardsi Sm.

1067 villosa Grt.

pauxillus Grt.

form sexata Sm.
1068 subatra $S \mathrm{~S}$.

1069 persimilis $\mathrm{Grt}$.

favidenta Sm.

1070 triolata $S m$.

1071 dubitans Tepper.

1072 perminuta $H y . E d w$.

1073 suets Grt.

a californica Grt.

1074 setheria $B$. \& $M c D$.

1075 honesta Grt.

kasloa Sm.

Melaporphyria Grt.

1076 immortua Grt.

Dysocnemis Grt.

1077 prorupta Grt. venusta Hy. Edw. fimbria Williams.

1078 belladonna $\mathrm{Hy}$. Edw.

1079 borealis Hamp.

1080 oregonica $\boldsymbol{H} y . \mathbf{E} d w$.

Incita Grot.

Pyrocleptria Hamp.

1081 aurantiaca $H y$. Edw. californica Hamp.

a tenuimargo $B$. \& $M C D$. 
Pseudotamila Sm.

1082 vaccinire $H y . E d w$. vanella Grt.

1083 carminatra Sm. 1084 avemensis Dyar.

Heliothis Ochs.

1085 chloropha $\mathrm{Hbn}$. lupata Grt.

1086 turbata Wlk. albidentina Wlk.

1087 paradoxa Grt.

1088 ononis Schiff.

1089 phloxiphaga $G$. \& $R$. phlogophagus G. \& R. acesias Feld. interjacens Grt. form luteitinctus Grt. 1090 obsoleta Fabr. armigera $\mathrm{Hbn}$. form umbrosa Grt.

1091 virescens $F a b r$. rhexia A. \& S. spectanda Stkr. a subflexa $G n$.

Oxylos Grt.

1092 citrinellus $G$. \& $R$.

Chlorocleptria Hamp.

1093 simplex $\mathrm{Sm}$.

1094 felicitata $S m$. imperialis B. \&. McD.

Dasypoudxa Sm.

1095 lucens Morr. $a b$. luxuriosa Grt.

1096 meadi Grt:

Rhodophora Gn.

1097 gaurx A. \& S. matutina $\mathrm{Hbn}$.

1098 florida $G n$.
Manruta Sm. 1099 elingua $S m$.

Thyreion Sm.

1100 rosea $\mathrm{Sm}$. stena Sm.

1101 snowi Grt. 1102 ligere $S m$.

Rhododipsa Grt. 1103 miniana Grt. 1104 pallicincta $\mathrm{Sm}$. 1105 volupia Fitch.

1106 masoni Sm. 1107 aden Stkr.

Podagra Sm.

1108 crassipes $\mathrm{Sm}$.

Prochloridea B. \& MeD. 1109 modesta $B$. \& $M c D$.

Lygranthocia $G$. \& R.

1110 bimatris Harv.

1111 carolinensis $B . \& M c D$. 1112 concinna $S m$.

1113 mortua Grt. form packardi Grt. form nobilis Grt.

1114 labe Stkr.

1115 errans $S m$.

1116 meskeana Grt. fastidiosa Stkr.

1117 rufimedia Grt.

1118 siren Stkr.

1119 tuberculum $\mathrm{Hbn}$. dorsilutea Wlk.

1120 obscurata Stkr.

1121 bicuspida Sm. 1122 brevis Grt.

\% atrites Grt.

1123 approximata Stkr.

1124 septentrionalis Wlk. 1125 biforma Sm. 
1126 inclara Stkr.

1127 thoreaui $G$. \& $R$.

1128 ultima Stkr.

1129 hanga Stkr.

1130 marginata Haw. rivulosa $\mathrm{Gn}$. contracta WIk. designala WIk. ab. constricta $\mathrm{Hy} . \mathrm{E} d w$.

1131 intrabilis $S m$.

1132 nubila Stkr.

1133 dolosa Stkr.

1134 lora Stkr.

1135 bifascia $H b n$. divergens Wlk.

1136 imperspicua Stkr. digitalis Sm.

Schinia Hbn.

1137 sara Sm.

1138 buta $S m$.

1139 ernesta Sm.

1140 luxa Grt.

1141 velutina $B$. \& $M c D$.

1142 neglecta Stkr.

1143 cumatilis Grt. oulmula Stkr.

1144 hulstia Tepper.

1145 tenuescens Grt.

1146 reniformis $S m$.

1147 oculata Sin.

1148 macroptica $S m$.

1149 aleucis Harv.

1150 chrysella Grt. conchula Feld.

1151 ciliata $S m$.

1152 trifascia $H b n$. lineata Wlk.

1153 oleagina Morr.

1154 gracilenta $H b n$.

1155 accessa $S m$.

1156 sexplagiata Sm. pyraloides Stkr.

1157 tobia Sm.
1158 biundulata Sm.

1159 espea Sm.

1160 separata Grt.

1161 acutilinea Grt.

1162 parmeliana $\boldsymbol{H} y . E d w$.

1163 nundina Dru. nigrirena Haw.

1164 arefacta $H y . E d w$.

1165 coercita Grt.

1166 obliqua $\mathrm{Sm}$.

1167 alensa $S m$.

1168 cupes Grt. crotchi Hy. Edw. navarra Dyar.

$a$ deserticola $B$. \& $M c D$.

1169 velaris Grt. ochreifascia Sm.

1170 lanul Stkr.

1171 argentifascia $B . \& M c D$.

1172 illustra $\mathrm{Sm}$.

1173 tertia Grt.

a megarena $\mathrm{Sm}$.

1174 albafascia $S m$.

a erosa $S m$.

1175 brunnea $B$. \& $M c D$.

1176 balba Grt. a brucei $S m$.

1177 diffusa $\mathrm{Sm}$.

1178 walsinghami $H y . E d w$.

1179 unimacula Sm.

1180 lynx Gn.

1181 sordida $S m$.

1182 petulans $H y . E d w$.

1183 ar Stkr.

1184 tanena Stkr.

1185 regia Stkr.

1186 niveicosta $S m$.

1187 gloriosa Stkr.

1188 sanguinea Geyer. carmosina Neum.

1189 saturata Grt. rubiginosa Stkr.

1190 roseitineta Harv. exaltata Hy. Edw. 
1191 jaguarina $G n$.

1192 arcigera $G n$.

\& spraguei Grt. a ferricosta $S m$.

1193 limbalis Grt.

1194 olivacea Sm.

Eupanychis Grt.

1195 spinosæ $G n$. hirtella G. \& R.

1196 crenilinea $\mathrm{Sm}$.

1197 camina $S m$.

Grotella Harv.

1198 septempunctata Harv.

1199 sampita Barnes.

1200 blanca Barnes.

1201 parvipuncta $B . \& M c D$.

1202 dis Grt.

1203 calora Barnes.

1204 grisescens $B . \& M c D$.

1205 olivacea $B . \& M c D$.

1206 binda Barnes.

1207 tricolor Burnes.

1208 soror B. \& $M c D$.

1209 spaldingi $B$. \& $M c D$.

1209,1 citronella $B$. \& $M c D$.

Canthylidia Butl.

1210 scutosa Schiff. nuchalis Grt.

Timora Wlk.

1211 julia Grt.

1212 toralis Grt. grandis Druce. rosurio Barnes.

Copablepharon Harv.

1213 grandis Stkr. subflavidens Grt.

1214 viridisparsa $D o d$.

1215 longipennis Grt.

1216 absida Harv.

1217 sanctre-monicre Dyar.
1218 alba Harv.

Agrotiphila Grt.

1219 quieta $H b n$. schoenherri Zett. constricta Wlk. rigida Wlk.

1220 alaskæ Grt.

1221 staudingeri Moesch.

1222 maculata $S m$.

1223 colorado $\mathrm{Sm}$.

1224 kyune Barnes.

Barrovia B. \& MeD.

1225 fasciata Skin.

Orosagrotis Hamp.

1226 incognita $\mathrm{Sm}$.

1227 montana Morr. rigida $\mathrm{Sm}$.

Porosagrotis Sm.

1228 dædalus Sm.

1229 dolli Grt.

1230 longidens $S m$.

1231 patula Wlk. snrfentrionalis Moesch.

1232 milleri Grt.

1233 orthogonia Morr. $a$ delorata $\mathrm{Sm}$.

1234 vetusta Wlk. murcenula G. \& R. $a$ catenuloides $S m$.

Euxoa IIbn.

1235 edictalis $\mathrm{Sm}$.

1236 obesula $\mathrm{Sm}$.

1237 siccata $\mathrm{Sm}$.

1238 wilsoni Grt.

$a$ specialis Grt.

$b$ rqualis Harv.

1239 riversi Dyar.

1240 olivia Morr. lacunosa Grt. segregata Sm. 
vanidica $5 \mathrm{~m}$.

- fieldi Dyar. form anncosto Sm. form enteridis Sm. zembla Sm.

1241 brevipennis Sm. ab. anguilirens $\mathrm{Sm}$. a brevistriga Sm.

1242 cicatricosa $G$. \& $R$. teplia Sm.

a recula Harv.

b neomexicana Sm.

1243 cinereopallida Sm.

1244 ducalis Sm.

1245 dargo Stkr. rumatona $\mathrm{Sm}$.

1246 niveilinea Grt. a rabiata $S m$.

1247 quadridentata $(F . \&$. $R$. pugionis Sm.

$a$ fiutea $\mathrm{Sm}$.

1248 Aavidens Sm.

1249 latificans Sm. masculinus Sm.

1250 plagigera Morr. olivalis Grt.

1251 oblongistigma $\mathrm{S} m$. ageme Stkr.

1252 ridingsiana Grt. maimes Sm.

1253 hollemani Grt.

1254 andera Sm.

1255 atristrigata $\mathrm{Sm}$. a collocata Sm.

1256 nevada $\mathrm{Sm}$. Roramina Sm.

1257 xasta $B$. \& $M C D$.

1258 citricolor Grt. tronellus $\mathrm{Sm}$.

1259 catenula Grt. contugionis Sm.

1260 pallinennis $\mathrm{Sm}$. alcesta Sm.

1261 satiens Sm.
1262 misturata Sm.

a perturbata $S m$. cardida $\mathbf{S m}$.

$b$ gian Stkr.

1268 vertesta Sm.

1264 mitis Sm.

a ura Sm. uramina Sm.

8 colla Stkr.

1265 moerens Grt. luteola Sm.

1266 orbicularis Sm. 1267 sotnia Sm.

1268 cona Stkr. falerina Sm.

1269 mercedes $B$. \& $M C D$.

1270 feniseca Harv.

1271 conjuncta Sm.

1272 detersa W'lk.

pitychrous Grt.

a personata Morr.

$b$ azif Stkr.

1273 intrita Morr.

form strigilis Grt.

titubatis Sm.

form reuda Stler.

a alticola Sm.

1274 rufula $\mathrm{Sm}$.

$a$ basiflava $\mathrm{Sm}$.

1275 infracta Morr. rubefactalis Grt.

1276 infausta Wlk. numa Stkr.

1277 micronyx Grt.

1278 satis Harv.

1279 sponsa Sm.

1280 holoberba Sm.

1281 bicollaris Grt. loya $\mathrm{Sm}$. monteclara Sm.

1282 perfusca Grt.

a compressipennis Sim.

$b$ corklei $5 m$.

1283 excogita Sm. 
1366 silens Grt.

1367 pimensis $B$. \& $M c D$.

1368 tristicula Morr. form nesilens $\mathrm{Sm}$.

1369 brocha Morr. bochus Morr.

1370 sculptilis Harv. xyliniformis Sm.

1371 vetusa Wlk. tetra Wlk. euroides Grt. perpura Morr.

Chorizagrotis Sm.

1372 auxiliaris Grt. form introferens $\mathrm{Grt}$.

1373 soror $\mathrm{Sm}$.

1374 agrestis Grt. mercenaria Grt.

1375 inconcinna Harv. differens Druce.

1376 balinitis Grt. 1377 perolivalis $S m$.

1378 terrealis Grt. 1379 thanatologia Dyar. form sordida $\mathrm{Sm}$. form boretha $\mathrm{Sm}$. form perfida Dod.

1380 socorro Barnes.

1381 salina Barnes.

1382 albicosta $S n$.

Rhizagrotis Sm.

1383 apicalis Grt. 1384 acclivis Morr. reclivis Dyar.

1385 opaca Harv.

1386 proclivis $S m$. oaxacana Schaus

1387 capota $\mathrm{Sm}$.

1388 flavicollis $\mathrm{Sm}$.

1389 biclavis Grt. demutabilis Sm.

1390 lagena Grt.
1391 querula Dod. 1392 albalis Grt. $a$ actona $S m$. 1393 insertans $\mathrm{Sm}$. 1394 cloanthoides Grt.

Feltia W7k.

1395 gladiaria Morr. morrisoniana Riley.

1396 robustior $\mathrm{Sm}$. 1397 venerabilis Wlk. $a$ arida $\mathrm{Ckll}$.

1398 circumdata Grt. 1399 evanidalis Grt. 1400 edentata Sm. 1401 hudsoni Sm. 1402 ducens Wlk. radiata Harr. subgothicał Auct.

1403 subgothica Haw. jaculifera $\mathrm{Gn}$. tricosa Lint.

1404 herilis Grt.

1405 pectinicornis $\mathrm{Sm}$.

1406 gravis Grt. vapularis Grt.

1407 musa Sm.

1408 vancouverensis Grt. hortulana Morr. agilis Grt. a semiclarata Grt.

1409 volubilis Harv. stigmosa Morr. form dentilinea $S m$.

1410 æneipennis Grt. clodiana Grt.

1411 obliqua $S m$.

1412 atha Stkr.

1413 annexa Treit. anteposita $\mathrm{Gn}$. decernens Wlk.

1414 malefida $G n$. inspinosa Gn. consueta Wlk. 
Agrotis Ochs.

1415 badinodis Grt.

1416 geniculata $G$. \& R.

1417 docilis Grt. ingeniculata $\mathrm{Sm}$.

1418 prefixa Morr.

1419 violaris $G$. \& $R$.

1420 opacifrons Grt.

1421 aurulenta Grt.

1422 ypsilon Rott. su ff usa Schiff. telifera Harr.

1423 collaris $G$. \& $R$.

1424 e-nigrum $L$.

1425 bicarnea $G n$. plagiate Wlk.

1426 treati Grt.

1427 conchis Grt.

1428 normaniana Grt. obtusa Speyer.

1429 ditrapezium Schiff. tristigma Treit.

1430 baja Fabr. smithi Snell.

1431 tenuicola Morr.

1432 acarnea $\mathrm{Sm}$.

1433 bolteri $\mathrm{Sm}$.

1434 plecta $L$. vicaria Wik.

1435 rosaria Grt.

1436 calgary $\mathrm{Sm}$.

1437 cynica Sm.

a perumbrosa Dyar. umbrosa Dyar.

1438 rubifera Grt.

1439 dislocata Sm.

1440 trumani Sm.

1441 hospitalis Grt.

1442 plebeia $S m$.

1443 jucunda Wlk. perconflun Grt. eriensis Grt.

1144 phyllophora Grt.

1445 esurialis Grt.
1446 fennica Tauscl. ineracta WIk.

1447 oblate Morr. hilliuna Harv.

1448 flavotincta Sm.

1449 substrigata $\mathrm{Sm}$.

1450 juncta Grt. a patefacta Sm.

1451 subporphyrea $W l k$.

1452 larga $S m$.

1458 atrifrons $\mathrm{Grt}$.

1454 piscipellis Grt.

a amia Dyar.

$b$ exculpatrix Dyar.

$c$ corrodera Sm.

1455 einereicollis Grt. pallidicollis Grt. congrua Sm.

$a$ vocalis Grt. form invenusta Grt.

$b$ planifrons $S m$.

1456 inopinatus $\mathrm{Sm}$.

1457 sierræ Harv.

1458 unimacula Morr. hamespica Grt. grandis Speyer.

1459 atrata Morr.

1460 clemens $S m$.

1461 unicolor Wik. clandestinn Harr. nigriceps Wlk.

1462 havilæ Grt.

Metalepsis Grt.

1463 cornuta Grt.

\section{Eucoptocnemis Grt.}

1464 fimbriaris $\mathrm{Gn}$. obvia WIk. form sordida Grt.

1465 dapsilis Grt. 1466 tripars WIk. u'orthingtoni Grt. 
1366 silens Grt.

1367 pimensis $B$. \& $M c D$.

1368 tristicula Morr.

1369 brocha Morr. bochus Morr.

1370 sculptilis Harv. xyliniformis $\mathrm{Sm}$.

1371 vetusa Wlk. tetra Wik. euroides Grt. perpura Morr.

Chorizagrotis Sm.

1372 auxiliaris Grt. form introferens Grt.

1373 soror $\mathrm{Sm}$.

1374 agrestis Grt. mercenaria Grt.

1375 inconcinna Harv. differens Druce.

1376 balinitis Grt. 1377 perolivalis $S m$.

1378 terrealis Grt. 1379 thanatologia Dyar. form sordida $\mathrm{Sm}$. form boretha $\mathrm{Sm}$. form perfida Dod.

1380 socorro Barnes. 1381 salina Barnes. 1382 albicosta $S n$.

Rhizagrotis Sm.

1383 apicalis Grt. 1384 acclivis Morr. reclivis Dyar.

1385 opaca Harv. 1386 proclivis $S m$. oaxacana Schaus

1387 capota $S m$.

1388 flavicollis $S m$.

1389 biclavis $G r t$. demutabilis Sm.

1390 lagena Grt.
1391 querula Dod. 1392 albalis Grt. a actona $S m$. 1393 insertans $\mathrm{Sm}$. 1394 cloanthoides Grt.

Feltia W7k.

1395 gladiaria Morr. morrisoniana Riley.

1396 robustior $\mathrm{Sm}$. 1397 venerabilis Wlk. a arida $\mathrm{Ckll}$.

1398 circumdata Grt. 1399 evanidalis Grt. 1400 edentata Sm. 1401 hudsoni Sm. 1402 ducens Wlk. radiata Harr. subgothica $\ddagger$ Auct.

1403 subgothica Haw. jaculifera $\mathrm{Gn}$. tricosa Lint.

1404 herilis Grt.

1405 pectinicornis Sm.

1406 gravis Grt. vapularis Grt.

1407 musa Sm.

1408 vancouverensis Grt. hortulana Morr. agilis Grt.

a semiclarata Grt.

1409 volubilis Harv. stigmosa Morr. form dentilinea $S m$.

1410 æneipennis Grt. clodiana Grt.

1411 obliqua $\mathrm{Sm}$.

1412 atha Stkr.

1413 annexa Treit. anteposita $\mathrm{Gn}$. decernens WIk.

1414 malefida $G n$. inspinosa Gn. consueta Wlk. 
Agrotis Oehs.

1415 badinodis Grt.

1416 geniculata $G$. \& R.

1417 docilis Grt. ingeniculata $\mathrm{Sm}$.

1418 preefixa Morr.

1419 violaris G. \& $R$.

1420 opacifrons $\mathrm{Grt}$.

1421 aurulenta Grt.

1422 ypsilon Rott. sufusa Schiff. telifera Harr.

1423 collaris $G$. \& $R$.

1424 c-nigrum $L$.

1425 bicarnea $G n$. plagiata Wik.

1426 treati Grt.

1427 conchis Grt.

1428 normaniana Grt. obtusa Speyer.

1429 ditrapezium Schiff. tristigna Treit.

1430 baja Fabr. smithi Snell.

1431 tenuicola Morr.

1432 acarnea Sm.

1433 bolteri $\mathrm{Sm}$.

1434 plecta $L$. ricarin Wlk.

143.5 rosaria Grt.

14:36 calgary Sm.

1437 cynica Sim.

a perumbrosa Dimer. umbrosa $\ddagger$ Dyar.

1438 rubifera $\mathrm{Grt}$.

14:39 dislocata Sim.

1440 trumani Sm.

1441 hospitalis Cirt.

1442 pleheia Sim.

1443 jucunda Wlk. perconfiun Grt. eriensis Grt.

1144 phyllophora Grt.

1445 esurialis Grt.
1446 fennica Tauseh. ineracta Wik.

1447 oblata Morr. hilliane Harv.

1448 flavotincta $\mathrm{Sm}$.

1449 substrigata $\mathrm{Sm}$.

1450 juncta Grt. n patefacta $S m$.

1451 subporphyres Wlk.

1452 larga $S m$.

1453 atrifrons $\mathrm{Grt}$.

1454 piscipellis Grt.

a amis Dyar.

$b$ exculpatrix Dyar.

$c$ corrodera $\mathrm{Sm}$.

1455 cinereicollis Grt. pallidicollis Grt. congrua $\mathrm{Sm}$.

$a$ vocalis Grt. form invenusta Grt.

$b$ planifrons $S m$.

1456 inopinatus $\mathrm{Sm}$.

1457 sierre Harv.

1458 unimacula Morr. hamespica Grt. grandis Speyer.

1459 atrata Morr.

1460 clemens $S m$.

1461 unicolor Wlk. clandestina Harr. nigriceps Wlk.

1462 havilæ Grt.

Metalepsis Grt.

1463 cornuta Grt.

\section{Eucoptocnemis Grt.}

1464 fimbriaris $G n$. obvia WIk. form sordida Grt.

1465 dapsilis Grt.

1466 tripars WIk. worthingtoni Grt. 
Onychagrotis Hamp.

1467 rileyana Morr.

Pseudorthosia Grt.

1468 variabilis $G r t$.

\section{Epipsilia Hbn.}

1469 interclusa $W l k$.

1470 hilaris Grt.

bolli Grt.

1471 fungorum $G$. \& $R$.

1472 hæsitans Wlk.

1473 manifesta Morr.

1474 salicarum Wlk.

claviformis Morr. orilliana Grt.

1475 monochromatea Morr.

1476 elevata $\mathrm{Sm}$.

1477 littoralis Pack.

$a$ pectinata Grt. ferruginoides Sm.

1478 fishi Grt.

1479 cinerascens $S m$.

1480 wockei Moesch. seropulana Morr.

1481 cinerea Staud. okakensis Pack.

1482 tecta $\mathrm{Hbn}$. carneał Auct.

$a$ roosta $S n$.

1483 terrifica $S m$.

1484 quadrangula $Z$ ett. rava $\mathbf{H}$. $\mathbf{S}$. umbratus Pack.

Lycophotia Hbn.

1485 radiola Hamp. radiatus $\mathrm{Sm}$.

1486 scandens Riley. elata Sm.

1487 astricta Morr. a subjugata Dyar.

1488 nigra $\mathrm{Sm}$.

1489 occulta $L$.
1490 margaritosa Haw. inermis Harris. form saucia $\mathrm{Hbn}$.

1491 serano $S m$.

1492 grandipennis Grt. 1493 rudens Harv. 1494 pellucidalis Grt. 1495 infecta Ochs. incivis $\mathrm{Gn}$. alabamae Grt.

1496 simplicia Morr. simplaria Morr.

1497 tenuescens $S m$.

1498 digna Morr. nigrovittata Grt.

1499 tepperi $S m$. atricincta Sm.

1500 bairdi $S m$.

1501 exuberans $\mathrm{Sm}$.

1502 lubricans $G n$. spreta $\mathrm{Sm}$. associans Wlk.

a illapsa $W l k$.

$b$ beata Grt.

1503 nanalis Grt. mantalini Sm.

Adita Grt.

1504 chionanthi A. \& S.

Aplectoides Butl.

1505 speciosa $H b n$. perquiritata Morr. baileyana Grt.

$a$ arctica $Z$ ett.

$b$ mixta Wlk.

1506 imperita $\mathrm{Hbn}$. comparata Moesch. saxigena Morr. discitincta Wlk.

1507 occidens Hamps.

1508 livalis $\mathrm{Sm}$.

1509 pressus Grt.

1510 fales $S m$.

1511 beddeci Hamp. 
1512 arufa Sm.

1513 condita $G n$. trabalis Grt.

1514 discolor Sm.

1515 abbea Sm.

Apharetra Grt.

1516 dentata Grt.

1517 pyralis Sm.

Ufous Grt.

1518 sagittarius Grt.

1519 electra Sm.

1520 hulsti $S m$.

1521 faunus Stkr.

1522 plicatus Grt. barometricus Goos.

1523 satyricus Grt.

1524 unicolor Grt.

Anytus Grt.

1525 evelina French. $a$ hanhami $S m$.

1526 discors Grt. vinela $\mathrm{Sm}$.

1527 cupola Hamp.

1528 yosemita Grt.

1529 enthea Grt.

1530 instruta $S m$. derelieta Hamp.

1531 exhilarata $\mathrm{Sm}$.

1532 betsia Sm.

1533 privata Wik. monstrata WIk. sculpto Grt. $a$ plana Grt.

1534 teltowa $S m$.

1535 atristrigata $\mathrm{Sm}$.

1536 tenuilinea $\mathrm{Sm}$.

1537 profunda $S m$. a obscura Sm.

Anomogyna Staud.

1538 sincera $\boldsymbol{H}$. S.
1539 letabilis Zell.

1540 infimatis Grl. a dernariu Sm.

1541 vernilis Grt. flive Sm.

Richia frrt.

1542 parentalis Grt. form decipiens Grt.

1543 distichoides Grt.

1544 chortalis Harv. form aratrix Harv.

Trichorthosia Grt.

1545 parallela Grt. terminatissima Dyar.

1546 spinosa $B$. \& $M C D$.

Mimobarathra B. \& MeD.

1547 antonito Barnes.

Mythimna Oehs.

1548 olivata Harv. blanda Grt. taedata Grt. decepta Grt.

1549 lobato Barnes.

1550 oxalina $H b n$. intexta Harv.

Matuta Grt.

1551 youngi $S m$.

1552 elimata $G n$.

a dilucida Morr.

$b$ badicollis Grt.

c janualis Grt.

1553 tenebrifera Wlk. catherina Grt. manifestolabes Morr.

1554 grisatra Sm.

1555 atoma Sm.

1556 indeterminata WIk. vashingtoniensis Grt. a innotabilis Grt. 
1557 stellaris Grt.

1558 apposita $G r^{\circ}$.

1559 quarta Grt.

1560 prasina Fabr.

herbacea Gn.

Abagrotis $\mathrm{Sm}$.

1561 erratica $S m$.

1562 ornatus $S m$.

1563 hero Morr.

1564 alcandola $\mathrm{Sm}$. tristis B. \& McD.

Rynchagrotis Sm.

1565 rufipectus Morr.

1566 brunneicollis Grt.

1567 morrisonistigma Grt. crenulata Sm.

1568 binominalis $\mathrm{Sm}$.

1569 confusa $S m$.

1570 formalis Grt. form facula Grt.

1571 insularis Grt.

1572 exsertistigma Morr. observabilis Grt.

1573 niger $S m$.

1574 meta Sm.

1575 lætula Grt. distracta Sm.

1576 cupidissima Grt. 1577 emarginata Grt.

1578 inelegans $\mathrm{Sm}$.

1579 carissima Harv.

1580 vittifrons $G r t$.

1581 bimarginalis Grt.

1582 mirabilis Grt.

1583 discoidalis Grt.

1584 negascia $\mathrm{Sm}$.

1585 placida Grt.

1586 trigona Sm.

1587 sambo $\mathrm{Sm}$.

1588 alternata Grt.

1588,1 orbipuncta $B$. \& $M C D$.

1589 anchocelioides $G n$.

velata Wlk.
1590 cupida Grt.

a brunneipennis $G r t$.

1591 duanca $\mathrm{Sm}$.

1592 belfragei $S m$.

1593 minimalis Grt.

1594 variata Grt.

form varix Grt. form orbis Grt.

1595 scopeops Dyar.

1596 gilvipennis Grt.

Euretagrotis Sm.

1597 sigmoides Grt.

1598 perattenta Grt.

1599 attenta Grt.

1600 inattenta $\mathrm{Sm}$.

Pronoctua Sm.

1601 typica $S m$.

1602 pyrophiloides Harv.

a peabodyæ Dyar.

Protagrotis Hamp.

1603 niveivenosa Grt. viralis Grt.

1604 nichollæ Hamp.

1605 obscura $B$. \& $M c D$.

\section{Hadeninæ}

Barathra Hbn.

1606 configurata Wlk. occidenta Grt.

1607 curialis Sm.

Miodera Sm.

1608 stigmata $\mathrm{Sm}$.

Discestra Hamp.

1609 florida Sm.

1610 chartaria Grt.

1611 hadeniformis Sm.

Craterestra Hamp.

1612 yakima $\mathrm{Sm}$. 
Scotogramma \$m.

1618 orida Sm.

1614 nevada B. \& MCD.

1615 trifolii Rott. inquieta Wlk. glaucovaria WIk. major Speyer.

$a$ albifusa $W l k$.

1616 mutata Dod.

1617 oregonica $W L k$.

a morana $\mathrm{Sm}$.

1618 ineoneinna $S m$.

1619 fervida B. \& MeD.

1620 gatei $\mathrm{Sm}$.

1621 castre B. \& McD.

1622 impolita Morr.

1628 repentina Morr.

1624 ptilodonta Grt.

1625 defessa Grt.

1626 submarina Grt.

Anarta Ochs.

1627 staudingeri Auriv.

a moeschleri Staud.

1628 richardsoni Curt. septentrionis Wlk.

a lanuginosa $S m$.

1629 leucocycla Staud.

1630 guadrilunata Grt.

1631 etacta $S m$.

1632 membrosa Morr.

1638 myrtilli $L$.

a acadiensis Beth.

1634 cordigera Thun. luteola G. \& R.

1635 mimuli Behr.

1636 impingens Wik.

curta Morr.

nivaria Grt.

perpura Morr.

1637 phea Hamp.

1638 melanops Thur.

1639 laerta $\mathrm{Sm}$.
1699,1 sierre B. \& MCD.

a laertidia $B$. \& $M C D$.

1610 flanda Sm.

1641 aquarn $S m$.

1642 hampa $S m$.

1643 mimula Grt.

1644 discolor $\mathrm{Sm}$.

Lasiestra Hamp.

1645 subdita Mosech.

1646 albinuda $S m$.

1647 phoca Moesch.

a luteola Sm.

1648 promulsa Morr. infuscata $\mathrm{Sm}$.

1649 uniformis $S m$.

1650 perplexa $S m$.

Lasionycta Auriv.

1651 rainieri Sm.

1652 subfuscula Grt.

1653 sedilis $S m$.

1654 conjugata $S m$.

1655 arietis Grt.

1656 ochracea Riley.

Polia Ochs.

Mamestra Ochs.

1657 lustralis Grt. cervina Sm.

a suffusa $\mathrm{Sm}$.

1658 nevia $S m$.

1659 detracta Wlk. claviplena Grt.

a neoterica $\mathrm{Sm}$.

1660 discalis Grt.

1661 imbrifera $G n$.

1662 leomegra $\mathrm{Sm}$.

1663 nugatis $\mathrm{Sm}$.

a tufa $S m$.

1664 languida $S m$.

1665 purpurissata Grt.

a juncimacula $\mathrm{Sm}$.

$b$ erydina Dyar.

$C$ apurpura $B$. \& $M C D$. 
1666 crotchi Grt. a fusculenta $S m$.

1667 lepidula $S m$.

1668 griseata $S m$.

1669 determinata $\mathrm{Sm}$.

1670 distincta $\mathrm{Hbn}$. vitis French.

1671 columbia Sm.

1672 leucogramma Grt.

1673 meditata Grt.

1674 brachiolum Harv.

1675 rubrifusa Hamp.

1676 umbrosa $S m$.

1677 hanhami $B$. \& $M c D$.

1678 gnata Grt.

1679 selama Stkr.

1680 insolens Grt. earina Harv.

1681 plicata $S m$.

1682 negussa $\mathrm{Sm}$.

1683 tristis $B . \& M c D$.

1684 purpurea $B$. \& $M c D$.

1685 grandis $B d v$.

libera Wlk.

1686 subjuncta $G$. \& $R$.

1687 nevadæ Grt.

$a$ canadensis $S m$.

1688 ingravis $\mathrm{Sm}$.

1689 obesula $S m$.

$a$ ortruda $S m$.

1690 passa Morr.

1691 vau-orbicularis $S m$.

1692 invalida $\mathrm{Sm}$.

1693 cristifera Wlk.

1694 lubens Grt. rufula Morr.

a glaucopis Hamp.

1695 latex $G n$. demissa Wlk.

1696 nimbosa $G n$.

1697 rogenhoferi Moesch.

1698 carbonifera Hamp.

1699 mystica $\mathrm{Sm}$.

1700 adjuncta $B d v$.
1700,1 brenda $B . \& M c D$.

1701 dilatata $S m$.

1702 variolata $S m$.

1703 glaciata Grt.

1704 chunka Sm.

1705 farnhami $G r t$.

1706 prodeniformis Sm.

1707 liquida Grt.

1708 meodana $S m$.

1709 tacoma Stkr.

1710 atlantica Grt. discolor Speyer.

1711 quadrata $\mathrm{Sm}$.

1712 radix Wlk. dimmocki Grt. desperata Sm.

1713 sutrina Grt.

1714 cuneata Grt. a gertana $\mathrm{Sm}$.

1715 legitima $G r t$.

1716 dodi Sm.

1717 lilacina Harv. form illabefacta Morr. $a$ luski $B$. \& $M c D$.

1718 goodelli-Grt.

1719 acutermina Sm.

1720 ectypa Morr. bella Grt.

1721 bolteri $S m$.

1722 obscura $S m$.

1723 assimilis Morr.

a pulverulenta $\mathrm{Sm}$.

1724 noverca Grt.

1725 tuana Sm.

1726 stretchi $H u$. Edw.

1727 francisca $S m$.

1728 mania Stkr.

1728,1 delecta $B$. \& $M c D$.

1729 megæra Sm.

1730 densa $S m$.

1731 canities Hamp.

1732 anguina Grt.

1733 imbuna $S m$. 
1734 vicina Grt.

teligera Morr.

1735 acutipennis Grl.

1736 pensilis Grt.

1737 doira Stkr. ascula $\mathrm{Sm}$.

1738 sareta Sm.

1739 larissa Sin.

1740 agnata $\mathrm{Sm}$.

1741 segregata Sm.

1742 gussata Sm.

1743 beani Grt.

1744 mutilata Sm.

1745 palilis Harv.

1746 bicolor B. \& MCD.

1747 stenotis Hamp.

1748 longiclava $S m$.

1749 ferrealis Grt.

1750 renigera Steph.

herbimacula Gn. infecte WIk.

1751 stricta Wlk. forrea Grt.

a cinnabarina Grt.

$b$ tenisca $\mathrm{Sm}$.

1752 circumcincta $S m$.

1753 spiculosa Grt.

1754 lorea $G n$.

lignta W1k.

dodgei Mort.

1755 olivaces Morr.

a comis Grt.

b obscurior $\mathbf{S m}$.

c lucina Sm.

d megarena $S m$.

e obnigra Sm.

$f$ altua $S m$.

$g$ davens Sm.

$h$ rectilinea $S m$.

i petita $\mathrm{Sm}$.

j vau-media $S m$.

1756 laudabilis $G n$. indirans WIk.

1757 illaudabilis Grt.
1758 atrigicollis Wall.

1759 alboguttata Gre.

1760 restora $S m$.

1761 marinitincta Harv.

1762 quadrilineata Grt.

1768 rugosa Morr.

1764 lunolacta Sm.

1765 erects $W i k$.

constipata W1k. innexa Grt.

1766 basiplaga $S m$.

1767 uliginosa $\mathrm{Sm}$.

1768 vittula Grt.

1769 intentata $S m$.

1770 roseosuffusa $\mathrm{Sm}$.

1771 falsa Grt.

1772 secedens $W l k$.

1773 basivirida $B$. \& $\mathrm{Mel}$.

1774 incurva $\mathrm{Sm}$.

1775 nipana $\mathrm{Sm}$.

1776 montara Sm.

1777 alfkeni Grt.

perplexa Grt.

latens $\mathrm{Sm}$.

occluna Sm.

Neuris Gn.

1778 procincta Grt.

Tholera Hbn.

1779 americana Sm.

Epia Hbn.

1780 capsularis $G n$. propulsa Wlk.

1781 minorata $\mathrm{Sm}$.

1782 ectrapela $S m$.

1783 circumvadis $\mathrm{Sm}$.

Cardepia Hamp.

1784 nova $\mathrm{Sm}$.

Trichoclea Grt.

1785 decepta Grt. 
1786 antica $\mathrm{Sm}$. 1787 postica $S m$. 1788 edwardsi $\mathrm{Sm}$. 1789 fuscolutea $\mathrm{Sm}$. 1790 u-scripta .Sm. 1791 artesta $S m$. 1792 ruisa Forbes.

Admetovis Grt.

1793 oxymorus Grt. 1794 similaris Barnes.

Lophoceramica Dyar.

1795 artega Barnes. pallicauda Sm.

Chabuata Wlk.

1796 elsinora Barnes. erebus $\mathrm{Sm}$.

1797 palmillo Barnes. 1798 signata Wlk. semiaperta Morr.

1799 notata Stkr. syrissa Stkr.

1800 baranca Barnes. 1801 fistula Harv. ulamora Sm.

1802 chipeta Barnes. 1803 endiva $S m$.

1804 inconspicua Grt. pectinicornis $\mathrm{Sm}$.

1805 velutina $S m$. lutina $\mathrm{Sm}$.

Hyssia Gn.

1806 senatoria $\mathrm{Sm}$. 1807 modesta Morr. 1808 fasciata $S m$. 1809 disticha Morr. 1810 dilecta $H y . E d w$. 1811 orbiculata $S m$. 1812 niveiguttata Grt.

Eriopyga Gn. 1813 curtica Sm.
1814 akalus Stkr. 1815 rufula Grt. 1816 indra $S m$. 1817 utahensis $S m$. 1818 perforata Grt. 1819 incincta Morr. 1820 saturnus Stkr. 1821 oviduca $G n$. capsella Grt. 1822 melanopis Hamp. 1823 orobia Harv. 1824 antennata $B$. \& $M c D$. 1825 serrata $S m$. dubiosa B. \& McD.

1826 jocosa $B$. \& $M c D$.

1827 reliqua $S m$. 1828 bostura $\mathrm{Sm}$. 1829 trifascia $\mathrm{Sm}$. 1830 planalis Grt. 1831 agrotiformis Grt. 1832 catalina $B . \& M c D$. 1833 euxoiformis $B$. \& $M C D$. 1834 alamosa Barnes. 1835 culea $G n$. modifica Morr.

1836 consopita Grt. 1837 hueco Barnes. 1838 crenulata Butl. 1839 eynica $G n$. nimia $\mathrm{Gn}$. candens Gn. tecta Wlk.

1840 intractata Morr. fidelis Grt.

1841 irrorata $S m$.

1842 vecors $G n$. enervis Gn. prodeuns Wlk. togata Wlk. velata Wlk. nitens Grt. a griseocincta Harv. 1843 calceolaria Stkr. 1844 imora Stkr. 1845 virgula Grt. 
$18 \$ 6$ keela $5 m$.

1847 gigas Sm.

1848 gigantoides $B$. \& $M c D$.

1849 dubia $B$. \& $M c D$.

1849, 1 discreta $B$. \& $M C D$.

18.50 puerilis Grt.

1851 contrahens Wik. thecala Morr.

1852 conar sikr. quadristigma $\mathrm{Sm}$.

1858 infidelis Dyar.

1854 rectiflava $S m$.

1855 flosea Sm.

1856 perbrunnea Grt.

1857 uniformis Sm.

1858 furfurata Grt. peredia Grt.

1859 communis Dyar. affurata Hamp.

1860 fractura Sm.

1861 smithi Dyar.

1862 mecrona Sm.

Ursogastra Sm.

1863 Iunata $S m$.

\section{Nephelodes Gn.}

1864 pectinata $S m$.

1865 tertialis $\mathrm{Sm}$.

1866 emmedonia Cram.

minians Gn.

expansa Wlk.

subnotata Wlk.

form violans $\mathrm{Gn}$. subdolens WIk.

Trichopolia Grt.

1867 dentatella Grt. obtua $\mathrm{Sm}$.

1868 ursina Sm.

Stretcbia Hy. Ealw.

1869 muricina Grt.

1870 inferior $S m$.
1871 plusiwformin $\mathrm{H} y . \mathbf{E} d w$. 1872 variabilis Sm.

Morrisonia Grt.

1878 mucens $\mathrm{H} b$ s. multifaris Wlk. spoliata Wlk.

1874 sectilis $G n$. rileyana $\mathrm{Sm}$.

1875 albidior B. \& $\mathrm{McD}$.

1876 bisulea Grt.

1877 evicta Grt. form vomerina Grt. form infidelis Grt.

1878 confusa $\mathrm{H} b n$. infructuosa Wilk.

Xylomyges $\mathrm{Gn}$.

1879 behrensiana Grt. 1880 erythrolita Grt. form apicata $S m$. form acutangula Sm.

1881 hiemalis Grt. californica Behr.

1882 simplex Wlk. pallidior Sm.

1883 erucialis Harv. a peritalis $\mathrm{Sm}$.

1884 cognata $S m$.

1885 curialis Grt.

a indurata $\mathrm{Sm}$.

$b$ nicalis $\mathrm{Sm}$.

c tantiva $S m$.

d argus $\mathrm{Sm}$.

1886 candida $\mathrm{Sm}$. 1887 dolosa Grt. 1888 rubrica Harv. 1889 mustelina Sm. 1890 pulchella $S m$. 1891 perlubens Grt. subapicalis Sm.

1892 patalis Grt. fletcheri Grt.

1893 alternans W/k. tabulata Grt. 
Perigrapha Led.

1894 prima $S m$.

1895 normalis Grt.

1896 pulchella Harv.

1897 terminata $\mathrm{Sm}$.

1898 pectinata $S m$.

1899 addenda Sm.

1900 algula $S m$.

1901 achsha Dyar.

1902 hepatica $B$. \& $M c D$.

1903 transparens Grt. hamifera Grt. fringata Sm.

1904 præses Grt. form saleppa $S m$.

Orthosia Ochs.

1905 mys Dyar.

$a$ caloramica $B . \& M c D$.

1906 ferrigera $S m$. agravens B. \& McD.

1907 macona Sm.

1908 flaviannula $S m$.

1909 annulimacula $S m$.

1910 rubrescens Wlk.

venata $\mathrm{Sm}$.

1911 tenuimacula $B$. \& $M c D$.

1912 carminata $S m$.

1913 arthrolita Harv.

1914 styracis $G n$.

1915 garmani Grt.

1916 pacifica Harv.

1917 revicta Morr. subterminata Sm.

1918 alurina $S m$.

1919 hibisci $G n$.

aliał 'Auct.

confluens Morr. form norm. insciens

a latirena Dod.

$b$ proba $S m$.

$c$ quinquefasciata $\mathrm{Sm}$.

$d$ inherita $S m$.

$e$ inflava $\mathrm{S} m$. $f$ nubilata $S m$.

$g$ malora $\mathrm{Sm}$.

$h$ brucei $S m$.

Perigonica Sm.

1920 fulminans $\mathrm{Sm}$.

1921 tertia Dyar.

1922 punctilinea $\mathrm{Sm}$.

1923 eldana $S m$.

1924 fermata $S m$.

1925 angulata $S m$.

Sideridis Hbn.

1926 rosea Harvv.

1927 normani Grt.

1928 congermana Morr.

1929 rubefacta Morr. vindemialis Grt.

Ceramica Gn.

1930 picta Harr.

exusta Gn.

contraria Wlk.

Xanthopastis Hbn.

1931 timais Cram. regnatrix Grt.

Cirphis Wlk.

1932 pilipalpis Grt.

1933 pseudargyria $G n$. form callida Grt.

1934 multilinea Wlk. solita Wlk.

lapidaria Grt.

1935 commoides $G n$.

1936 phragmatidicola $G n$.

1937 roseola $\mathrm{Sm}$.

1938 farcta Grt.

Wlk.1939 scirpicola Gn.

calpota Sm.

1940 imperfecta $S m$.

1941 insueta $G n$.

adonea Grt.

mimica Stkr. 
a heterodoxa Sm.

6 dia Grt.

c megadia Sin.

1942 anteroclara Sw.

1943 pendens Sm.

1944 oregona Sm.

1945 calgariana Sm.

1946 palliseca Sm.

1947 juncicola $G$. adjuta Grt.

1948 latiuscula $H$. S. subpunctate Harv. complicnta Stkr.

1949 dissimilis $B$. \& MCD.

1950 unipuncta Haw. extranen $\mathrm{Gn}$.

1951 quadrannulata Morr.

Borolia Moore.

1952 extincta $\mathrm{Gn}$.

1953 texana Morr. ligata Grt.

1954 rimosa Grt.

1955 flabilis Grt.

1956 amygdalina Harv.

1957 linita Gn.

\section{Neleucania Sm.}

1958 rubripennis $G$. \& $\boldsymbol{R}$.

1959 albilines $\mathrm{Hbn}$. moderata WIk. harveyi Grt.

a diffuss Wlk.

$b$ obscurior $\mathrm{Sm}$.

c neptis Sm.

d limitata Sm.

1960 tetera Sm.

1961 ferricola Sm.

1962 stolata Sm.

1963 patricia Grt.

1964 bicolorata Grt. $a$ citronella $\mathrm{S}$.

1965 niveicosta $S m$.

1966 pregracilis Grt.
Zosteropoda Grt.

1967 hirtipes Grt.

Leucania Ochs.

1968 rubripallens Sm.

1969 oxygala Grt.

1970 suavis $B$. \& $M c D$.

1971 minorata $S m$.

1972 luteopallens $S m$.

1973 pertracts Morr.

Faronta Sm.

1974 aleada $\mathrm{Sm}$.

Cucullina

Copicucullia Sm.

1975 antipoda Stkr.

1976 propinqua $S m$.

1977 eulepis Grt.

bistriga Sm. mala Sm.

1978 incresa $S m$.

1979 astigma $S m$.

1980 alfarata Stkr.

1981 luteodisca Sm.

Rancora Sm.

1982 strigata Sm.

1983 solidaginis Behr.

1984 matricaria Behr.

1985 serraticornis Lint.

1986 brucei $S m$.

1987 albicinerea Sm.

Cucullia Schrank.

1988 albids Sm.

1989 dentilinea Sm.

1990 obtusa Sm.

1991 dorsalis $\mathrm{Sm}$.

1992 speyeri Lint.

1993 latifica Lint. cita Grt.

hartmanni French.

1994 phila Sm. 
1995 minor $B$. \& $M c D$.

1996 cinderella Sm.

1997 intermedia Speyer.

1998 montanæ Grt.

1999 florea $G n$. indicta Sm. $a$ obscurior $\mathrm{Sm}$.

2000 lilacina Schaus. agua Barnes.

2001 omissa Dod. 2002 postera $G n$. 2003 asteroides $G n$. 2004 similaris $S m$. 2005 convexipennis $G$. \& $R$. 2006 aribac Barnes. 2007 arizona $\mathrm{Sm}$. strigata $\ddagger$ Schaus. perstrigata Hamp.

Nycterophæta Sm.

2008 luna Morr. magdalena Hlst. notatella Grt.

Pseudanthœcia Sm.

2009 tumida $G r^{2}$.

Euros Hy. Fdw.

Protophana Hamp.

2010 cervina $H y$. $E d w$.

2011 proprius $H y . E d w$.

Calophasia Steph.

2012 strigata Sm.

Lepipolys Gn.

2013 behrensi Grt.

2014 perscripta $G n$.

Oncocnemis Led.

2015 mirificalis Grt.

2016 dayi Grt.

2017 euta $S m$.

2018 hayesi Grt.
2019 regina $S m$.

2020 corusca $S m$.

2021 exemplaris $S m$.

2022 albifasciata Hamp. fasciata Sm.

2023 melantho Sm.

2024 pudorata $S m$.

2025 tenuifascia $S m$.

2026 balteata $\mathrm{Sm}$.

2027 terminalis $\mathrm{Sm}$.

2028 linda $B$. \& $M c D$.

2029 iricolor $S m$.

2030 levis Grt.

2031 sanina $\mathrm{Sm}$.

2032 simplex $\mathrm{Sm}$.

2033 meadiana Morr.

2034 saundersiana $G r t$.

2035 pernotata $\mathrm{Grt}$.

2036 polingi Barnes.

2037 fasciata $H y$. Edw.

2038 occata Grt.

2039 viriditincta $S m$.

2040 laticosta Dyar.

2041 flagrantis $\mathrm{S} m$.

2042 singularis $B$. \& $M C D$.

2043 augustus Harv.

2044 nita $S m$.

2045 glennyi Grt.

2046 riparia Morr.

2047 lacticollis $\mathrm{Sm}$.

2048 cibalis Grt.

2049 astrigata $B$. \& $M C D$.

2050 melalutea $S \mathrm{~m}$.

2051 extremis $\mathrm{Sm}$.

2052 chorda Grt. refecta $\mathrm{Sm}$.

2053 rosea $\mathrm{Sm}$.

2054 simplicia $\mathrm{Sm}$.

2055 deserta $\mathrm{Sm}$.

2056 punctilines Hamp.

2057 bakeri Dyar.

2058 pohono $S m$.

2059 homogena Grt.

2060 umbrifascia $S m$.

2061 atrifasciata Morr. 
2062 barnesi $5 \mathrm{sm}$.

2063 figurata Harr.

2064 major Cirt. a aqualis firt. b curvicollis lirt.

2065 ciliata 5 im.

2066 intruda $\mathrm{Sm}$.

2067 obscurata B. \& . VCD.

2068 semicollaris S $5 m$.

2069 chandleri Grit. poliuchron Hamp.

2070 colorado Sim. chandlerit Hamp.

2070, 1 sagittata $B$. \& $M C D$.

2071 extranea $\mathrm{Sm}$.

2072 tetrops Dyar.

2073 dunbari Harv. definita B. \& McD.

2074 griseicollis Grt. gerdis Sm.

2075 nigrocaput Sm.

2076 atricollaris Harv.

Cerapoda Sm.

2077 oblita Grt. deserta Grin.

2078 stylata $\mathrm{Sm}$.

Homoncocnemis Hamp.

2079 fortis Grt. vorax Behr. $a$ picins Grt.

Homohadena Grt.

2080 badistriga Grt. a rayata $S m$.

2081 infixa Wlk.

kappa Grt.

retroversa Morr.

a fifia Dyar. dinalda Sm.

2082 induta Harv.

2083 loculosa Grt.

2084 rustica $B$. \& $M C D$.

2085 incomitata Harv.
2086 inconstans Grt.

2087 stabilis Sm.

Pseudanarta Grt.

2088 flava Grt.

dupla Sm.

2089 croces $H y . E d w$.

2090 actura Sm.

2091 creca Dod.

2092 singula Grt.

2093 flavidens $\mathrm{Grt}$.

2094 Palcata Nerm.

2095 peralto Barnes.

Feralia Grt.

2096 jocosa $G n$. $a$ furtiva $S m$.

2097 major Sm.

Momophana Grt.

2098 comstocki Grt. a columbiana $S m$.

2099 februalis $G$ rt.

2100 brillians Barnes.

2101 sylvia Dyar.

Brachionycha Hbn.

2102 borenlis $\mathrm{Sm}$.

Lathosea Grt.

2103 pulla Grt. pullata Grt.

Psaphida Wlk.

2104 resumens $W l k$.

viridescens Wlk. muralis Grt.

2105 grotei Morr.

2106 thaxteriana Grt. 2107 electilis Morr.

Eutolype Grt.

2108 grandis $5 m$.

2109 depilis Grt.

bombyciformis Sm. 
2110 rolandi Grt. vernalis Morr.

2111 damalis Grt.

Copipanolis Grt.

2112 cubilis Grt.

form borealis $S m$. form fasciata $\mathrm{Sm}$. form stigma $\mathrm{Sm}$.

Provia B. \& McD.

2113 argentata $B$. \& $M C D$.

\section{Epidemas Sm.}

2114 cinerea Sm.

2115 obscura Sm.

2116 melanographa Hamp.

Bombycia Steph.

2117 curvifascia Sm.

2118 thula Stkr.

2119 elda French.

2120 rectifascia $S m$.

2121 onychina $G n$.

Brachylomia Hamp.

2122 populi Stker.

2123 discolor Sm.

Litholomia Grt.

2124 napæa Morr.

Hillia Grt.

2125 discinigra Wlk.

2126 algens Grt.

2127 iris Zett.

crasis H. S.

semisigna Wlk.

erdmanni Moesch. senescens Grt.

form vigilans Grt.

Dryotype Hamp.

2128 opina Grt.
Lithomoia Hbn.

2129 solidaginis $H b n$. germana Morr.

Graptolitha Hbn.

2130 semiusta Grt.

2131 bethunei $G$. \& $R$.

2132 patefacta Wlk.

2133 innominata Sm. signosał Grt.

2134 hemina Grt. 2135. oriunda Grt. 2136 disposita Morr. 2137 ferrealis Grt. 2138 petulca Grt. 2139 signosa Wlk. 2140 gausapata Grt. 2141 amanda $S m$. 2142 longior Sm.

2143 contenta Grt. pomona Sm.

2144 baileyi Grt.

2145 vivida Dyar.

2146 viridipallens Grt. 2147 pruena Dyar.

2148 puella Sm.

2149 querquera Grt.

2150 unimoda Lint.

2151 laticinerea Grt.

2152 winnipeg $S m$.

2153 antennata $W l k$. cinerea Riley.

2154 cinerosa Grt. grotei Riley.

2155 georgii Grt.

$a$ emarginata $S m$.

b holocinerea $\mathrm{Sm}$.

$c$ ancilla $\mathrm{Sm}$.

$d$ vertina $S m$.

e fletcheri $S m$.

$f$ oregonensis Harv.

2156 fagina Morr.

2157 itata $S m$.

2158 nigrescens Engel. 
2159 merceda Sm.

2160 tepida Grt.

a atincta Sw.

2161 torrida Sm.

2162 laceyi $B$. \& MCD.

2163 pexata Grt. a washingtonia Grt.

2164 dilatocula Sm.

2165 nasar Sm.

2166 atara Sm.

2167 lepida Lint.

2168 thaxteri Grt.

Xylena Ochs.

2169 nupera $L i n t$.

2170 mertena $\mathrm{Sm}$.

2171 curvimacula Morr.

2172 brillians Ottol.

2173 cineritia Grt.

2174 thoracica Put.-Cram.

2175 brucei $\mathrm{Sm}$.

\section{Behrensia Grt.}

2176 conchiformis Grt.

Xylotype Hamp.

2177 capax $G$. \& $R$.

Eurotype Hamp.

2178 confragosa Morr. medialis Grt. form acutissima Grt.

2179 contadina $\mathrm{Sm}$.

Pachypolia Grt.

2180 atricornis Grt.

\section{Pleroma Sm.}

2181 apposita Sm.

2182 conserta Grt.

2183 bonuscula Sm.

2184 obliquata $\mathrm{Sm}$.

2185 cinerea $S m$.
Eumichtis I11n.

2186 sommeri Lef.

2187 dueta Grt.

2188 versuta Sm. moilena Stkr.

2189 miniota Sm.

2190 loda Stkr. albiserrata Sm.

2191 maida Dyar.

2192 connecta Sm.

Bryomima Staud.

2193 fallax Hamp. form uintara $S m$.

2194 muscosa Hamp. 2195 distans $B$. \& $M C D$. 2196 pulverulenta $\mathrm{Sm}$. 2197 chryselectra Grt. benigna Hy. Edw.

Sympistis $\mathrm{Hbn}$.

2198 melaleuca Thun. bicycla Pack.

2199 kelloggi $H y$. Edw. 2200 lapponica Thun. tenebricosa Moesch.

2201 zetterstedti Staud.

a labradoris Staud.

2202 funesta $P a y k$. funebris $\mathrm{Hbn}$.

a cocklei Dyar.

Harpaglæa Hamp.

2203 sericea Morr. venustula Grt.

2204 tremula Harv. 2205 pastillicans Morr.

Epiglæa Grt.

2206 decliva Grt. deleta Grt.

2207 apiata Grt. 
Psectraglæa Hamp.

2208 carnosa Grt.

Conistra Hbn.

2209 inulta Grt.

2210 viatica Grt.

2211 signata Frch.

2212 tristigmata Grt.

2213 sidus $G n$.

walkeri Grt.

$a b$. vinulenta Grt.

a colorado $\mathrm{Sm}$.

2214 morrisoni Grt.

2215 devia Grt.

2215, 1 fringata $B . \& M c D$.

2216 indirecta $W l k$.

graefiana Grt. moffatiana Grt.

2217 pettiti Grt.

2218 ceromatica Grt.

Parastichtis Hbn.

2219 purpurea Grt. fornica Sm. form crispa Harv.

$a$ antapica $S m$.

2220 bicolorago $G n$.

spurcata Wlk.

form ferrugineoides $G n$.

$a$ verberata $S m$.

2221 acta $\mathrm{Sm}$.

2222 straminea $\mathrm{Sm}$.

2223 decipiens Grt.

2224 ralla $G . \& R$.

2225 puta $G$. \& $R$.

euroa G. \& R.

$a$ dusca $S m$.

2226 inops Grt.

insipida Stkr.

2227 aggressa $S m$.

2228 americana Morr.

2229 immaculata Morr.

Atethmia Hbn.

2230 pampina $G n$.
Iodia $\mathrm{Hbn}$.

2231 rufago $\mathrm{Hbn}$. honesta Wlk.

Brachycosmia Hamp.

2232 digitalis Grt.

Xanthia Ochs.

2233 lutea Strom. flavago Fabr. 2234 pulchella $S m$.

Homoglæa Morr.

2235 carbonaria Harv.

2236 dives ' $S m$.

2237 hircina Morr.

2238 californica $S m$. insinuata Sm.

Acronyctinæ

Amphipyra Ochs.

2239 pyramidoides $G n$.

$a b$. inornata Grt. $a b$. conspersa Riley.

2240 tragopoginis $L$. repressus Grt.

2241 glabella Morr.

Magusa Wlk.

2242 orbifera Wlk. form divaricata Grt.

Dipterygia Steph.

2243 scabriuscula $L$.

2244 patina Harv.

minorata Barnes.

Septis Hbn.

Xylophasia Steph.

2245 cuculliformis $\mathrm{Grt}$.

2246 verbascoides $G n$.

2247 nigrior $S m$.

2248 cariosa $G n$.

idonea Grt.

cluna Stkr. 


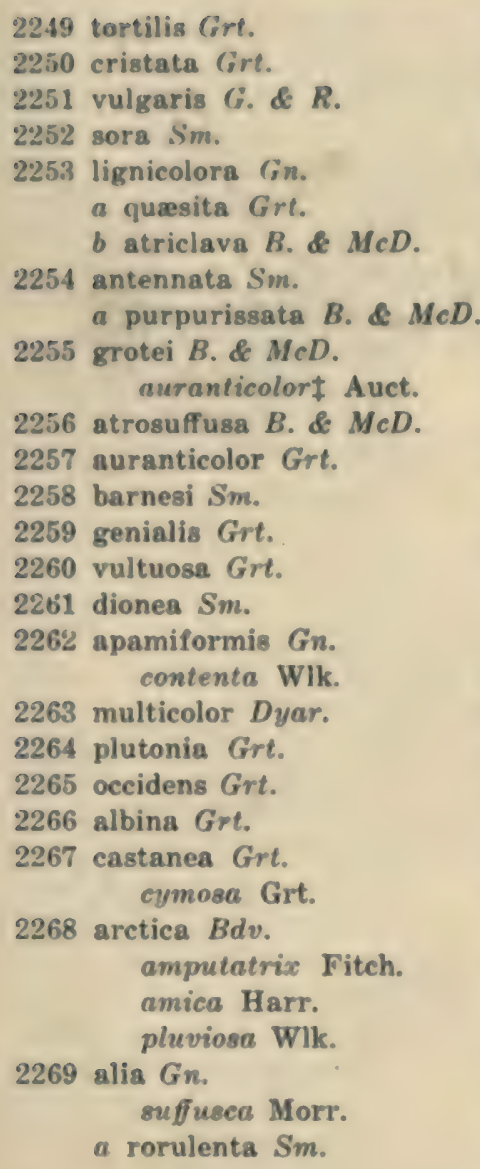

Trachea Ochs.

2270 illocata Wlk. stigmata Grt.

2271 delicata Grt. interna Grt.

2272 smaragdina Neum.

2273 marina Grt.

2274 semilunata Grt.

2275 inordinata Morr. a montana $S m$. -

2276 miselioides $G n$.

a miscellus $\mathrm{Sm}$.
2277 macerata $S m$.

2278 einefacta Grt.

2279 parcata $S m$.

2280 unita $S m$.

2281 spaldingi $S m$. umbrifacta Hamp.

2282 centralis $S m$.

2283 turbulente $\boldsymbol{H b n}$. arcuata WIk.

2284 tusa Grt.

2285 indocilis $W l k$. remissał Auct.

2286 mactata $G n$. a allecto $\mathrm{Sm}$.

2287 ferida $\mathrm{Sm}$.

2288 separans Grt. ferens Sm.

a lona Stkr. ninata Sm.

2289 perpenoa $\mathrm{Grt}$.

2290 divesta Grt.

2291 finitima $G n$.

a cerivana $S m$.

2292 fumosa Grt.

2293 fumeola Hamp. probata B. \& MeD.

2294 binotata $W l k$. extersa WIk.

a curvata Grt.

2295 adnixa Grt.

2296 pavise Behr. inconspicua $\mathrm{Sm}$.

2297 pausis $\mathrm{Sm}$.

2298 genetrix Grt.

2299 dilara Stkr.

2300 cara B. \& $M C D$.

2301 indirecta $\mathrm{Grt}$.

2302 catalina Sm.

2303 characts Grt.

$a$ erica $\mathrm{Sm}$.

2304 jocasta Sm.

2305 luteocinerea $\mathrm{Sm}$.

2306 susquesa $\mathrm{Sm}$.

2307 bultata Sm. 
2308 modica $G n$. subcedens Wlk.

2309 mustelina $S m$.

2310 ethnica $S m$.

2311 commoda Wlk. $a$ alberta $S m$.

2312 enigra $\mathrm{Sm}$. 2313 illustra $\mathrm{Sm}$.

2314 geminimacula Dyar.

2315 impulsa $G n$.

2316 mixta Grt.

Euplexia Steph.

2317 lucipara $L$.

2318 brillians $B . \& M c D$.

\section{Viridemas Sm.}

2319 galena $\mathrm{Sm}$.

2320 minuta $B$. \& $M c D$.

\section{Cropia W1k.}

2321 connecta Sm. striata Druce.

\section{Perigea Gn.}

2322 apameoides $G n$. $a$ icole Grt.

2323 xanthoides $G n$. $a$ enixa Grt.

2324 cupentia Cram. epopea Cram. confederata Grt. infelix $\mathrm{Gn}$.

2325 mersa Morr.

2326 albolabes Grt.

2327 vecors $G n$.

remissa Wlk. luxa Grt.

2328 orta B. \& McD.

2329 concisa Wlk. consocia Wlk. centralis Wlk.

2330 sutor $G n$. claufacta Wlk. fabrefacta Morr. cervina Sm.

2331 proxima Morr.

Oligia Hbn.

2332 violacea Grt.

2333 bridghami G. \& $R$.

2334 egens Wlk.

$a$ transfrons Neum.

2335 fractilinea Grt. form vulgivaga Morr. form modiola Grt. mactatoides B. \& McD. form una Stkr.

$a$ albescens $B . \& M c D$.

2336 arbora $B$. \& $M c D$.

2337 misera Grt.

2338 minuscula Morr.

2339 diversicolor Morr.

2340 semicana Wlk. latireptana Wlk. hausta Grt.

2341 exhausta $S m$. .

2342 tonsa Grt. $a b$. fasciata $B . \& M C l$. $a$ subjuncta $S m$.

$b$ lævigata $\mathrm{Sm}$.

2543 includens $W l k$. norma Morr. penita Morr. mariae Grt.

2343, 1 rubiginosa $W l k$.

Agroperina Hamn .

2344 dubitans Wlk. insignata Wlk. sputator Grt.

2345 cogitata $\mathrm{Sm}$.

2346 lateritia $H u f n$. 2347 obliviosa Wlk. 2348 satina $S t k r$. 2349 conradi Grt. 2350 citima Grt. 2351 morna Stkr. hulsti Grt. 
2352 pendina Snt.

2353 lineosa Sm.

2354 indela Sm.

2355 inficita Wlk.

$$
\text { belangeri Mort. }
$$

2356 lutosa $A n d r$.

2357 helva Grt.

Eremobia Steph.

2358 hilli Grt.

2359 claudens Wik: lencoscelis Grt. fibulata Morr.

a albertina Hamp.

2360 unicincta $\mathrm{Sm}$.

2361 tenera $\mathrm{Sm}$.

2362 alticola Sm.

2363 maillardi Gey.

a exulis Lef.

Tæniosea Grt.

2364 discivaria Wu.

perbellis Grt.

form gentilis Grt.

Macronoctua Grt.

2365 onusta Grt.

Sidemia Staud.

2366 longula Grt.

2367 devastator Brace.

abjecta $\mathrm{Gn}$.

ordinaria Wlk.

contenta Wik.

form speciosa Morr.

\section{Luperina Bdv.}

2368 stipata Morr.

2369 burgessl Morr.

2370 ona Sm.

2371 relicina Morr. migrate Sm.

2372 posticats Harv. veterata Sm.

2373 popofensis Sm.
2374 venose Sm.

2375 trigona $\mathrm{Sm}$.

2376 birnata Sm.

2377 extenss Sm.

2378 innota $S m$.

2379 obtusa Sm.

2380 passer $G n$.

incallida WIk.

loculata Mortr.

form conspicua Morr.

Trichoplexia Hamp.

2381 exornata Morsch.

2382 virguncula $S m$.

2383 contradicta Sm.

Phlogophora Tr.

2384 iris $G n$.

Chutapha Moore.

2385 periculosa $\mathrm{Gn}$.

form v-brunneum Grt.

Conservula Grt.

2386 anodonta $G n$.

Cobaliodes Dyar.

2387 angelica $S m$.

2388 franciscana $\mathrm{Sm}$.

2389 accurata $\boldsymbol{H} y$. Edw.

Callopistria Hbn.

2390 floridensis $G n$. strena Grt.

2391 mollissima Gn. mibicunda WIk.

2392 monetifera $\mathrm{G} n$.

2393 argentilines $W / k$.

2394 granitosa $\mathrm{Gn}$.

Acherdoa Wik.

2395 ferraria $W$ Wk.

ornata Neum. 
Fagitana Wlk.

2396 littera $G n$.

lucidata Wlk. niveicostatus Grt.

Phuphena Wlk.

2397 u-album $G n$. purpuripennis Grt. baliola Morr.

2398 obliqua $S m$.

Chytonix Grt.

2399 chlorostigma Harv. viridimusca $\mathrm{Sm}$.

2400 palliatricula $G n$. form iaspis $G n$.

2401 sensilis Grt.

2402 parvimacula $S m$.

2403 laticlava $S m$.

Harrisimemna Grt.

2404 trisignata $W l k$. sexguttata Harr.

Cerma Hbn.

2405 cora Hbn. festa $\mathrm{Gn}$.

2406 flavidior $B$. \& $M c D$.

2407 oaklandiæ $B . \& M c D$.

2408 nana $B . \& M c D$.

2409 sarepta Barnes.

2410 fascia $S m$.

2411 marina $S m$.

2412 cuerva Barnes.

2413 albipuncta $B$. \& McD.

2414 olivacea $S m$.

2415 galva Stkr.

Metachrostis Hbn.

2416 semifascia $\mathrm{Sm}$.

Polygrammate Hbn.

2417 hebræicum $\mathrm{Hbn}$.
Leuconycta Hamp.

2418 diphteroides $G n$.

form obliterata Grt.

Agriopodes Hamp.

2419 fallax $H$. $S$.

2420 geminata $\mathrm{Sm}$.

2421 tybo Barnes.

2422 viridata Harv.

2423 lepidula Grt.

$a$ avirida $S m$.

2424 teratophora $H$. S. inscripta Wlk.

2425 corticosa $G n$.

Acronycta Ochs.

2426 theodora Schaus.

2427 mansueta $S m$.

2428 vinnula Grt.

2429 paupercula Grt.

2430 lepetita $S m$.

2431 parallela Grt.

2432 albarufa Grt. walkeri And.

2433 connecta Grt.

2434 exilis Grt.

2435 modica $W l k$.

2436 ovata Grt.

2437 hæsitata Grt.

2438 pruni Harr.

. smithi Butl.

2439 marmorata Sm.

2440 fragilis $G n$.

spectans Wlk.

2441 minella Dyar.

2442 clarescens $G n$.

2443 tristis $S m$.

2444 inclara $\mathrm{Sm}$.

$a$ inconstans $S m$.

2445 liturata $\mathrm{Sm}$.

2446 brumosa $G n$.

$a$ persuasa Harv.

2447 superans $G n$. 
2448 subochrea firt.

2449 noctivaga live.

2450 afflicta $(i r t$. dolens Druce.

2451 hanamelis Gin.

2452 increta.$/ 0 r r$.

2453 retardata $W / k$. dissectu G. \& R.

.2454 casarea Sim.

2455 impleta WIk. lutricoma G. \& R.

2456 illita Sim.

2457 quadrata Grt.

2458 strigulata $\mathrm{Sm}$.

2459 thoracica Grt.

2460 lretifice Sm.

2461 hasta $G n$.

a telum $G n$.

2462 manitoba $S m$.

2463 furcifera $G n$.

2464 lobelix $G n$. grotei Butl.

2465 tritona $H b u$.

2466 elizabeta $S m$.

2467 falcula Grt.

2468 tartarea Sm.

2469 grisea Wlk.

pudorata Morr.

a revellata $S m$.

2470 radcliffei Harv.

2471 tota Grt.

2472 Puneralis $G$. \& $R$.

2473 morula $G$. \& $R$.

uni Harris.

2474 interrupta $G n$. occidentalis G. \& R.

2475 spinigera $G n$. harveyana Grt.

2476 felins Grt.

a cyanescens Hamp.

$b$ metra Sm. turpis $\mathrm{Sm}$.

e amicora Sm.

2477 frigida $S m$. pacifica Sm.
2478 similans Sm.

2479 tonitra Sm.

2480 lepusculina $G n$.

2481 cinderella $\mathrm{Sm}$.

2482 canadensis $\mathrm{Sm}$.

2483 populi Riley.

2484 transversata Sm.

2485 chionochron Hamp.

2486 leporina $L$. rulpina Grt. saneta $\mathrm{Hy}$. Edw.

2487 moesta Dyar.

2488 cretata $S m$.

2489 innotata $G n$. graef Grt. ab. griseor Dyar.

2490 betula Riley. 2491 americana Harris. acericola Gn.

a obscura $H y$. Edw.

$b$ eldora $S m$.

2492 dactylina Grt.

2493 insita Wlk.

a denvera $\mathrm{Sm}$.

2494 hesperida $S m$.

2495 hastulifera $A$. \& $S$.

2496 impressa $W l k$.

fasciata Wik. verrilli G. \& R.

a emaculata $S m$.

2497 distans Grt.

a dolorosa Dyar.

2498 rubricoma Gn.

2499 longa $G n$.

xylinoides Gn.

xyliniformis Gn.

pallidicoma Grt.

2500 extricata Grt.

2501 sperata Grt.

a speratina $\mathrm{Sm}$.

2502 lithospila Grt.

2503 gasta Stkr.

2504 othello Sm.

2505 edolata Grt.

2506 perdita Grt. 
2507 barnesi $S m$.

2508 oblinita $A$. \& S. salicis Harr.

2509 lanceolaria Grt.

2510 insolita Grt.

Merolonche Grt.

2511 lupini Grt.

2512 spinea Grt.

2513 ursina $S m$.

Simyra Oehs.

2514 henrici Grt.

form evanida Grt. form fumosa Morr.

Delta Saalm.

2515 ramosula $G n$.

2516 stewarti Grt.

Andropolia Grt.

2517 diversilineata Grt. illepida Grt. resoluta $\mathrm{Sm}$. form submissa $S m$.

2518 contacta Wlk.

aspera Morr.

diffusilis Harv.

a pulverulenta $\mathrm{Sm}$.

2519 pallifera Grt.

2520 sansar Stkr.

2521 ochracea $S m$.

2522 dispar $\mathrm{Sm}$.

2523 olorina Grt.

2524 ædon Grt.

2525 theodori Grt.

a epichysis Grt.

2526 olga $\mathrm{Sm}$.

2527 extincta $\mathrm{Sm}$.

2528 maxima Dyar.

2529 acera $S m$.

2530 lichena $B . \& M c D$.
Нурра Dup.

2531 xylinoides $G n$. contraria Wlk. ancocisconensis Morr.

2532 brunneicrista $\mathrm{Sm}$. 2533 indistincta $S m$.

Fota Grt.

2534 armata Grt.

2535 minorata Grt.

Oxycnemis Grt.

2536 advena Grt. baboquavaria Sm.

2537 fusimacula $S m$. 2538 orbicularis $B . \& M c D$. 2539 gustis $S m$.

2540 subsimplex Dyar.

2541 gracillima Grt. yита $\mathrm{Sm}$.

2542 acuna Barnes. adusta $\mathrm{Sm}$.

2543 grandimacula $B . \& M c D$. 2544 erratica B. \& McD.

Leucocnemis Hamp.

2545 sectilis $\mathrm{Sm}$.

2546 subtilis $B$. \& $M c D$.

2547 perfundis $S m$.

2548 nivalis $S m$.

2549 barbara $B$. \& $M c D$.

2549, 1 obscurella $B$. \& $M c D$.

Fala Grt.

2550 ptychophora Grt.

Crimona Sm.

2551 pallimedia $\mathrm{Sm}$.

Prothrinax Sm.

2552 luteomedia $\mathrm{Sm}$.

2553 ocellata $B . \& M c D$. 
Stilbia Steph.

2554 fotelloides B. \& MCD.

Copibryophila Sm.

2555 angelica $S m$.

Escaria Grt.

2556 clauda Grt.

Aleptina Dyar.

2557 inca Dyar.

a. texana $B$. \& $M c D$.

Prorachis Hamp.

2558 daria Druee.

Hadenella Grt.

2559 pergentilis $\mathrm{Grt}$.

Fotella Grt.

2560 notalis Grt.

2561 cylindrica Grt.

2562 olivia $B$. \& $M c D$.

2563 fragosa Grt. cervoides B. \& McD.

Catabena W/k.

2564 lineolata Wik.

- petrrea Wlk. miscellus Grt.

2565 sagittata $B . \& M c D$.

2565,1 pronuba $B$. \& $M c D$.

2566 vitrina Wlk.

terminella Grt. candida Sm.

2567 esula Druce.

Prodenia Gn.

2568 dolichos Fabr. commelinae A.\& $\mathbf{S}$. 2569 ornithogalli $G n$. lineatella Harv.
2570 eudiopta $G n$. Aavimedia Harv.

2571 profica Grt.

2572 latifascia $\boldsymbol{W} \mid k$.

2573 eridania Cram.

phytolnceas A. \& 8. derupta Morr.

nigrofascia Hlst.

Laphygma Gn.

2574 frugiperda A. \& S.

maera Gn.

inepta WIk.

signifera Wlk.

autumnalis Riley.

form fulvosa Riley.

form obscura Riley.

2575 exigua $\mathrm{Hbn}$.

Aavimaculata Harv.

Caradrina Ochs.

2576 triquetra Grt.

2577 multifera $W / k$.

fidicularia Morr.

2578 extima Wlk.

civica Grt.

2579 meralis Morr.

bilunata Grt.

2580 mona $B$. \& $M c D$.

2581 tarda $G n$.

prima $\mathrm{Sm}$.

2582 minuscula $B$. \& $M c D$.

2583 atrostriga $B$. \& $M c D$.

Acopa Harv.

2584 pacifica $H y$. Edw.

2585 carina Harv.

2586 perpallida $\mathrm{Grt}$.

2587 incana $H y . E d w$.

Platyperigea Sm.

2588 anotha Dyar.

2589 camina $\mathrm{Sm}$. 
Proxenus H. S.

2590 miranda Grt. a nitens Dyar.

2591 mindara $B$. \& $M c D$.

Galgula Gn.

2592 partita $G n$.

subpartita Gn.

ferruginea Wlk.

vesca Morr.

bias Druce.

mandane Druce.

form hepara $G n$.

interna Wlk.

hippotamada Druce.

Micrathetis Hamp.

2593 triplex Wlk. spilomela Wlk. conviva Harv. form subaquila Harv.

2594 costiplaga Sm.

Crambodes $\mathrm{Gn}$.

2595 talidiformis $G n$. conjungens Wlk.

2595, 1 lunata $B$. \& $M c D$.

Platysenta Grt.

2596 temecula Barnes.

2597 discistriga $\mathrm{Sm}$.

2598 videns $G n$.

$$
\begin{aligned}
& \text { indigens Wlk. } \\
& \text { atriciliata Grt. } \\
& \text { meskei Speyer. } \\
& \text { a albipuncta Sm. }
\end{aligned}
$$

Gonodes Hamp.

2599 liquida Moesch.

violascens Schaus.

leada Druce.

Balsa Wlk.

2600 malana Fitch. obliquifera Wlk.
2601 tristrigella $W l k$. zelleri Grt.

2602 labecula Grt.

\section{Monodes Gn.}

2603 fuscimacula $\mathrm{Grt}$.

2604 nucicolora $G n$. unisignata Wlk. paginata Morr. form clara Harv.

2605 agrotina $G n$. trientiplaga Wik.

2606 versicolor Grt. 2607 chalcedonia $\mathrm{Hbn}$. arna Gn. expuncta Wlk. vincta Wlk. irresoluta Wlk. tracta Grt.

2608 festivoides $G n$. cephalica Butl. form varia Wlk.

2609 exesa $G n$. floridana Wlk.

2610 ensina Barnes. 2611 grata $H b n$. rasilis Morr. subusta Druce.

Hypenopsis Dyar. 2612 macula Druce.

Menopsimus Dyar. 2513 caducus Dyar. fractilinea $\mathrm{Sm}$.

Ruacodes Hamp. 2614 tela $\mathrm{Sm}$.

Xylomœa Staud. 2615 didonea $\mathrm{Sm}$. Namangana Staud. 2616 lætabilis $S m$. 2617 licentiosa $S m$. 
2618 continens $H y . E d w$. a tapeta $\mathrm{Sm}$.

2619 suffusa B. $M c D$. a rubida $B$. \& $M c D$.

2620 perolivalis $B$. \& $M c D$.

2621 niveirena Harv.

2622 albimacula B. \& MCD.

2623 texans $S m$. a consors $\mathrm{Sm}$.

2624 variabilis $B$. \& MCD.

2625 epipaschia Grt.

2626 preacuta $S m$.

2527 canoa Barnes.

2628 vulnerea Grt.

2629 leucorena $S m$.

2630 andrena $S m$.

2631 morsa Sm.

2632 begallo Barnes.

2633 egestis Sm. abalas Sm.

Helotropha Led.

2634 caduca Grt.

2635 retis Grt.

2636 reniformis Grt.

form atra Grt.

Apamea Ochs.

2637 velata Wik. sera G. \& R.

2638 nictitans $L$.

a americana Speyer. lusea $\mathrm{Sm}$. atlantica $\mathrm{Sm}$.

$b$ interoceanica $S m$.

c pacifica $S m$.

2639 senilis Sm.

2640 lunata $S m$. albilunnta $\mathrm{Sm}$.

2641 erepta Grt. a ryensis Bird.

Achatodes Gn.

2642 zexe Harris. sandix Gn.
Xantbocia Hamp.

2643 buffaloensis Grt. latia Stkr. form simplicissima Bird.

Gortyna Ochs.

2644 serrata Grt. a ochrimacula $B$. \& $M C D$.

2645 repleta Bird.

2646 pallescens $S m$.

2647 medialis $\mathrm{Sm}$.

2648 obliqua Harv.

2649 immanis $G n$.

2650 micacea $E$ sp.

2651 perobliqua Hamp.

2652 stramentosa $\mathrm{Gn}$. diplocyma Hamp.

2653 juvenilis Grt.

Rhodoecia Hamp.

2654 aurantiago $G n$. illiterata Grt. differta Morr. illinoisensis French.

Pyrrhia Hbn.

2655 umbra $H u f n$.

a experimens $W l k$. angulata Grt.

$b$ stilla Grt.

Erythrocia Hamp.

2656 suavis $H y . E d w$.

Emboløcia Hamp.

2657 sauzalitze Grt.

Papaipema Sm.

2658 cerina Grt.

2659 appassionata Harv.

2660 stenoscelis Dyar.

2661 inquasita $G . \& R$.

2662 speciosissima $G$. \& $R$.

2663 marginidens $G n$.

birdi Dyar. 
2664 baptisir Bird.

2665 nephrasyntheta Dyar.

2666 nepheleptena Dyar. moeseri Bird.

2667 furcata $S m$.

2668 circumlucens $\mathrm{Sm}$.

2669 humuli Bird.

2670 rutila $G n$.

2671 arctivorens Hamp.

2672 ochroptena Dyar.

2673 insulidens Bird.

2674 merriccata Bird.

2675 angelica $S m$.

2676 harrisi Grt.

ab. rubiginosa Bird.

2677 astuta Bird.

2678 verona $S m$.

2679 anargyrea Dyar.

2680 erubescens Bird.

2681 impecuniosa Grt.

2682 limata Bird.

2683 purpurifascia $G$. \& $R$.

2684 lysimachir Bird.

2685 pterisii Bird.

triorthia Dyar.

2686 cataphracta Grt.

$a b$. fluxa Bird.

2687 duovata Bird.

2688 imperspicua Bird.

2689 rigida Grt.

2690 unimoda $\mathrm{Sm}$.

2691 errans $B . \& M c D$.

2692 aweme Lyman.

2693 nelita Stkr. form linda Bird.

2694 sciata Bird.

2695 frigida $S m$. perobsoleta Lyman. form thalictri Lyman.

2696 cerussata Grt.

2697 limpida $G n$.

aerata Lyman.

2698 nebris $G n$.

form nitela $G n$.
2699 duplicata Bird.

2700 necopina Grt.

imperturbata Bird.

2701 maritima Bird.

2702 silphii Bird.

2703 eupatorii Lyman.

Ogdoconta Butl.

2704 cinereola $G n$. atomaria Wlk.

2705 carneola Sm. 2706 moreno Barnes. 2707 altura Barnes. 2708 sexta $B$. \& McD. 2709 tacna Barnes.

Selicanis Sm.

2710 cinereola $S m$.

Stibadium Grt.

2711 spumosum Grt.

2712 mavina $B$. \& $M c D$.

2713 aureolum $\boldsymbol{H} y$. $E d w$.

2714 manti Barnes.

2715 ochoa Barnes. dolli Sm.

2716 olvello Barnes.

2717 curiosum Neum.

2718 navium Harv.

Emarginea Gn.

2719 percara Morr.

2720 quadrate $S m$.

2721 ochracea $S m$.

2722 pallida $S m$.

Bryolymnia Hamp.

2723 viridimedia $\mathrm{Sm}$.

Ipimorpha Hbn.

2724 pleonectusa Grt. aequilinea Sm.

2725 nanaimo Barnes.

2726 subvexa Grt.

2726-1 viridipallida $B . \& M C D$. 
Trichocosmia Girt.

2727 inornata $\mathrm{Grt}$. form demacula sirand. 2728 drasteroides Sm.

Bagisara Wlk.

2729 subusta Hbn.

a inusta Gn.

2730 rectifascia Grt.

2731 buxea Grt.

2732 delicia Dyar.

Elydna Wlk.

2733 tristicts Hamp. amoratu Barnes.

2734 gulnare Stkr.

Cosmia Orehs.

2735 orina $G n$.

canescens Behr.

form calami Harv.

Cirrhophanus Grt.

2736 triangulifer Grt. pretiosa Morr. .

2737 duplicatus $\mathrm{Sm}$.

2738 papago Barnes.

2739 dyari Ckll.

Basilodes Gn.

2740 pepita $G n$. chrysopasa Wlk.

2741 chrysopis Grt.

2742 eatharops Dyar.

2743 mirabilis Neum.

Chamæclea Grt.

2744 pernana Grt. 2744, 1 basiochrea $B$. \& $M c D$.

Lythrodes Smith.

2745 radiatus $S m$.

2746 venatus $S m$.

2747 tripuncta $B . \& M c D$.
Hoplolythra Hamp.

2748 dimcistriga $S m$.

2749 arivaca Barnes.

Chalcopasta Hamp. 2750 territans $H y . E d w$. arizona French.

2751 howardi $H y$. Edw. ornata Ottol.

2752 fulgens $B$. $M c D$. 2753 koebelei Riley.

Neumoegenia Grt.

2754 poetica Grt. smithi Druce. sagittalba Ottol.

2755 albavena Ottol. 2756 coronides Druce. pendula Ottol.

Enargia Hbn.

2757 decolor Wlk.

form infumata Grt. punctirena Sm.

Derrima W'lk.

2758 stellata $W l k$.

form henrietta Grt. form cinocentralis Strand.

Stiria Grt.

2759 rugifrons Grt. 2760 sulphurea Neum. 2761 consuela Stkr. 2762 hutsoni Sm.

fuliginosa $\mathrm{Sm}$. 2762,1 olivalis $B$. \& $M c D$.

Stiriodes Hamp.

2763 obtusa H. S. obtusula Zell.

2764 perflava Harv. 2764, 1 vivida $B$. \& $M C D$. 
Polenta Morr.

2765 tepperi Morr. richi Grt.

2766 gladiola Barnes.

Plagiomimicus Grt.

2767 pityochromus Grt. media Morr.

2768 triplagiatus $\mathrm{Sm}$. 2769 expallidus Grt.

Nocloa Sm.

2770 plagiata $S m$.

2771 rivulosa $\mathrm{Sm}$. 2772 pallens Tepper. nesaea $\mathrm{Sm}$.

2773 cordova Barnes.

2774 nanata Neum. macula Sm.

2775 pilacho Barnes.

2776 aliaga Barnes.

2777 dissimilis $B$. \& $M c D$.

2778 contrasta $B$. \& $M c D$.

2778,1 torniplaga $B . \& M c D$.

Oslaria Dyar.

2779 viridifera $G r t$.

2780 pura $B$. \& $M c D$.

2781 diffusa Barnes.

Perania B. \& MeD.

2782 dissociata $B . \& M c D$.

Minofala Sm.

2783 instans $S m$.

Arzama Wlk.

2784 obliqua Wlk. obliquata Grt.

2784, 1 brehmei $B . \& M c D$. 2785 densa Wlk.

oecogenes Dyar.

2786 gargantua Dyar. 2787 anoa Dyar.
Bellura Wlk.

2788 gortynoides Wlk. vulnifica Grt.

2789 melanopyga Grt.

2790 diffusa Grt.

Archanara Wlk.

2791 oblonga Grt. permagna Grt. subcarnea Kell.

2792 subflava Grt. 2793 alameda $\mathrm{Sm}$. 2794 læta Morr.

Cea Grt.

2795 immacula Grt. 2796 colorada Sm. leucanidia Hamp.

2797 cirphidia Hamp.

Calamia Hbn.

2798 inquinata $G n$. orientalis Grt.

2799 orphnina Dyar.

2800 variana Morr.

2801 defecta Grt.

Hypocœna Hamp.

2802 rufostrigata Pack. punctivena Sm.

Amolita Grt.

2803 fessa Grt. 2804 obliqua $\mathrm{Sm}$. 2805 roseola $\mathrm{Sm}$. 2806 fratercula $B$. \& $M c D$. 2807 delicata $B . \& M c D$.

Cilla Grt.

2808 distema Grt.

Senta Steph.

2809 enervata $G n$. fodiens $\mathrm{Gn}$. 
Redingtonia B. \& MED. 2810 albn B. \& MeD.

Aleptinoides B. \& MCD.

2811 ochren B. \& MCD.

Ommatostola Grt.

2812 lintneri Grt.

Luceria Hein.

2813 tranquilla Grt. sambuci Behr. form viridula Grt.

Northecophora sm.

2814 pulverea Sm.

Azenia Grt.

2815 implora Grt.

2816 edentata Grt.

Antaplaga Grt.

2817 atrolinea $B$. \& $M C D$.

2818 composita Hy. Edw.

2819 dimidiata Grt.

2820 hachita Barnes.

2821 sexseriata Grt.

2822 biundulalis Z $Z$ ell.

2823 thoracica $\boldsymbol{H} y$. Edw.

Tristyla $\mathrm{Sm}$.

2824 alboplagiata $S m$.

Triocnemis GM.

2825 saporis Grt.

\section{Xerociris Ckll.}

2826 wilsoni Grt.

Euthisanotia IIhn.

2827 grata $\mathrm{Fabr}$ : assimilis Bdv. 2828 brevipennis Stretch. 2829 unio $\mathbf{H b n}$.
Gerra Wik.

2830 sevoras Grt. aedesaa Druce.

Gerrodes IIamp. 2831 longipes Druce.

Psychomorpha Harila.

2832 epimenis Dru. 2832, 1 euryrhoda Hamp.

Microhelia Hamp.

2833 angelica Sm.

2834 restrictalis $\mathrm{Sm}$. ab. immacula Strand.

Heliothodes Hainp.

2835 diminutiva Grt. form suffusana Strand. form macromacula Strand.

form bifida Strand.

2836 fasciata $H y$. Edw.

Eutricopis Morr.

2837 nexilis Morr. elaborata Hy. Edw.

Baptarma Sm.

2838 felicita $S m$.

Palada Sm.

2839 scarletina $S m$.

Xanthothrix Hy. Edw.

2840 neumoegeni $H y . E d w$.

2841 ranunculi $H y$. $E d w$.

Axenus Grt.

2842 arvalis Grt.

form ochraceus $\mathrm{Hy}$. Edve. form amplus $\mathrm{H} y$. Edw. 
Annaphila Grt.

2843 arvalis $\boldsymbol{H} y$. Edw. salicis Hy. Edw.

2844 danistica Grt.

2845 mera Harv.

2846 domina $H y . E d w$. 2847 pustulata $\boldsymbol{H} y$. $E d w$.

2848 divinula Grt.

2849 germana $H y$. Edw.

2850 decia Grt.

amicula Hy. Edw.

2851 depicta Grt.

2852 lithosina $H y$. Edw. variegata $\mathrm{Sm}$.

2853 miona $\mathrm{Sm}$.

2854 diva Grt. form yosemitensis Strand. Sigela Hulst.

2855 casta $H y . E d w$.

2856 superba $H y$. Edw.

Pseudacontia Sm.

2857 louisa $S m$.

2858 crustaria Morr.

2859 cansa $\mathrm{Sm}$.

2860 aterrima Grt.

2861 groteana Dyar.

2862 anxia $\mathrm{Sm}$.

2863 cephalica $S m$.

2864 basifugens Dyar.

Copanarta Grt.

2865 aurea Grt.

$a$ nigerrima $S m$.

2865,1 sexpunctata $B . \& M c D$.

Erastriinæ

Eupseudomorpha Dyar.

2866 brillians Neum.

Cydosia Westw.

2867 nobilitella Cram.

2868 aurivitta $G . \& R$.

form imitella Stretch.

2869 majuscula $H y$. Edw.
Acidaliodes Hamp.

2870 eoides $B$. \& $M c D$.

Proroblemma Hamp.

2871 testa $B$. \& $M c D$.

Eublemma Hbn.

2872 minima $G n$. carmelita Morr.

2873 cinnamomea $H . S$. laphyra Druce.

2874 obliqualis Fabr. flammicineta Wlk. patula Morr. patruelis Grt.

Pseuderaspedia Hamp.

2875 penumbrata Hlst.

2876 basipunctaria Wlk. melanosticta Hamp.

Homocerynea B. \& MeD. 2877 cleoriformis $B . \& M c D$.

Phobolosia Dyar.

2878 anfracta $\boldsymbol{H} y$. $\boldsymbol{E} d w$. reincarnata Dyar.

2879 brimleyana Dyar. 2879,1 bilineata $B . \& M c D$.

Cobubatha Wlk.

2880 limbata $\boldsymbol{H} y$. $\boldsymbol{E} d w$. anaea Druce.

2881 flavofasciata Grt. versutus $\mathrm{Hy}$. Edw.

2882 luda Druce.

2883 balteata $\mathrm{Sm}$.

2884 dimidata $S m$.

2885 luxuriosa $\mathrm{Sm}$.

2886 quadrifera $Z$ ell. catiena Druce.

Ozarba Wlk.

2887 æria Grt. 
Amyna Gn.

2888 bullula Grt.

2889 acto $\mathrm{Gn}$. orbica Morr. tecta Grt.

Argillophora Grt.

2890 furcilla Grt.

Chamyris Gn.

2891 cerintha Treit.

Erastroides Hamp.

2892 propera Grt.

Paracretonia Dyar.

2893 aleptivoides B. \& McD. xithon Dyar.

Lithacodia Hbn.

2894 synochitis G. \& $\boldsymbol{R}$. 2895 albidula $G n$. intractabilis Wlk. cretiferana Wlk.

2896 parvimacula Grt. 2897 concinnimacula $G n$. 2898 bellicula $\boldsymbol{H b n}$. semichalcea Wlk.

2899 musta $G$. \& $R$.

2900 carneola $G n$. biplaga Wik.

2901 apicosa Hax. nigritula $\mathrm{Gn}$. undulifera Wlk.

2902 muscosula $G n$. 2903 distincta Grt.

Amiana Dyar.

2904 niama Dyer.

Cryphia Hhn.

Hyperstrotia Hamp.

2905 nana Hbn. aetheria Grt.
Erastria Ochs.

2906 lixiva Grt.

2907 basicinerea Grt.

2908 antonita Dyar.

2909 orthozona Hemp. santa rita Dyar.

2910 dividua Grt. form opipara $H y . E d w$.

2911 catilina Druce.

2912 cnossia Druce.

2913 flaviguttate Grt.

2914 secta Grt.

Cerathosia Sm.

2915 tricolor $\mathrm{Sm}$.

Diastema Gn.

2916 tigris $G n$.

lineata WIk.

Exyra Grt.

2917 rolandiana Grt.

2918 fax Grt.

2919 ridingsi Riley.

nigrocaput Morr.

2920 semicrocea $\mathrm{Gn}$. form hubbardiana $D_{y / a r}$.

Homolagoa B. \& MeD.

2921 grotelliformis B. \& MeD.

Phonicophanta Hamp. 2921, 1 bicolor B. \& $M c D$.

Xanthoptera Gn.

2922 nigrofimbria $\mathrm{Gn}$.

Heliocontia Hamp.

2923 apicella Grt.

truncatula Zell. accepta Hy. Edw. $a b$. obliquella Strand.

2924 margana Fabr. subapicana Wlk.

\& inorata Grt.

\& sordida Grt. 


\section{Spragueia Grt.}

2925 idella Barnes.

2926 guttata Grt.

2927 funeralis $G r t$.

2928 magnifica Grt.

2929 onagrus $G n$.

2930 leo $G n$.

2931 dama $G n$.

trifariana Wlk.

transmutata Wlk. pardalis Grt.

2932 jaguaralis Hamp.

2933 obatra Morr.

plumbifimbriata Grt. velata Stkr.

Græperia Grt.

2934 tripartita $S m$. mediatrix Dyar.

2035 sutor Hamp.

2936 sutrix $\mathrm{Grt}$.

2937 nuicola $\mathrm{Sm}$.

carcharodonta Hamp. form meskei $\mathrm{Sm}$.

2938 altera $S m$. conocharodes Hamp.

2939 megocula $\mathrm{Sm}$.

2940 costalis Wlk. magnifica Neum.

2941 indubitans $W l k$. citrina Druce.

Fruva Grt.

2942 fasciatella Grt.

Conochares Sm.

2943 acuta $S m$.

2944 catalina $\mathrm{Sm}$.

2945 elegantula Harv.

semiopaca Grt.

2946 arizonæ $H y . E d w$. seminivealis Hlst. interrupta $\mathrm{Sm}$.

2947 hutsoni Sm.
Tarachidia Hamp.

2948 parvula Wlk. georgica Grt.

2949 bicolorata $B . \& M c D$.

2949, 1 albitermen B. \& $M c D$.

2950 tortricina $Z$ ell. form obsoleta Grt. form modesta $H y$. Edw. form deleta $\boldsymbol{H} y$. Edw.

2951 fumata $\mathrm{Sm}$.

2952 erastrioides $G n$.

2953 nannodes Hamp.

2954 tenuicula Morr. phecolisca Druce.

2955 libedis $S m$.

2956 candefacta $\mathrm{Hbn}$. debilis Wlk.

a neomexicana $\mathrm{Sm}$.

2957 huita $\mathrm{Sm}$.

2958 virginalis Grt.

2959 binocula Grt.

2960 tenuescens $S m$.

2961 cuta $S m$.

2962 alata $S m$.

2963 venustula $W l k$.

discoidalis Wlk.

$q$ fortunata Grt.

of perita Grt. subcitrinalis Hlst.

2964 clausula Grt.

2965 semiflava $G n$.

2965, 1 albimargo $B . \& M c D$.

Neptunia B. \& MeD.

2966 pulchra $B . \& M c D$.

Euaontia B. \& McD.

2967 semirufa $B . \& M c D$.

2967, 1 clarki $B . \& M c D$.

Conacontia Sm.

2968 huachuca $S m$.

2969 flavicosta $S m$.

2970 orba $\mathrm{Sm}$.

2971 angustipennis Grt. 
Trichotarache Grt.

2972 assimilis Grt.

Tarache IIbn.

2973 lactipennis Harv.

2974 expolita Grt.

2975 lucasi Sm.

d pima $\mathrm{Sm}$.

\& aniluna Sm.

2976 tetragona Wlk.

\& quadriplnga Sm.

2977 major Sm.

\& alessandra Sm.

2978 Janceolata Grt.

2979 disconnecta Sm.

2980 sedata $\boldsymbol{H}$ y. Edw.

gonella Stkr. niveicollis $\mathrm{Sm}$.

a cacola $\mathrm{Sm}$.

2981 areloides $B$. \& $M C D$.

2982 areli Stkr.

2989 aprica $H b n$. biplaga $\mathbf{G n}$.

a ceyvestensis Dyar.

2984 abdominalis Grt.

2985 favipennis Grt.

2986 arida $S m$.

2987 semiatra Sm.

2988 acerba $\boldsymbol{H} y$. $\boldsymbol{E} d w$.

2989 terminimaculata Grt.

2990 dacia Druce.

2991 behri Sm.

\& curvilinea B. \& McD.

2992 delecta Wlk.

metallica Grt.

2993 cretata $G$. \& $R$.

form neocula $\mathrm{Sm}$.

form schwarzi Sm.

2994 coquilletti $\mathrm{Sm}$.

2995 eudryada $\mathrm{Sm}$.

2996 dorneri $\boldsymbol{B}$. \& $\mathrm{McD}$.
Euteliina

Eutelia IIbn.

2997 pulcherrima Grt. dentifera WIk.

2998 furcata Wlk. distracta WIk.

Marathyssa Wlk.

2999 basalis Wlk. ventilator Grt.

3000 inficita Wlk. histrio Grt.

A on Veum.

3001 noctuiformis Neum.

Pæctes Hhn.

3002 oculatrix $G n$.

3003 flabella Grt.

3004 burserve Dyar.

3005 pygmæa $\boldsymbol{H b n}$. abrostolella WIk. praepilata Grt.

3006 declinata Grt.

3007 abrostoloides $\mathrm{Gn}$. producta Wlk.

3008 delineata $G n$.

3009 acutangula Hamp.

3010 nubifera Hamp.

Sarrothripina

Characoma W1k.

3011 nilotica Rogenh.

chamaeleon Moesch.

proteella Dyar.

Sarrothripus Curtis.

3012 revayana Scop.

a lintnerana Speyer.

$b$ columbiana $\boldsymbol{H} y$. Edw.

c cinereana $N . \& D$. 
Casandria Wlk. Lussa Grt.

3013 abseuzalis Wlk. nigroguttata Grt. smithi Moesch. tumidicosta Hamp.

3014 filifers Wlk. inflexa Morr.

Baileya Grt.

3015 doubledayi $G n$. 3016 ophthalmica $G n$. 3017 australis Grt. 3018 dormitans $G n$. latebricola Grt. 3019 levitans Sm.

\section{Catocalinæ}

Catocala Schrank.

3020 innubens $G n$.

\& hinda Frch.

$a b$. flavidalis Grt.
3021 piatrix Grt.

a dionyza $\boldsymbol{H} y, E d w$.

3022 consors $A$. \& S.

3023 epione Drur.

3024 muliercula $G n$. $a b$. peramans $\mathrm{Hlst}$. 3025 antinympha $\mathrm{Hbn}$. affinis West. melanympha $\mathrm{Gn}$.

3026 coelebs Grt.

3027 badia $G$. \& $R$.

form phœbe $H y . E d w$.

5028 habilis Grt.

if basalis Grt.

S029 denussa $E h r$.

3030 serena $E d w$.

3031 robinsoni Grt.

form curvata Frch.

form missouriensis

Schwarz.
3032 judith Stkr. levettei Grt.

3033 flebilis Grt.

3034 angusi Grt. ㅇ edna Beut. form lucetta $H y$. $E d w$.

3035 obscura Stkr. simulatilis Grt.

3036 residua Grt.

3037 sappho Stkr.

3038 agrippina Stkr. form subviridis Harv. barnesi Frch.

5039 retecta Grt. form luctuosa Hlst.

3040 dejecta Stkr. 3041 insolabilis $G n$. 3042 vidua $A$. \& $S$. desperata $\mathrm{Gn}$.

3043 viduata $G n$. maestosa Hlst. moderna Grt. guenei Grt.

3044 lacrymosa $G n$.

form ulalume Stkr. form evelina Frch. emilia Hy. Edw. form zelica Frch. form paulina $H y$. $E d w$. 3045 palæogama $G n$. form annida Fager. form phalanga Grt.

3046 nebulosa $E d w$. ponderosa Grt.

3047 subnata Grt. 3048 neogama $A$. \& $S$. communis Grt. a snowiana Grt. 3049 euphemia Beut. 3050 aholibah Stkr. $a$ coloradensis Beut. 3051 ilia Cram. obsoleta Worth. duplicata Worth. 
ambrose Worth. confusa Worth. decorata Worth.

form conspicua Worth. uxort $\mathrm{Gn}$.

a zoe Belir.

ogruluta Hlst.

.3052 cerogama ( $\mathrm{m}$.

aurella Fisch.

elizu Fisch.

form bunkeri Grl.

3053 relicts WIk.

bianca Hy. Edw.

form phrynia $\mathrm{H} y$. Edw. form clara Beut.

a elda Behr.

3054 marmorata Edu.

3055 parta $G n$.

perplexa Stkr.

petulans Hlst.

3056 luciana Stkv.

nebrnskae Dodge.

form somnus Dodge.

3057 verecunda $\boldsymbol{H}$ lst. form diantha Beut.

3058 irene Behr.

- form virgilia $H y$. Edw.

a valeria $\boldsymbol{H}$ y. Edw.

$b$ volumnia $H y$. Edw.

3059 allusa $H / s t$.

frenchi Poling.

3060 faustina Stkr.

form zillah Stkr.

form carlota Beut.

form Iydia Beut.

a crerules Beut.

3061 cleopatra Stkr. perdifa Stkr.

3062 hermia Hu. Edw.

3063 californica $E d u$.

marianat Stkr.

edvardsi Kus.

eldoradensis Beut.
3064 francisea $H_{U}$. Edw.

3065 briseis Edw.

form albida Beut.

3066 grotiana Beiley.

3067 meskei Grt. rosalinda $\mathbf{H y}$. Edw.

3068 unijuga WIk.

Incilla Worth.

form agatha Beut.

ab. fletcheri Beut.

form beaniana Grt.

3069 semirelicts Grt.

3070 pura Hlst.

3071 nevadensis Beut.

form montana Beut.

3072 texans Frch.

3073 junctura $W / k$. uralshi Edw.

3074 julietta Frch.

3075 arizonse Grt.

form babavaga Stkr.

3076 jessica $H y$. Edw.

3077 electilis WIk.

caseandra Hy. Edw,

3078 hippolyts Stkr.

3079 stretchi Behr.

portia Hy. Edw.

form sierre Beut.

3080 aspasia Stkr.

a augusta $\boldsymbol{H} y \cdot \mathbf{E} d w$.

b sara Frch.

3081 cara $G n$.

a carissima Hlat. sulvia Hy. Edw.

3082 concumbens Wlk.

ab. hilli Grt.

ab. diana $H y . E d u$.

3083 amatrix $H b n$. nurus Wlk.

form selecta WIk.

$a$ editha $E d w$.

form pallida Poling. 
3084 delilah Stkr. adoptiva Grt. ab. calphurnia $H y . E d w$. $a$ desdemona $\boldsymbol{H} y . E d w$.

3085 andromache $\boldsymbol{H} y . \mathbf{E} d w$. 3086 frederici Grt. 3087 chelidonia Grt. 3088 illecta Wlk. magdalena Stkr.

3089 abbreviatella Grt. 3090 nuptialis $W l k$. myrrha Stkr.

3091 whitneyi Dodge. 3092 amestris Stkr. anna Grt. form westcotti Grt.

3093 messalina $G n$. belfragiana Harv. jocasta Stkr.

3094 gracilis $E d w$.

form sordida Grt.

3095 andromedæ $G n$. tristis Edw.

3096 herodias Stkr.

3097 coccinata Grt.

a circe Stkr.

$b$ sinuosa Grt.

3098 verrilliana Grt.

$a b$. wernerj Bied.

$a b$. votiva $H$ lst.

$a$ beutenmuelleri $B . \& M c D$. 2099 violenta $\boldsymbol{H} y$. $\boldsymbol{E} d w$.

ㅇ chiricahua Poling.

3100 ophelia $H y$. Edw.

a dolli Beut.

3101 miranda $H y$. Edw.

3102 orba Kus.

3103 ultronia $\mathrm{Hbn}$. mopsa Hy. Edw. form lucinda Beut. form adriana $\mathrm{H} \%$. $\mathrm{Edw}$. form celia $H y$. Edw.

3104 cratægi Saund.

form pretiosa Lint.
3105 mira Grt.

3106 grynea Cram. polygama Gn. nuptula Wlk. $a b$. constans Hulst. 3107 præclara G. \& $R$. 3108 manitoba Eeut. 3109 blandula Hlst. 3110 alabamæ Grt. 3111 olivia $H y$. Edw.

3112 titania Dodge.

:113 dulciola Grt.

3114 clintoni Grt.

3115 similis $E d w$. formula G. \& R. form aholah Stkr. form isabella $\boldsymbol{H} y$. $\mathrm{E} d w$.

3116 minuta $E d w$. form parvula $E d w$. form mellitula Hlst.

3117 micronympha $\mathrm{Gn}$. form fratercula $G$. \& $R$. jacquenetta Hy. Edw. timandra Hy. Edw. helene Pilate. form gisela Meyer. form hero $\boldsymbol{H} y$. Edw. form atarah Stkr. ouwah Poling.

3118 cordelia $H y$. Edw. amasiał A. \& S.

3119 connubialis $\mathrm{Gn}$. sancta Hlst. virens Frch.

3120 amica $\mathrm{Hbn}$. lineella Grt. form androphila $G n$. form nerissa $H y . E d w$. form suffusa Beut.

3121 jair Stkr.

\section{Euparthenos Grt.}

3122 nubilis $\mathrm{Hbn}$. 8 fasciata Beut. $a$ apache Poling. 
Allotria Hbn.

3128 elonymphn Hbn.

Parallelia Hbn.

3124 consobrina $G n$. redditura Wlk.

3125 similis $G n$. concolor Grt. form apicalis $G n$.

3126 smithi $G$ n.

3127 bistriaris $\mathrm{Hbn}$. amplissima WIk.

Euclidimera Hamp.

3128 annera $H y$. Edw. conspicus Sm.

3129 crerulea Grt. aquamarina Feld. livida Letcher.

Euclidia Ochs.

3130 euspidea Hbn.

Cænurgia Wlk.

3131 convalescens $\mathrm{Gn}$. purgata Wlk. socors Wik.

3132 togataria Wlk. adversa Grt.

3133 intercalaris Grt. dyari $\mathrm{Sm}$.

3134 diagonalis Dyar.

3135 erechtea Cram. patibilis Wlk. narrata Wlk. agricala $\mathrm{G}$. \& $\mathrm{R}$. mundula G. \& R. 3136 erassiuscula Haw. erichto $\mathrm{Gn}$. sobria Wlk. form ochrea Grt. 3137 distincts Neum.
Pelamia Gn.

3138 marcida Gn. perlats Wlk.

3139 texana Morr. hexastylus Harv。

3140 disseverans $W l k$. acuta Wlk.

$31 \$ 1$ munda Wlk.

3142 latipes $G n$. $a b$. indentata Harv.

Phurys An.

3148 vinculum $G n$. 3144 lima $G n$. obversa Wlk. dissocians Wlk.

3145 herbarum $G n$. bifasciata Bates.

3146 bistrigata $\mathrm{Hbn}$.

3147 ovalis Grt.

3148 flavistriaria $\mathrm{Hbn}$. perplexa $\mathrm{Gn}$. perspieua Wik. glans Grt.

3149 carolina Sm.

3150 bucetum Grt. campanilis Sm.

Doryodes $\mathrm{Gn}$.

3151 bistrialis Gey. acutaria H. S.

3152 spadaria $G n$. divisa Wlk. promptella Wlk.

Celiptera Gn.

3153 frustulum $G n$. elongatus Grt. discissa Wik.

Focillidia Hamp. 3154 texana Hamp. 
Argyrostrotis Hbn.

3155 pacalis $W l k$. irrorata Grt.

3156 diffundens Wlk.

3157 herbicola $G n$.

3158 sylvarum $G n$.

3159 erasa $G n$.

3160 contempta $G n$. placata Grt.

3161 deleta $G n$.

3162 obsoleta Grt.

3163 quadrifilaris $H b n$.

3164 anilis $D r u$. sesquistriaris $\mathrm{Hbn}$.

3165 revoluta Wlk,

Cutina Wlk.

3166 albopunctella $W l k$. strigulataria Sm.

Safia $\mathrm{Gn}$.

3167 amella $G n$. integervima Wlk. stylobata Harv.

3168 bilineata $\mathrm{Sm}$.

Zale $\mathrm{Hbn}$.

3169 exhausta $G n$. vernifera Wlk.

3170 viridans $G n$. viridisquama Wlk.

3171 fictilis $G n$. guadulpensis Gn. gradata Wlk.

3172 posterior Wlk.

3173 sexplagiata $W l k$.

3174 salicis Behr. rosae Behr.

3175 lunata Dru. of edusa Dru. putrescens Guer. form saundersi Beth.
3177 declarans $W l k$.

우 uniformis Morr.

3178 galbanata Morr.

3179 edusina Harv. ㅇ atritincta Harv.

3180 æruginosa $G n$. plenipennis Wlk.

3181 undularis Dru. nigricans Beth.

form umbripennis Grt.

3182 coracias $G n$. cornix Gn. cinerea Morr.

3183 insuda $S m$. 3184 minerea $G n$. involuta Wlk. $a b$. albofasciata Beth. $a$ norda $S m$.

3185 lunifera $H b n$. lineosa Wlk.

3186 penna Morr. 3187 benesignata Harv. a largera $\mathrm{Sm}$.

3188 duplicata Beth. 3189 helata $S m$. 3190 bethunei $S m$. 3191 squamularis Dru. 3192 obliqua $G n$. $a$ metata $S m$. $b$ curema $S m$. 3193 rubiata $S m$. 3194 rubi $H y$. Edw. 3195 yavapai $\mathrm{Sm}$. 3196 colorado $\mathrm{Sm}$. 3197 cingulifera $W l k$. intenta Wlk. woodi Grt.

3198 calycanthata $A . \& S$. 3199 horrida $H b n$.

Eubolina Harv. 3200 impartialis Harv.

3176 unilineata Grt. 
Matigramma Grt.

3201 pulverilinea Grt. 3202 rubrosuffusa $\mathrm{Grt}$. 3203 lsena Grt.

3204 metaleuca Hamp.

Pantheina

Colocasia Ochs.

3205 flavicornis Sm. infanta Sm. 3206 propinquilinea Grt.

Panthea Hbn.

3207 acronyetoides Wlk. leucomelana Morr

3208 portlandia Grt. 3209 virginaria Grt. electa Sm.

3210 gigantea French.

3211 furcilla Pack.

3212 palata Grt.

Charadra Wlk.

3213 pata Druce.

3214 ingenua $\mathrm{Sm}$.

3215 deridens $G n$. circulifera Wlk. contigua Wlk.

3216 gudena $\mathrm{Sm}$. 3217 dispulsa Morr.

Lichnoptera H. S. 3218 decora Morr. 3219 illudens $W l k$. pythion Druce.

\section{Plusînx}

Syngrapha Hbn. 3220 parilis $\mathrm{Hbn}$. quadriplaga Wik. 3221 devergens $\mathrm{Hbn}$. 3222 alticola Wik.
3223 hochenwarthi Hoehen. 3224 ignea Grt.

Autographa Hbn.

3225 microgamma $\mathrm{Hbn}$. 3226 orophila Hamp.

3227 diasema $B d v$. borea Auriv.

3228 sackeni Grt. 3229 snowi $H y . E d w$. 3230 falcifera Kirby. form simplex $\mathrm{Gn}$.

3231 simplicima Ottol. 3232 speciosa Ottol. 3233 epigres Grt. 3234 selecta $W l k$. viridisigma Grt. viridisignata Grt.

3235 celsa $\boldsymbol{H}$ y. Edw. 3236 rectangula Kirby. ab. mortuorum $\mathrm{Gn}$.

3237 alias Ottol. u. aureum $\ddagger$ Auct.

3238 u. aureum $G n$. vaccini $\mathrm{Hy}$. Edw.

3239 octoseripta Grt. $a$ epsilon Ottol.

$b$ zeta Ottol.

- variana Ottol.

d pallida Ottol.

3240 arctica Moesch.

3241 ottolenguii $D$ yar. areticat Ottol.

3242 excelsa Ottol.

3243 angulidens $\mathrm{Sm}$. 3244 altera Ottol. 3245 v-alba Ottol. 3246 surens Grt. 3247 egena $G n$. 3248 verruca Fabr. omega $\mathrm{Hbn}$. rutila Wik.

*3249 brassicx Riley. echinocystis Behr. 
3251 basigera $W l k$. laticlavia Morr.

3252 rubida Ottol.

3253 abrota Druce.

3254 oxygramma Geyer. indigna Wlk.

3255 putnami Grt.

3256 nichollæ Hamp.

3257 contexta Grt.

3258 bonaerensis Berg. solida Ottol.

3259 biloba Steph.

3260 oo Cram.

rogationis $\mathrm{Gn}$.

dyaus Grt. culta Lint.

3261 precationis $G n$.

3262 corusca Stkr.

3263 labrosa Grt.

3264 bimaculata Steph. u-brevis Gn.

3265 mappa $G$. \& $\boldsymbol{R}$.

3266 pseudogamma Grt. russea Hy. Edw.

3267 californica Speyer.

3268 flagellum $W l k$.

monodon Grt.

insolita Sm.

3269 ou $G n$.

fratella Grt. $a b$. pedalis Grt.

3270 pasiphria Grt.

3271 sansoni Dod.

3272 metallica Grt.

scapularis $\mathbf{H y}$. Edw.

lenzi Frch.

3273 albavitta Ottol.

$a$ hutsoni Sm.

3274 ampla Wlk. alterna Stkr.

Plusia Ochs.

3275 rereoides Grt.

3276 ærea $\mathrm{Hbn}$.

3277 balluea Geyer.
Chrysoptera Latr.

3278 moneta Fabr.

$a$ esmeralda Obert. trabea Sm.

3279 morigera $\boldsymbol{H y}$. Edw.

Eosphoropteryx Dyar.

3280 thyatyroides $G n$.

Pseudeva Hamp.

3281 purpurigera $W l k$.

3282 palligera Grt.

3283 rubigera Hamp.

Palæoplusia Hamp.

3284 venusta $W l k$. striatella Grt.

Abrostola Ochs.

3285 formosa $G r t$. 3286 ovalis $G n$.

3287 urentis $G n$.

3287,1 parvula $B$. \& $M C D$.

Erebinæ

Raphia Hbn.

3288 abrupta Grt.

3289 frater Grt. personata Wlk. flexuosa Wlk.

3290 coloradensis Put.

3291 elbea $\mathrm{Sm}$.

3292 pallula $H y$. $E d w$.

3293 cinderella Sm.

Cissusa Wlk.

3294 spadix Cram.

vegeta Morr.

3295 remigipila Gn.

3296 mucronata Grt.

3297 inepta $\boldsymbol{H} y$. Edw. , morbosa Hy. Edw.

3298 biformata $\boldsymbol{H} y$. $\boldsymbol{E} d w$.

3299 sabulosa $\boldsymbol{H y}$. Edw.

3300 serupulosa $H y . E d w$. 


\section{Ulosyneda Sm.}

3301 cervina Hy. Edw. 3302 indiscrete $H y$. Edw. 3303 subtermina $\mathrm{Sm}$. 3304 valens $\boldsymbol{H}$ y. Edw. insperate Grt.

Panula Gu.

3305 inconstans $G n$.

Melipotis Hbn.

3306 fasciolaris $H b n$. lunearis Gn.

8307 stygialis Grt.

3308 nigrescens $G$. \& $\boldsymbol{R}$. os ochreipennis Harv.

3309 famelica $G n$.

3310 pallescens $G$. \& $R$.

3311 fumosa Stkr.

of tenella Hy. Edw.

3311, 1 brunneifasciata $B$. \& $M c D$. 3312 jucunda $\mathrm{Hbn}$. cinis Gn.

3313 versabilis Harv.

3314 hadeniformis Behr.

3315 agrotipennis Harv.

3316 acontioides $\mathrm{Gn}$. sinualis Harv.

Cirrhobolina Grt.

3317 deducta Morr. pavitensis Morr.

3318 mexicana Behr. incandescens Grt. a vulpina $H y$. Edw. ab. albina Stkr.

3819 tetrica $H y . E d w$.

Drasteria Hbn.

3320 perlseta $H y$. Edw.

\& aegrotata Hy. Edw.

3321 favofasciata Stkr. 3522 mirifica $H y$. Edw.
3323 hastingai $\boldsymbol{H} y . \mathbf{E d w}$. a perpallida $\boldsymbol{H y}$. Edw.

3324 ingeniculata Morr.

3325 oceulta Hy. Edw.

3326 graphica Hbr.

3827 capticols Wlk. media Morr. faceta Hy. Edw.

Syneda $\mathrm{Gn}$.

3328 limbolaris Geyer. grandirena Haw.

3329 divergens Behr.

3330 adumbrata Behr.

3331 petricola $W$ Ik.

3332 perplexa $\boldsymbol{H} y, E d w$.

3333 alleni Grt.

a saxea $H y$. Edw.

3334 socia Behr.

3335 ochracea Behr.

3336 athabasca Neum.

3337 hudsonica $G$. \& $R$.

3338 seposita Hy. Edvo.

3339 nubicola $B$ ehr.

3340 maculosa Behr.

3341 stretchi Behr.

3342 howlandi Grt.

3343 tejonica Behr.

3344 edwardsi Behr.

3345 perfecta $H y$. Edw.

\& decepta Stkr.

\& nigromarginata Stkr.

Litocala Harv.

3346 sexsignata Harv.

$a$ deserta $H y$. Edw.

Hypocala Gn.

3347 andremona Cram. hilli Lint.

Homophoberia Morr.

3347, 1 cristata Mors. 
Toxocampa Gn.

3348 victoria $\mathrm{Grt}$.

Phoberia Hbn.

3349 atomaris $\mathrm{Hbn}$. orthosioides Gn. porrigens Wlk. ingenua Wlk.

Siavana Wlk.

3350 repanda Wlk. auripennis Grt.

3351 rigida Sm.

Panopoda Gn.

3352 rufimargo $\mathrm{Hbn}$.

cressoni Grt.

form carneicosta $G n$. scissa Wlk. combinata Wlk. form roseicosta $G n$. form rubricosta $G n$.

Palindia Gn.

3353 dominicata $G n$.

3354 merricki Holl.

3355 micca Druce.

Peosina Gn.

3356 pandrosa Cram.

Epidromia Gn.

3357 delinquens $W l k$.

Massala Wlk.

3358 obvertens Wlk.

Anticarsia $\mathrm{Hbn}$.

3359 gemmatilis $\mathrm{Hbn}$.

3360 ferruginea $S m$.

Antiblemma $\mathrm{Hbn}$.

3361 inexacta $W l k$.

canalis Grt.

form guttula $H y$. Edw. form minorata $\mathrm{Sm}$. form fasciata $\mathrm{Sm}$.

Litoprosopus Grt.

3362 futilis $G . \& R$.

3363 confligens Wlk.

Ophideres Bdv.

3364 materna $L$.

hybrida Fabr.

calaminea Cram.

Strenoloma Grt.

3365 lunilinea Grt.

Heteranassa Sm.

3366 mima Harv.

3367 minor $\mathrm{Sm}$.

3368 fraterna $\mathrm{Sm}$.

Cœnipeta Hbn.

3369 bibitrix $\mathrm{Hbn}$. meskei Hy. Edw.

Bendis Hbn.

Trama Harv.

3370 detrahens Wlk. arrosa Harv.

3371 hinna Geyer. pulverosa Wlk.

3372 griseipennis Grt.

3373 formularis $H b n$.

Coxina Gn.

3374 einctipalpis $\mathrm{Sm}$. 3375 umbrina Grt. protea $\mathrm{Sm}$.

3376 paresa $S m$.

3377 termina Grt.

Selenis Gn.

3378 monotropa Grt. 3379 agna Druce. 
Capnodes Gn.

3380 rufinans $G$ m. punetivena $\mathrm{Sm}$. form discerpta Wlk. 3381 ealifornica Behr.

Tyrissa Wlk.

3382 multilines $B$. \& $M C D$.

Egryrlon Sm.

3388 filaria $\mathrm{Sm}$.

Kakopoda Sm.

3384 cincta $S m$.

Yrias Gn.

8385 clientis Grt. pardalis Sm.

3386 strigalis $\mathrm{Sm}$.

3387 volucris Grt.

a terminalis $\mathrm{Sm}$.

3388 repentis Grt.

3389 crudelis Grt.

3390 albiciliatus $\mathrm{Sm}$. bifasciata B. \& McD.

Erebus Latr.

3391 odora $L$.

agarista Cram.

Thysania Dalm.

3392 zenobia Cram.

Latebraria Gn.

3393 amphipyroides $G n$.

Noropsis Gn.

3394 hieroglyphica Cram. festiva Fabr. elegans $\mathrm{Hbn}$. fastuosa $\mathrm{Gn}$.

Gonodonta Hbn.

3395 unica Neum.
3396 pyrgo Cram.

3397 nutrix Cram.

Calpe Treit.

3398 eanadensis Beth. purpurascens Wlk. sobria Wik.

Scoliopteryx Germ. 3399 libatrix $L$.

Plusiodonta Gn.

3400 compressipalpis $G n$. insignis Wlk.

3401 amado Bames.

Sylectra Hbn.

3402 ericata Cram. mirandalis $\mathrm{Hbn}$.

Hypsoropha $\mathrm{Hbn}$.

3403 monilis Fabr.

3404 hormos Hbn.

Rhescipha Wlk.

3405 obtusa Wlk. snowi Skin.

Alabama Grt. 3406 argillacea $\mathrm{Hbn}$. xylina Say. grandipuncta $\mathrm{Gn}$. bipunetina $\mathrm{Gn}$

Anomis IIhn. 3407 erosa $H b n$. 3408 fimbriago Steph. serrata B. \& McD. 3408,1 luridula $G n$. 3409 texana Riley. 3410 exacta $\mathrm{Hbn}$. conducta WIk. hostia Harv. 


\section{Hypeninæ}

Scolecocampa Gn.

3411 liburna Geyer. ligni Gn.

3412 atriluna $\mathrm{Sm}$.

Reabotis $\mathrm{Sm}$.

3413 immaculalis Hlst.

Pseudorgyia Harv.

3414 versuta Harv.

3415 russula Grt. pectinicornis Sm.

Gabara Wlk.

Eucalyptra Morr.

3416 obscura Grt.

3417 gigantea $\mathrm{Sm}$.

3418 stygialis $\mathrm{Sm}$.

3419 subnivosella $W l k$. nivealis $\mathrm{Sm}$.

3420 bipuncta Morr.

3420,1 pulverosalis $W l k$.

3421 apicalis $S m$.

3422 strigata $\mathrm{Sm}$.

3423 umbonata $\mathrm{Sm}$.

3424 humeralis $\mathrm{Sm}$.

3425 minorata $\mathrm{Sm}$.

Isogona $\mathrm{Gn}$.

Parora Sm.

3426 natatrix $G n$.

3427 tenuis Grt.

3428 texana $\mathrm{Sm}$.

3429 snowi $\mathrm{Sm}$.

3430 punctipennis Grt. acuna Barnes.

3431 segura Barnes.

3432 æolia Druce.

3433 agilaria Druce. reniformis Sm.

Rivula Gn.

3434 propinqualis $G n$.
Zelicodes Grt.

3435 linearis Grt.

Phiprosopus Grt.

3436 callitrichoides Grt. nasutaria Zell.

3437 pallens $B$. \& $M c D$.

Agassizia Behr.

3438 urbicola Behr.

Mycterophora Hlst.

3439 monticola Hlst.

3440 longipalpata Hlst.

3441 inexplicata Wlk. slossoniae Hlst.

Prosoparia Grt.

3442 perfuscaria Grt. anormalis B. \& $\mathrm{McD}$.

Diallagma Sm.

3443 latiorella Wlk. lutea $\mathrm{Sm}$.

3444 angustiorata Grt.

Pleonectyptera Grt.

3445 pyralis $H b n$. irrecta Wlk. floccalis Zell. form geometralis Grt.

3446 punitalis $S m$.

3447 secundalis $S m$.

3448 reversalis $\mathrm{Sm}$.

3449 habitalis $W l k$. phalaenalis Grt.

3450 historialis Grt. form rectalis $\mathrm{Sm}$.

3451 subflavidalis Grt.

3452 finitima $S m$. cumulalis Dyar.

$a$ tenalis $\mathrm{Sm}$. serena $\mathrm{Sm}$.

3453 incusalis Grt. 3454 parallela $S m$. 
3455 immaculalis Harv.

$\$ 456$ obliqualis $\boldsymbol{H}$ y. $\boldsymbol{E} d w$.

$\$ 457$ abbocostaliata Pack.

3458 noetuidalis Dyar.

Phytometra Haw.

3459 rhodarialis W/k.

a coceineifascia Grt.

3460 semipurpurea $W l k$. confinisalis WIk. roanlba Grt.

3461 plans Grt.

3461, 1 apicata $B$. \& $M c D$.

3461,2 curvata $B$. \& $M c D$.

3462 orgyise Grt.

subolivacea Harv.

Ommatochila Butl.

3463 mundula Zell.

Hormoschista Moesch.

3464 latipalpis Wlk. orba Grt. pagenatecheri Moesch.

Oxycilla Grt.

3465 tripla Grt. mecyanalis Druce.

3466 ondo Barnes.

3467 malaca Grt. 3468 mitographa Grt. 3469 panatela $S m$.

3469,1 basipallida $B . \& M c D$.

Phalænostola Grt.

3470 larentioides Grt. a citima Grt.

Pangrapta Hhn.

3471 decoralis $\boldsymbol{H b n}$. geometroides $\mathbf{G n}$. epionoides $\mathrm{Gn}$. elegantalis Fitch. recusans Wik.
Spargaloma Grt.

3472 sexpunctata $\mathrm{Grt}$.

3473 perditalis $W I k$. semilineata Wlk. umbrifascis Grt.

Melanomma Grt.

3474 auricinctaria Grt.

Dyspyralis Warr.

3475 illocata Warr. humerata Sm.

3476 puncticosta $S m$. 3476,1 noloides $B$. \& $M e D$. 3477 nigellus Stkr. immuna Sm.

Metalestra Hbn. Homopyralis Grt.

3478 discalis Grt. 3479 quadrisignata $W l k$. contracta Wik. zonata Wik. tactus Grt.

3480 bigallis $S m$.

3481 tantillus Grt. monodia Dyar.

3482 miserulata Grt. irentis Sm.

3483 edilis $S m$. 3484 cincta $S m$.

Epireuxis Hbn.

3485 americalis $G n$. scriptipennis Wik.

3486 æmula $\mathrm{Hbn}$. mollifera WIk. herminioides Wik. effusalis Wik. concise WIk.

3487 scobialis Grt.

3488 laurenti $S m$. 3489 suffusalis $\mathrm{Sm}$. 
3490 rotundalis $W l k$. borealis Sm.

3491 forbesi Frch.

3492 terrebralis $B$. \& $M c D$.

3493 merricki $S m$.

3494 denticulalis Harv.

3495 gopheri $S m$.

3496 lubricalis Geyer. phaealis Gn. surrectalis Wik.

a occidentalis $\mathrm{Sm}$. form intensalis $\mathrm{Sm}$.

$b$ partitalis $S m$.

c cobeta $S m$.

3497 parvulalis $B$. \& $M c D$.

3498 majoralis $\mathrm{Sm}$.

3498,1 punctalis $B . \& M c D$.

Zanclognatha Led.

3499 lituralis $H b n$.

3500 theralis $W l k$.

deceptricalis Zell.

a gypsalis Grt.

3501 minoralis $S m$.

3502 inconspicualis Grt.

3503 levigata Grt. form modestalis Fitch. form reversata Dyar. form obsoleta $\mathrm{Sm}$.

3504 atrilineella Grt.

3505 obscuripennis Grt.

3506 pedipilalis $G n$.

3507 protumnussalis $W l k$. minimalis Grt.

3508 cruralis $G n$.

3509 jacchusalis $W l k$. marcidilinea Grt.

3510 ochreipennis Grt.

3511 lutalba $\mathrm{Sm}$.

Hormisa Wlk.

3512 absorptalis $W \| k$. nubilifascia Grt.

3513 litophora Grt.
3514 bivittata Grt.

3515 orciferalis $W l k$.

a pupillaris Grt. harti Frch.

Philometra Grt.

3516 metonalis $W l k$. gaosalis WIk. longilabris Grt.

3517 eumelusalis Wlk. serraticornis Grt.

3518 hanhami $S m$.

Chytolita Grt.

3519 morbidalis $\mathrm{Gn}$.

3520 petrealis Grt. punetiformis Sm.

3521 fulicalis $S m$.

Renia Gn.

3522 salusalis Wlk. brevirostralis Grt.

3523 factiosalis W/k. clitosalis WIk. centralis Grt. plenilinealis Grt. alutalis Grt. tilosalis $\mathrm{Sm}$.

3524 discoloralis $\mathrm{Gn}$. fallacialis W1k. generalis Wlk. thraxalis Wlk.

3525 pulverosalis $S m$.

3526 hutsoni $\mathrm{Sm}$. 3527 rigida $S m$.

3528 flavipunetalis Geyer. phalerosalis WIk. heliusalis Wlk. pastoralis Grt. belfragei Grt.

3529 exserta $S m$. 3530 atrimacula $S m$. 3531 fraternalis $S m$. 3532 sobrialis WIk. 
3593 larvalis Grt. restrictalis Grt.

3534 subterminalis $B$. \& $M C D$.

3535 mortualis $B$. \& $M C D$.

\section{Bleptina Gn.}

3536 caradrinalis $\mathrm{Gn}$. cloniasnlis Wlk.

3537 medialis $\mathrm{Sm}$.

3538 inferior Grt.

3539 sangamonia $\boldsymbol{B}$. \& $\boldsymbol{M} \boldsymbol{C D}$.

3540 flaviguttalis $B$. \& $M C D$.

3541 minimalis B. \& $\mathbf{M c D}$.

Tetanolita Grt.

3542 mynesalis Wik. lixalis Grt.

3543 palligera $\mathrm{Sm}$. greta $\mathrm{Sm}$.

3544 floridana $S m$. a fulata $S m$.

3545 negalis $B$. \& $M C D$.

Phalrenophana Grt.

3546 pyramusalis $W L$. gyasalis Wik. rurigena Grt.

3547 extremalis $B$. \& $M c D$.

Hypenula Grt.

3548 cacuminalis Wlk. biferalis Wik. opacalis Grt.

3549 caminalis $\mathrm{Sm}$.

Lascoria Wlk.

Gaberasa Wlk.

3550 ambigualis $W l k$. \& bifidalis Grt. $\%$ indivisalis Grt.

3551 son Druce.

Rejectaria $\mathrm{Gn}$.

3552 albisinuata Sm.
Palthis Hlın.

3553 angulalis $\boldsymbol{H b n}$. aracinthusalis Wlk.

3554 asopialis Gn. insignalis WIk.

Capis Grt.

3555 curvata Grt.

Dercetis Grt.

3556 vitrea Grt.

3557 pygmae Grt.

3558 bilinealis $B$. \& $M C D$.

Salia Hbn.

3559 interpuncta Grt. saligna Zell.

a rufa Grt.

Bomolocha Hbn.

3560 manalis $W l k$.

3561 baltimoralis $G n$. benignalis Wlk. laciniosa Zell.

3562 bijugalis $W U k$. pallialis Zell. of fecialis Grt.

3563 palparia Wlk. scutellnris Grt.

3564 abalienalis Wlk.

3565 deceptalis Wik. perangulalis Harv.

3566 madefactalis $\mathrm{Gn}$. achatinalis Zell. o damnosalis Wlk. \& cadrecalis Wlk.

3567 sordidula Grt.

3568 henloa $\mathrm{Sm}$.

3569 atomaria $\mathrm{Sm}$. chicagonis Dyar. a perpallida Dyar.

3570 vega $S m$.

3571 toreuta Grt.

albisignalis Zell. 
3572 edictalis Wlk.

우 vellifera Grt.

ô lentiginosa Grt.

3573 umbralis $\mathrm{Sm}$.

3574 citata Grt. trituberalis Zell.

3575 annulalis Grt.

Lomanaltes Grt.

3576 eductalis Wlk. laetulus Grt.

Anepischetos Sm. 3577 lividalis $\mathrm{Hbn}$. abjuralis Wlk. scissalis Wlk. bipartita $\mathrm{Sm}$.

Plathypena Grt. 3578 scabra Fabr. erectalis Gn. palpalis Haw. obesalis Steph. $a b$, subrufalis $G r t$.

Hypena Schrank. 3579 humuli Harr. evanidalis Rob. germanalis Wlk. form olivacea Grt. form albopunctata

3580 californica Behr.

Tepper.

3582 modesta Sm.

Hyblaina

Hyblæa Fabr.

3583 puera Cram.

saga Fabr.

mirificum Stkr.

\section{PERICOPIDE}

Daritis Wlk.

3584 howardi $H y . E d w$.

Composia Hbn.

3585 fidelissima $H$. 'S. olympia Butl. $a$ discreta Stretch. arizonae French. morrisoni Druce.

$b$ vermiculata $G . \& R$. continua Hy. Edw.

3587 clappiana Holl.

Gnophæla Wlk.

3586 latipennis $B d v$. hopfferi G. \& R.

\section{DIOPTIDFE}

Phryganidia Pack.

3588 californica Pack.

\section{NOTODONTIDEE}

Ichthyura Hbn.

3589 apicalis Wik. vau Fitch. indentata Pack. $a$ ornata $G . \& R$. incarcerata Bdv. $b$ astoriæ $H y . E d w$. c bifiria $H y . E d w$. 
3590 inornata Neum.

3591 inclusa $H b n$. americana Harris.

$a$ inversa Pack. palla Freh.

b. jocoea $H y . E d w$.

3592 strigosn $\mathrm{Grt}$.

a luculenta $\boldsymbol{H}$. Edw.

3593 albosigma Fitch.

a specifiea Dyor.

3594 brucei $\boldsymbol{H y}$. Edw.

a multnoma Dyar. alethe N. \& D.

Datana Wlk.

3595 ministra Dru. a californica Dyar.

3596 angusi $G$. \& $R$.

3597 drexeli $\boldsymbol{H} y$. Edw.

3598 major $G$. \& $R$.

3599 palmi Beut.

3600 floridana Graef.

3601 modesta Beut.

3602 robusta Stkr.

3603 perspicua $G$. \& $R$.

$a$ mesilla Ckll.

3604 integerrims $G$. \& $\boldsymbol{R}$. a cochise Dyar.

3605 neomexicana Doll.

3606 chiriquensis Dyar.

3607 contracta Wlk.

Fyperæschra Butl.

3608 stragula Grt. scitipennis Wlk.

a manitou $N$. \& $D$.

$b$ pacifica Behr.

C ochreata $B$. \& $M c D$.

3609 georgica $\boldsymbol{H} . \mathrm{S}$.

3610 tortuosa Tepp.

Odontosia Hbn.

3611 elegans Stkr.

a grisea Stkr. notaria Hy. Edw.
Lophopteryx Steph.

3612 americana Harv.

Notodonta Ochs.

3613 basitriens Wlk.

3614 aimplaria Graef.

Pheosia Hbn.

3615 rimosa Pack. californica Stretch.

3616 portlandia $H y . E d w$. descherei Neum.

Lophodonta Pack.

3617 ferruginea Pack.

3618 angulosa $A$. \& $S$.

Eunystalea Grt.

3619 indiana Grt.

Nadata Wlk.

3620 gibbosa $A$. \& $S$.

a doubledayi Pack.

$b$ rubripennis $N$, \& $D$.

c oregonensis Butl.

$d$ behrensi $\boldsymbol{H} y$. Edw.

Nerice Wilk.

3621 bidentata $W l k$.

Symmerista N. \& D.

3622 albifrons $A$. \& $S$.

$a$ albicosta $\mathrm{Hbn}$.

b suavis Barnes.

Hippia Moesch.

3623 packaxdi Morr.

3624 insularis Grt.

Dasylophia Pack.

3625 anguine $A$. \& $S$.

cuculifera H. S. punctata Wik. cana Wlk.

signata Wlk. 
a puntagorda Slos.

b saturata Barnes.

3626 thyatiroides $W l k$. interna Pack. tripartita Wlk.

3627 seriata Druce. melanopa Barnes.

Ursia B. \& McD.

3628 noctuiformis $B$. \& $M c D$.

Litodonta Harv.

3629 hydromeli Harv. $a b$. fusca Harv. $a$ contrasta $B . \& M c D$. 3630 wymola Barnes.

Heterocampa Dbldy.

3631 lineata Druce.

3632 picta Feld.

3633 astarte Dbldy. menas Harr.

\& chapmani Grt.

3634 varia Wlk.

ô obliqua Pack.

form trouveloti Pack. brunnea G. \& R.

form perolivata Pack.

3635 ditta $B . \& M c D$.

3636 ruficornis Dyar.

3637 subrotata Harv. superba Hy. Edw. a celtiphaga Harv.

3638 belfragei $G r t$.

3639 lunata $\boldsymbol{H} y$. $E d w$. plumosa Hy. Edw.

3640 umbrata Wlk. athereo Harris. $a$ pulverea $G$. \& $R$.

$b$ averna $B$. \& $M c D$. 3641 manteo Dbldy. cinerascens Wlk. subalbicans Grt.

3642 biundata $W l k$. semiplaga Wlk. olivata Pack. mollis Wlk.

3643 guttivitta Wlk. albiplaga Wlk. mucorea H. S. cinereus Pack. harrisi Pack. indeterminata Wlk. doubledayi Scud.

3644 bilineata Pack. turbida Wlk. associata Wlk. ulmi Harris. $a$ exsanguis Dyar.

Misogada Wlk.

3645 unicolor Pack. marina Pack. sobria Wlk.

Euhyparpax Beut.

3646 rosea Beut.

Didugua Druce.

3647 argentilinea Druce.

Theroa Schaus.

3648 zethus Druce.

Ianassa Wlk.

3649 lignicolor $W l k$.

virgata Pack.

lignigera Wlk.

$a$ delicata Dyar.

3650 coloradensis $H y . E d w$.

3651 pallida Stkr.

3652 semirufescens $W l k$. eximia Grt.

3653 perangulata $H y$. Edw. 
Schisura Dbldy.

3654 ipomes Dbldy.

biguttatue Peck.

confusa Wik.

ducens Wlk.

corticea Wik.

compta Wlk.

nigrosignata W1k.

form telifer Grt.

form cinereofrons Pack.

rstipennis Wik.

3655 concinna A.\& S. nitida Pack.

3656 badia Pack. significata Wlk.

3657 unicornis $A$. \& $S$. edmandsi Pack.

humilis Wlk.

a conspecta $\boldsymbol{H} y . E d w$.

3658 errucata Dyar.

3659 apicalis $G$. \& $R$.

3660 leptinoides Grt. mustelina Pack.

3661 biedermani $B . \& M c D$.

Hyparpax Hbn.

3662 aurora $A$. \& $S$. rosea Wlk. venusta Wlk.

3663 venus Neum:

3664 perophoroides Stkr. tyria Sloss.

a aurostriata Graef.

3665 aonides Stkr.

Cerura Sehrank.

3666 seitiseripta Wlk.

a multiscripta Riley.

3667 candida Lint.

3668 rarata $W \mid k$.

3669 borealis $B d v$.

3670 occidentalis Lint.

3671 cinerea $W l k$. a einereoides Dyer.

$b$ paradoxa Behr.

- placida Dyar.

3672 nives Neum.

a meridionalis Dyar.

3678 scolopendrina $B d v$. aquilonaris Lint.

a modesta $\mathrm{H}$ ud.

b albicoma Stkr.

Fentonia Butl.

3674 marthesia Cram. tesella Pack.

turbida WIk.

\& elongata G. \& R.

3675 dorothea Dyar.

3676 miranda Dyar.

Notela Schaus.

3677 jaliscana Schaus.

3678 angustiora $B$. \& $M c D$.

Afilia Schaus.

3679 oslari Dyar.

moqui Barnes.

antennata $\mathrm{Sm}$.

Gluphisia Bdv.

3680 septentrionalis Wlk. clandestina Wlk. trilineata Pack.

$a$ ridenda $\boldsymbol{H} y . E d w$. form rupta $\boldsymbol{H} y$. Edw.

- b albofascia $H y$. Edu. form formosa $\mathrm{Hy}$. Edw.

c wrighti $H y . E d w$.

d quinquelinea Dyar.

3681 lintneri Grt.

form avimacula $\mathrm{Hud}$. form slossoni Pack.

a' severa Hy. Edw. form danbyi Neum. form normalis Dyar. 
Ellida Grt.

3682 caniplaga $W l k$. transversata Wlk. gelida Grt.

Hemiceras Gn.

3683 cadmia $G n$. obliquilinea Wlk.
Pseudhapigia Schaus. 3684 estrella Barnes.

Gargida Schaus. 3685 pyrrha Druce.

Crinodes H. S. 3686 biedermani Skin.

\section{THYATIRID $\mathbb{E}$}

Habrosyne Hbn.

3687 scripta Gosse. abrasa $\mathrm{Gn}$. 3688 chatfeldi Grt. derasa Hy. Edw. 3689 rectangulata Ottol. $a$ arizonensis $B$. \& $M c D$.

Pseudothyatira Grt. 3690 cymatophoroides Gn. form expultrix Grt.

Thyatira Ochs. 3691 mexicana $H y$. Edw. superba Barnes.
Euthyatira Sm.

3692 lorata Grt. 3693 pudens $G n$.

$a$ anticostiensis Grt. $b$ pennsylvanica $S m$. 3694 candida $S m$. 3695 semicircularis Grt. tema Stkr. $a$ griseor $B . \& M c D$.

Cymatophora Tr.

3696 improvisa $H y$. Edw. 3697 tearli $H y$. Edw. 3698 fasciata $B . \& M c D$.

\section{LYMANTRIIDEE}

Gynæphora Hbn. 3699 rossi Curt. 3700 gronlandica Hom.

Notolophus Germ.

3701 antiqua $L$. nova Fitch. $a$ badia $H y$. $E d w$.

Hemerocampa Dyar. 3702 oslari Barnes. 3703 libera Stkr. 3704 vetusta $B d v$. a cana $H y . E d w$. $b$ gulosa $H y . E d w$.
3705 leucostigma $A . \& S$. leucographa Geyer. intermedia Fitch. borealis Fitch. obliviosa Hy. Edw.

3706 inornata Beut. falcata Schaus. 3707 plagiata Wlk. definita Pack.

Animomyia Dyar. 3708 morta Dyar.

Olene Hbn. 3709 achatina $A . \&$ S. 
3710 basiflava Peck. elintoni G. \& R. a meridionalis $B$. \& MoD. 3711 kerrvillei $B$. $\& C D$. 3712 vagans $B . \& M C D$. plagiatn $\$$ Auet. a grisea $B$. \& $M c D$. $b$ willingi $B$. \& $M C D$.

3713 leucophea $A$. \& $S$. 3714 atrivenosa $\mathrm{Palm}$. 3715 atomaria Wlk. obliqueatn G. \& R. form parallela $G$. $\& R$. 3716 cinnamomen $G$. \& $R$. 3717 manto Stkr. a interposita Dyar. b montana Beut.
3718 plagiata Wik. pinicole Dyar.

3710 griefacta Dyar.

8720 pini Dyar.

3721 tyx B. \& McD.

Porthetria Hbn.

3722 dispar $L$.

Euproctis Hbn.

3728 chrysorrhoea $L$.

Doa N. \& D.

3724 ampla Grt.

Leuculodes Dyar.

3725 lacteolaria Hlst.

\section{EUPTEROTIDF}

Apatelodes Pack.

3726 torrefacta A. \& S.

a floridana $H y . E d w$.

3727 pudefacta Dyar.
3728 angelica $\mathrm{Grt}$.

hyalinopuneta Pack. $a$ indistineta $H y . E d w$.

b seraphica Dyar. uv'rda Barnes.

\section{LASIOCAMPIDE}

Gloveria Pack.

3729 arizonensis Pack. form dolores $N$. \& $D$. 3730 sphingiformis $B$. \& $M e D$. 3731 gargamelle Stkr. \& medusa Stkr.

3732 howardi Dyar.

Eutachyptera B. \& MeD. 3733 psidii Sallé.

Quadrina Grt.

3734 diazoms Grt. oweni Barnes.
Caløcia B. \& MeD.

3735 juvenalis $B$. \& $M c D$.

Dicogaster B. \& MeD.

3736 coronada Barnes. form valens Dyar.

Artace Wlk.

3737 punctistriga $W l k$. rubripalpis Feld.

Tolype Hbn.

3738 velleda Stoll. 3739 glenwoodi Barnes. 
3740 minta Dyar.

3741 brevicrista Dyar.

3742 distincta French.

3743 laricis Fitch. minuta Grt.

Hypopacha N. \& D.

3744 grisea Neum.

Malacosoma Hbn.

3745 americana Fabr. decipiens Wlk. frutetorum Bdv.

3746 fragilis Stretch. a incurva $H y . E d w$. discolorata Neum.

$b$ mus Neum.

c constrictina $N$. \& $D$. lutescens N. \& D. $d$ perlutea $N . \& D$.

3747 tigris Dyar. $a$ inducta $D$ yar.

3748 californica Pack. ? californica Wlk. pseudoneustria Bdv.

3749 ambisimilis Dyar.
3750 pluvialis Dyar.

3751 constricta Stretch. a strigosa Stretch.

3752 disstria $\mathrm{Hbn}$. drupacearum Bdv. a sylvatica Harris.

$b$ thoracicoides $N . \& D$.

c erosa Stretch.

$d$ sylvaticoides $N . \& D$.

$e$ thoracica Stretch.

$f$ perversa $N . \& D$. $a b$. astriata Reiff. $a b$. anita Reiff.

Heteropacha Harv.

3753 rileyana Harv.

Epicnaptera Ramb.

3754 americana Harris. occidentis Wlk. carpinifolia Bdv. form ferruginea Pack. : a californica Pack. form mildei Stretch. roseata Stretch. $b$ dyari Rivers.

\section{BOMBYCIDFE}

Bombyx L.

3755 mori $L$.

\section{DREPANID平}

Eudeilinia Pack.

3756 herminiata $\mathrm{Gn}$. albata Gn. a biseriata Pack.

Oreta Wlk.

3757 rosea Wlk. americana H. S. formula Grt. form marginata Wlk.
3758 irrorata Pack. 3759 adona Stkr.

Drepana Schrank. 3760 arcuata Wlk. fabula Grt. form genicula Grt. a siculifer Pack.

3761 bilineata Pack. form levis $\boldsymbol{H} u d$. 


\section{GEOMETRIDE:}

\section{Brephinse}

Brephos Zinck.

3762 infans Moesch. hamadryas Harris.

- 3768 melanis $B d v$.

-3764 californicus $B d v$.

3765 fletcheri $S m$.

Leucobrephos Grt.

3766 brephoides Wik. resoluta Zell.

hoyi Grt.

\section{Oenochromina}

Alsophils Hbn.

3767 pometaria Harris. restituens Wik. autumnata Pack.

Ametris $\mathrm{Hbn}$.

3768 nitocris Cram. nitocritaria $\mathrm{Hbn}$. peninsularia Grt. schausaria Hy. Edw.

\section{Almodes Gn.}

3769 terraria Gn. stellidaria Gn. rivularia Grt. subaustralis Hlst. pedicellata Hlst.

\section{Hemitheina}

Racheospila Gn.

3770 lixaria $G n$. inclusaria Wlk.

8771 catachloa Hlst. 3772 extremaris Wlk. 3773 rubromarginaria Pack. 3774 texana $\boldsymbol{H l s t}$.
3775 rubrolinearia Pack. 3776 festaria Hlst. 3777 obliqua Hlst.

a bellonaria Sikr. 3778 gerularia $\mathrm{Hbn}$. ocellata Stoll. - tollaria Gn. xysteraria Hist.

3779 sitellaria $G n$. congruata Wik. hulatiana Dyar. 3780 cupedinaria Grt. louisa Hlst.

Nemoria Hhn.

3781 bistriaria $H b n$. bifilata W1k.

3782 brunnearia Pack. 3783 mimosaria $G n$. tractaria Wlk. venustus Walsh. approximaria Pack. latiaria Pack. coniferaria Pack. 3784 rubrifrontaria Pack. packardaria Grt.

3785 pistaciaria Pack. unistrigata Gump.

3786 delicataria Dyar. 3787 strigataria Grossb. 3788 arizonaria Grt. 3789 latirosaria Pears. 3790 zygotaria Hlst. 3791 darwiniata Dyar. 3792 junctolinearia Graef. 3793 hudsonaria Tayl. 3794 unilinearia Tayl. 3795 splendidaria Grossb. 3796 viridicaria Hlst. $a$ albaria Grt.

3797 intensaria Pears. 3798 crerulescens Prout.

- Pombly helone in Genus Ieptarmia stretch. 
Synchlora Gn.

3799 liquoraria $G n$.

tricoloraria Pack. rubrifrontaria Dyar.

3800 ærata Fabr. glaucaria Gn. mimicata Wlk. mubivora Riley. albolineata Pack. gracilaria Pack.

3801 denticularia $W l k$. excurvaria Pack.

3802 rubrifrontaria Pack. rufofrontaria Gump.

Annemoria Pack.

3803 unitaria Pack.

Chlorosea Pack.

3804 nevadaria Pack.

3805 proutaria Pears.

3806 roseitacta Prout.

3806,1 pulcherrima $B . \& M c D$.

Cheteoscelis Prout.

3807 bistriaria Pack. undinaria Stkr.

3808 pectinaria Grossb.

Phrudocentra Warr.

3809 centrifugaria $\boldsymbol{H}$. S. protractaria H. S. hollandaria Hlst. jaspidiaria Hlst. viridipurpurea Hlst.

Dichorda Warr.

3810 iridaria $G n$.

remotaria Wlk. consequaria Hy. Edw. latipennis Hlst. perpendiculata Warr.

3811 rectaria Grt.

iridariał Hlst.
3812 illustraria Hlst.

Chlorissa Steph.

3813 pistasciaria $G n$. insecutata Wlk. superata Wlk.

3814 euchloraria $G n$. 3815 subcroceata Wlk. auranticolorata Stkr. dyari Hlst.

Mesothea Warr.

3816 incertata $W l k$. oporaria Zell. gratata Pack.

3817 viridipennata $\mathrm{Hlst}$. marinaria Stkr.

Chlorochlamys Hlst.

3818 chloroleucaria $G n$. indiscriminata Wlk. densaria Wlk. deprivata Wlk. rectilinea Zell.

3819 phyllinaria $Z$ ell. vertaria Pears.

3820 zelleraria Pack. phyllinariał Hlst.

3821 viridipallens $H$ lst.

3822 masonaria Schaus. volantaria Pears.

3823 inveterascaria Swett. 3824 appellaria Pears. 3825 curvifera Prout. 3826 triangularis Prout.

Chloropteryx Hlst. 3827 tepperaria Hlst.

Eueana Prout.

3828 niveociliaria $H . S$. saltusaria Hist.

Oospila Warr.

3829 lesteraria Grossb. 
Dichordophora Prout.

3830 phanix Prout.

Merochlora l'rout.

3831 faseolaria $\mathrm{Gn}$. perviridaria Pack.

3832 graetiaria Hlat. eutraphes Prout.

Eucrostes Hhn.

3833 dominicaria $G n$.

\section{Acidaliinæe}

Euacidalia Prok.

3834 sericearia Puck.

3835 ossularia $\mathrm{Hbn}$. temuaria Gn. subletaria $\mathrm{Gn}$. magniferaria Wlk. favillifera Wlk. repletaria Wlk.

3836 granitaria Pack. 3837 balistarie $G n$. perirrorata Pack.

a lanceolata $\mathrm{H}$ lst.

3838 rufescens Hlst.

3839 peralbata Pack. longipennata Pack.

Scelolophia Hlst.

3840 pannaria Gn. tremularia Wlk. purpurissata Grt. formosa Hlst.

3841 erossi Hlst. 3842 purpurascens Hlst. 3843 rubrotincta $\mathrm{H} / \mathrm{st}$. 3844 domeraria $B$. \& $M c D$. 3845 levitaria $\mathrm{Hbn}$. floridata Pack.
Dysephyra Ware.

Charommataea Hist.

3846 ella Hlat. form ellatina Hlat.

Dasycosymbia Grossl. 3847 gracilata Groseb.

Xystrota Hlst.

3848 hepaticaria $G n$. volucrata Hlst.

form rubromarginaria Pack. ferruminaria Zell.

3849 roseicosta $B$. \& $M c D$. 3850 suavata Hlst.

Arcobara Wik.

3851 multilineata Hlst. 3852 siculodaria Schaus. albiserpentata Pears.

Acidalia Tr.

3853 purata $G n$. cacuminaria Morr.

3854 æmulata $H$ lst.

3855 nigrodiscalis $\boldsymbol{H}$ lst.

3856 junctaria Wlk. quadrilineate Pack. persimilis Hlst.

3857 enucleata $G n$. restrictata Wlk. reconditaria Wlk. continuaria Wlk. form mensurata Wik. form relevata Swett. form adornata Prout. alabastariat Hlst.

3858 vestalialis $B$. \& $M c D$.

3859 quinquelinearia Pack.

3860 impunctata Worr. 3861 sideraria $G n$. 
3862 frigidaria Moesch. defixaria Wlk. impauperata Wlk. arcticaria Wlk. okakaria Pack.

3863 subfuscata Tayl. 3864 ancellata $\mathrm{Hlst}$. 3865 fuscata $H$ lst. 3866 luteolata Hlst. 3867 qursitata Hlst. 3868 timandrata Wlk. rufilineata W1k. 3869 ordinata $W l k$. puraria Wlk. candidaria Pack.

3870 compensata Wlk. obluridata Hlst.

3871 umbilicata $G n$. indoctaria Wlk.

3872 benitaria $B . \& M c D$. 3873 plantagenaria Hlst. 3874 nigrocandida $H$ lat. 3875 lautaria $H b n$. myrmidonata $\mathrm{Gn}$. minutularia Hlst.

3876 roseotincta $\mathrm{Hlst}$. 3877 placidaria $G n$.

Holaretias Prout.

3878 sentinaria Geyer. spuraria Christ. gracilior Butl.

3879 magnetaria $G n$. rubrolinearia Pack.

3880 californiaria Pack. pacificaria Pack.

Euphenolia Grossb.

3881 skinnerata Grossb. 3882 pallimedia Grossb.

Ptychopoda Curt. 3883 minuta Schaus. 3884 parvularia Hlst.
3885 bonifata Hlst. ptelearia Riley. delicata Hist. 3886 microphysa Hlst. 3887 scintillularia $\boldsymbol{H}$ lst. 3888 demissaria $\boldsymbol{H b}$. ferrugata Pack. russata Hlst.

$a$ inclusaria Wlk.

3889 eremiata $\boldsymbol{H}$ lst. flavescens Hlst.

3890 gemmata Pack.

3891 occidentaria Pack. 3892 hilliata Hlst. 3893 brauneata Swett. 3894 violacearia Wlk. nimbicolor Hlst. floridaria Hlst.

3895 micropterata Hlst. 3896 ostentaria Wlk. 3897 lacteola Lint. pallida Hlst.

3898 tacturata Wlk. albidula Hlst.

3899 obfusaria Wlk. punctofimbriata Pack. 3900 rotundopennata Pack. hanhami Hlst.

3901 inductata $G n$. anticaria Wlk. sobria Wlk. consecutaria Wlk. suppressaria Wlk.

3902 productata Pack. 3903 australis $\mathrm{Hlst}$. 3904 retractaria Wlk. *3906 sparsaria Wlk. psilogrammaria Zell.

Synomila Hlst.

3907 subochreata Hlst.

Ptenopoda Hlst. 3908 miranda Hlst.

*No number 3905. 
Goniacidalia IIlst.

3909 furciferata Pack.

Lophosis Hlst.

3910 labeculata Hlat.

Paota IIlst.

3911 fultaria Grt.

Hæmatopis $\mathrm{Hbn}$.

3912 grataria Fabr. saniaria Hbn. successaria Wik. form annettearia Haim.

Timandra Dup.

3913 amaturaria $W l k$.

Pleuroprucha Moesch.

3914 insulsaria $G n$. asthenaria WIk. invariata Wlk. imparata WIk. persimilata Grt.

Cosymbia Hbn.

3915 culicaria $G n$. 3916 dataria Hlst. 3917 myrtaria $G n$. ignotaria Wlk.

3918 lumenaria Hbn. pendulinaria Gn. quadrannulata Wlk. 3919 serrulata Pack.

\section{Larentiinæ}

\section{Stamnodes Gn.}

3920 gibbicostata WIk. costinotata Wik. strigularia Minot. oeneiformis Harv.

3921 blackmorei Swett. 3922 albiapicata Groseb. 3923 rickseckeri Pears.
3924 affliata Pears. 3925 delicata Groseb. 3926 ululata Pears. 3927 annellata $H$ lat. 3928 ceenonymphata $\boldsymbol{H}$ lot. 3929 splendorata Pears. 3930 formosata Stkr. dryadata Hlst. sponsate Grt.

3931 franckata Pears. 3932 seiferti Neum. 3933 topazata Stkr. $a$ albida $B$. \& $M c D$.

3934 pearsalli Swett. 3935 fervifactaria $\mathrm{Grt}$. 3936 morrisata $\mathrm{Hlst}$. volucer Hlst. 3937 rubrosuffusa Grossb. 3938 costimacula Grossb.

Marmopteryx Pack.

3939 marmorata Pack. a odontata Hlst.

3940 tessellata Pack. 3941 animata Pears.

Trichodesia Warr. 3942 albovittata $G n$. propriaria Wlk. reciprocata WIk.

3943 albofasciata Grt. 3944 californiata Pack.

Carsia Hbn.

3945 paludata Thun. a alpinata Pack. boreata Pack.

b labradoriensis Som.

Lithostege Hbn.

3946 rotundata Pack. arizonate Grt. 3947 Puscata Groseb. 3947 , 1 marcata $B$. \& MeD. 3948 elegans Grossb. 
3948, 1 deserticola $B . \& M c D$.

Scelidacantha Hlst.

3949 triseriata Pack. virginata Graef.

Acasis Dup.

3950 viridata Pack. eborata Hlst.

Nyctobia Hulst.

3951 limitaria $W l k$.

lobophorata W1k. fusifasciata Wlk. longipennis Wlk. form reiffi Swett.

3952 anguilineata.G. \& $R$. vernata Pack. (part.) form eastmani Reiff.

3953 nigroangulata Stkr.

Nothopteryx Prout.

3954 veritata Pears.

- Cladara Hist.

3955 atroliturata $W l k$. geminata G. \& R. vernata Pack. (part.)

Lobophora Curt.

3956 nivigerata $W l k$. a tabulata Hlst.

3957 montanata Pack. nocticolata Hlst. a magnoliatoidata Dyar. 3958 canavestita Pears.

Heterophleps H. S. 3959 refusata $W l k$. harveiata Pack. 3960 morensata Hlst. 3961 triguttaria $H$. S. quadrinotata Wlk. hexaspilata Wlk.
Dyspteris Hbn. 3962 abortivaria $H$. S.

Paraptera Hlst. 3963 danbyi Hlst.

Rachela Hist. 3964 bruceata Hlst. 3965 hyperborea Hlst. 3966 pulchraria Tayl. 3967 occidentalis Hlst. form latipennis $\mathrm{Hlst}$.

Triphosa Steph.

3968 affirmaria Wlk. indubitata Grt. dubitata Pack.

3969 hæsitata $G n$. a pustularia $\boldsymbol{H} y . \mathbf{E} d w$. 3970 californiata Pack. packardata Grossb.

Calocalpe Hbn.

3971 undulata Linn.

Coryphista Hlst. 3972 meadi Pack. 3973 badiaria $H y$. Edw. optimata Stkr.

Monotaxis Hist. 3974 semipectinata Hlst.

Eustroma Hbn. 3975 nubilata Pack. semiatrata Hlst.

Lygris Hbn.

3976 luteolata Hlst. 3977 diversilineata $\mathrm{Hbn}$. form gracilineata $G n$. 3978 propulsata $W l k$. packardata Lint. 
3979 testata Linn.

echatinata Hbn.

3980 molliculata $W / k$.

3981 destinata Moesch.

remulate Wik.

a lugubrata Moeach.

$b$ triangulata Pack. montureatn Pack.

c schistacea Warr.

3982 gimilis Wik.

a harveyata Tayl.

3983 explansta Wlk.

form cunigerata $W l k$. diejunctaria Pack.

$a b$. brunneomaculata

Bates.

3984 atrifasciata $\boldsymbol{H}$ lst. delimitata Warr.

3985 xylina Hlst. ab. speciosa Hlat.

Diactinia Warr.

3986 atrocolorata Grt.

3987 silaceata $\boldsymbol{H b n}$.

a albolineata Pack.

b deflavata Staud.

Thera Steph.

3988 variata Schif.

3989 contractata Pack.

3990 otisi Dyar.

3991 georgii Hlst.

Dysstroma Hbn.

3992 truncata $\boldsymbol{H} u \boldsymbol{f} n$.

? transversata Kell.

a mulleolata Hlst.

$b$ brunneata Pack.

c suspectata Mosach.

3993 citrata Linn.

a immanata Haw.

3994 glacialis Hlst.

a longula Hlst.
3995 walkerata Pears.

3996 casloata Tayl.

3997 ethela Hlst.

3998 hersiliata $G n$. Rammifera Wik.

a mirandata Tayl.

3999 cervinifascia Wlk.

4000 occidentata Tayl.

form mutata Tayl.

4001 decorata Tayl.

4002 formosa Hlst.

a boreata Tayl.

4003 hulstata Tayl.

4004 leoninata Pack.

4005 mancipata Gn. subochreata Pack.

Eurhinosea Pack. 4006 flavaria Pack.

Hydriomena Hbn. 4007 furcata Thun. elutata Hbn.

a quinquefasciata Park.

$b$ viridata Pack.

c sordidata Fabr.

d resecta Swett.

e periclata Swett.

$f$ albifasciata Pack.

4008 reflata Grt. abacta Hlst.

4009 nubilofasciata Pack.

a raptata Swett.

b scalata Warr.

c cupidata Swett.

$d$ cumulata Swett.

c vulnerata Swett.

$f$ sparsimacula Hlat.

4010 henshawi Surett.

4011 manzanita Tayl.

4012 cochiseata Suett.

4013 irata Suett. 
4014 cœrulata Fabr. autumnalis Strom. pluviata $\mathbf{G n}$. divisaria Wlk. $a$ perfracta Swett.

$b$ crokeri Swett. c columbiata Tayl. $d$ constricta Strand. $e$ nigrescens Hoyn.

4015 frigidata Wlk.

4016 transfigurata Swett. 4017 renunciata $W l k$. 4018 californiata Pack. $a$ niveifascia Swett. 4019 glaucata Pack. 4020 banavahrata Stkr. 4021 similaris Hlst. glenwoodata Swett.

4021, 1 terminipunctata $B . \& M c D$. 4022 chiricahuata Swett.

4023 mediodentata $B$. \& $M c D$. 4024 ruberata Frey. 4025 edenata Swett. 4026 bistriolata Zell. 4027 regulata Pears. 4028 barnesata Swett. 4029 speciosata Pack. $a$ agassizi Swett.

$b$ taylori Surett.

$c$ ameliata Swett.

4030 magnificata Tayl.

4031 costipunctata $B$. \& $M c D$.

Ersephila Hlst.

4032 indistincta Hlst.

4033 grandipennis Hlst.

Carptima Pears.

4034 hydriomenata Pears.

Catoclothis Hlst.

4035 frondaria Grt.
Grossbeckia B. \& MeD.

4036 semimaculata $B . \& M c D$.

Xanthorhoe Hbn.

4037 lacustrata $G n$.

4038 designata Hufn.

$a$ emendata Pears.

form gynandrata Pears.

4038,1 alticolata $B . \& M c D$.

4039 ferrugata Clerk. inclinataria Wlk. form unidentaria Haw.

4040 dentilinea $B$. \& $M c D$. 4041 munitata $\mathrm{Hbn}$.

strigata Pack.

anticostiata Stkr.

a labradorensis Pack. 4042 convallaria $G n$.

form defensaria $G n$. californiata Pack. amorata Hlst. thanataria Swett. form mephistaria Swett. suppuraria Swett. form conciliaria Swett. form gigantaria Swett.

4043 incursata $\mathrm{Hbn}$.

4044 borealis Hlst.

4045 nemorella $\mathrm{Hlst}$. illocata Hlst.

4046 montanata Schiff. a lapponica Staud.

4047 algidata Moesch. 4048 pontiaria Tayl. 4049 fossaria $T a y l$. 4050 iduata $G n$. planata Tayl.

4051 columelloides $B$. \& $M c D$. 4052 spaldingaria Grossb. 4053 abrasaria $H$. S.

a congregata Wik. nigrofasciata Pack.

$b$ trilineata Warr. salvata Pears. 
Orthonama IIbn.

Perenoptilote Hlat.

$\$ 054$ obstipata Fabr. Auviate Hbn. peracutata Wik. obruptata Wlk. alternata Wlk. pigrate WIk. brunueipennis Hlst.

Calostigia Hbn.

4055 turbata $\mathrm{Hbn}$.

a circumvallaria Tayl.

Loxofidonia Pack.

4056 acidaliata Pack.

Psychophora Kirby.

4057 sabini Kirby.

a polaris Hlst.

4058 phocata Moesch.

Dasyuris Gn.

4059 polata Dup.

punctipes Curt.

$a$ brullei Lef.

b immaculata Skin.

Entephria Hbn.

4060 aurata Pack.

4061 multivagata Hlst.

4062 takuata Tayl.

4063 lagganata Tayl.

4064 nigrescens Hlst.

4065 inventaria Grt.

\section{Spargania Gn.}

4066 viridescens Grossb.

4067 aurata Grt. daira Druce.

4068 magnoliata $G n$. ineommodata Wlk.

placidnta Wik.

zicznentn Wlk.

cumatilis G. \& R. a pernotata $\mathrm{H} l s t$.

b quadripunctata Pack.

Oporinia Hbn.

4069 dilutata Schiff. impluviata Bork. inseriptata Don. fimbriata Haw. form autumnata $G$.

Euphyia Hbn.

4070 implicata $G n$.

a multilineata Pack. villiamsi Swett.

b grandiosa Hlat.

4071 lacteata Pack.

4072 intermediata $G u$. ideata WIk.

4073 mirabilate Grt.

4074 centrostrigaria Woll. latirupta Wlk. luscinate Zell.

4075 immediats Grt. 4076 multiferata Wlk. 4077 luctuata Sehiff. kodiakata Pack.

a obductata Moesch. concordata Wik.

Hammaptera H. S. 4078 parinotata Zell. 4079 densata Grossb.

Camptogramma Steph. 4080 neomexicana $H$ lst. 4081 indefinita Grossb. 4082 multipunctata Tayl. 4083 floridata Wlk. 4084 australata Hlst. 4085 stellata $G n$. impauperata Wlk. albosignata Pack.

Mesoleuca Hbn.

4086 ruficillata $G n$. 4087 gratulata $W i k$. brunneiciliata Pack. 
Eulype Hbn.

4088 hastata $L$.

a gothicata $G n$. furcifascia Wlk. hecate Butl.

$b$ subhastata Nolc. ab. mostata Nolc.

Epirrhoe Hbn.

4089 tristata Linn. limbopunctata Nolc.

4090 plebeculata $G n$. rubrosuffusata, Pack.

4091 medeifascia Grossb.

4092 alternata Mull. sociata Bork.

Perizoma Hbn.

4093 explagiata Wlk.

4094 basaliata Wlk. a grandis Hlst. form saawichata Swett.

4095 alaskæ Hlst. 4096 actuata Pears. 4097 interrupta Grossb. 4098 ochreata Grossb. 4099 custodiata $G n$. gueneeata Pack.

4100 carnata Pack.

4101 polygrammata $\mathrm{Hlst}$. 4101, 1 tahoensis $B$. \& $M c D$. 4102 curvilinea Hlst. occidens Hlst. 4103 costiguttata Hlst. 4104 herbicolata Hlst. 4105 osculata Hlst. 4105, 1 epictata $B$. \& $M c D$. 4106 ablata Hlst. 4107 oxygramma Hlst.

Ceratodalia Pack. 4108 gueneata Pack. excurvata Grt.
Earophila Gumpb.

4109 vasiliata $G n$. spilosaria Wlk.

- rigidata Wlk. form niveifasciata $\mathrm{H} l s t$.

Eutrepsia H.-S.

4110 inconstans Geyer. cephisaria Grt.

Zenophleps Hlst.

4111 lignicolorata Pack.

4112 victoria $T$ ayl. 4113 obscurata Hlst.

Venusia Curt.

4114 cambrica Curt. erutaria Dup: nebulosaria Frey. scitularia Wlk.

4115 duodecemlineata Pack. unipecta Pears. $a$ secunda Pears. 4115, 1 obsoleta Swett. 4116 comptaria Wlk. perlineata Pack. salienta Pears.

4117 pearsalli Dyar.

Hydrelia $\mathrm{Hbn}$.

4118 lucata $G n$. condensata Wlk.

4119 inornata Hlst. exhumata Pears.

4120 albifera $W l k$. albogilvaria Morr. triseriata Pack.

4121 brunneifasciata Pack.

Eudule Hbn.

4122 mendica Wlk. biseriata H. S. 4123 meridiana Sloss. 4124 helveta Barnes. 
4125 unicolor Rob. hyaline Hlat.

Gymnocelis Mab.

4126 minuta Hlat.

Nasusina Pears.

4127 inferior Hlat.

4128 gypsata Grt.

4129 leucata $H$ lst.

4130 remorata Grossb.

4131 discoidalis Grossb.

4132 artestata Groseb.

4133 desperata Hlst.

4134 mellisa Grossb. insipidata Pears.

4135 opinata Pears.

4136 niveifascia Hlst.

\section{Eupithecia Curt.}

4137 albipunctata Haw.

4138 misturata Hlot.

4139 huachuea Grossb.

4140 miserulata Grt. nebulosa Hist. grossbeckiata Swett.

4141 frostiata Swett. conformata Pears.

4142 russeliata Swett.

4143 vaporata Pears.

4144 affinata Pears.

4145 filmata Pears.

4146 insignificata Tayl.

4147 sublineata Tayl.

4148 obumbrata Tayl.

4149 cimicifugata Pears.

4150 modesta Tayl.

4151 plumbaria Hlst.

4152 bifasciata $D_{y / a r}$.

4153 catskillata Pears.

4154 minorata Tayl.

$\$ 155$ chagnoni Swett.

4156 palpata Pack.

4157 luteata Pack.

4158 columbiata Dyar.
4159 erpata Pears.

4160 ornata Hlet.

4161 edna Hlat.

4162 laisata Stkr.

4163 unicolor Hlat.

4164 rotundopuncta Pacl.

4165 sierre Hlst.

4166 bivittata Hlst.

4167 obscurior Hlst.

4168 coagulata $G n$. fumosa Hlst.

4169 geminata Pack. packardata Tayl. meritata Pears.

4170 cocoata Pears.

4171 casloata Dyar.

4172 niphadophilata Dyar.

4173 strattonata Pack.

4174 multiscripta $H$ lot.

4175 perillata Pears.

4176 cretaceata Pack.

4177 zygadæniata Pack.

tenebrescens Hist.

4178 grata Tayl.

4179 multistrigata $\mathrm{H}$ lst.

4180 lagganata Tayl.

4181 compactata Tayl.

4182 spaldingi Tayl.

4183 longidens Hlst.

4184 dyarata Tayl.

4185 scelestata Tayl.

4186 castigata $\mathrm{Hbn}$.

4187 latipennis $\mathrm{Hlst}$.

4188 winnata $T a y l$.

4189 alberta Tayl.

4190 regina Tayl.

4191 youngata Tayl.

4192 interruptofasciata Pack.

4193 dodata Tayl.

4194 adornata Tayl.

4195 lachrymosa Hlst.

4196 perfusea $H$ lst.

4197 limnata Pears.

4198 olivacea $T a y l$.

4199 terminata Tayl. 
4200 coloradensis $\mathrm{Hlst}$. 4201 subcolorata Hlst. 4202 seriptaria $H$. S. 4203 gelidata Moesch. 4204 hyperboreata Staud. 4205 californiata Gump. 4206 insolabilis Hlst.

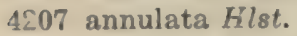
4208 albicapitata Pack. 4209 mutata Pears. 4210 borealis Hlst. 4211 cretata $\mathrm{Hlst}$. 4212 nimbicolor Hlst. 4213 plenoseripta Hlst. 4214 longipalpata Pack. 4215 cupressata Pears. 4216 mrestosa Hlst. 4217 nimbosa Hlst. 4218 stellata $\mathrm{Hlst}$. 4219 bolteri Hlst. 4220 flavigutta Hlst. 4221 flebilis Hlst. 4222 behrensata Pack. 4223 scabrogata Pears. 4224 subapicata $G n$. occidentalis Pack.

4225 acutipennis Hlst. 4226 nevadata Pack. 4227 cestata Hlst. 4228 ravocostaliata Pack. 4229 implorata Hlst. 4230 helena Tayl. 4231 togata $H b n$. 4232 agnesata Tayl. 4233 perbrunneata Tayl. 4234 placidata Tayl. 4235 slocanata Tayl. 4236 fletcherata Tayl. 4237 bryanti Tayl. 4238 harveyata Tayl. 4239 hanhami Tayl. 4240 intimata Pears. 4241 quakerata Pears. 4242 conceptata Peurs. 4243 usurpata Pears.
4244 classicata Pears. 4245 exudata Pears. 4246 cognizata Pears. 4247 valariata Pears. 4248 incresata Pears. 4249 adequata Pears. 4250 dolorosata Pears. 4251 bindata Pears. 4252 piccata Pears. 4253 segregata Pears. 4254 subfoveata Dyar. 4255 cootenaiata Dyar. 4256 harlequinaria Dyar. 4257 emmedonia Grossb. 4258 pretansata Grossb. 4259 purpurissata Grossb. 4260 swetti Grossb. 4261 indistincta Tayl. 4262 carolinensis Grossb. 4263 taylorata Swett. 4264 brauneata Swett. 4265 promulgata Pears. 4266 albigrisata Pears. 4267 fortunata Pears. 4268 suspiciosata Dietz. 4269 subvirens Dietz. 4270 chlorofasciata Dietz. 4271 gibsonata Tayl. 4272 fasciata Tayl. 4273 quebecata Tayl. 4274 fumata Tayl. 4275 tenuata $\mathrm{Hlst}$. 4276 anticaria $W l k$. implicata Wlk. explanata Wlk.

4277 penumbrata Pears.

4278 graefi Hlst.

4279 vancouverata Tayl.

Horisme $\mathrm{Hbn}$.

4280 intestinata $G n$. impleta Wlk. indoctrinata Wlk.

4281 vitalbata $D$. \& $S$. 4282 rectilineata Tayl. 4283 gillettei Hlst. 
Cysteophori Hist.

$\$ 284$ pervertipennis Hist.

Geometrina

Fernaldella IIIst.

4285 fimetaria $G$. \& $R$. halesaria Zell.

a partitaria Grt.

4286 stalachtaria Stkr. alternaria Grt.

Isturgia Hbn.

4287 truncataria W/k.

4288 amitaria $G n$. faxoni Minot.

Eufidonia Pack.

4289 notataria WIk.

of fidoniata W1k.

guadripnenctata Morr. ab. famulata Hlst.

a convergaria Wlk.

bicoloraria Minot.

b discospilata $W l k$.

Orthofidonia Pack.

4290 tinctaria Wlk.

distinctaria D'Urban. exornata Wik.

albifusata Wik.

deceptata Hlst.

a flavivenata Hlst.

Dasyfidonia Pack.

4291 avuncularia $G n$.

Bapta Steph.

4292 semiclarata Wlk. viatica Harv.

4293 vestaliata $G n$.

4294 elsinora Hlat.

4295 glomeraria Grt.
Heliomata Grt.

4296 infulata Gr.

4297 elaborata Grt.

4298 cycladata Grt.

Mellilla Grt.

4299 inextricata Wlk.

foridensis Hlst.

4300 xanthometata Wlk.

chamaechrysaria Grt. rilevaria Pack.

form snoviaria Pack.

Chloraspilates Pack.

4301 bicoloraria Pack.

a arizonaria Grt.

4302 minima Hlst.

Physosteganis Warr.

4303 pustularia $G n$.

Gueneria Pack.

4304 basiaria Wlk.

incoloraria Whk.

congrua WIk.

Cabera Tr.

Deilinia Hbn.

4305 borealis Hlat. a solamata Hlst.

4306 quadrifasciaria Pack. elimaria Hlst.

4307 variolaria $G n$. intentata W1k.

4308 undularia $B$. \& $M C D$.

4309 erythemaria Gn. similaria Wlk.

4310 pacificaria Pack.

4311 bryantaria Tayl.

Drepanulatrix Gump.

4312 rectifascia $H / s t$.

$a$ erubescens Warr. 
4313 bifilata Hlst.

o perpallidaria Grt. carneata Warr.

4314 monicaria $G n$. celataria Hlst. indurata Dyar. mercedulata Stkr.

4315 fominaria $G n$. ferruginosaria Pack.

4316 californiaria Pack. 4316, 1 secundaria $B$. \& $M c D$. 4317 falcataria Pack. electa Hlst.

4318 nevadaria Hlst. 4319 ella Hlst. 4320 carnearia $H$ lst. 4320,1 lutearia $B$. \& $M c D$. 4321 pulveraria $\boldsymbol{H}$ lst. 4322 verdiaria Grossb.

4323 litaria Hlst. fumosa Hlst.

4324 hulsti Dyar. lenitaria Gross.

4325 liberaria Wlk. integraria Wlk. lintneraria Pack. ab. helena Hlst.

4326 quadraria Grt. 4327 unicalcararia $G n$. behrensaria Hlst. form cervinicolor Hlst. 4328 ida Hlst.

\section{Episemasia Hlst.}

4329 morbosa Hlst. 4330 cervinaria Pack.

Philobia Dup.

4331 remulataria Wlk. sectomaculata Morr.

4332 ulsterata Pears.

4333 perplexata Pears.

4334 aspirata Pears.

4335 versitata Pear 8.
Macaria Curt.

4336 æquiferaria $W l k$. postrema Wlk. morosaria Wlk. festa Hlst.

4337 atrimacularia $B$. \& $M c D$. 4338 minorata Pack.

$a$ incolorata Dyar.

4339 bisignata Wlk. galbineata Zell.

4340 inaptata Wlk.

4341 bicolorata Fabr. praeatomata Haw. consepta Wlk. consimilata Zell. grassata Hlst.

4342 punctolineata Pack. simulata Hlst.

4343 distribuaria $\mathrm{Hbn}$. oppositaria $\mathrm{Gn}$. proseanthata Wlk. antaurata Wlk.

$a$ transitaria Wlk. 4344 multilineata Pack. patriciata Grt.

4345 granitata $G n$. contemptata Gn. haliata Gn.

? retractaria Wlk.

$a$ irregulata Wlk.

$b$ quadrisignata Wlk.

c dispuncta Wlk. inordinaria. Wlk. subapiciaria Wlk. haliata Wlk. fissinotata Wlk.

d sexmaculata Pack. labradoriata Moesch.

e retinotata Wlk.

$f$ exnotata Wlk. $g$ submarmorata Wlk.

4346 succosata $Z$ Zell.

4347 unimodaria Morr. 4348 abruptata Wlk. 
4349 purcellata Tayl.

4350 oweni Swett.

4351 denticulata Grt. a sexpunctata Bates.

4352 quadrinotaria $H .-5$. septemfluaria Grt. septemlinearia Grt.

Phasiane Dup.

4353 sublacteolata Hlot. Iapitaria Stkr.

4354 colorata Grt. conarata Grossb.

4355 parcata Grossb. 4356 pallidata Pack. 4357 ealiforniaria Pack. 4358 delectata $\mathrm{Hlat}$. 4359 maricopa $H$ lst. 4360 respersata Hlat. subacuta Hlst. a teucaria Stkr. 4361 dislocaria Pack. 4362 museariata $\mathrm{Gn}$. 4363 pictipennata $\mathrm{Hlst}$. 4364 nubiculata Pack. 4365 pervolata $H$ lst. 4366 nigrocomina Warr. heliothidatał Auct.

4367 ocellinata $G n$. duplicata Pack.

4368 stipularia $B . \& M c D$. 4369 flaviterminata $B$. \& $M c D$. 4370 subterminata $B . \& M c D$. 4371 irrorata Pack. 4372 neptaria $G$ n. Ravofasciata Pack. cinereata Bates. a trifasciata Pack. b sinuata Pack. 4373 mellistrigata Grt. 4374 hebetsta $H$ lst. a decorata Grossb. sinuatat Warr. 4375 yavapai Grossb. 4376 subminiata Pack.
4377 moviata Pack. enbminiatat Pack.

4378 gillettenta Dyer.

4379 meadiaria Puck.

4380 infimate $G n$. gnophosaria $\mathrm{Gn}$. infectata Wik. reductaria Wik. caesiaria Hlst.

4380,1 fieldi Swett. 4380, 2 grossbecki Swett. 4381 puertata Grossb. 4382 minuta Hlst. 4383 s-signata Pack. 4384 hyprethrata Grt. 4385 continuats Wlk. a strigularia Wlk. 4386 atrofasciata Pack. 4387 orillata $W l k$.

$a$ curvata Grt. cruciata Grt. 4388 excurvata Pack. cinereola HIst. spodopterata Hlst.

4389 eremiata $G n$. retectata Wlk. gradata Wik. retentata Wik. subcinctaria WIk.

4390 ordinata $W l k$. aucillaria Stkr.

4391 maculifascia $H$ lst. 4392 quadrifasciata Tayl. 4393 sabularia $G n$. 4394 mendicata $H / s t$. 4395 tenebrosata Hlst. 4396 octolineata Hlst. 4397 flumenata Pears.

Hesperumia Pack.

4398 sulphuraria Pack.

a ochreata Pack. form baltearia $H$ lst. form unicoloraria Hlst. 
Itame Hbn.

Diastictisł Hbn.

4399 ribearia Fitch. sigmaria $\mathrm{Gn}$. annisaria Wlk. aniusaria Wlk. grossulariata Saund.

4400 sulphurea Pack. sulphuraria Pack. 4401 occiduaria Pack. 4402 subfalcata Hlst. 4403 flavicaria Pack. 4404 fascioferaria Hlst. 4405 evagaria $\mathrm{Hlst}$. 4405, 1 andersoni Swett. 4406 inceptaria $W l k$. argyllacearia Pack. modestaria Hlst. olivalis Hlst.

4407 brunneata Thun. ferruginaria Pack.

4408 subcessaria Wlk. perarcuata Wlk. 4408, 1 perornata $B . \& M c D$. 4409 coortaria Hlst. 4410 quadrilinearia Pack. inquinaria Hlst.

4411 anataria Swett. 4412 pallescens Grossb. 4413 exauspicata Wlk. 4414 trilinearia Grossb. 4415 coloradensis Hlst. aegaria Stkr. disparata Warr.

4416 guenearia Pack. 4417 umbriferata Hlst. umbrifasciata Hlst.

4418 benigna Hlst. 4419 correllata Hlst. 4420 colata Grt. sericeata Hlst. 4421 bitactata Wlk. atrosignata Wlk. 4422 packardaria Moesch.
4423 denticulodes $\mathrm{Hlst}$. 4424 decorata Hlst. 4425 pustularia $\mathrm{Hbn}$. latifermigata Wlk. brunneata Pack.

4426 graphidaria Hlst. 4427 matilda Dyar. 4428 subalbaria Pack. 4429 hulstiaria Tayl. subalbaria Hlst.

4430 virginalis Hlst. 4431 varadaria $W l k$. florida Hlst.

4432 pallipennata $B . \&$ McI). 4433 grossbecki $B$. \& McD. 4434 donataria $W l k$. 4435 crassata $\mathrm{Hlst}$. 4436 pallidula Hlst. 4437 particolor Hlst. 4438 crocearia Hist. 4439 deleta Hlst. 4440 gausaparia $G r t$. 4441 nicetaria $G n$. vagaria Wlk. 4442 solitaria Wlk. 4443 nervata $G n$. tractata Wlk.

Nyctiphanta Hlst. 4444 lætula Hlst.

Apolema Hlst. 4445 carata Hlst.

Euaspilates Pack. 4446 spinataria Pack.

Anthelia Hlst.

4447 taylorata Hlst. angulata Warr. 4448 hyperborea Hlst. 4449 nigroseriata Pack. fautaria Hlst. 
Elpiste Gump.

Gladela Grossb.

4450 marcescaria $G n$. cinerarie Pack.

4451 lorquinaria $G n$. tripunctaria Pack.

4452 metanemaria Hlst.

Dysmigia Warr.

4453 loricaria Evere. julia Hlst. nubilata Warr.

Homochlodes Hlst.

4454 fritillaria $G n$. disconventa W1k. lactispargaria Wlk. immergata Wlk.

Apæcasia Hlst.

4455 detersata $G n$.

4456 atropunctata Pack. fernaldi Grt. 4457 subsequaria $W l k$. defuata Wlk.

4458 extremaria Wlk. ordinata Wlk.

4459 graefiaria Hlat.

Catopyrrha Hbn.

4460 coloraris Fabr. accessaria Hbn. cruentaria Hbn.

form aest. dissimilaria $\boldsymbol{H b n}$.

olenusaria Wlk.

4461 sphæromacharia Harv. $a$ perolivata $H$ lat.

Sericosema Warr. Euemera Hist.

4462 juturnaria $G n$. a californiaria Pack.

$b$ immaculata $B$. \& McD.
4468 simularia Tayl. 4464 viridirufaria Neum.

Caripeta WIk.

4465 divisata $W 1 k$. albopuretata Morr.

1466 piniata Paek. seductaria Stkr.

4467 angustiorata $W l k$. criminosa Swett.

4468 latiorata $W l k$. 4469 aretaria $W l k$. subochrearia Grt.

4470 sequaliaria Grt. interalbicans Warr.

Phengommatæa Hlst.

4471 edwardsata Hlat. 4472 spoliata Grossb. 4473 niveostriata $\mathrm{Ckll}$. gertruda Hlst. 4474 dissimilis Hlat.

Platrea H. S.

4475 californiaria $H$. $S$. personaria Hy. Edw.

4476 lessaria Pears.

4477 uncanaria $G n$.

4478 trilinearia Pack. $a$ dulciaria Grt.

4479 diva $H$ lst. 4479,1 triangularia $B . \& M C D$.

Enypia Hlst.

4480 venata Grt.

4481 perangulata $H$ lst.

4482 griseata Grossb.

4483 packardata $T a y l$.

Philedia Hlst.

4484 punctomacularia $\mathrm{H}$ lat.

Nepytia Hlst.

4485 umbrosaria Pack.

a nigrovenaria Pack.

4485,1 regulata $B . \& M c D$. 
4486 canosaria Wlk. pulchraria Minot. piniaria Pack.

4487 phantasmaria Stkr. 4488 semiclusaria Wlk. fumosaria Stkr. a pellucidaria Pack.

Pygmæna Bdv.

4489 simplex Dyar.

Paraphia Gn.

4490 unipuncta Haw. deplanaria $\mathbf{G n}$. triplipunctaria Fitch.

4491 guttata Hlst. 4492 subatomaria Wood. nubecularia Gn. mammuraria Gn. impropriata Wlk. fidoniata Wlk. exsuperata Wlk. piniata Pack.

Spodolepis Hlst.

4493 substriataria $H$ lst. a danbyi Hlst. demorsaria Stkr.

Stenotrachelys Gn.

4494 approximaria $H b n$. 4495 permagnaria Pack.

Lytrosis Hlst. 4496 unitaria $H$. S.

Meris Hlst.

4497 alticola Hlst.

4498 speciosa Hlst.

Pterotæa Hlst.

4499 cariosa Hlst.

4500 memoriata Pcars. 4500,1 tremularia $B . \& M c D$. 4500,2 serrataria $B . \& M C D$.
Ixala Hlst.

4501 desperaria Hlst. a unicoloraria $\mathrm{Hlst}$. 4502 adventaria Pears.

Pterospoda Dyar.

4503 nigrescens $H$ lst. 4504 opuscularia Hlst. 4505 kunzei Hlst.

Tornos Morr. 4506 scolopacinarius $\mathrm{Gn}$. pervelata Wlk. robiginosus Morr. abjectarius Hlst.

4507 erectarius Grossb. 4508 cinctarius Hlst. 4509 fieldi Grossb.

Exelis Gn.

4510 pyrolaria $G n$. tensaria Wlk. approximaria Pack.

Glaucina Hlst.

4511 escaria Grt. 4512 erroraria Dyar. 4513 macdunnoughi Grossb. 4514 eupetheciaria Grt. pygmeolaria Grt.

4515 puellaria Dyar. 4516 elongata Hlst. 4517 pearsalli Grossb. 4518 abdominalis Grossb. 4519 bilineata Grossb. 4520 epiphysaria Dyar. 4521 golgolata Stkr. 4522 magnifica Grossb. 4523 hulstinoides Grossb. 4524 mormonaria Dyar.

Cœnocharis Hlst.

4525 interruptaria Grt. 4526 ochrofuscaria Grt. 4527 indistincta Grossb. 
4528 obseura Groesb.

4529 infumataria Grt. ignavaria Pears.

4530 denticuleria Dyar.

4531 eureka Groseb.

4532 imperdata Dyar.

4538 alboceptata Dyar.

Synglochis IIlst.

4534 perumbraria Hlat.

Morins Grossb.

4535 coniferaria Grossb.

4535,1 curvata $B . \& M c D$.

Stenocharis Grossb.

4536 permagnaria Grossb.

Holochros Hist.

4537 dissociarius Hlst.

Barnesia Grossb.

4538 ritaria Grossb.

Hulstina Dyar.

4539 packardaria Hlst. lachrymosa Hlst. homopteroides Hlst.

Parexcelsa Pears.

4540 ultraria Pears.

4540,1 inconspicuaria $B$. \& $M C D$.

Chesiadodes Hlst.

4541 morosata Hlst.

Cleora Curt.

4542 wrightiaria Hlst.

4548 inconspicus Hlst. aethalodaria Dyar:

4544 lamiaria Stkr.

4545 formosata Hlst. terlineata Dyar.

4546 agrestaria Groseb.

4547 quinquelinearia Pack.
4548 Jacumbaria Dyar.

4549 obliquarie Grt. rufaria Grt.

4550 depromaria Grt.

4551 dissonaria Hlat.

4552 addendaria Groseb.

4553 excelsaria Stkr.

4554 albescens Hlat.

4555 anastomosaria Groseb.

4556 nigricaria $B$. \& $M e D$.

4557 separataria Grt.

4558 purpuraria $B$. \& $M C D$.

4559 dejecta Hlst.

4560 umbrosaria $\mathrm{Hbn}$. porcellaria $\mathbf{G n}$.

a gnopharia Gn.

4561 grisearia Grt.

4562 pulchella Grossb.

4563 furfuraria $H$ lat.

4564 pexata Swett.

4565 indicataria $W l k$. filaria Wik. maestosa Hlst. ab. fuliginaria Hlst.

4566 cribrataria $G n$.

4567 sublunaria Gn.

? transfixaria Wlk. atrolinearia Hlst. areataria Broad.

4568 manitoba Grossb.

4569 configurata Hlst.

4570 pampinaria $\mathrm{Gn}$.

frugaliaria $\mathbf{G n}$. collecta WIk.

fraudulentaria Zell.

a erosiata Wlk.

b nubiferaria Suett.

4571 atristrigaria $B$. \& $M c D$.

4572 polygrammaria Pack.

4573 newcombi Swett.

4574 humaria $\mathrm{Gn}$.

intraria Gn.

intractaria WIk.

illaudata WIk.

4575 vellivolata $\boldsymbol{H}$ lst. 
4576 plumosaria Pack. 4577 pergracilis Hlst.

? momaria $\mathrm{Gn}$.

4578 dataria Grt.

4579 pulmonaria Grt.

4580 defectaria $G n$.

albigenaria Wlk.

4581 emasculata Dyar.

4582 clivinaria $G n$.

4583 fragilaria Grossb.

4584 ephyraria $W l k$.

expressaria Wlk.

takenaria Pears.

4585 larvaria $G n$.

4586 opacaria $\mathrm{Hlst}$.

4587 lixaria Grt.

4588 lallata Hlst.

4589 glaucaria Grossb.

4590 lineata Hlst. incopriaria Hlst.

4591 spododea Hlst.

4592 lichenaria Pears.

4593 californiaria Pack.

form latifasciaria Pack.

4594 latipennis Hlst.

4595 fumida Warr.

4595,1 perpictaria $B . \& M c D$.

Mericisca Hlst.

4596 gracea Hlst.

Somatolophia Hlst.

4597 haydenata Pack. umbripennis Hlst.

4598 obliterata Hlst.

Melanolophia Hlst.

4599 canadaria $G n$. signataria Wlk. imperfectaria Wlk. ejectaria Wlk. patularia D’Urban.

4600 imitata $W l k$.

subgenericata Dyar.
Aethaloptera Hlst. 4601 anticaria $W l k$. submuraria Wlk.

Glena Hlst.

4602 cognataria $\mathrm{Hbn}$. infixaria Wlk. umatillaria Stkr. insaria Dyar. muricolor Hlst. luridula Hlst.

4603 texanaria Hlst.

Ectropis Hbn.

4604 crepuscularia $D . \& S$. occiduaria Gn. signaria Wlk. spatiosaria Wlk. intrataria Wlk. cineraria Wlk. cunearia D'Urban. $a b$. fumataria Minot. form abraxaria Wlk. divisaria Wlk. fernaldaria Hlst.

Stergamatæa Hlst. 4605 inornata $\mathrm{Hlst}$. 4606 delicata $\mathrm{Hlst}$. $a$ dolliata Grossb.

Epimecis Hbn.

4607 virginaria Cram. hortaria Fabr. liriodendraria A. \& S. disserptaria Wlk. amplaria Wlk.

form dendraria $G n$. gravilinearia And. form carbonaria Haim.

Lycia Hbn.

4608 ursaria Wlk. 
Amphidasis $\mathrm{Tr}$.

4609 cognataria $\mathrm{Gn}$.

Cochisea B. \& McD.

4609, 1 rigidaria $B . \& M C D$.

4609, 2 sinuaria $B$. \& $M c D$.

Phæoura Hlst.

4610 mexicanaria Grt.

4611 cristifera Hlst.

Gabriola Tayl.

4612 minima Hlat. bidisata Dyar.

4613 dyari Toyl.

Nacophora Hlst.

4614 ypsilon Forbes. carlotta Hlst.

4615 quernaria A. \& S. of phigaliaria $\mathbf{G n}$. sperataria WIk.

of paenulataria Grt.

\& cupidaria Grt.

form atrescens Hlst.

\section{Ceratonyx Gn.}

4616 satanaria $\mathrm{Gn}$.

\section{Pacilopsis Harrsn.}

4617 rachelæ Hlat.

Coniodes Hlst.

4618 plumogeraria Hlot.

Graefia Pears.

4619 smithi Pears.

\section{Phigalia Dup.}

4620 olivacearia Morr. ab. mephistaria Reiff.

4621 denticulata Hlst.

4622 titea Cram. revocata Wlk. strigataria Minot. cinetaria Frch.

a nevadaria Hlat.
Palæacrita Riley.

4623 longiciliate Hlst.

4624 vernata Peek.

sericeiferata Wik. autumuata Pack.

4625 merriccata Dyar. 4626 speciosa Hlot.

Erannis Hbn.

4627 vancouverensis Hlot. 4628 tiliaria Harris. 4629 coloradata Hlst.

Cingilia Wlk.

4630 catenaria Dru. devinctaria $\mathrm{Gn}$. humeralis WIk. $a b$. immacularia Swett. 4631 rubiferaria Swett.

Eucaterva Grt. 4632 variaria Grt. lymax Druce. form \& labesaria Grt.

Philtræa Hlst. 4633 elegantaria $H y . E d w$.

Acanthophora Hlst. 4634 graefi Hlat.

Tracheops Hlst. 4635 bolteri Hlst.

Aspilates $\mathrm{Tr}$.

4636 orciferaria Wlk.

a labradoriata Moesch. aberrata Hy. Edw. taylori Butl.

Lychnosea Grt.

4637 helveolaria Hlst. aulularia Grt. \& runciraria Stkr.

4638 intermicata Wlk. pervaria Pack. form interminaria Gre. 
Melemæa Hlst.

4639 magdalena Hlst. 4640 virgata Tayl.

Anagoga $\mathrm{Hbn}$.

4641 pulveraria Linn. form occiduaria Wlk. plagifasciata Wlk. californiaria Pack.

Neoterpes HIst.

4642 ephelidaria Hlst. form kunzei Hlst. 4643 edwardsata Pack.

Sicya Gn.

4644 macularia Harris. sublimaria Gn.

$a$ solfataria $G n$. calipusaria Wlk. faustinaria Stkr.

$b$ truncataria $G n$. agyllaria Wlk. crocearia Pack.

e lewisi Swett.

4645 snoviaria Hlst. 4646 morsicaria Hlst. 4646, 1 olivata $B . \& M c D$.

\section{Ellopia Tr.}

4647 vitraria $G r t$. 4648 punctata Hlst. 4648, 1 phantoma B. \& McD. 4649 læta Hlst. favilinearia B. \& McD. 4650 hyalinaria Grossb. 4651 pellucidaria $G . \& R$. 4652 athasaria $W l k$. siccaria Wlk. seminudata Wlk. aequaliaria Wlk. bibularia G. \& R. 4653 endropiaria $G$. \& $R$. fatuaria Stkr.
4654 fiscellaria $G n$. form flagitiaria $\mathrm{Gn}$. form johnsoni Swett. form peccataria Swett. a lugubrosa Hlst. 4655 fervidaria $\mathrm{Hbn}$. 4656 pultaria $G n$. scitata Wlk. \& invexata Wlk.

4657 somniaria Hlst. 4658 brunneolineata Hlst.

Campæa Lam. 4659 perlata $G n$. viridoperlata Pack.

Slossonia Hlst.

4660 rubrotincta Hlst.

Eugonobapta Warr. 4661 nivosaria $G n$.

Sericoptera H. S.

4662 virginaria $\mathrm{Hlst}$. form vestalis $\mathrm{H} l s t$.

Himera Dup.

4663 pennaria $L$.

Ennomos Tr.

4664 subsignarius $H b n$. niveosericeatus Jones.

4665 magnarius $G n$. lutarius Wlk. $a$ ochreatus Hlst.

Xanthotype Warr.

4666 crocataria Fabr. citrina Hbn. ab. crlaria Hlst.

Plagodis Hbn.

4667 serinaria $H$. S. subprivata Wlk. floscularia Grt. form rosaria Grt. 
4668 kuetzingi Cirt.

nigreacuria Hlst.

4669 altruaria Pears. keutaingaria Pack.

4670 approximaria Dyar.

4671 purpuraria Peurs.

4672 fervidaria $H$. $\lesssim$. emargataria Gn. esenearia Morr.

4673 schuylkillensis Groseb.

4674 alcoolaria $\mathrm{Gn}$.

4675 kempi Hlat.

4676 phlogosaris Gn.

4677 arrogaria Hlat.

\section{Hyperetis Gn.}

4678 amicaria $H . S$. insinuaria Gn.

a alienaria $\boldsymbol{H}$. S. nyssaria $\mathbf{G n}$. persinuaria $\mathrm{Gn}$. subsinuaria Gn. nesionaria Wlk.

$b$ exsinuaria $G n$. neonaria WIk. laticincta Wlk.

e nepiasaria Wlk.

d mollicularia Zell.

4679 trianguliferata Pack. notataria Hlst. a costinotata Warr.

\section{Nematocampa Gn.}

4680 limbata Haw.

vestitaria H. S.

resistaria $\mathbf{H}$. $\mathbf{S}$.

flamentaria Gn.

form expunctaria Grt. ab. chagnoni Swett.

4681 brehmeata Grosab.

\section{Gonodontis Hhn.}

4682 ocellaria Grossb.

4682, 1 macularia $B$. \& $M C D$.

4683 hilumaria Hlst.
4684 canidiaria Stkr. 4685 hypochraria $H . S$. refractaria $\mathrm{Gn}$. mestusata WIk.

a lateritiaria $\mathrm{Gn}$. foedaria WIk. indeclinata W1k.

$b$ homuraria Grt. amethyotaria Stkr.

4686 apiciaria Pack.

4687 warneri Harv.

4688 pilosaria Pack.

4689 duaria $G n$.

hamaria Gn. panisaria Wlk. amyrisaria Wlk. agreasaria Wlk. adustaria Wlk.

4690 ectrapelaria Grosab.

4691 formosa $H$ lst.

4692 obfirmaria $H b n$.

4693 antidiscaria Wlk. lentaria Hlst.

Euchlæna Hbn.

4694 sesquilinearia Grt. 4695 cavillaria Hlst. \& novellata Hlst.

4696 falcata Pack. 4697 argillaria Hlst. 4698 galbanaria Hlst. 4698, 1 detractaria $B$. \& $M c D$. 4699 serrata Dru. concisaria Wlk.

4700 obtusaria $\mathrm{Hbn}$. decisaria Wlk. muzaria Wik. incisaria Wlk.

4701 effecta WIk.

4702 johnsonaria Fiteh. bilinearia Pack. a minoraria Hlst. 4703 mollisaria Hlat. occantaria Hlst. 
4704 amœenaria $G n$. arefactaric G. \& $\mathbf{R}$. 4705 vinulentaria $G$. \& $R$. 4706 astylusaria Wlk. madusaria Wlk. vinosaria G. \& R. a oponearia Wlk. tiviaria Wlk.

$b$ deplanaria $W l k$. 4707 marginata Minot. 4708 pectinaria $D$. \& $S$. deductaria Wlk.

4709 manubiaria Hlst. 4710 tigrinaria $G n$. propriaria Wlk. a sirenaria Stkr. $a b$. abnormalis Hlst.

Selenia Hbn.

4711 alciphearia $W l k$. perangulata Hlst. form aest. ornata $B . \&$ $M c D$.

4712 kentaria $G$. \& $R$.

Lycimna Wlk.

4712, 1 peccataria $B . \& M c D$.

\section{Epiplatymetra Grt.}

4713 coloradaria $G$. \& $R$. 4714 grotearia Pack. q vidularia Grt. angularia Grossb.

\section{Synaxis Hlst.}

4715 fuscata Hlst. 4716 jubararia Hlst. 4717 pallulata Hlst.

Pherne Hlst. 4718 placeraria $G n$. mellitularia Hlst.

4719 parallelia Pack. 4720 subpunctata Hlst.

\section{Metanema Gn.}

4721 inatomaria $G n$.

4722 determinata $W l k$. carnaria Pack.

4723 excelsa Stkr. a simpliciaria Grt.

4724 barnesi Hlst. 4725 hirsutaria $B . \& M c D$. 4726 quercivoraria $G n$. aeliaria Wlk. trilinearia Pack. textrinaria G. \& R. $a b$. $q$ incongruaria $\mathrm{Hlst}$. 4727 brunneilinearia Grossb.

\section{Priocycla Gn.}

4728 armataria $H$. S. 4729 decoloraria Hlst. jucundaria Stkr.

Apicrena Pears. 4730 calcaria Pears.

Snowia Neum.

4731 montanaria Neum.

Stenaspilates Pack.

4732 zalissaria $W l k$. 4733 inviolata $\mathrm{Hlst}$. 4734 radiosaria $\mathrm{Hlst}$. 4735 meskaria Pack. muricolor Hlst. $a$ albomacularia $H y . E d w$. \& arizonaria Hy. Edw.

4736 levisaria Grossb. 4737 albidula Hlst. 4738 flavisaria Grossb. 4739 smithi Grossb. 4740 apapinaria $D y a r$. 4741 metzaria Dyar. 4742 astapa Druce. egregiata Pears. 4743 atrocolorata Hlst. 
Pero H. S.

4744 honestarius Wik. st ygiurius Wik.

4745 giganteus Groseb. 4746 peplarioides $\mathrm{H}$ lal. 4747 behrensarius Puck. 4748 occidentalis $\mathrm{Hlst}$. 4749 modestus Groseb. a grossbecki Pears.

4750 morrisonarius $H y$. Edw. 4751 colorado Grossb. 4752 marmoratus Grossb.

\section{Syssaura IIbn.}

4753 hamulata $G n$. perizomaria Hlst.

4754 olyzonaria Wlk. syzygiavia Hlst.

4755 puber $G . \& R$. \& varus G. \& R. form of sesquilinea Grt. form \& aquosus G. \& $R$. juniperaria Pack.

\section{Apicia Gn.}

4756 fundaria $G n$.

\& juncturaria Gn. effascinaria Hlst. subflevaria Pears.

4757 distycharia $\mathrm{Gn}$. a geniculata Hlst.

$b$ subcineraria Grossb. 4758 confusaria $\mathrm{Hbn}$. remissario Gn. imbraria Gn. superaria $\mathrm{Gn}$. majoraria Gn. ineffusaria $\mathrm{Gn}$. Roridaria Gn. phasianaria Gn. interlinearia $\mathrm{Gn}$. pandaria Wlk. form metrocamparia $G n$. ab. subochrearia Hlst.
4759 irraria B. \& MeD. majoraria $\ddagger$ Pack.

Miorogonia H. S.

4760 zonulatn Hlst. 4761 vesulia Cram.

Tetracis Gn.

4762 erocallata $G n$. form. vern. aspilatata $G n$.

Sabulodes Gin.

4763 arcasaria Wlk.

depontanata Grt. sulphuraria Pack. form. vern. furciferata Pack.

4764 indiscretata $H y$. Edw. vanusaria Stkr.

4765 imitata $H y . E d w$. costinotata Tayl.

4765,1 triangulata $B . \& M c D$.

4766 constans Hlst. 4767 ochreata $H$ lst. 4768 arizonata Tayl. dyari Grossb. form ligata Grossb.

4769 lorata Grt.

4770 truxaliata $G n$. 4771 oblentaria Grt. 4772 cervinaria Pack. \& aurantiacaria Pack. 4773 caberata $G n$. arsesaria Wlk. form ægrotata Gn.

4774 nonangulata Stkr. 4775 forficaria $G n$. nubilata Pack.

a catenulata Grt. anfractata Hlst.

4776 amplicineraria Pears. 4777 transversata D Mury. goniata $\mathrm{Gn}$. contingens Wlk. 
form incurvata $\mathbf{G n}$. transposita Wlk.

a transvertens Wlk.

transmutans Wlk.

4778 politia Cram.

floridata Grt.

Destutia Grossb.

4779 novata Grossb.

Abbottana Hlst.

4780 clemataria $A$. \& S. transducens Wlk. transferens Wlk. transfingens Wlk.
Phrygionis Hbn.

4782 argentata Dru. argentistriata Stkr. cerussaria Grt. obrussata Grt.

Melanchroia Hbn. 4783 regnatrix Grt. 4784 chephise Cram. 4785 geometroides $W l k$. mors Lucas.

Sphacelodes Gn. 4786 vulneraria $H b n$. floridensis Holl.

Palyas Gn.

4781 auriferaria Hlst.

\section{EPIPLEMIDE}

Philagraula Hlst.

4787 slossoniæ Hlst.

Callizzia Pack.

4788 amorata Pack.

4789 certiorata Pears.
Calledapteryx Grt. 4790 dryopterata Grt. erosiata Pack.

Schidax Hbn.

4791 coronaria Stkr.

\section{Superfamily TINEOIDEA}

LACOSOMIDE

Cincinnus Blanch.

4792 melsheimexi Harr. egenaria Wlk.
Lacosoma Grt. 4793 chiridota Grt. 4794 arizonicum Dyar.

\section{PSYCHID\#E}

Oiketicus Guild.

4795 abboti Grt. 4796 townsendi Ckll.

4797 davidsoni Hy. Edw.
Thyridopteryx Steph. 4798 ephemeræerormis Haw. coniferarum Pack.

4799 alcora Barnes. 4800 meadi $H y$. $E d w$. 
Platoceticus Pack.

4801 gloveri Pack. edwardei Heyl.

4802 tracyi Jones.

\section{Eurycyttarus Hamp.}

4803 confederats Grt.

4804 carbonaria Pack.

4805 fragmentella $H y$. Edw. coniferella $\mathbf{H y}$. Edw.

Manatha Moore.

4806 nigrita $B . \& M c D$.

Chalia Moore.

Prochalia B. \& MeD.

4808 pygmea $B$. \& $M C D$.

Apterons Mill.

4808, 1 fragilis B. \& MeD.

Solenobia Zell.

4809 walshella Clem.

Hyaloscotes Butl.

4810 fumosa Butl.

Oedonia Kirby.

4811 exigua $H y$. Edw.

4807 rileyi Heyl.

\section{LIMACODID\&}

Sibine H. S.

4812 stimulea Clem. ephippiotus Harr.

Parasa Moore.

4813 indetermina $B d v$. vernata Pack.

4814 chloris $H$. S. viridus Reak. fraterna Grt. a huachuea Dyar.

Euclea Hbn.

4815 nanina Dyar. nanał Dyar. 4816 delphinii $B d v$. strigata Bdv. quercicola H. S. tardigrada Clem. ferruginea Pack. argentatus Weth. form querceti $\boldsymbol{H}$. $\boldsymbol{S}$. bifida Pack. form interjecta Dyar. form viridiclava Wlk. monitor Pack. form ellioti Pears. form prenulata Clem. exeisa Wlk.

4817 incisa Harv. mira Dyar. 4818 flava $B$. \& $M c D$. 4819 dolliana Dyar. a spadicis Grossb.

Monoleuca G. \& R. 4820 subdentosa Dyar. 4821 semifascia Wlk. $a$ sulfurea Grt. 4822 obliqua $H y . E d w$. 4823 occidentalis B. \& MCD.

Adoneta Clem.

4824 spinuloides $\boldsymbol{H}$. S. voluta Clem. ferrigera Wik. reptilinea WIk. nebulosus Weth. form leucosigma Pack. 
4825 bicaudata Dyar.

4826 рудтæа $G$. \& $R$.

4827 gemina Dyar.

Sisyrosea Grt.

4828 textula $H$. S. inornata G. \& $\mathbf{R}$. 4829 schæfferana Dyar.

Natada Wlk.

4830 nasoni Grt. daona Druce. mude Hy. Edw.

4831 nigripuncta $B . \& M c D$.

Phobetron Hbn.

4832 pithecium $A . \& S$. abbotana Hubn. nigricans Pack. hyalinus Walsh. tetradactylus Walsh. nondescriptus Weth.

Isochætes Dyar.

4833 beutenmuelleri $\boldsymbol{H} y$. $E d w$.

Cryptophobetron Dyar. 4834 oropeso Barnes.

Alarodia Moesch.

4835 slossoniæ Pack.

Cnidocampa Dyar.

4836 flavescens Wlk.

Prolimacodes Schaus.

4837 badia $H b n$. scapha Harr. undifera Wlk. $a$ argentimacula $B . \& M c D$. 4838 trigona $H y$. Edw. telligii Barnes.
Limacodes Latr.

4839 biguttata Pack. tetraspilaris Wlk.

4840 rectilinea $G$. $\& R$. a latomia Harv.

4841 y-inversa Pack. a parallela $H y$. Edw.

Lithacodes Pack.

4842 fasciola $H$. S. laticlavia Clem. divergens Wlk.

Packardia G. \& R. 4843 elegans Pack. nigripunctata Goodell. a fusca Pack. 4844 ceanothi Dyar. 4845 geminata Pack. 4846 albipunctata Pack. goodelli Grt. $a$ ocellata Grt.

Heterogenea Krioch. 4847 shurtleffi Pack.

Kronæa Reak. 4848 minuta Reak.

Slossonella Dyar. 4849 tenebrosa Dyar.

Tortricidia Pack. 4850 fiskeana Dyar. 4851 græfi Pack. 4852 flexuosa Grt. form cæsonia Grt.

4853 pallida $H$. $S$. a flavula $H$. S.

4854 testacea Pack. a crypta Dyar. 


\section{MEGALOPYGID $\mathbb{E}$}

Norape WIk.

4855 ovina Sepp. cretata Grt.

4856 virgo Butl.

4857 tener Druce. achriogelos Dyar.

Megalopyge Hbn.

4858 opercularis A. \& S. lanuginosa Clem. subeitrina WIk.
4859 bissesa Dyar. 4860 lapena Schares.

Iagoa Harris. 4861 erispata Pack. ab. grisen B. \& McD. 4862 laceyi $B$. \& $M c D$. 4863 pyxidifera $A$. \& $S$.

Trosia Hbn.

4864 obsolescens Dyar.

DALCERID开

Dalcerides N. \& D.

4865 ingenita $H y . E d w$.

\section{EPIPYROPIDEE}

Epipyrops Westw.

4866 barberiana Dyar.

\section{ZYGENID FE}

Malthaca Clem.

4867 dimidiata $H$. S. perlucidula Clem.

4868 centralis Wlk. nothe Hy. Edw.

4869 rata $H y . E d w$.

4870 fusea $H y . E d w$. landia Druce.

4871 marteni Frch. barnea Druce.

Tetraclonia Jordan. 4872 latercula $H y$. Edwo 4873 dyari Jord. laterculaet Dyar.

Triprocris Grt.

4874 smithsonianus Clem.
4875 yampai Barnes.

Acoloithus Clem.

4876 falsarius Clem. sanborni Pack. ruficollis Druce. 4877 novarius $B . \& M C D$. 4878 rectarius Dyar.

Seryda Wlk.

4879 constans $H y, E d v$. form saneta $N$. \& $D$. 4880 basirei Druce.

Harrisina Pack.

4881 americana Guer.

a texana Stretch.

b australis Streteh. 
4882 metallica Stretch.

4883 coracina Clem. nigrina Graef.

4884 brillians $B . \& M c D$.
4885 lustrans Beut. 4886 cyanea $B$. \& McD. 4887 aversus $H y . E d w$.

\section{THYRIDIE}

Thyris Lasp.

4888 maculata Harris. perspicua Wlk.

4889 lugubris $B d v$. sepulchralis Bdv. nevadae Oberth.

Dysodia Clem.

4890 vitrina $B d v$.

4891 oculatana Clem. plena Wlk. fasciata G. \& R. montana Hy. Edw. aurea Pag.

4892 granulata Neum.
4893 speculifera Sepp. aequalis Wlk.

4894 flagrata $W l k$. floridana Hlst.

Hexeris Grt. 4895 enhydris Grt. reticulina Beut.

Belnoptera H. S. 4896 fratercula Pag.

Meskea Grt. 4897 dyspteraria Grt.

Thyridopyralis Dyar. 4898 gallærandialis Dyar.

\section{PYRALIDAE}

Pyraustinæ

Glaphyria Hbn.

4899 glaphyralis $G n$. stipatalis Wlk. albolineata G. \& R.

4900 sesquistrialis $H b n$. dimotalis Wlk.

4901 invisalis $G n$. lentiflualis Zell.

4902 psychialis Hlst.

4903 reluctalis Hlst. form remellalis Druce.

4904 fulminalis Led. 4905 peremptalis Grt. 4906 basiflavalis $B$. \& $M c D$. 4907 dualis $B . \& M c D$. 4908 periculosalis Dyar.
Scybalista Led. 4909 restionalis Led.

Egesta Rag.

Symphysa Hamp.

4910 reniculalis Z $Z$ ell. 4911 simplicialis Kearf. 4912 eripalis Grt. $a$ salutalis Hlst. ochralis Haim.

Lipocosma Led.

4913 sicalis Wlk. perfusalis Wlk.

4914 fuliginosalis Fern. 4915 intermedialis $B . \& M c D$. 4916 adelalis Kearf. 4917 albibasalis $B . \& M c D$. 
Sufetula Wik.

4918 diminutalis Wik. dematrialis Druee.

Hymenia Hbn.

4919 perspectalis $\mathrm{Hbn}$.

4920 fascialis Cram. recurvalis Fabr.

Desmia West.

4921 funeralis $\mathrm{Hbn}$. maculalis West. Q subdivisalis Grt.

4922 tages Cram. propinqualis Moesch.

Syuclera Led.

4923 traducalis Zell. jarbusalis Wik. cottalis Wlk.

Ercta Wlk.

4924 ornatalis Dup. 4925 desmialis $B$. \& $M C D$. kaeberalis Haim.

Marasmia Led.

4926 cochrusalis WLk. azionalis WIk. ruptalis Wik.

4927 trapezalis $G n$. creonalis W1k. bifurcalis Snell.

4928 floridalis Fern.

Anania Hbn.

4929 florella Cram.

Eurrhyparodes Snell. 4930 lygdamis Druce.

Samea Gn.

4931 ecclesialis $\mathrm{Gn}$. castellalis Gn. luceusalis Wlk. disertulis Wik.
4932 multiplicalis $\mathrm{Gn}$. discessalis WIk. nicaenasalis WIk.

Disstictis IIhn.

4938 argyralis $\mathrm{Hbn}$. form ventralis G. \& $\boldsymbol{R}$.

4934 fracturalis Zell. 4935 crecalis Warr. 4936 talis Grt.

Pilocrocis Led.

4937 ramentalis Led. perfuscalis Hlst.

4938 tripunctata Fabr. campalis $\mathrm{Gn}$. cubanalis Gn. memmialis Wlk. 4939 plumbicostalis Grt. 4940 inguinalis $G n$. thoosalis Wlk. anticostalis Grt. levalis Hist.

4941 tristigmalis Hamp.

Conchylodes Gn.

4942 diphteralis Gey. 4943 salamisalis Druce. 4944 ovulalis $G n$. concinnalis Hamp.

Dichogama Led. 4945 redtenbacheri Led. 4946 amabilis Moesch. 4947 bergi Moesch.

Lamprosema Hbn. 4948 lunulalis $H b n$.

Hedylepta Led. 4949 indicata $\mathrm{Fabr}$. vulgalis $\mathrm{Gn}$. connexalis Wlk. 4950 futilalis B. \& McD. 
Blepharomastix Led.

4951 ebulealis $G n$.

4952 aplicalis $G n$. xeniolalis Hlst.

4953 acutangulalis Snell. santatalis B. \& McD.

4954 magualis $G n$.

medealis Wlk.

belusalis Wlk.

curtalis Wlk.

4955 ranalis $G n$.

archasialis Wlk.

ofellusalis Wlk.

olliusalis Wlk.

strictalis Wlk.

gracilis $\mathbf{G}$. \& $\mathbf{R}$.

$a$ datisalis Druce. occidentalis Haim.

4956 stenialis $\mathrm{Gn}$.

acestealis Wlk.

phaerusalis Wlk.

Nacoleia Wlk.

4957 hampsoni $B$. \& $M C D$.

Asciodes Gn.

4958 gordialis $\mathrm{Gn}$. quietalis Wlk. confusalis Hlst.

4959 anormalis $G n$.

alvinalis Gn.

helcitalis Wlk.

orphnealis Wlk.

dracusalis Wlk.

subaequalis W1k.

Pantographa Led.

4960 limata $G$. \& $R$.

Sylepta Hbn.

4961 penumbralis Grt.

4962 fluctuosalis Led.

4963 obscuralis Led.

4964 masculinalis $B$. \& $M c D$.

4965 brumalis $B$. \& $M c D$.

4966 elevata $\mathrm{Fabr}$.
Lygropia Led.

4967 rivulalis Hamp. nymphulalis Haim.

4968 stictigramma Hamp.

4969 chromalis $G n$. principalis Led.

Agathodes Gn.

4970 designalis $\mathrm{Gn}$. floridalis Hlst. 4971 monstralis $G n$.

Paradosis Zell.

4972 flegia Cram. phantasmalis $\mathbf{G n}$. villosalis Zell.

Diaphania Hbn.

4973 olealis Feld.

4974 nitidalis Stoll. vitralis $\mathrm{Hbn}$. fumosalis $\mathbf{G n}$. praxialis Druce.

4975 infimalis $G n$. 4976 arguta Led. 4977 hyalinata Linn. marginalis Stoll. lucernalis $\mathrm{Hbn}$. $a b$. niveocilia Hamp).

4978 quadristigmalis $G n$. 4979 sibillalis Wlk. batesi Feld. alitalis Hlst.

Metrea Grt.

4980 ostreonalis Grt. urticaloides Fyles.

Ommatospila Led.

4981 narcæusalis Wlk. nummulalis Led.

Hellula Gn.

4982 undalis Fabr. rogatalis Hlst. 4983 aqualis $B$. \& $M C D$. 
Sameodes Snell.

4984 subcostalis Hamp.

4985 elealis Wik.

tredialia Wlk.

phyllisalis Wik. adipaloides G. \& R.

4986 trimaculalis (irt.

4987 mopsalis Wlk.

Mimorista Warr.

4988 flavidissimalis Grt.

Terastia Gn.

4989 meticulosalis $\mathrm{Gn}$. subjectalis Led. coeligenalis Hlst.

Laniifera IIamp.

4990 cyclades Druce.

Evergestis Ilbn.

4991 funalis Grt.

4992 insulalis $B . \& M c D$.

4993 obliqualis Grt.

4994 subterminalis $B$. \& $M c D$.

4995 triangulalis $B$. \& $M c D$.

4996 simulatalis Grt. brunneogrisea Hy. Edw.

4997 vinctalis $B$. \& $M c D$.

4998 eurekalis $B$. \& $M C D$.

4999 obscuralis B. \& MCD.

5000 rimosalis $G n$.

5001 consimilis Warr.

5002 aridalis $B$. \& $M c D$.

5003 lunulalis $B$. \& $M c D$.

5004 straminalis $\mathrm{Hbn}$. eunusalis Wlk.

Crocidophora Led.

5005 serratissimalis Z Zell. subdentalis Grt.

5006 pustuliferalis Led.

5007 palindialis Gn. dyaralis Fern.

5008 tuberculalis Led.
Stenophyes Led.

5009 huronalis $\mathrm{Gn}$. zinghalis Wlk. serinalis WIk.

\section{Polygrammodes Gn.}

5010 capitalis Grt. 5011 hirtalis Gn. Iybialis WIk. amatalis Wlk.

Nomophila Hbn.

5012 noctuella $D . \& S$.

Pachyzancla Meyr.

5013 periusalis Wlle.

5014 bipunetalis Fabr. detritalis $\mathbf{G n}$. lycialis WIk. repetitalis Grt.

5015 phseopteralis $\mathrm{Gn}$. cellatalis WIk.

Loxostege Hbn.

5016 dasconalis Wik.

5017 coloradensis $G$. \& $R$. pergilvalis Hlst.

5018 chortalis $\mathrm{Grt}$.

5019 aureolalis Hlst.

cyralis Druce.

5020 obliteralis Wlk. marculenta G. \& R.

5021 mancalis Led.

5022 helvialis $W / k$.

thycesalis Wik.

apertalis Wik. eitrina G. \& R.

5023 bifidalis $\mathrm{Fabr}$. inornatalis Wlk.

5024 flavalis Fern.

5025 similalis $G n$.

licealis Gn.

crinisntis Wlk.

diotimenlis WIk.

communis Grt. 
form rantalis $G$ n. siriusalis Wlk. murcialis Wlk. intractella Wlk. posticata G. \& R. occidentalis Pack.

5026 typhonalis $B$. \& $M c D$.

5027 allectalis Grt. perplexalis Fern.

5028 lepidalis $\mathrm{Hlst}$.

5029 roseiterminalis $B . \& M c D$. 5030 baccatalis $\mathrm{Hlst}$. 5031 sticticalis Linn. 5032 commixtalis $W l k$. indotatellus Wlk. cereralis Zell.

5033 offumalis $\mathrm{Hlst}$.

5034 thallophyllalis $\mathrm{Hlst}$.

5035 indentalis Grt.

5036 albiceralis Grt. $a$ floridalis $B . \& M c D$.

5037 oberthuralis Fern. 5038 labeculalis Hlst. 5039 annaphilalis Grt. 5040 anartalis Grt. a lulualis Hlst.

5041 maclurae Riley. 5042 fiavifimbrialis Warr. 5043 oblectalis Hlst. 5044 succandidalis Hlst. simplex Warr.

5045 ophionalis Wlk. nasonialis Zell.

5046 sesquialteralis $Z$ ell. 5047 vibicalis $Z$ ell.

\section{Diasemia Hbn.}

5048 ramburialis $D$ up. leodocusalis Wlk.

5049 nigralis Fern. 5050 janassialis Wlk. hariolalis Hlst. 5051 plumbosignalis Fern. 5052 zephyralis $B$. \& $M c D$.
5053 roseopennalis Hlst. 5054 magdalena Fern. 5055 elegantalis Warr. argalis Fern.

5056 leucosalis $B$. \& $M c D$. 5057 fenestralis $B$. \& $M c D$.

Gonocausta Led. 5058 sabinalis Dyar.

Antigastra Led. 5059 catalaunalis Dup.

Liopasia Moesch. 5060 teneralis Led.

Condylorrhiza Led. 5061 vestigialis $G n$. $a$ tritealis $W l k$. mestoralis Wlk. oratalis Hlst.

Microcausta Hamp. 5062 flavipunctalis $B$. \& $M c D$. 5063 bipunctalis $B . \& M c D$.

Tholeria Hbn. 5064 reversalis $G n$.

Calamochrous Led. 5064, 1 straminea Warr.

Cybalomia Led. 5065 extorris Warr. quadristrigalis Fern.

Gyros Hy. Edw. 5066 muiri $H y, E d w$. rubralis Warr. 5067 atripennalis $B . \& M c D$.

Autocosmia Warr. 5068 concinna Warr. 5069 nexalis Hlst. 
Maroa B. \& MeD.

5070 unicoloralis B. \& McD.

Edis Dyar.

5071 semiluna Sm.

bidentalis B. \& McD. miorostagma Dyar.

5072 minutissima Sm.

\section{Orenais Dup.}

5073 coloradalis $B$. \& $M C D$.

5074 trivialis $B$. \& $M c D$.

Titanio Hbn.

5075 pollinalis Schiff. Ravinotalis Grt.

5076 ephippialis Zett.

5077 alticolalis $B$. \& $M c D$.

5078 dapalis Grt.

5079 proximalis Fern.

5080 triumphalis Grt.

5080, 1 immerens Harv.

5081 helianthialis Murt.

5082 belialis Druce.

5083 lutosalis B. \& McD.

5084 letalis B. \& McD.

Chrismania B. \& MeD.

5085 pictipennalis $B$. \& $M C D$.

Polingia B. \& MeD.

5086 qusestoralis $B$. \& $M c D$.

Perispasta Zell.

5087 creculalis Zell.

\& immixtalis Grt.

Phlyctænia Ithn.

5088 ferrugalis $\mathrm{Hbn}$. rubigalis $\mathrm{Gn}$. oblunalis Led. harveyana Grt.

5089 profundalis Pack.

5090 desistalis $W l k$.

5091 inquinatalis Zell. glacialis Pack.
5092 rusticalis B. \& McD.

5093 itysalis Wlk.

variegats Wik. turmalis Grt.

- hyperborealis Moesch. tillialis Dyar.

5094 washingtonalis Grt. invinctalis Hlst.

5095 radiosalis Moesch.

5096 externalis Warr.

5097 indistinctalis Warr.

5098 acutella $W l k$.

venalis Grt.

5099 terrealis $\mathrm{Tr}$. mysippusalis Wlk. humilalis Led.

5100 extricalis $G n$. dionalis Wlk. nisoeecalis WIk. intricatalis Led. oppilalis Grt.

5101 helvalis Wlk. oscitalis Grt. gyralis Hist.

5102 tertialis $G n$. plectilis G. \& R. ryringicola Pack.

Cindaphia Led.

5103 bicoloralis $G n$. julialis Wlk. incensalis Led. amiculatalis Berg. pulchripietalis Hamp.

5104 angustalis Feld.

Epicorsia $\mathrm{Hbn}$.

5105 mellinalis $\mathrm{Hbn}$. oedipodalis $\mathrm{Gn}$. butyrosa Butl.

Pyrausta Schrank. 5106 sartoralis $B$. \& $M C D$. 5107 pilatealis $B$. \& $M C D$. 5108 naprealis Hiot. 5109 linealis Fern. 
5110 vacunalis Grt.

5111 pertextalis Led. gentilis Grt. thesealis Zell.

5112 fissalis Grt.

5113 æglealis $W l k$. quinquelinealis Grt.

5114 thestealis Wlk. magistralis Grt. gulosalis Hlst.

5115 abdominalis Zell.

5116 theseusalis Wlk. feudalis Grt.

5117 langdonalis Grt.

5118 oxydalis $\mathrm{Gn}$.

5119 flavidalis $G n$. lacoalis WIk. cinctipedalis Wlk.

5120 fuscimaculalis Grt. flavicoloralis Grt. confovealis Hlst.

5121 submedialis Grt. dissectalis Grt. pilalis Hlst.

5122 gracilalis Hlst. atrisquamalis Hamp.

5123 hædulalis Hlst.

5124 illibalis $\mathrm{Hbn}$.

5125 arsaltealis Wlk. euphaesalis Wlk. guttulosa Wik. fascialis Wlk. subjectalis Led. magniferalis W1k.

5126 pseudoranalis $B$. \& $M C D$.

5127 potentalis $B$. \& $M c D$.

5128 venalalis $H$ lst.

5129 penitalis Grt. nelumbialis $\mathrm{Sm}$.

5130 obumbratalis Led.

5131 beddeci Dyar.

5132 fumalis $G n$. orasusalis Wlk. badipennis Grt.
5133 inconcinnalis Led. crocotalis Grt. festalis Hlst.

5134 futilalis Led. erectalis Grt.

5135 fumoferalis Hlst.

5136 singularis Led. 5137 octosignalis Hlst. 5138 mustelinalis Pack. catenulalis Grt. monulalis Hlst.

5139 luscitialis $B$. \& $M c D$.

5140 unifascialis Pack. obnigralis Hlst. $a$ subolivalis Pack. hircinalis Grt.

5141 torvalis Moesch. gelida McLach.

5142 fodinalis Led. socialis Grt.

5143 semirubralis Pack.

5144 perrubralis $P$ ce -7 . a scurralis $\mathrm{H}^{\prime} \mathrm{s}$. postrubralis Hamp.

5145 phœnicealis $H b n$. flegialis Wlk. noraxalis Wlk.

5146 tæniolalis $\mathrm{Gn}$.

5147 onythesalis Wlk.

5148 acrionalis Wlk. acuphisalis WIk. proceralis Led. sumptuosalis Wlk. haruspica G. \& R. form rufifimbrialis Grt.

5149 rubricalis $\mathrm{Hbn}$. similalis Led. nescalis Wlk. californicalis Pack.

5150 tatalis Grt. 5151 borealis Pack. madetesalis Wlk. efficitalis Wlk. repletalis Wlk. 
5152 subsequalis $G n$. insequalis $\mathrm{Gn}$. matronalis Grt. a plagalis Haim.

5153 orphisalis WIk.

5154 generosa G. \& $\boldsymbol{R}$.

5155 ochosalis Dyar.

5156 flavofascialis Grt.

5157 laticlavia $G . \& R$.

form cinerosa $C$. . \& $R$.

5158 tyralis Gin.

ervancalis Wik.

difisen G. \& R.

bellulalis Hist.

5159 coccinea Warr.

5160 costimaculalis Fern.

5161 inornatalis Fern. rosa Druce.

5162 signatalis Wlk. vinulenta G. \& R.

5163 volupialis Grt.

5164 corinthalis $B$. \& $M c D$.

5165 augustalis Grt.

5166 nicalis Grt. u.roreulalis Hlst.

5167 subnicalis Warr.

5168 morenalis Dyar.

5169 atropurpuralis $\mathrm{Grt}$.

5170 lethalis Grt.

5171 unimacula $G$. \& $R$.

$\$ 172$ commortalis Grt.

5173 versicolor Warr.

5174 marginalis WUk. stenopteralis Grt.

5175 niveicilialis Grt.

5176 funebris Strom. octomaculata Linn. glomerulis Wilk.

5177 xanthocrypta Dyar.

Eustixia Iflm.

5178 pupula $\mathrm{Hbn}$.

¿179 octonalis Zell. secmucululis Grt.
Cornifrons Led.

5180 sinalis Grt.

5181 pulveralis Warr.

Noctuelia Gn.

5182 thalialis $W I k$.

a novalis Grt.

form decorata Druce.

$b$ nuchalis Grt.

5183 castanealis Hlst. jativa Barnes:

5184 elautalis Grt. magnalis Hlst.

5185 puertalis $B$. \& $M c D$.

5186 pandoralis $B$. \& $M c D$.

5187 bububattalis Hlst. tectalis B. \& McD.

5188 achemonalis $B$. \& $M c D$.

Heliothela Gn.

5189 costipunctalis $B$. \& $M c D$.

5190 unicoloralis B. \& McD.

\section{Lineodes Gn.}

5191 contortalis Gn.

5192 integra Zell.

5193 interrupta Zell.

5194 triangulalis Moesch.

Stenoptycha Zell.

5195 solanalis $B$. \& $M c D$.

Chlorobapta B. \& MeD.

5196 rufistrigalis $B$. \& $M c D$.

Scissolia B. \& MeD.

5197 harlequinalis $B$. \& $M c D$.

\section{Nymphulinæ}

Nymphula Schrank.

5198 maculalis Clem. seminivella Wlk.

dispar Grt.

ab. of foeminalis Dyar.

ab. \& masculinalis $D$ yar. 
5199 allionealis Wlk. aptalis Led. plenilinealis Grt. $a b$. itealis Wlk. cretacealis Led.

5200 obscuralis Grt.

5201 seminealis Wlk. tedyuscongalis Clem.

5202 badiusalis Wlk. curviferalis Wlk. albalis Rob.

5203 vittatalis Dyar. 5204 obliteralis $W l k$. obscuralis Moesch. proprialis Fern.

5205 nomophilalis Dyar.

5206 gyralis $\mathrm{Hlst}$. dentilinea Hamp.

5207 nebulosalis Fern. 5208 icciusalis Wlk. faulalis Wlk. formosalis Clem. genuialis Led. pacalis Grt.

5209 elsthlipsis Grt.

Ambia Wlk.

5210 striatalis Dyar.

Cataclysta Hbn.

5211 brunnildalis Dyar.

5212 bifascialis $R o b$. $a$ kearfottalis Dyar.

5213 drumalis Dyar.

5214 claudialis $W l k$. a medicinalis Grt.

5215 slossonalis Dyar. 5216 magnificalis $\mathrm{Hbn}$. lamialis Wlk. helopalis Clem.

5217 fulicalis Clem. angulatalis Led.

5218 confusalis Wlk.

5219 cronialis Druce.

5220 schaefferalis Dyar.
5221 avernalis Grt. confusalis B. \& $\mathrm{McD}$. 5222 dxmonalis Dyar. 5223 cryptalis Druce.

Geshna Dyar. 5224 cannalis Quaint. 5225 primordialis Dyar.

Diathrausta Led. 5226 reconditalis Wlk. minualis Wlk. octomaculalis Fern. $a$ harlequinalis Dyar. montana Haim.

5227 drckealis Haim.

Steniodes Snell.

5228 gelliasalis Wlk. lutealis Snell.

Piletocera Led. 5229 bufalis $G n$. stercoralis Moesch. 5230 simplicialis $B . \& M c D$.

Eurrhypara Hbn. 5231 urticata Linn.

\section{Scopariinæ}

\section{Scoparia Haw.}

5232 rectilinea $\mathbb{Z}$ ell. refugalis Hlst.

5233 expallidalis Dyar. 5234 rigidalis $B$. \& $M c D$. 5235 centuriella $D . \& S$. caecalis Wlk. caliginosalis Wlk. a ninguidalis $\mathrm{Hlst}$. 5236 normalis Dyar. 5237 delphusa Druce. 5238 penumbralis Dyar. 5239 cinereomedia Dyar. 5240 schwarzalis Dyar. 
5241 strigalis Dyar.

5242 lugubralis $W U$. nominatalis Hist.

5243 spaldingalis $B$. M $M C D$.

5244 basalis Wlk.

biplagiulis Wlk. libella Grt.

a palloralis Dyar.

b fernaldalis Dyar.

- obispalis Dyar.

5245 torniplagalis Dyar.

5246 alialis $B$. \& $M c D$.

5247 sabura Druce.

5248 tricoloralis Dyar.

Pyralinæ

Aglossa Latr.

5249 cuprealis $H b n$. domalis $\mathrm{Gn}$. enthealis Hlst.

5250 euprina Zell.

5251 acallalis Dyar.

5252 baba Dyar.

Pyralis Linn.

5253 farinalis Linn.

5254 costiferalis Wlk. costigeralis Wik.

5255 disciferalis Dyar.

5256 electalis $\mathrm{H} l s t$.

5257 cacamica $D$ yar.

5258 manihotalis $\mathrm{Gn}$.

Hypsopygia Hbn.

5259 costalis $\mathrm{Fabr}$.

fimbrialis D. \& S.

$a$ hyllalis Wik.

Herculia WIk.

5260 planalis Grt. anniculalis Hlst. occidentalis Hlst.

5261 intermedialis Wlk. sodalis WIk. squamealis Grt.
5262 phoezalis Dyar.

5263 cohortalis Grt.

5264 thymetusalie $W l k$. devialis Grt.

5265 binodulalis Zell.

5266 sordidalis B. \& MCD.

5267 infimbrialis Dyar.

5268 olinalis $G n$. trentonalis Led.

$a b$. himonialis Zell.

Uscodys Dyar.

5269 cestalis Hlat.

5270 atalis Dyar.

Omphalocera Led.

5271 cariosa Led.

5272 dentosa Grt.

\section{Chrysauginse}

Galasa Wlk.

5273 nigrinodis Zell.

5274 nigripunctalis $B$. \& $M c D$. fulvusana Haim.

Negalasa B. \& MeD.

5275 fumalis $B$. \& $M c D$.

5276 rubralis $B$ \& $M c D$.

Tosale Wlk.

5277 oviplagalis $W u k$. nobilis Grt. anthoecioides G. \& R.

5278 aucta Hamp.

Chalinitis Rag. 5279 olealis Rag. 5280 viridalis $B$. \& $M c D$. 5281 obliqquata $H y . E d v$. albistrigalis B. \& $\mathrm{MeD}$.

Salobrens Wlk.

5282 sincers Zell.

Clydonopteron Riley.

5283 tecoma Riley. 
Xantippides Dyar.

5284 descansalis Dyar.

Arta Grt.

5285 statalis Grt.

5286 epiconalis $R a g$.

5287 olivalis Grt.

Heliades Rag.

5288 mulleolella Hlst. huachucalis Haim.

Caphys Wlk.

5289 bilinea Wlk.

Satole Dyar.

5290 ligniperdalis Dyar.

Parachma Wlk.

Artopsis Dyar.

5291 ochracealis Wlk. auratalis Wlk.

a culiculalis Hlst. nua Dyar.

5292 borregalis Dyar.

Acallis Rag.

5293 gripalis Hlst.

fernaldi Rag.

angustipennis Warr.

5294 centralis Dyar.

5295 mitchelli Dyar.

Polloccia Dyar.

5296 alticolalis Dyar.

Condylolomia Grt.

5297 participialis Grt.

Penthesilea Rag.

5298 sacculalis Rag.
Schœenobiinæ

Patissa Moore.

5299 xantholeucalis $G n$. fasciella Fern.

5300 flavicostella Fern. 5301 flavifascialis $B$. \& $M c D$. 5302 sordidalis $B$. \& $M c D$. 5303 vestaliella $Z$ ell.

Scirpophaga Tr. 5304 albinella Cram. nivea Wlk.

5305 perstrialis $H b n$. semiradiellus Wlk. macrinellus Zell.

5306 repugnatalis Wlk. consortalis Dyar.

Storteria B. \& MeD. 5307 unicolor $B . \& M C D$.

Schœnobius Dup.

5308 sordidellus $\boldsymbol{Z}$ inck. 5309 unipunctellus $R o b$. 5310 tripunctellus $R o b$. 5311 melinellus Clem. $a$ dispersellus $R o b$. $b$ albicostellus Fern. 5312 clemensellus Rob. aquilellus $\ddagger$ Clem. 5313 pallulellus $B$. \& $M c D$. 5314 forficellus Thun. longirostrellus Clem. 5315 maximellus Fern.

\section{Crambinæ}

Uinta Hlst.

5316 oreadella Hlst. 
Surattha Wilk.

5317 santella Kearf.

5318 indentella Kenrf.

Mesolia Rag.

5819 baboquivariella Kearf.

5320 araculella Kearf.

5321 huachucaella Kearf.

Prionapteryx Steph.

5322 nebulifera Steph.

5323 achatina Zell.

5324 cuneolalis Hlst.

5325 serpentella Kearf.

\section{Eugroten Fern.}

5326 incertella Z inck. dentella Fern.

5327 olivella Grt.

5328 yavapai Kearf.

\section{Pseudoschønobius Fern.}

5329 opalescalis Hlst. griseosparsa Hamp. saganella Hlst.

\section{Raphiptera Hamp.}

5330 minimella Rob.

5331 argilliceella Pack.

Crambus Fabr.

5332 satrapellus Zinek. aculeilellus WIk. elegantellus Wik.

5333 hastiferellus Wlk.

50.34 quinquareatus Zell. ertorralis HIst.

5335 occidentalis (jirt. 5336 hamellus Thun. ensigerella $\mathrm{Hbn}$.

5337 cypridalis $\mathrm{H} / \mathrm{st}$. 5338 pascuellus Linn. foridus Zell. 5339 lyonsellus Haim.
5340 dreckellus Haim.

5841 girardellus Clem. nivihumellus Wlk.

5342 leachellus Zinck. pulchellue Zell.

5348 unistriatellus Pack. exesus Grt.

5344 prwfectellus Zinek. involutellus Clem.

5345 carpenterellus Pack. oslarellus Haim.

5346 youngellus Kearf.

5347 bidens Zell.

5348 labradoriensis Christ.

5349 dissectus Grt.

5350 dumetellus $\mathrm{Hbn}$. pratella $\mathrm{Hbn}$.

5351 trichusalis Hlst.

5352 cockleellus Kearf.

5353 alboclavellus $\mathbb{Z}$ ell.

5354 carolinellus Haim.

5355 agitatellus Clem. saltuellus Zell.

5356 myellus $\boldsymbol{H b n}$. latiradiellus Wlk. interruptus Grt.

5357 luctuellus $\boldsymbol{H}$. S. 5358 laqueatellus Clem. semifusellus Wlk.

5359 gausapalis Hlst.

5360 multilinellus Fern.

5361 hortuellus $\mathrm{Hbn}$.

$a$ topiarius Zell.

b vachellellus Kearf.

5362 albellus $\mathrm{Clem}$.

5363 pusionellus Zell.

5364 innotatellus $W / k$. sericinellus Zell. inornatellus Clem.

5365 turbatellus W/k. bipunctellus Zell.

5366 elegans Clem.

5367 polingi Kearf. 
5368 vulgivagellus Clem. aurifmbrialis Wlk. chalybirostris Zell.

5369 plumbifimbriellus Dyar.

5370 dorsipunctellus Kearf.

5371 ruricolellus Zell.

5372 teterrellus Zinck. camurellus Clem. terrellus Zell.

5373 decorellus Zinck. polyactinellus Zell. goodellianus Grt. bonusculalis Hlst.

5374 coloradellus Fern.

5375 bolterellus Fern.

5376 hulstellus Fern.

5377 attenuatus Grt.

5378 biothanatalis $\mathrm{Hlst}$. behrensellus Fern.

5379 albilineellus Fern.

5380 truncatellus Zett. abtrusellus Wlk. rufinalis Wlk.

5381 trichostomus Christ. albisinuatella Pack.

5382 oregonicus Grt.

5383 bonifatellus $\mathrm{Hlst}$.

5384 mutabilis Clem. fuscicostellus Zell.

5385 murellus Dyar.

5386 hemiochrellus Z $\mathrm{Z}$ ell.

5387 haytiellus Zinck.

5388 intermedius Kearf.

5389 nevadellus Kearf.

5390 undatus Grt.

5391 anceps Grt.

5392 trisectus Wlk.

interminellus Wlk. exsiccatus Zell. biliturellus Zell.

5393 inornatellus Wlk.

5394 laciniellus $\mathrm{Grt}$.

5395 simpliciellus Kearf.

5396 dimidiatellus Grt.

5397 caliginosellus Clem.
5398 zeellus Fern. refotalis Hlst.

5399 luteolellus Clem. duplicatus Grt. a ulae Ckll. 5400 chiloidellus $B$. \& $M C D$.

Haimbachia Dyar. 5401 placidella Haim.

Thaumatopsis Morr.

5402 magnificus Fern. 5403 pexellus Zell.

ㅇ macropterellus Zell. longipalpus Morr. $a$ coloradellus Kearf.

5404 gibsonellus Kearf. 5405 edonis $G r t$.

5406 fernaldellus Kearf. $a$ nortellus Kearf.

5407 floridellus $B$. \& $M c D$. 5408 lagunellus Dyar.

5409 atomosellus Kearf.

5410 fieldellus $B$. \& $M c D$.

5411 repandus Grt.

5412 crenulatellus Kearf.

5413 striatellus Fern.

5414 dæckeellus Kearf.

5415 pectinfer Zell.

Eufernaldia Hlst.

5416 cadarella Druce. argenteonervella Hist.

Ommatopteryx Kirby.

5417 ocellea Haw. texana Rob. californicalis Pack.

5418 virescens $H$ lst.

Argyria $\mathrm{Hbn}$.

5419 nivalis Dru. argentata Emm. michrochrysella Wlk. nummulalis Zell. 
5420 argentana Martyn. พummulalis $\mathrm{Hbn}$. subeenescens WIk. fuscipes Zell.

5421 auratella Clem. pulchella WIK.

5422 lacteella Fabr. pussillalis Hbn. rufieignella Zell.

\section{Platytes Gn.}

5423 dentilineatella $B$. \& $M c D$. 5424 punctilineella $B$. \& $M C D$. 5425 multilineatella $\mathrm{H}$ lst. 5426 squamulella Zell.

Eoreuma Ely.

5427 densellus ZZell.

Chilo Zinek.

5428 multipunctellus Kearf. 5429 puritellus Kearf. 5430 plejadellus Zinek. sabulifera Wik. prolatella Grt. oryzacellus Riley.

5431 comptulatalis Hlst. 5432 forbesellus Fern. 5433 prosenes Dyar. 5434 epia Dyar.

Iesta Dyar.

5435 lisetta Dyar.

Diatræa Guild.

5436 saccharalis Fabr. a crambidoides Grt.

5437 zeacolella Dyar.

5438 lineolata $W l k$.

5439 parallela Kearf.

5440 alleni Fern.

5441 differentialis Fern.

5442 idalis Fern.
Chalcoela Zell.

5443 iphitalis $W$ Ik. aurifora Zell.

Dicymolomia Zell.

5444 julianalis WIk. decora Zell.

5445 opuntialis Dyar. 5446 metalliferalis Puck. sauberi Hed.

5447 pegasalis Wlk. principalis Wlk. egressalis Wlk. robinsoni Grt.

Galleriina

Galleria Fabr.

5448 mellonella Linn. cereana Linn. obliquella Wlk.

Aphomia Hbn.

5449 sociella Linn. colonella Linn. tribunella D. \& S.

Paralipsa Butl.

5450 terrenella Zell. furellus Zell.

5451 fulminalis $Z$ ell. 5452 decorella $\mathrm{H}$ lst.

Melissoblaptes Zell.

5453 fuscolimbellus Rag.

Achroia Hbn.

5454 gxisella $\mathrm{Fabr}$. cinereola $\mathrm{Hbn}$.

Tineopsis Dyar.

5455 theobromse Dyar. 


\section{Macrothecinæ}

Macrotheca Rag.

5456 interalbicalis Rag.

5457 unicoloralis $B$. \& $M c D$.

5458 unipuncta Dyar.

5459 ponda Dyar.

5460 nigrocinereella $\mathrm{Hlst}$.

5461 flexilinealis Dyar.

Alpheias Rag.

5462 vicarilis Dyar.

5463 querula Dyar.

5464 oculiferalis $R a g$.

Alpheioides B. \& MeD.

5465 parvulalis $B . \& M c D$.

Decaturia B. \& MeD.

5466 pectinalis $B . \& M c D$.

Epipaschiinæ

Epipaschia Clem.

5467 superatalis Clem. borealis Grt. olivalis Hlst.

5468 zelleri Grt.

Cacozelia Grt.

5469 basiochrealis Grt.

5470 alboplagialis Dyar.

Jocara Wlk.

5471 incrustalis Hlst.

5472 perseella $B$. \& $M c D$.

5473 breviornatalis $G r t$.

5474 trabalis Grt. adulatalis Hlst.

5475 interruptella $R a g$. dentilineella Hlst.

Oneida Hlst.

5476 lunulalis Hlst. 5477 luniferella Hlst.
Tallula Hlst.

5478 atrifascialis $\mathrm{Hlst}$. 5479 fieldi $B . \& M c D$.

Tetralopha Zell.

5480 robustella $Z$ ell. diluculella Grt.

5481 scortealis Led.

5482 slossoni Hlst.

5483 melanogrammos Zell.

5484 texanella $\mathrm{Rag}$.

5485 callipeplella $\mathrm{Hlst}$.

5486 speciosella $\mathrm{Hlst}$.

5487 floridella Hlst.

5488 subcanalis Wlk. taleolalis Hlst. querciella B. \& McD.

5489 militella Zell.

? platanella Clem.

5490 asperatella Clem. expandens Wlk. form nephelotella Hlst. clemensalis Dyar.

5491 aplastella Hlst. 5492 fuscolotella $R a g$. 5493 tiltella Hlst. 5494 humerella Rag. formosella Hlst.

5495 tertiella Dyar.

5496 baptisiella Fern.

5497 euphemella Hlst. variella Rag. melanographella Rag.

Dyaria Neum.

5498 singularis Neum.

\section{Endotrichiinæ}

Neodavisia B. \& MeD. Davisiał B. \& McD. 5499 singularis $B$. \& $M c D$. 
Phycitine

Trachycera Rag.

5500 pallicornella Rag.

Myelois Hihn.

5501 transitella Wth. wotatalis Wlk.

5502 duplipunctella $R a g$.

5503 fragilella $D$ yar.

5504 subtetricella $R a g$.

5505 obnupsella Hlat.

5506 minutularia Hlst.

5507 oporedestella Dyar.

5508 zonulella $\boldsymbol{R a g}$.

5509 bistriatella $\mathrm{Hlst}$. bilineatella Rag.

5510 immundella $\boldsymbol{H}$ lst.

5511 grossipunctella $R$ ag.

5512 alatella $\boldsymbol{H}$ lst.

5513 coniella Rag. rectistrigella Rag.

5514 caliginoidella Dyar.

\section{Rhodophæa $\mathrm{Gn}$.}

5515 hystriculella Hlst.

5516 annuliferella $D$ yar.

5517 intransitella $D$ yor.

5518 exsulella Zell.

Acrocaula Hlst.

5519 comacornella Hlst.

\section{Acrobasis Zell.}

5520 kearfottella Dyar.

5521 feltella Dyar.

5522 caryalbella Ely.

5523 palliolella Rag. albocapitella Hlst.

5524 secundella Ely.

5525 ostryella Ely.

5526 hebescella Hlst.

5527 evanescentella Dyar.

5528 earyivorella Rag.
5529 minimella $R a g$. nigrosignella Hlst.

5530 stigmella Dyar.

5531 aurorella $E$ ly.

5532 angusella $G r t$.

eliella Dyar.

5538 demotella Grt.

5534 malipennella Dyar.

5535 sylviella Ely.

5536 irrubriella Ely.

5537 latifasciella Dyar.

5538 normella Dyar.

5539 coryliella Dyar.

5540 dyarella Ely.

5541 carys Grt.

5542 betulella $\mathrm{H}$ lst.

5543 rubrifasciella Pack.

5544 comptoniella Hlst.

5545 slossonella Hlst. tenuella B. \& McI).

5546 cirroferella Hlst.

\section{Mineola Hlst.}

5547 scitulella Hlst.

5548 tricolorella $\mathrm{Grt}$.

5549 amplexella $\boldsymbol{R a g}$.

5550 caliginella Hlst. comptella Rag.

5551 juglandis Le Bar.

5552 vaccinii Riley.

5553 indigenella Zell. nebulo Walsh. zelatella Hlst.

a nebulella Riley.

Ulophors Rag.

5554 grotei Rag.

5555 tephrosiella Dyar.

5556 carica Dyar.

Sarasota Hlst.

5557 plumigerelia Hlat.

5558 subrufella Hlst.

5559 filiolella $H$ lst. 
Physitopsis Rag.

5560 flavicornella $\mathrm{Rag}$.

Dioryctria Zell.

5561 clarioralis $W l k$. brunneella Dyar.

5562 auranticella Grt. a miniatella $\mathrm{Rag}$.

5563 xanthœenobares Dyar.

5564 erythropasa Dyar.

5565 abietella $D$. \& $S$. abietivorella Grt. a elegantella Hlst.

5566 ponderosæ Dyar. 5567 reniculella Grt. 5568 actualis Hlst.

Pinipestis Grt.

5569 zimmermanni Grt. austriana Cosens.

5570 delectella $\mathrm{H}$ lst.

5571 amatella $\mathrm{Hlst}$.

5572 pygmæella $R a g$.

5573 cambiicola Dyar.

5574 albovittella $\mathrm{Hlst}$.

5575 gulosella Hlst.

Bertelia B. \& MeD.

5576 grisella $B$. \& $M c D$.

Monoptilota Hist.

5577 pergratialis Hlst. nubilella Hist. grotella Rag.

Tacoma Hist.

5578 feriella Hlst.

5579 texanella Hlst.

5580 dulciella Hlst.

5581 submedianella Dyar.

Dasypyga Rag.

5582 alternosquamella $R a g$. $a$ stictophorella Rag.
Promylea Rag.

5583 lunigerella $R a g$. 5584 glendella Dyar.

Glyptocera Rag.

5585 consobrinella Zell. busckella Dyar.

Ortholepis Rag. 5586 jugosella Rag.

Polopeustis Rag.

5587 annulatella $Z$ ett. altensis Wocke.

Ambesa Grt.

5588 walsinghami $R a g$.

5589 monodon Dyar.

5590 mirabella Dyar.

5591 lætella Grt.

5592 niviella $\mathrm{Hlst}$.

Nephopteryx Hbn.

5593 lallatalis Hlst. denticulella Rag.

a brucei Hlst.

5594 scobiella Grt. decimerella Hlst.

5595 decipientella Dyar. 5596 ovalis Pack. + latifasciatella Pack. modestella HIst.

a geminipunctella Rag.

5597 rhypodella Hlst. 5598 curvatella Rag. 5599 fasciolalis Hlst. 5600 hypochalciella Rag. 5601 rubrisparsella Rag. rufibasella Rag. croceella Hlst.

5602 crassifasciella $\mathrm{Rag}$. 5603 inquilinella $R a g$. 5604 gilvibasella Hlst. lacteella Hlst. 
5605 rubescentella $\mathrm{H} / \mathrm{sl}$.

5606 nyssnecolella Dyar.

5607 subtinctella Rag.

Tlascala IIlst.

5608 finitella WIk. melanella Hist.

5609 umbripennis Hlst. gillettella Dyar.

5610 reductella $W I k$. gleditschiella Fern.

\section{Meroptera Grt.}

5611 mirandella Rag.

5612 cviatella Dyar.

5613 uvinella Rag.

5614 pravella Grt.

5615 unicolorella Hlst. leucophaeella Hlst.

5616 canescentella Hlst.

\section{Immyrla Dyar.}

5617 nigrovittella Dyar.

5618 bumeliella $B$. \& $M c D$.

Salebria Zell.

5619 pumilella $R a g$.

5620 nubiferella Rag.

5621 engeli $D$ yar.

5622 turpidella $\mathrm{Rag}$.

5623 annulosella Rag.

5624 ademptandella Dyar.

5625 tenebrosella $\boldsymbol{H}$ lst. quercicolella Rag.

5626 afflictella $\mathrm{H} / \mathrm{st}$.

liquidambarella Dyar.

5627 nigricans $H$ lst.

5628 ochripunetella Dyar.

5629 fructetella Hlat. rectistrigella Dyar.

5630 carneella Hlat.

5631 virgatella Clem.

quinquepunctella Grt.

a subcresiella Clem. contatella Grt.

b inconditella Rag.
5632 celtidella Hlat.

5638 levigatella $\boldsymbol{H}$ lat.

5634 pudibundella $\mathrm{Rag}$.

5635 basilaris Zell.

5636 georgiella Hlat.

5637 robustella Dyar.

5638 semiobscurella Hlst.

5639 subfuscella Rag.

5640 bifasciella $\boldsymbol{H}$ lot.

5641 nogalesella Dyar.

5642 furciferella $D$ yar.

5643 aliculella $\boldsymbol{H}$ lst. oberthuriella Rag.

5644 yumaella Dyar.

5645 odiosella Hlst.

5646 bakerella Dyar.

Passadena Hlst.

5647 flavidorsella Rag. constantella Hlst.

5648 cinctella $\mathrm{Hlst}$.

\section{Myrlæa Rag.}

5649 tarmitalis Hlst.

5650 vetustella $D$ yar.

5651 delassalis $H$ lst. purpurella Hlst.

5652 fernaldi Rag.

Laodamia Rag.

5653 fusca Haw.

moestella Wlk. cacabella Hlst.

triplngiella Dyar.

a frigidella Pack.'

Elasmopalpus Blanch.

5654 decoloralis $W / k$. metagrammalis Wlk.

5655 petrellus Zell.

rubiginella WIk.

rufinalis Wik.

hapsella Hlst.

obsipella Hlst.

5656 furfure'lus $H$ lst.

floridellue Hlst. 
5657 lignosellus $Z$ ell. angustellus Blanch. form incautellus Z Zll. form tartarellus Zell. carbonellus Hist.

Selagia Hbn.

5658 australella Hlst.

5659 lithosella Rag. luteella Hlst.

Pyla Grt.

5660 scintillans Grt. 5661 metalicella Hlst. 5662 æneoviridella Rag. 5663 bistriatella Hlst. 5664 hanhamella Dyar. 5665 incorruscella $\mathrm{Hlst}$. 5666 criddlella Dyar. 5667 æneella Hlst. 5668 rainierella Dyar.

Epischnia Hbn.

5669 ruderella $R a g$. 5670 albiplagiatella Pack. $a$ fosterella Hlst. 5671 albocostalis Hlst. 5672 subcostella $\mathrm{Rag}$. 5673 fulvirugella $R a g$. 5674 incanella $\mathrm{Hlst}$. 5675 granitella $R a g$. piperella Dyar.

Megasis Gn.

5676 edwardsialis Hlst. polyphemella Rag.

5677 excantalis $\mathrm{Hlst}$. pullatella Rag.

5678 caudellella Dyar.

5679 aridella Dyar.

5680 atrella $\mathrm{H}$ lst.

5681 rubrithoracella $B . \& M c D$.

Sarata Rag.

5682 nigrifasciella $\mathrm{Rag}$.
5683 rhoiella Dyar.

5684 tephrella Rag. 5685 perfuscalis $H$ lst. dnopherella Rag.

5686 umbrella Dyar. 5687 cinereella Hlst.

Lipographis Rag.

5688 fenestrella Pack. a humilis Rag. 5689 leoninella Pack. pallidella Dyar.

Etiella Zell.

5690 zinckenella $T r$. a rubribasella $\mathrm{Hlst}$. $b$ schisticolor Z Zell. villosella Hlst.

Hypochalcia Hbn.

5691 hulstiella Rag. 5692 truncatella Wright.

Melitara Wlk.

5693 prodenialis Wlk. bolli Zell. 5694 dentata Grt.

Olyca Wlk.

5695 junctolineella Hlst. pectinatella Hamp.

Cactobrosis Dyar.

5696 fernaldialis Hlst. gigantella Rag. cinereella Hlst.

5697 strigalis $B$. \& $M c D$.

Ozamia Rag.

5698 lucidalis Wlk.

Yosemitia Rag.

5699 graciella $H$ lst. a longipennella $\mathrm{Hlst}$.

5700 maidella Dyar. 5701 mysiella Dyar. 
5702 aureomaculella Dyar.

5708 fuscatella Hlat. 5704 pallidipennella Hlot.

Zophodia Hbn.

5705 grossularia Riloy. turbatella Grt.

5706 franconiella $\mathrm{Hlat}$. bellu Hist.

5707 packardella Rag. 5708 orobanchella Dyer. 5709 dilatifasciella Rag. 5710 perdubiella $D$ yar. 5711 fieldiella Dyar. 5712 glaucatella $\boldsymbol{H}$ lst. 5713 polingella Dyar. 5714 epischnioides Hlat. 5715 bidentella Dyar. 5716 stigmella Dyar. a maculicula Dyar.

\section{Euzophera Zell.}

5717 placidella Dyar.

5718 nigricantella Rag.

5719 aglwella Rag.

5720 semifuneralis Wlk. impletella Zell. pallulella Hlst.

5721 ochrifrontella Zell. ferruginella Rag.

5722 ostricolorella $\mathrm{H}$ lst.

5723 fuscomaculella Wright.

\section{Hyphantidium Scott.}

5724 tumidulella Rag.

Mescinia Rag.

5725 estrella $B . \& M c D$.

Vitula Rag.

5726 edmandsi Pack. dentosella Rag.

5727 serratilineella Rag. 5728 basimaculatella Rag.
Lotilia Rug.

5729 ephestiella Rag. luatrella Dyar.

5730 coccidivora Comst. pallida Comst.

$a$ hulsti Ckil.

5731 eremiella Dyar.

5732 myersella Dyar.

5788 fiskeella Dyar.

Canarsia Hlst.

5734 ulmiarrosorella Clem. pnoumatella Hlst. ulmella Rag. fuseatella Hist.

5735 gracilella Hlat. 5736 feliculella Dyar.

Psorosina Dyar.

5737 hammondi Riley. 5738 angulella Dyar.

Staudingeria Rag.

5739 albipenella $H$ lst. a olivacella Dyar.

b perluteella Dyar.

Heterographis Rag.

5740 ignistrigella Rag.

5741 morrisonella Rag. olbiella Hlst. form coloradensis Rag.

5742 arizonella Hlst.

Hulstia Rag.

5743 undulatella Clem. propriella WIk.

5744 texanella $\mathrm{H}$ lst.

Honora Grt.

5745 mellinella Grt. ochrimceulella Rag.

5746 dotella Dyar. 5747 sciurella Rag. 
5748 subsciurella $R a g$. 5749 montinatatella Hlst. canicostella Rag.

5750 dulciella $H$ lst.

5751 fumosella $H$ lst.

Dolichorrhinia Rag.

5752 aureofasciella $R a g$.

Valdivia Rag.

5753 lativittella $R a g$.

5754 mirabilicornella Dyar.

5755 albocostella $\mathrm{Hlst}$.

Divitiaca B. \& MeD.

5756 parvulella $B$. \& $M c D$.

5757 simulella $B$. \& $M c D$.

5758 ochrella $B$. \& $M c D$.

Diviana Rag.

5759 eudoreella $R a g$.

Ocala Hlst.

5760 dryadella Hlst.

Palatka Hlst.

5761 nymphæella Hlst.

Eurythmidia Rag.

5762 ignidorsella $R a g$.

Dannemora IIlst.

5763 edentella Hlst.

Homøosoma Curt.

5764 impressale Hlst.

5765 uncanale $\mathrm{Hlst}$.

5766 elongellum Dyar.

5767 illuviellum $R$ ag. candidellum Hlst.

5768 striatellum Dyar.

5769 albescentellum $R a g$.

5770 inornatellum Hlst.

5771 mucidellum $R a g$.

5772 stypticellum Grt.
5773 reliquellum $D_{l} a r$.

5774 oslarellum Dyar.

5775 electellum Hlst.

$a$ texanellum $R a g$. differtellum B. \& McD.

5776 opalescellum $\mathrm{Hlst}$. tenuipunctellum Rag.

Paudellia Dyar.

5777 apyrelia Dyar.

5778 albovittella Dyar.

Unadilla HIst.

5779 erronella Zell. nasutella Hlst.

Ephestioides Rag.

5780 gilvescentella $R a g$.

5781 infimella $R a g$.

5782 mignonella Dyar.

5783 nigrella Hlst.

5784 benjaminella Dyar.

5785 erythrella $R a g$.

Eurythmia Rag.

5786 hospitella Zell. quantulella Hlst.

5787 parvulella Ely.

5788 angulella Ely.

5789 diffusella Ely.

5790 furnella Ely.

5791 coloradella Hlst.

5792 spaldingella Dyar.

5793 yavapaella Dyar.

Moodna IIlst.

5794 lugubrella $R a g$.

5795 ostrinella Clem. obtusangulella Rag. pelviculella HIst.

5796 nigrella Hlst.

Ephestia Gn.

5797 fuscofasciella $R a g$. 5798 nonparilella Dyar. 
5799 kuchniella Zell. gitonella Druce.

5800 amarella Dyar. 5801 cautella W1k. 5802 figulileila Greg. 5803 rileyella $\mathrm{Rag}$. 5804 elutella $H b n$.

Varneria Dvar.

5805 postremella Duar. 5806 atrifasciella $B$. \& $M C D$.

Plodis Gn.

5807 interpunctella $\mathrm{Hbn}$. zeae Fitch.

\section{Anerastiina}

Ragonotia Grt.

5808 dotalis Hlst. discigerella Rag.
olivella Hlst.

Aurora Rag.

5809 longipalpella Rag.

\section{Tolima Rag.}

5810 roseopennella Hlst.

5811 opacella Hlst.

5812 cincaidella Dyar.

Pectinigeria Rag.

5813 ardiferella Hlst. nigramaculella Hlst.

5814 pamponerella Dyar.

5815 gemmatella Hlst.

5816 bistriatella $H$ lst. discostrigella Dyar.

Poujadia Rag.

5817 glareosella Zell. bicolorella Hlst.

5818 pimella Dyar.

5819 quadricolorella Dyar.

5820 floscella Hlst.
Ollia Dyar.

5821 holoponerella Dyar.

5822 santaritella Dyar.

5823 parvella Dyar.

Cabnia Dyar.

5824 myronella Dyar.

Saluria Rag.

5825 ostreella $R a g$.

5826 tetradella Zell.

5827 rostrella Rag.

5828 dichroella Rag.

Martia Rag.

Urula Hlst.

5829 arizonella $\mathbf{R a g}$. incongruella Hist.

Navasota Rag.

5830 hebetella Rag.

Alamosa Rag.

5831 piperatella $R a g$.

5832 bipunctella $B$. \& $M C D$.

Hypsotropa Zell.

5833 luteicostella $\mathrm{Rag}$. nodosella $\mathrm{Hlst}$.

Peoria Rag.

5834 bipartitella Rag.

5835 approximella $W U$. haematica Zell. roseatella Pack.

5836 albidella $\mathrm{H} l s t$.

Barberia Dyar.

5837 affinitella Dyar.

Anerastia $\mathrm{Hbn}$.

5838 ella Hlst.

5839 conspersella Rag. 
Bandera Rag.

5840 binotella $Z$ eil.

5841 carneella $B$. \& $M c D$.

5842 virginella Dyar.

5843 cupidinella Hlst.

5844 subluteella Rag.

Tampa Rag.

5845 dimediatella $R a g$.

Statina Rag.

5846 gaudiella $\mathrm{Hlst}$.

5847 roseotinctella $\mathrm{Rag}$.
5848 bifasciella Hamp.

Calera Rag.

5849 punctilimbella $R a g$.

Cœnochroa Rag.

5850 illibelia $\mathrm{Hlst}$. puricostella Rag.

5851 inspergella $R a g$.

5852 californiella $R a g$.

Chipeta Hlst.

5853 perlepidella $\mathrm{Hlst}$.

\section{PTEROPHORIDEE}

Trichoptilus Wlshm.

5854 congrualis $W l k$. ochrodactylus Fish. centetes Meyr.

5855 pygmæus $W l s h m$.

5856 lobidactylus Fitch. californicus Wlshm. $a$ wrighti Grinnell.

\section{Oxyptilus Zell.}

5857 periscelidactylus Fitch. 5858 delawaricus Z Zell.

5859 ningoris Wlshm.

5860 tenuidactylus Fitch. nigrociliatus Zell.

5861 raptor Meyr. bernardinus Grinnell. finitimus Grinnell.

Platyptilia $\mathrm{Hbn}$.

5862 edwardsi Fish.

5863 cosmodactyla $\mathrm{Hbn}$. punctidactyla Haw. ulodactyla Zett.

5864 acanthodactyla $\mathrm{Hbn}$. 5865 marmarodactyla Dyar. 5866 pica Wlshm.
5867 crenulata $B . \& M c D$. 5868 pasadenensis Grinnell. 5869 carduidactyla Riley. 5870 tesseradactyla Linn. 5871 percnodactyla $\mathrm{Wlshm}$. 5872 monticola Grinnell. 5873 orthocarpi Wlshm. 5874 fragilis Wlshm. 5875 albidorsella Wlshm. 5876 williamsi Grinnell. 5877 shastæ Wlshm. 5878 hesperis Grinnell. 5879 albida Wlshm. 5880 albicans $F i s h$. 5881 pallidactyla Haw. marginidactylus Fitch. nebulaedactylus Fitch. bertrami Roess. bischoffi Zell. cervinidactylus Pack.

5882 grandis Wlshm.

5883 cooleyi Fern. 5884 albiciliata $W l s h m$. 5885 adusta Wlshm. 5886 xylopsamma Meyr. 5887 carolina Kearf. 5888 modesta Wlshm. 5889 petrodactyla $W l k$. 
Alucita Linn.

5890 montana Wlohrs. 5891 walsinghami Fern. 5892 belfragei Fish. 5893 cinerascens IVlshm.

Adains Tutt.

5894 bjpunctatus Moeseh.

\section{Pterophorus Geof.}

5895 rhynchosix Dyar. 5896 stramineus Wlshm. 5897 helianthi Wlshm. 5898 mathewianus Zell. 5899 cineraceus Fish. 5900 baroni Fish. 5901 gratiosus Fish. 5902 lugubris Fish. 5903 barberi Dyar. 5904 integratus Meyr. 5905 caudelli Dyar. 5906 agraphodactylus Wlk, aspilodactylus Wik.

5907 homodactylus Wlk. 5908 elliotti Fern. 5909 paleaceus Zell. sericidactylus Murt. 5910 angustus Wlshm. 5911 inconditus Wlshm. 5912 baccharides Grin. 5913 subochraceus Wlshm. a australis Grin.

5914 lacteodactylus Cham. 5915 sulphureodactylus Pack.

5916 chlorias Meyr. 5917 unicolor B. \& $M c D$. 5918 cervinicolor $B$. \& $M c D$. 5919 pictipennis Grin. 5920 grandis Fish. 5921 aquila Meyr. 5922 rileyi Fern. 5923 kellicotti $F$ ish.
5924 cretidactylus Fitch. occidentali Wlshm.

5925 eupatorii Fern. cretidactylust Zell.

5926 guttatus Wlshm.

5927 fishi Fern.

5928 inquinatus Zell. ambrosiae Murt.

5929 gorgoniensis Grin.

5930 acrias Meyr.

5931 chionastes Meyr.

5932 brucei Fern.

5933 behri Grin.

5934 grisescens Wlshm.

5935 hilda Grin.

5936 declivis Meyr.

5937 citrites Meyr.

5938 parvus Wlshm.

5939 cataline Grin.

5940 serenus Meyr.

5941 balanotes Meyr.

5942 monodactylus Linn. pterodactyla $\mathbf{H b n}$. cineridactylus Fitch. naevosidactylus Fitch. pergracilidactylus Pack.

Stenoptilia Hbn.

5943 schwarzi Dyar. 5944 mengeli Fern. 5945 pumilio Zell. gilvidorsis Hed.

5946 exclamationis Wlshm. 5947 semicostata Zell. 5948 gorgoniensis Grin. 5949 pterodactyla Linn. ptilodactyla Hbn.

5950 coloradensis Fern. 5951 californica Grin. 5952 pallistriga $B$. \& MeD.

Agdistis Hbn. 5958 adactyla $\mathrm{Hbn}$. 


\section{ORNEODIDAE}

Orneodes Latr.

5954 hexadactyla Linn. polydactyla $\mathrm{Hbn}$. mantana Ckll.

\section{COSMOPTERYGID王}

Idioglossa Wlshm.

5955 miraculosa $F$. \& $B$. americella Wlshm.

Cosmopteryx Hbn.

5956 pulcherrimella Cham. 5957 clemensella Staint. 5958 gemmiferella Clem. 5959 attenuatella $W l k$. lespedezae Wlshm.

5960 ipomøæ Busck. 5961 unicolorella Wlshm. 5962 quadrilineella Cham. 5963 chalybrella Wlshm. 5964 delicatella $W l s h m$. 5965 minutella Beut. 5966 fernaldella Wlshm. floridanella Beut. nigrapunctella Busck.

5967 montisella Cham.

5968 nitens Wlshm.

5969 clandestinella Busck.

Chrysoclista Staint. 5969,1 villella Busck.

Erineda Busck. 5970 elyella Busck.

Synallagma Busck. 5971 busckiella Engel.

Theisoa Cham.

5972 constrictella Zell. bifasciella Cham.

5973 multifasciella Cham. 5974 permolestella Busck.
Aetia Cham.

5975 bipunctella Cham.

Ithome Cham.

Eriphiał Cham.

5976 concolorella Cham. 5977 albalineella Cham. 5978 nigrilineella Cham.

Chrysopeleia Cham. 5979 ostryæella Cham. 5980 purpuriella Cham. 5981 quadricristatella Cham.

Cystiœcetes Braun. 5982 nimbosus Braun.

Pyroderces H. S. 5983 rileyi $W l s h m$.

Stilbosis Clem. 5984 tesquella Clem. quinquecristatella Cham.

Walshia Clem.

5985 amorphella Clem. miscecalonella Cham.

Blastodacna Wocke. 5986 curvilineella Cham. bicristatella Cham.

Perimede Cham. 5987 erransella Cham. unomaculella Cham.

5988 particornella Busck.

Lymnæcia Staint. 5989 phragmitella Staint. 
Cyphophora H. S.

5990 tricristatella Cham. grandisella Cham. subiridescens Wlshm.

Homaledra Busck.

5991 heptathalama Busck. 5992 sabalella Cham.

Psacaphora H. S.

5993 cambiella Busck.

5994 passerella Busck.

5995 purpuriella Busck.

5996 terminella Westw. engelella Busck.

5997 argentimaculella Murt. 5998 luciferella Clem.

\section{Stagmatophora H. S.}

5999 ceanothiella Cosens.

6000 iridella Busek.

6001 sexnotella Cham.

6002 gleditschiæella Cham.

Mompha Hbn.

6003 albella Cham.

6004 albopalpella Cham.
6005 bifasciella Cham.

6006 brevivittella Clem. oenotherivorella Cham. oenotheraceeminella

Cham.

6007 stellella Busck.

6008 eloisella Clem.

magnatella Zell.

oenotheraeella Cham.

Iyonetiella Cham.

6009 circumseriptella Zell.

6010 pecosella Busck.

6011 coloradella Cham.

6012 definitella Zell. unicristatella Cham.

6013 ignobilisella Cham.

6014 claudiella Kearf.

6015 cephalanthiella Cham.

6016 minimella Cham.

6017 murtfeldtella Cham. albocapitella Cham. grissaeella Cham. obseurusella Cham. parvicristatella Cham.

6018 unifasciella Cham. 6019 rufocristatella Cham. 6020 punctiferella Busck.

\section{GELECHIIDIE}

Metzneria Zell.

6021 lappella Linn.

\section{Paltodora Meyr.}

6022 striatella $\mathrm{Hbn}$.

6023 pallidistrigella Cham.

6024 magnella Busck.

6025 cilialineella Cham.

6026 pennella Busok.

6027 dietriella Busck.

6028 canicostella $W / s / \mathrm{m}$.

6029 anteliella Busck.

6030 sabulella Wlshm.
6031 similiella Cham. solaniellał Cham. piscipellis Zell.

6032 tophella Wlshm. 6033 pallidella Cham. 6034 modesta Wlshm.

Ptycerata Ely.

6035 busckella Ely.

Sophronia Hbn:

6036 primella Busck.

6037 roseicrinella Busck. 
Sitotroga Hein.

6038 cerealella Oliv.

Megacraspedas Zell.

Autoneda Busck.

6039 plutella Cham.

\section{Glauce Cham.}

6040 pectenalæella Cham.

\section{Aristotelia $\mathrm{Hbn}$.}

6041 roseosuffusella Clem. bellela Wlk.

6042 pudibundella Z ell. intermediella Cham.

6043 molestella Zell.

6044 rubidella Cham. rubensella Cham.

6045 fungivorella Clem. 6046 salicifungiella Clem. 6047 ivæ Busck.

6048 bifasciella Busck. 6049 elegantella Cham. superbella Cham.

6050 argentifera Busck. 6051 cockerella Busck. 6052 absconditella $W l k$. palpiannulella Cham.

6053 minimella Cham. 6054 physaliella Cham. 6055 disconotella Cham. 6056 gilvolineella Cham. 6057 angustipennella Clem. kearfottella Busck. 6058 quinquepunctella Busck. 6059 placidella $Z$ ell. natalella Busck. 6060 harrisonella Busck.

\section{Helice Cham.}

6061 pallidochrella Cham. gleditschiaeella Cham.
Evippe Cham.

6062 prunifoliella Cham. 6063 leuconota Z $\mathrm{ell}$. plutella Cham. 6064 pollostella Busck.

Eucordylea Dietz.

6065 atrupictella Dietz. 6066 gallicola Busck.

Enchrysa Zell.

6067 dissectella Z Zell. youngella Kearf.

Chrysopora Clem. 6068 lingulacella Clem. hermannella Cham. armeniella F. \& B.

6069 hermannella $F a b r$. 6070 versicolorella Kearf.

Telphusa Cham.

6071 longifasciella Clem. curvistrigella Cham. obliquifasciella Cham.

6072 acaciella Busck.

6073 quinquecristatella Cham. 6074 latifasciella Cham. 6075 quercinigracella Cham. fragmentella Zell. 6076 fuscopunctella Clem. 6077 palliderosacella Cham. 6078 querciella Cham. 6079 basistrigella Zell. 6080 basifasciella Z ell. 6081 velatella Busck. 6082 betulella Busck. 6083 belangerella Cham. oronella Wishm. 6084 glandiferella Zell. sella Cham. 6085 alexandrixella Cham. 6086 fasciella Cham. 
Agrippo Cham.

6087 biscolorella Cham.

6088 fuscopulvella Cham.

6089 evippeella Busck.

Numata Busck.

6090 bipunctella Bunck.

Nealyda Dietz.

6091 pisonize Busck:

6092 bifidella $D$ ietz.

6093 kinzelella Busck.

Leuce Cham.

6094 fuscocristatella Cham. belfragesella Cham.

Gnorimoschema Busck.

6095 dudiella Busck.

6096 tetradymiella Busck.

6097 chiquitella Busck.

6098 washingtoniella Busck.

6099 baccharisella Busck.

6100 gemicyclionella Busck.

6101 coquillettella Busele.

6102 albimarginella Cham.

6108 pallidochrella Cham.

6104 splendoriferella Busck.

6105 saphirinella Cham.

6106 serratipalpella Cham.

6107 faustella Busck.

6108 pedmontella Cham.

6109 banksiella Busck.

6110 triocellella Cham.

6111 florella Busck.

6112 radiatella Busck.

6113 octomaculella Cham.

6114 ochreostrigella Cham. henshawiella Busck.

6115 collinusella Cham.

6116 lavernella Cham. physalivorella Cham.

6117 batanella Busele.

6118 terracottella Busek.
6119 nrtemisielln Kearf.

6119,1 chenopodiella Busck.

6120 scutellarisella Cham.

6121 detersella Clem.

brackenridgella Busck.

6122 gallæsolidaginis Riley.

6123 alaricella Busck.

6124 busckiella Kearf.

6125 gallædiplopappi Fyles.

6126 gallwasteriella Kell. caesiella Brodie.

6127 princeps Busck.

6128 atriplex Busck.

6129 septentrionella Fyles.

6130 laguna Busck.

6131 salinaris Busek.

6132 subterranea Busck.

6133 gibsoniella Busck.

6134 petrella Busck.

Recurvaria Haw.

6135 apicitripunctella Clem. abietisella Pack.

6136 variella Cham.

6137 colubrinæ Busck.

6138 piceaella Kearf.

form obscurella Kearf. nigrat Kearf.

6139 obliquistrigella Cham.

6140 graphicella Busck.

6141 thujaella Kearf.

6142 juniperella Kearf.

6143 cratægella Busek.

6144 robiniella Fitch.

fuscopallidella Cham. robininefoliella Cham.

6145 quercivorella Cham. gilviscopella Zell.

6146 dorsivittella Zell. vagatioella Cham.

6147 eristatella Cham.

6148 eitriella Cham.

6149 nigra Busck.

6150 invictella Busck.

6151 elachistella Busck. 
6152 alnifructella Busck.

6153 pinella Busck.

6154 milleri Busck.

6155 coniferella Kearf.

6156 gibsonella Kearf.

Trypanisma Clem.

6157 prudens Clem. quinqueannulella Cham.

6158 fagella Busck.

Besciva Busck.

6159 longitudinella Busck.

Epithectis Meyr.

6160 attributella $W l k$. difficilisella Cham.

6161 bicostomaculella Cham. thoracella Wlshm.

6162 sylvicolella Busck.

6163 subsimella Clem.

6164 saundersella Cham.

6165 gallægenitella Clem. geminella Riley.

Paralechia Busck.

6166 pinifoliella Cham.

Arogalea Wlshm.

6167 cristifasciella Cham. inscripta Wlshm.

6168 californica Busck.

Phthorimæa Meyr.

6169 operculella Z $\mathrm{ell}$. solanella Bdv. tabacella Rag.

6170 minor Busck.

6171 glochinella $Z$ ell. solaniella Cham. cinerella Murt. inconspicuella Murt.

6172 striatella Murt. 6173 marmorella Cham.
Neodactylota Busck.

6174 snellenella Cham. 6175 barberella Busck.

Deoclona Busck.

6176 yuccasella Busck.

Prostomeus Busck.

6177 brunneus Busck.

Polyhymno Cham.

6178 luteostrigella Cham. fuscostrigella Cham.

6179 acaciella Busck. 6180 sexstrigella Cham.

Aproærema Durr.

6181 crotalariella Busck. 6182 palpilineella Cham. 6183 nigrella Cham. 6184 kearfottella Busck.

Duvita Busck. 6184,1 vittella Busck. 6185 nigratomella Clem. apicilinella Clem. apicistrigella Cham. 6186 concinusella Cham.

Anacampsis Curt. 6187 fragariella Busck. 6188 innocuella $Z$ ell. 6189 rhoifructella Clem. consonella Zell. quadrimaculella Cham. ochreocostella Cham. 6190 crescentifasciella Cham. 6191 niveopulvella Cham. 6192 argyrothamniella Busck. 6193 lagunculariella Busck. 6194 cyclella Busck. 6195 nonstrigella Busck. 6196 paltodoriella Busck. 
6197 fullonella Zell. mujusella Cham. rubercens Wlshm. subruberella Chan.

6198 Iupinella Buscli.

6199 agrimonielia Clem. aduncella Zell. aderusella Riley.

6200 tristrigella Wlahm.

6201 levipedella Clem.

6202 coverdalella Kearf.

Gelechia IIbn.

6203 cercerisella Cham. olympiadella Zell.

6204 quinella Zell.

6205 arizonella Busck.

6206 lipatiella Busck.

6207 coloradensis Buscle.

6207, 1 paralogella Busck.

6208 trialbamaculella Cham. epigaeella Cham.

6209 confusella Cham. persicaeella Murt.

6210 bimaculella Cham. ternariella Zell. sylvaecolella Cham.

6211 continuella Zell. trimaculella Pack. albomaculella Cham.

6212 periculella Busck.

6213 ribesella Cham.

6214 trophella Busek.

6215 dromicella Busck.

6216 lugubrella Fabr.

6217 viduella $\mathrm{Fabr}$. labradoriella Clem.

6218 albilorella Zell. trifnscielln Cham.

6219 dentella Busck. 6220 sistrella Busck. 6221 unifasciella Busck. 6222 paulella Busck. 6223 catalinella Busek.
6224 packardella Cham. 6225 morenella Buack. 6226 aristella Busck. 6227 abdominella Busok. 6228 leucanieella Busck. 6229 paraplutella Busele. 6230 basquella Cham. costipunctella Moesch.

6231 kincaidella Busck. 6232 coticola Busck. 6233 texanella Cham. chambersella Dyar.

6234 variabilis Busck.

6235 invariabilis Kearf.

6236 fluvialella Busck. 6237 striatella Busch. 6238 figurella Busck. 6239 ochreostrigella Cham. 6240 hibiscella Busck. 6241 trilineella Cham. 6242 argentipunctella Ely. 6243 bianulella Cham. ocellella Cham.

6243,1 notandella Busck. 6244 cockerelli Busck.

6245 discoocellella Cham. violaceofusea Zell.

6246 grisella Cham.

6247 anarsiella Cham. 6248 monella Busck. 6249 inquilinella Busck. 6250 pravinominella Cham. quadrimaculella Cham.

6251 barnesiella Busck. 6251,1 puertella Busck. 6251, 2 diversella Busck. 6252 lindenella Busck. 6253 albisparsella Cham. platanella Cham. 6254 ornatifimbriella Clem. unctulella Zell.

6255 malindella Busck. 6256 rileyella Cham. 6257 ceanothiella Busck. 
6258 ochreosuffusella Cham. depressostrigella Cham.

6259 thoracealbella Cham. 6260 minimaculella Cham. 6261 triangulella Busck. 6262 obscuroocelella Cham. 6263 versutella $\mathrm{Z}$ ell. 6264 lynceella $Z$ ell. 6265 bicostomaculella Cham. quercifoliella Cham. 6266 nigrimaculella Busck. 6267 dyariella Busck. 6268 maculimarginella Cham. 6269 gilvomaculella Clem. biminimaculella Cham.

6270 bigella Busck.

6271 mandella Busck. 6272 pullifimbriella Clem. 6273 pseudoacaciella Cham. caecella Zell.

6274 serotinella Busck. 6275 vernella Murt. formosella $\$$ Murt. 6276 brumella Clem. 6277 occidentella Cham. 6278 mediofuscella Clem. vagella Wlk. fuscoochrella Cham. liturosella Zell.

6279 fondella Busck.

6280 walsinghami Dietz.

6281 scabrella Busck.

6282 pennsylvanica Dietz.

6283 conclusella $W l k$. grissefasciella Cham.

6284 nundinella $Z$ ell. beneficentella Murt. 6285 monumentella Cham. 6286 obscurosuffusella Cham. 6287 petasitis $P$ faff. 6288 panella Busck. 6289 abella Busck. 6290 fuscotæniaella Cham. 6291 laudatella Wlshm.
6292 terminimaculella Kearf. 6293 alternatella Kearf. 6294 tephriasella Cham. 6295 branella Busck. 6296 pseudofondella Busck. 6297 biforella Busck. 6298 depuratella Busck. 6299 fulmenella Busck. 6300 nanodella Busck. 6301 inrequalis Busck. 6302 sedulitella Busck. 6303 lophosella Busck. 6305 adaptella Wlk. 6306 ambrosiæella Cham. 6307 amorphæella Cham. 6308 argentialbella Cham. 6309 attritella Wlk. 6310 discomaculella Cham. aurimaculella Cham.

6311 badiomaculella Cham. 6312 bistrigella Cham. 6313 canopulvella Cham. 6314 capiteochrella Cham. 6315 caryævorella Pack. 6316 cassella Wlk. 6317 decemmaculella Cham. 6318 discoannulella Cham. 6319 discostrigella Cham. 6320 flavicorporella Wlshm. 6321 flexurella Clem. 6322 fuscoluteella Cham. 6323 fuscomaculella Cham. 6324 glycyrrhizæella Cham. 6325 griseaella Cham. 6326 griseochrella Cham. 6328 labradorica Moesch. 6329 lacteusochrella Chum. 6330 liturella Wlk. 6331 maculatusella Cham. 6332 milleriella Cham. 6333 mimella Clem. 6334 obscurella Cham. 6335 obscurusella Cham. fuscopulvella Cham. 6336 ocherfuscella Cham. 
6337 pallidegrisseella Cham. 6338 palpialbella Cham.

6339 parvipulvella Cham.

6341 pullusella Cham.

6342 simpliciella Cham.

6343 subalbusella Cham.

63.14 suffusella Chum.

6345 thoracestrigella Cham.

6346 thoraceochrella Cham.

6347 unistrigella $\mathrm{C} / \mathrm{Cm}$.

6348 versicolorella Cham.

6349 wacoella Cham.

-6350 subtractella Wilk.

\section{Anorthosia Clem.}

6351 punctipennella Clem. 6352 gracilella Cham.

Anarsia Zell.

6353 lineatella Zell. pruniella Clem.

6354 suffusella Cham.

\section{Trichotaphe Clem.}

6355 flavocostella Clem.

6356 inserrata Wlshm.

6357 serrativitella Z Zell. plutella Cham.

6358 barnesiella Busck.

6359 simpliciella Busck.

6360 costarufoella Cham.

6361 alacella Clem. ochripalpella Zell. goodellella Cham.

6362 purpureofusen Wlshm.

6363 nonstrigella Cham.

6364 juncidella Clem. pallipalpis WIk. dubitella Cham.

6365 trinotella Busck.

6366 leuconotella Busck. 6367 setosella Clem. costolutella Cham. bilobella Zell.
6368 levisella Fyles.

6369 washingtoniella Busck.

6370 inversella Zell.

6371 condaliavorella Busck.

6372 melantherella Busck.

6373 chambersella Murt. inaequepulvella Cham.

6374 lactiflosella Cham.

6375 bidiscomsculella Cham.

6376 fernaldella Busck.

6377 trimaculella Cham.

Dichomeris Hbn.

6378 punctidiscella Clem. stramineella Cham.

6379 eupatoriella Cham. dolabella Zell. setosella Wishm.

6380 ligulella $H b n$. contubernatella Fitch. malifoliella Fitch. favivittella Clem. quercipomonella Cham. form pometella Harris. pauciguttella Clem. reedella Cham.

6381 delotella Busck.

6382 trimaculella Cham. touceyella Busck.

6383 citrifoliella Cham.

6384 georgiella Wlk.

roseocostella Wlshm.

6385 deflecta Buscle.

6386 trinotella Coq.

6387 hirculella Busek.

6388 bipunctella Wlshm.

6389 ventrella Fitch. unicipunctella Clem. quereicella Cham.

6390 earyæeoliella Cham.

6391 bimaculella Cham.

6392 grisseella Cham.

6393 rustica Wlshm.

6394 vacciniella Busck.

- For 6ses. 6327 and 6340 have Doen omfled. 
Menesta Clem.

6395 tortriciformella Clem. liturella Wlk. coryliella Cham.

6396 melanella Murt. 6397 albaciliæella Cham.

Strobisia Clem.

6398 emblemella Clem. venustella Cham.

6399 irridipennella Clem. aphroditella Cham. proserpinella F. \& B.

Untomia Busck.

6400 untomiella Busck.

6401 albistrigella Cham.
Durrantia Busck.

6402 piperatella $\mathrm{Z}$ ell.

6403 obiterella Busck. chambersella Dyar.

Glyphidocera Wlshm.

6404 floridanella Busck. 6405 xquepulvella Cham.

6406 septentrionella Busck.

6407 aberratella Busck.

6408 meyrickella Busck.

6409 dimorphella Busck.

6410 speratella Busck.

Symmoca Hbn.

6411 novimundi Busck.

\section{OECOPHORIDE}

Martyringa Busck.

6412 latipennis Wlshm.

Pleurota Hbn.

6413 albastrigulella Kearf.

Eumeyrickia Busck.

6414 trimaculella Fitch. albapulvella Cham. haustellata Wlshm.

Eido Cham.

6415 albapalpella Cham.

Gerdana Busck.

6416 caritella Busck.

Cryptolechia Zell.

6417 cretacea Zell.

6418 obscuromaculella Cham.

6419 concolorella Beut.

6420 humata Meyr.

6421 huachucella Busck.

6422 canariella Busck.
6423 ciliella Busck.

6424 tentoriferella Clem. confertella Wlk. fernaldella Cham.

Psilocorsis Clem.

6425 quercicella Clem.

6426 obsoletella Zell.

6427 reflexella Clem. cressonella Cham.

6428 faginella Cham.

6429 dubitatella $Z$ ell.

6430 ferruginosa Zell.

6431 fletcherella Gibson.

6432 cryptolechiella Cham.

Agnopteryx Hbn.

6433 atrodorsella Clem.

6434 umbraticostella Wlshm.

6435 thoracenigræella Cham.

6436 gracilis Wlshm.

6437 canella Busck.

6438 nebulosa $Z$ ell.

6439 scabella $Z$ ell.

6440 ciniflonella $Z$ ell. 
6441 Klamathiana Wlohm.

6442 psoradiella Wlshm.

6443 fernaldella Wlshm. walsinghamiella Busck.

6444 nigrinotella Busck.

6445 curvilineella Bcut.

6446 amyrisella Busck.

6447 clemensella Cham.

6448 rosaciliella Busck.

6449 pulvipennella Clem. eupatoriella Cham. solidaginis Wlshm.

6450 gelidella Busck.

6451 thoracefasciella Cham.

6452 arnicella Wlshm.

6453 fulva Wlshm.

6454 novimundi Wlshm.

6455 muricolorella Busck.

6456 plummerella Busck.

6457 hyperella Ely.

6458 sanguinella Buscle.

6459 argillscen Wlshm.

6460 amissella Busck.

6461 pallidella Busck.

6462 pergandiella Busak.

6463 flavicomella Engel.

6464 senicionella Busck.

6465 sabulella Wlshm.

6466 arenella Schiff.

6467 canadensis Busck.

B468 lythrella Wlshm.

6469 posticella Wlshm.

6470 nubiferelle Wlshm.

6471 robiniella Pack. hilarella Zell.

6472 lecontella Clem.

Depressaria Haw.

6473 alienella Busck.

6474 togata Wlshm.

6475 juliella Busck.

6476 nervosa Haw.

6477 barberella Buscle.
6478 heraclians De Geer.

ontariella Beth.

6479 groteella Rob.

6480 betulella Busck.

6481 maculatella Busck.

6482 cinereocostella Clem. clausella Wik.

Inga Busck.

6483 sparsiciliella Clem. contrariella Wlk. atropicta Zell.

Semioscopis Hbn.

6484 packardella Clem. eruditella Grt.

6485 merriccella Dyar.

6486 aurorella Dyar.

6487 megamicrella Dyar.

6488 inornata $W l s h m$.

6489 allenella Wlshm.

Epicallima Dyar.

6490 argenticinctella Clem.

6491 edithella Busck.

6492 coloradella Wlshm.

6493 dimidiella Wlshm.

6495 quadrimaculella Cham.

6495 lucidella Busck.

6496 antidectis Meyr.

Decantha Busck.

6497 borkhauseni Zell. boreasella Cham.

Fabiola Busck.

6498 shalleriella Cham.

Oecophora Latr.

6499 newmanella Clem.

Triclonella Busck.

6500 pergandeella Busck. 
6501 determinatella Z ell. australisella Cham.

Endrosis Hbn.

6502 lacteella Schiff. kennicottella Clem.

Chambersia Riley.

6503 haydenella Cham.
Borkhausenia Hbn.

6504 pseudospretella Staint. 6505 ascriptella Busck. 6506 episcia Wlshm. 6507 conia Wlshm. 6508 fasciata Wlshm. 6509 orites Wlshm.

\section{BLASTOBASIDIE}

Blastobasis Zell.

6510 guilandinæ Busck.

6511 hulstella Dietz.

6512 yuccæcolella Dietz.

6513 plummerella Dietz.

form fuscopurpurella

Dietz. 6535 nigrostriata Wlshm.

form simplicella Dietz.

6514 sagitella Dietz.

6515 distinctella Dietz.

6516 villella Busck.

6517 eriobotyræ Busck.

Zenodochium Wlshm.

6518 citricolella Cham.

6519 coccivorella Cham.

Valentinia Wlshm.

6520 glandulella Riley. nubilella Zell.

6521 fractilinea $Z$ ell.

6522 quaintancella Dietz.

6523 nothrotes Wlshm.

6524 retectella $Z$ ell.

6525 floridella Dietz.

6526 confectella Zell.

6527 repartella Dietz.

Euresia Dietz.

6528 pulchella Dietz.

Calosima Dietz.

6529 argyrosplendella Dietz.

6530 dianella Dietz.
Holcocera Clem.

6531 dives Dietz. form basipallidella Dietz.

6532 aphidiella Wlshm.

6533 estriatella Dietz.

6534 gigantella Cham.

6536 purpurocomella Clem.

6537 ochrocephala Dietz.

6538 crassicornella Dietz.

6539 modestella Clem.

6540 chalcofrontella Clem. form minorella Dietz. form fumerella Dietz. $a$ quisqueliella Z $\mathrm{Z}$ ell.

6541 confluentella Dietz.

6542 busckiella Dietz.

6543 sciaphilella Zell. triangularisella Cham.

6544 plagiatella Dietz.

6545 livorella $Z$ ell.

6546 iceryæella Riley.

6547 maligemmella Murt.

6548 gilbociliella Clem.

6549 funebra Dietz. form reductella Dietz.

6550 inconspicua $W l s h m$.

6551 stygna Wlshm.

6552 boreasella Dietz.

6553 nucella Wlshm.

6554 nana Dietz.

6555 elyella Dietz.

6556 insulatella Dietz.

6557 inclusa Dietz. 
6558 simulelia Dietz.

6559 messelinella Dietz.

form spoliatella Diets.

6560 morrisoni $W$ lshu.

6561 fluxella ZeU.

6562 clemensella Cham.

6563 irenica Wlahm.

6564 illibella Dietz.

6565 crescentella Dietz. a annulipes Dietz.

6566 spretella Dietz.

6567 ursells Wlshm.

6568 punctiferella Clem. form subsenella Zell. form texanella Wlshm.

6569 zelleriella Dietz. form annectella Dietz.

6570 vestaliella Dietz.

6571 tartarella Dietz.

6572 pusilla Dietz.

6578 rufopunctella Dietz.

6574 melanostriatella Dietz.

6575 interpunctella Dietz.

Eubolepia Dietz.

6576 anomalella Dietz.

Ploiophora Dietz.

6577 ampla Dietz.

6578 fidella Dietz.

Pigritia Clem.

6579 confusella Dietz.

6580 purpurella Dietz.

6581 basilarella Dietz.
6582 laticapitella Clem. aufugella Zell. luteopuivella Cham.

6583 mediofasciella Dietz.

6584 ornatella Dietz.

6585 angustipennella Dietz.

6586 tristella Dietz.

6587 arizonella Dietz.

6588 spoliatella Dietz.

6589 obscurella Dietz.

Epigritia Dietz.

6590 ochrocomella Clem. pallidotinctella Dietz. form heidemannella Dietz.

Dryoperia Cool. Dryopeł Cham.

6591 occidentella Dietz.

6592 tenebrella Dietz.

6593 minnicella Dietz.

6594 grisella Dietz.

6595 fuscosuffusella Dietz.

6596 murtfeldtella Cham. erratella Dietz.

6597 canariella Dietz.

6598 discopunctella Dietz.

6599 ochreella Clem.

6600 fenyesella Dietz.

Pseudopigritia Dietz.

6601 dorsomaculella Dietz.

6602 equitella Dietz.

6603 fraternella Dietz.

6604 argyreella Dietz.

\section{STENOMIDE}

Menestomorpha Wlshm.

6605 oblongata Wlshm.

Stenoma Zell.

6606 schlægeri Zell.

6607 furcata Wlshm.
6608 crambitella Wlshm.

6609 algidella Wlk. leucillana Zell.

6610 humilis Zell. nubeculosa Zell. canusella Cham. 
6611 mistrella Busck. 6612 unipunctella Clem. tortricella Cham. lithosina Zell.
6613 vestalis Z Zell. albella Cham.

6614 quercicella Busck. 6615 decorosella Busck. 6616 osseella Wlshm.

\section{ETHMIIDEE}

\section{Ethmia Hbn.}

6617 albitogata Wlshm.

6618 coquillettella Busck.

6619 umbrimarginella Busck.

6620 monachella Busck.

6621 lassenella Busck.

6622 albistrigella Wlshm. chrysurella Dietz.

6623 caliginosella Busck.

6624 monticola Wlshm.

6625 fuscipedella Wlshm.

6626 macelhosiella Busck.

6627 arctostaphylella Wlshm.

6628 obscurella Beut.

6629 mediella Busck.

6630 subcærulea Wlshm.

6631 discostrigella Cham.

6632 semitenebrella Dyar.

6633 confusella Wlk.

6634 josephinella Dyar.
6635 trifurcella Cham.

6636 marmorea $W l s h m$.

6637 semilugens $\mathrm{Z}$ ell. multipunctella Cham. semiopaca Grt. plumbeella Beut.

6638 semiombra Dyar.

6639 hagenella Cham.

6640 mirusella Cham. albicostella Beut.

6641 apicipunctella Cham.

6642 longimaculella Cham. walsinghamella Beut.

6643 coranella Dyar.

6644 zelleriella Cham. texanella Cham.

6645 delliella Fern.

6646 bittenella Busck. 6647 zavaliella Busck.

6648 prattiellả Busck.

\section{AEGERIID压}

Bembecia Hbn.

6649 marginata Harr. pleciaeformis. Wlk. odyneripennis Wlk. mubi Riley. flavipes Hlst. form albicoma Hlst.

Alcathoe Hy. Edw.

6650 caudata Harris. a walkeri Neum.

6651 korites Druce.

6652 apiformis Clerck.
Aegeria Fabr.

6653 pacifica $H y . E d w$. californicum Neum.

6654 tibialis Harris. flavitibia Wlk. minimum Neum.

$a$ dyari Ckll.

Synanthedon $\mathrm{Hbn}$.

6655 exitiosa Say. persica Thom. pepsidiformis $\mathrm{Hbn}$. xiphiaeformis Bdv. 
form fitchi $H y . E d w$. form edwardsi Beut. form luminosa Neum.

6656 opalescens $H y$. E'dw. pacifica Riley.

6657 graefi $H y . E d u$.

$a$ barnesi Beut.

6658 mellinipennis Bdv. resplendens $\mathrm{Hy}$. Edw. artemisiue $\mathrm{Hy}$. Edw. senecivides Hy. Edw.

6659 giliæ $H y$. Eidu. vitrina Neum. deceptiva Beut.

6660 rileyana $H y . E d w$. brunneipennis Hy. Edw. hyperici Hy. Edw.

6661 rutilans $H y . E d w$. aureola Hy. Edw. hemizonae Hy. Edw. lиріпі Hy. Edw. perplexa Hy. Edw. impropria By. Edw. washingtonia $\mathrm{Hy}$. Edw.

6662 madariæ $H y . E d w$.

6663 aretica Beut.

6664 florissantella Ckll. 6665 mimuli $H y . E d w$.

6666 neglecta $H y . E d w$. 6667 refulgens $H y$. $E d w$. 6668 bassiformis Wlk. lustrans Grt. consimilis $\mathrm{Hy} . \mathrm{Edw}$. bolli Hy. Edw. eupatorii Hy. Edw. sexfasciata Hy. Edw. infirma Hy. Edw. imitata Hy. Edw.

6669 tipuliformis Linn.

6670 arizonse Beut.

6671 morula $H y . E d w$.

6672 novaroensis $H y$. Edw.

6673 brunneri Busck.

6674 rubrofascia $H y . E d w$.
6675 bolteri Hy. Edw. 6676 rubricincta Beut. 6677 fulvipes Harris. 6678 saxifragw $H y$. Edw. henehawi Hy. Edw.

6679 pictipes G. \& $R$. inusitata Hy. Edw.

6680 castanes Busck.

6681 albicornis $H y, E d w$. proxima Hy. Edw. modesta Kell.

6682 americana Beut.

6683 culiciformis Linn.

6684 tepperi $H y . E d w$.

6685 acerni Clem.

acericolum Germ.

6686 corni Hy. Edw.

6687 aureopurpura $\boldsymbol{H} y$. Edw.

6688 pyri Harris.

koebelei Hy. Edw.

6689 rhododendri Beut.

6690 seitula Harris.

gallivorum Westw.

hospes Walsh. aemula Hy. Edw.

6691 ithace Beut.

6692 corusea $\boldsymbol{H} y . E d w$.

6693 decipiens $H y$. Edw。 imperfecta Hy. Edw. nicotianas Hy. Edw.

6694 rubristigma Kell.

6695 querei $H y$. Edw.

6696 prosopis Hy. Edw.

6697 tecta $H y . E d w$.

6698 candescens $\boldsymbol{H} y . E d w$.

6699 sigmoidea Beut.

6700 tacoma Beut.

6701 texana $H y . E d v$. wittfeldi Hy. Edw.

6702 seminole Beut.

6703 palmi Beut.

6704 marcia Beut.

6705 prestans $H y . E d u$. 
6706 fragariæ $H y . E d w$. orthocarpi Hy. Edw. $a$ semiprestans Ckll.

6707 helianthi $H y$. Edw. behrensi Hy. Edw. elda Hy. Edw.

6708 mariona Beut.

6709 polygoni $H y$. Edw. meadi Hy. Edw.

6710 achillæ $H y . E d w$. eremocarpi Hy. Edw.

6711 floridensis Grt.

6712 geliformis $W l k$.

6713 sapygæformis $W l k$.

6714 animosa $H y$. Edw.

6715 pyralidiformis Wlk. nigella Hlst.

6716 sanborni $H y$. Edw.

6717 ruficornis $H y$. Edw. minuta Hy. Edw.

6718 subærea $H y$. Edw.

6719 nigra Beut.

6720 verecunda $H y$. $E d w$.

6721 edwardsi Beut.

6722 utahensis Beut.

Parharmonia Beut.

6723 pini Kellic.

6724 piceæ Dyar.

Sannina Wlk.

6725 uroceriformis Wlk. quinquecaudata Ridings.

Podosesia Moesch.

6726 syringæ Harris. longipes Moesch.

6727 fraxini Lugger.

Vespamina Beut.

6728 вequoiæ $H y . E d w$. pinorum Behrens.

Palmia Beut.

6729 præcedens $H y, E d w$.
Gæa Beut.

6730 emphytiformis Wlk. 6731 solituda $H y$. Edw. 6731,1 arizonensis Beut.

Euhagena Hy. Edw.

6732 nebraskæ Hy. Edw.

Zenodoxus G. \& R.

6733 maculipes $G . \& R$. 6734 heucheræ $H y$. Edw. potentillae $\mathrm{Hy}$. Edw.

6735 palmi Neum.

6736 canescens $H y . E d w$. 6737 mexicanus Beut.

Calasesia Beut.

6738 coccinea Beut.

Melittia Hbn.

6739 satyriniformis $\mathrm{Hbn}$. cucurbitae Harris. ceto Westw. amoena $\mathrm{Hy}$. Edw.

6740 snowi $H y$. $E d w$.

6741 gloriosa $H y . E d w$.

6742 grandis Stkr.

6743 magnifica Beut.

Memythrus Newm.

6744 pyramidalis $W l k$.

hylotomiformis Wlk. vancouverensis $\mathrm{Hy}$. Edw. form montana $H y$. Edw. tanaceti Hy. Edw. form rubrescens $H$ lst. form coloradensis torva Hy. Edw. Hy. Edw.

6745 fraxini $H y$. $E d w$. morrisoni Hy. Edw. 6746 beutenmülleri Skin.

Paranthrene Hbn.

6747 tricincta Harris. 
6748 denotata $H y$. Edw.

6749 robinia $H y$. Edw.

6750 cupresel Hy. Edw.

6751 polistiformis Harris.

6752 scepsiformis $\boldsymbol{H y} . \mathbf{E} d w$.

6758 aimulans Grt.

luggeri Hy. Edw.

6754 palmi $H$ y. Edw.
6755 asilipennis $\boldsymbol{B d v}$.

denudatum Harris. vespipenne H. $\mathbf{S}$. bombyciformis Wlk.

6756 seminole Neum.

6757 dolli Neum.

a castaneum Beut.

6758 perlucida Busck.

6759 admiranda $H y . E d w$.

\section{EUCOSMIDEE}

\section{Evetria Hbn.}

6760 frustrana Comst.

6761 rigidana Fern.

6762 comstockiana Fern.

6763 subcervinana Wlshm.

6764 neomexicana. Dyar.

6765 gemistrigulana Kearf.

6766 siskiyouana Kearf.

6767 monophylliana Kearf.

6768 sabiniana Kearf.

6769 zozana Kearf. matutina Meyr.

6770 colfaxiana Kearf.

6771 pasadenana Kearf.

6772 burkeana Kearf.

6773 bushnelli Busck.

6774 virginiana Busck.

6775 taxifoliella Busck.

6776 metallica Busck.

6777 montana Busck.

6778 albicapitana Busck.

Polychrosis Rag.

6779 viteana Clem. vitivorana Pack.

6780 liriodendrana Kearf.

6781 magnoliana Kearf.

6782 slingerlandana Kearf.

6783 aruneans Kearf.

6784 yaracana Kearf. signifera Meyr.

6785 carduans Busel.
6786 rhoifructana Kearf. 6787 vernoniana Kearf. 6788 ambrosiana Kearf.

Bactra Steph.

6789 lanceolana Hbn. dibeliana Hbn.

a verutana Zell.

6790 furfurana Haw.

6791 inquadrana Wlshm.

Exartema Clem.

6792 fagigemmeanum Cham.

6793 nitidanum Clem.

6794 ferrugineanum Riley.

6795 monetiferanum Riley.

6796 sericoranum Wlshm.

6797 zellerianum Fern.

6798 permundanum Clem.

meanderana WIk.

6799 olivaceanum Fern.

6800 concinnanum Clem. foedana Clem.

6801 punctanum Wlshm. 6802 atrodentanum Fern. 6803 versicoloranum Clem. appendiceum Zell.

6804 fasciatanum Clem. decisana Wik. albofasciatum Zell.

6805 corylanum Fern. 6806 exoletum Zell. 
6807 inornatanum Clem. clavana Wlk. quadrifidum Zell.

6808 malanum Fern.

6809 footianum Fern.

6810 merrickanum Kearf.

6811 ferriferanum $W l k$. gratiosana Clem. usticana Zell.

6812 hippocastanum Kearf.

Argyroploce Hbn.

Olethreutes Hbn.

(Tent. ined.)

6813 intermistana Clem. tessellana Pack.

6814 septentrionana Curt. primariana Wlk. fulvifrontana Pack.

6815 griseoalbana Wlshm. 6816 albeolana Zell.

6817 frigidana Pack.

6818 capreana $H b n$. moestana Wocke.

6819 deceptana Kearf. 6820 wellingtoniana $K e a r f$. 6821 nimbatana Clem. contrariana Wlk. a montanana Kearf.

6822 mana Kearf. thaliastis Meyr.

6823 rubipunctana Kearf. 6824 consanguinana Wlshm. 6825 separatana Kearf. dimidianat Auct. 6826 conditana Wlshm. 6827 hebesana Wlk. inexpertana Wlk. fullerea Riley.

6828 cyanana Murt.

6829 dxckeana Kearf. 6830 auricapitana Wlshm. 6831 agilana Clem. 6832 albiciliana Fern.
6833 chalybeana Wlshm. 6834 coruscana Clem. ferrolineana Wlk. argyroelana Zell.

6835 constellatana Zell. 6836 galaxana Kearf. 6837 glitranana Kearf. 6838 coronana Kearf. 6839 major Wlshm. 6840 mengelana Fern. 6841 rivulana Scop. conchana $\mathrm{Hbn}$.

6842 urticana $\mathrm{Hbn}$.

6843 astrologana Zell. 6844 puncticostana Wlk. 6845 gogana Kearf. crepuscularis Meyr. 6846 costimaculana Fern. 6847 chionosema Zell. 6848 devotana Kearf. 6849 interruptolineana Fern. 6850 osmundana Fern. 6851 ochromediana Kearf. 6852 rosaochreana Kearf. 6853 abietana Fern. 6854 malachitana Zell. 6855 roseomaculana $H$. S. lienigiana Hein.

6856 hemidesma Zell. 6857 murina Pack. 6858 melanosticta Wlshm. 6859 duplex Wlshm. 6860 removana Kearf. 6861 nubilana Clem. $a$ vetulana $W l s h m$. 6862 instrutana Clem. poana Zell. ? flavofasciana Westw.

6863 turfosana $H$. S. 6864 campestrana Zell. 6864,1 piceæ Busck. 6865 fuscalbana Zell. 6866 glaciana Moesch. 6867 dealbana Wlk. 6868 dilutifuscana Wlshm. 
6869 bipartitana Clem. cacrialbano Zell.

6870 similisans $W(k$.

6871 impudens Wlshm.

6872 inquietana W/k.

6873 niveiguttana firt. 6874 schulziana Fubr. 6875 provana Kearf.

6.876 islandana Kearf. insulicola Meyr.

\section{Phrcasiophora Grt.}

6877 confixana Wlk. perductana Wlk. mutabilana Clem.

Pseudogalleria Rag. 6878 inimicella $Z$ ell.

Eucosma Hbn.

6879 quinquemaculana $R o b$. 6880 robinsonans $\mathrm{Grt}$. quintrenu Zell. a tryonana Kearf. 6881 bolanderana Wlshm. 6882 agassizi Rob. 6883 gilletteans Dyar. 6884 adamantana $D$ yar. 6885 argenteana Wlshm. 6886 morrisoni Wlahm. 6887 ragonoti Wlshm. a barnesiana Dyar. 6888 serpentans Wlshm. 6889 fandana Kearf. argyraula Meyr.

6890 canariana Kearf. 6891 optimana Dyor. 6892 idahoana Kearf. 6893 spaldingana Kearf. 6894 ridingsana $R o b$. a argentifurcatana Grt. $b$ hipeana Grt. 6895 fernaldana Grt. 6896 crambitana Wlshm.
6897 gandana Kearf. chloroleuca Meyr.

6898 sandiego Kearf.

6899 caniceps Wlshm.

6900 immaculane Kearf.

6901 circulana $H$ br. a scintillana Clem.

6902 dodecana Zell.

6903 randann Kearf. paraglypta Meyr.

6904 sandana Kearf. gryphodes Meyr.

6905 handana Kearf. ceramitis Meyr.

6906 subflavana Wlshm. 6907 grandiflavana Wlshm. 6908 monogrammana Zell. 6909 galenapunctana Kearf. 6910 costastrigulana Kearf. 6911 kandana Kearf. argullacea Meyr.

6912 floridana Kearf.

6913 graciliana Kearf. 6914 mandana Kearf. amanda Meyr.

6915 larana Wlshm. 6916 luridana Wlshm. 6917 pergandeana Fern. a flavana Fern.

6918 argentialbana Wlshm. 6919 albiguttana Zell. 6920 pallidipalpana Kearf. 6921 atomosana Wlshm. 6922 agricolana Wlshm. 6923 basipunctana Wlshm. 6924 cataclystiana Wlk. ochreana Clem.

6925 ochraceana Fern. 6926 comatulana Zell. 6927 occipitana Zell. 6928 totana Kearf. spodias Meyr. 6929 popana Kearf. carcharias Meyr. 
6930 heathiana Kearf.

6931 rorana Kearf. sceletopa Meyr.

6932 sosana Kearf. pelina Meyr.

6933 numerosana Z ell.

6934 perdricana Wlshm.

6935 denverana Kearf.

6936 glomerana Wlshm. 6937 fulminana Wlshm.

6938 irroratana Wlshm.

6939 fraternana Busck. 6940 palousana Kearf. filiana Busck.

6941 womonana Kearf. semnitis Meyr.

6942 reversana Kearf. 6943 pandana Kearf.

6944 nandana Kearf. chersaea Meyr.

6945 rusticana Kearf. 6946 vandana Kearf. pholas Meyr.

6947 palpana Wlshm.

6948 radicana Wlshm.

6949 passerana Wlshm.

6950 wandana Kearf. eumaea Meyr.

6951 canana Wlshm.

6952 lineana Fern.

6953 culminana Wlshm.

6954 miscana Kearf. semalea Meyr.

6955 subplicana Wlshm.

6956 nigralbana Wlshm.

6957 suffusana $Z$ ell.

6958 grotiana Kearf.

6959 lolana Kearf.

leucomalla Meyr.

6960 nonana Kearf. carphologa Meyr.

6961 vovana Kearf. typicodes Meyr.

6962 matutina Grt.
6963 biquadrana Wlshm. 6964 rectiplicana Wlshm. 6965 terrococtana Wlshm. 6966 albangulana Wlshm. 6967 juncticiliana Wlshm. 6968 pulveratana Wlshm. 6969 edemoidana Dyar. 6970 abbreviatana Wlshm. 6971 constrictana Zell. 6972 solicitana Wlk. packardiana Clem. tephrinana Zell.

6973 zomonana Kearf. explosa Meyr.

6974 transmissana Wlk. 6975 boxcana Kearf. aspista Meyr.

6976 tomonana Kearf. limigena Meyr. 6977 brightonana Kearf. 6978 engelana Kearf. 6979 walkerana Kearf. 6980 abruptana Wlshm. 6981 strenuana $W l k$. exvagana Wlk. flavocellana Clem. subversana Zell.

6982 minutana Kearf. 6983 perplexana Fern. 6984 vertumnana $Z$ ell. 6985 celtisana Riley. 6986 nisella $\mathrm{Cl}$.

6987 medioviridana Kearf. 6988 pulsatillana Dyar. 6989 cercocarpana Dyar. 6990 hirsutana Wlshm. 6991 domonana Kearf. efficax Meyr.

6992 improbana Wlk. diffinana Wlk.

6993 yandana Kearf. nothrodes Meyr.

6994 zandana Kearf. peristicta Meyr. 
6995 xandana Kearf. atacta Meyr.

6996 crenana Hbn. 6997 vomonana Kearf. serangias Meyr.

6998 gomonana Kearf. discipula Meyr.

6999 trigeminana Steph. argyrana Steph. poecilana Gn.

7000 bobana Kearf. antichroma Meyr.

7001 cocana Kearf. rhodophaea Meyr.

7002 sonomana Kearf.

7003 dodana Kearf. sphilophora Meyr.

7004 fofana Kearf. annulata Meyr.

7005 hohana Kearf. syrtodes Meyr.

7006 kokana Kearf. chortaea Meyr.

7007 landana Kearf. isospora Meyr.

7008 picicolana Dyar. 7009 johnsonana Kearf. 7010 hopkinsana Kearf. 7011 fuscana Kearf. 7012 momana Kearf. metaschista Meyr.

7013 illotana Wlshm.

7014 scudderiana Clem. saligneana Clem. affusana Zell.

7015 desertana Zell. 7016 obfuscata Riley. 7017 dorsisufusana $K r a r f$. 7018 invicta Wlshm. 7019 subinvicta Kearf. 7020 carolinans Wlshm. 7021 giganteana Riley. 7022 bipunctella $W U$. worthingtoniana Fern. 7023 biplagats Wlahm.
7024 primulans Wlshm.

7025 tripartitane Zell.

7026 otiosana Clem. inclinana Zell.

7027 hamptonana Kearf.

7028 similana $\mathrm{Hbn}$.

7029 dorsisignatana Clem. similane Clem. distigmana Wlk. clavana Zell. graduatana Wlshm. form confluana Kearf. form diffusana Kearf.

7030 solandriana Linn.

7031 aspidana Wlshm.

7032 tandana Kearf. trapezitis Meyr.

7033 maculatana Wlshm.

7034 snyderana Kearf. 7035 fiskeana Kearf.

7036 sombreana Kearf.

7037 castaneana Wlshm.

7038 madderana Kearf.

7039 bilineana Kearf.

7040 corosana Wlshm.

7041 dilatana Wlshm.

7042 emaciatana Wlshm.

7043 fuscosparsa Wlahm.

7044 hyponomeutana Wlshm.

7045 shastana Wlshm.

7046 mediostriata Wlshm.

7047 smithiana Wlshm.

7048 radiatana Wlshm.

7049 essexana Kearf.

7050 umbrastriana Kearf.

7051 roseoterminana Kearf.

7052 awemeana Kearf.

7058 ferruginana Fern.

7054 formosana Clem. sagittana Whk. stercoreana Zell.

7055 aspidiscana $\mathrm{Hbn}$.

7056 corculana Zell.

7057 ochreicostana Wlshm. 7058 annetteana Kearf. 
7059 refusana Wlk. 7060 amphorana Wlshm. 7061 olivaceana Riley. 7062 imbridana Fern. 7063 ochroterminana Kearf. 7064 spiculana Zell. 7065 striatana Clem. albicepsana Wlk. trivittana Zell.

7066 tenuiana Wlshm. 7067 clavana Fern. 7068 pallidicostana Wlshm. 7069 griseocapitana Wlshm. 7070 grindeliana Busck. 7071 taleana Grt. 7072 perangustana Wlshm. 7073 minimana Wlshm. 7074 kiscana Kearf. speculigera Meyr.

7075 raracana $K$ earf. fastidiosa Meyr. 7076 stramineana Wlshm. 7077 daracana Kearf. profana Meyr. 7078 ochrocephala Wlshm. 7079 dorsiatomana Kearf. 7080 decempunctana $W l s h m$. 7081 helianthana Riley. 7082 columbiana Wlshm. 7083 scalana Wlshm. 7084 signatana Clem. variana Clem. aceriella Clem. subnisana Zell.

7085 lapidana Wlshm. 7086 sublapidana Wlshm. 7087 parvana Wlshm. 7088 succedana $D$. \& S. a gallicana $\boldsymbol{H}$. S. b conjunctana Moesch. 7089 roessleri Z ell. 7090 stygiana Dyar. 7091 vestaliana $Z$ ell. 7092 elongana Wlshm.
7093 tarandana Moesch. 7094 infuscana Wlshm. 7095 offectalis $\mathrm{Hlst}$. bucephaloides Wlshm.

7096 perstructana Wlk. 7097 artemisiana Wlshm. $a$ infimbriana Dyar. 7098 argenticostana Wlshm. 7099 apacheana Wlshm. 7100 montanana Wlshm. 7101 obliterana Wlshm. 7102 octopunctana Wlshm. 7103 transversa Wlshm. 7104 triangulana Kearf. 7105 asphodelana Kearf. 7106 granulatana Kearf. 7107 baracana Kearf. oxyleuca Meyr. 7108 caracana Kearf. famosa Meyr.

Episimus Wlshm. 7109 augmentanus Zell. 7110 argutanus Clem. allutana Zell.

Exentera Grt.

7111 apriliana Grt.

Hystricophora Wlshm.

7112 leonana Wlshm. a aurantiana Wlshm.

Proteopteryx Wlshm.

7113 emarginana Wlshm. 7114 oregonana Wlshm. 7115 cressoniana Clem. 7116 deludana Clem. 7117 haracana Kearf. resoluta Meyr.

7118 laracana Kearf. navalis Meyr.

7119 faracana Kearf. ultrix Meyx. 
7120 maracana Kearf. praeseripta Meyr.

7121 spoliana Clem.

7122 resumptana $W I k$.

7123 columbia Kearf.

a albidorsana Kearf.

$b$ mediostriana Kearf.

7124 eriddleana Kearf.

7125 willingana Kearf.

7126 bolliana Sling.

7127 momonana Kearf. sanifica Meyr.

7128 marmontana Kearf.

7129 ilicifoliana Kearf.

\section{Proteoteras Riley.}

7130 sesculana Riley.

7131 arizonae Kearf.

7132 crescentana Kearf.

7133 torontana Kearf.

7134 naracana Kearf. praesinospila Meyr.

7135 moffatiana Fern.

\section{Enarmonia IIbn.}

7136 septemberana Kearf.

7137 arctostaphylana Kearf.

7138 virginiana Clem.

7139 costomaculana Clem. bipnestulana Wlk.

7140 lindana Fern.

7141 pennsylvaniana Kearf.

7142 albicapitana Kearf.

7143 pseudotsugana Kearf.

7144 pinicolana Zell. occultana WIk.

7145 incarnana Haw.

7146 fasciolana Clem. blakeana Grt.

7147 dietziana Kearf.

7148 haimbachians Kearf.

7149 lagopans Wlehm.

7150 liturana $W l s h m$.

7151 purpuriciliana Wlshm.
7152 erispana Clem.

7158 augustana $\boldsymbol{H b n}$. direptane WIk. vilisana WIk.

7154 biangulana Wlahm.

7155 nebulosana Pack.

7156 claypoleana Riley. instrutana Clayp.

7157 ratzeburgiana Sex.

7158 pyricolana Murt.

7159 salicicolana Clem.

7160 saliciana Clem.

7161 medioplagata Wlshm.

7162 plumbolineana Kearf.

7163 piceafoliana Kearf.

7164 kennebecana Kearf.

7165 favillana Dyar.

7166 cornutana Dyar.

7167 watchungana Kearf.

7168 fortunana Kearf.

7169 normanana Kearf.

Tmetocera Led.

7170 ocellana $D$. \& $S$. pyrifoliana Clem. oculana Harris. a lariciana Hein.

Phthinolophus Dyar.

7171 indentanus Dyar.

Eudemis Hbn.

7172 vacciniana Pack.

? luctiferana Wlk.

Ancylis $\mathrm{Hbn}$.

7173 mediofasciana Clem.

7174 nubeculana Clem.

7175 subsequana Zell.

7176 discigerana $W l k$.

7177 lundana Fabr.

7178 semiovans Zell.

7179 murtfeldtiana Riley.

7180 angulifasciana Zell. 
7181 pulchellana Clem.

7182 intermediana Kearf. 7183 spireæfoliana Clem. metamelana Wlk. discoferana Wlk.

7184 maritima Dyar. 7185 comptana Froel. conflexana Wlk. fragariae Wlsh 7186 burgessiana Z Zell. 7187 dubiana Clem. 7188 amblygona Zell. 7189 floridana $Z$ ell. 7190 laciniana Z ell. 7191 platanana Clem. marcidana Zell.

7192 divisana $W l k$.

7193 apicana $W l k$.

7194 cornifoliana Riley. 7195 muricana Wlshm. 7196 uncana $\mathrm{Hbn}$. 7197 biarcuana Steph. 7198 goodelliana Fern. 7199 plagosana Clem. 7200 pacificana Wlshm. 7201 cometana Wlshm. 7202 loricana Grt. 7203 tineana $\mathrm{Hbn}$. ocellana Clem. leucophaleratana Pack. 7204 kincaidiana Fern. 7205 diminuatana Kearf. 7206 albacostana Kearf.

Laspeyresia Hbn.

Grapholitha $\ddagger$ Auct.

7207 earyana Fitch. caryae Shimer.

7208 prunivora Wlsh. 7209 shawiana Kearf. 7210 interstinctana Clem. scitana Wlk. distema Grt. 7211 maculana Fern.
7212 vancouverana Kearf. 7213 fana Kearf. oenochroa Meyr. 7214 angleseana Kearf. 7215 saundersana Kearf. 7216 eclipsana Zell. 7217 dyarana Kearf. 7218 edwardsiana Kearf. \& Riley. 7219 lana Kearf. chrysotypa Meyr.

7220 tristrigana Clem. 7221 placerana Kearf. 7222 bracteatana Fern. 7223 conversana Wlshm. 7224 albimaculana Fern. 7224, 1 mathesoni Busck. 7225 Iunatana Wlshm. 7226 wana Kearf. cupida Meyr.

7227 vana Kearf. eoleuca Meyr.

7228 zana Kearf. xanthospora Meyr.

7229 cæruleana Wlshm. 7230 vitrana Wlshm. 7231 lautana Clem. perfiuana Zell.

7232 youngana Kearf. 7233 dana Kearf. aequorea Meyr.

7234 dandana Kearf. ratifera Meyr.

7235 nigricana Steph. 7236 gallæsaliciana Riley. 7236, 1 populana Busck. 7237 trossulana Wlshm. 7238 packardi Z ell. 7239 parmatana Clem. 7240 duodecemstriata Wlshm. 7241 sebastianiæ Riley. 7242 larimana Wlshm. 7243 cockleana Kearf. 7244 americana Wlshm. 7245 walsinghami Kearf. 
7246 fletcherana Kearf.

7247 prosperana Kearf.

7248 tana Kearf. cirrhas Meyr.

7248, 1 leucobasis Busck.

7249 garacana Kearf. sppticoln Meyr.

7250 multilineana Kearf. 7251 articulatana Kearf.

7251, 1 laricana Busck.

Gymnandrosoma Dyar.

7252 punctidiscanum Dyar.

Ecdytolopha Zell.

7253 insiticiana Zell.

Hemimene Hbn.

7254 incanana Clem.

7255 simulans Clem. aurisignana Zell.

7256 bittana Busck.

7257 radicicolana Wlshm.

7258 leopardana Busck.

7259 plummeriana Busck.

7260 sedatana Busck.

7261 piperana Busck.

7262 capitana Busck.

7263 britana Busck.
7264 nigromaculana Kearf.

7265 albolineana Kearf.

7266 lana Bueck. planilogua Meyr.

7267 banana Busck. sordescens Meyr.

Pammene Hbn.

7268 texanana Wlshm.

Melissopus Riley.

7269 latiferreanus Wlshm.

Carpocapsa 'Tr.

7270 pomonella Linn. a simpsoni Busck.

7271 saltitans Westw. deshaisiana Luc.

7272 toreuta Grt. 7273 ninana Riley. 7274 piperana Kearf. 7275 cupressana Kearf. 7276 inquilina Kearf. 7277 colorana Kearf.

Catastega Clem. 7278 aceriella Clem. 7279 hamameliella Clem. 7280 timidella Clem.

\section{TORTRICIDAE}

Colostathma Clem.

7281 discopunctana Clem.

Adoxophyes Meyr.

7282 furcatana Wlk.

Homona Wlk.

7283 fervidana Wlk. rileyana Grt.

7284 patulane Wlk. audaculana Busck.
Amorbia Clem.

7285 cuneana Wlshm. a adumbrana Wlshm.

7286 humerosana Clem.

Synnoma Wlshm.

7287 lynosyrana Wlshm.

Sparganothis IIbn.

7288 pettitana $R o b$. 7289 albicaudana Busek. 7290 groteana Fern. 
7291 niveana Wlshm.

7292 pulcherrimana $W$ lshm.

7293 karacana Kearf.

7294 reticulatana Clem. subauratana Wlk. directana Wlk. a mesospila Zell.

7295 ferreana Busck.

7296 diluticostana Wlshm. quercana Fern.

7297 testulana Zell.

7298 distincta Wlshm.

7299 saracana Kearf.

7300 demissana Wlshm.

7301 chambersana Kearf.

7302 cana $R o b$.

7303 irrorea $R o b$.

7304 xanthoides Wlk.

a breviornatana Clem.

7305 flavibasana Fern.

7306 violaceana $R o b$.

7307 unifasciana Clem. puritana Rob.

7308 vocaridorsana Kearf.

7309 inconditana Wlshm.

7310 rudana Wlshm.

7311 senecionana Wlshm.

7312 californiana Wlshm.

7313 tunicana Wlshm.

7314 pilleriana Schiff. luteolana $\mathrm{Hbn}$.

7315 caryæe Rob.

7316 sulfureana Clem. gratana Wlk. fulvoroseana Clem. virginiana Clem. gallivorana Clem. gracilana Wlshm.

$\boldsymbol{a}$ belfrageana Z $\mathrm{Z}$ ll.

7317 lycopodiana Kearf.

7318 taracana Kearf.

7319 yumana Kearf.

7320 striata Wlshm.

7321 bistriata Kearf.
7322 tristriata Kearf.

7323 flavedana Clem. concursana Wlk. laterana Rob.

7324 tinctana Wlk.

7325 semiustana Wlshm.

7326 labiosana Zell.

7327 rostrana Wlk.

7328 nigrocervina Wlshm.

7329 wenzelana Haim.

7330 metallicana Wlshm.

7331 exasperatana Zell.

7332 idæusalis Wlk. sentana Clem.

Pandemis Hbn.

7333 canadana Kearf.

7334 pyrusana Kearf. pyrana Meyr.

7335 albaniana Wlk. obliquana Kearf.

7336 limitata $R o b$.

7337 lamprosana $R o b$.

Chrysoxena Meyr.

7338 auriferana Busck.

Capua Steph.

7339 lentiginosana Wlshm.

Cacocia Hbn.

7340 rosana $L$.

7341 dissitana Grt.

7342 persicana Fitch. blandana Clem. fragariana Pack. conigerana Zell. ? schreberiana Steph.

7343 infumatana Zell.

7344 brauniana Kearf.

7345 obsoletana Wlk. transiturana Wlk. vesperana Clem. sanbornana Rob. 
7346 seminolana Kearf.

7347 zapulata $R o b$.

7948 fervidana Clem. paludena Rob.

7349 cerasivorana Fitch.

7350 georgiana Wlle.

7351 houstonana Grt. relana Wlshm.

7352 retiniana $W$ lahm.

7353 argyrospila $W t k$. furvana Rob. v-signatana Pack.

a vividana Dyar.

b mortuana Kearf.

7354 semiferana Wlk. Raccidana Rob.

7355 negundana $D$ yar. 7355, 1 lambertiana Busck.

7356 fractivittana Clem. frumosa Rob.

7357 melaleucana $W l k$. invexana Wlk. semifuscana Clem. ? biustulana Steph. 7358 purpurana Clem. gurgitana Rob. lintneriana Grt.

7359 magnoliana Fern. 7360 parallela $R o b$.

7361 rosaceana Harris. vicariana Wlk. gossypiana Pack. areticane Moesch.

7362 striana Fern. 7363 conflictana Wlk.

\section{Tortrix Linn.}

7364 quercifoliana Fitch.

7365 lata $R o b$. trifurculana Zell.

7366 pallorana $R o b$. 7367 clemensiana Fern. nervosana Kearf.

7368 citrana Fern.
7369 alleniana Fern.

7370 lomonana Kearf. veneratrix Meyr.

7871 triangulana Kearf.

7372 packardiana Fern.

7373 ivana Fern.

7374 peritana Clem. inconclusana Wik.

7375 baboquavariana Kearf. 7376 moeschleriana Wocke. algidana Moesch. gelidana Moesch.

7377 virescana $\mathrm{Clem}$. sescuplana Zell.

7378 glaucana Wlshm. 7379 franciseana Wlshm. 7380 cockerellana Kearf. 7381 dorsalana Dyar. 7382 fucana Wlshm. 7383 semicirculana Fern. 7384 kukakana Kearf. 7385 symphoricarpana Kearf. 7386 musculana $\mathrm{Hbn}$. 7387 grisea $R o b$. 7388 afflictana $W l k$. fuscolineana Clem.

Eulia Hbn.

7389 ministrana Linn. ferrugana Hbn. subfascianus Steph.

7390 velutinana Wlk. triferana Wlk. lutosana Clem. incertana Clem.

7391 amatana Dyar. 7392 coloradana Fern. 7393 gloverana Wlshm. 7394 pinatubana Kearf. 7395 niscana Kearf. camerata Meyr.

7396 mariana Fern. 7397 alisellana $R o b$. 7398 quadrifasciana Fern. 7399 juglandana Fern. 
Harmologa Meyr.

7400 fumiferana Clem. nigridia Rob.

Cnephasia Curt.

7401 osseana Scop. niveosana Pack.

7402 argentana $\mathrm{Cl}$. georgiella Hlst.

7403 fernaldana Wlshm. 7404 arizonana Wlshm. 7405 horariana $W l s h$. 7406 basiplagana Wlshm. 7407 indivisana $W l k$.

7407,1 oleraceana Gibson.

Argyrotoxa Steph.

7408 chioccana Kearf. chiococcana Meyr.

7409 albicomana Clem. form semipurpurana

Kearf. form dorsipurpurana form curvalana Kearf. 7410 bergmanniana Linn. rosana $\mathrm{Hbn}$.

\section{Peronea Curt.}

7411 foliana Wlshm. 7412 caudana $F a b r$. effractana $\mathrm{Hbn}$. indecorana Zett.

7413 ferrugana Schiff. gallicolana Clem. semiannula Rob.

7414 britannia Kearf.

7415 oxycoccana Pack. 7416 cervinana Fern. 7417 comandrana Fern. 7418 hypericana Ely. 7419 minuta $R o b$. malivorana Le Bar. vacciniivorana Pack. variolana Zell. form cinderella Riley.
7420 schalleriana Linn. latifasciana Haw. erutana H. S. 7421 americana Fern. 7422 trisignana $R o b$. 7423 logiana Schiff. tristana $\mathrm{Hbn}$. trigonana Steph. form viburnana Clem. form famula Zell.

7424 permutana Dup.

7425 nivisellana Wlshm.

7426 variana Fern.

7427 angusana Fern.

7428 pulverosana $W l k$. implexana Wlk.

7429 heindelana Fern.

7430 fragariana Kearf.

7431 maculidorsana Clem. inana Rob.

7432 maccana $T r$.

7433 celiana $R o b$.

7434 brewsteriana $R o b$.

7435 nigrolinea $R o b$.

senescens Zell.

7436 albilineana Kearf.

7437 fishiana Fern.

7438 ferruginiguttana Fern.

7439 hastiana Linn.

logiana Fabr.

form divisana $\mathrm{Hbn}$. form flavivittana Clem. form perspicuana $R o b$. form albistriana Haw. form ptychogrammos Zell. form hudsoniana Wlk. form caliginosans Wlk.

7440 chalybeana Fern.

7441 niveana Fabr.

7442 simpliciana Wlshm.

7443 subnivana $W l k$. deflectana Rob.

7444 boscana Fabr. cerusana $\mathrm{Hbn}$.

a parisiana $G n$. placidana Rob.

7445 peculiana Zell. 


\section{PHALONIIDEE}

Phalonia Hbn.

7446 floccosana Wik. confusana Rob.

7447 atomosana Busck.

7448 spartinana $B$. \& $M c D$.

7449 straminoides Grt.

7450 zaracana Kearf.

7451 schwarziana Busek.

7452 hospes Wlshm.

7453 seissana $W(k$.

7454 smeathmanniana $\mathrm{Fabr}$.

7455 romonana Kearf. officiose Meyr.

7456 louisiana Busck.

7457 formonana Kearf. myrinitis Meyr.

7458 kindermannana $\boldsymbol{T r}$.

7459 deutschiana Zett. fuscostrigana Clem. chalcana Pack.

7460 felix $W l s h m$.

7461 rutilana $\mathrm{Hbn}$.

7462 dorsimaculana $R o b$.

7463 angustana Clem. promiptana Rób.

7464 seriatana $Z$ ell.

7465 intactana Wlshm.

7466 angulatana $R o b$.

7467 argentilimitana $R o b$.

7468 labeculana $R o b$.

7469 parallelana Wlshm.

7470 transversana $W l s h m$.

7471 pimana Busck.

7472 lepidana Clem.

7473 maiana Kearf.

7474 hollandana Kearf.

7475 voxeana Kearf. omphacitis Meyr.

7476 viscana Kearf. peganitis Meyr.

7477 interruptofasciats $R o b$.

7478 bunteana $R o b$.

7479 aureana Busck.
7480 gunniann Busck.

7481 marloffiana Busck.

7482 lavana Busek.

7483 nonlavana Keurf.

7484 punctadiscana Kearf.

7485 toxcana Kearf. baryzela Meyr.

7486 zoxcana Kearf. telifera Meyr.

7487 œenotherana Riley.

7488 latipunctana Wlshm.

7489 dilutana Wlshm.

7490 nana $\mathrm{Haw}$.

carneana Gn. ochreoalbana Wlk.

7491 campicolana Wlshm. 7492 parvimaculana Wlghm.

7493 vachelliana Kearf.

7494 hubbardana Busck.

7495 leguminana Busck.

7496 bana Kecrf.

rhodites Meyr.

7497 glaucofuscana Zell.

7498 dorsistriatana Wlshm.

7499 edwardsiana Wlshm.

7500 fulvotinctana Wlshm.

7501 nomonana Kearf. voluntaria Meyr.

7502 plummeriana Busck.

7503 listerana Kearf.

7504 temerana Busck.

7505 cincinnatana Kearf.

7506 discana Kearf. cricota Meyr.

7507 Poxeana Kearf. liquida Meyr.

7508 aurorana Kearf.

7509 albidana $W l k$. winniana Kearf.

7510 rana Busck. funcsta Meyr.

7511 grandis Busck.

7512 yuecatana Busek. 
7513 biscana Kearf. ixeuta Meyr. a giscana Kearf. 7514 bomonana Kearf. cyamitis Meyr.

7515 ziscana Kearf. fabicola Meyr.

7516 sublepidana Kearf.

7517 carmelana Kearf. $a$ obispoana Kearf.

7518 wiscana Kearf. acropeda Meyr.

7519 elderana Kearf. helonoma Meyr.

7520 basiochreana Kearf.

7521 ednana Kearf.

7522 obliquana Kearf.

7523 hoffmanana Kearf.

Pharmacis $\mathrm{Hbn}$.

7524 sartana $\mathrm{Hbn}$.

7525 bimaculana Rob.

7526 erigeronana Riley.

7527 deceptana Busck.

7528 mexicana Busck.

7529 vitellinana $Z$ ell.

Commophila $\mathrm{Hbn}$.

7530 macrocarpana Wlshm.

7531 comes Wlshm.
7532 fuscodorsana Kearf.

7533 saxicolana Wlshm.

7534 contrastana Kearf. 7535 huachucana Kearf.

7536 umbrabasana Kearf.

Hysterosia Steph.

7537 inopiana Haw.

7538 ægrana $W l s h m$.

7539 fulviplicana Wlshm.

7540 aureoalbida Wlshm.

7541 homonana Kearf. refuga Meyr.

7542 komonana Kearf. fermentata Meyr.

7543 waracana Kearf. dicax Meyr.

7544 villana Busck. cartwrightana Kearf.

7545 merrickana Kearf.

7546 terminana Busck.

7547 birdana Busck.

7548 riscana Kearf. vincta Meyr.

7549 pecosana Kearf.

7550 baracana Busck.

7551 tiscana Kearf. vigilans Meyr.

7552 modestana Busck.

CARPOSINID正

Bondia Newm.

7553 fidelis Meyr.

7554 crescentella Wlshm.
Carposina H. S.

7555 ottawana Kearf. 7556 fernaldana Busck. 7557 comonana Kearf. euryleuca Meyr.

HELIODINID E

Cycloplasis Clem.

7558 panicifoliella Clem.
Scelorthus Busck.

7559 pisoniella Busck. 
Lamprolophus Busck.

7560 lithella Busck.

Lithariapteryx Cham.

7561 abronimella Cham.

Heliodines Staint.

7562 bella Cham.

7563 extraneella Wlshm.

7564 sexpunctella Wlshm.

7565 tripunctella Wlshm.

7566 unipunctella Wlshm.
7567 metallicella Busek.

7568 albaciliella Busck.

7569 nyetaginella Gibson.

7570 perichalca Meyr.

Schreckensteinia Hbn.

7571 felicella Wlalım.

7572 festaliella $H b n$.

7573 erythriella Clem.

Euclemensia Grt.

7574 bassettella Clem.

7575 schwarziella Busck.

\section{HELIOZELID AE}

Heliozela H. S.

7576 æsella Cham.

7577 gracilis Zell.

Antispila Hbn.

7578 ampelopsiella Cham.

7579 cornifoliella Clem.

7580 eugeniella Busck.

7581 hydrangixella Cham.

7582 isabella Clem.

7583 nyssefoliella Clem.

7584 viticordifoliella Clem.

7585 major Kearf.

7586 aurirubra Braun.
Coptodisca Wlshm.

7587 diospyriella Cham.

7588 condalire Busck.

7589 ella Cham.

7590 lucifluella Clem.

7591 juglandiella Cham.

7592 magnella Braun.

7593 ostryæfoliella Clem.

7594 saliciella Cham.

7595 splendoriferella Clem. pruniella Clem. saccatella Pack.

7596 arbutiella Busck.

\section{GLYPHIPTERYGID}

Setiostoma Zell.

7597 xanthobasis Zell.

7598 fernaldella Riley.

Thelethia Dyar.

7599 extranea $\boldsymbol{H} y . E d w$.

Tortyra Wlk.

7600 diva Riley.

7601 slossonia Fern.

7602 biferana Wlk.

dyari Busek.
Simæthis Leech.

7603 fabriciana Linn. a alpinella Busck.

Allononyma Busek. 7604 vicarialis Z Zell. betuliperda Dyar.

7605 diana $\mathrm{Hbn}$. luridana Wik.

Abrenthia Busck.

7606 cupren Busck. 
Brenthia Clem.

7607 pavonacella Clem. amphicarpeoeana Cham.

Choreutis Hbn.

7608 inflatella Clem. a virginiella Clem.

7609 dyarella Kearf.

7610 extrincicella Dyar.

7611 carduiella Kearf.

7612 busckiella Kearf.

7613 gemmalis Hlst.

7614 piperella Busck.

7615 sororculella Dyar.

7616 silphiella Grt.

7617 gnaphaliella Kearf.

7618 onustana Wlk.

ohioensis Zell.

7619 balsamorrhizella Busck.

7620 coloradella Kearf.
7621 occidentella Dyar. 7622 schausiella Busck. 7623 leucobasis Fern.

Glyphipteryx Hbn.

7624 impigritella Clem. exoptatella Cham.

7625 montisella Cham.

7626 californix Wlshm.

7627 saurodonta Meyr.

7628 quadragintapunctata Dyar.

7629 quinqueferella $W l s h m$.

7630 unifasciata Wlshm.

7631 bifasciata Wlshm.

7632 regalis Wlshm.

7633 circumscriptella Cham.

Lotisma Busek.

7634 trigonana Wlshm. kincaidiella Busck.

\section{PLUTELLIDFE}

Eucalantica Busck.

7635 polita Wlshm.

Pliniaca Busck.

7636 bakerella Busck.

7637 sparsisquamella Busck.

Euceratia Wlshm.

7638 securella Wlshm.

7639 castella Wlshm.

Aræolepia Wlshm.

7640 subfasciella $W l s h m$.

Abebæa Hbn.

7641 subsylvella Wlshm.

7642 sublucella Wlshm.

7643 querciella Busck.

7644 gerdanella Busck.

7645 delicatella Busck.

7646 cockerella Busck.
7647 nella Busck.

7648 cervella Wlshm.

7649 electropa Meyr. .

Trachoma Wall.

7650 falciferella Wlshm.

7651 walsinghamiella Busck. instabilella末 Wlshm.

7652 ordinalis Meyr.

7653 senex Wlshm.

koebelella Dyar.

Harpipteryx Hbn.

7654 dentiferella $W l s h m$.

7655 frustrella Wlshm.

7656 canariella Wlshm.

Cerostoma Latr.

7657 rubrella Dyar.

7658 undulatella Busck.

7659 oliviella Busck.

7660 arizonella Busck. 
7661 unicipunetella Busck.

7662 radiatella Don. ochrella Cham.

7668 aleutianella Beut.

7664 schwarziella Busck.

7665 Aavistrigella Buack.

7666 barberella Busck.

7667 manella Busck.

7668 striatella Busck.

7669 vintrella Busck.

7670 maculatella Busck.

7671 angelicella Busck.

7672 dorsimaculella Kearf.

Acrolepia Curt.

7678 incertella Cham.

doraimaculella Cham.
Plutella Selır.

7674 vanella Wlahm.

7675 porrectelia Linn.

vigilacella Clem.

7676 monochiora Meyr.

7677 poulella Busck.

7678 omisen Wlshm.

7679 notabilis Busck.

7680 albidorsella Wlshm.

7681 interrupta Wlahm.

7682 armoracise Busck.

7683 maculipennis Curt. cruciferarum Zell. brassicella Fitch. limbipennella Clem. mollipedella Clem. xylostella Pack. dubiosella Beut.

\section{YPONOMEUTIDFE}

Argyresthia Hbn.

7684 quadristrigeila $Z$ ell. 7685 trifascia Braun.

7686 thoracella Busck.

7687 alternatella Kearf. 7687, 1 libocedrella Busck. 7688 cupressella Wlehm. 7689 freyella Wlshm. abdominalisł Zell.

7690 quercicolella Cham. 7691 annettella Busck. 7692 calliphanes Meyr. 7693 goedartells Linn. 7694 pygmaella $\mathrm{Hbn}$. 7695 oreasella Clem. andereggiellat Zell.

7696 castancella Busek. 7697 franciscella Busek. 7698 inscriptella liusck. 7699 apicimaculella Cham. visaliella Cham.

7700 subreticulata Wlshm. 7701 deletella Z Zell. 7701, 1 eugeniella Busek.
7702 altissimella Cham.

7703 conjugelia Zell. 7704 belangerella Cham. 7705 montella Cham. 7706 plicipunctella Wlshm. 7707 rileiella Busck. 7708 pedmontella Cham. 7708, 1 furcatella Busek. 7709 bolliella Busek. 7710 mesocausta Meyr. 7711 undulatella Cham. 7712 austerella Zell. 7713 thuiella Pack. 7714 laricella Kearf. 7715 media Braun. 7716 pilatella Brantn. 7716,1 arceuthobiella Busek.

Podiasa Busck.

7717 chiococcella Busck.

Zelleria Staint.

7718 gracilariella Busck. form ribesella Busek. 
7719 haimbachi Busck.

Swammerdamia Hbn.

7720 castaneæ Busck.

Xyrosaria Meyr.

7721 celastrusella Kearf.

Eucatagma Busck.

7722 amyrisella Busck.

Yponomeuta Latr.

7723 multipunctella Clem. ordinatella Wlk.

7724 orbimaculella Cham. euonymellał Cham.

7725 atomocella Dyar. diaphorus Wlshm.

7726 semialba Meyr.

7727 leucothorax Meyr.

7728 padella Linn.
Atteva Wlk.

7729 floridana Neum. 7730 edithella Busck. 7731 aurea Fitch. compta Clem.

Lactura Wlk. Mieza Wlk.

7732 pupula $H b n$. laeta Gey. igninix Wlk. crassivenella Zell.

7733 subfervens Wlk. $a$ psammitis Z $Z$ ell.

7734 basistriga $B . \&$ McD. rhodocentra Meyr.

7735 atrolinea $B . \& M c D$.

Urodus H. S.

Trichostibas Zell.

7736 parvula $H y$. Edw.

\section{HAPLOPTILIIDE}

Haploptilia Hbn.

7752 alniella Heinr.

Coleophora Hbn. (Tent.ined.) 7753 castipennella Wlshm.

7754 albiantennaella Wild.

7737 accordella Wlshm.

7755 lentella Heinr.

7738 æneusella Cham.

7756 gaylussaciella Heinr.

7739 acutipennella $W l s h m$.

7757 cerasivorella Pack.

7740 albacostella Cham.

7741 argentialbella Cham.

7742 chambersella Dyar. artemisicolella Cham.

7743 atriplicivora Ckll.

7744 basistrigella Cham.

7745 bella Wlshm.

7746 biminimaculella Cham.

7747 bipunctella $W l s \mathrm{hm}$.

7748 bistrigella Cham.

7749 conosipennella Clem.

7750 carpinella Heinr.

7751 caryæfoliella Clem. rufoluteella Cham.

7758 cespititiella Zell. ciliaeochrella Cham.

7759 cinerella Cham.

7760 concolorella Clem.

7761 cornella Wlshm.

7762 coruscipennella Clem. auropurpurella Cham.

7763 corylifoliella Clem.

7764 cratipennella Clem. gigantella Cham.

7765 cretaticostella Clem. 7766 currucipennella Zell. 7767 discostriata Wlshm. 7768 fletcherella Fern. 
7769 fagicosticella Cham。

7770 fuscostrigella Cham. 7771 glaucella Wlshm.

7772 infuscatella Clem.

7773 inornatella Cham.

7774 irroratella Wlshm.

7775 laricella $\mathrm{Hbn}$.

7776 laticornella Clem.

7777 leucochrysella Clom.

7778 lineapulvella Cham.

7779 lynosyridella Wlshm.

7780 luteocostella Cham. argentella Cham. argentialbella Cham.

7781 malivorella Riley. multipulvella Cham.

7782 nigralineella Cham.

7783 nigrostriata Wlshm.

7784 ochrella Cham.

7785 pruniella Clem. occidentis Zell.

7786 ochrostriata Wlshm.

7787 octagonella Wlshm.

7788 ostrywe Clem.

7789 portulacæ Ckll.

7790 quadrilineella Cham.

7791 querciella Clem.

7792 rosacella Clem.

7793 rossefoliella Clem.

7794 sacramenta Heinr.

7795 shaleriella Chnm.

7796 sparsipulvella Cham.
7797 suxdicola Ckll.

7798 tenuis Wlshm.

7799 texanella Cham.

7800 tilixfoliella Clem.

7801 trilineella Cham.

7802 unicolorella Cham.

7803 veronisella Cham.

7804 viburnella Clem.

7805 viridicuprella Wlshm.

7806 viscidiflorella Wlshm.

7807 wythis Wlshm.

7808 zelleriella Cham.

7809 elæagnisella Kearf.

7810 lapidicornis Wlshm.

7811 vagans $W$ lahm.

7812 quadristrigella Busck.

7813 entoloma Busck.

7814 acamtopappi Busck.

7815 suædæ Busck.

7816 manitoba Busck.

7817 demissella Braun.

7818 umbratica Braun.

7819 atromarginata Braun.

Batrachedra Staint.

7820 preangusta Haw.

7821 salicipomonella Clem.

7822 striolata Zell. pulvella Cham. clemensella Cham.

7823 trichella Busck.

7824 placendiella Busck.

\section{ELACHISTIDE}

Elachista Tr.

7825 albapalpella Cham.

7826 bicristatella Cham. duplicatella Dyar.

7827 orestella Busck.

7828 brachyelytrifoliella Clem.

7829 concolorella Cham.

7830 cristatella Cham.

7831 illectella Clem.

7832 inornatella Cham.
7833 maculosella Clem.

7834 madarella Clem.

7835 metallifera W/shm.

7836 orichalcella Clem.

7837 parvipulvella Cham.

7838 prematurella Clem.

7839 pusilla $F$. \& $B$.

7840 staintonella Cham.

7841 texanella Cham.

7842 texanica $F$. \& $B$. 
7843 unifasciella Cham.

7844 albicapitella Engel.

7845 prælineata Braun.

Aphigalia Dyar.

7846 albella Cham.

7847 ochreomaculella Cham.

Tinagma Zell.

7848 obscurofasciella Cham. crenulellum Engel.
Eulyonetia Cham.

7849 inornatella Cham.

Cœlopoeta Wlshm.

7850 glutinosi Wls'ım.

Busckia Dyar.

7851 albella Cham.

7852 luteella Cham.

\section{TISCHERIID $\mathbb{E}$}

Tischeria Zell.

7853 sulphurea $F$. \& $B$.

7854 clemensella Cham.

bicolor F. \& B.

7855 concolor Z Zell.

7856 castaneæella Cham. castanella Wlshm.

7857 citrinipennella Clem.

7858 quercitella Clem. quercivorella Cham.

7859 badiiella Cham. purinosella Cham. albostraminea Wlshm.

7860 fuscomarginella Cham.

7861 tinctoriella Cham.

7862 helianthi $F$. \& $B$.

7863 solidaginifoliella Clem.
7864 pulvella Cham.

7865 heteroteræ $F$. \& $B$.

7866 heliopsisella Cham. nolckeni F. \& B.

7867 longeciliata $F$. \& $B$.

7868 ambrosiæella Cham. 7869 bifurcata Braun.

7870 ambigua Braun.

7871 immaculata Braun.

7872 ceanothi Wlshm.

7873 malifoliella Clem.

7874 ænea $F$. \& $B$.

7875 roseticola $F$. \& B .

Coptotriche Wlshm.

7876 zelleriella Clem. complanoides F. \& B. latipenniella Cham.

\section{GRACILARIID无}

Lithocolletis Hbn.

7877 fitchella Clem. quercifoliella Fitch. quercetorum F. \& B.

7878 leucothorax Wlshm.

7879 hageni $F . \& B$. necospinusella Cham.

7880 arbutusella Braun.

7881 insignis $W$ lshm.

7882 trinotella Braun.
7883 quercialbella Fitch. quercipulchella Cham.

7884 clemensella Cham.

7885 argentifimbriella Clem. longestriata F. \& B. fuscocostella Cham.

7886 lucidicostella Clem. 7887 albanotella Cham. subaureola F. \& B.

7888 caryæalbella Cham. 7889 olivæformis Braun. 
7890 obscuricostella Clem. virgimilla Cham.

7891 ostryweloliella Clens. mirifica $\mathbf{F}$. \& B.

7892 rileyella Cham. tenuistrigata F. \& B.

7893 kearfottella Braun.

7894 basistrigella Clem. intermedia F.\& B.

7895 obsoleta $F . \& B$.

7896 aeriferella Clem.

7897 sexnotella Cham.

7898 propinquinells Braun.

7899 cratregella Clem.

7900 malimalifoliella Braun.

901 occitanica $\boldsymbol{F}$. \& $\boldsymbol{B}$.

7902 argentinotella Clem.

7903 populiella Cham.

7904 incanella Wlshm.

7905 alni Wlshm.

alnivorella $\neq$ Cham.

7906 alnicolella Wlshm.

7907 deceptusella Cham.

7908 apicinigrella Braun.

7909 salicivorella Braun.

7910 ledella WLshm.

7911 scudderella $\vec{F}$. $\&$ B .

7912 minutella $F$. \& $B$.

7913 diaphanella $F$. \& $B$.

7914 auronitens $F$. \& $B$.

7915 robiniella Clem.

pseudacaciella Fitch.

7916 uhlerella Fitch. amorphaeella Cham. amorphae F. \& B.

7917 morrisella Fitch.

texanella Zell.

amphicarpaeella Cham.

7918 gemmea $F$. \& $B$.

7919 martiella Braun.

7920 lucetiella Clem. aenigmatella F. \& B.

7921 tiliacella Cham.

7922 symphoricarpella Cham. symphoricarpella F. \& bolliella Dyar.
7923 ostensackenella Fitch. ormatella Cham.

7924 tritanianella Cham. consimilella F. \& B.

7925 afhinis $F$. \& B.

7926 oregonensis Wlshm.

7927 mariseella Cham.

7928 Iragilella $\boldsymbol{F}$. \& $\boldsymbol{B}$.

7929 lysimachisella Cham.

7930 celtifoliella Cham.

7931 celtisella Cham. nonfasciella Cham. pusillifoliella F. \& B.

7932 salicifoliella Cham. atomariella Zell.

7933 tremuloidiella Braun.

7934 bataviella Braun.

7935 gaultheriella Wlshm.

7936 nemoris Wlshm.

7937 caryæfoliella Clem. juglandiella Clem.

7938 lentella Braun.

7939 saccharella Braun.

7940 macrocarpella $F$. \& B.

7941 cincinnatiella Cham.

7942 hamadryadella Clem. alternatella Zell.

7943 umbellularix Wlshm.

7944 agrifoliella Braun.

7945 conglomeratella Zell. bicolorella Cham. obturilobae F.\& B.

7946 ulmella Cham. modesta F. \& B.

7947 quereivorella Cham.

7948 mediodorsella Braun.

7949 australisella Cham.

7950 chambersella W/shm.

quinquenotellat Cham.

7951 cervina Wlshm.

7952 platanoidiella Braun.

7953 fletcherella Braun.

7954 arcuella Braun.

7955 betulivora Wlshm.

B. 7956 eppelsheimi $F$. \& $B$. 
7957 bethunella Cham. lebertella F. \& B.

7958 picturatella Braun.

7959 fasciella Wlshm. unifasciellał Cham.

7960 castaneæella Cham.

7961 guttifinitella Clem. toxicodendri F. \& B.

7962 obstrictella Clem. bifasciella Cham. ceriferae Wlshm.

7963 corylisella Cham. bifasciella: Wlshm.

7964 rsculisella Cham. 7965 ostryarella Cham. 7966 aceriella Clem.

7967 hamameliella Busck. 7968 tubiferella Clem. 7969 desmodiella Clem. gregariella Murt. 7970 diversella Braun.

Cremastobombycia Braun.

7971 grindeliella $\mathrm{Wlshm}$. 7972 solidaginis $F . \& B$. 7973 ambrosiella Cham. amoena F. \& B.

7974 ignota $F$. \& $B$. bostonica F. \& B. helianthisella Cham. helianthivorella Cham. elephantopodella F. \& B. actinomeridis F. \& B.

7975 verbesinella $B u s c k$.

Apophthisis Braun.

7976 pullata Braun.

Marmara Clem.

7977 arbutiella Busck. 7978 salictella Clem.

7979 guilandinæ Busck. 7980 opuntiella Busck. 7981 smilacisella Cham. 7982 pomonella Busck.
7983 serotinella Busck. 7984 auratella Braun. 7985 apocynella Braun.

Leucanthiza Clem.

7986 amphicarpeæfoliella Clem. saundersella Cham.

7987 dircella Braun.

Acrocercops Wall.

7988 pnosmodiella Busck. 7989 sebastianiella Busck. 7990 gunniella Busck. 7991 venustella Clem. eupatoriella Cham.

7992 fasciella Cham. quinquenotella Cham.

7993 albinatella Cham. 7994 interpositella $F$. \& $B$. 7995 boreasella Clem. 7996 strigosa Braun. 7997 strigifinitella Clem. duodecemlineella Cham. quercifoliella Cham.

7998 sideroxylonella Busck.

Parectopa Clem.

7999 texanella Busck.

8000 plantaginisella Cham. geiella Cham. erigeronella Cham.

8001 astericola $F$. \& $B$.

8002 lespedezæfoliella Clem. mirabilis F. \& B.

8003 robiniella Clem. 8004 thermopsella Cham. 8005 bosquella Cham. 8006 pennsylvaniella Eng. 8007 randiella Busck. 8008 quinquestrigella Cham. 8009 rhombiferella $F$. \& $B$. 8010 serotinella Ely. 8011 salicifoliella Cham. 8012 paradoxa $F$. \& B. 
Ornix $\mathrm{Tr}$.

8013 guttea Haw.

a solitariella Dietz.

8014 kalmiella Dietz.

8015 preciosella Dietz.

8016 innotata Wlshm.

5017 trepidella Clem.

8018 eratregifoliella Clem.

8019 festinella Clem.

8020 dubitella Dietz.

8021 conspicuella Dietz.

8022 arbitrella Dietz.

8023 inusitatumelis Cham.

8024 melanotella Dietz.

8025 geminatella Pack. prunivorella Cham.

8026 quadripunctella Clem.

form albifnciella Distz.

8027 vicinella Dietz.

8028 strobivorella Dietz.

8029 arbutifoliella Dietz.

8030 obliterella Dietz.

Chilocampyla Busck.

8031 dyariella Busck.

Gracilaria Haw.

8032 fulgidella Clem.

8033 elotella Busck.

8034 atomosella Zell.

8035 behrensella Cham.

S036 strictella WLk.

8037 aceriella Cham.

8038 juglandiella Cham. juglandisnigraeella

Cham.

8039 acerifoliella Cham.

8040 negundella Cham.

8041 sassafrasella Cham.

8042 rhoifoliella Cham.
8043 reticulata Braun.

8044 palustriella Braun.

8045 sauzalitmella Cham.

8046 populiella Cham.

8047 auriferella $k$. \& $B$.

8048 alnivorella Cham.

a alnicolella Chnm.

b pulchella Cham.

- sanguinella Beut.

d nigristrigella Beut. ruptistrigella Beut.

c shastella Beut.

$f$ fuscoochrella Beut.

8049 burserella Busck.

8050 obscuripennella $F$. $\&$ B .

8051 murtfeldtella Busck.

8052 blandella Clem.

8053 violacella Clem. desmodifoliella Clem.

8054 superbifrontella Clem.

8055 belfrageella Cham.

8056 zachrysa Meyr. azalene Busck.

8057 burgessiella Zell.

8058 coroniella Clem.

8059 packardella Cham. elegantella F. \& B. irornatella Cham.

8060 consimilella $F$. \& $B$.

8061 purpuriella Cham.

8062 ribesella Cham.

8063 ostryreella Cham.

8064 vacciniella $E l y$.

8065 bimaculatella Ely.

8066 cornusella Ely.

8067 glutinella Ely.

8068 flavella Ely.

8069 flavimaculella Ely.

8070 minimella Ely.

8071 fraxinella Ely.

8072 quercinigrella Ely.

\section{SCYTHRIDIDFE}

Scythris Hbn.

8073 eburnes Wlshm. arizoniella Kearf.
8074 abapennella Cham.

8075 sponsella Busck.

8076 albilineata Wlshm. 
8077 aterrimella Wlk.

8078 basilaris $\mathrm{Z}$ ell. flavifrontella Clem.

8079 impositella Z Zell. matutella Clem. monstratella Wlk. dorsipallidella Cham. buristriga Cham. brevistriga Cham. immaculatella Cham.

8080 eboracensis $Z$ ell.

8081 fuscicomella Clem. 8082 ochristriata Wlshm. 8083 perspicelella $\mathrm{Wlshm}$.
8084 pilosella $\mathbf{Z}$ ell. 8085 magnatella Busck. 8086 plausipennella Cham. 8087 suffusa Wlshm. 8088 trivinctella $\mathbf{Z}$ ell.

Epermenia Hbn.

8089 ramapoella Kearf. 8090 albapunctella Busck. 8091 canicinctella Clem. 8092 pimpinella Murt. 8093 cicutaella Kearf. 8094 imperialella Busck.

\section{LYONETIID正}

Leucoptera Hbn.

8095 erythrinella Busek.

8096 guettardella Busck:

8097 pachystimella Busck.

Corythophora Braun.

8098 aurea Braun.

Proleucoptera Busck.

8099 albella Cham.

8100 smilaciella Busck.

Philonome Cham.

8101 clemensella Cham.

Euprora Busek.

8102 argentiliniella Busck.

Caconome Dyar.

8103 fuscoscapulella Cham.

Lyonetia Hbn.

8104 alniella Cham.

8105 latistrigella Wlshm.

8106 candida Braun.

8107 speculella Clem. nidifincansella Pack. gracilella Cham. apicistrigella Cham.
8108 saliciella Busck.

Phyllocnistis Zell.

8109 ampelopsiella Cham.

8110 populiella Cham.

8111 vitifoliella Cham.

8112 vitigenella Clem.

8113 liriodendrella Clem.

8114 magnoliella Cham.

8115 liquidambarisella Cham.

8116 intermediella Busck.

8117 erechtiisella Cham. insignis F. \& B.

8118 magnatella Zell.

Metriochroa Busck.

8119 psychotriella Busck.

Bedellia Staint.

8120 somnulentella $\mathrm{Z}$ ell. staintonella Clem.

8121 minor Busck.

Bucculatrix Zell.

8122 albicapitella Cham.

8123 agnella Clem.

8124 angustata $F$. \& $B$.

8125 canadensisella Cham.

8126 capitialbella Cham.

8127 coronatella Clem. 
8128 immaculatella Chom.

8129 ivella Busck.

8130 litigiosella Zell.

8131 luteella Cham.

8132 magnella Cham.

81 sิ niveella Cham.

8134 packardella Cham.

8135 pomifoliella Clem. pomonella Pack. curvilineatella Pack.

8136 quinquenotella Cham.

8137 rileyi $F . \& B$.

8138 staintonella Cham. albellat Cham.

8139 trifasciella Clem. obscurofasciella Cham.
8140 ambrosiafoliella Cham.

8141 ainsliella Murt.

8142 ochristrigella Braun.

8143 abaciliella Braun.

8144 tetrella Braun.

8145 variabilis Braun.

8146 transversata Braun.

8147 sporobolella Busck.

8148 koebelella Busck.

8149 albertiella Busck.

8150 eurotiella Wlshm.

8151 thurberiella Busck.

8152 ilecella Busck.

8153 crescentella Braun.

\section{ACROLOPHIDAE}

Acrolophus Poey.

8154 fureatus Wlshm.

8155 persimplex Dyar.

8156 leopardus Busel.

8157 punctellus Busck.

8158 diversus Busck.

8159 antonellus $B$. \& $M c D$.

8160 cressoni Wlshm.

8161 flavicomus Busek.

8162 hirsutus Busck.

8163 pyramellus $B$. \& $M c D$.

8164 maculifer $W / s h m$.

8165 occidens Busck.

8166 kearfotti $D$ yar.

8167 cockerelli $D$ yar.

8168 griseus Wlshm.

8169 mortipennellus Grt.

quadripunetellus Dyar.

8170 plumifrontellus Clem. bombycina Zell.

8171 eervinus $W / s h m$.

angustipennellus Beut.

8172 texanellus Cham.

8173 hulstellus Beut.

8174 arizonellus Wlshm.
8175 simulatus Wlshm. 8176 laticapitanus Wlshm. 8177 coloradellus $W$ lshm.

8178 unistriganus Dyar.

8179 barnesi Dyar.

8180 morrisoni Wlshm.

8181 propinquus Wlshm.

8182 confusellus Dyar.

8183 busckellus Haim.

8184 popeanellus Clem. scardina Zell.

8185 agrotipennellus Grt.

8186 tenuis $W l s h m$. violaceellus Beut.

8187 macrogaster Wlshm.

8188 variabilis Wlshm.

8189 piger $D$ yar.

8190 filicicornis Wlshm. mexicanellus Beut.

8191 dorsimacula $D$ yar.

8192 arcanellus Clem.

8193 davisellus Beut. a minor Dyar.

8194 quadrellus $B$. \& $M c D$. 8195 mora Grt. 


\section{TINEID平}

Amydria Clem.

8196 brevipennella Dietz.

8197 effrenatella Clem. 8198 coloradella Dietz.

8199 arizonella Dietz.

8200 apachella Dietz.

8201 curvistrigella Dietz.

8202 pandurella Dietz.

8203 confusella Dictz.

8204 clemensella Cham.

8205 onagella Dietz.

$a$ occidentella Dietz.

8206 obliquella Dietz.

8207 dyarella Dietz.

8208 crescentella Kearf.

8209 margorieella Dietz.

Hypoplesia Busck.

Paraplesiat Dietz.

8210 busckiella Dietz.

8211 dietziella Busck.

Paraneura Dietz.

8212 simulella Dietz.

8213 ehrhornella Dietz.

8214 cruciferella Dietz.

Setomorpha Zell.

8215 insectella $F a b r$. operosella Zell. inamoenella Zell. ruderella Zell. multimaculella Cham. majorella Dietz. transversestrigella Dietz. fractiliniella Dietz.

8216 sigmoidella Dietz.

Epilegis Dietz.

8217 cariosella Dietz.

Apreta Dietz.

8218 paradoxella Dietz.
Epichæta Dietz.

8219 nepotella Dietz.

Dorata Busck.

8220 lineata Wlshm. virgatella Busck.

8221 medioliniella Kearf. 8222 inornatella Busck.

Scardia Tr.

8223 anatomella Grt. 8224 coloradella Dietz. 8225 fiskeella Busck. 8226 gracilis Wlshm. 8227 fuscofasciella Cham. 8228 pravatella Busck. 8229 caryophyllella Busck. 8230 burkerella Busck. 8231 approximatella Dietz. 8232 errandella Busck.

Dyotopasta Busck. 8233 yumaella Kearf. angustella Wlshm.

Xylestia Clem.

8234 pruniramiella Clem. congeminatella Zell. clemensella Cham. kearfottella Dietz.

Dietzia Busck.

Abacobia Dietz.

8235 martinella Wlk. afflictella Wlk. carbonella Dietz.

Kearfottia Fern. 8236 albifasciella Fern.

Monopis $\mathrm{Hbn}$.

8237 rusticella $\mathrm{Hbn}$.

8238 biflavimaculella Clem. $a$ insignisella $W l k$. 
8239 marginistrigella Cham. 8240 monachella $\mathrm{Hbu}$.

8241 dorsistrigella Clem. subjunctella Wik.

8242 crocicapitella Clom. 8243 irrorella Dielz.

\section{Trichophaga Rag.}

8244 tapetiella Linn.

Phryganeopsis W'shm.

8245 brunnea Wlshm.

\section{Cyane Cham.}

8246 visaliella Cham.

\section{Tinen Linn.}

8247 misella Zell.

8248 obscurostrigella Cham.

8249 fuscipunctella Haw. nubilipenuella Clem. frigidella Pack.

8250 apicimaculella Cham. 8251 orleansella Cham. 8252 straminiella Cham. 8253 bimaculella Cham. 8254 trimaculella Cham. 8255 carnariella Clem. 8256 griseella Cham. 8257 grumella Zell. 8258 pellionella Linn. 8259 misceella Cham. 8260 behrensella Cham. 8261 occidentella Cham. 8262 sparsipunctella Wlshm. 8263 tuscanella Dietz.

8264 croceoverticella Cham. 8265 thoracegtrigella Cham. 8266 vicinella Dietz. 8267 unomaculella Cham. 8268 niveocapitella Cham. 8269 leucocapitella Busck. 8270 seminolella Beut. 8271 ophrionella Dietz. 8272 xanthostictella Dietz.
8273 imitatorella Cham. 8274 mandarinella Dietz. 8275 roburella Dietz. 8276 oregonella Busck.

8277 rileyi Diots.

8278 multistriatella Dielz. 8279 molybdanella Diets. 8280 geniculatella Dietz. 8281 arcella Fabr. 8282 auropulvella Cham. 8283 acapnopennella Clem. minutipulvella Cham.

8284 defectella Zell.

8285 apicisignatella Dietz.

8286 angulifasciella Dietz.

8287 marmorella Cham.

8288 fuscomaculella Cham. 8289 granella Linn. variatella Clem. costrotristrigella Cham.

8290 cloacella Haw.

8291 interstitiella Dietz.

8292 fulvisuffusella Dietz.

8293 maculabella Cham.

8294 marginimaculella Cham. maculimarginella Cham.

8295 fuscopulvella Cham.

8296 nigroatomella Dieta.

Homostinea Dietz. 8297 curviliniella Dietz.

Tineola H. S.

8298 biselliella Hum. lanariella Clem. 8299 dietziella Kearf.

Tenaga Clem.

8300 pomiliella Clem.

Hybroma Clem.

8301 servulella Clem. auroenffusella Cham. 
Tryptodema Dietz.

8302 sepulchrella Dietz.

Mea Busck.

Progona $\ddagger$ Dietz.

8303 skinnerella Dietz.

8304 bipunctella Dietz.

8305 floridella Dietz.

Diachorisia Clem.

8306 velatella Clem.

Homosetia Clem.

8307 argentistrigella Cham.

8308 argentinotella Cham.

8309 cristatella Cham.

bifasciella Cham.

8310 obscurella Dietz.

8311 chrysoadspersella Dietz.
8312 costisignella Clem.

8313 fasciella Cham.

8314 maculatella Dietz.

8315 miscecristatella Cham. aurocristatella Cham. fuscocristatella Cham.

8316 tricingulatella Clem.

8317 ornatella Dietz.

8318 auriferella Dietz.

8319 septemstrigella Cham.

Pelates Dietz.

8320 heteropalpella Dietz.

Leucomela Dietz.

8321 miriamella Dietz.

Oenoe Cham.

8322 hybromella Cham.

\section{COSSID开}

Inguromorpha Hy. Edw.

Pomeria B. \& McD.

8323 itzalana Stkr.

8324 arcifera Dyar.

8325 basalis Wlk.

slossoni Hy. Edw.

Givira Wlk.

8326 mucida $H y$. Edw.

8327 arbeloides Dyar.

$a$ rufescens $B . \& M c D$.

8328 theodori Dyar.

8329 cornelia $N$. \& $D$.

8330 lucretia $B$. \& $M c D$.

8331 ethela $N$. \& $D$.

8332 anna Dyar.

8333 marga B. \& McD.

8334 lotta $B$. \& $M c D$.

8335 francesca $D$ yar.

8336 minuta $B . \& M c D$.

8337 cleopatra $B . \& M c D$.
Hypopta Hbn.

8338 palmata $B$. \& $M c D$.

Zeuzera Latr.

8339 pyrina $L$.

Hamilcara B. \& McD.

8340 ramuscula Dyar.

8341 atra $B$. \& $M c D$.

8342 gilensis $B . \& M c D$.

Cossula Bailey.

8343 magnifica Stkr. magnifica Bailey.

Toronia B. \& MeD.

8344 perplexa $N . \& D$.

8345 luzena Barnes.

Fania B. \& MeD.

8346 nanus Stkr. 
Comadia B. \& MeD.

8347 bertholdi Grt.

8348 edwardi N.\& D.

8349 manfredi Neum.

8350 henrici Grt.

Acossus Dyar.

8351 centerensis Lint.

8352 populi Wlk.

a angrezi Bailey.

$b$ ore Stkr.
8353 undosus Lint.

brueei Frch.

8353,1 connectus B. \& McD.

Prionoxystus Grt.

8354 robinise Peek.

8 plagiatu Wlk.

of crepera Hart.

zabolicus Stkr.

ab. \& reticulatus Lint.

ab. \& quercus Ehrm.

8355 macmurtrei Guer.

querciperda Fitch.

\section{NEPTICULID}

Nepticula v. Heyd.

8356 amelanchierella Clem.

8357 anguinella Clem.

8358 apicialbella Cham.

8359 badiocapitella Cham.

8360 belfrageella Cham.

8361 bifasciella Clem.

8362 castanexfoliella Cham.

8363 carysefoliella Clem.

8364 clemensella Cham.

8365 condaliafoliella Busck.

8366 corylifoliella Clem.

8367 cratægifoliella Clem.

8368 dallasiana $F$. \& $B$.

8369 fuscocapitella Cham.

8370 fuscotibiella Clem. cilliaefuscella Cham. furcotibiella Riley.

8371 grandisella Cham.

8372 juglandifoliella Clem.

8373 latifasciella Cham.

8374 maculosella Cham.

8375 maximella Cham.

8376 minimella Cham.

8377 myricafoliella Busck.

8378 nigriverticella Cham.

8379 nyssefoliella Cham.

8380 ostryæefoliella Clem.

8381 platanella Clem.
8382 platea Clem.

8383 pomivorella Pack.

8384 slingerlandella Kearf.

8385 populetorum $F$. \& $B$.

8386 prunifoliella Clem.

8387 pteliæella Cham.

8388 quercicastanella Cham.

8389 quercipulchrella Cham.

8390 resplendensella Cham.

8391 rosæefoliella Clem.

8392 rubifoliella Clem.

8393 saginella Clem.

8394 serotinæella Cham.

8395 thoracealbella Cham.

8396 unifasciella Cham.

8397 villosella Clem.

8398 virginiella Clem.

8399 variella Braun.

8400 ceanothi Braun.

8401 diffasciæ Braun.

8402 punctulata Braun.

8403 pallida Braun.

8404 discolorella Braun.

8405 ulmella Braun.

8406 leucostigma Braun.

8407 tiliella Braun.

8408 rhoifoliella Braun.

8409 obscurella Braun.

8410 rhamnicola Braun. rhamnella $\ddagger$ Braun. 
8411 saccharella Braun.

8412 argentifascieila Braun.

8413 trinotata Braun.

8414 flavipedella Braun.

8415 chalybeia Braun.

8416 altella Braun.

8417 opulifoliella Braun.

8418 terminella Braun.

Opostega Zell.

8419 albogaleriella Clem. 8420 nonstrigella Cham.
8421 quadristrigella Cham. accessoriella F. \& B.

Ectodemia Busck.

8422 obrutella Zell. bosquella Cham.

8423 populella Busck. 8424 castaner Busck. 8425 phleophaga Busck. 8426 heinrichi Busck.

Obrussa Braun.

8427 ochrefasciella Cham.

\section{INCURVARIIDE}

Incurvaria Haw.

8428 oregonella Wlshm.

8429 taylorella Kearf.

8430 russatella Clem.

tripunctella Wlshm.

8431 ænescens Wlshm.

8432 politella Wlshm.

8433 labradorella Clem.

8434 aureovirens Dietz.

8435 gillettella Busck.

8436 itoniella Busck.

8437 cyanella Busck.

8438 cockerelli Busck.

8439 sedella Busck.

Greya Busck.

8440 humilis Wlshm.
8441 solenobiella $W l s h m$.

8442 punctiferella Wlshm. piperella Busck.

Paraclemensia Busck.

8443 acerifoliella Fitch. iridella Cham.

Isocorypha Dietz.

8444 mediostriatella Clem. flavistrigella Wlshm. auristrigella Cham.

8445 chrysocomella Dietz.

Eudarcia Clem.

8446 simulatricella Clem.

7447 comitariella Cham.

\section{ADELIDAE}

Nemotois Hbn.

8448 bellela Wlk.

Adela Latr.

8449 ridingsella Clem. schlaegeri Zell. corruscifasciella Cham.

8450 trifasciella Cham.

8451 trigrapha Zell. fasciella Cham.

8452 septentrionella Wlshm.
8453 flammeusella Cham. lactimaculella Wlshm.

8454 purpurea Wlk. biviella Zell.

8455 singulella Wlshm. 8456 punctiferella Wlshm. 8457 bella Cham. chalybeis Zell. iochroa Zell. 8458 ruginosella W/shm. 8459 simpliciella Wlshm. 


\section{PRODOXIDES}

Prodoxus Riley.

8460 quinquepunctellus Cham. decipien Riley. paradoxica Cbam.

8461 intermedius Riley. 8462 marginatus Riley. 8463 y-inversus Riley. 8464 reticulatus Riley. 8465 coloradensis Riley. rheumapterella Dietz. form lautus Ckll. form confluens Ckll. 8466 pulverulentus Riley.
8467 sordidus Riley.

8468 cinereus Riley.

8469 znescens Riley.

8470 barberellus Busek.

Tegeticula Zell.

Promuba Riley.

8471 alba Zell. yuccesella Riley.

8472 maculata Riley. form apicella Dyar.

8473 aterrims Treal.

8474 paradoxa Riley. synthetica Riley.

\section{MICROPTERYGIDE}

\section{Mnemonica Meyr.}

8475 luteiceps Wlk.

8476 cyanosparsella Willms.

8477 auricyanea Wlshm.

8478 aurosparsella Wlshm.
8479 griseocapitella Wlshm.

Epimartyria Wlshm.

8480 pardella Wlshm.

8481 auricrinella Wlshm.

\section{HEPIALIDE}

Sthenopis Pack.

8482 argenteomaculatus Harris. argentata Pack. alni Kell.

8483 purpurascens Pack. form quadriguttatus $\mathrm{Grt}$. semiauratus N. \& D.

ab. los Stkr.

ab. perdita Dyar.

8484 thule Stkr.

8485 auratus Grt.

Hepialus Fabr.

8486 hyperboreus Moesch.

8487 pulcher Grt.

a macglashani $H y . E d w$.

8488 mathewi Hy. Edw. roseicaput N. \& D.

a confusus $H y, E d w$ 。
8489 mustelinus Pack.

labradoriensis Pack.

8490 gracilis Grt. furcatus Grt.

8491 lemberti Dyar.

8492 behrensi Stretch.

mendocinolus Behrens.

form sequoiolus Behrens.

a tacomae $H y$. Edw.

8493 montanus Stretch.

desolatus Stkr.

baroni Behrens. anceps $\mathrm{Hy}$. Edw. rectus Hy. Edw.

8494 californicus $B d v$.

8495 hectoides $B d v$.

modestus Hy. Edw. inutilis $\mathrm{Hy}$. Edw.

form lenzi Behrens. sangaris Stkr. 



\section{Species Omitted as European or Foreign}

absinthiata Clerk. (Eupithecia) adelina Staudinger (Chlosyne) albovenosa Goeze (Arsilonche) alchimiella Seopoli (Gracilaria) alniella Zeller (Lithocolletis) alpinana Treitschke (Hemimene) ancetaria Hubner (Pero) anglicella Stainton (Ornix) anguliferellum Ragonot (Homøesoma)

applana Fabricius (Agnopteryx) ardoris Hubner (Tarache) arioch Strecker (Acronycta) atropicta Hampeon (Scoparia) atropunctaria Walker (Apaecasia) basilinea Schiffermuller (Trachea) blancardella Fabricius (Lithocolletis)

boreata Hubner (Opheroptera)

botrana Schiffermuller (Polychrosis)

caesiata Schiffermuller (Entephria)

calligera Zeller (Trichostibas)

carnea Thunberg (Epipsilia)

cervinaria Blanchard (Itame)

ciliella Stainton (Depressaria)

confine $H$. S. (Eucereon)

cristatrix Guenee (Paectes)

cuculipennellum Hubner (Coriscium)

dara Kollar (Potanthus)

decorella Stephens (Mompha)

deductaria Walker (Apaecssia)

defoliaria Clerk (Erranis)

dentalis Smith (Trileuca) derosa Morrison (Caradrina)

dimidiata $H$. S. (Pheosia)

dolis Grote (Euxos)

dubitana Hubner (Phalonia)

eburneata Guenee (Eois)

electra Lintner (Thorybes)

elisata Strecker (Ortholitha)

elongella Linné (Gracilaria)

emeritella Stainton (Depressaria)

enotata Guenee (Philobia)

exanthemata Scopoli (Cabera)

expressa $H_{y}$. Edwards (Euchaetias)

extimalis Scopoli (Evergestis)

faginella Zeller (Lithocolletis)

falconipennella Hubnor (Gracilaria)

fauna Morrison (Euxoa)

ferruginella Hubner (Monopis)

fervens Walker (Caradrina)

fluctuata Linné (Petrophora)

fulla Eversmann (Oeneis)

fusea Boisduval (Porosagrotis)

gemmata Grote (Atteva)

hartmanniana Linne (Olethreutes)

heliothidata Guenee (Macaria)

inæequaliata Packard (Lobophora)

infensata Guence (Drepanodes)

intertexta Walleer (Aethaloptera)

islandica Staudinger (Euxoa)

juncealis Guenes (Oligostigma)

ladon Cramer (Cyaniris)

laidion Borkhausen (Cœnonympha)

lucetta Smith (Perigea)

lycens $G$. \& S. (Archonias)

mendica Haworth (Argyresthia) 


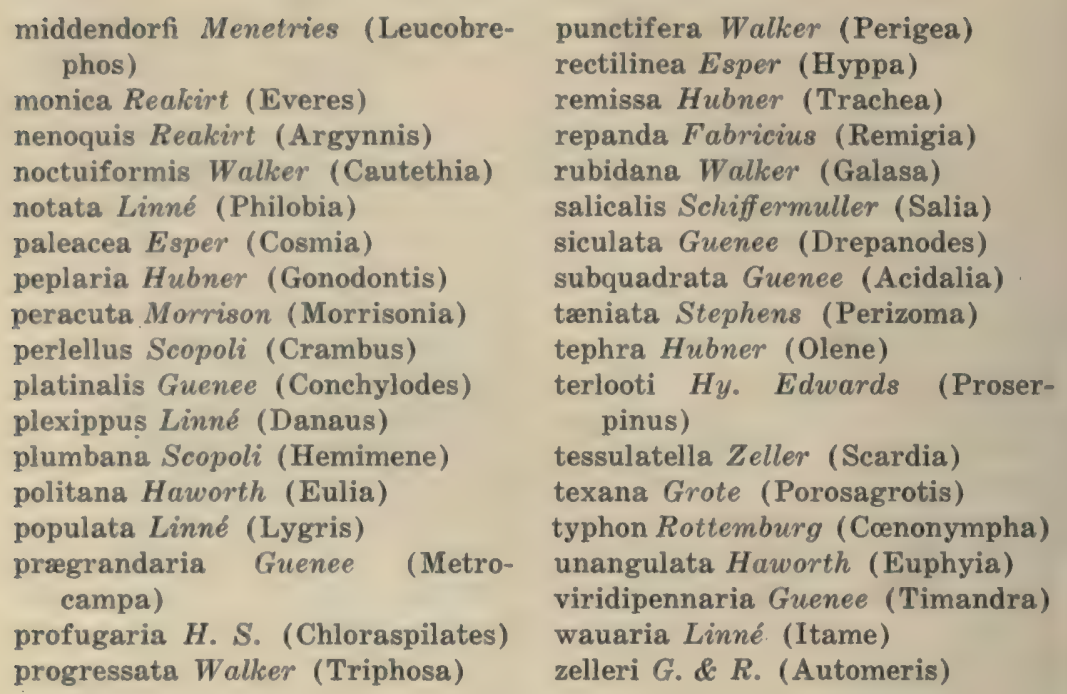




\section{N D E X}

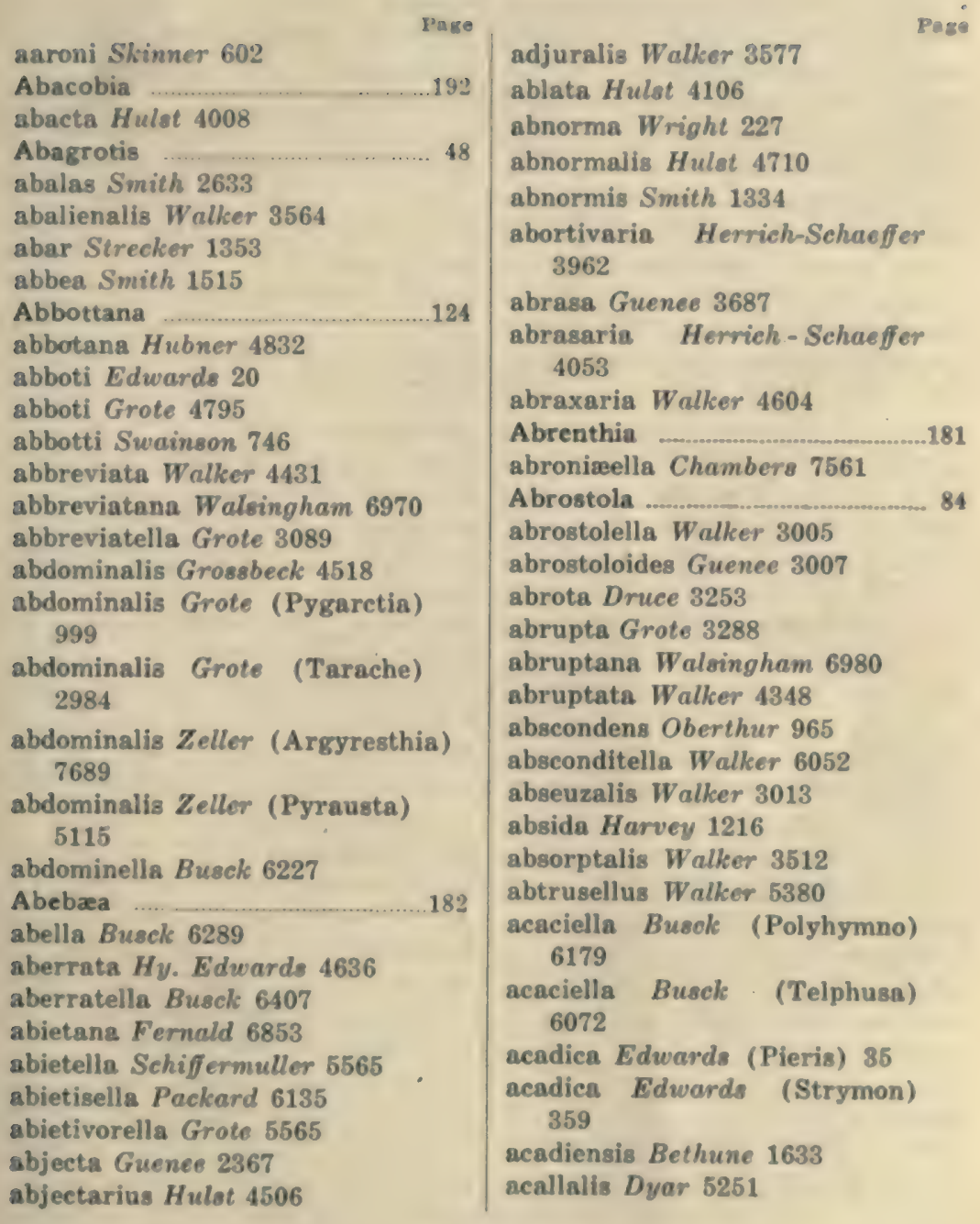




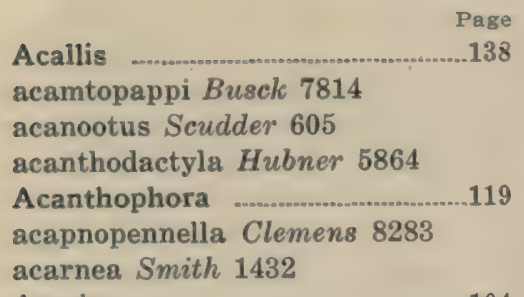

Acasis .104

acastus Edwards 224

acauda Oberthur 1

accepta $H y$. Edwards 2923

accessa Smith 1155

accessaria Hubner 4460

accessoriella Frey \& Boll 8421

accius Smith \& Abbot 642

acclivis Morrison 1384

accordella Walsingham 7737

accurata $\boldsymbol{H y}$. Edwards 2389

acera Smith 2529

acerba $\boldsymbol{H}$. Edwards 2988

acericola Guenee 2491

acericolum Germar 6685

aceriella Chambers 8037

aceriella Clemens (Catastega) 7278

aceriella Clemens (Lithocol-

letis) 7966

aceriella Clemens (Eucosma)

7084

acerifoliella Chamber 88039

acerifoliella Fitch 8443

acerni Clemens 6685

acesias Felder 1089

acestealis Walker 4956

achaia Grote \& Robinson 978

Achalarus

achatina Smith \& Abbot 3709

achatina Zeller 5323

achatinalis Zeller 3566

achatinata Hubner 3979

Achatodes

achemon Drury 738

achemonalis $B$. \& $M c D .5188$

Acherdoa acheronta Fabricius 316

achillæ $H y$. Edwards 6710

achor Strecker 1295

achriogelos Dyar 4857

Achroia 141

achsha Dyar 1901

Acidalia 101

acidaliata Packard 4056

Acidaliinæe 101

Acidaliodes

acis Drury 341

acmon Doubleday \& Hewitson 436

Acolastus 17

Acoloithus 127

acontioides Guenee 3316

Acopa 67

acornis Smith 1292

Acossus .195

acrza Drury 960

acrias Meyrick 5930

acrionalis Walker 5148

Acrobasis 143

Acrocaula 143

Acrocercops .188

Acrolepia 183

Acrolophida 191

Acrolophus 191

Acronycta 64

Acronyctina 60 acronyctoides Walker 3207

acropeda Meyrick 7518 acta Smith 2221

actinomeridis Frey \& Boll 7974 actona Smith 1392

actualis Hulst 5568

actuata Pearsall 4096

actura Smith 2090

aculeilellus Walker 5332

acuna Barnes (Isogona) 3430

acuna Barnes (Oxycnemis)

2542

acuphisalis Walker 5148

acuta Smith 2943 
acuta Walker 8140

acutangula Hampson 8009

acutangula Smith 1880

acutangulalis Snellen 4953

acutaria Herrich-Schae ff er 8151

scutella Walker 5098

acutermina Smith 1719

acultifrons Smith 1329

acutilinea Grote 1161

acutipenella Walsingham 7739

acutipennis Grote 1735

acutipennis Hulst 4225

acutissima Grote 2178

adactyla Hubner 5953

Adaina

adamantana Guenee 6884

adaptella Walker 6305

addenda Smith 1899

addendaria Grossbeck 4552

Adela

adelalis Kearfott 4916

Adelida

Adelocephala

ademptandella Dyar 5624

aden Strecker 1107

adenostomatis $\boldsymbol{H y}$. Edwards 369

adequata Pearsall 4249

aderusella Riley 6199

adiante Boisduval 177

adiaste Behr 177

adipaloides Grote \& Robinson 4985

Adita

adjuncta Boisduval 1700

adjuta Grote 1947

adjutrix Scudder 263

Admetovis

admiralis Retzins 282

admiranda $\boldsymbol{H}$ y. Edwards 6759

adnata Boisduval 941

adnixa Grote 2295

adona Strecker 3759

adonea Grote 1941

Adoneta
Adopaes

I'tasen-

adoptiva Grote 3084

adornata Prout 3857

adornata Taylor 4194

Adoxophyes 175

adrians Hy. Edwarde 8103

adulatalis Hulst 5474

adumbrana Walsingham 7285

adumbrata Behr 3330

aduncella Zeller 6199

adusta $B$. \& McD. 1354

adusta Smith 2542

adusta Walsingham 5885

adustaria Walker 4689

advena Grote 2536

advena Packard 763

adventaria Pearsall 4502

adversa Grote 3132

rdessa Druce 2830

adon Grote 2524

ægaria Strecker $\mathbf{4 4 1 5}$

Aegeria 164

Aegeriida 164

aglealis Walker 5113

ægrana Walsingham 7538

agrotata $\boldsymbol{H} y$. Edwards 3320

ægrotata Guence 4773

æhaja Behr 430

æliaria Walker 4726

Aellopos 25

smilea Skinner 484

Aemilia 32

æmula Hy. Edwards 6690

semula Hubner 3486

semulata Hulst 3854

æmulataria Walker 4381

senea Frey \& Boll. 7874

meella Hulst 5667

seneipennis Grote 1410

seneoviridella Ragonot 5662

ænescens Walsingham 8431

renescens Riley 8469

wneusella Chamber 7738 
ænigmatella Frey \& Boll $7920^{\text {Page }}$ ænus Edwards 629

æolia Druce 3432

xqualiaria Grote 4470

æqualiaria Walker 4652

requalis Walker 4893

æqualis Harvey 1238

æquepulvella Chambers 6405

aquiferaria Walker 4336

æquilinea Smith 2724

æquorea Meyrick 7233

ærata Lyman 2697

ærata Fabricius 3800

ærea Hubner 3276

æreoides Grote 3275

æria Grote 2887

æriferella Clemens 7896

æruginosa Guenee 3180

æruginosella Walsingham 8458

æsculana Riley 7130

æsculisella Cmambers 7964

æsella Chambers 7576

æsionaria Walker 4678

astiva Edwards (Phyciodes) 246

æstiva Edwards (Pieris) 35

æthalodaria Dyar 4543

Aethaloptera

ætheria B. \& McD. 1074

ætheria Grote 2905

æthra Strecker 735

Aetia .152

ætna Scudder 590

affiliata Pearsall 3924

affinata Pearsall 4144

affinis Edwards 390

affinis Frey \& Boll 7925

affinis Goeze 662

affinis Grote 906

affinis Westwood 3025

affinitella Dyar 5837

affirmaria Walker 3968

afficta Grote 2450

afflictana Walker 7388 afflictella Hulst 5626

afflictella Walker 8235

affurata Hampson 1859

affusana Zeller 7014

Afilia

afra $E d w a r d s ~ 449$

afranius Limertner 521

Afrida ........................................ 30

Agapema …................................. 27

agarista Cramer 3391

Agaristidæ

agarithe Boisduval 50

Agassizia

agassizi Packard 918

agassizi Robinson 6882

agassizi Swett 4029

agatha Beutenmuller 3068

Agathodes 130

Agdistis 151

agema Strecker 1251

Ageronia

aggressa Smith 2227

agilana Clemens 6831

agilaria Druce 3433

agilis Grote 1408

agitatellus Clemens 5355

aglæella Ragonot 5719

Aglais

Aglossa 137

agna Druce 3379

agnata Smith 1740

agnella Clemens 8123

agnesata Taylor 4232

Agnippe .155

Agnopteryx 160

agraphodactylus Walker 5906

agravens $B$. \& McD. 1905

agreasaria Walker 4689

agrestaria Grossbeck 4546

agrestis Grote 1374

Agriades 441

agricola Boisduval 574

agricola Grote \& Robinson 3135

agricolana Walsingham 6922 
agrifoliella Braun 7944

agrimoniella Clemene 6199

Agriopodes

agrippina Streeker 3038

Agroperina

agrotiformis Grote 1831

agrotina Guenee 2605

Agrotinx

agrotipennellus Grote 8185

agrotipennis Harvey $\mathbf{3 3 1 5}$

Agrotiphila

Agrotis

agua Bornes 2000

Agylla

agyllaria Walker 4644

ahaton Harris 576

aholah Strecker 3115

aholibah Streekor $\mathbf{3 0 5 0}$

ainsliella Murtfeldt 8141

ajax Linnaeus 13

akalus Strecker 1814

Alabama

alabamse Grote (Lycophotia) 1495

alabams Grote (Catocala) 3110

alabastaria Hulst 3857

alacella Clemens 6361

alameda Smith 2793

Alamosa

alamosa Burnes 1834

alaricella Busck 6123

Alarodia

alascensis Streteh 1024

alaskæ Grote 1220

alaskse Hulst 4095

alaskensis Holland (Brenthis) 194

alaskensis Holland (Erebia) 146

alaskensis Holland (Oeneis) 123

alaskensis Lehmann 197

alata Smith 2962

alatella Hulst 5512

alba $B$. \& $M c D .2810$ alba Edwarde 503

alba Grote 802

alba Harvey 1218

alba Strecker (Eurema) 78

alba Streeker (Eurymus) 65

alba Zeller 8471

albacilizella Chamber 6397

albaciliella Braun 8143

albaciliella Busek 7568

albacostana Kearfott 7206

albacostella Chamber 7740

albafascia Smith 1174

albalineella Chambers 5977

abbalis Robinson 5202

albalis Grote 1392

albangulana Walsingham 6966

albaniana Walker 7355

albanotella Chambers (Lithocolletis) 7887.

albanus Felder 16

albapalpella Chambers (Eido) 6415

albapalpella Chambers (Elachista) 7825

albapennella Chambers 8074

albapulvella Chambers 6414

albapunctella Busck 8090

albaria Grote 3796

albarufa Grote 2432

albastrigulella Kearfott 6413

albata Guenee 3756

albata Packard 892

albavena Ottolengui 2755

albavitta Ottolengui 3273

albella Chambers (Aphigalia) 7846

albella Chamber (Bucculatrix) 8138

albella Chambers (Busckia) 7851

albella Chambers (Mompha) 6003

albella Chambers (Proleucoptera) 8099 


\section{albella Chambers (Stenoma) 6613}

albellus Clemens 5362

albeolana Zeller 6816

alberta Edwards 201

alberta Elwes 126

alberta Smith 2311

alberta Taylor 4189

albertæ Dyar 939

albertiella Busck 8149

albertina Hampson 2359

albescens B. \& McD. 2335

albescens $H$ ulst 4554

albescens Tepper 688

albescentellum Ragonot 5769

albiantennaella Wild 7754

albiapicata Grossbeck 3922

albibasalis $B . \& M c D .4917$

albicans Fish 5880

albicapitana Busck 6778

albicapitata Packard 4208

albicapitella Chambers 8122

albicapitella Engel 7844

albicaudana Busck 7289

albicepsana Walker 7065

albiceralis Grote 5036

albiciliana Fernald 6832

albiciliata Walsingham 5884

albiciliatus Smith 3390

albicilla Herrich-Schaeffer 454

albicinerea Smith 1987

albicollis Oberthur 965

albicoma Hulst 6649

albicoma Strecker 3673

albicomana Clemens 7409

albicornis $\boldsymbol{H} y$. Edwards 6681

albicosta Smith 1382

albicosta Walker 1011

albicosta Hubner 3622

albicostella Beutenmuller 6640

albicostellus Fernald 5311

albida $B$. \& $M c D .3933$

albida Beutenmuller 3065

albida Smith 1988 albida Stretch 961

albida 'Walker 892

albida Walsingham 5879

albidana Walker 7509

albidella Hulst 5836

albidentina Walker 1086

albidior B.\& McD. 1875

albidorsana Kearfott 7123

albidorsella Walsingham (Platyptilia) 5875

albidorsella Walsingham (Plutella) 7680

albidula Hulst . (Ptychopoda) 3898

albidula $\boldsymbol{H}$ ulst (Stenaspilates) 4737

albidula Guenee 2895

albifaciella Dietz 8026

albifascia French 941

albifasciata Hampson 2022

albifasciata Packard 4007

albifasciella Fernald 8236

albifera Walker 4120

albifrons Smith \& Abbot 3622

albifusa Walker 1615

albifusata Walker 4290

albigenaria Walker 4580

albigrisata Pearsall 4266

albiguttana Z eller 6919

albilinea Hubner 1959

albilineana Kearfott 7436

albilineata Walsingham 8076

albilineellus Fernald 5379

albilorella Zeller 6218

albilunata Smith 2640

albimacula $B$. \& McD. 2622

albimaculana Fernald 7224

albimarginella Chambers 6102

albimargo $B . \& M c D .2965,1$

albina Grote 2266

albina Poey 81

albina Strecker 3318

albinatella Chambers 7993

albinella Cramer 5304 
albinuda Smith 1646

albipenella Hulat 5739

albipennis Grote 1341

albiplaga A aron 239

albiplaga Walker 3653

albiplagiatella Packard 5670

albipuneta $B$. \& $M$ CD. 2418

albipuncta Smith 2598

albipunctata Haworth 4137

albipunctata Packard 4846

albiserpentata Pearsall 3852

albiserrata Swith 2190

albisignalis Zeller 3571

albisinuata Smith 3552

albisinuatella Packard 5381

albisparsella Chambers 6253

albistriana Haworth 7439

albistrigalis $B$. \& $M C D .5281$

albistrigata $B$. \& $M c D .1005$

albistrigella Chambers 6401

albistrigella Walsingham 6622

albitermen $B$. \& $M c D .2949,1$

albitogata Walsingham 6617

albocapitella Chambers 6017

albocapitella Hulst 5523

alboceptata D yar 4533

albociliata Mabille 475

alboclavellus Z Zeller 5353

albocostaliata Packard 3457

albocostalis Hulst 5671

albocostella Hulst 5755

albofascia $H y$. Edwards 3680

albofasciata Bethune 3184

albofasciata Grote 3943

albofasciata Neweomb 304

albofasciatum Zeller 6804

albofaseiatus Hewitson 464

albogaleriella Clemens 8419

albogilvaria Morrison 4120

alboguttata Grote 1759

albolabes Grote 2326

albolineana Kearfott 7265

albolineate Grote \& Robinson (Adelocephals) 808
Page

albolineata Grote \& Robinson (Glaphyria) 4899

albolineata Packard (Synchlora) 3800

albolineata Packard (Diactinia) 3987

albomacularia $H y$. Edwards 4735

albomaculata Stoll 1042

albomaculella Chambera 6211

albopalpella Chambere 6004

alboplagialis Dyar 5470

alboplagiata Smith 2824

albopunctata Morrison 4465

albopunctata Tepper 3579

albopunctella Walker $\mathbf{3 1 6 6}$

albosigma Fitch 3593

albosignata Packard 4085

albostraminea Walsingham 7859

albovittata Guenee 3942

albovittella Dyar 5778

albovittella Hulst 5574

alcreus Hewitson 462

alcandola Smith 1564

Alcathoe 164

alce Edwards 423

alcesta Smith 1260

alcestis Edwards (Argynnis)

159

alcestis Edwards (Strymon)

356

alcidamus Cramer 13

alcina Skinner $\mathbf{5 7 8}$

alcionea Cramer 149

alciphearia Walker 4711

alcoolaria Guenee 4674

alcora Barnes 4799

aleada Smith 1974

alensa Smith 1167

Aleptina ....nas...... 67

Aleptinoides ..._. 73

aleptivoides $\boldsymbol{B}$. \& $\boldsymbol{M c D} .2893$

alessandra Smith 2976

alethe Neumoegen \& Dyar 3594 
aleucis Harvey 1149

aleutianella Beutenmuller 7663

alexandra Edwards 65

alexandriæella Chambers 6085

alfarata Strecker 1980

alfkeni Grote 1777

algens Grote 2126

algidana Moeschler 7376

algidata Moeschler 4047

algidella Walker 6609

algula Smith 1900

alia Guenee 2269

aliaga Barnes 2776

alialis $B . \& M c D .5246$

alias Ottolengui 3237

aliaska Scudder 9

alicia Edwards 311

aliculella Hulst 5643

alienaria Herrich-Schaeffer 4678

alienella Busck 6473

alinda Druce 796

alisellana Robinson 7397

alitalis Hulst 4979

alko Strecker 1294

allectalis Grote 5027

allecto Smith 2286

allegheniensis Holland 865

allenella Walsingham 6489

alleni Fernald 5440

alleni Grote 3333

alleniana Fernald 7369

allionealis Walker 5199

Allononyma

Allotria

allusa Hulst 3059

allutana Z eller $\mathbf{7 1 1 0}$

alma Strecker 236

Almodes

alni $H y$. Edwards 918

alni Kellicott 8482

alni Walsingham 7905

alnicolella Chambers 8048

alnicolella Walsingham 7906

alniella Chambers 8104 alniella Heinrich $\mathbf{7 7 5 2}$

alnifructella Busck 6152

alnivorella Chambers (Lithocolletis) 7905

alnivorella Chambers (Gracilaria) 8048

alope Fabricius 111

alope Drury 713

Alpheias 142

Alpheioides 142

alpheus Edwards 509

alpina Quensel 943

alpinata Packard 3945

alpinella Busck 7603

also Moeschler 133

Alsophila 99

altaurus Dyar 24

altella Braun 8416

altensis Wocke 5587

altera Ottolengui 3244

altera Smith 2938

alterna Strecker 3274

alternans Walker 1893

alternaria Grote 4286

alternata Grote \& Robinson 635

alternata Grote $\mathbf{1 5 8 8}$

alternata Muller 4092

alternata Walker 4054

alternatella Kearfott (Gelechia) 6293

alternatella Kearfott (Argyresthia) 7687

alternatella Zeller 7942

alternosquamella Ragonot 5582

alticola $B . \& M c D .189$

alticola Smith (Eremobia) 2362

alticola Smith (Euxoa) 1273

alticola Hulst 4497

alticola Walker 3222

alticolalis B.\& McD. 5077

alticolalis Dyar 5296

alticolata B. \& McD. 4038, 1

altissimella Chambers 7702

altruaria Pearsall 4669 
altua Smith 1755

altura Barnes 2707

Alucita

alunats Skinner \& Aaron 4

alurina Smith 1918

alutalis Grote $\$ 523$

alvinalis Guenee 4959

Alypia

Alypiodes 37

amabilis. Moeschler 4946

amadis Horrich-Schaeffer 579

amado Barnes 3401

amanda Meyrick 6914

amanda Smith 2141

amarella Dyar 5800

amaryllis Fabricius 29

amaryllis Smith $\mathbf{1 0 5 5}$

amasia Smith \& Abbot 3118

amatalis Walker 5011

amatana Dyar 7391

amatella Hulst 5571

amatrix Hubner 3083

amaturaria Walker 3913

Ambesa 144

Ambia 136

ambigua Braun 7870

ambigua Strecker 928

ambigualis Walker 3550

ambisimilis Dyar 3749

amblygona Zeller 7188

Amblyscirtes

ambrosise Murtfeldt 5928

ambrosixella Chambers (Gelechia) 6306

ambrosisella Chambera (Tischeria) 7868

ambrosixfoliella Chambers 8140

ambrosiana Kearfott 6788

ambrosiella Chambers 7973

amelaina Dyar 950

amelanchierella Clemens 8356

ameliata Swett $\mathbf{4 0 2 9}$

amells Guenee $\$ 167$ americalis Guonec 8485

americana Beutenmuller 6682

americana D'Urban 410

americana Fabriciue 8745

americana Fornald $\mathbf{7 4 2 1}$

americana Guevin 4881

americana Harris (Acronycta)

2491

americana Harris (Aretia)

1026

americana Harria (Epicnaptera) 3754

americana Harris (Ichythura) 3591

americana Harvey 3612

americana Herrich-Schaeffer 3757

americana Morrison 2228

americana Smith 1779

americana Speyer 2638

americana Strand 196

americans Walsingham 7244

americella Walsingham 5955

americus Kollar 4

amestris Strecker 3092

amethystaria Strecker 4685

Ametris

amia Dyor 1454

Amiana 75

amica Edwards 431

amics Harris 2268

amica Hubner 3120

amicaria Herrich-Schaeffor 4678

amicora Smith 2476

amicula $\boldsymbol{H y}$. Edwards 2850

amiculatalis Berg $\mathbf{5 1 0 3}$

amissella Busck 6460

amisus Hewitson $\mathbf{4 5 9}$

amitaria Guenee 4288

Ammalo 31

ammon Lucas 465

ammoni Behr 14

amcena Frey \& Boll 7973

amona $H_{y}$. Edwards 6739 
amœenaria Guenee 4704

Amolita

amorata Barnes 2733

amorata Hulst 4042

amorata Packard $\mathbf{4 7 8 8}$

Amorbia

amorphæ Frey \& Boll 7916

amorphæ Hy. Edwards 54

amorphæella Chambers (Gelechia) 6307

amorphæella Chambers (Lithocolletis) 7916

amorphella Clemens 5985

Ampelœca

ampelopsiella Chambers (Antispila) 7578

ampelopsiella Chambers (Phyllocnistis) 8109

ampelos Edwards 104

amphicarpæella Chambers 7917

amphicarpeoeana Chambers 7607

amphicarpeæfoliella Clemens 7986

Amphidasis

amphidusa Boisduval 59

Amphion 26

Amphipyra

amphipyroides Guenee 3393

amphorana Walsingham 7060

ampla Dietz 6577

ampla Grote 3724

ampla Walker 3274

amplaria Walker 4607

amplexella Ragonot 5549

ampliata Menetries 4

amplicineraria Pearsall 4776

amplissima Walker 3127

amplus $H y$. Edwards 2842

amputatrix Fitch 2268

Amydria

amygdalina Harvey 1956

amymone Menetries 266

Amyna 75

72
Amynthia

Page

amyntor Hubner 674

amyntula Boisduval 425

amyrisaria Walker 4689

amyrisella Busck (Agnopteryx) 6446

amyrisella Busck (Eucatagma) 7722

Anacampsis 156

anacosta Smith 1240

Anæa

anæa Druce 2880

Anagoga 120

Anania .129

ananius Ploetz 484

anargyrea Dyar 2679

Anarsia 159

anarsiella Chambers 6247

Anarta

anartalis Grote 5040

Anartia

anastomosaria Grossbeck 4555 .

anataria Swett 4411

anatomella Grote 8223

ancellata Hulst 3864

anceps Grote 5391

anceps $H y$. Edwards 8493

anchemolus Cramer 736

anchocelioides Guenee 1589

ancilla Smith 2155

ancocisconensis Morrison 2531

Ancylis .173

Ancyloxypha 20

andera Smith 1254

andersoni Dyar 188

andersoni Swett 4405, 1

andremona Cramer 3347

andrena Smith 2630

andria Scudder 317

Androloma 36

andromacha Hubner 90

andromache $\boldsymbol{H} y$. Edwards 3085

andromedæ Guenee 3095

androphila Guenee 3120 
Andropolia
Anepischetos
Anerastia
Anerastiine

anfracta $H y$. Edwards 2878

anfractata Hulst 4775

angelica Grote 8728

angelica Smith (Cobaliodes) 2387

angelica Smith (Copibryophila) 2555

angelica Smith (Papaipema) 2675

angelica Smith (Microhelia) 2838

angelicella Busck 7671

angelina Boisduval 41

angelus Dyar 883

angleseana Kearfott $\mathbf{7 2 1 4}$

angrezi Bailey 8352

anguilineata Grote \& Robinson 3952

anguina Grote 1732

anguina Smith \& Abbot 3626

anguinella Clemens 8357

angulalis Hubner 3553

angularia Grossbeck 4714

angulata Smith 1925

angulata Warren 4447

angulata Grote 2655

angulatalis Lederer 5217

angulatana Robinson $\mathbf{7 4 6 6}$

angulella Dyar 5738

angulella $E$ ly 5788

angulidens Smith 3243

angulifasciana Zeller $\mathbf{7 1 8 0}$

angulifasciella Dietz 8286

angulifera Walker (Callosamia) 772

angulifera Walker (Thauma) 781

angulifera Walker (Halisidota) 918

angulirena Smith 1241 angulosa Smith \& Abbot 3618 anguasna Fernald 7427

angurella Grote 5532

angusi Grote 3034

angusi Grote \& Robinson 8596

angustalis Felder 5104

angustana Clemens 7463

angustata Frey \& Boll 8124

angustella Wolsingham 8233

angustellus Blanchard 5657

angustifascia $B$. $M c D .306$

angustiora $\mathrm{B} . \& \mathrm{MeD} .3678$

angustiorata Grote 3444

angustiorata Walker $\mathbf{4 4 6 7}$

angustipennella Clemens 6057

angustipennella Dietz 6585

angustipennellus Beutenmuller

8171

angustipennis Warren 5293

angustipennis Grote 2971

angustus Walsingham 5910

anicia Doubleday \& Hewitson 219

anilis Drury 3164

aniluna Smith 2975

animata Pearsall 3941

Animomyia 96

animosa $H y . E d w a r d s ~ 6714$

Anisota 28

anita Reiff 3752

aniusaria Walker 4399

anna Dyar 8332

anna Edwards 428

anna Grote (Apantesis) 979

anna Grote (Catocala) 3092

Annaphila

annaphilalis Grote 6039

annectella Dietz 6569

annellata Hulst 3927

Annemoria 100

annetta Mead 426

annetteans Kearfott 7058

annettearia Haimbach 3912

annettella Busek 7691 
annexa $\boldsymbol{H y}$. Edwards 3128

annexa Treitschke 1413

anniculalis Hulst 5260

annida Fager 3045

annir Strecker 1323

annisaria Walker 4399

annulalis Grote 3575

annulata Hulst 4207

annulata Meyrick 7004

annulatella Zetterstedt 5587

annulifascia Walker 915

annuliferella Dyar 5516

annulimacula Smith 1909

annulipes Dietz 6565

annulipes Smith 1324

annulosa Walker 925

annulosella Ragonot 5623

annulosum Swainson 731

anoa Dyar 2787

anodonta Guenee 2386

anomalella Dietz 6576

Anomis

Anomogyna

anona Ottolengui 775

anormalis $B$. \& $M c D .3442$

anormalis Guenee 4959

Anorthosia

anotha Dyar 2588

antægon Boisduval 436

antreus Drury 663

antapica Smith 2219

Antaplaga

antaurata Walker 4343

anteliella Busck 6029

antennata $B . \& M c D .1824$

antennata Smith (Afilia) 3679

antennata Smith (Septis) 2254

antennata Walker 2153

anteposita Guenee 1413

anteroclara Smith 1942

Anthanassa

Anthelia

anthocioides Grote \& Robinson 5277
Page

Anthocharis 3 anthyale Hubner 60 antiacis Boisduval 450

Antiblemma 86

antibubastus Hubner 420

antica Smith 1786

antica Walker (Ammalo) 905

antica Walker (Euchaetias) 1010

anticaria Walker (Ptychopoda) 3901

anticaria Walker (Eupithecia) 4276

anticaria Walker (Aethaloptera) 4601

Anticarsia

antichroma Meyrick 7000

anticostalis Grote 4940

anticostiata Strecker 4041

anticostiensis Grote 3693

anticostiensis Strecker 4

antidectis Meyrick 6496

antidiscaria Walker 4693

Antigastra .132

antigone Strecker 963

antilochus Linnaeus 13

antinympha $\mathrm{Hubner} 3025$

antiopa Linnaeus 281

antiphola Walsh 921

antipoda Strecker 1975

antiqua Linnaeus 3701

Antispila .181

antonellus $B$. \& $M c D .8159$

antonia Edwards 311

antonio Smith 1062

antonita Dyar 2908

antonito Barnes 1547

anxia Smith 2862

Anytus 47

Aon 77 aon Druce 3551

aonides Strecker 3665

apache Poling 3122

apacheana Walsingham 7099 
apachella Diet: 8200

A paccasia

apama Educerds 392

Apamea

apameoides (inenee 2322

apamiformis Guenee 2262

Apantesis

apapinaria Dyar 4740

A patelodes

apera Druce 848

apertalis Wulker 5022

Apharetra

aphidiella Walsingham 6532

Aphigalia

aphirape Linnaeus 189

Aphomia

aphrodite Fabricius 159

aphroditella Chambers 6399

aphyla Hampson 840

apiata Grote 2207

apicalis Grote 1383

apicalis Grote \& Robinson 3659

apicalis Guenee 3125

apicalis Smith 3421

apicalis Walker 3589

apicana Walker 7193

apicata $B$. \& $M c D .3461,1$

apicata Smith 1880

apicella Dyar 8472

apicella Grote 2923

Apicia

apicialbella Chambers 8358

apiciaria Packard 4686

spicilinella Clemens 6185

apicimaculella Chambers (Tinea) 8250

apicimaculella Chambera (Argyresthia) 7699

apicinigrella Braun 7908 apicipunctella Chambers 6641 apicisignatella Dietz 8285 apicistrigella Chambers (Aproserema) 6185 apicistrigella Chambers (Lyonetia) 8107

apicitripunctella Clemens 6135

apicosa Haworth 2901

A picrena 122

apiformis Linnaeus 6652

aplastella Hulot 5491

Aplectoides

aplicalis Guenee 4952

apocynella Braun 7985

Apodemia

Apolema ……‥ 114

Apophthisis ………….................188

appassionata Harvey 2659

appellaria Pearsall 3824

appendiceum Zeller 6803

Appias

apposita Grote 1558

apposita Smith 2181

approximaria Dyar 4670

approximaria Hubner 4494

approximaria Packard (Exelis)

4510

approximaria Packard (Nemoria) 3783

approximata Strecker (Lygranthoecia) 1123

approximata Strecker (Melitrea) 241

approximata Stretch 973

approximatella Dietz 8231

approximella Walker 5835

Apreta

aprica Hubner 2983

apricatus Stichel 25

apriliana Grote 7111

Aprozerema

aptalis Lederer 5199

Apterona 125

apurpura $B$. \& $M c D .1665$

apyrella Dyar 6777

aqualis B. \& MeD. 4983

aqualis Grote 2064

aquamarina Felder 3129 
aquila Meyrick 5921

aquilellus Clemens 5312

aquilo Boisduval 429

aquilonaris Lintner 3673

aquosus Grote \& Robinson 4755

ar Strecker 1183

arabus Edwards 644

arachne Edwards 240

Arachnis

aracinthusalis Walker 3553

Arzolepia

aratrix Harvey 1544

araxes Hewitson 453

arbeloides Dyar 8328

arbitrella Dietz 8022

arbora $B . \& M c D .2336$

arbutiella Busck (Coptodisca) 7596

arbutiella Busck (Marmara) 7977

arbutifoliella Dietz 8029

arbutusella Braun $\mathbf{7 8 8 0}$

arcanellus Clemens 8192

arcasaria Walker 4763

arcella Fabricius 8281

arceuthobiella Busck 7716,1

Archanara

archasialis Walker 4955

archesilea Felder 259

archippus Cramer 308

archippus Fabricius 84

arcifera Dyar 8324

arcigera Guenee 1192

Arcobara

Arctia

arctica Bentenmuller 6663

arctica Boisduval 2268

arctica Moeschler 3240

arctica Ottolengui 3241

arctica Verity 35

arctica Zetterstedt (A plectoides) 1505

arctica Zetterstedt (Brenthis)

193 arcticana Moeschler 7361

arcticaria Walker 3862

arcticus Skinner 13

Arctiidæ

Arctinæ

arctivorens Hampson 2671

Arctonotus 26

arctostaphylana Kearfott 7137

arctostaphylella Walsingham 6627

arcuata Walker (Drepana) 3760

arcuata Walker (Trachea) 2283

arcuella Braun 7954

ardea $\boldsymbol{E}$ dwards 433

ardiferella Hulst 5813

areataria Broadwell 4567

arefacta $H y$. Edwards 1164

arefactaria Grote \& Robinson 4704

areli Strecker 2982

areloides $B . \& M c D .2981$

arene Edwards 546

arenella Schiffermuller 6466

areolatus Smith \& Abbot 94

ares $\boldsymbol{E} d w a r d s 325$

ares $F$ elder 648

Aresia

aretaria Walker 4469

arethusa $\operatorname{Dod} 410$

argalis Fernald 5055

argante Fabricius 49

arge Drury 977

arge Strecker 184

argentana Clerck 7402

argentana Martyn 5420

argentata $B . \& M c D .2113$

argentata Drury 4782

argentata Emmons 5419

argentata Fletcher 452

argentata Packard (Halisidota)

913

argentata Packard (Sthenopis)

8482

argentatus Wetherby 4816 
argenteana Walsingham 6885 srgentella Chamber 7780 argenteomaculatus Harris 8482 argenteonervella Hulat 5416 argentialbana Walsingham 6918 argentialbelia Chambere (Gelechia) 6308

argentialbella Chambers (Haploptilia) $\mathbf{7 7 4 1}$

argentialbelln Chambers (Haploptilia) $\mathbf{7 7 8 0}$

argenticinctella Clemens 6490 argenticostana Walsingham 7098

argentifascia $B$. \& $M c D .1171$ argentifasciella Braun 8412 argentifera Busck 6050 argentifimbriella Clemens $\mathbf{7 8 8 5}$ argentifurcatana Grote 6894 argentilimitana Robinson $\mathbf{7 4 6 7}$ argentilinen Druce $\$ 647$ argentilinea Walker 2393 argentiliniella Busck 8102 argentimacula B. \& McD. 4837 argentimaculella Murtfeldt 5997 argentinotella Chambers 8308 argentinotella Clemens 7902 argentipunctella Ely 6242 argentistriata Strecker $\mathbf{4 7 8 2}$ argentistrigella Chambers 8307 argillacea Hubner 3406 argillacea Packard 855 argillacea Rothschild 910 argillacea Wolsingham 6459 argillaceella Paokard 5331 argillaria Hulat 4697

Argillophora 75 argus Neumoegen \& Dyar 780 argus Smith 1885 arguta Lederer 4976 argutanus Clemens $\mathbf{7 1 1 0}$ argyllaceu Meyriek 6911 argyllacearia Packard $\mathbf{4 4 0 6}$
Prese

Argynnis

argyralis Hubner 4933

argyrana Stephene 6999

argyraula Meyrick 6889

argyreelln Dietz 6604

Argyresthia 183

Argyria 140)

argyroelana Zeller 6834

Argyroploce 168

argyrospila Walker 7353

agryrosplendella Dietz 6529

Argyrostrotis 82

argyrothamniella Busek 6192

Argyrotoxa 178

argyrotoxus Behr 428

ariadne $B . \& M c D .735$

ariadne Edwards 59

ariane Boisduval 111

aribac Barnes 2006

aricye Cramer 48

arida Cockerell 1397

arida Skinner 873

arida Smith 2986

aridalis B. \& McD. 5002

aridella Dyar 5679

arietis Grote 1655

aristella Busck 6226

aristodemus Esper 12

Aristotelia 154

arivaca Barnes 2749

arizona French 2750

arizons Smith 2007

arizonæ Beutenmuller 6670

arizona $\boldsymbol{H y}$. Edwards 2946

arizonæ French 3586

arizone $G . \&$ S. 453

arizone Grote 3075

arizone Kearfott 7131

arizonana Walsingham $\mathbf{7 4 0 4}$

arizonaria Grote (Nemoria) 3788

arizonaria Grote (Chloraspilates) 4801
Inase 7 
arizonaria $H y$. Edwards 4735

arizonata Grote 3946

arizonata Taylor 4768

arizonella Busck (Cerostoma)

7660

arizonella Busck (Gelechia)

6205

arizonella Dietz (Amydria)

8199

arizonella Dietz (Pigritia) 6587

arizonella Hulst 5742

arizonella Ragonot 5829

arizonellus Walsingham 8174

arizonensis $B . \& M c D .3689$

arizonensis Beutenmuller 6731, 1

arizonensis Edwards (Basilarchia) 304

arizonensis $E d w a r d s$ (Lycænopsis) 452

arizonensis Edwards (Papilio) 14

arizonensis Packard 3729

arizonensis Rothschild (Estigmene) 960

arizonensis Rothschild (Ophar-

us) 908

arizonensis Skinner (Acolastus) 457

arizonensis Skinner (Heterop-

ia) 472

arizonensis Strecker 794

arizonicum Dyar 4794

arizoniella Kearfott 8073

arizoniensis Stretch 983

armata Grote 2534

armataria Guenee 4728

armeniella Frey \& Boll 6068

armigera Hubner 1090

armillata $H y$. Edwards 940

armoraciæ Busck 7682

arna Guenee 2607

arnicella Walsingham 6452

Arogalea 156

arogos Boisduval \& LeConte 599 arota Boisduval 397

arpa Boisduval \& LeConte 606 arrogaria Hulst 4677

arrosa Harvey 3370

arsace Boisduval \& LeConte 381

arsaltealis Walker 5125

arsesaria Walker 4773

Arta 138

Artace 97

artega Barnes 1795

artemis Packard 786

artemisiæ Hy. Edwards 6658

artemisiana Walsingham 7097

artemisicolella Chambers 7742

artemisiella Kearfott 6119

artesta Smith 1791

artestata Grossbeck 4132

arthechippus Scudder 305

arthemis Fabricius 305

arthrolita Harvey 1912

articulatana Kearfott 7251

artonis Edwards 186

Artopsis 138

arufa Smith 1512

aruncana Kearfott 6783

arvalis $H y$. Edwards 2843

arvalis Grote 2842

aryxna Dyar 660

Arzama

Asciodes

130

ascula Smith 1737

asellus $R$. \& J. 690

ashtaroth Fisher 156

ashtaroth Strecker 109

asilipennis Boisduval 6755

asopialis Guenee 3554

aspasia Strecker 3080

aspera Morrison 2518

asperatella Clemens 5490

aspersa Grote 959, 1

asphodelana Kearfott $\mathbf{7 1 0 5}$

aspidana Walsingham 7031

aspidiscana Hubner 7055 
aspilatata Guence 4862

Aspilates

aspilodactylus Walker 5906

aspirata Peorsall 4834

aspista Meyrick 6975

assimilans Wolker 949

assimilis Boisduval 2827

assimilis Butler 134

assimilis Grote 2972

assimilis Morrison 1723

assiniboia Lyman 562

associans Walker 1502

associata Walker 3644

astapa Druce 4742

astarte Doubleday \& Hevitson 202

astarte Doubleday 3633

astarte Fischer 153

astarte Sirecker 706

aster Edwards 426

astericola Frey \& Boll 8001

asterioides Reak. 4

asterius Cramer 4

asteroides Guenee 2003

asthenaria Walker 3914

astigma Smith 1979

astinous Drury 1

astoria $\boldsymbol{H}$. Edwards 3589

astrea Edwards 64

astragalus Wright 421

astriata Reiff 3752

astricta Morrison 1487

astrigata $B$. \& McD. 2049

astrologana Zeller 6843

astur Cramer 908

astuta Bird 2677

astyanax Fabricius 304

astylus Drury 709

astylusaria Walker 4706

astymone Olivier 800

asychis Cramer 497

atacta Meyrick 6995

ataln Pocy 881

atalanta Linnaeus 282 atalis Dyar 5270

Atalopedes

Pㄸำ

atarn Smith 2166

atarah Strecker 8117

ate Strecker 284

atergatis Doubleday \& Hewitson 86

aterrima Grote 2860

aterrima Trealease 8473

aterrimella Walker 8077

Atethmia 60

atha Strecker 1412

athabasea Neumoegen 3336

athasaria Walker 4652

Athena $1: ?$

athene Strecker 963

athereo Harris 3640

atincta Smith 2160

atlantica Grote 1710

atlantica Smith 2638

atlantis Edwards 162

Atlides 13

atoma Smith 1555

atomaria Smith 3569

atomaria Walker (Ogdoconta) 2704

atomaria Walker (Olene) 3715

atomariella Zeller 7932

atomaris Smith 1333

atomaris Hubner 3349

atomosana Busel $\mathbf{7 4 4 7}$

atomosana Walsingham 6921

atomosella Dyar $\mathbf{7 7 2 5}$

atomosella Zeller $\mathbf{8 0 3 4}$

atomosellus Kearfott $\mathbf{5 4 0 9}$

atossa Edwards 176

atra $B . \&$ M D. 8341

atra Grote 2636

atrata $H_{y}$. Edwards 1051

atrata Morrison 1459

atrella Hulst 5680

atrescens $\mathrm{Hulst} 4615$

Atreus

atriciliata Grote 2598 
atricincta Smith 1499

atriclava B. \& McD. 2253

atricollaris Harvey 2076

atricornis Grote 2180

atrifascialis Hulst 5478

atrifasciata Hulst 3984

atrifasciata Morrison 2061

atrifasciella $B . \& M c D .5806$

atrifera Grote 1365

atrifrons Grote 1453

atrilineella Grote 3504

atriluna Smith 3412

atrimacula Smith 3530

atrimacularia $B . \& M c D .4337$

atripennalis $B . \& M c D .5067$

atripennis Grote 839

atriplex Busck 6128

atriplicivora Cockerell 7743

atrisquamalis Hampson 5122

atristrigaria $B, \& M c D .4571$

atristrigata Smith (Euxoa)

1255

atristrigata Smith (Anytus)

$$
1535
$$

atrites Grote 1122

atritincta Harvey 3179

atrivenosa Palm 3714

atrocolorata Grote 3986

atrocolorata Hulst 4743

atrodentanum Fernald 6802

atrodorsella Clemens 6433

atrofasciata Packard 4386

atrofusca Smith 1289

atrolinea B. \& McD. (Anta-

plaga) 2817

atrolinea $B . \& M c D$. (Lactura)

7735

atrolinearia Hulst 4567

atroliturata Walker 3955

atromarginata Braun 7819

atropicta Z eller 6483

atropulverea Smith 1310

atropunctata Packard 4456

atropurpuralis Grote 5169
Page

atropurpurea Grote 1329

atrosignata Walker 4421

atrostriga $B$. \& McD. 2582

atrosuffusa $B . \& M c D .2256$

atrupictella Dietz 6065

Atrytone ...................................... 21

Atrytonopsis …............................ 22

attalus Edwards 565

attenta Grote 1599

attenuatella Walker 5959

attenuatus Grote 5377

Atteva 184

attributella Walker 6160

attritella Walker 6309

auburniana Harris 377

aucillaria Strecker 4390

aucta Hampson 5278

audaculana Busck 7284

audentis Smith 1322

aufugella Zeller 6582

augias Hubner 571

augmentanus Zeller 7109

augusta $H y$. Edwards 3080

augusta Edwards 209

augustalis Grote 5165

augustana Hubner 7153

augustina Wright 209

augustinus Westwood 379

augustus Kirby 379

augustus Harvey 2043

aulæa Geyer 967

aulularia Grote 4637

aurantiaca $H y$. Edwards 1081

aurantiaca Hewitson 542

aurantiaca Hubner 934

aurantiacaria Packard $\mathbf{4 7 7 2}$

aurantiago Guence 2654

aurantiana Walsingham 7112

auranticella Grote 5562

auranticolor Grote 2257

auranticolorata Strecker 3815

aurata Butler 906

surata Grote 4067

aurata Packard 4060 
auratalis Walleer 5291

auratolla Braun 7984

auratella Clemens 5421

auratus Grote 8485

auren Braun 8098

surea Fitch 7731

aurea Grote 2865

auren Pagenstecher 4891

aureana Busck 7479

surelia Druce 774

aurella Fischer 3052

aureoalbida Walsingham 7540

aureofasciella Ragonot 5752

aureola $H y$. Edwards 6661

aureolalis Hulst 5019

aureolum Hy. Edwards 2713

aureomaculella Dyar 5702

aureomarginata Cockerell 278

aureopurpura .Hy. Edwards 6687

aureovirens Dietz 8434

auretorum Boisduvel 368

auricapitana Walsingham 6830

auricinctaria Grote 3474

auricrinella Walsingham $\mathbf{8 4 8 1}$

auricristatella Chambers 8315

auricyanen Walsingham $\mathbf{8 4 7 7}$

aurifera Zeller 5443

auriferana Busck 7338

auriferaria Hulat 4781

auriferella Dietz 8318

auriferella Frey \& Boll 8047

aurifimbrialis Walker 5368

aurimaculella Chambers 6310

auripennis Grote 3350

aurirubra Braun 7586

aurisignana Zeller 7255

auristrigella Chambers $\mathbf{8 4 4 4}$

aurivitta Grote \& Robinson 2868

auronitens Frey \& Boll 7914

auropulvella Chamber 8282

suropurpurella Chambers 7762

Aurora
Pase.

aurora Smith \& Abbot 3662

aurorana Kearfott 7508

aurorella Ely 5531

aurorella Dyar 6486

aurosea Neumoegen 778

aurosparsella Walsingham 8478

aurostriata Graef $\mathbf{3 6 6 4}$

aurosuffusella Chamber 8301

aurulenta Smith 1421

susonides Boisduval 40

ausonius Lintner 524

austerella Zeller 7712

australata Hulet 4084

australella Hulst 5658

australis Edwards 330

australis Grinnell (Anthocharis) 42

australis Grinnell (Pterophorus) 5913

australis Grote 3017

australis Hulst 3903

australis Maynard 18

australis Stretch 4881

australisella Chamber (Triclonella) 6501

australisella Chambers (Lithocolletis) 7849

austriana Cosens 5569

autholea Boisduval 983

autocles $R$. \& J. 10

Autocosmia 132

Autographa

autolycus Edwards 354

Automeris

Autoneda

autumnalis Cockerell 59

autumnalis Stromeyer $\mathbf{4 0 1 4}$

autumnalis Riley 2574

autumnata Guenee 4069

autumnata Packard (Palseacrita) 4624

autumnata Packard (Alsophi-

la) 3767

suxiliaris Grote 1372 
avalona Wright 350

avemensis Dyar 1084

averna $B . \& M c D .3640$

avernalis Grote 5221

aversus $H y$. Edwards 4887

avia Hubner 943

avimacula Hudson 3681

avirida Smith 2423

avuncularia Guenee 4291

aweme Lyman 2692

awemeana Kearfott 7052

Axenus

axillaris Grote \& Robinson 735

axius Ploetz 553

azaleæ Busck 8056

azaleæ Smith \& Abbot 745

Azenia

azia Hewitson 348

azif Strecker 1272

azionalis Walker 4926

baal Strecker 158

baba Dyar 5252

babayaga Strecker 3075

baboquavaria Smith 2536

baboquavariana Kearfott 7375

baboquivariella Kearfott 5319

baccatalis Hulst 5030

baccharides Grinnell 5912

baccharisella Busck 6099

bachmani Kirtland 320

Bactra

badia Grote \& Robinson 3027

badia $H y$. Edwards 3701

badia Hubner 4837

badia Packard 3656

badiaria $H y$. Edwards 3973

badicollis Grote 1552

badiiella Chambers 7859

badinodis Grote 1415

badiocapitella Chambers 8359

badiomaculella Chambers 6311

badipennis Grote 5132

badistriga Grote 2080

badiusalis Walker 5202
Page

Bagisara

Baileya

baileyana Grote 1505

baileyi Grote 2144

bairdi Edwards 5

bairdi Smith 1500

baja Fabricius 1430

bakerella Busck 7636

bakerella Dyar 5646

bakeri Dyar 2057

balanotes Meyrick 5941

balba Grote 1176

balder Boisduval 123

baldur Edwards 24

balinitis Grote 1376

baliola Morrison 2397

balistaria Guenee 3837

balluca Geyer 3277

Balsa 68

balsamorrhizella Busck 7619

baltearia Hulst 4398

balteata Kirtland 731

balteata Smith (Cobubatha) 2883

balteata Smith (Oncocnemis) 2026

baltimoralis Guenee 3561

bana Kearfott 7496

banana Busck 7267

banavahrata Strecker 4020

Bandera 150

banksiella Busck 6109

Bapta 111

Baptarma 73

baptisiæ Bird 2664

baptisiella Fernald 5496

baracana Busck 7550

baracana Kearfott 7107

baracoa Lucas 579

baranca Barnes 1800

Barathra

barbara $B . \& M c D .2549$

barbara $H y . E d w a r d s ~ 61$ 
barberella Buscle (Neodactylota) 6175

barberella Busck (Depressaria) 6477

barberella Buselk (Cerostoma) 7666

barberellus Busck 8470

barberi Dyar 5903

Barberia

barberiana Dyar 4866

barda Hy. Edwarde 978

barnea Druee 4871

barnesata Swett 4028

barnesi Beutenmuller 6657

barnesi Dyar (Acrolophus) 8179

barnesi Dyar (Illice) 883

barnesi French 3038

barnesi Hulst 4724

barnesi Skinner 255

barnesi Smith (Acronycta) 2507

barnesi Smith (Oncocnemis) 2062

barnesi Smith (Septis) 2258

Barnesia

barnesiana Dyar 6887

barnesiella Busck (Gelechia) 6251

barnesiella Busck (Trichotaphe) 6358

barometricus Goossens 1522

baroni Behrens 8493

baroni Edwards (Argynnis) 179

baroni Edwards (Cercyonis) 111

baroni Edwards (Euphydryas) 208

baroni Fieh 5900

Barrovia

bartschi Reiff 158

baryzela Meyrick 7485

basaliata Walker 4094

basalis Grote (Catocala) 8028

basalis Grote (Euxoa) 1361 basalis Walker (Inguromorpha) 8.325

basalis Walker (Marathysa) 2999

basalis Walker (Scoparia) 5244

basiaria Walker 4304

basicinerea Grote 2907

basifasciella Zeller 6080

basiflava Packard 3710

basiflava Smith 1274

basiflavalis $B$. \& McD. 4906

basifugens Dyar 2864

basigera Walker 3251

Basilarchia

basilarella Dietz 6581

basilaris Zeller (Salebria) 5635

basilaris Zeller (Scythris) 8078

Basilodes

Basilona

basimaculatella Ragonot 5727

basiochrea $B$. \& $M c D .2744,1$

basiochrealis Grote 5469

basiochreana Kearfott 7520

basipallida $B$. \& $M C D .3469,1$

basipallidella Dietz 6531

basiplaga Smith 1766

basiplagana Walsingham 7406

basipunctana Walsingham 6923

basipunctaria Walker 2876

basirei Druce 4880

basistriga $B$. \& McD. 7734

basistrigella Chambers $\mathbf{7 7 4 4}$

basistrigella Clemens 7894

basistrigella Zeller 6079

basitriens Walker 3613

basivirida $B$. \& $M c D .1773$

basquella Chambers 6230

bassettella Clemens 7574

bassiformis Walker 6668

b-ata Goeze 989

batabano Lefebre 456

batanella Busck 6117

bataviella Braun 7934

batesi Felder 4979 
batesi Reakirt 248
bathyllus Smith \& Abbot 485
Batrachedra ........................
battoides Behr 442
beani Elwes 135
beani Grote 1743
beani Neumoegen 946
beani Skinner 211
beaniana Grote 3068
beata Grote 1502
beckeri Edwards 31
beddeci Dyar 5131
beddeci Hampson 1511
Bedellia

befana Skinner 818

begallo Barnes 2632

behrensaria Hulst 4327

behrensarius Packard 4747

behrensata Packard 4222

behrensella Chambers (Gracilaria) 8035

behrensella Chambers (Tinea) 8260

behrensellus Fernald 5378

behrensi Edwards 167

behrensi Hy. Edwards (Nadata) 3620

behrensi $H y$. Edwards (Synanthedon) 6707

behrensi Grote 2013

behrensi Stretch 8492

Behrensia 59

behrensiana Grote 1879

behri Edwards (Callipsyche) 394

behri Edwards (Glaucopsyche) 449

behri Edwards (Eurymus) 71

behri Edwards (Papilio) 25

behri Grinnell (Cercyonis) 116

behri Grinnell (Ptorophorus) 5933

behri Smith 2991 behri Stretch 985

belæ Grote 820

belangerella Chambers (Telphusa) 6083

belangerella Chambers (Argyresthia) 7704

belangeri Morrison 2355

belfrageana Zeller 7316

belfrageella Chambers (Nepticula) 8360

belfrageella Chambers (Gracilaria) 8055

belfragei $F$ ish 5892

belfragei Grote (Heterocampa) 3638

belfragei Grote (Renia) 3526

belfragei Stretch 934

belfragei Smith 1592

belfragesella Chambers 6094

belfragiana Harvey 3093

belialis Druce 5082

belioides Verity 39

bella Chambers (Adela) 8457

bella Chambers (Heliodines) 7562

bella Grote 1720

bella Hulst 5706

bella Linnaeus 1029

bella Walsingham $\mathbf{7 7 4 5}$

belladonna Hy. Edwards 1078

bellela Walker (Aristotelia) 6041

bellela Walker (Nemotois) 8448

bellicula Hubner 2898

bellona Fabricius 199

bellonaria Strecker 3777

bellulalis Hulst 5158

Bellura

bellus Edwards 638

belmaria Ehrman 937

Belnoptera 128

belusalis Walker 4954

Bembecia .164 
Bendis 86

beneficentella Murtfeldt 6284

benesignata Harvey $\$ 187$

benigna Hulat $\mathbf{4 1 8}$

benigna $\boldsymbol{H y}$. Edwards 2197

benignalis Walker 3561

benitaria B. \& McD. 3872

benjaminella Dyar 5784

berenice Cramer 85

bergi Moeschler 4947

bergmanniana Linnceus $\mathbf{7 4 1 0}$

bernardino $B$. \& MCD. 442

bernardino Educurds 54

bernardinus Grinnell 5861

Bertelia

bertholdi Grote 8347

Bertholdia

bertrami Roessler 5881

Besciva 156

bethunei Grote \& Robinson 2131

bethunei Smith 3190

bethunella Chambers 7957

betsia Smith 1532

betula Riley 2492

betulella Hulat 5542

betulella Busck (Telphusa) 6082

betulella Busck (Depressaria) 6480

betuliperda Dyar 7604

betulivora Walsingham 7955

beutenmuelleri B. \& McD. 3098

beutenmuelleri $\mathrm{H} y$. Edwards 4833

beutenmulleri Skinner 6746

bialbe Smith 1341

bianea $H y$. Edwards 3053

biangulana Walsingham $\mathbf{7 1 5 4}$

bianulella Chambers 6243

biareuana Stephens 7197

bias Druce 2592

bibitrix Hubner 3369

bibularia Grote \& Robinson 4652 bicarnea Guenee 1425

I'a

bicaudnta Dyar 4825

biclavis Grote 1389

bicollaris Grote 1281

bicolor B. \& MCD. (Phonicophanta) 2921, 1

bicolor B. \& MCD. (Polia) 1746

bicolor Frey \& Boll $\mathbf{7 8 5 4}$

bicolor Grote 855

bicolor Harris 803

bicolor Walker 955

bicolorago Guever 2220

bicoloralis Guenee 5103

bicoloraria Minot 4289

bicoloraria Packard 4301

bicolorata B. \& McD. 2949

bicolorata Fabricius 4341

bicolorata Grote 1964

bicolorella Hulst 5817

bicolorella Chambers 7945

bicostomaculella Chambers

(Epithectis) 6161

bicostomaculella Chambers

(Gelechia) 6265

bicristatella Chambers (Blastodacna) 5986

bicristatella Chamber (Elachista) 7826

bicuspida Smith 1121

bicycla Packard 2198

bidens Zeller 5347

bidentalis $B . \&$ MCD. 5071

bidentata Walker 3621

bidentella Dyar 5715

bidiscomaculella Chambers 6375

bidisata $D$ yor 4612

biedermani B. \& MCD. 3661

biedermani Skinner 3686

bifascia Hubner 1135

bifascialis Robinson 5212

bifasciata $B$. \& MeD. 3390

bifasciata Bates 3145

bifasciata Dyar 4152 
bifasciata Smith 1311

bifasciata Walsingham 7631

bifasciella Busck 6048

bifasciella Chambers (Homosetia) 8309

bifasciella Chambers (Lithocolletis) 7962

bifasciella Chambers (Mompha) 6005

bifasciella Chambers (Theisoa) 5972

bifasciella Clemens 8361

bifasciella Hampson 5848

bifasciella Hulst 5640

biferalis Walker 3548

biferana Walker 7602

bifida Packard 4816

bifida Strand 2835

bifidalis Grote 3550

bifidalis Fabricius 5023

bifidella Dietz 6092

bifilata Hulst 4313

bifilata Walker 3781

bifiria $H y$. Edwards 3589

biflavimaculella Clemens 8238

biforella Busck 6297

biforma Smith 1125

biformata Hy. Edwards 3298

biformata Smith 1298

bifurcalis Snellen 4927

bifurcata Braun 7869

bigallis Smith 3480

bigella Busck 6270

biguttata Packard 4839

biguttatus Packard 3654

bijugalis Walker 3562

bilinea Walker 5289

bilinealis $B . \& M c D .3558$

bilineana Kearfott 7039

bilinearia Packard 4702

bilineata $B$. \& $M c D .2877,1$

bilineata Grossbeck 4519

bilineata Packard (Drepana) 3761 bilineata Packard (Heterocampa) 3644 .

bilineata Smith 3168

bilineatella Ragonot 5509

biliturellus Zeller 5392

biloba Stephens 3259

bilobella Zeller 6367

bilunata Grote 2579

bimacula Grote \& Robinson 605

bimaculana Robinson 7525

bimaculata Gmelin 1042

bimaculata Herrich-Schaeffer 1049

bimaculata Stephens 3264

bimaculata Saunders 934

bimaculatella Ely 8065

bimaculella Chambers (Gelechia) 6210

bimaculella Chambers (Dichomeris) 6391

bimaculella Chambers (Tinea) 8253

bimarginalis Grote 1581

bimatris Harvey 1110

biminimaculella Chambers (Gelechia) 6269

biminimaculella Chambers (Haploptilia) 7746

bina Guenee 1054

binda Barnes 1206

bindata Pearsall 4251

binocula Grote 2958

binodulalis Zeller 5265 .

binominalis Smith 1568

binotata Walker 2294

binotella Zeller 5840

biothanatalis Hulst 5378

bipartita Smith 3577

bipartitana Clemens 6869

bipartitella Ragonot 5834

biplaga Guenee 2983

biplaga Walker 2900

biplagata Walsingham 7023

biplagialis Walker 5244 
bipuneta Morrison 3420

bipunctalis B. \& MCD. 5063

bipunctalis Fabricius 5014

bipunetatus Moeachler 5894

bipunetella B. \& MeD. 8832

bipunctella Busck 6090

bipunctella Chambers 5975

bipunctella Dietz 8304

bipunctella Walker 7022

bipunctella Walsingham (Dichomeris) 6388

bipunctella Waloingham (Haploptilia) $\mathbf{7 7 4 7}$

bipunctellus Zeller 5365

bipunctina Guenee 3406

bipustulana Walker $\mathbf{7 1 3 9}$

biquadrana Waloingham 6963

birdana Busek 7547

birdi $D$ yar 2663

birnata Smith 2376

biscana Kearfott 7513

bischoff Edwards 185

bischoff Zeller 5881

biscolorella Chambers 6087

bisecta Lintner 807

biselliella Hummel 8298

biseriata Packard 3756

biseriata Herrich-Schaeffer 4122

bisignata Walker 4339

bissesa Dyar 4859

bistrialis Geyer 3151

bistriaria Hubner 3781

bistriaria Pacleard 3807

bistriaris Hubner 8127

bistriata Kearfott 7321

bistriatella Hulst (Myelois) 5509

bistriatella $\mathrm{Hulat}$ (Pyla) 5663

bistriatella Hulat (Pectinigeria) 5816

bistriga Smith 1977

bistrigata Hubner 3146

bistrigella Chambers (Gelechia) 6312 bistrigella Chamber (Haploptilia) 7748

bistriolata Zeller 4026

bisulea Grote 1876

bitactats Walker 4421

bittana Busek 7256

bittenelia Busck 6646

biundata Walker 3642

biundulalis Zeller 2822

biundulata Smith 1158

biustulana Stephens 7357

biviella Zeller 8454

bivittata Clemens 1026

bivittata Grote 3514

bivittata Hulat 4166

blackmorei Swett 3921

blakeana Grote 7146

blakei Grote 987

blakei Maynard 82

blanea Barnes 1200

blanda Grote 1548

blandana Clemena 7342

blandella Clemens 8052

blandula Hulst 3109

Blastobasidx $\quad . . \ldots \ldots \ldots 162$

Blastobasis .............................. 162

Blastodacna ………… 152

blenina Hewitson 376

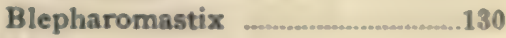

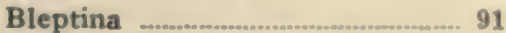

bobana Kearfott 7000

bochus Morrison 1369

boisduvali Butler 941

boisduvali Duponchel 193

boisduvali Harris 100

boisduvali Oberthur 794

boisduvaliana Felder 75

boisduvaliella Guenee 5810

bolanderana Walsingham 6881

bolanderi Stretch 986

Bolla

bolli Edwards 238

bolli Hy. Edwards 6668

bolli Grote 1470 
bolli Zeller 5693

bolliana Slingerland 7126

bolliella Busck 7709

bolliella Dyar 7922

bolterellus Fernald 5375

bolteri $H y$. Edwards (Aemilia) 928

bolteri Hy. Edwards (Synanthedon) 6675

bolteri Hulst (Eupithecia) 4219

bolteri Hulst (Tracheops) 4635

bolteri Smith (Agrotis) 1433

bolteri Smith (Polia) 1721

bolteri Stretch 1012

Bombycia

Bombycida 98

bombyciformis Smith 2109

bombyciformis Walker 6755

bombycina Zeller 8170

Bombycoidea

bombycoides Walker 701

Bombyx 98

Bomolocha 91

bomonana Kearfott 7514

bonaerensis Berg 3258

Bondia .180

bonifata Hulst 3885

bonifatellus Hulst 5383

bonuscula Smith 2183

bonusculalis Hulst 5373

boopis Behr 111

bootes Boisduval 129

boothi Curtis 58

borea Aurivillius 3227

borealis Boisduval 3669

borealis Cary 526

borealis Fitch 3705

borealis Grote (Epipaschia) 5467

borealis Grote (Pieris) 35

borealis Grote \& Robinson (Calephilis) 329

borealis Hampson 1079 borealis Hulst (Cabera) 4305

borealis Hulst (Eupithecia)

4210

borealis Hulst (Xanthorhoe)

4044

borealis Moeschler 942

borealis Packard 5151

borealis Smith (Brachionycha) 2102

borealis Smith (Copipanolis)

2112

borealis Smith (Epizeuxis) 3490

borealis Staudinger 948

boreasella Chambers 6497

boreasella Clemens 7995

boreasella Dietz 6552

boreata Packard 3945

boreata Taylor 4002

boretha Smith 1379

Borkhausenia 162

borkhauseni Z eller 6497

Borolia 55

borregalis Dyar 5292

borus Boisduval 360

boscana Fabricius 7444

bosquella Chambers (Ectodemia) 8422

bosquella Chambers (Parecto-

pa) 8005

bostonica Frey \& Boll 7974

bostoniensis Grote 1318

bostura Smith 1828

boucardi Druce 778

boxcana Kearfott 6975

brachiolum Harvey 1674

Brachionycha 57

Brachycosmia 60

Brachylomia 58

brachyelytrifoliella Clemens 7828

brackenridgella Busck 6121

bracteatana Fernald 7222

branella Busck 6295 
brannani Streteh 1041

brassica Riley 3249

brassicella Fitch $\mathbf{7 6 8 3}$

brauneata Swett (Eupithecia) 4264

brauneata Swett (Ptychopoda) 3893

brauniana Kearfott 7344

bredowi Hubner 310

brehmeata Grossbeck 4681

brehmei $B$. \& MeD. 2784, 1

bremneri Edwards 166

brenda $B$. \& MCD. 1701,1

brenda Edwards 105

brennus Godman \& Salvin 515

Brenthia 182

Brenthis 8

Brephidium 15

Brephine 99

brephoides Walker 3766

Brephos 99

brettoides Eduards 587

brettus Boisducul 587

brevicauda Saunders 4

brevicornis Walker 934

brevicrista Dyar 3741

brevimargo Butler 669

breviornatalis Grote 5473

breviornatana Clemens 7304

brevipennella Diets 8196

brevipennis Streteh 2825

brevipennis Smith 1241

brevirostralis Grote 3522

brevis Grote 1122

brevistriga Chambers 8079

brevistriga Smith 1241

brevivitella Clemens 6006

brewsteriana Robinson 7434

bridghami Grote \& Robinson 23.33

brightonana Kearfott 6977

brillians Bames $2100^{\circ}$

brillians $B$. \& $M c D$. (Euplexia)

2318 brillians B. \& McD. (Harrisina) 4884

brillians Neumoegen 2867

brillians Ottolengui 2172

brimleyana Dyar 2879

briseis Edwards 3065

britana Busck 7263

britannia Kearfott $\mathbf{7 4 1 4}$

brizo Boisduval \& LeConte 518

brocha Morrison 1369

brontes Drury 667

browningi Skinnor 46

bruceata Hulst 3964

brucei Edwards (Euphydryas)

219

brucei Edwards (Oeneis) 135

brucei Edwards (Papilio) 5

brucel Hy. Edwards (Ichthyura) 3594

brucei $H y$. Edwards (Neoaretia) 945

brucei Elwes 142

brucei Fernald 5932

brucei French (Acossus) 8353

brucei French (Høemorrhagia) 734

brucei Hulst 5593

brucei Smith (Orthosia) 1919

brucel Smith (Schinia) 1176

brucei Smith (Rancora) 1986

brucei. Smith (Xylena) 2175

Bruceia

brullei Lefebre 4059

brumalis B. \& McD. 4965

brumella Clemens 6276

brumosa Guenee 2446

brunnea B. \& $M c D .1175$

brunnea Grote \& Robinson 3634

brunnea Herrich-Schaeffer 516

brunnea Strecker 959

brunnea Stretch 835

brunnes Walsingham 8245

brunnearia Packard 3782 
Page

brunneata Packard (Dysstroma) 3992

brunneata Packard (Itame) 4425

brunneata Thunberg 4407

brunneella Dyar 5561

brunneiciliata Packard 4087

brunneicollis Grote 1566

brunneicrista Smith 2532

brunneifasciata $B . \& M c D$. 3311,1

brunneifasciata Packard 4121

brunneigera Grote 1309

brunneilinearia Grossbeck 4727

brunneipennis Hy. Edwards 6660

brunneipennis Grote 1590

brunneipennis Hulst 4054

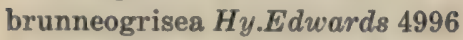

brunneolineata Hulst 4658

brunneomaculata Bates 3983

brunneri Busck 6673

brunneus Busck 6177

brunnildalis Dyar 5211

bryantaria Taylor 4311

bryanti Taylor 4237

Bryolymnia

Bryomima

bryoniæ Ochsenheimer 36

bububattalis Hulst 5187

Bucculatrix

bucephaloides Walsingham 7095

bucephalus Stephens 571

bucetum Grote 3150

budea Hubner 959

bufalis Guenee 5229

buffaloensis Grote 2643

buffaloensis Grote \& Robinson 732

bulenta Boisduval\&Leconte 596

bullula Grote 2888

bultata Smith 2307

bumeliella $B . \& M c D .5618$

bunkeri Grote 3052 bunteana Robinson 7478

burgessi Morrison 2369

burgessi Skinner 519

burgessiana Zeller 7186

burgessiella Zeller 8057

buristriga Chambers 8079

burkeana Kearfott 6772

burkerella Busck 8230

burrisoni Maynard 307

burseræ Dyar 3004

burserella Busck 8049

burnsi Watson 790

busckella Dyar 5585

busckella Ely 6035

busckellus Haimbach 8183

Busckia .186

busckiella Dietz (Holcocera) 6542

busckiella Dietz (Hypoplesia) 8210

busckiella Engel 5971

busckiella Kearfott (Choreutis) 7612

busckiella Kearfott (Gnorimoschema) 6124

bushnelli Busck 6773

buta Smith 1138

butleri Edwards 193

butyrosa Butler 5105

buxea Grote 2731

byssus $E d w a r d s 596$

Cabares 18

cabelus Edwards 560

Cabera 111

caberata Guenee 4773

cablei Reizenstein 711

Cabnia

cacabella Hulst 5653

cacamica Dyar 5257

Cacceia 176

cacola Smith 2980

Caconome 190

Cacozelia

Cactobrosis 146 
cacuminalis Walker 3548

cacuminaria Morrison $\mathbf{3 8 5 3}$

cadarella Druce 5416

cadaverosa Grote 906

cadaverosa Strecker 901

eadmia Guenee 3683

caduca Grote 2634

caducalis Walker 3566

cadueus Dyar 2613

cueca Cockerell 771

creca Dod 2091

creca Reiff 410

caeca Strecker 1021

crecalis Warren 4935

crecalis Walker 5235

crecella Zeller 6273

creculalis Zeller 5087

calaria Hulst 4666

caenis Grote 1303

Canurgia

carulea Beutenmullor 3060

crerulea Grote 3129

crrulea Godman \& Salvin 293

cxruleans Walsingham 7229

cærulescens Prout 3798

crerulescens Holland 156

cresarea Smith 2454

cesia Smith 1353

cesialbana Zeller 6869

cæsiaria Hulst 4380

cresiella Brodic 6126

cæsonia Grote 4852

cresonia Stoll 55

cespitalis Boisduval 489

crespitis Grote \& Robinson 1024

exspititiella Zeller 7758

caia Linnaeus 1025

caicus Cramer 721

eaicus Herrich-Schaeffer $\mathbf{4 8 0}$

eajona Reakirt 428

calais Seudder 122

calami Harvey 2735

Calamia
Pיom

Calamochrous

I.as:

calanus Hubner 365

Calasesia 166

calcaria Pearsall 4730

calceolaria Strecker 184:3

calchas Behr 435

calchas Herrich-Schaefer 476

Calephelis

Calera

calera Barnes 933

calgariana Smith 1945

calgary Smith 1436

Calidota 35

caliente Wright 45

californis Westwood \& Heuitson 101

california Wright 559

californix Walsingham 7626

californix Walker 941

californiana Menetries 59

californiana Walsingham 7312

californiaria Herrich-Schaeffer 4475

californiaria Packard (Anagoga) 4641

californiaria Packard (Cleora) 4593

californiaria Packard (Drepanulatrix) 4316

californiaria Packard (Holaretias) 3880

californiaria Packard (Phasiane) 4357

californiaria Packand (Sericosena) 4462

californiata Gumppenberg 4205

californiata Packard (Hydriomena) 4018

californiata Packard (Trichodesia) 3944

californiata Packard (Tripho8a) 3970

californiata Peckard (Xanthorhoe) 4042 
californica Behr (Capnodes) 3381

californica Behr (Hypena) 3580 californica Behr (Xylomyges) 1881

californica Boisduval (Aglais) 279

californica Boisduval (Oeneis) 121

californica Busck 6168

californica Butler 310

californica Dyar 3595

californica $E d w a r d s$ (Catocala) 3063

californica $E d w a r d 8$ (Strymon) 360

californica Grinnell 5951

californica Grote (Melicleptria) 1073

californica Grote (Samia) 769

californica Hampson 1081

californica Menetries 6

californica Packard (Epicnaptera) 3754

californica Packard (Estigmene) 960

californica Packard (Isia) 957

californica Packard (Malacosoma) 3748

californica Packard (Phryganidia) 3588

californica Reakirt 87

californica Smith 2238

californica Speyer 3267

californica Stretch 3615

californica Walker (Halisidota) 918

californica Walker (Malacosoma) 3748

californica Wright (Chlosyne) 264

californica Wright (Hemileuca) 786

californicalis Packard (Pyraus- ta) 5149

Page

californicalis Packard (Ommatopteryx) 5417

californicum Neumoegen 6653

californicus Boisduval (Brephos) 3764

californicus Boisduval (Hepialus) 8494

californicus Walsingham 5856

californiella Ragonot 5852

caliginella Hulst 5550

caliginoidella Dyar 5514

caliginosana Walker 7439

caliginosalis Walker 5235

caliginosella Busck 6623

caliginosellus Clemens 5397

calipusaria Walker 4644

Calledapteryx

calleta Westwood 770

callias Edwards 145

Callicore 11

callida Grote 1933

callidus Grinnell 523

callina Boisduval 229

callipeplella Hulst 5485

calliphanes Meyrick 7692

callippe Boisduval 178

Callipsyche

callitrichoides Grote 3436

Callizzia 124

Callophrys _................................. 15

Callopistria …...................................... 63

Callosamia …............................... 27

Calocalpe .............................................104

Calcecia ............................................ 97

Calophasia ………………….... 97

calora Barnes 1203

caloramica $B . \& M c D .1905$

Calosaturnia …......................................... 27

Calosima …n.................................162

Calostigia _.......................................107

Calpe ......................................... 87

calphurnia $H y$. Edwards 3084

Calpodes 23 
calpota Smith 1939

calverleyi Grote 1

calycanthata Smith \& Abbot 3198

calyce Edwards 38

cambiella Busek 5993

cambiicola $D$ yor 5573

cambrica Curtis 4114

camerata Meyrick 7895

eamerta Cramer 96

camertus Cramer 727

camillus Edwards 250

camina Smith (Eupanychis)

1197

camina Smith (Platyperigea) 2589

caminalis Smith 3549

Campaea

campalis Guenee 4938

campanilis Smith 3150

campestrana Zeller 6864

campestris Behr 249

campestris Boisduval 589

campestris Grote 1339

campicolana Walsingham 7491

Camptogramma

camurellus Clemens 5372

cans $H_{y}$. Edwards 3704

cane Martyn 700

cans Robinson 7302

cana Walker (Clemensia) 892

cana Walker (Dasylophia) 3625

canace Edwerds 253

canadana Kearfott 7333

canadaria Guence 4599

canadensis Bethure 3398

canadensis Boisduval 691

canadensis Buscle 6467

canadensis Guence 761

canadensis $R$. \& J. 13

canadensis Smith (Acronyeta) 2482

canadensis Smith (Polia) 1687

canadensisella Chambers 8125 canalis Grote 3361

canana Walsingham 6951

canariana Kearfott 6890

canariella Busck 6422

canariella Dietz 6597

canariella Walsingham 7656

Canarsia

canavestita Pearsall 3958

candefacta Hubnor 2956

candens Guenee 1839

candescens $\boldsymbol{H}$ y. Edwards 6698

candida Braun 8106

candida $\boldsymbol{H}$. Edwards 860

candida Lintner 3667

candida Smith (Catabena) 2566

candida Smith . (Euthyatira)

3694

candida Smith (Euxoa) 1262

candida Smith (Xylomyges) 1886

candida Walker 958

candida Wright 542

candidaria Packard 3869

candidellum Hulst 5767

canella Busck 6437

canescens Behr 2735

canescens Hy. Edwards 6736

canescentella Hulat 5616

caniceps Walsingham 6899

canicinctella Clemens 8091

canicostella Ragonot 5749

canicostella Walsingham 6028

canidiaria Strecker 4684

caniplaga Walker 8682

canities Hampson 1731

cannalis Quaintance 5224

canoa Barnes 2627

canopulvella Chambers 6313

canosaria Walker 4486

cansa Smith 2859

canthus Linnaeus 100

canusella Chamber 6610

Canthylidia 40

capax Grote \& Robinson 2177 


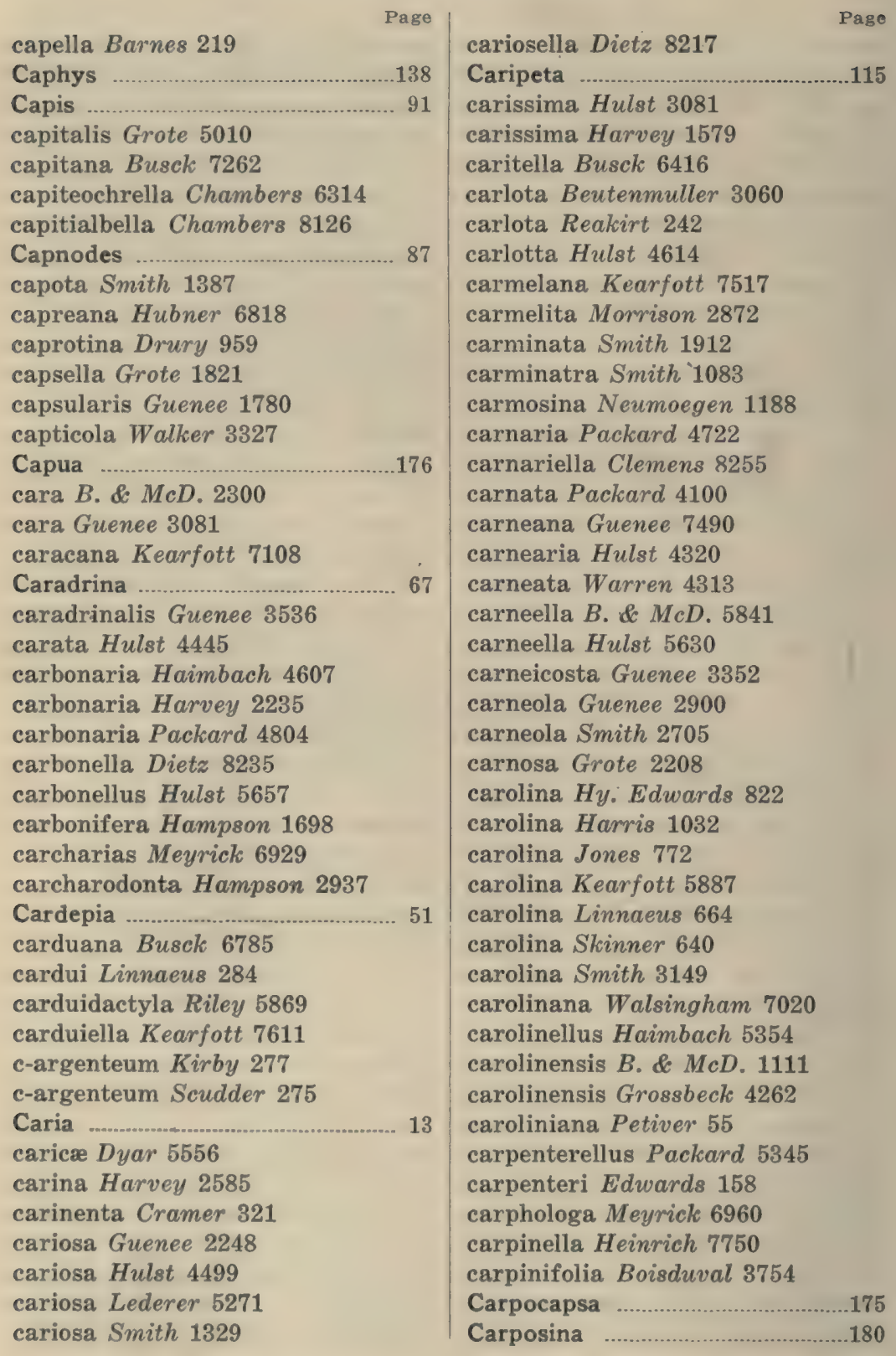




$\begin{array}{lr}\text { Carposinidge } & \begin{array}{r}\text { Paze } \\ \text { Carptima }\end{array} \text {. } \\ \text { Carsia } & 180\end{array}$

Carterocephalus 20

cartwrightana Kearfott 7544

carus Eduards 551

carywe Grote 5541

caryw Harris 915

caryve Robinson 7315

caryse Shimer 7207

caryealbella Chambers 7888

carysefoliella Chambers (Dichomeris) 6390

caryrefoliella Clemens (Haploptilia) 7751

caryafoliella Clemens (Lithocolletis) 7937

carywefoliella Clemens (Nepticula) 8363

carysevorella Packard 6315

caryalbella Ely 5522

caryana Fitch 7207

earye Hubner 285

caryi Dyar 127

caryivorella Ragonot 5528

caryophyllella Busck 8229

Casandria

casloata Dyar 4171

casloata Taylor 3996

cassandra $\mathrm{Hy}$. Edwards 3077

cassella Walker 6316

cassius Morrison 470

cassus Edwards 630

casta $H y$. Edwards 2855

casta Kirby 35

casta Sanborn 860

castalis Edwards 377

castalla B. \& McD. 1017

castanea Grote 2267

castanese Buscle (Ectcedemia)

8424

castanese Busck (Swammerdamia) 7720 castanea Busck (Synanthedon) 6680

castanexella Chamber (Lithocolletis) 7960

castanexella Chambers (Tischeria) 7856

castanesefoliella Chamber 8362

castanealis Hulst 5183

castaneana Walsingham 7037

castaneella Busck 7696

castanella Walsingham (Tischeris) 7856

castaneum Beutenmuller 6757

castella Walsingham 7639

castellalis Guenee 4931

castigata Hubner 4186

castipennella Walsingham 7753

castoria Reakirt 35

castra $B$. \& $M c D, 1621$

castro Reakirt 407

Catabena

catachloa Hulst 3771

Cataclysta

cataclystiana Walker 6924

catalaunalis Duponchel 5059

catalina B. \& $M c D .1832$

catalina Druce 2911

catalina Fabricius 419

catalina Reakirt 448

catalina Smith (Conochares) 2944

catalina Smith (Trachea) 2302

catalins Grinnell 5939

catalinella Busck 6223

catalpr Boisduval 676

cataphracta Grote 2686

Catastega

catenaria Drury 4630

catenula Grote 1259

catenulalis Grote 5138

catenulata Grote 4775

catenuloides Smith 1234

catharops Dyar 2742

catherina Grote 1553 


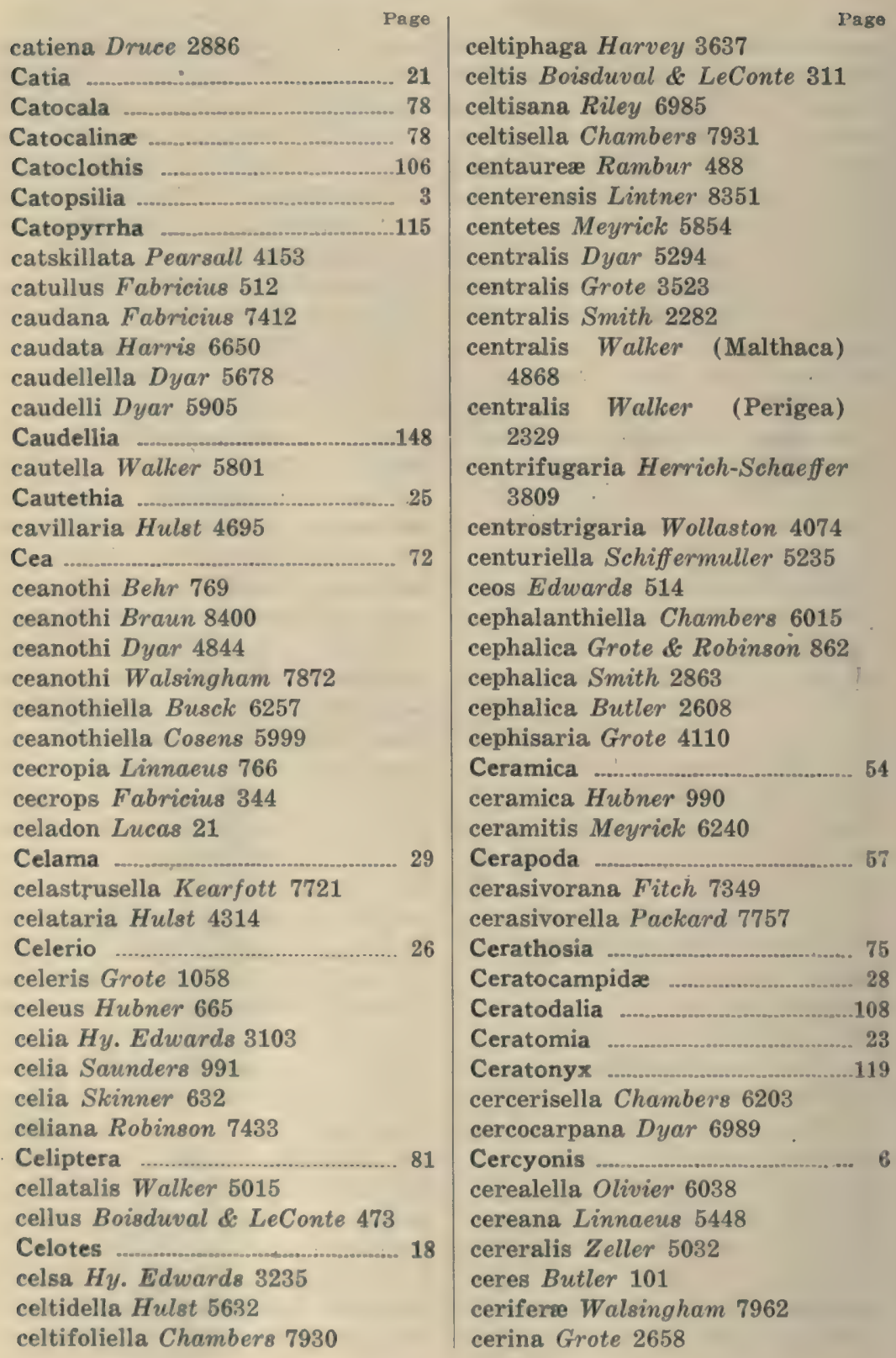


cerintha Treitschke 2891

Charephon

cerisyi Kirby 706

cerivana Smich 2291

Cerma

cernes Boiaduval \& LeConte 576

cernes Harris 577

cerogama Guence 3052

ceromatica Grote 2218

Cerostoma

cerrita Wright 296

certiorata Pearsall 4789

cerulea Ehrman 304

Cerura

cerusana Hubner $\mathbf{7 4 4 4}$

chagnoni Swett (Eupithecia)

4155

chagnoni Swott (Nematocampa) $\mathbf{4 6 8 0}$

chalcana Packard 7459

chalcas Felder 19

chalcedona Doubleday \& Howitson 204

chalcedonia Hubner 2607

chalcis Behr 371

Chalccela

chalcofrontella Clemens 6540

Chalcopasta

Chalia

Chalinitis

chalybsella Walsingham 5963

chalybeana Fernald $\mathbf{7 4 4 0}$

chalybeana Walsingham 6833

chalybeia Braun 8415

chalybeis Zeller 8457

chalybirostris Zeller 5368

chamsehrysaria Grote $\mathbf{4 3 0 0}$

Chamaclea

chamseleon Moeschler 3011

chamanerii Harris 761

chambersella Dyar (Durrantia) 6403

chambersella Dyar. (Gelechia) 6233

chambersella Dyar (Haploptilia) 7742

chambersella Murtfeldt 6373

chambersella Walsingham 7950

Chambersia .............................. 162

Chamyris ................................... 75

chandleri Grote 2069

chapmani Grote 3633

chara Edwards 232

Characoma ................................. i7

characta Grote 2303

Charadra …………… 83

chariclea Schneider 193

charie Blanchard 285

Chabuata 
charithonia Linnaeus 148 charlotti Barnes 157

Charommatrea

charon Edwards 119

chartaria Grote 1610

chatfeldi Grote 3688

chelidonia Grote 3087

chemnis Fabricius 647

chephise Cramer 4784

chersæa Meyrick 6944

chersis Hubner 687

Chesiadodes

Cheteoscelis

chicagonis Dyar 3569

chicorii Grote \& Robinson 1024

Chilo

Chilocampyla

chiloidellus B. \& McD. 5400

chioccana Kearfott 7408

chiococcana Meyrick 7408

chiococcella Busck 7717

Chiomara

chionanthi Smith \& Abbot (Protoparce) 666

chionanthi Smith \& Abbot (Adita) 1504

chionastes Meyrick 5931

chione Curtis 58

chionochroa Hampson 2485

chionosema Zeller 6847

chipeta Barnes 1802

Chipeta

chippewa $E$ dwards 68

chiquitella Busck 6097

chiricahua Poling 3099

chiricahuata Swett 4022

chiridota Grote 4793

chiriquensis Dyar 3606

chiron Fabricius 301

chironias Hubner 301

chispa Wright 584

chitone Edwards 165

Chlanogramma

Chloraspilates

chlorias Meyrick 5916

Page chlorina Skinner 439

chlorinda Martyn 745

Chlorippe

chloris Herrich-Schaeffer 4814

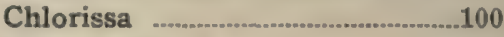

Chlorobapta …........................... 135

Chlorochlamys ...............................100

Chlorocleptria

chlorofasciata Dietz 4270

chloroleuca Meyrick 6897

chloroleucaria Guenee 3818

chloropha Hubner 1085

Chloropteryx

Chlorosea

chlorostigma Harvey 2399

Chlosyne

chœrilus Cramer 745

chorda Grote 2052

Choreutis

choriona Reakirt 934

choris Harvey 1295

Chorizagrotis'

chortæa Meyrick 7006

chortalis Grote 5018

chortalis Harvey 1544

Chrismania

christina Edwards 64

chromalis Guenee 4969

Chrysaugina

chryseis Olivier 964

chryselectra Grote 2197

chrysella Grote 1150

chrysoadspersella Dietz 8311

Chrysoclista

chrysocomella Dietz 8445

chrysomelas $H y$. Edwards 61

chrysopasa Walker 2740

Chrysopeleia 152

Chrysophaninze

chrysopis Grote 2741

Chrysopora

Chrysoptera 84

chrysoptera Wright 270 
chrysorrhoes Linnaeus 8723

chrysothemoides Verity 59

chrysotypa Meyriek 7219

Chrysoxena 176

chrysurella Dietz 6622

chryxus Doubleday \& Hewitson 122

chunka Smith 1704

chusea Edwardo 585

Chutapha

Chytolita

Chytonix 64

cibalis Grote 2048

cicatricosa Grote \& Robinson 1242

cicutaella Kearfott 8093

cilixeochrella Chambers 7758

cilialineella Chambers 6025

ciliata Smith (Schinia) 1151

ciliata Smith (Oncocnemis) 2065

eilicoides Grote 842

ciliella Busck 6423

\section{Cilla}

cilla Behr 429

cilliæfuscella Chambers 8370

cimbiciformis Stephens 732

cimicifugata Pearsall $\mathbf{4 1 4 9}$

cincaidella Dyar 5812

cincinnatana Kearfott 7505

cincinnatiella Chambers 7941

Cincinnus

cincta Edwards 260

eincta Smith (Kakopoda) 3384

cincta Smith (Metalestra) 3484

cinctaria French 4622

cinctarius Hulst 4508

cinctella Hulst 5648

cinctipalpis Smith $\mathbf{3 3 7 4}$

cinctipedalis Walker 5119

einctipes Grote 920

cinctus Tepper 765

Cindaphia .133

cinderella Riley 7419

cinderella Smith (Cucullia) 1996 cinderella Smith (Acronycta) 2481

cinderella Smith (Raphia) 3293

cinefacta Grote 2278

cineraceus $F$ ish 5899

cineraria Walker 4604

cineraria Packard 4450

cinerascens Smith 1479

cinerascens Welsingham 5893

cinerascens Walker 3641

cinerea Edwards 452

cinerea Harris 687

cinerea Morrison 3182

cinerea Riley 2153

cinerea Smith (Epidemas) 2114

cinerea Smith (Pleroma) 2185

cinerea Staudinger 1481

cinerea Walker 3671

cinereana Neumoegen \& Dyar 3012

cinereata Bates 4372

cinereella Hulst (Sarata) 5687

cinereella Hulat (Cactobrosis) 5696

cinereicollis Grote 1455

cinerella Chambers 7759

cinerella Murtfeldt 6171

cinereocostella Clemens 6482

cinereofrons Packard 3654

cinereoides Dyar 3671

cinereola Guenee 2704

cinereola Hubner 5454

cinereola Hulst 4388

cinereola Smith 2710

cinereomacula Morrison 1363

cinereomedia Dyor 5239

cinereopallida Smith 1243

cinereus Packard 3643

cinereus Riley 8468

cineridactylus Fitch 5942

cineritia Grote 2173

cinerosa Grote (Erinnyis) 718

cinerosa Grote (Graptolitha)

2154 
cinerosa Grote \& Robinson $5157^{\text {Page }}$

Cingilia .119

cingulata Fabricius 662

cingulifera Walker 3197

ciniflonella Zeller 6440

cinis Guenee 3312

cinna Ploetz 590

cinnabarina Grote 1751

cinnamomea Boisduval 929

cinnamomea Grote \& Robinson 3716

cinnamomea Herrich - Schaeffer 2873

cinocentralis Strand 2758

circa Stretch 973

circa Hy. Edwards 750

circe Strecker 3097

circulana Hubner 6901

circulifera Walker 3215

circumcincta Smith 1752

circumdata Grote 1398

circumlucens Smith 2668

circumscriptella Chambers 7633

circumscriptella Zeller 6009

circunvadis Smith 1783

circumvallaria Taylor 4055

cirphidia Hampson 2797

Cirphis

cirxhas Meyriek 7248

Cirrhobolina

Cirrhophanus

cirroferella Hulst $\mathbf{5 5 4 6}$

Cissia

Cissusa

Cisthene 30

cita Grote 1993

citata Grote 3574

Citheronia 28

citima $H$ y. Edwards 335

citima Grote (Agroperina) 2350

citima Grote (Phalænostola) 3470

citra Neumoegen \& Dyar 968 citrana Fernald 7368

citrata Linnaeus 3993

citricolella Chambers 6518

citricolor Grote 1258

citriella Chambers 6148

citrifoliella Chambers 6383

citrina Druce 2941

citrina Hubner 4666

citrina Grote \& Robinson 5022

citrinaria Neumoegen \& Dyar 971

citrinellus Grote \& Robinson 1092

citrinipennella. Clemens 7857

citrites Meyrick 5937

citronella $B . \& M c D .1209,1$

citronella Smith 1964

civica Grote 2578

Cladara .104

clandestina Harris 1461

clandestina Walker 3680

clandestinella Busck 5969

clappiana Holland 3587

clappi Maynard 78

clara Beutenmuller 3053

clara $H y$. Edwards 414

clara Harvey 2604

clarescens Guenee 2442

clarioralis Walker 5561

clarki B. \& McD. 2967, 1

clarkiæ Boisduval 752

clarkiæ Franck 705

claromonta Smith 1284

clarus Grote \& Robinson 867

clarus Cramer 468

classicata Pearsall 4244

clauda Grote 2556

claudens Walker 2359

claudia Cramer 151

claudialis Walker 5214

claudianus Stichel 24

claudiella Kearfott 6014

claufacta Walker 2330

clausella Walker 6482 
elausula Grote 2964

clavana Fernald 7067

clavans Walker 6807

clavana Zeller 7029

claviformis Morrison 1474

clavipes $R . \&$ J. 729

claviplena Grote 1659

claypoleana Riley $\mathbf{7 1 5 6}$

cleis Edwards 325

clemataria Smith \& Abbot 4780

elemens Smith 1460

clemensalis Dyar $\mathbf{5 4 9 0}$

clemensella Chambers (Agnopteryx) 6447

clemensella Chambers (Holcocera) 6562

clemensella Chambers (Batrachedra) 7822

clemensella Chambers (Tischeria) 7854

clemensella Chambers (Lithocolletis) 7884 . :

clemensella Chambers (Philonoma) 8101

clemensella Chambers (Amydria) 8204

clemensella Chambers (Xylestia) 8234

clemensella Chambers (Nepticula) 8364

clemensella Stainton 5957

clemensellus Robinson 5312

Clemensia

clemensiana Fernald 7367

cleobrea Godart 86

cleomes Boisduval 30

cleopatra B. \& MCD. 8337

cleopatra Strecker 3061

Cleora

cleoriformis B. \& MeD. 2877

clethre Dyar $\mathbf{8 4 7}$

clientis Grote 3385

clintoni Grote $\mathbf{3 1 1 4}$

clintoni Grote \& Robinson 8710 clio Edwards 186

clio Packard 1027

clitosalis Walker 3523

clitus Edwards 533

clivinaria Guenee 4582

cloacella Haworth 8290

cloanthoides Grot6 1394

clodiana Grote 1410

clodius Menetries 24

cloniasalis Walker 3536

clorinde Godart 52

clothilde Edwards 393

clotho Fabricius $\mathbf{7 4 2}$

cluna Strecker 2248

Clydonopteron 137

elymena Cramer 297

clymene Brown 1031

clytie Edwards 347

clyton Boisduval \& LeConte 313

Cnephasia 178

cnidia Godart 49

Cnidocampa 126

c-nigrum Linnaens 1424

cnossia Druce 2912

cnotus Hubner 744

coagulata Guenee 4168

Cobaliodes

cobeta Smith 3496

Cobubatha

cocana Kearfott 7001

cocandicides Verity 69

Cocceius 18

coccidivora Comstock 5730

coccinata Grote 3097

coccinea Beutenmuller 6738

coccinea $\boldsymbol{H y}$. Edwards 870

coccinea Warren 5159

coccineifascia Grote 3459

cocciniceps Schaus 932

coccivorella Chambers 6519

cochise Dyar 3604

Cochisea 119

cochiseata Swett 4012

cochrani Riley 1304 


cochrusalis Walker 4926
cockerella Busck (Aristotelia)
6051 Pago
cockerella Busck (Abebra) 7646
cockerelli Busck (Incurvaria)
8438
cockerelli Busck (Gelechia)
6244 cockerelli Dyar 8167
cockerellana Kearfott 7380
cockleana Kearfott 7243
cockleellus Kearfott 5352
cocklei Dyar (Scepsis) 825
cocklei Dyar (Sympistis) 2202
cocklei Smith 1282
cocles Lintner 312
cocoata Pearsall 4170
cocyta Cramer 247

Cocytius 23

cœlebs Grote 3026

colebs Martyn 977

coligenalis Hulst 4989

Colopoeta

Colostathma .186

coemitariella Chambers 8447

coenia Hubner 286

Conipeta

Conocharis 86

Conochroa

Cononympha 116

cononymphata Hulst 3928

conosipennella Clemens 7749

coercita Grote 1165

cœerulata Fabricius 4014

cofaqui Strecker 656

Cogia

cogitans Smith 1295

cogitata Smith 2345

cognata Smith 1884

cognataria Guenee 4609

cognataria Hubner 4602

cognizata Pearsall 4246

cohortalis Grote 5263

Colænis colata Grote (Itame) 4420

colata Grote (Euxoa) 1342

Coleophora 184

colfaxiana Kearfott 6770

colla Strecker 1264

collaris Fitch 905

collaris Grote \& Robinson 1423

collecta Walker 4570

collina Behr 254

collinusella Chambers 6115

collocata Smith 1255

Colocasia 83

colona Hubner 1032

colonella Linnaeus 5449

colonia Wright 211

coloro Wright 6

colorada Smith 2796

coloradalis $B$. \& McD. 5073

coloradana Fernald 7392

coloradaria Grote \& Robinson

4713

coloradata Hulst 4629

coloradella Chambers 6011

coloradella Dietz (Amydria)

8198

coloradella Dietz (Scardia) 8224

coloradella Hulst 5791

coloradella Kearfott 7620

coloradella Walsingham 6492

coloradellus Fernald 5374

coloradellus Kearfott 5403

coloradellus Walsingham 8177

coloradensis Beutenmuller 3050

coloradensis Busck 6207

coloradensis Cockerell 780

coloradensis $\boldsymbol{H} y$. Edwards

(Euchloe) 40

coloradensis $\boldsymbol{H}$. Edwards (Ianassa) 3650

coloradensis $\boldsymbol{H} y$. Edwards

(Memythrus) 6744 
Page

coloradensis Grote Robinson 5017

coloradensis Hulet (Eupithecia) $\$ 200$

coloradensis Hulet (Itame) 4415

coloradensis Putnam-Cramer 3290

coloradensis Ragonot 5741

coloradensis Riley (Megathymus) 654

coloradensis Riley (Prodoxus) 8465

Coloradia

colorado Grossbeck 4751

colorado Seudder 562

colorado Smith (Agrotiphila) 1223

colorado Smith (Oncocnemis) 2070

colorado Smith (Zale) 3196

colorado Smith (Conistra) 2213

coloradus Smith 697

colorana Kearfott 7277

coloraria Fabricius 4460

colorata Grote 4354

colorata Walker 996

colubrinæ Busck 6137

columbia $\boldsymbol{H}$ y. Edwards 159

columbia Rearfott 7123

columbia Scudder 559

columbia Smith (Samia) 768

columbia Smith (Polia) 1671

columbiana Hy. Edwards 3012

columbiana Smith 2098

columbiana Walsingham 7082

columbiata Dyar 4158

columbiata Taylor 4014

columbina Godart 151

columella Fabricius 345

columelloides B. \& McD. 4051

Comacla

comacornella Hulst 5519

Comadia 195 comatulana Zeller 6926

combinata Walker 3352

comes Walsingham 7531

cometana Walsingham 7201

comis Grote 1755

comma Hurris 269

comma Linnaeus 562

comma Walker 1031

commelinse Smith \& Abbot 2568

commixtalis Walker 5032

commoda Walker 2311

commoides Guenee 1935

Commophila 180

commortalis Grote 5172

communis Dyar 1859

communis Grote (Catocala) 3048

communis Grote (Loxostege) 5025

communis Grote (Pyrgus) 495

comonana Kearfott 7557

comosa Morrison 1314

compactata Taylor 4181

somparata Moeschler 1506

compensata Walker 3870

complanoides Frey \& Boll 7876

complicata Walker 980

complicata Strecker 1948

Composia 92

composita $H y$. Edwards 2818

compressipalpis Guenee 3400

compressipennis Smith 1282

compta Clemens 7731

compta Walker 3654

comptana Froelich 7185

comptaria Walker $\mathbf{4 1 1 6}$

comptella Ragonot 5550

comptoniella Hulst $\mathbf{5 5 4 4}$

comptulatalis Hulst 5431

comstocki Grote 2098

comstockiana Fernald 6762

comus Edwards 633

comyntas Godart 424

cona Strecker 1268 


Conacontia
conar Strecker 1852
conarata.................... 76
conceptata Pearsall 4242
conchana Hubner 6841
conchiformis Grote 2176
conchis Grote 1427
conchula Felder 1150

Conchylodes .129 conciliaria Swett 4042 concinna Smith 1112 concinna Smith \& Abbot 3655 concinna Warren 5068 concinnalis Hampson 4944 concinnanum Clemens 6800 concinnimacula Guenee 2897 concinusella Chambers 6186 concisa Walker (Perigea) 2329 concisa Walker (Epizeuxis) 3486

concisaria Walker 4699

conclusella Walker 6283

concolor Grote 3125

concolor Zeller 7855

concolorella Beutenmuller 6419 concolorella Chambers (Ithome) 5976

concolorella Chambers (Elachista) 7829

concolorella Clemens $\mathbf{7 7 6 0}$ concordata Walker 4077 concumbens Walker 3082 concursana Walker 7323 condaliæ Busck 7588 condaliafoliella Busck 8365 condaliavorella Busck 6371 condensata Walker 4118 condita Guenee 1513 conditana Walsingham 6826 conducta Walker 3410

Condylolomia 138

Condylorrhiza 132 confederata Grote (Perigea) 2324

confederata Grote (Euryoyttarus) 4803 confertella Walker 6424 configurata Hulst 4569 configurata Walker 1606 confinis Walker 1033 confinisalis Walker 3460 confixana Walker 6877 conflexana Walker 7185 conflictana Walker 7363 confligens Walker 3363 confluana Kearfott 7029 confluens Cockerell 8465 confluens Morrison 1919 confluens Oberthur 964 confluentella Dietz 6541 conformata Pearsall 4141 confovealis Hulst 5120 confracta Morrison 1304 confragosa Morrison 2178 confusa Hubner 1878 confusa Lyman 1034 confusa Smith 1569 confusa Walker 3654 confusa Worthington 3051 confusalis $B . \& M c D .5221$ confusalis Hulst 4958 confusalis Walker 5218 confusana Robinson 7446 confusaria Hubner 4758 confusella Chambers 6209 confusella Dietz (Amydria) 8203

confusella Dietz (Pigritia) 6579 confusella Walker 6633 confusellus Dyar 8182 confusus $\boldsymbol{H} y$. Edwards 8488 congeminatella Zeller 8234 congermana Morrison 1928 conglomeratella Zeller 7945 congregata Walker 4053 


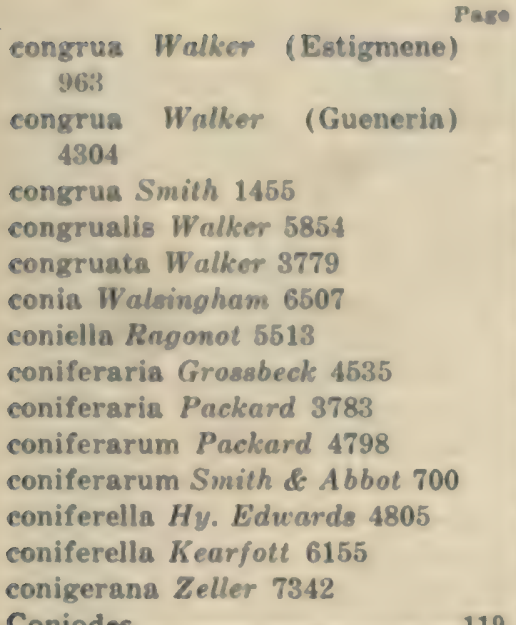

Coniodes 60

conjugata Smith 1654

conjugella Zeller 7703

conjuncta $B$. \& McD. 888

conjuncta $\boldsymbol{H y}$. Edwards 1039

conjuncta Smith 1271

conjuncta Watson 790

conjunctana Moeschler 7088

conjungens Walker 2595

connecta Grote 2433

connecta Smith (Eumichtis)

2192

connecta Smith (Cropia) 2321

connectus $\boldsymbol{B}$. \& $\mathbf{M c D} .8353,1$

connexalis Walker 4949

connubialis Guenee 3119

Conochares

conocharodes Hampson 2938

conradi Grote 2349

consanguinana Walsingham 6824

conscita Walker 1032

consecutaria Walker 3901

consepta Walker 4341

consequaria $\boldsymbol{H}$. Edwards 3810

conserta Grote 2182
Conservula 63

consimilata Zeller $43 \$ 1$

consimilella Frey \& Boll (Lithocolletis) 7924

consimilella Frey \& Boll (Gracilaria) 8050

consimilis $\boldsymbol{H} y$. Edwards 6668

consimilis Warren 5001

consobrina Guenee 3124

consobrinella Zeller 5585

consocia Walker 2329

consonella Zeller 6189

consopita Grote 1836

consors Smith \& Abbot 3022

consors Smith 2623

consortalis Dyar 5306

conspecta $\boldsymbol{H}$. Eduards 3657

conspersa Riley 2239

conspersella Ragonot 5839

conspicua Dyar 854

conspicua Edwards 610

conspicua Morrison 2380

conspicus Neumoegen 1003

conspicua Smith 3128

conspicua Stretch 974

conspicua Worthington 3051

conspicuella Dietz 8021

constans $\boldsymbol{H} y$. Edwards 4879

constans Hulst (Catocala) 3106

constans Hulst (Sabulodes)

4766

constantella Hulst 5647

constellatana Zeller 6835

constipata Walker 1765

constricta $\mathrm{Hy}$. Edwards 1130

constricta Strand 4014

constricta Stretch 3751

constricta W'alker 1219

constrictana Zeller 6971

constrictella Zeller 5972

constrictina Neumoegen \& Dyar

3746

consuela Strecker 2761 
consueta Walker 1414

consularis Dyar 799

contacta Walker 2518

contadina Smith 2179

contagionis Smith 1259

contatella Grote 5631

contempta Guenee 3160

contemptata Guenee 4345

contenta Grote 2143

contenta Walker (Septis) 2262

contenta Walker (Sidemia) 2367

contexta Grote 3257

contigua Walker (Haploa) 1035

contigua Walker (Charadra) 3215

continens $\boldsymbol{H y}$. Edwards 2618

contingens Walker 4777

continua $\boldsymbol{H y}$. Edwards 3586

continuaria Walker 3857

continuata Walker 4385

continuella Zeller 6211

contortalis Guenee 5191

contracta Walker (Lygran-

thocia) 1130

contracta Walker (Metalestra) 3479

contracta Walker (Datana) 3607

contractata Packard 3989

contradicta Smith 2383

contrahens Walker 1851

contraria Walker (Ceramica) 1930

contraria Walker (Нyppa) 2531

contrariana Walker 6821

contrariella Walker 6483

contrasta B. \& McD. (Nocloa) 2778

contrasta $B$. \& $M C D$. (Litodonta) 3629

contrastana Kearfott $\mathbf{7 5 3 4}$

contubernatella Fitch 6380

convalescens Guenee 3131
Page

convallaria Guenee 4042

convergaria Walker 4289

conversana Walsingham 7223

convexipennis Grote \& Robinson .... 2005

conviva Harvey 2593

cooleyi Fernald 5883

cooperi Behr (Anthocharis) 44

cooperi Behr (Euphydryas) 206

coortaria Hulst 4409

cootenaiata Dyar 4255

Copablepharon 40

Coprodes 20

Copanarta 74

Copibryophila 67

Copicucullia 55

Copidryas 36

Copipanolis 58

Coptodisca 181

Coptotriche 186 coquillettella Busck (Gnorimo- :

schema) 6101

coquillettella Busck (Ethmia) 6618

coquilletti Smith 2994

cora Hubner 2405

coracias Guenee 3182

coracina Clemens 4883

coranella Dyar 6643

coras Cramer 583

corbis Godman \& Salvin 1

corculana Zeller 7056

cordelia $H y$. Edwards 3118

cordigera Thunberg 1634

cordova Barnes 2773

coresia Godart 300

corinthalis $B . \& M c D .5164$

cornelia Edwards 164 .

cornelia Neumoegen \& Dyar

8329

cornelius Godart 92

cornella Walsingham 7761

corni Hy. Edwards 6686 
cornifoliana Riley 7194

cornifoliella Clemens 7579

Cornifrons

cornix Guenee $\mathbf{8 1 8 2}$

cornusella Ely 8066

cornuta Grote 1463

cornutana Dyar 7166

corollaria Perry 780

coronada Barnes 3736

coronana Kearfott 6838

coronaria Strecker $\mathbf{4 7 9 1}$

coronatella Clemens 8127

coronides Druce 2756

coroniella Clemens 8058

coronis Behr 179

corosana Walsingham $\mathbf{7 0 4 0}$

correllata Hulat 4419

corrodera Smith 1454

corruscifasciella Chambers 8449

corruscipennella Clomens 7762

corticea Walker 3654

corticosa Guenee $\mathbf{2 4 2 5}$

corusca Hy. Edwards 6692

corusca Smith 2020

corusca Strecker 3262

coruscana Clemens 6834

corvina Boisduval 837

corylanium Fernald 6805

coryliella Chambers (Menesta)

6395

coryliella Dyar 5539

corylifoliella Clemen (Haploptilia) 7763

corylifoliella Clemens (Nepticula) 8366

corylisella Chamber 7963

Coryphista

Corythophora

coscinia Herrich-Schaeffor 648

Cosmia

cosmodactyla Hubner 5863

Cosmopterygidse

Cosmoptery $x$

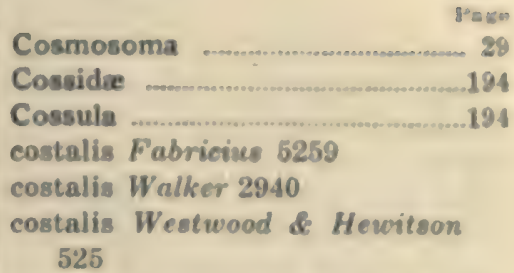

costarufoella Chambers 6360

costastrigulana Kearfott 6910

costata Grote 1358

costata Stretch 936

costiferalis Walker 5254

costigeralis Walker 5254

costiguttata Hulst 4103

costimacula Grossbeck 3938

costimaculalis Fernaid 5160

costimaculana Fernald 6846

costinotata Taylor 4765

costinotata Warren 4679

costinotata Walker 3920

costiplaga Smith 2594

costipunctalis B. \& $\mathbf{M c D}$. 5189

costipunctata B. \& MCD. 4031

costipunctella Moeschler 6230

costisignella Clemens 8312

costolutella Chambers 6367

costomaculana Clemens 7139

costotristrigella Chambers 8289

Cosymbia

cosyra Druee 1038

coticola Busck 6232

cottalis Walker 4923

cottlei Grinnell 436

couperi Grote 449

coverdalella Kearfott 6202

Coxina

coyote Skinner 475

Crambidia 30

crambidoides Grote 5436

Crambina 138

crambis Freyer 133

crambitans Walsingham 6896

crambitella Walsingham 6608 


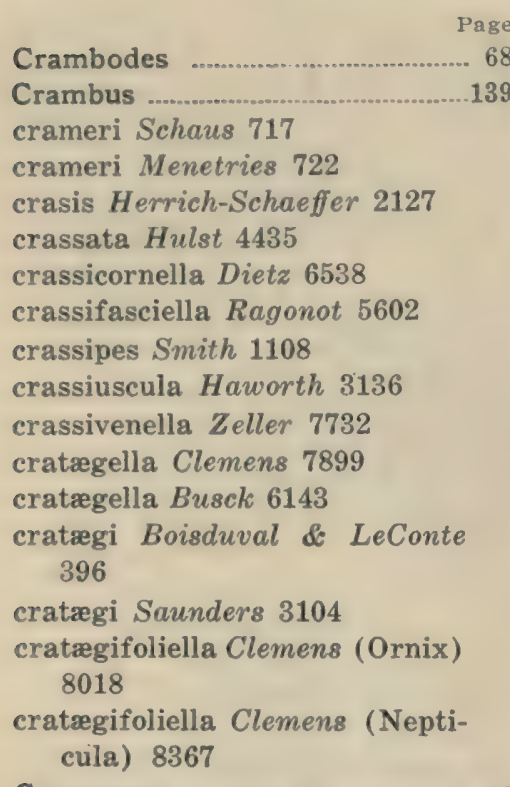

Craterestra

cratipennella Clemens 7764

Cremastobombycia .188

crenana Hubner 6996

crenilinea Smith 1196

crenulata B. \& McD. 5867

crenulata Butler 1838

crenulata Smith 1567

crenulatellus Kearfott $\mathbf{5 4 1 2}$

crenulellum Engel 7848

creola Skinner 91

creonalis Walker 4927

crepera Harris 8354

crepuscularia Schiffermuller 4604

crepuscularis Meyrick 6845

crescens Walker 1049

crescentana Kearfott 7132

crescentella Braun 8153

crescentella Dietz 6565

crescentella Kearfott 8208

crescentella Walsingham 7554 crescentifasciella Chambers 6190

cresina Smith 1064

cresphontes Cramer 11

cresphontinus Martyn 12

cressonana Grote 834

cressonella Chambers 6427

cressoni Grote 3352

cressoni Walsingham 8160

Cressonia 24

cressoniana Clemens 7115

cretacea Zeller 6417

cretacealis Lederer 5199

cretaceata Packard 4176

cretata Grote 4855

cretata Grote \& Robinson 2993

cretata Hulst 4211

cretata Smith 2488

cretaticostella Clemens 7765

cretidactylus Fitch 5924

cretidactylus Zeller $\mathbf{5 9 2 5}$

cretiferana Walker 2895

creusa Doubleday 39

cribrataria Guenee 4566

cricota Meyrick 7506

criddleana Kearfott 7124

criddlei Smith 1288

criddlella Dyar 5666

criminosa Swett 4467

Crimona 66

crinisalis Walker 5025

Crinodes 96

crispa Harvey 2219

crispana Clemens 7152

crispata Packard 4861

cristata Grote 2250

cristata Morrison 3347,1

cristatella Chambers (Recurvaria) 6147

cristatella Chambers (Elachista) 7830

cristatella Chambers (Homosetia) 8309

cristifasciella Chambers 6167 
l'ase

criatifera Walker 1693

cristifera Hulst 4611

critola Hewitson 339

crocale Educurds 263

erocallata Guenee 4862

erocataris Fabricius 4666

crocea $\boldsymbol{H}$. Eduards 2089

crocearia Hulat $\mathbf{4 4 3 8}$

crocearia Packard 4644

croceella Hulst 5601

croceoverticella Chumbers 8264

crocicapitella Clemens 8242

Crocidophora

erocotalis Grote 5133

croesus Dalman 757

crokeri Swett 4014

cronialis Druce 5219

Cropia

crossi Hulst 3841

crotalariella Busck 6181

crotehi $\mathrm{Hy}$. Edwards (Pseuda)ypia) 1051

crotchi Hy. Edwards (Schinia) 1168

crotchi Grote 1666

crucialis Harvey 1883

cruciata Grote 4387

eruciferarum Zeller 7683

cruciferarum Boisduval 35

cruciferella Dietz 8214

crudelis Grote 3389

cruentaria Hubner 4460

eruralis Guenee 3508

crustaria Morrison 2858

crydina Dyar 1665

Cryphia

erypta Dyar 4854

cryptalis Druce 5223

Cryptolechia 160

cryptolechiella Chambers $\mathbf{6 4 3 2}$

Cryptophobetron 126

erysalus Edwards 335

crysothemoides Verity 57

Ctenucha cubanalis Guenee 4938

cubensis Grote (Calidota) 1022

cubensis Grote (Protoparce) 667

cubilis Grote 2112

euculifera Herrich-Schaeffer 3625

Cucullia

cuculliformis Grote 2245

Cucullinine 5is

cucurbitre Harris 7009

cuerva Bames 2412

culea Guenes 1835

culicaria Guenee 3915

culiciformis Linnoens 6683

culiculalis Hulst 5291

culminara Walsingham 6953

culta Lintner 3260

cumatilis Groted Robinson 4068

cumatilis Grote 1143

cumulalis Dyor 3452

cumulata Swett $\mathbf{4 0 0 9}$

cunea Drury 959

cuneana Walsingham 7285

cunearia D'Urban 4604

cuneata Goeze 995

cuneata Grote 1714

cuneolalis Hulat 5324

cunigerata Walker 3983

cunninghami Owen 184

cupedinaria Grote 3780

cupentia Cramer 2324

cupes Grote 1168

cupida Grote 1590

cupida Meyrick 7226

cupidaria Grote 4615

cupidata Swett 4009

cupidinella Hulst 5843

cupidissima Grote 1576

cupola Hampson 1527

cuprea Busck 7606

cuprealis Hubner 5249

cupressana Kearfott 7275

cupressatn Pearsall 4215 


\begin{tabular}{|c|c|}
\hline 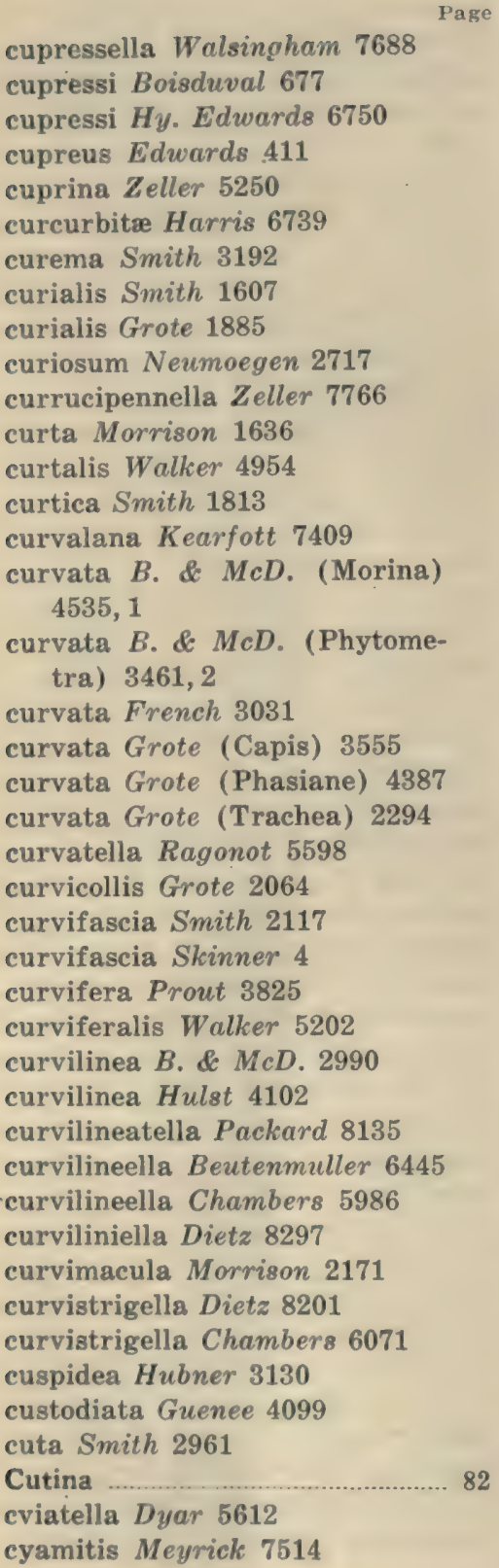 & 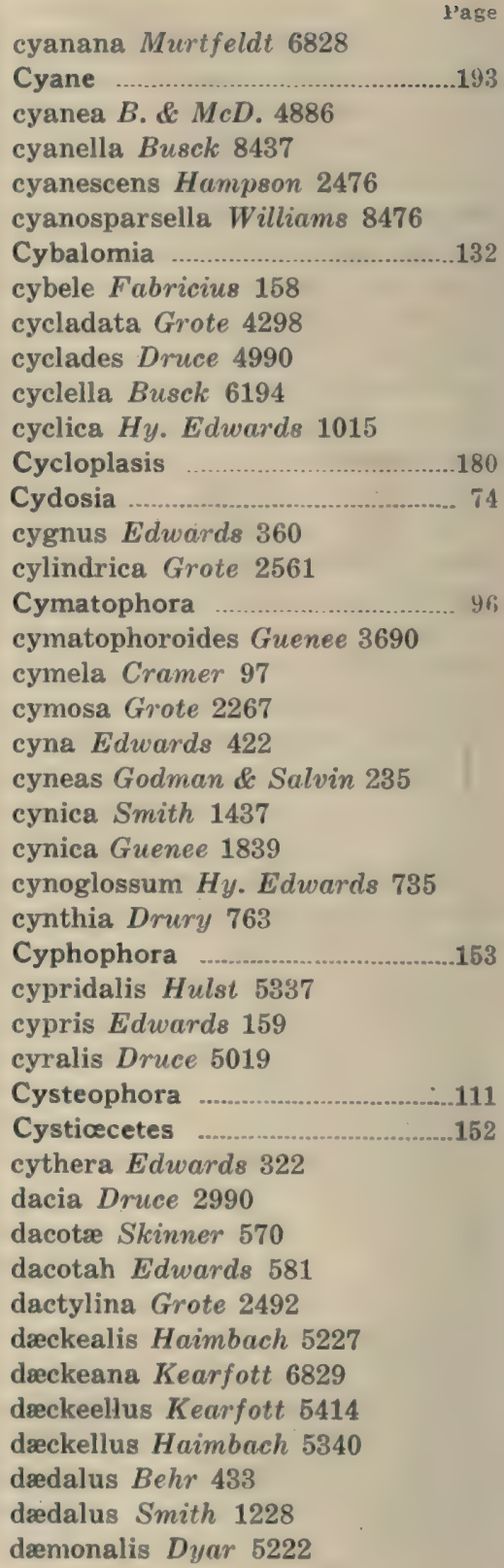 \\
\hline
\end{tabular}




\begin{tabular}{|c|c|}
\hline $\begin{array}{r}\text { Yhane } \\
\quad 29\end{array}$ & Dasefidonis \\
\hline Dahana & Dasylidonsa in \\
\hline daira Druee 4067 & Dasylophia . _ _... 93 \\
\hline daira Godart 80 & Dasypyga .... \\
\hline dakota Smith 1321 & Dasyspoudrea \\
\hline Dalcerida & $\begin{array}{lllll}\text { Dasyuris } & \ldots & \ldots & \ldots & \ldots\end{array}$ \\
\hline Dalcerides ……........................... & Datana ...... \\
\hline Dalla & dataria Grote 4578 \\
\hline dallasiana Frey \& Boll 8368 & dataria Hulst 3916 \\
\hline dama Guevee 2931 & datisalis Druec 4955 \\
\hline damalis Grote 2111 & daucus Cramer 762 \\
\hline damaris Felder 74 & daunia Edwards 448 \\
\hline damastus Godart 377 & daunus Boisduval 15 \\
\hline damnosalis Walker 3566 & daunus Cramer 485 \\
\hline damoetas Skinner 225 & daunus Herbat $157^{\circ}$ \\
\hline damon Cramer 377 & dauria Strecker 130 \\
\hline dana Kearfott 7233 & davena Smith 1755 \\
\hline dana Cramer 315 & davidsoni $H y$. Edwards 4797 \\
\hline Danaidx & davisellus Beutenmuller 8193 \\
\hline Danaus . & Davisia \\
\hline danbyi Hulat (Paraptera) 3963 & davisi $H y$. Edwards 920 \\
\hline danbyi Hulst (Spodolepis) $\mathbf{4} 493$ & dawsoni $B$. \& $M c D .189$ \\
\hline danbyi Neumoegen (Diacrisia) & dayi Grote 2016 \\
\hline 956 & dealbana Walker 6867 \\
\hline danbyi Neumoegen (Gluphisia) & debilis Walker 2956 \\
\hline 3681 & Decantha \\
\hline dandana Kearfott 7234 & Decaturia ................. 142 \\
\hline danistica Grote 2844 & decemmaculella Chambers 6317 \\
\hline Dannemora & decempunctana Walsingham \\
\hline danum Cramer 727 & 7080 \\
\hline daona Druce 4830 & decepta Grote (Mythimna) 1548 \\
\hline dapalis Grote 5078 & decepta Grote (Trichoclea) 1785 \\
\hline daphnis Cramer 158 & decepta Strecker 3345 \\
\hline daphnis Groy 12 & deceptalis Walker 3565 \\
\hline daphnis Martyn 159 & deceptana Busck 7527 \\
\hline dapsilis Grote 1465 & deceptana Kearfott 6819 \\
\hline daracana Kearfott 7077 & deceptata Hulst 4290 \\
\hline Darapsa & deceptiva Beutenmuller 6659 \\
\hline dargo Streeker 1245 & deceptricalis Zeller 3500 \\
\hline daria Druce 2558 & deceptusella Chamber 7907 \\
\hline Daritis 92 & decernens Walker 1413 \\
\hline darwiniata Dyar 8791 & decia Grote 2850 \\
\hline dasconalis Walker 5016 & decia Boisduval 941 \\
\hline Dasycosymbia . . _ _ & decimerella Hulat 5594 \\
\hline
\end{tabular}


decipiens Grote (Parastichtis) 2223

decipiens Grote (Richia) 1542

decipiens $\boldsymbol{H}$. Edwards 6693

decipiens Riley 8460

decipiens Walker 3745

decipientella Dyar 5595

decisana Walker 6804

decisaria Walker 4700

declarans Walker 3177

declarata Wrulker 1338

declinata Grote 3006

decliva Grote 2206

declivis Meyrick 5936

decolor Mabille 454

decolor Morrison 1338

decolor Walker 2757

decolora Hy. Edwards 662

decoloralis Walker 5654

decoloraria Hulst 4729

decora Morrison 3218

decora Zeller 5444

decoralis Hubner 3471

decorata Druce $\mathbf{5 1 8 2}$

decorata Grossbeck 4374

decorata Hulst 4424

decorata Saunders 996

decorata Smith 3581

decorata Taylor 4001

decorata Worthington 3051

decorella Hulst $\mathbf{5 4 5 2}$

decorellus Zincken 5373

decorosella Busck 6615

deducta Morrison 3317

deductaria Walker 4708

defecta Grote 2801

defectaria Guenee $\mathbf{4 5 8 0}$

defectella Zeller 8284

defensaria Guenee 4042

defessa Grote 1625

definita Aaron 239

definita B. \& McD. 2073

definita Packard 3707 definitella Zeller 6012

defixaria Walker 3862

deflavata Staudinger 3987

deflecta Busck 6385

deffectana Robinson 7443

deflorata Fabricius 964

defloriana Martyn 995

defluata Walker 4457

Deidamia 26

Deilinia 111

dejecta Hulst 4559

dejecta Strecker 3040

delassalis Hulst 5651

delaware Eduards 597

delawaricus Zeller 5858

delecta $B$. \& McD. 1728, 1

delecta Walker 2992

delectata Hulst 4358

delectella Hulst 5570

deleta Hy. Edwards 2950

deleta Grote 2206

deleta Guenee 3161

deleta Hulst 4439

deletella Zeller 7701

delia Cramer 80

delicata $B . \& M C D$. (Amolita) 2807

delicata $B$. \& McD. (Euxoa) 1355

delicata Dyar 3649

delicata Grossbeck 3925

delicata Grote 2271

delicata Hulst (Ptychopoda) 3885

delicata Hulst (Stergamatæa) 4606

delicataria Dyar 3786

delicatella Walsingham 5964

delicatella Busck $\mathbf{7 6 4 5}$

delicia Dyar 2732

delila Fabricius 149

delilah Strecker 3084

delimitata Warren 3984 
delineata Guenee 3008

delinquens Walker 3957

delliella Fervald 6645

delorata Smith 1233

delotella Busck 6381

delphinii Boisduval 4816

delphuse Drues 5237

del-sud Wright 399

Delta

deludana Clemene $\mathbf{7 1 1 6}$

demacula Strand 2727

dematrialis Druce 4918

demissa Walker 1695

demissana Walsinghem 7300

demissaria Hubner 3888

demissella Braun 7817

demoditas Hubner 80

demonica Hubner 291

demorsaria Strecker 4493

demotella Grote 5533

demutabilis Smith 1389

dendraria Guenee 4607

denotata $\boldsymbol{H}$. Edwards 6748

densa Smith 1730

densa Walker 2785

densaria Walker 3818

densata Grossbeck 4079

densellus Zeller 5427

dentata Dyar 853

dentata $\boldsymbol{H}$. Edwards 3851

dentata Grote (A pharetra) 1516

dentata Grote (Melitara) 5695

dentatella Grote 1867

dentella Busck 6219

dentella Fernald 5326

denticulalis Horvey 3494

denticularia Dyar $\mathbf{4 5 3 0}$

denticularia Walker 3801

denticulata Hulst 4621

denticulata Grote 4351

denticulella Ragonot 5593

denticulodes Hulst 4423

dentifera Walker 2997 dentiferella Wa!singham 7654

dentilines Hampsom 5206

dentilines B. \& McD. 4040

dentilinea Smith (Cucullis)

1989

dentilines Smith (Feltia) 1409

dentilineatells B. \& MCD. 5423

dentilineella Hulst 5475

dentosa Grole 5272

dentosella Ragonot 5725

denuba Ploetz 454

denudata Slosan 964

denudata Neumoegen 794

denudatum Harris 6755

denussa Ehrman 3029

denvera Smith 2493

denverana Kearfott 6935

Deoclona .156

depauperata Oberthur 965

depicta Grote 2851

depilis Grote 2109

deplanaria Walker 4706

deplanaria Guenee $\mathbf{4 4 9 0}$

depontanata Grote $\mathbf{4 7 6 3}$

Depressaria

161

depressostrigella Chambers 6258

deprivata Waller 3818

depromaria Grote 4550

depuiseti Boisduval 74

depuratella Busck 6298

derasa $\boldsymbol{H}$. Edwards 3688

Dercetis

derelicta Hampson 1530

deridens Guenee 3215

dernarius Smith 1540

Derrima 71

derupta Morrison 2573

descansalis Dyar 5284

descherei Neumoegen 3616

desdemona $\boldsymbol{H}$. Edwards 3084

degerta Felder 890

deserta Grinnell 2077

deserte $\boldsymbol{H} y$. Edwarde 3346 
deserta Smith 2055

desertana Zeller 7015

deserti Wright 44

deserticola $B$. \& $M c D$. (Lithostege) 3948,1

deserticola B. \& McD. (Schinia) 1168

desertus Hy. Edwards 832

deshaisiana Lucas 7271

designalis Guenee 4970

designata Hufnagel $\mathbf{4 0 3 8}$

designata Walker 1130

desistalis Walker 5090

Desmia

desmialis $B$. \& McD. 4925

desmodiella Clemens 7969

desmodifoliella Clemens 8053

desolatus Strecker 8493

desperaria Hulst 4501

desperata Guenee 3042

desperata Hulst 4133

desperata Smith 1712

destinata Moeschler 3981

Destutia

determinata Neumoegen 988

determinata Smith 1669

determinata Walker 4722

determinatella Zeller 6501

detersa Walker 1272

detersata Guenee 4455

detersella Clemens 6121

detesta Smith 1333

detracta Walker 1659

detractaria $B$. \& $M c D$. 4698, 1

detrahens Walker 3370

detritalis Guenee 5014

deutschiana Zetterstedt $\mathbf{7 4 5 9}$

deva Edwards 617

devastator Brace 2367

devergens Hubner 3221

devia Grote 2215

devialis Grote 5264

devilliers Godart 2

devinctaria Guenee 4630 devotana Kearfott 6848

dia Grote 1941

Diachorisia 194

Diacrisia 33

Diactinia .105

diagonalis Dyar 3134

Diallagma

diana Cramer 154

diana Hy. Edwards 3082

diana Hubner 7605

diana Packard 788

dianella Dietz 6530

diantha Beutenmuller 3057

diaphanella Frey \& Boll 7913

Diaphania 130

diaphorus Walsingham 7725

diasema Boisduval 3227

Diasemia 132

Diastema

Diastictis

Diathrausta 136

Diatraa 141

diazoma Grote 3734

dibeliana Hubner 6789

dicax Meyrick 7543

Dichogama 129

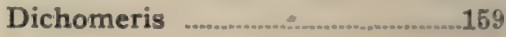

Dichorda 100

Dichordophora 101

dichrœella Ragonot 5828

Dicogaster

dicospilata Walker 4376

dictynna Walker 773

Dictyosoma 24

Dicymolomia

Didasys 29

didonea Smith 2615

Didugua 94

didyma Beauvois 813

diecki Neumoegen 987

Dietzia .192

dietziana Kearfott 7147

dietziella Busck (Hypoplesia) 8211 
dietriella Busck (Paltodora)

Pmern 6027

dietziella Kearfott 8299

diffascin Braun 8401

differens Druce 1375

differentialis Fernald 5441

differta Morrison 2654

differtellum B. \& MCD. 5775

difficilisella Chamber 6160

diftinana Walker 6992

diffinis Boieduval 785

diffissa Grote \& Robinson 5158

difformis Smith 1306

diffundens Walker 3156

diffusa Barnes 2781

diffusa Grote 2790

diffusa Smith 1177

diffusa Walker 1959

diffusana Kearfott .7029

diffusella Ely 5789

diffusilis Harvey 2518

digitalis Grote 2232

digitalis Smith 1136

digna Morrison 1498

dilara Streeker 2299

dilatana Walsingham 7041

dilatata Smith 1701

dilatifasciella Ragonot 5709

dilatocula Smith 2164

dilects $H y$. Edwards 1810

dilucids Morrison 1552

diluculella Grote 5480

dilutana Walaingham $\mathbf{7 4 8 9}$

dilutata Schiffermuller $\mathbf{4 0 6 9}$

diluticostana Walsingham 7296

dilutifuscana Walsingham 6868

dimediatella Ragonot 5845

dimidata Smith 2884

dimidiata Grote 2819

dimidiata Stretch 941

dimidiata Herrich-Schaeffer 4867

dimidiatellus Grote 5396

dimidiella Walsingham 6493

diminutalis Wulker 4918

diminuatana Kearfott 7205

diminutiva Graef 984

diminutiva Grote $28: 35$

dimmocki Grote 1712

dimorphella Busck 6409

dimotalis Walker 4900

dina Geyer 77

dinalda Smith 2081

dion Edwards 609

dionalis Walker 5100

Dione

dione Fabricius 977

dione Seudder 402

dionea Smith 2261

dionysius Scudder 109

dionyza $H y$. Edwards 3021

Dioptidx

Dioryctria

diospyriella Chambers 7587

diotimealis Walker 5025

diphteralis Geyer 4942

diphteroides Guenee 2418

diplocyma Hampson 2652

dipsaci Grote \& Robinson 1045

Dipterygia 60

dircella Braun 7987

Dircenna

directana Walker 7294

direptana Walker 7153

dis Grote 1202

disa Thunberg 140

discalis Grote (Polia) 1660

discalis Grote (Metalestra) 3478

discana Kearfott 7506

discerpta Walker 3380

discessalis Walker 4932

Discestra 48

disciferalis Dyar 5255

discigerana Walker 7176

discigerella Ragonot 5808 
discinigra Walker 2125

discipula Meyrick 6998

disippe Godart 308

disjunctaria Packard 3983

discissa Walker 3153

discistriga Smith (Hoplolythra) 2748

discistriga Smith (Platysenta) 2597

discitincta Walker 1506

discivaria Walker 2364

discoannulella Chambers 6318

discoferana Walker 7183

discoidalis Grossbeck 4131

discoidalis Grote 1583

discoidalis Kirby 137

discoidalis Skinner 377

discoidalis Walker 2963

discolor Smith (Aplectoides)

1514

discolor Smith (Anarta) 1644

discolor Smith (Brachylomia) 2123.

discolor Speyer 1710

discoloralis Guenee 3524

discolorata Neumoegen 3746

discolorella Braun 8404

discomaculella Chambers 6310

disconnecta Smith 2979

disconotella Chambers 6055

disconventa Walker 4454

discoocellella Chambers 6245

discopunctana Clemens 7281

discopunctella Dietz 6598

discors Grote 1526

discospilata Walker 4289

discostriata Walsingham 7767

discostrigella Chambers (Ethmia) 6631

discostrigella Chambers (Gelechia) 6319

discostrigella Dyar 5816

discreta B. \& McD. 1825, 1

discreta Stretch 3586

disertalis Walker 4931

dislocaria Packard 4361

dislocata Smith 1439

Dismorphia

dispar Grote 5198

dispar Linnaeus 3722

dispar Smith 2522

disparata $H y$. Edwards 1040

disparata Warren 4415

dispersellus Robinson 5311

displiciens Walker 1304

disposita Morrison 2136

dispulsa Morrison 3217

dispuncta Walker (Macaria) 4345

dissecta Grote \& Robinson 2453

dissectalis Grote 5121

dissectella Z eller 6067

dissectus Grote 5349

disserptaria Walker 4607

disseverans Walker 3140

dissimilaria Hubner 4460

dissimilis $B . \& M c D$. (Cirphis)

1949

dissimilis $B$. \& $M c D$. (Nocloa) 2777

dissimilis Hulst 4474

dissitana Grote 7341

dissocians Walker 3144

dissociarius Hulst 4537

dissociata $B . \& M c D .2782$

dissona Moeschler 1350

dissonaria Hulst 4551

disstria Hubner 3752 .

distans B. \& McD. 2195

distans Grote 2497

distema Grote (Cilla) 2808

distema Grote (Laspeyresia)

7210

disticha Morrison 1809

distichoides Grote 1543

distigma Walsh 803 
distigmana Walker 7029

distincta Freneh 3742

distincta Grote 2903

distincte Hubner 1670

distineta Neumoegen 8137

distincta Walsingham 7298

distinctaria $D^{\prime}$ Urban 4290

distinctella Diels 6515

distracts Smith 1575

distracta Walker 2998

distribuaria Hubner 4343

distycharia Guenee 4757

ditrapezium Schiffermuller 1429

ditta B. \& MeD. 3635

diva Grote 2854

diva Hulst 4479

diva Riley 7600

divaricata Grote 2242

divergens Walker (Lygranthocia) 1135

divergens Walker (Lithacodes) 4842

divergens Walker (Euxoa) 1353

divergens Behr 3329

diversella Braun 7970

diversicolor Morrison 2339

diversilineata Grote 2517

diversilineata $\mathrm{Hubner} 3977$

diversus Busck 8158

dives Smith 2236

dives Dietz 6531

divesta Grote 2290

Diviana 148

dividua Grote 2910

divinula Grote 2848

divisa Walker 3152

divisana Hubner $\mathbf{7 4 3 9}$

divisana Walker 7192

divisaria Walker (Hydriomena) 4014

divisaria Walker (Ectropis) 4604

divisata Walker 4465
I'ane

Divitiaca

3105

dnopherella Ragonot 5685

Doa 97

dobla Smith 1065

docilis Grote 1417

docta Walker 98.3

dodana Kearfott 7003

dodata Taylor 4193

dodecana Zeller 6902

dodgei Butler 989

dodgei Morriaon 1754

Dodia 32

dodi Smith 1716

doira Strecker 1737

dolabella Zeller 6379

Dolba 23

Dolbogena 23

dolens Druce 2450

dolens Smith 1320

Dolichorrhinia 148

dolichos Fabricius 2568

dolichus Hubner 334

dolli Beutenmuller 3100

dolli Dyar 900

dolli Grote 1229

dolli Neumoegen (Paranthrene) 6757

dolli Neumoegen (Sphinx) 697

dolli Smith 2715

dolliana Dyar 4819

dolliata Grossbeck 4606

dolores Neumoegen \& Dyar 3729

dolorosa Dyar 2497

dolorosata Pearsall 4250

dolosa Grote 1887

dolosa Strecker 1133

domalis Guenee 5249

domicella Erichson 499

domina $H y$. Edwards 2846

domingonis Butler 719

dominicaria Guenee 3833

dominicata Guenee 3353 
domitianus Fabricius 327

domonans Kearfott 6991

donataria Walker 4434

dorantes Stoll 459

Dorata

dorcas Kirby 408

doris Boisduval 977

doris Barnes 781

dormitans Guenee 3018

dorneraria B. \& McD. 3844

dornerì B. \& McD. 2996

dorothea Dyar 3675

dorsalana Dyar 7381

dorsalis Smith 1991

dorsiatomana Kearfott 7079

dorsilutea Walker 1119

dorsimacula Dyar (Illice) 889

dorsimacula Dyar (Acrolophus)

8191

dorsimaculana Robinson 7462

dorsimaculella Chambers 7673

dorsimaculella Kearfott 7672

dorsipallidella Chambers 8079

dorsipunctellus Kearfott 5370

dorsipurpurana Kearfott 7409

dorsisignatana Clemens $\mathbf{7 0 2 9}$

dorsistriatana Walsingham 7498

dorsistrigella Clemens 8241

dorsisuffusana Kearfott 7017

dorsivittella Zeller 6146

dorsomaculella Dietz 6601

dorus Edwards 465

Doryodes

dotalis Hulst 5808

dotella Dyar 5746

doubledayi Guenee 3015

doubledayi Packard 3620

doubledayi Scudder 3643

draco Edwards 586

dracusalis Walker 4959

Drasteria

drasteroides Smith 2728

Drepana 98

Drepanida

Drepanulatrix

drewseni Staudinger 1347

drexeli Hy. Edwards 3597

dromicella Busck 6215

drumalis Dyar 5213

drupacearum Boisduval 3752

drupiferarum Smith \& Abbot 696

druræi Donovan 662

drury Latreille 590

drusius Edwards (Cocceius) 482

drusius Edwards (Phyciodes) 243

dryadata Hulst 3930

dryadella Hulst 5760

dryas Edwards 269

Dryope 163

dryope Edwards 361

Dryoperia 163

dryopterata Grote 4790

Dryotype 58

dualis B. \& McD. 4907

duanca Smith 1591

duaria Guenee 4689

dubia $B$. \& $M c D .1849$

dubia Walker 960

dubiana Clemens 7187

dubiosa $B$. \& McD. 1825

dubiosella Beutenmuller 7683

dubitans Walker 2344

dubitans Tepper 1071

dubitatella Zeller 6429

dubitella Chambers 6364

dubitella Dietz 8020

ducalis Smith 1244

ducens Walker (Schizura) 3654

ducens Walker (Feltia) 1402

ducta Grote 2187

dudiella Busck 6095

dugesi Cockerell 1049

dulciaria Grote 4478

dulciella Hulst (Tacoma) 5580 
dulciella Hulet (Honora) 5750 dulciola Grote $\mathbf{3 1 1 3}$

dumetellus Hubner 5350

dumeti Behr 322

dumetorum Boieduval 389

dunbari Harvey 2073

duodecemlineata Packerd $\mathbf{4 1 1 5}$

duodecemlineella Chamber 7997

duodecemstriata Wulsingham 7240

duovata Bird 2687

dupla Smith 2088

duplex Walsingham 6859

duplicata Bethune 3188

duplicata Bird 2699

duplicata Neumoegen \& Dyar 1032

duplicata Packard 4367

duplicata Worthington 3051

duplicatella Dyar 7826

duplicatus Grote 5399

duplicatus Smith 2737

duplipunctella Ragonot 5502

Durrantia

duryi Edwards 322

dyari Rivers 8754

ronge

dyari Swith 3133

dyari Taylor 4613

Dyaria

dyariella Busck (Gelechia) 6267

dyariella Busck (Chilocampyla)

8031

dyaus Grote 8260

dymas Eduards 231

Dynamine

Dynothea

dyonis Hubner 296

Dyotopasta

Dysephyra

Dysmigia

Dysocnemis

Dysodia

dyspteraria Grote 4897

Dyspteris

Dyspyralis

Dysstroma

Eantis

earina Morrison 1680

Earophila 108

eastmani Reiff 3952

eavesi $\boldsymbol{H} y$. Edwards 307

eavesi Stretch 997

eboracensis Zeller 8080

eborata Hulst 3950

ebriola Poey 81

ebulealis Guenee 4951

eburnea Walsingham 8073

ecclesialis Guenee 4931

Ecdytolopha 175

echinocystis Behr 3249

echo Edwards 452

echo Smith \& Abbot 1020

eclipsana Zeller 7216

Ecpantheria

Ectcedemia

ectrapela Smith 1782

ectrapelaria Grossbeck 4690

Ectropis

dyari Merricle 1033 


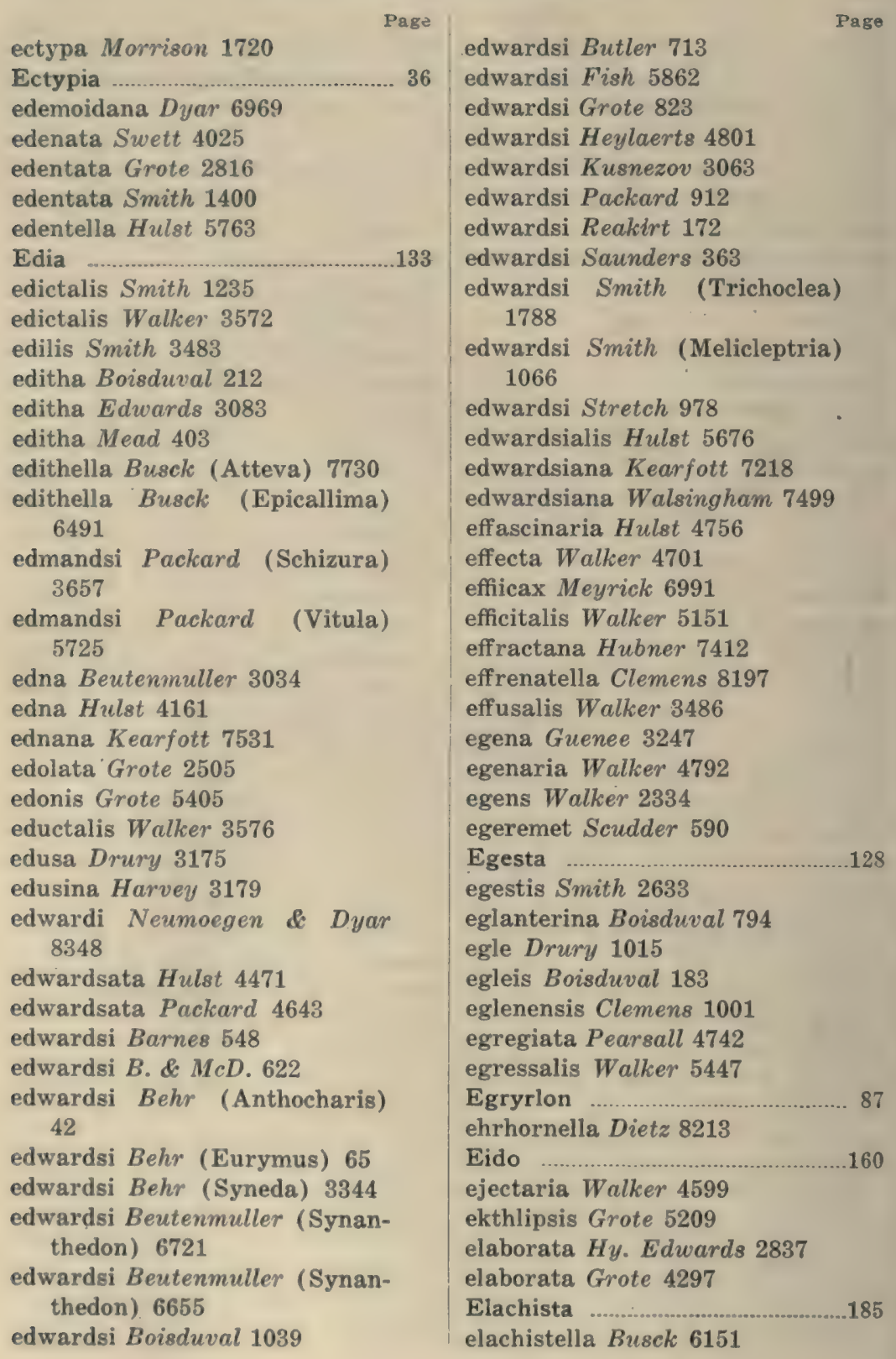




\begin{tabular}{|c|c|}
\hline 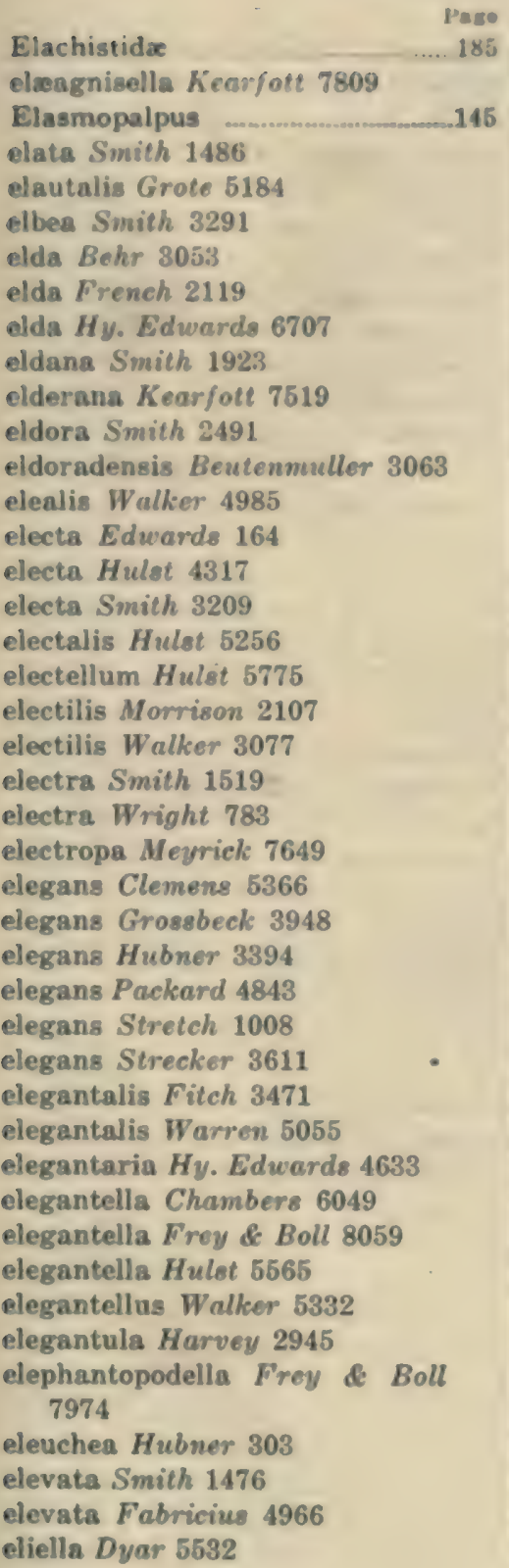 & 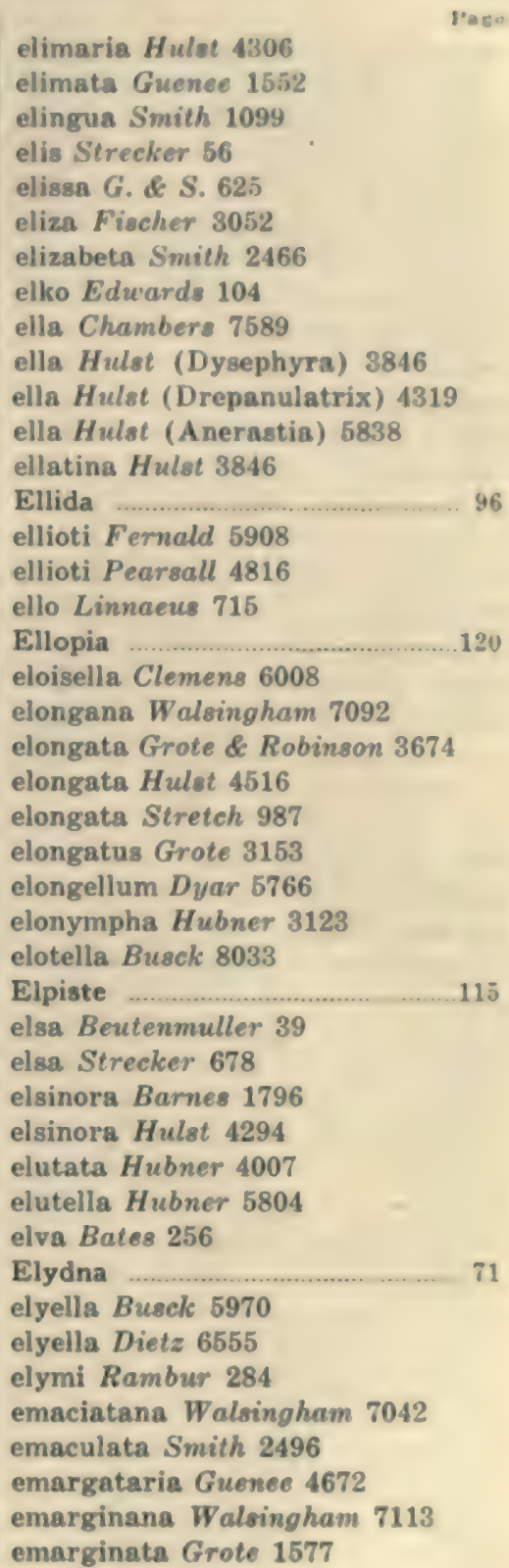 \\
\hline
\end{tabular}




\begin{tabular}{|c|c|}
\hline emarginata Smith 2155 & 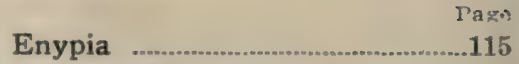 \\
\hline Emarginea ............................ & eodamus Cramer 316 \\
\hline emasculata Dyar 4581 & eoides $B . \& M c D .2870$ \\
\hline mblemella Clemens 6398 & eoleuca Meyrick 7227 \\
\hline $\begin{array}{l}\text { Emboløcia } \\
\text { emendata Pearsall } 4038\end{array}$ & $\begin{array}{l}\text { Eoreuma } \\
\text { eos Edwards } 631\end{array}$ \\
\hline Emesis & Eosphoropteryx ....................... 84 \\
\hline migdionis Grinnell 440 & Epargyreus ............. \\
\hline ia $H y . E d w a n$ & Epermenia \\
\hline Edwards 6 & idaria Hulst 4642 \\
\hline dwards & orth 4798 \\
\hline edonia Cra & Ephestia \\
\hline ia Gro & ephestiella Ragonot 5729 \\
\hline emphytiformis & .148 \\
\hline 71 & epl \\
\hline Enarmonia & tedt 5076 \\
\hline Enchrysa ........................................... & iatus $H a n$ \\
\hline is $G . \&$ S. 265 & ia $W a l$ \\
\hline Smith 1803 & Dyar 5434 \\
\hline otrichiinæe & Epia \\
\hline endropiaria Grote \& Robinson & 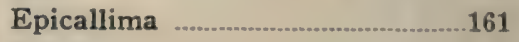 \\
\hline 4653 & hata \\
\hline Endrosis & epichysis Grote 2525 \\
\hline enervata Guenee 2809 & Epicnaptera \\
\hline s Guenee 1842 & onalis Ragonot 5286 \\
\hline engelana Kearfott 6978 & Epicorsia \\
\hline lla Busck 5996 & ata $B . \& M c D .4105,1$ \\
\hline li Dyar 5621 & epi \\
\hline Grote & 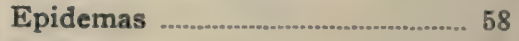 \\
\hline a Smith 2312 & Epidromia \\
\hline Grote 2323 & epigæa Grote 3233 \\
\hline us Scudder \& Burgess 525 & epigæella Chambers 6208 \\
\hline Ennomos & na Butler 481 \\
\hline Enodia & Epiglæa \\
\hline enoptes Boisduval 444 & epigona Herrich-Schaeffer 481 \\
\hline gerella Hubner 5336 & 163 \\
\hline a Barn & ep \\
\hline Entephria & Epilegis ....... \\
\hline enteridis Smith 1240 & Epimartyria .... \\
\hline enthea Grote 1529 & 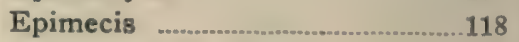 \\
\hline enthealis Hulst 5249 & epimenis Drury 2832 \\
\hline loma Busck 7813 & ep \\
\hline aloto $C$ u & \\
\hline nyo & 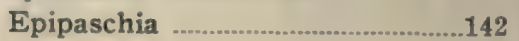 \\
\hline
\end{tabular}




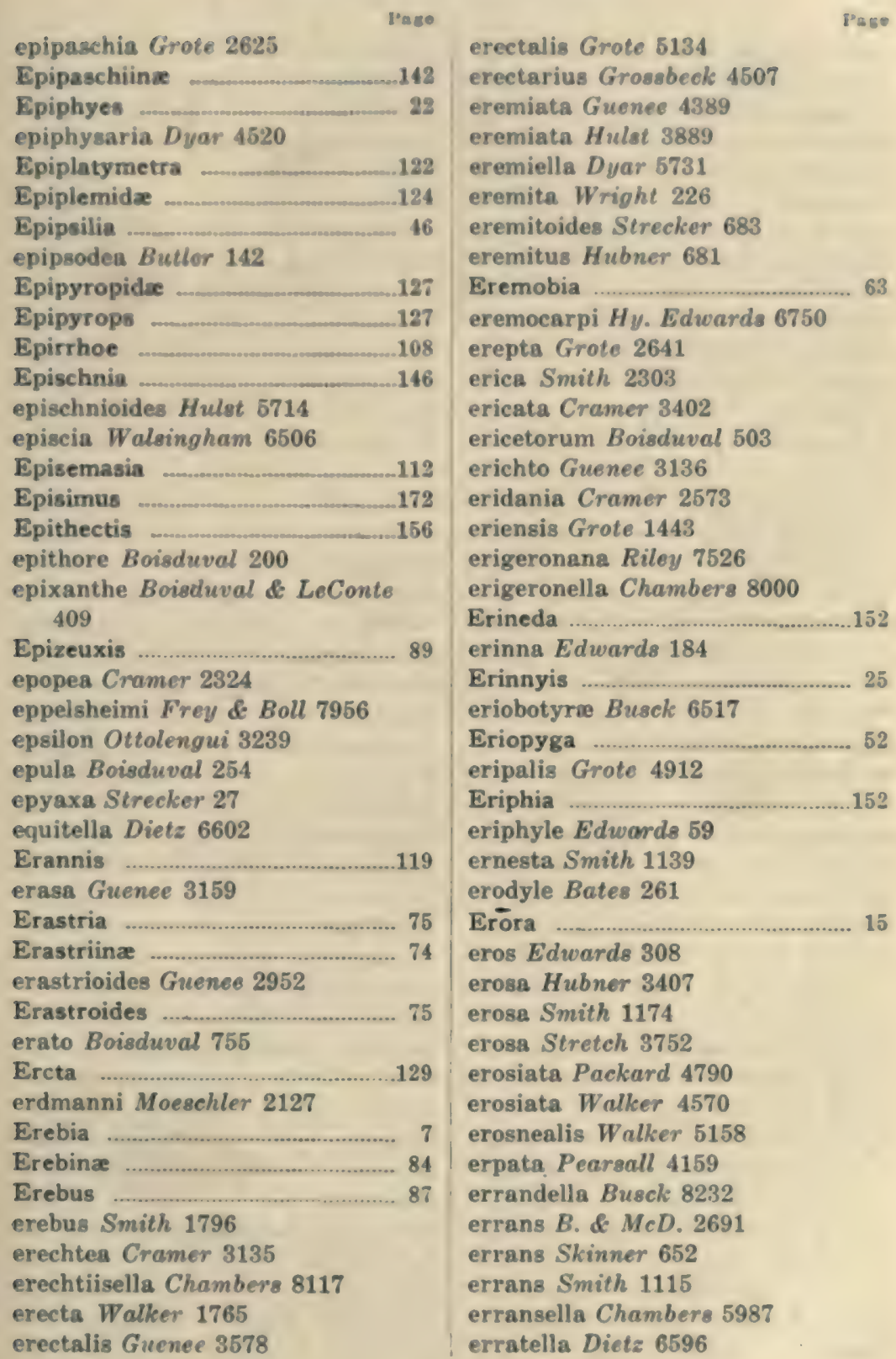




\begin{tabular}{|c|c|}
\hline Page & \\
\hline rratica $B \cdot \& M c D \cdot 2544$ & etolus Boisduval 732 \\
\hline & Euacidalia \\
\hline erronella Zeller 5779 & Euaontia \\
\hline erroraria Dyar 4512 & Euaspilates ................ \\
\hline errucata Dyar 3658 & Eubaphe \\
\hline Ersephila .......................... & Eublemma .......................... \\
\hline erubescens Bird 2680 & Eubolepia \\
\hline erubescens Warren 4312 & Eubolina \\
\hline eruditella Grote 6484 & eubule Linnaeus 47 \\
\hline erutana Herrich-Schaeffer 7420 & 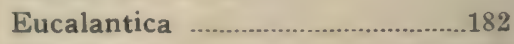 \\
\hline erutaria Duponchel 4114 & Eucalyptra \\
\hline erymus Boisduval 451 & Eucatagma ............................. \\
\hline eryngii $H y$. Edwards 101 & Eucaterva \\
\hline erynnioides Dyar 559 & Euceratia \\
\hline eryphon Boisduval 387 & Eucereon \\
\hline erythemaria Guenee 4309 & 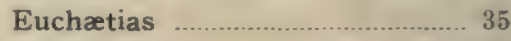 \\
\hline erythrella Ragonot 5785 & Euchlæna \\
\hline $\begin{array}{l}\text { erythriella Clemens } 7573 \\
\text { erythrinella Busck } 8095\end{array}$ & $\begin{array}{l}\text { Euchloe } \\
\text { euchloraria Guenee } 3814\end{array}$ \\
\hline 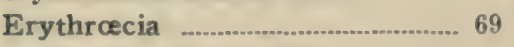 & 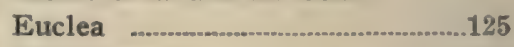 \\
\hline erythrolita Grote 1880 & Euclemensia \\
\hline erythropasa Dyar 5564 & Euclidia ..................... \\
\hline Escaria & Euclidimera \\
\hline escaria Grote 4511 & Eucoptocnemis \\
\hline esmeralda Oberthur 3278 & Eucordylea \\
\hline espea Smith 1159 & ssma $\ldots \ldots \ldots$ \\
\hline essexana Kearfott 7049 & Eucosmidæ \\
\hline esta Smith 1332 & Eucrostes .................... \\
\hline Estigmene & Eudarcia \\
\hline estrella Barnes 3684 & Eudeilinia .............. \\
\hline estrella $B . \&$ McD. 5728 & Eudemis \\
\hline estriatella Dietz 6533 & eudiopta Guenee 2570 \\
\hline esula Druce 2567 & eudora $D$ yar 869 \\
\hline esurialis Grote 1445 & eudoreella Ragonot 5759 \\
\hline etacta Smith 1631 & eudryada Smith 2995 \\
\hline ethela Edwards 146 & Eudule \\
\hline ethela Hulst 3997 & 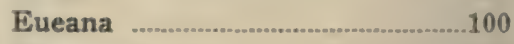 \\
\hline ethela Neumoegen \& Dyar 8331 & Euemera ......................... \\
\hline ethlius Cramer 647 & Euerythra \\
\hline Ethmia .............................. & eufala $E d w a r d s 645$ \\
\hline Ethmiidx & Eufernaldia \\
\hline ethnica Smith 2310 & Eufidonia \\
\hline ethusa Boisduval 294 & eugeniella Busck (Antispila) \\
\hline 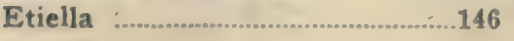 & 7580 \\
\hline
\end{tabular}




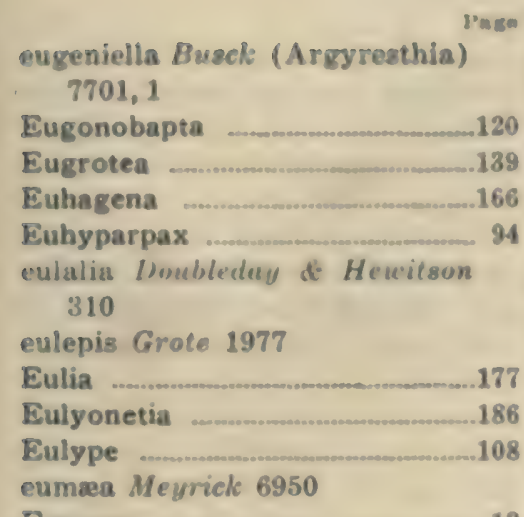

Eumzeus 13

eumelusalis Walker 3517

Eumeyrickia 160

Eumichtis 69

Eunica 11

eunus Edwards 540

eunusalis Walker 5004

Eunystalea

euonymella Chambers 7724

Eupackardia

Eupanychis

Euparthenos

eupatoriella Chambers (Dichomeris) 6379

eupatoriella Chambers (Agnopteryx) 6449

enpatoriella Chambers (Acrocercops) 7991

eupatorii $\boldsymbol{H} y$. Eduards 6668

eupatorii Fernald 5925

eupatorii Lyman 2703

eupetheciaria Grote 4514

euphasalis Walker 5125

euphemella Hulst 5497

euphemia Beutenmuller 3049

Euphenolia

euphrosyne Linnaeu 188

Euphydryas

Euphyes

Euphyia
Eupithecia

1 แะแ

Euplexia 109

Euproctis 62

Euprora

Eupseudomorpha _............... 74

Euproserpinus _. 26

Eupseudosoma _. 31

Eupterotida 97

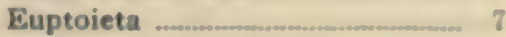

eureka Dyar 918

eureka Grossbeck 4531

eurekalis B. \& MCD. 4998

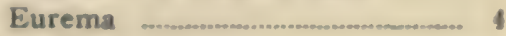

Euresia . 162

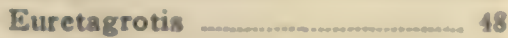

Eurhinosea ..opos 105

euridice Johannis 103

euroa Grote \& Robinson 2225

euroides Grote 1371

europome Stephens 60 .

Euros 56

eurotiella Walsingham 8150

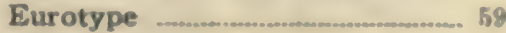

Eurrhypara _. 136

Eurrhyparodes _......... 129

euryalus Boisduval 769

eurycles Latreille 461

Eurycyttarus 125

eurydice Boisduval 54

eurydice Johann 100

euryleuca Meyrick 7557

eurymedon Lucas 16

Eurymus

eurynome Edwarda 186

euryrhoda Hampson 2832, 1

eurytheme Boisduval 59

Eurythmia 148

Eurythmidia 148

eurytris Fabricius 97

eurytulus Hubrer 345

eurytus Fabricius 97

Eustixia .135

Eustroma 


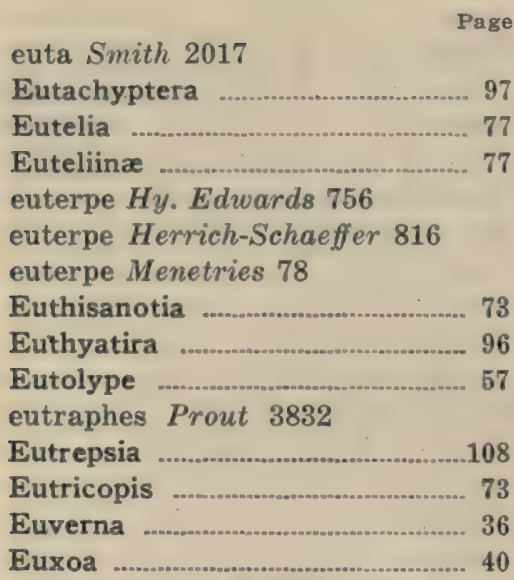

euxoiformis B.\& McD. 1838

Euzophera 147

evagaria Hulst 4405

evanescentella Dyar $\mathbf{5 5 2 7}$

evanida Grote 2514

evanidalis Grote 1399

evanidalis Robinson 3579

evelina French (Anytus) 1525

evelina French (Catocala) 3044

Everes

Evergestis

eversmanni Menetries 22

Evetria

evicta Grote 1877

Evippe

evippeella Busck 6089

evius Boisduval 433

exacta Hubner 3410

exadeus Cramer 469

exaltata $H y$. Edwards 1190

Exartema

exasperatana Z Zller 7331

exauspicata Walker 4413

excreata Smith \& Abbot 707

excantalis Hulst 5677

excavaria Morrison 4672

excellens Grote 1364

excelsa Neumoegen 990

excelsa Ottolengui 3242 excelsa Strecker 4723

excelsaria Strecker 4553

excisa. Walker 4816

exclamationis Walsingham 5946

excogita Smith 1283

exculpatrix Dyar 1454

exculta Smith 1288

excurvaria Packard 3801

excurvata Grote 4108

excurvata Packard 4388

Exelis 116

exemplaris Smith 2021

Exentera

exesa Guenee 2609

exesus Grote 5343

exhausta Smith 2341

exhausta Guenee 3169

exhilarata Smith 1531

exhumata Pearsall 4119

exigua Hubner 2575

exigua $H y$. Edwards 4811

exilis Boisduval 417

exilis Grote 2434

eximia Grote 3652

exitiosa Say 6655

exnotata Walker 4345

exoleta $\mathrm{Hy}$. Edwards 374

exoletum Zeller 6806

exoptatella Chambers 7624

exornata Moeschler 2381

exornata Walker 4290

expallidalis Dyar 5233

expallidus Grote 2769

expandens Walker 5490

expansa Walker 1866

explagiata Walker 4093

explanata Walker (Lygris)

3983

explanata Walker (Eupithecia)

4276

explosa Meyrick 6973

expolita Grote 2974

exposita Dyar 848

expressaria Walker 4584 
exprimens Wulker 2655

Page expulaa Walker 1304 expultrix Grote 3690 expuncta Walker 2607 expunctaria Grote 4680 exsanguis Dyar 3644 exserta Smith 3529 exsertistigma Morrison 1572 exsiccatus Zeller 5392 exsinuaria Guenee 4678 exsulella Zeller 5518 exsuperata Walleer 4492 extensa Smith 2377 externa Walker 2592 externalis Warren 5096 extersa Walker 2294 extima Walker 2578 extincta Guenee 1952 extincta Smith 2527 extorralis $\mathrm{Hulst} 5334$ extorris Warren 5065 extranea $\boldsymbol{H} y$. Edwards 7599 extranea Guenee 1950 extranea Smith (Euxoa) 1345 extranea Smith (Oncocnemis) 2071

extraneella Walsingham 7563

extremalis $B$. \& $M c D .3547$

extremaria Walker (Racheospila) 3772

extremaria Walker (Apæcasia) 4458

extremis Smith 2051

extricalis Guenee 5100

extricata Grote 2500

extrincicella $D_{\text {yar }} 7610$

exuberans Smith 1501

exudata Pearsall 4245

exulis Lefebre 2363

exusta Guenee 1930

exvagana Walker 6981

Exydrium

Exyra

Pabicola Meyrick $\mathbf{7 5 1 5}$

I'ome

Fabiola .161

fabrefacta Morrison 2330

fabricians Linnaeus 7603

fabricil Boisduval 780

fabricii Edwards 268

fabricii Kirby 363

fabula Grote 3760

faceta $H y$. Edwarde 3327

factiosalis Walker 3523

factoris Smith 1353

facula Grote 1570

fadus Cramer 731

fagella Busck 6158

fagicosticella Chambere 7769

fagigemmeanum Chambers 6792

fagina Morrison 2156

faginella Chambers 6428

Fagitana 64

Fala 66

falacer Godart 365

falcata Neumoegen 2094

falcata Packard 4696

falcata Schaus 3706

falcataria Packard 4317

falcifera $K i r b y 3230$

falciferella Walsingham 7650

falco Walker 759

falcula Grote 2467

falerina Smith 1268

fales Smith 1510

fallacialis Walker 3524

fallax Hampson 2193

fallax Herrich-Schneffer 2419

falsa Grote 1771

falsarius Clemens 4876

famelica Guenee 3309

famosa Meyrick 7108

famula Zeller 7423

famulata $H$ ulst 4289

fana Kearfott 7213

fandana Kearfott 6889

Fania 194 
fantasia Butler 53

faracana Kearfott 7119

farcta Grote 1938

farinalis Linnaeus 5253

farnhami Grote 1705

Faronta 55

fascia Smith 2410

fascialis Cramer 4920

fascialis Walker 5125

fasciata $B$. \& McD. (Cymatophora) 3698

fasciata $B . \& M c D$. (Oligia) 2342

fasciata Beutenmuller 3122

fasciata Butler 138

fasciata Hy. Edwards (Heliothodes) 2836

fasciata $H y$. Edwards (Oncocnemis) 2037

fasciata Grote \& Robinson 4891

fasciata Smith (Antiblemma) 3361

fasciata Smith (Copipanolis) 2112

fasciata Smith (Hyssia) 1808

fasciata Smith (Oncocnemis) 2022

fasciata Skinner 1225

fasciata Strecker $\mathbf{4 1 0}$

fasciata Swainson 713

fasciata Taylor 4272

fasciata Walker 2496

fasciata Walsingham 6508

fasciatanum Clemens 6804

fasciatella Grote 2942

fasciatus Sulzer 741

fasciella Chambers (Acrocercops) 7992

fasciella Chambers. (Adela) 8451

fasciella Chambers (Homosetia) 8313
Page

fasciella Chambers (Telphusa) 6086

fasciella Fernald 5299

fasciella Walsingham 7959

fascioferaria Hulst 4404

fasciola Herrich-Schaeffer 4842

fasciolalis Hulst 5599

fasciolana Clemens 7146

fasciolaris Hubner 3306

faseolaria Guenee 3831

fastidiosa Meyrick 7075

fastidiosa Strecker 1116

fastuosa Guenee 3394

fatima Fabricius 289

fatuaria Strecker 4653

faulalis Walker 5208

faunus Edwards 271

faunus Strecker 1521

faustella Busck 6107

faustina Strecker 3060

faustinaria Strecker 4644

faustinula Boisduval 891

fautaria Hulst 4449

favillana Dyar 7165

favillifera Walker 3835

favonius Boisduval \& LeConte 352

favonius Smith \& Abbot 353

favorita Neumoegen 984

fax Grote 2918

faxoni Minot 4288

fea $E$ dwards 417

februalis Grote 2099

fecialis Grote 3562

fegens Cramer 726

feildeni McLachlan 410

felicella Walsingham 7571

felicita Smith 2838

felicitata Smith 1094

feliculella Dyar 5736

felina Grote 2476

felix Walsingham 7460

feltella Dyar 5521 
Feltia $\quad$ Phag

fenestra Perry 774

fenestralis B. \& McD. 5057

fenestrella Packard 5688

Feniseca 15

feniseca Harvey 1270 .

fennica Truscher 1446

Fentonia

fenyesella Dietz 6600

Feralia 57

ferens Smith 2288

ferida Smith 2287

Periella Hulst 5578

fermata Smith 1924

fermentata Meyrick 7542

fernaldalis Dyar $\mathbf{5 2 4 4}$

fernaldana Busck 7556

fernaldana Grote 6895

fernaldana Wulsingham 7403

fernaldaria Hulst 4604

Fernaldella

fernaldella Busck 6376

fernaldella Chambers 6424

fernaldella Riley 7598

fernaldella Walsingham (Agnopteryx) 6443

fernaldella Walsingham (Cosmopteryx). 5966

fernaldellus Kearfott 5406

fernaldi Grote 4456

fernaldi Morrison 1346

fernaldi Ragonot (Acallis) 5293

fernaldi Ragonot (Myrima)

5652

fernaldialis Hulst 5696

feronia Linnucus 299

ferox Walker 816

ferraria Walker 2395

ferrea Grote 1751

ferrealis Grote (Polia) 1749

ferrealis Grote (Graptolitha)

2137

ferreana Busck 7295 ferricosta Smith 1192

ferricola Smith 1961

ferriferanum Walker 6811

ferrigera Smith 1906

ferrigera Walker $\mathbf{4 8 2 4}$

ferrolineana Walker 6834

ferrugalis Hubver 5088

ferrugana Schiffermuller $\mathbf{7 4 1 3}$

ferrugana Hubner 7389

ferrugata Packard 3888

ferrugata Clerele 4039

ferruginana Fernald 7053

ferruginaria Packard 4407

ferruginea Packard (Epicnaptera) 3754

ferruginea Packard (Euclea) 4816

ferruginen Packard (Lophodonta) 3617

ferruginea Smith 3360

ferruginea Walker 2592

ferrugineanum Riley 6794

ferruginella Ragonot 5721

ferrugineoides Guenee 2220

ferruginiguttana Fernald $\mathbf{7 4 3 8}$

ferruginoides Smith 1477

ferruginosa Walker 934

ferruginosa Zeller 6430

ferruginosaria Packard 4315

ferruminaria Zeller 3848

fervida $B$. \& MCD. 1619

fervidana Clemens 7348

fervidana Walker 7283

fervidaria Herrich-Schaeffer 4672

fervidaria Hubner 465:5

fervifactaris Grote 3935

fessa Grote 2803

festa Guenes 2405

festa Hulst 4336

festa $\boldsymbol{H y}$. Edwards $\mathbf{7 1 9}$

festaliella Hubner 7572

festalis Hulst 5133 
festaria Hulst 3776

festinella Clemens 8019

festiva Fabricius 3394

festivoides Guenee 2608

festus Hubner 473

feudalis Grote 5116

fibulata Morrison 2359

fictilis Guenee 3171

ficus Linnaeus 722

fidelis Grote 1840

fidelis Meyrick 7553

fidelissima Herrich-Schaeffer 3585

fidella Dietz 6578

fidicularia Morrison 2577

fidoniata Walker (Eufidonia) 4289

fidoniata Walker (Paraphia) 4492

fieldellus $B . \& M c D .5410$

fieldi $B$. \& $M c D .5479$

fieldi $D$ yar 1240

fieldi Grossbeck 4509

fieldi Swett 4380,1

fieldiella Dyar $\mathbf{5 7 1 1}$

fifia Dyar 2081

figulilella Gregson 5802

figurata Drury 990

figurata Harvey 2063

figurella Busck 6238

filamentaria Guenee 4680

filaria Smith 3383

filaria Walker 4565

filenus Poey 420

filiana Busck 6940

filicicornis Walsingham 8190

filifera Walker 3014

filiis Smith 1541

filiolella Hulst 5559

filmata Pearsall $\mathbf{4 1 4 5}$

fimbria Williams 1077

fimbrialis Schiffermuller 5259

fimbriago Stephens 3408 fimbriaris Guenee 1464

fimbriata Haworth 4069

fimetaria Grote \& Robinson 4285

finis Smith 1329

finitella Walker 5608

finitima Guenee 2291

finitima Smith 3452

finitimus Grinnell 5861

fiscellaria Guenee 4654

fishiana Fernald 7437

fishi Fernald 5926

fishi Grote 1478

fiskeana Dyar 4850

fiskeana Kearfott 7035

fiskeella Busck 8225

fiskeella Dyar 5733

fissalis Grote 5112

fissinotata Walker 4345

fistula Harvey 1801

fitchella Clemens 7877

fitchi Hy. Edwards 6655

flabella Grote 3003

flabilis Grote 1955

flaccidana Robinson 7354

flagellum Walker 3268

flagitiaria Guenee 4654

flagrantis Smith 2041

flagrata Walker 4894

flammans Dyar 871

flammea Neumoegen 994

flammeusella Chambers 8453

flammicincta Walker 2874

flammifera Walker 3998

flanda Smith 1640

flava $B$. \& $M c D .4818$

flava Edwards 35

flava Grote (Pseudanarta) 2088

flava Grote (Telea) $\mathbf{7 7 4}$

flava Strecker 76

flavago Fabricius 2233

flavalis Fernald 5024

flavana Fernald 6917

flavaria Packard 4006 
Aavedana Clemens 7323

Paso

fiavella Eily 8068

Alavisaria Cirusabeck 4738

inge

favescens $\mathrm{Hulst} 3889$

flavescens Rothachild 917

flaviscapula Smith 1329

flavistriaria Hubner 3148

flavistriga Smith 2377

flavistrigella Busck 7665

flavistrigella Walsingham $\mathbf{8 4 4 4}$

flaviterminata B. \& . McD. 4369

flavitibia Walker 6654

flavitincta Nixon 705

flavivenata $\mathrm{Hulst} 4290$

flavivittana Clemens 7439

flavivittella Clemens 6380

flavocellana Clemens 6981

flavocostella Clemens 6355

flavofascialis Grote 5156

flavofasciana Westuood 6862

flavofasciata Grote 2881

flavofasciata Packard 4372

flavofasciata Strecker 3321

flavofasciata Walker 753

flavotincta Cockerell 66

flavotincta Smith 1448

flavula Herrich-Schaeffer 4853

flebilis Grote 3033

flebilis Hulst 4221

flegia Cramer 4972

flegialis Walker $\mathbf{5 1 4 5}$

fletcherana Kearfott 7246

fletcherata Taylor 4236

fletcherella Braun 7953

fletcherella Fernald 7768

fletcherella Gibson 6431

fletcheri Beutenmuller 3068

fletcheri Grote 1892

fletcheri Kemp 13

fletcheri Smith (Brephos) 3765

fletcheri Smith (Graptolitha)

2155

flexilinealis Dyar 5461

flexuosa Walker 3289

flexuosa Grote 4852

flexurella Clemens 6321

floccalis Zeller 3445 
floccosana Walker 7446

flora $E$ dwards 313

flora Wright 46

floramina Smith 1256

florea Guenee 1999

florella Busck 6111

florella Cramer 4929

florencia Clemens 421

florestan Stoll 669

florida Guenee 1098

florida Hulst 4431

florida Smith 1609

floridæ Mamille 645

floridalis $B . \& M c D .5036$

floridalis Fernald 4928

floridalis Hulst 4970

floridana $H y$. Edwards 3726

floridana Graef 3600

floridana Hulst 4894

floridana Kearfott 6912

floridana Neumoegen 7729

floridana Smith 3544

floridana Strecker 266

floridana Walker 2609

floridana Z eller 7189 .

floridanella Beutenmuller 5966

floridanella Busck 6404

floridaria Guenee $\mathbf{4 7 5 8}$

floridaria Hulst 3894

floridata Grote 4778

floridata Packard 3845

floridata Walker 4083

floridella Dietz (Valentinia) 6525

floridella Dietz (Mea) 8305

floridella Hulst (Tetralopha) 5487

floridellus $B . \& M c D .5407$

floridellus Hulst 5656

floridensis Grote 6711

floridensis Grote \& Robinson 732

floridensis Guenee 2390
Page

floridensis Holland (Papilio) 20

floridensis Holland (Sphacelodes) 4786

floridensis Hulst 4299

floridensis Morrison 415

floridensis Neumoegen 51

floridensis Ploetz 697

floridensis Rober 50

floridensis Strecker 308

floridum Grote 903

floridus Z eller 5338

florissantella Cockerell 6664

florus Edwards 407

flosca Smith 1855

floscella Hulst 5820

floscularia: Grote 4667

fluctuosalis Lederer 4962

flumenata Pearsall 4397

flutea Smith 1247

fluvialella Busck 6236

fluviata Hubner 4054

fluxa Bird 2686

fluxella Zeller 6561

Focillidia 81

focina Smith 1329

fodiens Guenee 2809

fodinalis Lederer 5142

foedana Clemens 6800

foedaria Walker 4685

foeminalis Dyar 5198

foeminalis Smith 1360

foeminaria Guenee 4315

fofana Kearfott 7004

foliana Walsingham 7411

fondella Busck 6279

footianum Fernald 6809

forbesellus Fernald 5432

forbesi French 3491

forficaria Guenee 4775

forficellus Thunberg 5314

formális Grote 1570

formonana Kearfott 7457

formosa $H y$. Edwards 3680 
formosa Grote 3285

formosa Hulst (Scelolophia) 3840

formosa Hulst (Dystroma) 4002

formosa Hulst (Gonodontis) 4691

Lormosalis Walker 850

formosalis Clemens 5208

formosana Clemens 7054

formosata Hulat 4545

formosata Strecker 3930

formosella Murtfeldt 6275

formosella Hulat 5494

formula Grote \& Robinson 3115

formula Grote 3757

formularis Hubner 3373

fornax Hubner 298

fornica Smith 2219

fortis Grote 2079

fortunana Kearfott 7168

fortunata Pearsall 4267

fortunata Grote 2963

fossaria Taylor 4049

fosterella $\mathrm{Hulst} 5670$

Fota 66

Fotella 67

fotelloides B. \& McD. 2554

fotis Strecker 386

foxcana Kearfott 7507

1-pallida Strecker 992

fractilines Grote 2335

fractilinea Smith 2613

fractilinea Zeller 6521

fractiliniella Dietz 8215

fractivittana Clemens 7356

fractura Smith 1860

fracturalis Zeller 4934

fragariae $H y$. Edwards 6706

fragariae Walsh \& Riley 7185

Pragariana Kearfott $\mathbf{7 4 3 0}$

fragariana Packard 7342

fragariella Busck 6187 fragilaria Grossbeek 4583

fragilella Dyar 5503

fragilella Frey \& Boll 7928

fragilis $B$. \& $M C D$. 4808, 1

fragilis Guonee 2440

Pragilis Streeker 935

fragilis Stretch 3746

fragilis Walsingham 5874

fragmentella $H_{y}$. Edwards 4805

fragmentella Zeller 6075

fragosa Grote 2563

francesea Dyar 8335

francisea $H y$. Edwards 3064

francisca Ploetz 572

francisca Smith 1727

franciscana Smith 2388

franciscana Walsingham 7379

franciscella Busck 7697

franckata Pearsall 3931

francki Neumoegen 692

franconia $H y$. Edwards 990

franconica Slosson 949

franconiella Hulst 5706

franklini Curtis 429

fratella Grote 3269

frater Grote 3289

fratercula $B . \& M c D .2806$

fratercula Grote \& Robinson 3117

fratercula Pagenstecher 4896

fraterna Grote 4814

fraterna Smith 3368

fraternalis Smith $\mathbf{3 5 3 1}$

fraternana Busck 6939

fraternella Dietz 6603

fraudulentaria Zeller 4570

fraxinella $E l y ~ 8071$

fraxini $H y$. Edwards 6745

fraxini Lugger 6727

frederici Grote $\mathbf{3 0 8 6}$

freija Thunberg 195

frenchi Poling $\mathbf{3 0 5 9}$

freyella Walsingham 7689

friabilis Grote 1304 
frigga Thunberg 197

frigida Scudder 35

frigida : Smith (Acronycta) 2477

frigida Smith (Papaipema) 2695

frigidana Packard 6817

frigidaria Guenee 4185

frigidaria Moeschler 3862

frigidata Walker 4015

frigidella Packard (Laodamia) 5653

frigidella Packard (Tinea) 8249

fringata $B . \& M c D .2215,1$

fringata $S$ mith 1903

frisia Poey 257

fritillaria Guenee 4454

frondaria Grote 4035

frostiata Swett 4141

fructetella Hulst 5629

frugaliaria Guenee $\mathbf{4 5 7 0}$

frugiperda Smith \& Abbot 2574

frustella Walsingham 7655

frustrana Comstock 6760

frustulum Guenee 3153

frutetorum Boisduval 3745

Fruva 76

fucana Walsingham 7382

fucosa Hubner 900

fugax Boisduval 759

fulata Smith 3544

fulda Smith 1304

fulgens $B . \& M c D .2752$

fulgens $H y$. Edwards 829

fulgidella Clemens 8032

fulicalis Clemens 5217

fulicalis Smith 3521

fuliginaria Hulst 4565

fuliginosa $E$ dwards 395

fulginosa Linnaeus 948

fuliginosa Smith 2762

fuliginosalis Fernald 4914

fulla $E$ dwards 433 fullerea Riley 6827

fulliolus Hulst 410

fullonella Zeller 6197

fulmenella Busck 6299

fulminalis Lederer 4904

fulminalis Zeller 5451

fulminana Walsingham 6937

fulminans Smith 1920

fultaria Grote 3911

fulva Stretch 997

fulva Walsingham 6453

fulvescens $H y$ Edwards 370

fulvia Edwards 237

fulvia Dodge 283

fulvicollis Hubner 824

fulvicosta Clemens 1032

fulvifrontana Packard 6814

fulvipes Harris 6677

fulviplicana Walsingham 7539

fulvirugella Ragonot 5673

fulvisuffusella Dietz 8292

fulvofasciata Butler 941

fulvoflava Walker 918

fulvoroseana Clemens 7316

fulvosa Riley $\mathbf{2 5 7 4}$

fulvotinctana Walsingham 7500

fulvusana Haimbach 5274

fumalis $B . \& M c D .5275$

fumalis Grote 1312

fumalis Guenee 5132

fumata $B . \& M c D .908$

fumata Smith 2951

fumata Taylor 4274

fumataria Minot 4604

fumeola Hampson 2293

fumerella Dietz 6540

fumida Warren 4595

fumidus $H y$. Edwards 1011

fumiferana Clemens 7400

fumoferalis Hulst $\mathbf{5 1 3 5}$

fumosa Butler 4810

fumosa Grote 2292

fumosa Hulst (Drepanulatrix) 4323 
fumosa Hulet (Eupithecia) 4168

fumosa Morrison 2514

fumosa Robinson 7356

fumosa Strecleor (Diacrisia) 954

fumosa Strecker (Eurymus) 59

fumosa Strecker (Haemorrhagia) 735

fumosa Strecker (Melipotis) 3311

fumosalis Guenee 4974

fumosaria Strecker 4488

fumosella Hulst 5751

fumosus Hulst 84

fumosus Leussler 100

funalis Grote 4991

fundaria Guenee 4756

funebra Dietz 6549

funebris Hubner 2202

funebris Strom 5176

funeralis Grote (Acronycta) 2472

funeralis Grote (Spragucia) 2927

funeralis Hubner 4921

funeralis Scudder \& Burgess 535

funerea Grote 876

funesta Meyrick 7510

funesta Paykull 2202

fungivorella Clemens 6045

fungorum Grote \& Robinson 1471

furcata Smith 2667

furcata Thunberg 4007

furcata Walker 2298

furcata Walsingham 6607

furcatana Walker 7282

furcatella Busck 7708, 1

furcatus Grote 8490

furcatus Walsingham 8154

furcifascia Walker 4088

furcifera Guenee 2463

furciferata Packard (Goniacidalia) 3909 furciferata Packard (Sabulodes) $\mathbf{4 7 6 3}$

furciferella Dyar 5642

furcilla Grote 2890

furcilla Packard 3211

furcillata Say 280

furcotibiella Riley 8370

furellus Zeller 5450

furfurana Haworth 6790

furfuraria Hulat 4563

furfurata Grote 1858

furfurellus Hulst 5656

furnella Ely 5790

furtiva Smith (Feralia) 2096

furtiva Smith (Euxoa) 1858

furvana Robinson 7353

fusca $H y$. Edwards 4870

fusca Grote \& Robinson 641

fusca Harvey 3629

fusea Haworth 5653

fusca Packard 4843

fusca Rothschild 1007

fusca Stretch 891

fuscalbana Zeller 6865

fuscana Kearfott 7011

fuscata Grosebeck 3947

fuscata Hulst (Acidalia) 3865

fuscata Hulst (Synaxis) $\mathbf{4 7 1 5}$

fuscatella Hulst (Yosemitia) 5703

fuscatella Hulst (Canarsia) 5734

fuscicaudis Walker 732

fuscicomella Clemens 8081

fuscicostellus Zeller 5384

fuscigera Grote 1307

fuscimacula Grote 2603

fuscimaculalis Grote 5119

fuscipedella Walsingham 6625

fuscipes Grote 868

fuscipes Zeller 5420

fuscipunctella Haworth 8249

fuscocapitella Chamber 8369 
fuscocostella Chambers 7885 fuscocristatella Chambers

(Leuce) 6094

fuscocristatella Chambers

(Homosetia) 8315

fuscodorsana Kearfott 7532

fuscofasciella Chambers 8227

fuscofasciella Ragonot 5797

fuscolimbellus Ragonot 5453

fuscolineana Clemens 7388

fuscolotella Ragonot 5492

fuscolutea Smith 1789

fuscoluteella Chambers 6322

fuscomaculella Chambers (Gelechia) 6323

fuscomaculella Chambers (Tinea) 8288

fuscomaculella Wright 5723

fuscomarginella Chamber $\mathbf{7 8 6 0}$

fuscoochrella Beutenmuller 8048

fuscoochrella Chambers 6278

fuscopallidella Chambers 6144

fuscopulvella Chambers (Agnippe) 6088

fuscopulvella Chambers (Gelechia) 6335

fuscopulvella Chambers (Tinea) 8295

fuscopunctella Clemens 6076

fuscopurpurella Dietz 6513

fuscosa Neumoegen 946

fuscoscapulella Chambers 8103

fuscosparsa Walsingham 7043

fuscostrigana Clemens 7459

fuscostrigella Chambers (Polyhymno) 6178

fuscostrigella Chambers (Haploptilia) 7770

fuscosuffusella Dietz 6595

fuscotæniæella Chambers 6290

fuscotibiella Clemens 8370

fuscula Grote 854

fusculenta Smith 1666

fuscus Luther 780 fusifasciata Walker 3951

fusimacula Barnes 204

fusimacula Smith (Oxycnemis) 2537

fusimacula Smith (Euxoa) 1353

futilalis $B . \& M c D .4950$

futilalis Lederer 5134

futilis Grote \& Robinson 3362

Gabara

gabbi Behr 223

gabbi Edwards 114

Gaberasa 91

Gabriola 119

Gra 166

gagates Grote 1303

galactinus Boisduval 101

Galasa 137

galaxana Kearfott 6836

galbanaria Hulst 4698

galbanata Morrison 3178

galbineata $Z$ eller 4339

galbina Clemens 775

galena Smith 2319

galenapunctana Kearfott 6909

Galgula

galianna Burmeister 725

gallæasteriella Kellicott 6126

gallædiplopappi Fyles 6125

gallægenitella Clemens 6165

gallærandialis Dyar 4898

gallæsaliciana Riley 7236

gallæsolidaginis Riley 6122

gallatinus Stichel 24

Galleria

Galleriinae

141

gallicana Herrich - Schaeffer

7088

gallicola Busck 6066

gallicolana Clemens 7413

gallii Rottemburg 761

gallivorana Clemens 7316

gallivorum Westwood 6690

galva Strecker 2415

gamma Dyar 884 
gandana Kearfott 6897

gaosalis Walker 8516

garacana Kearfott 7849

gargamelle Strecker 8731

gargantua Dyar 2786

Gargida

garita Reakirt 547

garmani (irote 1915

gasta Strecker 2503

gatei Smith 1620

gaudiella Hulst 5846

gaultheriella Walsingham 7935

gaure Smith \& Abbot (Proserpinus) 750

gaure Smith \& Abbot (Rhodophora) 1097

gausapalis Hulst 5359

gausaparia Grote $\mathbf{4 4 4 0}$

gausapata Grote 2140

gaylussaciella Heinrich 7756

geddesi Neumoegen 1024

geiella Chambers 8000

Gelechia

Gelechiid $x$

gelida Grote 3682

gelida Moeschler 980

gelide MeLachlan 5141

gelidana Moeschler 7376

gelidata Moeschler 4203

geliformis Walker 6712

gelliasalis Walker 5228

gellidella Busck 6450

gemina $D$ yar 4827

geminata Smith 2420

geminata Grote \& Robinson 3955

geminata Packard (Packardia)

4845

geminata Packard (Eupithecia) 4169

geminatella Packard 8025

geminatus Say 705

geminella Riley 6165

geminimacula Dyar 2814 geminipunetella Ragonet 5596 geninus $R$. \& J. 682

gemistrigulana Kearfott 6765

gemma Hubner 92

gemmalis Hulst 7613

gemmats Packard 3890

gemmatella Hulst 5815

gemmatilis Hubner 3859

gemmen Frey \& Boll 7918

gemmiferella Clemens 5958

generalis Walker 3524

generosa Grote \& Robinson 5154

genetrix Grote 2298

geneura Streeker 985

genialis Grote 2259

genicula Grote 3760

geniculata Grote \& Robinson 1416

geniculata Hulat $\mathbf{4 7 5 7}$

geniculatella Dietz 8280

genoa Ploetz 584

genoveva Cramer 287

gentilis Grote (Tsniosea) 2364

gentilis Grote (Pyrausta) 5111

genuialis Lederer 5208

genutia Fabricius 48

geometralis Grote 3445

geometrica Grote 1024

Geometridae

Geometrinae

geometroides Guence 3471

geometroides Walker 4785

georgiana Walker 7350

georgica Grote 2948

georgica Herrich-Schaeffer 3609

georgiella Hulst (Salebria) 5636

georgiella Hulst (Cnephasia)

7402

georgiella Walker 6384

georgii Grote 2155

georgii Hulst 3991

Gerdana 160

gerdanella Busck 7644 
gerdis Smith 2074

germana $H y$. Edwards 2849

germana Morrison 2129

germanalis Walker 3579

geronimo Barnes 1050

Gerra 73

Gerrodes 73

gertana Smith 1714

gertruda Hulst 4473

gerularia Hubner 3778

Geshna 136

gesta Herrich-Schaeffer 498

gian Strecker 1262

gibbicostata Walker 3920

gibbosa Smith \& Abbot 3620

gibsonata Taylor 4271

gibsonellus Kearfott 5404

gibsonella Kearfott 6156

gibsoniella Busck 6133

gigantaria Swett 4042

gigantea B. \& McD. 1014

gigantea French 3210

gigantea Smith 3417

gigantea Strecker 64

giganteana Riley 7021

gigantella Chambers (Holcocera) 6534

gigantella Chambers (Haploptilia) 7764

gigantella Ragonot 5696

giganteus Grossbeck $\mathbf{4 7 4 5}$

gigantoides $B . \& M c D .1848$

gigas Smith 1847

gigas Butler 121

gila Ploetz 478

gilbociliella Clemens 6548

gilensis $B . \& M c D .8342$

giliæ $H y$. Edwards 6659

gilletteana Dyar 6883

gilletteata Dyar 4378

gillettei Hulst 4283

gillettella Busck 8435

gillettella Dyar 5609 gilletti Barnos 220

gilvescentella Ragonot 5780

gilvibasella Hulst 5604

gilvidorsis Hedemann 5945

gilvipennis Grote 1596

gilviscopella Zeller $\mathbf{6 1 4 5}$

gilvolinella Clemens 6056

gilvomaculella Clemens 6269

girardellus Clemens 5341

giscana Kearfott 7514

gisela Meyer 3117

gitonella Druce 5799

Givira .194

glabella Morrison 2241

glacialis Hulst 3994

glacialis McLachlan 57

glacialis Packard 5091

glaciana Moeschler 6866

glaciata Grote 1703

Gladela .115

gladiaria Morrison 1395

gladiola Barnes 2766

glandiferella Zeller 6084

glandulella Riley 6520

glans Grote 3148

glaphyralis Guenee 4899

Glaphyria 128

glareosella Zeller 5817

glaucana Walsingham 7378

glaucaria Guenee 3800

glaucaria Grossbeck 4589

glaucata Packard 4019

glaucatella Hulst 5712

Glauce 154

glaucella Walsingham 7771

Glaucina .116

glaucofuscana Zeller $\mathbf{7 4 9 7}$

glaucon Edwards 443

glaucopis Hampson 1694

Glaucopsyche 17

glaucovaria Walker 1615

glaucus Linnaeus 13 
gleditschixella Chambers (Stagmatophora) 6002

gleditschixella Chambere (Hel. ice) 6061

gleditschiella Fernald 5610

Glens .118

glendella Dyar 5584

glennyi Groto 2045

glenwoodata Swett 4021

glenwoodi Barnes 3739

glitranana Kearfott 6837

glochinella Zeller 6171

glomeralis Walker 5176

glomerana Walsingham 6936

glomeraria Grote 4295

gloriosa $H y$. Edwarde 6741

gloriosa Strecker 1187

gloverana Walsingham 7393

gloveri Grote \& Robinson 1036

gloveri Packard 4801

gloveri Strecker 767

Gloveria 97

Gluphisia

glutinella Ely 8067

glutinosi Walsingham 7850

glycyrrhizaeella Chambers 6324

Glyphidocera .160

Glyphipterygidae 181

Glyphipteryx 182

Giyptocera 144

gnaphaliella Kearfott $\mathbf{7 6 1 7}$

gnata Grote 1678

Gnophala 92

gnopharia Guenee 4560

gnophosaria Guenee 4380

Gnorimoschema .155

godarti Boisdural 332

godarti Perty 52

goedartella Linnaeus 7693

gogana Kearfott 6845

golgolata Strecker 4521

gomonana Kearfott 6998

gonella Strecker 2980
$8 \cdot \sin 5$

Goniacidalia 103

goniata Guenee 4777

Goniurus …… 1 1i

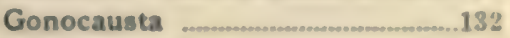

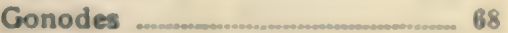

Gonodonta _. 87

Gonodontis _...n.n.

goodellella Chambers 6361

goodelli Grote (Polia) 1718

goodelli Grote (Packardia) 4846

goodelliana Fernald 7198

goodellianus Grote 5373

gopheri Smith 3495

gordialis Guenee 4958

gordius Cramer 694

gorgon Boisduval 400

gorgone Hubner 242

gorgoniensis Grinnell (Ptero-

phorus) 5929

gorgoniensis Grinnell (Stenop-

tilia) 5948

Gortyna 69

gortynoides Walker 2788

gossypiana Packard 7861

gothicata Guenee 4088

gozora Boisduval 452

gracea Hulst 4596

graciella Hulst 5699

gracilalis Hulst 5122

gracilana Walsingham 7316

gracilaria Packard 3800

Gracilaria

Gracilariidae

.186

gracilariella Busck 7718

gracilata Grossbeck $\mathbf{3 8 4 7}$

gracilella Chambers (Anorthos-

ia) 6352

gracilella Chambers (Lyonetia)

6107

gracilella $\mathrm{Hulst} 5735$

gracilenta Graef 1040

gracilenta Hubner 1154

graciliana Kearfott 6913 
gracilineata Guenee 3977

gracilior Butler 3878

gracilis Edwards 3094

gracilis Grote 8490

gracilis Grote \& Robinson (Blepharomastix) 4955

gracilis Grote \& Robinson (Hæmorrhagia) 733

gracilis Grote \& Robinson (Polygonia) 275

gracilis Walsingham (Agnopteryx) 6436

gracilis Walsingham (Scardia) 8226

gracilis Zeller 7577

gracillima Grote 2541

gradata Walker (Phasiane) 4389

gradata Walker (Zale) 3171

graduatana Walsingham 7029

græfi $H y$. Edwards 6657

græfi Grote 2489

græfi Hulst (Acanthophora) 4634

græfi Hulst (Eupithecia) 4278 græefi Packard 4851

Graefia

græfiana Grote 2216

græfiana Tepper 1060

græfiaria Hulst (Caripeta) 4459

græfiaria Hulst (Merochlora) 3832

Graperia

Grais

grandiflavana Walsingham 6907

grandimacula $B . \& M c D .2543$

grandiosa Hulst $\mathbf{4 0 7 0}$

grandipennis Grote 1492

grandipennis Hulst 4033

grandipuncta Guenee 3406

grandirena Haworth 3328

grandis B. \& McD. 193

grandis Boisduval 1685 grandis Busck 7511

grandis Druce 1211

grandis Ehrman 281

grandis Fish 5920

grandis Hulst 4094

grandis Smith 2108

grandis Speyer 1458

grandis Strecker (Melittia) 6742

grandis Strecker (Copablephar-

on) 1213

grandis Walsingham 5882

grandisella Chambers (Cyphophora) 5990

grandisella Chambers (Nepticula) 8371

granella Linnaeus 8289

granitaria Packard 3836

granitata Guenee 4345

granitella Ragonot 5675

granitosa Guenee 2394

granulata Neumoegen 4892

granulatana Kearfott 7106

graphica Hubner 3326

graphicella Busck 6140

graphidaria Hulst 4426

Grapholitha 174

Graptolitha

grassata Hulst 4341

grata Fabricius 2827

grata Hubner 2611

grata Taylor 4178

gratana Walker 7316

grataria Fabricius 3912

gratata Packard 3816

gratiosana Clemens 6811

gratiosus Fish 5901

gratulata Walker 4087

gravilinearia Andrews 4607

gravis Grote 1406

gravis Hy. Edwards 826

gregariella Murtfeldt $\mathbf{7 9 6 9}$

greta Smith 3543

Greya .196 
grindelians Busek 7070

grindeliella Walsingham 7971

gripalis Hulet 5293

grisatra Swith 1554

grisea B.\& MeD. (Lagoa) 4861

grisea B. \& McD. (Olene) 3712

grisea Coekerell 793

grisea Neumoegen 3744

grisea Packand 890

grisea Robinson 7387

grisea Streeker $\mathbf{8 6 1 1}$

grisea Walker 2469

griseaella Chambers 6325

grisearia Grote 4561

griseata Grossbeolk 4482

griseata Smith 1668

griseella Chambers 8256

grisefacte Dyar 3719

griseicollis Grote 2074

griseipennis Grote 3372

grisella $B . \& M c D .5576$

grisella Chambers 6246

grisella Dietz 6594

grisella Fabricius 5454

griseoalbana Walsingham 6815

griseocapitana Walsingham

7069

griseocapitella Walsingham 8479

griseochrella Chambere 6326

griseocincta Harvey 1842

griseopunctata $B . \& M c D .1017$

griseor B. \& MCD. $\mathbf{3 6 9 5}$

griseor Dyar 2491

griseosparsa Hampson 5329

grisescens B. MCD. 1204

grisescens Walsingham 5934

griseus Walsingham 8168

grisseella Chambers 6017

grisseella Chambera 6393

grissefasciella Chamber 6283

groenlandica Homeyer 3700

grossbecki B. \& McD. 4433 grossbecki Davis 1000

grossbecki Pearsall $\mathbf{4 7 4 9}$

grossbecki Suell 4380, 2

Grossbeckia 106

grossbeckiata Swett $\mathbf{4 1 4 0}$

grossipunctella Ragonot 5511

grossularie Riley 5705

grossulariata Saunder 4399

groteana Dyor 2861

groteana Fernald 7290

grotearia Packard 4714

groteella Robinson 6479

grotei B. \& McD. 2255

grotei Boisduval 1049

grotel Butler (Acronyeta) 2464

grotei Butler (Hsmorrhagia)

735

grotei $\boldsymbol{H}$. Edwards 728

grotei Grote \& Robinson 788

grotei Morrison 2105

grotei Packard 827

grotei Ploetz 615

grotei Ragonot 5554

grotei Riley 2154

Grotella 40

grotella Ragonot 5577

grotelliformis B. \& $M c D .2921$

grotiana Bailoy 3066

grotiana Kearfott 6958

grumella Zeller 8257

grundeli Coolidge 204

grumella Zeller 8257

grunus Boisduval 333

grynea Cramer 3106

gryneus Hubnor 377

gryphodes Meyrick 6904

guadeloupe Strecker 380

guadulpensis Guenee 8171

guenearia Packard 4416

gueneata Packard (Ceratodal-

ia) 4108

gueneeata Packard (Perizoma) 4099 


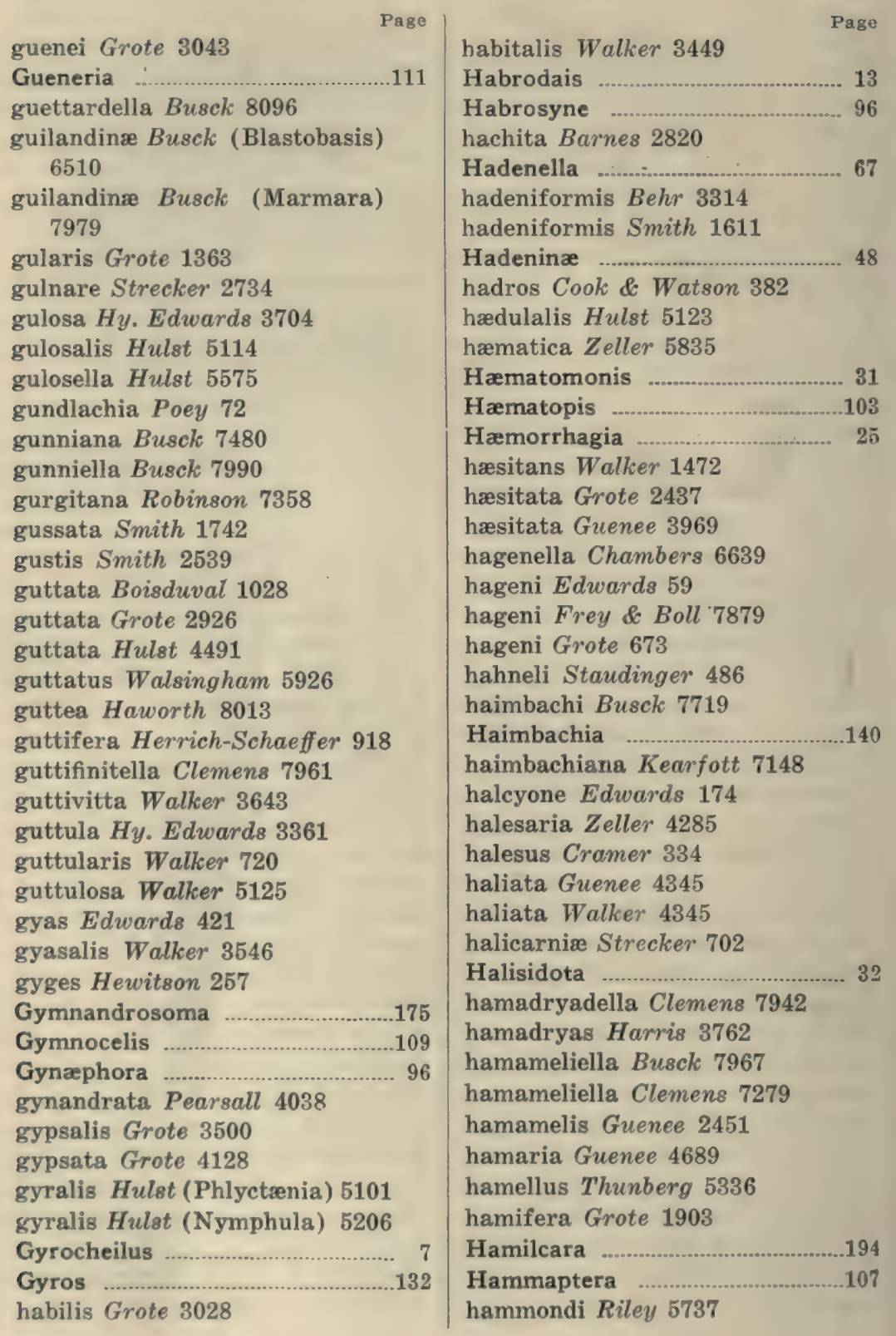


hampa Smith 1642

hampsoni Bames 818

hampsoni $B . \&$ McD. 4957

hampsoni Dyar 968

hamptonans Kearfott 7027

hamulata Guever 4753

handana Kearfott 6905

hanga Strecker 1129

hanhamella Dyar 5664

hanhami Fletcher 244

hanhami Taylor 4239

hanhami B. \& MCD. 1677

hanhami Hulst 3900

hanhami Smith (Anytus) 1525

hanhami Smith (Philometra) 3518

hanno Stoll 420

Haploa 36

Haploptilia

Haploptiliida 184

hapsella Hulst 5655

haracana Kearfott 7117

harfordi $H$ y. Edwards 62

hariolalis Hulst $\mathbf{5 0 5 0}$

harlequinalis $B . \&$ MCD. 5197

harlequinalis $D$ yar 5226

harlequinaria Dyar 4256

Harmologa 178

Harpaglxa

harpalus Edwards 561

Harpiptery $x$ 182

harrisi Boisduval 875

harrisi Clemens 701

harridi Dyar 1033

harrisi Eduards 269

harrisi Grote 2676

harrisi Oberthur 794

harrisi Packard 3643

harrisi Seudder 228

harrisi Walsh 922

Harrisimemna

Harrisina 127

harrisonella Busele 6060

harti French 3515 hartmanni French 1998

I"a 8

hartwegd Butler 672

haruspica Grote 1458

haruspica Grote \& Robinson 5148

harveiata Packard 3959

harveyana Grote (Acronycta)

2575

harveyana Grote (Phiyctenia) 5088

harveyata Taylor (Lygris) 3982

harveyata Taylor (Eupithecia) 4238

harveyi Grote 1959

hasdrubal Cramer 712

hasta Guenee 2461

hastata Linnaeus 4088

hastiana Linnaeus 7439

hastiferellus Walker 5333

hastingsi $\boldsymbol{H}$ y. Edwards 3323

hastulifera Smith \& Abbot 2495

hausta Grote 2340

haustellata Walsingham 6414

havilue Grote 1462

haydenata Packard 4597

haydenella Chambers 6503

haydeni Edwards 107

hayesi Grote 2018

hayhursti Edwards 513

haytiellus Zinckèn 5387

heathiana Kearfott 6930

heathi Fletcher 366

hebesana Wralker 6827

hebescella Hulst 5526

hebetella Ragonot 5830

hebetata Hulst 4374

hebraicum Hubner 2417

hecate Butler $\mathbf{4 0 8 8}$

hecla Lefebre 57

hectoides Boisduval 8495

Hedylepta 129

hegesin Cramer 152

hegon Seudder 635 
heidemannella Dietz 6590

heiligbrodti Harvey 809

heindelana Fernald 7429

heinrichi Busck 8426

hela Strecker 57

helata Smith 3189

helcita Boisduval 227

helcita Hubner 94

helcitalis Walker 4959

helena Edwards (Brenthis) 191

helena Edwards (Eurymus) 68

helena Hulst 4325

helena Pilate 3117

helena Reakirt 54

helena Taylor 4230

Heliades

helianthana Riley 7081

helianthi $H y$. Edwards 6707

helianthi Frey \& Boll 7862

helianthi Walsingham 5897

helianthialis Murtfeldt 5081

helianthisella Chambers 7974

helianthivorella Chambers 7974

Helice

Heliconiinæ

Heliconius

Heliocontia

Heliodines

Heliodinida

Heliolonche

Heliomata

Heliopetes

Heliophana

heliopsisella Chambers 7866

helios Edwards 433

Heliosea

Heliothela

Heliothis

Heliothodes

73

Heliozela 181

Heliozelida 181

heliusalis Walker 3528

helloides Boisduval 407

Hellula helonoma Meyrick 7519

helopalis Clemens 5216

Helotropha

helva Grote 2357

helvalis Walker 5101

helveolaria Hulst 4637

helveta Barnes 4124

helvia Scudder 214

helvialis Walker 5022

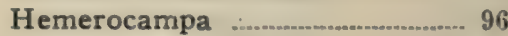

Hemeroplanes ….......................... 25

Hemiargus ................................ 15

Hemiceras .................................... 96

hemidesma Z eller 6856

Hemihyalea

Hemileuca ................................................ 28

Hemimene …...................................175

hemina Grote 2134

hemiochrellus Zeller 5386

Hemitheinae

hemizonæ Hy. Edwards 6661

henloa Smith 3568

henrici Grote (Simyra) 2514

henrici Grote (Comadia) 8350

henrici Grote \& Robinson 383

henrietta Grote 2758

henrietta Smith 1354

henshawi Edwards (Neonympha) 93

henshawi $H y$. Edwards 6678

henshawi Swett 4010

henshawiella Busck 6114

Heodes

hepara Guenee 2592

hepatica $B$. \& $M c D .1902$

hepaticaria Guenee 3848

hepburni G. \& S. 323

Hepialidx 197

Hepialus 197

heptathalama Busck 5991

hera Harris 795

heracliana De Geer 6478

herbacea Guenee 1560

herbarum Guenee 3145 


\begin{tabular}{|c|c|}
\hline herbicola Guense 3157 & Hexeris monomoment \\
\hline herbicolata $\mathrm{Hulat} 4104$ & hianna Seudder 615 \\
\hline herbimacula Guenee 1750 & hibigcella Busck 6240 \\
\hline Herculia ……137 & hibisel Guenee 1919 \\
\hline herilis Grote 1404 & hiemalis Edwarda 245 \\
\hline hermannella Fabricius 6069 & hiemalis Grote 1881 \\
\hline hermes Edwards 899 & hieroglyphica Cramer 8894 \\
\hline hermia Hy. Edwands 8062 & hilariella Zeller 6471 \\
\hline herminiata Guenee 8756 & hilaris Grote 1470 \\
\hline herminioides Walker 3486 & hilda Grinnell (Plebeius) 430 \\
\hline hermodur $\boldsymbol{H y}$. Edwarda 25 & hildn Grinnell (Pterophorus) \\
\hline hermosu Wright 217 & 5935 \\
\hline hero Morrison 1563 & hilli Grote (Eremobia) 2358 \\
\hline Hy. Edwards 3117 & hilli Grote (Catocala) 3082 \\
\hline dias Streeker 3096 & hilli Lintner 3347 \\
\hline herri Grinnell 424 & Hillia \\
\hline Herse & hillians Harvey 1447 \\
\hline hersiliata Guenee 3998 & hilliata Hula \\
\hline Hesperia _ _ $\quad 18$ & hilumaria Hulat \\
\hline hesperida Smith 2494 & Himera \\
\hline Hesperiid $x \quad \ldots \ldots \ldots$ & himonialis Zeller 5268 \\
\hline Hesperiina $\ldots \ldots \ldots$ & hinda Fren \\
\hline hesperis Edwards 163 & hinna Gey \\
\hline hesperis Grinnell 5878 & hipeana Grote 6894 \\
\hline Hesperopsis $\ldots .19$ & lus Edwarde 478 \\
\hline Hesperumia …… & Hippia \\
\hline $\begin{array}{l}\text { hesus Westuood \& Hewitson } \\
\quad 470\end{array}$ & $\begin{array}{l}\text { hippocastanum Kearfott } 6812 \\
\text { hippolita Lyman } 281\end{array}$ \\
\hline Heteranassa & hippolyta $E d w c$ \\
\hline Heterocampa … ............ 94 & hippolyta Strecker 3078 \\
\hline Heterochroa $\ldots 12$ & imada Druce 2592 \\
\hline heterodoxa Smith 1941 & hircina Morrison 2237 \\
\hline Heterogenea & lis Grote 5140 \\
\hline Heterographis ....................... 147 & hirculella Busck 6387 \\
\hline heteronea Boisduval $\mathbf{4 1 4}$ & hirsuta Skinner 1 \\
\hline Heteropacha & hirgutana Walsingham 6990 \\
\hline heteropalpella Dietz 8320 & hirsutaria $B . \&$ McD. 4725 \\
\hline Heterophleps & hirsutus Busok 8162 \\
\hline Heteropia & hirtalis Guenee 5011 \\
\hline heteroterw Frey \& Boll 7865 & hirtella Grote \& Robinson 1195 \\
\hline heucherse Hy. Edwards 6734 & hirtipes Groto 1967 \\
\hline hexadactyla Linnaeus 5954 & historalis Grote 3450 \\
\hline hexaspilata Walker 3961 & 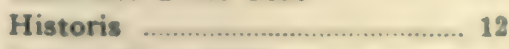 \\
\hline haxastylus Harvey 8189 & histrio Grote 3000 \\
\hline
\end{tabular}


hobomok Harris 592

Yage

hochenwarthi Hochenwarth 3223

hoffmanana Kearfott 7523

hoffmani Behr 227

hoffmani Strecker 113

hogei Druce 805

hohana Kearfott 7005

Holarctias 102

Holcocera

hollandana Kearfott $\mathbf{7 4 7 4}$

hollandaria Hulst 3809

hollandi Edwards 5

hollandi Rober 28

hollemani Grote 1253

holoberba Smith 1280

Holochroa

holocinerea Smith 2155

holoponerella Dyar 5821

Homaledra .153

Homocerynea

Homochlodes

homodactylus Walker 5907

Homœosoma

homogena Dyar 776

homogena Grote 2059

Homoglaea

Homohadena

Homolagoa

Homona

homonana Kearfott $\mathbf{7 5 4 1}$

Homoncocnemis 57

Homophoberia

homopteroides Hulst 4539

Homopyralis

Homosetia

Homostinea

homuraria Grote 4685

honesta Walker 2231

honesta Grote 1075

honestarius Walker 4744

Honora

hopfferi Grote \& Robinson 3586

hopkinsana Kearfott 7010

Hoplolythra

Horama ….................................... 29

horariana Walsingham 7405

horatius Scudder \& Burgess 527

Horisme

Hormisa

hormos Hubner 3404

Hormoschista

hornbeckiana Harris $\mathbf{7 4 0}$

horrida Hubner 3199

hortaria Fabricius 4607

hortuellus Hubner 5361

hortulana Morrison 1408

horus Edwards 643

hospes Walsingham $\mathbf{7 4 5 2}$

hospes Walsh 6690

hospita Denis \& Schiffermuller 1024

hospitalis Grote 1441

hospitella Zeller 5786

hostia Harvey 3410

houstonana Grote 7351

howardi Dyar 3732

howardi Hy. Edwards (Chalcopasta) 2751

howardi Hy. Edwards (Daritis) 3584

howardi Skinner 601

howlandi Grote 3342

hoyi Grote 3766

huachuca Dyar 4814

huachuca Grossbeck 4139

huachuca Smith 2968

huachucaella Kearfott 5321

huachucalis Haimbach 5288

huachucana Kearfott 7535

huachucella Busck 6421

hualapai Neumoegen 792

hubbardana Busck 7494

hubbardi Dyar (Adelocephala) 809

hubbardi Dyar (Bruceia) 897

hubbardiana Dyar 2920

hudsonaria Taylor 3793 
hudsoniana Walker 7439

hudsonica $H_{y}$. Edwards 1046

hudsonics Grote Robinson 3337

hudsoni Smith 1401

hueco Barnes 1837

hugo Doubleday \& Hewitson 336

hugon Godart 336

huita Smith 2957

hulda Edwards 35

hulstata Taylor 4003

hulstella Dietz 6511

hulstellus Beutenmuller 8173

hulstellus Fernald 5376

hulsti Cockerell 5730

hulsti Dyar 4324

hulsti Edwards 309

hulsti Grote 2351

hulsti Smith 1520

Hulstia

hulstia Tepper 1144

hulstiana Dyar 8779

hulstiaria Taylor 4429

hulstiella Ragonot 5691

Hulstina

hulstinoides Grossbeck 4523

humaria Guenes 4574

humata Meyrick 6420

humeralis Smith 3424

humeralis Walker 4630

humerata Smith 3475

humerella Ragonot 5494

humerosana Clemens 7286

humilalis Lederer 5099

humilis Ragonot 5688

humilis Walsingham 8440

humilis Walker 3657

humilis Zeller 6610

humuli Bird 2669

humili Harris (Strymon) 352

humili Harris (Hypena) 3579

huntera Fabricius 283

hunteri Hubner 283 huron Edwards 589

huronalis Guenee 5009

hutsoni Smith (Conochares)

2947

hutsoni Smith (Stiria) 2762

hutsoni Smith (Autographa)

3273

hutsoni Smith (Renis) 3526

hyalina Hulat 4125

hyalinaria Grossbeek 4650

hyalinata Linnaeus 4977

hyalinopuncta Packard 3728

hyalinus Waloh 4832

Hyaloscotes 125

hyantis Edwards 39

Hyblax ...................................... 92

Hyblaina ................................... 92

hybrida Butler 1029

hybrida Fabricus 3364

Hybroma 193

hybromella Chambers 8322

hydaspe Boisduval 169

hydrangiella Chambers 7581

Hydrelia 108

Hydriomena 105

hydriomenata Pearsall 4034

hydromeli Harvey 3629

hyemalis Streteh 845

hygias Heydenreich 281

hyleus Drury 671

hylas Edwards 272

hylax Edwards 547

Hylephila 20

Hylesia 28

hyllalis Walker 5259

hylotomiformis Walker 6744

Hymenia 129

hypathrata Grote 4384

Hypanartia

Hyparpax 95

Hypaurotis …......... 13

Hypena …………… 32

Hypeninae …… 88

Hypenopsis _...__............ 68 


\begin{tabular}{|c|c|}
\hline 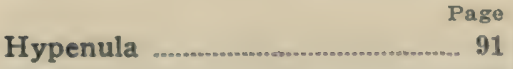 & $\begin{array}{r}\text { Page } \\
\text { Ianassa } \ldots \ldots \ldots \ldots \ldots\end{array}$ \\
\hline Hyperaschra & ianthe Edwards 406 \\
\hline hyperbola Slosson 710 & iaspis Guenee 2400 \\
\hline hyperborea Curtis 943 & icariodes Boisduval 433 \\
\hline $\begin{array}{l}\text { hyperborea Hulst (Rachela) } \\
3965\end{array}$ & $\begin{array}{l}\text { icciusalis Walker } 5208 \\
\text { icelus Lintner } 517\end{array}$ \\
\hline $\begin{array}{l}\text { hyperborea Hulst (Anthelia) } \\
4448\end{array}$ & $\begin{array}{l}\text { iceryæella Riley } 6546 \\
\text { Ichthyura }\end{array}$ \\
\hline hyperborealis Moeschler 5093 & icole Grote 2322 \\
\hline hyperboreata Staudinger 4204 & ida Hulst 4328 \\
\hline hyperboreus Moeschler 8486 & idæusalis Walker 7332 \\
\hline hyperella Ely 6457 & idaho Edwards 562 \\
\hline hypericana Ely 7418 & idahoana Kearfott 6892 \\
\hline $\begin{array}{l}\text { hyperici Boisduval \& LeConte } \\
352\end{array}$ & $\begin{array}{l}\text { idahoensis Grote } 1358 \\
\text { idalia Drury } 153\end{array}$ \\
\hline hyperici Hy. Edwards 6660 & idalis Fernald 5442 \\
\hline Hyperetis & idas Cramer 466 \\
\hline Hyperstrotia & ideata Walker 4072 \\
\hline 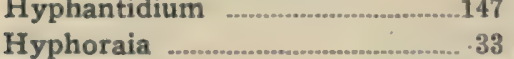 & $\begin{array}{l}\text { idella Barnes } 2925 \\
\text { Idioglossa }\end{array}$ \\
\hline 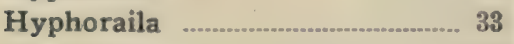 & idonea Grote 2248 \\
\hline Hypocala & iduata Guenee 4050 \\
\hline Hypochalcia & iduna Edwards 121 \\
\hline hypochaliciella Ragonot 5600 & Iesta \\
\hline $\begin{array}{l}\text { hypochraria Herrich-Schaeffer } \\
4685\end{array}$ & $\begin{array}{l}\text { ignavaria Pearsall } 4529 \\
\text { ignea Grote } 3224\end{array}$ \\
\hline 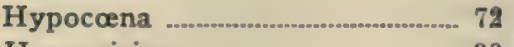 & ignidorsella Ragonot 5762 \\
\hline Hypocrisias & igninix Walker 7732 \\
\hline Hypolimnas & ignistrigella Ragonot 5740 \\
\hline $\begin{array}{l}\text { hyponomeutana } \\
7044\end{array}$ & $\begin{array}{l}\text { ignobilisella Chambers } 6013 \\
\text { ignota Frey \& Boll } 7974\end{array}$ \\
\hline Hypopacha ........................... 98 & ignotaria Walker 3917 \\
\hline $\begin{array}{l}\text { hypophleas Boisduval } 410 \\
\text { Hypoplesia }\end{array}$ & $\begin{array}{l}\text { ilaire Godart } 28 \\
\text { ilavia Beutenmuller } 354\end{array}$ \\
\hline Hypoprepia & $\begin{array}{l}\text { lavia Beutenmuller } 354 \\
\text { ilecella Busck } 8152\end{array}$ \\
\hline Hypopta & ilia Cramer 3051 \\
\hline 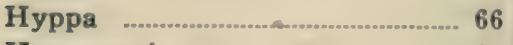 & ilicifoliana Kearfott 7129 \\
\hline Hypsopygia & ilioneus Smith \& Abbot 18 \\
\hline Hypsoropha .................................. 87 & illabefacta Morrison 1717 \\
\hline 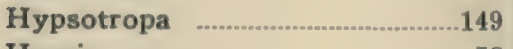 & illapsa Walker 1502 \\
\hline 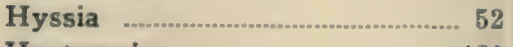 & illata Walker 1329 \\
\hline Hysterosia & illaudabilis Grote 1757 \\
\hline $\begin{array}{l}\text { Hystricophora } \\
\text { hystriculella Hulst } 5515\end{array}$ & $\begin{array}{l}\text { illaudata Walker } 4574 \\
\text { illecta Walker } 3088\end{array}$ \\
\hline
\end{tabular}


illectella Clemena 7831

illepida Grote 2517

illibalis Hubner 5124

illibella Dietz 6564

illibella Hulat 5850

Illice

illinois Dodge 605

illinoisensis French 2654

illita Smith 2456

illiterata Crote 2654

illocata Hulst 4045

illocata Walker 2270

illocata Warren 3475

illotana Walsingham 7013

illudens Walker 3219

illustra Smith (Schinia) 1172

illustra Smith (Trachea) 2313

illustraria Hulst 3812

illuviellum Ragonot 5767

ilma Watson 790

imbraria Guenee 4758

imbridana Fernald 7062

imbrifera Guenee 1661

imbuna Smith 1733

imitata Hy. Eduards (Sabulodes) 4765

imitata Hu. Edurards (Synanthedon) 6668

imitata Strecker 229

imitata Walker 4600

imitatorella Chambers 8273

imitella Stretch 2868

immacula Grote 2795

immacula Sirand 2834

immaculalis Harve/ 3455

immaculalis Hulst 3413

immaculana Kearfott 6900 .

immacularia Suett 4630

immaculata $B$. \& $M C D .4462$

immaculata Braun 7871

immaculata Corkle 380

immaculata Graef 903

immaculata Jeuett 803

immaculata Morrison 2229
Pare

immaculata Reakirt 938

immaculata Skinner (Cocceius) 483

immaculata Skinner (Dasyuris) 4059

immaculata Skinner (Parnassius) 24

immaculata Skinner \& Aaron

(Pieris) 37

immaculatella Chambers 8128

immaculatella Chambers (Scythris) 8079

immaculatus Williams 604

immaculosus Comstock 358

immanata Haworth 3993

immanis Guenee 2649

immediata Grote 4075

immerens Harvey 5080, 1

immersata Walker 4454

immixta Grote 1285

immixtalis Grote 5087

immortua Grote 1076

immuna Smith 3477

immundella Hulst 5510

Immyrla

imora Strecker 1844

imparata Walker 3914

impartialis Harvey 3200

impauperata Walker (Acidalia) 3862

impauperata Walker (Campto-

gramma) 4085

impecuniosa Grote 2681

imperator Strecker 711

imperatoria Smith \& Abbot 813

imperdata Dyar 4532

imperfecta Smith 1940

imperfecta $H_{y}$. Eduards 6693

imperfectaria Walker 4599

imperialella Busck 8094

imperialis B. \& MCD. 1094

imperalis Drury 813

imperita Hubner 1506

imperspicua Bird 2688 
imperspicua Strecker 1136 imperturbata Bird 2700 impigritella Clemens $\mathbf{7 6 2 4}$ impingens Walker 1636 impleta Walker (Acronycta) 2455

impleta Walker (Horisme) 4280 impletella Zeller 5720 implexana Walker 7428 implicata Guenee 4070 implicata Walker 4276 implora Grote 2815 implorata Hulst 4229 impluviata Borkhausen 4069 impolita Morrison 1622 impositella Zeller 8079 impressa Walker 2496 impressale Hulst 5764 improba Butler 197 improbana Walker 6992 impropria $H y$. Edwards 6661 impropriata Walker 4492 improvisa $H y$. Edwards 3696 impudens Walsingham 6871 impulsa Guenee 2315 impunctata Warren 3860 impura $B$. \& $M c D .864$ inæqualis Busck 6301 inæquepulvella Chambers 6373 inamonella Zeller 8215 inana Robinson 7431 inaptata Walker 4340 inatomaria Guenee 4721 inattenta Smith 1600 inea Dyar 2557 incallida Smith 1315 incallida Walker 2380 incana $H y$. Edwards 2587 incana $E d w a r d s 111$ incanana Clemens 7254 incandescens Grote 3318 incanella Hulst 5674 incanella Walsingham 7904 incarcerata Boisduval 3589 incarnana Haworth 7145 incarnata Stretch 996 incarnata Walker (Lerina) 895 incarnata Walker (Arachnis) 967

incarnatorubra Goeze 977 incautellus Z Zeller 5657 incensalis Lederer 5103 inceptaria Walker 4406 incertana Clemens 7390 incertata Walker 3816 incertella Chambers 7673 incertella Zincken 5326 incincta Morrison 1819 incisa Harvey 4817

Incisalia 14

incisaria Walker 4700

Incita 37

incivis Guenee 1495

inclara Smith 2444

inclara Strecker 1126

inclinana Zeller 7026

inclinataria Walker 4039

includens Walker 2342

inclusa Dietz 6557

inclusa Hubner 3591

inclusaria Walker (Racheospila) 3770

inclusaria Walker (Ptchopoda) 3888

incognita Smith 1226

incoloraria Walker 4304

incolorata Dyar 4338

incomitata Harvey 2085

incommodata Walker 4068

incompleta Butler 996

inconcinna Harvey 1375

inconcinna Smith 1618

inconcinnalis Lederer 5133 inconclusana Walker 7374

inconditana Walsingham 7309

inconditella Ragonot 5631

inconditus Walsingham 5911 
incongruaria Hulat 4726 incongruella Hulst 5829 inconspicua Grote 1804 inconspicua Hulst 4543 inconspicua Smeith 2296 inconspicua Walker 723 inconspicua Wulsingham 6550 inconspicualis Grote 3502 inconspicuaria $B$. \& $M c D$. 4540,1

inconspicuella Murtfeldt 6171 inconstans Smith 2444 inconstans Geyer 1110 inconstans Grote 2086 inconstans Guenee 3305 incopriaria $\boldsymbol{H}$ ulet $\mathbf{4 5 9 0}$ incorrupta $\boldsymbol{H y}$. Edwarde 985 incorruscella. Hulst 5665 incresa Smith 1978 incresata Pearsall 4248 increta Morrison 2452 incrustalis Hulst 5471 incubita Smith 1305 inculta $H y$. Edwards 902 incursata Hubner 4043 incurva $H y$. Edwards 3746 incurva Smith 1774

Incurvaria 196

Incurvariidae 196

incurvata Guenee 4777

incusalis Grote 3453

indeclinata Walker 4685

indecorana Zetterstedt $\mathbf{7 4 1 2}$

indefinata Grossbeck 4081

indela Smith 2354

indensa Smith 1341

indentalis Grote 5035

indentanus Dyar 7171

indentata Harvey 3142

indentata Packard 3589

indentella Kearfott 5818

indetermina Boisduval 4813

indeterminata Wolker (Matu. ta) 1556 indeterminata Walker (Heterocampa) 3643

indiana Smith 1053

indiana Grote 3619

indicans Wulker 1756

indicate Fubricius 4949

indicataria Walker 4565

indicta Smith 1999

indigenella Zeller 5553

indigens Walker 2598

indigna Walker 3254

indirecta Grote 2301

indirecta Walker (Conistra) 2216

indirecta Walker (Euxoa) 1304

indisereta $\mathrm{H}_{y}$. Edwards 3302

indiscretata $H_{y}$ Edwards 4764

indiscriminata Walker 3818

indistincta B. \& McD. 919

indistincta Grossbeck 4527

indistincta $\mathrm{Hy}$. Edwards 3728

indistincta Hulot 4032

indistincta Smith 2533

indistineta Taylor 4261

indistinctalis Warren 5097

indivisalis Grote 3550

indivisana Walker 7407

indocilis Walker 2285

indoctaria Walker 3871

indoctrinata Walker 4280

indotatellus Walker 5032

indra Reakirt 7

indra Smith 1816

indubitans Walker 2931

indubitata Grote 3968

inducta $D$ yar 3747

inductata Guenee 3901

indurata Dyar 4314

indurata Smith 1885

induta Harvey 2082

ineffusaria Guenee 4758

inelegans Smith 1578

inepta $\mathrm{Hy}$. Edwards $32 y 7$

inepta Walker 2574

J'a ge 
inermis Harris 1490

ines Edwards 349

inexacta Walker 3361

inexpertana Walker 6827

inexplicata Walker 3441

inextricata Walker (Mellilla) 4299

inextricata Walker (Euxoa) 1304

infans Moeschler 3762

infanta Smith 3205

infausta Walker 1276

infecta Ochsenheimer 1495

infecta Walker 1750

infectata Walker 4380

infelix Smith 1364

infelix Guenee 2334

inferior Grote 3538

inferior Hulst 4127

inferior Smith 1870

infernalis Strecker 810

inficita Walker (Agroperina) 2355

inficita Walker (Marathyssa) 3000

infidelis Dyar 1853

infidelis Grote 1877

infimalis Guenee 4975

infimata Guenee 4380

infimatis Grote 1540

infimbriana Dyar 7097

infimbrialis Dyar 5267

infimella Ragonot 5781

infirma Hy. Edwards 6668

infixa Walker 2081

infixaria Walker 4602

inflatella Clemens 7608

inflava Smith 1919

inflexa Morrison 3014

infracta Morrison 1275

infructuosa Walker 1878

infulata Grote 4296

infumata Grote 2757

infumata Oberthur 156 infumatana Z eller 7343

infumataria Grote 4529

infusa Smith 1356

infuscana Walsingham 7094

infuscata Smith 1648

infuscatella Clemens 7772

Inga .161

ingeniculata Smith 1417

ingeniculata Morrison 3324

ingenita $H y . E d w a r d s ~ 4865$

ingens $H y$. Edwards 914

ingenua Smith 3214

ingenua Walker 3349

ingrata Felder 75

ingravis Smith 1688

inguinalis Guenee 4940

Inguromorpha 194

inherita Smith 1919

inimicella Zeller 6878

injecta Dyar 884

innexa Grote 1765

innocuella Zeller 6188

innominata Smith 2133

innota Smith 2378

innotabilis Grote 1556

innotata Guenee 2489

innotata Walsingham 8016

innotatellus Walker 5364

innubens Guenee 3020

ino G. \& S. 327

inopiana Haworth 7537

inopinatus $H y$. Edwards 907

inopinatus Smith 1456

inops Grote 2226

Inopsis 30

inorata Grote (Spragueia) 2924

inorata Grote (Strymon) 3365

inordinaria Walker $\mathbf{4 3 4 5}$

inordinata Morrison 2275

inornata Beutenmuller 3706

inornata Edwards (Argynnis)

181

inornata Edwards (Cœnonympha) 103 
inornata Grote (Amphipyra) 2289

inornata Grote (Trichocosmia) 2727

inornatn Grote \& Robineon (Sisyrosen) 4828

inornata Hulst (Hydrelia) 4119 inornata Hulat (Stergamatrea) 4605

inornata Neumoegen 3590

inornata $W$ alsingham 6488

inornata Wolcott $\mathbf{3 1 1}$

inornatalis Fernald 5161

inornatalis Walker 5023

inornatanum Clemens 6807

inornatella Busck 8222

inornatella Chambers (Haploptilia) 7773

inornatella Chambers (Elachista) 7832

inornatella Chambers (Eulyonetia) 7849

inornatella Chambers (Gracilaria) 8059

inornatellum Hulst 5770

inornatellus Clemens 5864

inornatellus Walker 5393

inquadrana Waloingham 6791

inquxsita Grote \& Robinson 2661

inquasita Walker 1615

inquietsna Walker 6872

inquilina Kearfott 7276

inquilinella Busck 6249

inquilinella Ragonot 5603 .

inquinaria Hulat $\mathbf{4 4 1 0}$

inquinate Guenee 2798

inquinatalis Zeller 5091

inquinatus Zeller 5928

insaria Dyar 4602

insciens Walker 1919

inscripta Walker 2424

inscripta Walsingham 6167

inseriptata Donovan 4069

inscriptella Buscle 7698 inscriptum Harris $\mathbf{7 4 7}$

insectella Fabricius 8215

insecutata Walker 3813

insequalis Guenee 5152

inserrats Walsingham 6356

insertans Smith 1393

insignalis Walker 3554

insignata Walker (Agroperina) 2344

insignata Walker (Euxoa) 1329

insignata Walker (Euxon) 1363

insignificata Taylor 4146

insignis Frey \& Boll 8117

insignis Walker 3400

insignis Walsingham 7881

insignisella Walker 8238

insinuaria Guence 4678

insinuata Smith 2238

insipida Strecker 2226

insipidata Pearsall 4134

insita Walker 2493

insiticians Zeller 7253

insolabilis Guenee 3041

insolabilis Hulst 4206

insolens Grote 1680

insolita Grote 2510

insolita Litner 689

insolita Smith 3268

insperata Grote 3304

inspergella Ragonot 5851

inspinosa Guenee 1414

instans Smith 2783

instruta Smith 1530

instrutana Claypole $\mathbf{7 1 5 6}$

instrutana Clemens 6862

insuda Smith 3183

insueta Guenee 1941

insulalis B. \& McD. 4992

insularis Grote (Rynchagrotis) 1571

insularis Grote (Hippia) 3624

insularis Maynard 150

insulata Walker 906

insulatella Dietz 6556 


\begin{tabular}{|c|c|}
\hline tactana Walsingham 7465 & 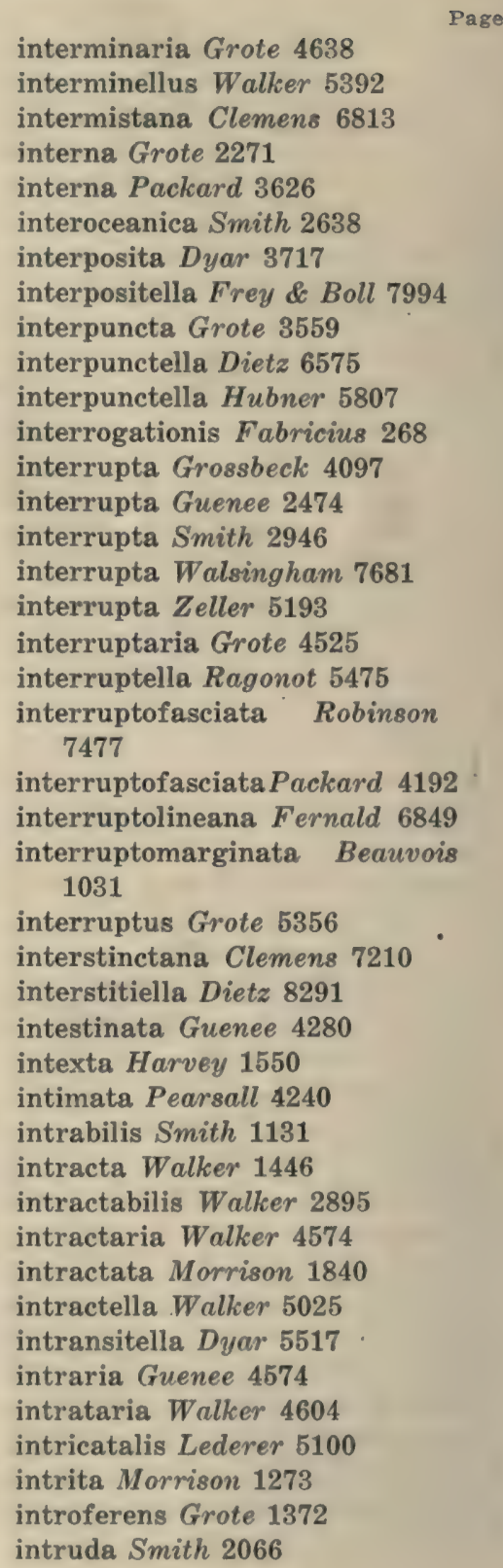 \\
\hline
\end{tabular}


intruse Smith 1831

inulta Grote 2209

inusitata $\boldsymbol{H y}$. Edwosds 6679

inusitatumnella Chambers 8023

inusta Guenee 2729

inutilis $\boldsymbol{H y}$. Edwarde 8495

invalida Smith 1692

invariabilis Kearfott 6235

invariata Walker 3914

inventaria Grote 4065

invenusta Grote $\mathbf{1 4 5 5}$

inversa Pachard 3591

inversella Zeller 6370

inveterascaria Swett 3823

invexana Walker 7357

invexata Walker 4656

invicta Walsingham 7018

invictella Busck 6150

invinctalis Hulst 5094

inviolata $\mathrm{Hulat} 4733$

invisalis Guenee 4901

involuta Dyar 848

involuta Walker 3184

involutellus Clemens 5344

involutum Sepp 903

jo Fabricius 779

ia Gray 709

jochros Zeller 8457

Iodia

jole Boisduval 38

iole Cramer 283

iowa Scudder 599

iphitalis Walker 5443

Ipimorpha T0

imomcere Busck 5960

ipomoere Doubleday 3654

ipomoese Harris 816

irata Swett 4013

irene Boisduval 170

irene Behr 3058

irene Fitch 38

irenics Walsingham 6563

irentis Smith 3482

iricolor Smith 2029 iridaris Guence 3810

iridella Busck 6000

iridella Chambers 8443

iris Guenee 2384

iris Zetterstedt 2127

iroides Boisduval 380

irraria B. \& McD. 4759

irrecta Walker 3445

irregulats Walker 4345

irresoluta Walker 2607

irridipennella Clemens 6399

irrorata $\boldsymbol{H}$ y. Edwards 892

irrorata Grote $\mathbf{3 1 5 5}$

irrorata Packard (Oreta) $\mathbf{3 7 5 8}$

irrorata Packard (Phasiane)

4371

irrorata Smith 1841

irroratana Walsingham 6938

irroratella Walsingham 7774

irrorea Robinson 7303

irrorella Dietz 8243

irrubriella Ely 5536

irus Godart 381

isabella Clemens 7582

isabella $\boldsymbol{H}$. Edwards 8115

isabella Smith \& Abbot 957

Isia

isias Boisduval 804

islandana Kearfott 6876

ismeria Boisduval \& LeConte 242

Isochates .126

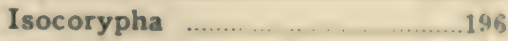

Isogona ........................................ 88

Isogramma ............................. 23

isola Reakirt 423

Isoparce 24

isophthalma Horrich-Schaeffer 418

isospora Meyriek 7007

istapa Reakirt $\mathbf{3 4 5}$

istar $R . \& J .685$

Isturgia

Itame 


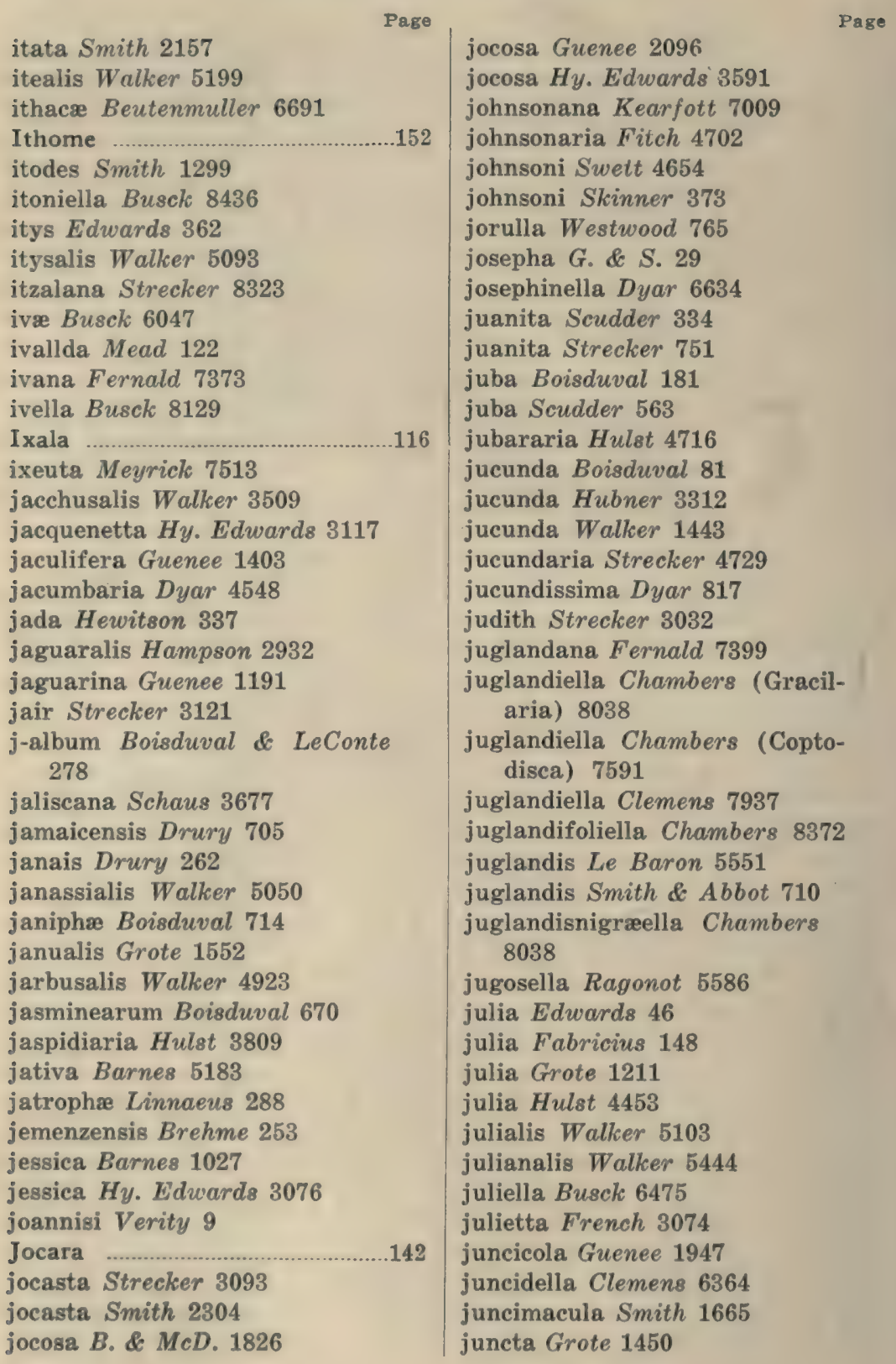


junctaria Walker 8856

juncticilians Walsingham 0967

junctolinearia Graef 3792

junctolineella $\mathrm{Hulst} 5695$

junctura Walker 3073

Juncturaria Guenee $\mathbf{4 7 5 6}$

juniperaris Packard 4755

juniperella Kearfott 6142

juno Pacleard 787

Junonia

jussieus Hubner 741

jutta Hubner 123

juturnaria Guenee 4462

juvenalis B. \& McD. 8735

juvenalis Fabricius 525

juvenilis Grote 2653

juvenis Hubner 525

kaeberalis Haimbach 4925

Kakopoda

kali Strecker 394

kalmise Smith \& Abbot 693

kalmiella Dietz 8014

kana Busck 7266

kandana Kearfott 6911

kappa Grote 2081

karacana Kearfott 7293

karwinski Hubner 314

kasion Dyar 955

kaslor Smith 1075

kasloensis Cockerell 769

katahdin Newcomb 128

kearfottalis Dyar 5212

kearfottella Braun 7893

kearfottella Busele (Aristotelia) 6057

kearfottella Busck (Aproærema) 6184

kearfottella Dietz 8234

kearfottella Dyar 5520

kearfotti Dyar 8166

Kearfottia 192

keela Smith 1846

keewaydin Edwards 59

kellicotti Fish 5923 kellogxi Hy. Edwards 2199

kempi Hulst 4675

kennebecana Kearfott 7164

kennicottella Clemens 6502

kentaria Grote 4712

kentuckiensis Dyar 882

kerrvillei $B$. \& $M c D .3711$

kerrvillei Smith 1301

keutzingaria Packard 4669

kincaidella Busck 6231

kincaidiana Fernald 7204

kincaidiella Busck 7634

kindermannana' Treitschke $\mathbf{7 4 5 8}$

kinzelella Busck 6093

kiowah Reakirt 604

kiscana Kearfott 7074

klagesi Ehrman 960

klamathiana Walsingham 6441

klugi Hubner 88

kodiak Edwards (Cononympha) 102

kodiak Edwards (Plebeius) 426

kodiakata Packard 4077

Kodiosoma 35

kœebelei Riley 2753

kœbelei Hy. Edwards 6688

kœbelella Busck 8148

kœbelella Dyar 7653

kokana Kearfott 7006

komonana Kearfott $\mathbf{7 5 4 2}$

kootenai Cockle 59

korites Druce 6651

kriemhild Strecker 190

Kricogonia

Kronaea 126

kuehniella Zeller 5799

kuetzingi Grote 4668

kukakana Kearfott 7884

kunzei Hulet (Neoterpes) $\mathbf{4 6 4 2}$

kunzei Hulat (Pterospoda) $\mathbf{4 5 0 5}$

kunzei $R$. \& J. 711

kyune Barnes 1224

labe Strecker 1114 
labecula Grote (Hemihyalea) 911

labecula Grote (Balsa) 2602

labeculalis Hulst 5038

labeculana Robinson 7468

labeculata Hulst 3910

labesaria Grote 4632

labiosana Zeller 7326

labradorella Clemens 8433

labradorensis Packard 4041

labradorensis Scudder 67

labradoriata Moeschler (Macaria) 4345

labradoriata Moeschler (Aspilates) 4636

labradorica Moeschler 6328

labradoriella Clemens 6217

labradoriensis Christoph 5348

labradoriensis Packard 8489

labradoriensis Staudinger 1349

labradoriensis Sommer 3945

labradoris Staudinger 2201

labrosa Grote 3263

labruscæ Linnaeus 742

laceyi $B . \& M c D$. (Strymon) 346

laceyi B. \& McD. (Graptolitha) 2162

laceyi B. \& McD. (Lagoa) 4862

lachrymosa Hulst (Eupithecia) 4195

lachrymosa Hulst (Hulstina) 4539

lacinia Geyer 263

laciniana Zeller $\mathbf{7 1 9 0}$

lacinellus Grote 5394

laciniosa Zeller 3561

lacoalis Walker $\mathbf{5 1 1 9}$

Lacosoma

Lacosomidae

lacrymosa Guenee 3044

lactata Smith 1032

lactea Stretch 893

lacteata Packard 4071

lacteella Fabricius 5422 lacteella Hulst 5604

lacteella Schiffermuller 6502

lacteodactylus Chambers 5914

lacteola Lintner 3897

lacteolaria $\mathrm{Hulst} 3725$

lacteusochrella Chambers 6329

lacticollis Smith 2047

lactiflosella Chambers 6374

lactimaculella Walsingham 8453

lactipennis Harvey 2973

lactispargaria Walker 4454

Lactura

lacunosa Grote 1240

lacustra Wright 518

lacustrata Guenee 4037

læna Grote 3203

laerta Smith 1639

laertidia B. \& McD. 1639,1

lata Edwards 393

læta Guerin 931

læta Geyer 7732

læta Hulst 4649

læta Morrison 2794

lætabilis Smith 2616

lætabilis Zetterstedt 1639

lætalis $B . \& M c D .5084$

lætella Grote 5591

lætifica Lintner 1993

lætifica Smith 2460

lætificans Smith 1249

Laetilia .147

lætula Grote 1575

lætula Hulst 4444

lætulus Grote 3576

lævigata Grote 3503

lævigata Smith 2342

lævitaria Hubner 3845

lagena Grote 1390

lagganæe Smith 1308

lagganata Taylor (Entephria) 4063

lagganata Taylor (Eupithecia) 4180

Lagoa 127 
lagopana Walsingham 7149

laguna Busck 6130

laguneularise Dyar 849

laguneulariella Busek 6193

lagunellus Dyar 5408

lagus Edwards 598

Iais Edwards 160

laisata Streoker 4162

lallata Hulst $\mathbf{4 5 8 8}$

lallatalis Hulst 5593

lambertiana Busck 7355, 1

lamialis Walker 5216

lamiaria Strecker 4544

lamina Fabricius 305

laminis Smith 1329

Lamprolophus

lamprosana Robinson 7337

Lamprosema 129

lana Kearfott 7219

lanariella Clemens 8298

lanceolana Hubner 6789

lanceolaria Grote 2509

lanceolata Boisduval 42

lanceolata Grote 2978

lanceolata Hulst 3837

landana Kearfott 7007

landia Druce 4870

langdonalis Grote 5117

langtoni Couper 1046

languida Smith 1664

languida $H y$. Edwards 1061

lanice Lintner 53

Laniifera

lanthanis Cook \& Watson 308

lanuginosa Clemens 4858

lanuginosa Smith 1628

Isnul Strecker 1170

Laodamia 145

Lapara

lapena Schares 4860

Laphygma 67

laphyra Druce 2873

lapidana Walsingham 7085 lapidaria Grote 1934

lapidicornis Walsingham 7810

Iapitaria Strecker $\mathbf{4 3 5 3}$

lappella Linnaeu 6021

lapponica Staudinger (Eurymus) 68

lapponica Staudinger (Xanthorhoe) 4046

lapponica Thunberg (Hyphoraia) 943

lapponica Thunberg (Sympistis) 2200

laqueata Hy. Edwards 1022

laqueatellus Clemens 5358

laracana Kearfott 7118

larana Walsingham 6915

Larentiinze

larentioides Grote 3470

larga Smith 1452

l-argenteum Seudder 277

largera Smith 3187

laricana Busck 7251, 1

laricella Kearfott 7714

laricella Hubner $\mathbf{7 7 7 5}$

lariciana Heinemann $\mathbf{7 1 7 0}$

laricis Fitch 3743

larimana Walsingham 7242

larissa Smith 1739

larra Fabricius 49

larunda Strecker 231

larvalis Grote 3533

larvaria Guenee $\mathbf{4 5 8 5}$

larvata Strecker 321

Lasaia

Lascoria

91

Lasiestra

Lasiocampidx _............................. 97

Lasionycta

Laspeyresia

lassauxi Boisduval 714

lassenella Busck 6621

lasus Edwards 554

lata Robinson 7365 


\begin{tabular}{|c|c|}
\hline $\begin{array}{r}\text { Page } \\
87\end{array}$ & Page \\
\hline 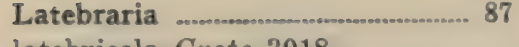 & latipennis Hulst (Eupithecia) \\
\hline atebricola Grote 3018 & 4187 \\
\hline latens Smith 1777 & latipennis Hulst (Rachela) 3967 \\
\hline laterana Robinson 7323 & latipennis Stretch 953 \\
\hline latercula $H y$. Edwards 4872 & latipennis Walsingham 6412 \\
\hline laterculæe Dyar 4873 & latipes Guenee 3142 \\
\hline lateritia Hubner 2346 & latipunctana Walsingham 7488 \\
\hline lateritiaria Guenee 4685 & latiradiellus Walker 5356 \\
\hline latex Guenee 1695 & latirena Dod 1919 \\
\hline Lathosea & latireptana Walker 2340 \\
\hline latia Strecker 2643 & latirosaria Pearsall 3789 \\
\hline latiaria Packard 3783 & latirupta Walker 4074 \\
\hline laticapitanus Walsingham 8176 & latistrigella Walsingham 8105 \\
\hline laticapitella Clemens 6582 & latiuscula Herrick-Schaffer 1948 \\
\hline laticincta Walker 4678 & lativittella Ragonot 5753 \\
\hline laticinerea Grote 2151 & latomia Harvey 4840 \\
\hline laticlava Smith 2403 & latreillana Kirby 838 \\
\hline laticlavia Clemens 4842 & laudabilis Guenee 1756 \\
\hline laticlavia Grote \& Robinson 5157 & laudatella Walsingham 6291 \\
\hline laticlavia Morrison 3251 & laura Edwards 181 \\
\hline laticornella Clemens 7776 & laurenti Skinner 190 \\
\hline latiscosta Dyar 2040 & laurenti Smith 3488 \\
\hline latifascia $B . \& M c D .785$ & laurentina Lyman 562 \\
\hline latifascia Walker 2572 & laurentina Scudder 63 \\
\hline latifasciana Haworth 7420 & laurina Wright 182 \\
\hline latisfasciaria Packard 4593 & lautana Clemens 7231 \\
\hline latifasciata Butler 941 & lautaria Hubner 3875 \\
\hline latifasciatella Packard 5596 & lautus Cockerell 8465 \\
\hline latifasciella Dyar 5537 & lavana Busck 7482 \\
\hline $\begin{array}{l}\text { latifasciella Chambers (Tel- } \\
\text { phusa) } 6074\end{array}$ & $\begin{array}{l}\text { lavernella Chambers } 6116 \\
\text { laviana Hewitson } 502\end{array}$ \\
\hline latifasciella Chambers (Nepti- & lavinia Fabricius 290 \\
\hline cula) 8373 & leachellus Zincken 5342 \\
\hline latiferreanus Walsingham 7269 & leada Druce 2599 \\
\hline latiferrugata Walker 4425 & leanira Boisduval 233 \\
\hline latiorata Walker 4468 & lebertella Frey \& Boll 7957 \\
\hline latiorella Walker 3443 & leca Butler 502 \\
\hline latipalpis Walker 3464 & lecontei Boisduval 1033 \\
\hline latipenella Chambers 7876 & lecontei $R . \&$ J. 20 \\
\hline latipennis Boisduval 3586 & lecontella Clemens 6472 \\
\hline latipennis Hulst (Cleora) 4594 & leda Edwards 349 \\
\hline latipennis Hulst (Dichorda) & ledella Walsingham 7910 \\
\hline 3810 & legitima Grote 1715 \\
\hline
\end{tabular}


leguminana Buack 7495

leilia Edwards 812

lemberti Dyar 8491

lena Boisduval 941

lena Edverds 511

lenitaria Grosebeck 4824

Jenola Smith 1803

lentaria Hulet 4693

lentella Braun 7938

lentella Heinrich 7755

lentiflualis Zeller 4901

lentiginosana Grote 3572

lentiginosana Walsingham 7339

lenzi Behrens 8495

lenzi French 3272

leo Guence 2930

leodocusalis Walker 5048

leomegra Smith 1662

leona Wright 233

leonana Walsingham 7112

leonardus Harris 566

leoninata Packard 4004

leoninella Packard 5689

leopardana Busck 7258

leopardus Busck 8156

lepetita Smith 2430

lepida Lintner 2167

lepidalis Hulst 5028

lepidana Clemens 7472

lepidula Grote 2423

lepidula Smith 1667

Lepipolys

leporina Linnaeu 2586

Leptarctia

leptinoides Grote 3660

Leptotes

Jepusculina Guense 2480

Lerema

Lerina

Lerodea

lespedezx Walsingham 5959

lespedersefoliella Clemens 8002

lessaria Pearsall 4476 lesteraria Groabeck 3829

I.:

lethalis Grote 5170

lethe Fabricius 291

letis Wright 157

leto Behr 157

Leucania 55

leucanidia Hampson 2796

leucanieella Busck 6228

Leucanthiza 188

leucata Hulat 4129

Leuce 155

leucillana Zeller 6609

leucobasis Busck 7248, 1

leucobasis Fernald 7623

Leucobrephos 99

leucocapitella Busck 8269

leucochrysella Clemens 7777

Leucocnemis 66

leucocycla Staudinger 1629

leucogramma Grote 1672

leucographa Geyer 3705

leucomalla Meyriele 6959

Leucomela .194

leucomelana Morrison 3207

leucomelas Herrich-Schaeffer 1033

leuconota Zeller 6063

leuconotella Busck 6366

Leuconycta 64

leucophra Smith \& Abbot 3713

leucophrata Clemens 686

leucophrella Hulst 5615

leucophaleratana Packard 7203

Leucoptera

leucorena Smith 2629

leucosalis $B . \& M c D .5056$

leucoscelis Grote 2359

leucosigma Packard 4824

leucostigma Braun 8406

leucostigma Smith \& Abbot 3705

leucothorax Meyriek 7727

leucothorax Walsingham 7878

Leuculodes 
- levalis Hulst 4940

levettei Grote 3032

levigatella Hulst 5633

levipedella Clemens 6201

levis Hudson 3761

levis Grote 2030

levisaria Grossbeck 4736

levisella Fyles 6368

levitans Smith 3019

lewisi Grote 1343

lewisi Swett 4644

Lexis

libatrix Linnaeus 3399

libedis Smith 2955

libella Grote 5244

libera Strecker 3703

libera Walker 1685

liberaria Walker 4325

liberomacula Dyar 889

libocedrella Busck 7687, 1

libocedrus $\boldsymbol{H} y$. Edwards 689

liburna Geyer 3411

libya Scudder 510

Libytheidæ

Libythea

licastus Stoll 725

licealis Walker 5025

licentiosa Smith 2617

lichena B. \& McD. 2530

lichenaria Pearsall 4592

Lichnoptera 83

licinus Edwards 555

lienigiana Heinemann 6855

ligata Grossbeck 4768

ligata Grote 1953

ligata Walker 1754

ligeæ Smith 1102

ligni Guenee 3411

lignicolor Walker 3649

lignicolora Guenee 2253

lignicolorata Packard 4111

lignigera Walker 3649

ligniperdalis Dyar 5290 lignosellus Zeller 5657

ligulella Hubner 6380

lilacina Harvey 1717

lilacina Schaus 2000

lilea Reakirt 454

liliana $H y$. Edwards 179

lilith Strecker 780

lilius Dyar 522

lima Guenee 3144

Limacodes .126

Limacodidæe

125

limata Bird 2682

limata Grote \& Robinson 4960

limbalis Grote 1193

limbata Haworth 4680

limbata $H y$. Edwards 2880

limbipennella Clemens 7683

limbolaris Geyer 3328

limbopunctata Nolcken 4089

limigena Meyrick 6976

limitaria Walker 3951

limatata Smith 1959

limitata Robinson 7336

limnata Pearsall 4197

limpida Guenee 2697

linda $B . \& M c D .2028$

linda Bird 2693

linda Edwards 79

lindana Fernald 7140

lindenella Busck 6252

linealis Fernald 5109

lineana Fernald 6952

lineapulvella Chambers 7778

linearis Grote 3435

lineata Druce 3631

lineata Fabricius 762

lineata Hulst 4590

lineata Walker. (Diastema) 2916

lineata Walker (Schinia) 1152

lineata Walsingham 8220

lineatella Harvey 2569

lineatella Zeller 6353

lineella Grote 3120 
lineifrons Smith 1922

Lineodes

lineolata Walker (Catabena) 2564

lineolata -Walker (Diatrea) 5438

lineosa Smith 2353

lineosa Walker $\$ 185$

lingulacelle Clemene 6068

linita Guence 1957

linnei Grote \& Robinson 740

lintnerana Speyer 3012

lintneraria Packard 4325

lintneri Fitch 281

lintneri Grote (Ommatostola) 2812

lintneri Grote (Gluphisia) 3681

lintneriana Grote 7358

Liopasia

liparops Boisduval \& Le Conte 367

lipatiella Busck 6206

Lipocosma

Lipographis

liquida Grote 1707

liquida Moeschler 2599

liquida Meyrick 7507

líquidambarella Dyar 5626

liquidambarisella Clemens 8115

liquoraris Guence 3799

liriodendrana Kearfott 6780

lidiodendraria Smith \& Abbot 4607

liriodendronella Clemens 8113

lisa Boisduval 78

lisetta Dyor 5435

listerana Kearfott 7503

litaria Hulat 4323

Lithacodes

Lithacodia

Lithariapteryx

lithella Busck.7560

Lithocolletis

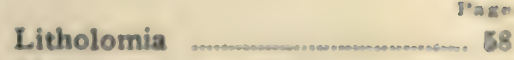

Lithomoia

lithosella Ragonot 5659

Lithosiin $x$ 80

lithosina Hy. Edu'urds 2852

lithosina Zeller 6612

lithosioides Dyar 857

lithospila Grote 2502

Lithostege .103

1itigiosella Zeller 8130

Litocala ………................... 85

Litodonta ................................ 94

litophora Grote 3513

Litoprosopus 86

littera Guenee 2396

littoralis Packard 1477

lituralis Hubner 3499

Jiturana Walsingham $\mathbf{7 1 5 0}$

liturata Menetries 980

liturata Smith 2445

liturella Walker (Gelechia) 6330

liturella Walker (Menesta) 6395

liturosella Zeller 6278

livalis Smith 1508

livida Letcher 3129

lividalis Hubner 3577

lividus Hubner 457

livorella Zeller 6545

lixalis Grote 3542

lixaria Grote 4587

lixaria Guenee 3770

lixiva Grote 2906

llano Dodge 498

loammi Whitney 614

lobato Barnes 1549

lobelise Guenee 2464

lobidactylus Fitch 5856

Lobophora 104

lobophorata Walker 3951

loculata Morrison 2380

loculosa Grote 2083 
locutia Hewitson 500

loda Strecker 2190

logan Edwards 597

logiana Fabricius 7439

logiana Schiffermuller $\mathbf{7 4 2 3}$

loiperda Dyar 781

lois Dyar 781

loki Skinner 378

lolana Kearfott 6959

Lomanaltes

lomonana Kearfott 7370

lona Strecker 2288

longa Guenee 2499

longa Grote 924

longeciliata Frey \& Boll 7867

longestriata Frey \& Boll 7885

longiciliata Hulst 4623

longiclava Snith 1748

longidens Hulst 4183

longidens Smith 1230

longifasciella Clemens 6071

longilabris Grote 3516

longimaculella Chambers 6642

longior Smith 2142

longipalpata Hulst 3440

longipalpata Packard 4214

longipalpella Ragonot 5809

longipalpus Morrison 5403

longipennata Packard 3839

longipennella Hulst 5699

longipennis Grote 1215

longipennis Walker 3951

longipes Druce 2831

longipes Moeschler 6726

longirostrellus Clemens 5314

longitudinella Busck 6159

longleyi French 545

longula Grote 2366

longula Hulst 3994

Lophoceramica

Lophodonta

Lophoptervx 93
Lophosis

Page

lora Strecker 1134

lorata Grote (Euthyatira) 3692

lorata Grote (Sabulodes) $\mathbf{4 7 6 8}$

lorata Grote \& Robinson (Strymon) 365

lorea Guenee 1754

loricana Grote 7202

loricari Eversmann 4453

lorquinaria Guenee 4451

lorquini $\mathrm{Behr} 448$

lorquini Boisduval (Basilarch-

ia) 307

lorquini Boisduval (Zerene) 54

lorquini Grote \& Robinson 1039

lorquini Oberthur (Melitæa)

204

lorquini Oberthur (Parnassius) 24

lorula Dyar 1009

los Strecker 8483

lotis Lintner 427

Lotisma .182

lotta B. \& McD. 8334

lotta Beutenmuller 39

louisa Hulst 3780

louisa Smith 2857

louisiana Busck 7456

Loxofidonia .107

Loxostege 131

loya Smith 1281

lubens Grote 1694

lubricalis Geyer 3496

lubricans Guenee 1502

lucasi Smith 4118

luccusalis Walker 4931

lucens Morrison 1095

Luceria 73

lucernalis Hubner 4977

lucetiella Clemens 7920

lucetta $H y$. Edwards 3034

lucia Kirby 452

luciana Strecker 3056 
lucida B. \& MCD. 1316 lucidalis Walker 5698 Iucidata Walker 2396 lucidella Buaok 6495 lucidicostella Clemens 7886 lucidus Boisduval 748 luciferella Clemens 5998 lucifluella Clemens 7590 lucilius Lintner 520 lucilla Worthington 3068 lucina $\boldsymbol{H}$ y. Edwards 785 lucina Smith 1755 lucinda Beutenmuller 3103 lucipara Linnaeus 2317 lucretia $B . \&$ McD. 8330 luctiferana Walker 7172 luetuata Schiffermuller 4077 luctuellus Herrich-Schaeffer 5857

luctuosa Hulst 3039

luctuosus Boisduval 726

luculenta $H y$. Edwards 3592 luda Druce 2882

lugens Walker 680

luggeri $\boldsymbol{H} y$. Edwards 6753

lugubralis Walker 5242

lugubrata Moeschler 3981

lugubrella Fabricius 6216

lugubrella Ragonot 5794

lugubris Boisduval 4889

lugubris Fish 5902

lugubris Hulst 990.

lugubris Linneeus 726

lugubrosa $\mathrm{Hulst} 4654$

lulualis Hulat 5040

lumbonigera $D$ yar 1035

lumenaria Hubner 3918

luminosa Neumoegen 6655

luna Linnaeus 773

luna Morrison 2008

lunata $B$. \& $M C D .2595,1$

lunata Drury 3175

lunata $\boldsymbol{H} y$. Edwards 3639
Page

lunata Smith (Apamea) 2640

lunata Smith (Urragastra) 1863

lunata Stretch 1048

lunatana Walsingham 7225

lundana Fabricius 7177

lunearis Guenee 3306

lunifera Hubner $\mathbf{3 1 8 5}$

Juniferella Hulat $\mathbf{5 4 7 7}$

lunigerella Ragonot 5583

lunilinea Grote 3365

lunolacta Smith 1764

lunulalis B. \& McD. 5003

lunulalis Hubnor 4948

lunulalis Hulst 5476

lunus Edwards 616

lupata Grote 1085

Luperina 63

lupinella Busck 6198

lupini Boisduval 437

lupini Grote 2511

lupini $H y$. Edwards 6661

lurida $\boldsymbol{H} y$. Edwards 926

luridana Walsingham 6916

luridana Walker 7605

luridula Hulst 4602

luridula Guenee 3408, 1

lusea Stichel 24

lusca Swith 2638

luscinata Zeller 4074

luscitialis B. \& McD. 5139

luscitiosa Clemens 695

luski B. \& McD. (Argynnis) 186

luski $B$. \& $M c D$. (Polia) 1717

lustralis Grote 1657

lustrans Beutenmuller 4885

lustrans Grote 6668

lustrella Dyar 5729

lutalba Smith 3511

lutarius Walker 4665

lutea Grote 834

lutea Reiff 785

lutea Smith 3443

lutea Ström 2233 
lutealis Snellen 5228

lutearia $B$. \& $M c D .4320,1$

luteata Packard 4157

luteella Chambers (Busckia) 7852

luteella Chambers (Bucculatrix) 8131

luteella Hulst 5659

luteiceps Walker 8475

luteicoma Grote \& Robinson 2455

luteicostella Ragonot 5833

luteitincta Wollcott 60

luteitinctus Grote 1089

luteocinerea Smith 2305

luteocostella Chambers 7780

luteodisca Smith 1981

luteola Grote \& Robinson 1634

luteola Smith (Lasiestra) 1647

luteola Smith (Euxoa) 1265

luteolana Hubner 7314

luteolata Hulst (Acidalia) 3866

luteolata Hulst (Lygris) 3976

luteolellus Clemens 5399

luteolus Reakirt 38

luteomedia Smith 2552

luteopallens Smith 1972

luteopulvella Chambers 6582

luteoscapus Neumoegen \& Dyar 836

luteosita Smith 1348

luteostrigella Chambers 6178

lutescens Neumoegen \& Dyar 3746

lutheri Cockerell 780

lutina Smith 1805

lutosa Andrews 2356

lutosalis $B . \& M C D .5083$

lutosana Clemens 7390

lutulenta Smith 1315

luxa Grote (Schinia). 1140

luxa Grote (Perigea) 2327

luxuriosa Grote 1095
Page

luxuriosa Smith 2885

luzena Barnes 8345

lybialis Walker 5011

Lycæna ........................................ 17

Lycænidx .................................. 13

Lycæninæ ................................... 15

Lycanopsis .............................. 17

lycaste Fabricius 89

lycea $E$ dwards 433

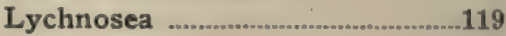

Lycia ...............................................118

lycialis Walker 5014

lycidas Smith \& Abbot 472

lycides Hubner 472

Lycimna .122

Lycomorpha

lycopersici Boisduval 664

Lycophotia 46

lycopodiana Kearfott 7317

Lycorea

lycus Hubner 342

lydia Beutenmuller 3060

lygdamas Doubleday 449

lygdamis Druce 4930

Lygranthocia .......................... 38

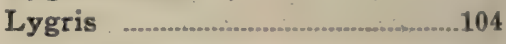

Lygropia 130

lymani Dyar 1034

Lymantriida 96

lvmax Druce 4632

Lymire 29

Lymnacia 152

lyncea Clemens 722

lynceella Zeller 6264

lynosyrana Walsingham 7287

lynosyridella Walsingham 7779

lynx Guenee 1180

Lyonetia 190

lyonetiella Chambers 6008

Lyonetiida 190

lyonsellus Haimbach 5339

lyside Godart 53

lysimachix Bird 2684

lysimachimella Chambers 7929 
Jythrella Walsingham 6468

Lythrodes

Lytrosis

macaira Reakirt 500

macareus Herrich-Scheeffer 623

Macaria

macaris Edwards 182

maccana Treitschle $\mathbf{7 4 3 2}$

mac-eullochi Kirby 1039

macdunnoughi Grosebeck 4513

macdunnoughi Oberthur 491

macerata Smith 2277

macelhosiella Busek 6626

macglashani $H_{y}$. Edwards 8487

macglashani Rivera 204

machaon Linnaeus 9

maclurxe Riley 5041

macmurtrei Guerin 8355

macona Smith 1907

macouni Edwards 120

macra Guenee 2574

macrinellus Zeller 5305

macrocarpana Walsingham 7530

macrocarpella Frey \& Boll 7940

macrogaster Welsingham 8187

macromacula Strand 2835

Macronoctua

macropterellus Zeller 5403

macroptica Smith 1148

Macrotheca

Macrothecinæ

142

mactata Guenee 2286

mactatoides $B$. \& McD. 2335

macula Druee 2612

macula Smith 2774

maculabella Chambers 8293

maculalis Clemens 5198

maculalis Westwood 4921

maculana Fernald 7211

macularia $B$. \& $M C D .4682,1$

macularia Harris 4644

maculata Edwards 646 maculata Harrie (Halisidota)

918

maculata Harris (Thyris) 4888

maculata Riley 8472

maculata Smith 1222

maculatana Walsingham 7033

maculatella Busck (Cerostoms)

7670

maculatella Buscle (Depressar-

ia) 6481

maculatella Dietz 8314

maculatusella Chambers 6331

maculicula Dyar 5716

maculidorsana Clemens $\mathbf{7 4 3 1}$

maculifascis Hulst 4391

maculifer Walsingham 8164

maculimarginella Chambers

(Gelechia) 6268

maculipennis Curtis 7683

maculipes Grote \& Robinson

6733

maculomarginella Chambers

8294

maculosa Behr 3340

maculosa Stretch 978

maculosella Chambers 8374

maculosella Clemens 7833

madarella Clemens 7834

madarix Hy. Edwards 6662

madderana Kearfott 7038

madefactalis Guenee 3566

madetesalis Walker 5151

Madoryx

madusaria Walker 4706

Mænas

mærula Hubner 52

mastosa Hulst (Catocala) $\mathbf{3 0 4 3}$

mestosa Hulst (Eupithecia)

4216

mæstosa Hulot (Cleora) 4565

mastus G. \& S. 533

magdalena Fernald 5054 
Page

magdalena Hulst (Melemæa) 4639

magdalena Hulst (Nycterophæta) 2008

magdalena Strecker (Catocala) 3088

magdalena Strecker (Erebia) 144

magistralis Grote 5114

magnalis Hulst 5184

magnarius Guenee 4665

magnatella Busck 8085

magnatella Zeller (Mompha) 6008

magnatella Zeller (Phyllocnistis) 8118

magnella Chambers 8132

magnella Braun 7592

magnella Busck 6024

magnetaria Guenee 3879

magniferalis Walker 5125

magniferaria Walker 3835

magnifica Bailey 8343

magnifica Beutenmuller 6743

magnifica Grossbeck 4522

magnifica Grote 2928

magnifica Neumoegen 2940

magnifica Strecker 8343

magnificalis Hubner 5216

magnificata Taylor 4030

magnificus Fernald 5402

magnoliana Fernald 7359

magnoliana Kearfott 6781

magnoliata Guenee 4068

magnoliatoidata Dyar 3957

magnoliella Chambers 8114

magnus Wright 25

magualis Guenee 4954

Magusa 60

maia Ottolengui 968

maia Drury 784

maiana Kearfott 7473

maida Dyar 2191 maidella Dyar 5700

maillardi Geyer 2363

maimes Smith 1252

maizi Fitch 1329

major Grote 2064

major Grote \& Robinson 3598

major Kearfott 7585

major Smith (Feralia) 2097

major Smith (Tarache) 2977

major Speyer 1615

major Walsingham 6839

majoralis Smith 3498

majoraria Guenee 4758

majorella Dietz 8215

majuscula $H y$. Edwards 2869

mala Smith 1977

malaca Grote 3467

malachitana Z eller 6854

Malacosoma 98

malana Fitch 2600

malanum Fernald 6808

m-album Boisduval \& LeConte 351

malefida Guenee 1414

malifoliella Clemens 7873

malifoliella Fitch 6380

maligemmella Murtfeldt 6547

malimalifoliella Braun 7900

malindella Busck 6255

malipenella Dyar 5534

malis Smith 1341

malivorana Le Baron 7419

malivorella Riley 7781

malora Smith 1919

Malthaca 127

Mamestra

mammuraria Guenee 4492

mana Kearfott 6822

manalis Walker 3560

manataaqua Scudder 577

Manatha 125

mancalis Lederer 5021

mancinus Doubleday \& Hewitson 140 
maneinus Herrich-Sehaeffor 456 mancipata Guence 4005 mandan Edwarde 536 mandana Kearfott 6914 mandane Druec 2592 mandarinella Dietz 8274 mandella Busek 6271 manella Busek 7667 manfredi Neumoegen 8349 mania Strecker 1728 manifesta Morrison 1473 manifestolabes Morrison 1553 manihotalis Guenes 5258 manitoba Beutenmuller 3108 manitoba Busek 7816 manitoba Grossbeck 4568 manitoba Smith 2462 manitoba Scudder 562 manitoboides Fleteher 557 manitou Neumoegen \& Dyar 3608

Manruta 38

mansueta Smith 2427

mantalini Smith 1503

manteo Doubleday 3641

manti Barnes 2714

manto Strecker 3717

manubiaria Hulst 4709

manzanita Taylor 4011

mappa Grote \& Robinson 3265

maracana Kearfott 7120

Marasmia .129

Marathyssa 77

mareata $B$. \& $M c D .3947,1$

marcata Neumoegen 795

marcellina Crumer 47

marcellus Cramer 20

marcescaria Guenee 4450

marcia Edwards 247

marcia Beutenmuller 6704

marcida Guenee 3138

marcidana Zeller 7191

marcidilinea Grote 3509 marculenta Grote \& Robinson 5020

mardon Edwards 582

marga B. \& MCD. 8333

margana Fabricius 2924

margarita Hubner 28

margarita Skinner 620

margaritosa Haworth 1490

marginalis Grote 785

marginalis Stoll 4977

marginalis Seudder 35

marginalis Walker 5174

marginata Edwards 452

marginata Harris 6649

marginata Haworth 1130

marginata Minot 4707

marginata Walker 3757

marginatus Harris 544

marginatus Riley 8462

marginidactyla Fitch 5881

marginidens Guenee 2663

marginimaculella Chambers

8294

marginistrigella Chambera 8239

margoricella Dietz 8209

maria Skinner 218

marie Grote 2343

marixella Chambera 7927

mariana Barnes 204

mariana Fernald 7396

mariana Strecker 3063

maricopa Hulst 4359

maricopa Reakirt 433

marina Grote 2273

marina Packard 3645

marina Reakirt 416

marina Smith 2411

marinaria Strecker 3817

marinitincta Harvey 1761

mariona Bentenmuller 6708

mariposa Grote \& Robinson 1048

mariposa Reakirt 405

maritima Bird 2701 
maritima Dyar 7184

maritima Edwards 111

marius Cramer 301

marloffiana Busck 7481

Marmara

marmarodactyla Dyar 5865

marmontana Kearfott 7128

Marmopteryx

marmorata Edwards 3054

marmorata Packard 3939

marmorata Smith 2439

marmoratus Grossbeck 4752

marmorea Walsingham 6636

marmorella Chambers (Phthor-

imæa) 6173

marmorella Chambers (Tinea) 8287

Maroa

mars Fabricius 341

marsyas Edwards 270

marteni French 4871

marthesia Cramer 3674

Martia

martialis Herrich-Schaeffer 340

martialis Scudder 524

martiella Braun 7919

martinella Walker 8235

Martyringa

masculinalis $B . \& M c D .4964$

masculinalis Dyar 5198

masculinus Smith 1249

masonaria Schaus 3822

masoni Cockerell 1310

masoni Smith 1106

Massala

massasoit Scudder 591

Mastor 22

mata Reakirt 254

materna Linnaeus 3364

mathesoni Busck 7824,1

mathewi $\boldsymbol{H} y$. Edwards Scep-

sis) 825

mathewi Hy. Edwards (Hepial-

us) 8488 mathewianus Z Zeller 5898

Matigramma

matilda Dyar 4427

matricaria Behr 1984

matronalis Grote 5152

Matuta 47

matuta $H y$. Edwards 1043

matutella Clemens 8079

matutina Grote 6962

matutina Hubner 1097

matutina Meyrick 6769

mavina $B . \&$ McD. 2712

maxima Dyar 2528

maxima Neumoegen 50

maximella Chambers 8375

maximellus Fernald 5315

Mea 194

meadi Edwards (Argynnis) 171

meadi Edwards (Cercyonis) 115 meadi $E d w a r d s$ (Eurymus) 56 meadi Hy. Edwards (Synanthedon) 6709

meadi $\boldsymbol{H} y$. Edwards (Thyridopteryx) 4800

meadi Grote 1096

meadi Packard 3972

meadiana Morrison 2033

meadiaria Packard 4379

meanderana Walker 6798

Mechanitis

mecrona Smith 1862

mecyanalis Druce 6345

medealis Walker 4954

medeifascia Grossbeck 4091

media Braun 7715

media Morrison (Drasteria) 3327

media Morrison (Plagiomimicus) 2767

medialis Grote 2178

medialis Smith (Hydroeia)

2647

medialis Smith (Bleptina) 8537

medialis Smith (Euxoa) 1301 
mediatrix Dyar 2934

medicinalis Grote 5214

mediella Busele 6629

mediodentata B. \& MeD. 4023

mediodorsella Braun 7948

mediofasciana Clemens 7173

mediofasciella Diots 6583

mediofuscella Clomens 6278

medioliniella Kearfott 8221

medioplagata Walsingham 7161

mediostriana Kearfott 7123

mediostriata Walaingham 7046

mediostriatella Clemene $\mathbf{8 1 4 4}$

medioviridana Kearfott 6987

meditata Grote 1673

medor Merian 663

medusa Strecker 3732

Megacraspedas

megadia Smith 1941

megrera Smith 1729

megalippe Hubner 84

Megalopyge 127

Megalopygidae

megamicrella Dyar 6487

megarena Smith (Schinia) 1173

megarena Smith (Polia) 1755

Megasis 146

megastigma Smith 1292

Megathyminz 23

Megathymus 23

Megistias 22

megocula Smith 2939

mejicanus Behr 322

mejicanus Reakirt 512

mela Strecker 422

melaleuca Thunberg 2198

melaleucana Walker $\mathbf{7 3 5 7}$

melalutea Smith 2050

melancholica Grote 716

Melanchroia 124

melane Edwards 595

melanella Hulst 5608

melanella Murtfeldt 6396 melanippe Cramer 48

melanis Boisduval 3763

melanogrammos Zeller 5483

melanographa Hampeon 2116

melanographella Ragonot $\mathbf{5 4 9 7}$

Melanolophia

Melanomma \$2

melanopa Barnes 3627

melanopa Thunberg 1638

melanopa Zeller 850

melanopis Hampson 1822

melanopyga Grote 2789

melanosticta Hampson 2876

melanosticta Walsingham 6858

melonostriatelia Dietz 6574

melanotella Dieta 8024

melantherella Busck 6372

melantho Smith 2023

Melanthus

melanympha Guenee 3025

Melaporphria

Melemaea

melicerts Schaus 327

Melicleptria 37

melimona Wright 440

melinellus Clemens 5811

melinus Hubner 352

Melipotis

melissa Edwards 427

Melissoblaptes

Melissopus

Melitaa

Melitara

melite Linnaeus 8 ?

Melittia

Mellilla

mellinalis Hubner 5105

mellinella Grote 5745

mellinipennis Boisduval 6658

mellisa Grossbeck 4134

mellistrigata Grote 4373

melitula Hulat 8116

mellitularia Hulst $\mathbf{4 7 1 8}$

mellonella Linnaeus 5448 
melon Godman \& Salvin 471 melsheimeri Harris 4792 membrosa Morrison 1632 memmialis Walker 4938 memoriata Pearsall 4500 Memythrus .166 menapia Felder 26 menas Harris 3633 mendica Walker 4122 mendica Stichel 25 mendicata Hulst 4394 mendocino Behrens 777 mendocinolus Behrens 8492

Menesta 160

Menestomorpha

menetriesi $H y$. Edwards 24 mengelana Fernald 6840 mengeli Fernald 5944 menisme Verity 67

Menopsimus

mensurata Walker 3857

menthrastrina Martyn 960

meodana Smith 1708

mephistaria Reiff 4620

mephistaria Swett 4042

mera Harvey 2845

meralis Morrison 2579

merceda Smith 2159

mercedes B. \& McD. 1269

mercedulata Strecker 4313

mercenaria Grote 1374

mercurius Fabricius 466

merianæ Grote 714

Mericisca

meridiana Slosson 4123

meridionalis $B . \& M c D .3710$

meridionalis Dyar (Amblyscirtes) 627

meridionalis Dyar (Cerura) 3672

Meris

meritata Pearsall 4169

Merochlora
Merolonche

\section{Meroptera} 66 merrickana Kearfott 7545 merriccata Bird 2674 merriccata Dyar 4625 merriccella Dyar 6485 merrickanum Kearfott 6810 merricki Holland 3354 merricki Smith 3493 mersa Morrison 2325 mertena Smith 2170 mertila Edwards 450 mesapano Scudder 536

Mescinia 147

mesillæ Cockerell 3603 meskaria Packard 4735

Meskea .128

meskeana Grote 1116 meskei Edwards 567 meskei Hy. Edwards 3369 meskei Grote 3067 meskei Speyer 2598 meskei Smith 2937 mesocausta Meyrick 7710

Mesoleuca 107

Mesolia .139

mesospila Zeller 7294

Mesothea 100

messalina Guenee 3093 messelinella Dietz 6559 messoria Harris 1304 mestoralis Walker 5061

Mestra 10 mestusata Walker 4685 meta Smith 1574

Metachrostis 64 metacomet Harris 604 metagrammalis Walker 5654

Metalepsis 45

Metalestra 89

metaleuca Hampson 3204 metalicella Hulst 5661 metallica Busck 6776 


\section{Page}

metallica Grote (Autographa) 3272

metallica Grote (Tarache) 2992

metallica Stretch 4882

metallicana Waloingham 7330

metallicella Busek 7567

metallifera Waleinghan 7835

metalliferalis Prekerd 5446

metamelana Walker 7183

Metanema

metanemaria Hulat 4452

metaschista Meyrick 7012

metata Smith 3192

metathetis Butler 735

meten Scudder 556

meticulosalis Guenee 498!

metonalis Walker 3516

metra Smith 2476

Metrea

130

Metriochroa

metrocamparia Guenee $\mathbf{4 7 5 8}$

metzaria Dyar 4741

Metzneria .153

mexicana Behr 3318

mexicana Boisduval $\mathbf{7 4}$

mexicana Busck $\mathbf{7 5 2 8}$

mexicana Druce 898

mexicana Erschoff $\mathbf{7 5 9}$

mexicana Grote \& Robinson

(Citheronia) 812

mexicana Grote \& Robinson

(Apantesis) 983

mexicana Herrich-Schacffer 484

mexicana $\boldsymbol{H}$ y. Edwards 3691

mexicanaria Grote 4610

mexicanellus Beutenmuller 8190

mexicanus Beutenmuller 6737

meyrickella Busck 6408

micacea Eeper 2650

micea Druce 3355

michabo Grote 976

Micrathetis
Microcausta

mierogamma Hubner $\mathbf{3 2 2 5}$

Microgonia

Microhelia 73

micronympha Guenee $\mathbf{3 1 1 7}$

micronyx Grote 1277

microphysa $\boldsymbol{H}$ ulat 3886

micropterata $\boldsymbol{H}$ ulet $\mathbf{3 8 9 5}$

Micropterygida 197

microstagma Dyar 5071

microsticta Godman \& Salvin 538

Microtia 10

midea Hubner 43

Mieza .184

mignonella Dyar 5782

migrata Smith 2371

milberti Godart 280

mildei Stretch 3754

militaris Harris 1033

militella Zeller 5489

milleri Busck 6154

milleri Grote 1232

milleriella Chamber 6332

milo Edwards 575

mima Harvey 3365

mimallonis Grote 1303

mimella Clemens 6333

mimica Strecker 1941

mimicata Walker 3800

Mimobarathra 47

Mimorista

mimosaria Guence 3783

mimula Grote 1643

mimuli Behr 1635

mimuli $\boldsymbol{H} y$. Edwards 6665

mindara $B$. \& $M c D .2591$

minea Slosson 976

minella Dyor 2441

Mineola .143

minerea Guenee 3184

miniana Grote 1103

minians Guenee 1866

miniata Kirby 899 
miniata Packard 831

miniatella Ragonot 5562

minima Edwards 542

minima Grote 819

minima Guenee 2872

minima Hulst (Chloraspilates) 4302

minima Hulst (Gabriola) 4612

minima Neumoegen 940

minimaculella Chambers 6260

minimalis $B$. \& $M c D .3541$

minimalis Grote (Rhynchagrotis) 1593

minimalis Grote (Zanclognatha) 3507

minimana Walsingham 7073 minimella Chambers (Mompha) 6016

minimella Chambers (Aristotelia) 6053

minimella Chambers (Nepticu-

la) 8376

minimella Ely 8070

minimella Ragonot 5529

minimella Robinson 5330

minimum Neumoegen 6654

miniota Smith 2189

minisni Bean 67

ministra Drury 3595

ministrana Linnaeus 7389

minna Butler 845

minnehaha Scudder 435

minnicella Dietz 6593

Minofala

minor $B . \&$ M $M .1995$

minor Busck (Bedellia) 8121

minor Busck (Phthorimæa) 6170

minor Cockerell 284

minor Dyar (Acrolophus) 8193

minor Dyar (Roeselia) 852

minor Smith 3367

minor Verity 25

minoralis Smith 3501

minoraria Hulst 4702 minorata Barnes 2244

minorata Grote 2535

minorata Packard (Macaria)

4338

minorata Smith (Antiblemma) 3361

minorata Smith (Epia) 1781

minorata Smith (Gabara) 3425

minorata Smith (Leucania)

1971

minorata Taylor 4154

minorelia Dietz 6540

minta Dyar 3740

mintha Edwards 433

minualis Walker 5226

minuscula $B$. \& $M c D .2582$

minuscula Morrison 2338

minuscula Zeller 851

minuta $B . \& M c D$. (Givira) 8336

minuta $B . \& M c D$. (Viridemas) 2320

minuta Druce 877

minuta Edwards (Catocala)

3116

minuta Edwards (Melitæa) 241

minuta $H y$. Edwards 6717

minuta Grote 3743

minuta Hulst (Gymnocelis) 4126

minuta Hulst (Phasiane) 4682

minuta Reakirt 4848

minuta Robinson 7419

minuta Schaus 3883

minutana Kearfott 6982

minutella Beutenmuller 5965

minutella Frey \& Boll 7912

minutipulvella Chambers 8283

minutissima Smith 5072

minutularia Hulst (Acidalia) 3875

minutularia Hulst (Myelois) 5506

minyas Hubner 332 
miona Smith 2853

mira Dyar 4817

mira Grote 3105

mira Verity 67

mirabella Dyar 5590

mirabelle Barnes 364

mirabilata Grote 4073

mirabilicornella Dyar 5754

mirabilis Wright 208

mirabilis Neumoegen 2743

mirabilis Frey \& Boll 8002

mirabilis Grote 1582

miraculosa Frey \& Boll 5955

miranda Dyar 3676

miranda Grote 2590

miranda $\mathrm{H} y$. Edwards 3101

miranda Hulst 3908

mirandalis Hubner 3402

mirandata Taylor 3998

mirandella Ragonot 5611

miriamella Dietz 8321

mirifiea Frey \& Boll 7891

mirifica $\mathbf{H y}$. Edwards 3322

mirificalis Grote 2015

mirificum Strecker 3583

mirusella Chambers 6640

Misa

miscana Kearfott 6954

miscecalonella Chambers 5985

miscecristatella Chambers 8315

misceella Chambers 8259

miscellus Grote 2564

miscellus Smith 2276

miscidice Scudder 60

miselioides Guenes 2276

misella Zeller 8247

misera Grote 2337

miserulata Grote (Metalestra) 3482

miserulata Grote (Eupithecia) 4140

misippus Linnaeus 267

Misogada

missouriensis Schwarz 3031

Page

mistrella Busek 6611

misturata Hulat $\mathbf{4 1 8 8}$

misturata Smith 1262

mitchelli Dyar 5295

mitchelli Freneh 95

mitis Grote 1056

mitis Smith 1264

mitographa Grote 8468

Mitoura

mixta Neumoegen 916

mixta Grote 2316

mixta Walker 1505

Mnemonica

moderata Walker 1959

moderna Grote 3043

modesta B. \& McD. 1109

modesta Bates 292

modesta Beutenmuller 3601

modesta $\boldsymbol{H y}$. Edwards 2950

modesta Frey \& Boll 7946

modesta Harris 711

modesta Hudson 3673

modesta Kellicott 6681

modesta Morrison 1807

modesta Maynard 345

modesta Packard 1024

modesta Smith 3582

modesta Taylor 4150

modesta Walsingham (Paltodora 6034

modesta Walsingham (Platyptilis) 5888

modestalis Fitch 3503

modestana Busck 7562

modestaria $\mathrm{Hulst} 4406$

modestella Clemens 6539

modestella $\mathrm{Hulst} 5576$

modestus Grossbeck 4749

modestus $\boldsymbol{H}$ y. Edwards $\mathbf{8 4 9 5}$

modica Guenee 2308

modica Walker 2435

modicella Grote 1052

modifica Morrison 1835 
modiola Grote 2335 modulata $\mathrm{Hy}$. Edwards 874 moerens Grote 1265 moeschleri Grum Grschmailo 67 moeschleri Staudinger 1627 moeschleriana Wocke 7376 moeseri Bird 2666 moesta Dyar 2487 moestana Wocke 6818 moestata Nolcken 4088 moestella Walker 5653 moffatiana Fernald 7135 moffatiana Grote 2216 moierra Dyar 993 moilena Strecker 2188 moina Strecker 70 molestella Zeller 6043 mollicularia Zeller 4678 molliculata Walker 3980 mollifera Walker 3486 mollipedella Clemens 7683 mollis Walker (Heterocampa) 3642

mollis Walker (Euxoa) 1346 mollis Wright 46 mollisaria Hulst 4703 mollisima Guenee 2391 molpodia Hubner 28 molybdanella Dietz 8279 momana Kearfott 7012 momaria Guenee $\mathbf{4 5 7 7}$ momonana Kearfott 7127

Momophana

Mompha 153

mona $B$. \& McD. 2580

monachella Busck 6620

monachella Hubner 8240

monella Busck 6248

moneta Fabricius 3278

monetifera Guenee 2392

monetiferanum Riley 6795

monicaria Guenee 4314

monilis Fabricius 3403

monima Cramer 292

monitor Packard 4816 monochlora Meyrick 7676 monochromatea Morrison 1475 monoco Scudder 642 monodactylus Linnaeus 5942 Monodes 68 monodia Dyar 3481 monodon Dyar 5589 monodon Grote 3268 monogrammana Zeller 6908

Monoleuca .125

monophylliana Kearfott 6767

Monopis 192

Monoptilota 144

Monotaxis 104

monotropa Grote 3378

monstralis Guenee 4971

monstrata Walker 1533

monstratatella Walker 8079

montana Behr 251

montana Beutenmuller (Catocala) 3071

montana Beutenmuller (Olene) 3717

montana Busck 6777

montana Cockerell 5954

montana Grote 2090

montana Haimbach 5226

montana $\mathrm{Hy}$. Edwards (Dysodia) 4891

montana Hy. Edwards (Memythrus) 6744

montana Morrison 1227

montana Skinner 569

montana Smith 2275

montana Verity 40

montana Walsingham 5890

montanæ Grote 1998

montanana Kearfott 6821

montanana Walsingham 7100

montanaria Neumoegen 4731

montanata Packard (Lobophora) 3957 
montanata Packard (Lygris) 3981

montanata Schiffermuller 4046 montanus Stretch 8493

montara Smith 1776 monteclara Smith 1281 montella Chambers 7705 monticola Behr 168 monticola Clemence 438 monticola Grinnell 5872 monticola Hulst 3439 monticola Walsingham 6624 montinatatella Hulat 5749 montinus Soudder 192 montis Edwards 311 montisella Chambers (Cosmopteryx) 5967

montisella Chambers (Glyphipteryx) 7625

montivaga Behr 183

montivagus Reakirt 493

monulalis Hulst 5138

monumentella Chambers 6285

monuste Linnaeus 30

Moodna

mopsa Hy. Edwards 3103

mopsalis Walker 4987

mopsus Hubner 358

moqui Barnes 3679

mora Grote $\mathbf{8 1 9 5}$

morana Smith 1617

morbidalis Guenee 3519

morbosa $\mathrm{Hy}$. Edwards 3297

morbosa Hulst 4329

morenalis Dyar 5168

morenella Busck 6225

moreno Barnes 2706

morensata $\mathrm{Hulat} 3960$

mori Linnaeus 3755

morigera $\boldsymbol{H y}$. Edwards 3279

Morina

mormo Felder 322

mormonaria Dyor 4524 mormonia Boisduval (Argynnis) 184

mormonia Boisduval (Apodemia) 322

mormonica Neumoegen 983

morna Streeker 2351

morosaria Walker 4336

morosata Hulst 4541

morpheus Fabricius 247

morrisata Hulst 3936

morrisella Fitch 7917

morrisonarius $\mathrm{Hy}$. Edwards 4750

morrisonella Ragonot 5741

morrisoni Druce 3586

morrisoni Edwards ((Anæea) 319

morrisoni Edwards (Anthocharis) 44

morrisoni Edwards (Pamphila) 558

morrisoni Grote 2214

morrisoni $H y$. Edwards 6745

morrisoni Walsingham (Acroolphus) 8180

morrisoni Walsingham (Eucosma) 6886

morrisoni Walsingham (Holcocera) 6560

Morrisonia

morrisoniana Riley 1395

morrisonistigma Grote 1567

morrissoni Ploetz 558

mors Lucas 4785

morsa Smith 2631

morsicaria Hulst 4646

morta Dyar 3708

mortipenellus Grote 8169

mortua Grote 1113

mortualis B. \& MeD. 3535

mortuana Kearfott 7853

mortuorum Guenee 3236

morula Grote \& Robinson 2473

morula $H y$. Edwards 6671 
moschus Edwards 480 mossi $H y$. Edwards 384 moxa Smith 1320

mucens Hubner 1873 mucida $H y$. Edwards 8326 mucidellum Ragonot 5771 mucorea Herrich-Schaeffer 3643 mucronata Grote 3296 muelleri Letcher 285 muiri $\mathrm{Hy}$. Edwards (Mitoura) 374

muiri Hy. Edwards (Gyros) 5066

muliercula Guenee 3024

mulleolata Hulst 3992

muelleolella Hulst 5288

multicolor Dyar 2263

multifaria Walker (Ctenucha) 836

multifaria Walker (Morrisonia) 1873

multifasciella Chambers 5973

multifera Walker 2577

multiferata Walker 4076

multilinea $B$. \& McD. 3382

multilinea Walker 1934

multilineana Kearfott 7250

multilineata Hulst 3851

multilineata Packard (Euphyia) 4070

multilineata Packard (Maca. ria) 4344

multilineatella Hulst 5425

multilinellus Fernald 5360

multimaculella Chambers 8215

multiplicalis Guenee 4932

multipulvella Chambers 7781

multipunctata Taylor 4082

multipunctella Chambers 6637

multipunctella Clemens 7723

multipunctellus Kearfott 5428

multiscripta Hulst 4174

multiscripta Riley 3666

multistriatella Dietz 8278

multistrigata Hulst 4179 multivagata Hulst 4061

multnoma Dyar 3594

munda Walker 3141

mundula Grote \& Robinson 3135

mundula Zeller 3463

munis Grote 1351

munitata Hubner 4041

murænula Grote \& Robinson

1234

muralis Grote 2104

murcialis Walker 5025

murdocki Smith 1328

murellus Dyar 5385

Murgaria 18

muricana Walsingham 7195

muricina Grote 1869

muricolor Dyar 1023

muricolor Hulst (Glena) 4602

muricolor Hulst (Stenaspilates)

4735

muricolorella Busck 6455

murina Packard 6857

murina Stretch 1005

murina Walker 867

murtfeldtella Busck 8051

murtfeldtella Chambers (Mom-

pha) 6017

murtfeldtella Chambers (Dryoperia) 6596

murtfeldtiana Riley 7179

mus Neumoegen 3746

musa Smith 1407

muscariata Guenee 4362

muscosa Grote 1303

muscosa Hampson 2194

muscosa $R$. \& J. 668

muscosula Guenee 2902

musculana Hubner 7386

musta Grote \& Robinson 2899

mustelina Packard 3660

mustelina Smith (Xylomyges)

1889

mustelina Smith (Trachea).

2309

mustelinalis Packard 5138 
mustelinus Packard 8489 mutabilana Clemene 6877 mutabilis Clemens 5384 mutata Dod 1616 mutata Peareall 4209 mutata Taylor 4000 mutilata Smith 1744 mutius Ploots 599 muzaria Walker $\mathbf{4 7 0 0}$ muzina Oberthur 965

Mycterophora 88 myellus Hubner 5856

Myelois 143

myersella Dyar 5732

mylitta Edwards 254

mynesalis Walker 3542

myops Smith \& Abbot 708

myricafoliella Busck 8377

myrina Cramer 187

myrina Martyn 199

myrinitis Meyriok 7457

myrissa Godart 187

Myrlaea

myrmidonata Guenee 3875

myrodora Dyar 814

myron Cramer $\mathbf{7 4 4}$

myronella Dyar 5824

myrrha Strecker 3090

myrta Godart 292

myrtaria Guenee 3917

myrtilli Linnaeus 1633

myrtis Eduards 546

mys Dyar 1905

Myscelia

mysia Godart 28

mysie Dyar $\mathbf{4 7 9}$

mysiella Dyar 5701

mysippusalis Walker 5099

mystic Scudder 581

mystica Smith 1699

Mythimna

myus French 579

Nacoleia

Nacophora
Nadata

I"m en

nævia Snith 1658

nevius Lintner 529

navosidactylus Fitch 5042

nevula Smith 1294

nahanni Dyar 125

nais Drury 995

nais Eduards 324

Namangana 68

nana $B . \&$ McD. 2408

nana Dietz 6554

nana Dyar 4815

nana Hubner 2905

nana Haworth 7490

nanaimo Barnes 2725

nanalis Grote 1503

nanata Neumoegen 2774

nandana Kearfott 6944

nanina Dyar 4815

nanno Edwards 624

naninodes Hampson 2953

nanodella Busek 6300

nanus Nermoegen 25

nanus Strecker 8346

napa Edwards 572

napæea Morrison 2124

napralis Hulst 5108

napi Linnaeus 35

naracana Kearfott 7134

narcsusalis Watker 4981

narrata Walker 3135

narses Staudinger 326

Narthecophora

nasar Smith 2165

Nascus 18

nasoni Grote 4830

nasonialis Zeller 5045

nastes Boisduval 69

nasturtii Boisduval 35

Nasusina 109

nasutaria Zeller 3436

nasutella Hulst 5779

Natada $126^{\circ}$

natalella Busck 6059 


natatrix Guenee 3426
Natbalis .a.n......... 8
nausicaa Edwards 161
navajo Skinner 654
navalis Meyrick 7118
navarra Dyar 1168
Navasota

navium Harvey 2718

Nealyda

nearchus Edwards 499

nebraskre Dodge 3056

nebraskæ $H$ y. Edwards 6732

nebris Guenee 2698

nebulædactylus Fitch 5881

nebulella Riley 5553

nebulifera Stephens 5322

nebulo Walsh 5553

nebulosa Edwards 3046

nebulosa Hulst 4140

nebulosa Neumoegen 807

nebulosa Zeller 6438

nebulosalis Fernald 5207

nebulosana Packard 7155

nebulosaria Freyer 4114

nebulosus Wetherby 4824

necopina Grote 2700

necospinusella Chambers 7879

Negalasa

negalis $B$. \& $M c D .3545$

negascia Smith 1584

neglecta Edwards 452

neglecta Hy. Edwards 6666

neglecta Strecker 1142

neglecta-major $\boldsymbol{T} u t t \mathbf{4 5 2}$

negreta Reakirt 89

negundana Dyar $\mathbf{7 3 5 5}$

negundella Chambers 8040

negussa Smith 1682

Neleucania

nelita Strecker 2693

nella Busck 7647

nelsoni Edwards 33

nelsoni Boisduval 374

nelumbialis Smith 5129
Nematocampa

nemesis Edwards 330

nemorella Hulst 4045

Nemoria

nemoris $\boldsymbol{E} d$ wards 635

nemoris Walsingham 7936

nemorum Boisduval 573

Nemotois .196

Neoarctia

neocula Smith 2993

Neodactylota 156

Neodavisia 142

neogama Smith \& Abbot 3048

neomexicana Barnes 1004

neomexicana Brehme 801

neomexicana Doll 3605

neomexicana Dyar 6764

neomexicana Hulst 4080

neomexicana Smith (Euxoa) 1242

neomexicana Smith (Tarachidia) 2956

Neominois

neonaria Walker 4678

Neonympha

Neophasia

Neoplynes

neotelis Smith 1329

neoterica Smith 1659

Neoterpes

nephele Kirby 111

nepheleptena Dyar 2666

Nephelodes 53

nephelotella Hulst $\mathbf{5 4 9 0}$

Nephopteryx

nephrasyntheta Dyar 2665

nepiasaria Walker 4678

nepotella Dietz 8219

neptaria Guenee 4372

Nepticula 195

Nepticulida 195

neptis Smith 1959

Neptunia 76

Nepytia 115 
nerea Boisduval 977

nereus Edurards 636

Nerice

nerissa $H_{y}$. Eduards 3120

Neritos

nero Fabriciua 649

nervata Guence $\mathbf{4 4 4 3}$

nervosa Neumoegen \& Dyar 977

nervosa Haworth 6476

nervosana Kearfott 7367

nesae Smith 2772

nescalis Walker 5149

nesilens Smith 1368

nessus Cramer 749

nessus Edwards 496

nestos Boisduval 429

neumoegeni Edwards 659

neumoegeni $\mathrm{Hy}$. Edwards

(Hemileuca) 789

neumoegeni $\boldsymbol{H y}$. Edwards (Xanthothrix) 2840

neumoegeni Skinner (Appias) 28 neumoegeni Skinner (Melitæa) 222

Neumoegenia

Neuria

neurona Skinner 441

nevada $B . \& M C D .1614$

nevada Seudder (Thorybes) 484

nevada Seudder (Pamphila) 562

nevada Smith 1256

nevadæ Grote 1687

nevada Oberthur 4889

nevadaria Packard 3804

nevadaria Hulet (Drepanulatrix) 4318

nevadaria Hulst (Phigalia) 4622

nevadata Packnrd 4226

nevadellus Kearfott 5389

nevadensis Beutenmuller 3071

nevadensis Edwards 171

nevadensis Felder 121 nevadensis (irote \& Robinson 985

nevadensis Stretch 786

newcombi Swett 4573

newmanella Clemens 6499

nexa Boisduval 890

nexalis Hulst 5069

nexilis Morrison 2837

nezahualcoyotl Streeker 1

niama Dyar 2904

nicreusalis Walker 4932

nicalis Grote 5166

nicalis Smith 1885

nicetaria Guenee 4441

nichollae Hampson (Protagrotis) 1604

nichollae Hampson (Autographa) 3256

nicippe Cramer 76

nicotianx $\boldsymbol{H}$ y. Edwards 6693

nictitans Linnaens 2638

nidificansella Packard 8107

nig Strecker 308

nigella Hulst 6715

nigellus Strecker 3477

niger Wright 25

niger Smith 1573

nigerrima Smith 2865

nigerrima Verity 25

Nigetia

nigra Beutenmuller 6719

nigra Busck 6149

nigra Cary 242

nigra Edwards (Lyesenopsis)

452

niera Edwards (Oeneis) 131

nigra Kearfott 6138

nigra Smith 1488

nigra Stretch 997

nigralbana Walsingham 6956

nigralineella Chambers 7782

nigralis Fernald 5049

nigrapunctella Busek 5966

nigratomella Clemens 6185 
nigrella Chambers 6183
nigrella Hulst (Ephestioides)
5783

nigrella Hulst (Moodna) 5796

nigrescaria Hulst 4668

nigrescens Engel 2158

nigrescens Fletcher 452

nigrescens Grote \& Robinson 3308

nigrescens Hoyne 4014

nigrescens Hulst (Entephria) 4064

nigrescens Hulst (Pterospoda) 4503

nigrescens Wright 263

nigricana Stephens 7235

nigricans Bethune 3181

nigricans Hulst 5627

nigricans Packard 4832

nigricans Reakirt 937

nigricans Riley 1329

nigricantella Ragonot $\mathbf{5 7 1 8}$

nigricaria $B . \& M c D .4556$

nigriceps Walker 1461

nigridia Robinson $\mathbf{7 4 0 0}$

nigridice Scudder 60

nigrifera Walker 937

nigrilineella Chambers 5978

nigrimaculella Busck 6266

nigrina Strecker 60

nigrina Graef 4883

nigrinodis Zeller 5273

nigrinotella Busck 6444

nigrior Smith 2247

nigripennis Grote 1341

nigripuncta $B$. \& $M c D .4831$

nigripunctalis $B . \& M c D .5274$

nigripunctata Goodell 4843

nigrixena Haworth 1163

nigristrigella Beutenmuller 8048

nigrita $B . \&$ McD. 4806

nigritula Guenee 2901

nigriverticella Chambers 8378 nigroangulata Strecker 3953

nigroatomella Dietz 8296

nigrocærulea Cockerell 156

nigrocandida Hulst 3874

nigrocaput Morrison 2919

nigrocaput Smith 2075

nigrocervina Walsingham 7328

nigrociliatus Zeller 5860

nigrocinereella Hulst 5460

nigrocomina Warren 4366

nigrodiscalis Hulst 3855

nigrofascia Hulst 2573

nigrofasciata Packard 4053

nigrofasciata $Z$ eller 841

nigrofasciella Ragonot 5682

nigrofimbria Guenee 2922

nigroflava Graef 1026

nigroguttata Grote 3013

nigrolinea Robinson 7435

nigrolunata Packard 1638

nigromaculana Kearfott 7264

nigromaculella Hulst 5813

nigromarginata Strecker 3345

nigroseriata Packard 4449

nigrosignata Walker 3654

nigrosignella Hulst 5529

nigrostriata Walsingham (Holcocera) 6535

nigrostriata Walsingham (Haploptilia) 7783

nigrosuffusa $B . \& M c D .286$

nigrovenaria Packard 4485

nigrovenosa Watson 790

nigrovittata Grote 1498

nigrovittella Grote 5617

nilotica Rogenhofer 3011

nilus Edwards 633

nimbatana Clemens 6821

nimbicolor Hulst (Ptychopoda) 3894

nimbicolor Hulst (Eupithecia) 4212

nimbosa Guenee 1696

nimbosa Hulst 4217 
nimbosus Braun 5982

nimia Guenee 1839

ninana Riley 7273

ningoris Walsingham 5859

ninguidalis $\mathbf{H u l e t} 5235$

ninonia Boisduval 26

ninus Eduards 372

niobe Strecker 1020

nipana Smith 1775

niphadophilata Dyar 4172

niphon Hubner 388

niscana Kearfott 7395

nisella Clerck 6986

nisceecalis Walker 5100

nita Smith 2044

nitela Guence 2698

nitens Dyar 2590

nitens Grote 1842

nitens Walsingham 5968

nitida Packard 3655

nitidalis Stoll 4974

nitidanum Clemens 6793

nitocris Cramer 3768

nitocris Edwarde 156

nitocritaria Hubner 3768

nitra Edwards 8

nivalis Boisduval 406

nivalis Drury 5419

nivalis Stretch 907

nivalis Smith 2548

nivaria Grote 1636

nivea Neumoegen 3672

niven Seudder 501

nivea Walker 5304

nivealis Smith $\mathbf{3 4 1 9}$

niveana Fabricius $\mathbf{7 4 4 1}$

niveana Walsingham 7291

niveella Chamber $\mathbf{8 1 3 3}$

niveicilialis Grote 5175

niveicollis Smith 2980

niveicoste Smith (Schinia) 1186

niveicosta Smith (Neleucania)

1965

niveicostatus Grote 2396 niveifascia $\boldsymbol{H}$ ulst 4136

niveifascia Swett 4018

niveifasciata Hulot $\mathbf{1 1 0 9}$

niveiguttann Grote (Argyroploce) 6873

niveiguttata Grote (Hyesia)

1812

niveilinea Grote 1246

niveirena Harvey 2621

niveivenosa Grote 1603

nivella Mabille 501

niveocapitella Chambers 8268

niveocilia Hampson 4977

niveociliaria Herrich-Schaeffer 3828

niveopulvella Chambers 6191

niveosana Packard $\mathbf{7 4 0 1}$

niveosericeatus Jones 4664

niveostriata Cockerell $\mathbf{4 4 7 3}$

niviella Hulat 5592

nivigerata Walker 3956

nivihumellus Walker 5341

nivisellana Walsingham 7425

nivium Boisduval 435

nivosaria Guence 4661

nobilis Neumoegen 813

nobilis Grote (Lygranthoecia)

1113

nobilis Grote (Tosale) 5277

nobilitella Cramer 2867

Nocloa

nocticolata Hulst 3957

noctivaga Grote 2449

Noctuelia

noctuella Schiffermuller 5012

Noctuida 87

noctuidalis Dyar 3458

noctuiformis $B$. \& $M C D .3628$

noctuiformis Moeschler 1037

noctuiformis Neumoegen 3001

noctuiformis Smith 1335

nodosella Hulst 5833

nogalesella Dyar 5641

nokomis Edwards 155 


\section{nokomis Brodie 768}

Nola

nolckeni Frey \& Boll 7866

Nolinæ

noloides $B . \& M c D .3476,1$

nominatalis Hulst 5242

nomion Fischer 23

nomonana Kearfott 7501

Nomophila

nomophilalis Dyar 5205

nonana Kearfott 6960

nonangulata Strecker 4774

nondescriptus Wetherby 4832

nonfasciella Chambers 7931

nonlavana Kearfott 7483

nonparilella Dyar 5798

nonstrigella Busck 6195

nonstrigella Chambers (Tricho-

taphe) 6363

nonstrigella Chambers (Opostega) 8420

Norape

noraxalis Walker 5145

norda Smith 3184

nordica Smith 1329

norma Morrison 2343

normalis Dyar (Gluphisia) 3681

normalis Dyar (Pseudohazis)

794

normalis Dyar (Scoparia) $\mathbf{5 2 3 6}$

normalis Grote 1895

normanana Kearfott 7169

normani Grote 1927

normaniana Grote 1428

normella Dyar 5538

norna Thunberg 127

Noropsis

nortellus Kearfott 5406

nortoni Edwards 642

norus Ploetz 646

nostra Smith 1296

notabilis Busck 7679

notabilis Strecker 496

notalis Grote 2560 notaria $H y$. Edwards 3611

notata Strecker 1799

notatalis Walker 5501

notataria Hulst 4679

notataria Walker 4289

notatella Grote 2008

Notela 95

notha $H y . E d w a r d s ~ 4868$

Nothopteryx 104

nothrodes Meyrick 6993

nothrotes Walsingham 6523

Notodonta 93

Nctodontidæ 92

Notolophus 96

nova Smith (Utetheisa) 1029

nova Smith (Cardepia) 1784

novalis Grote 5182

novangliæ Scudder 37

novarius $B$. \& $M c D .4877$

novaroensis $H y$. Edwards 6672

novata Grossbeck 4779

novellata Hulst 4695

noverca Grote 1724

novimundi Busck 6411

novimundi Walsingham 6454

nua Dyar 5291

nubeculana Clemens 7174

nubecularia Guenee 4492

nubeculosa Zeller 6610

nubicola Behr 3339

nubiculata Packard 4364

nubifera Hampson 3010

nubiferaria Swett 4570

nubiferella Ragonot 5620

nubiferella Walsingham 6470

nubigena Behr 211

nubila Strecker 1132

nubilana Clemens 6861

nubilata Smith 1919

nubilata Packard (Eustroma)

3975

nubilata Packard (Sabulodes)

4775 
nubilat Warren 4453

nubilella Hulst 5577

nubilella Zeller 6520

nubilifascia Grote $\$ 512$

nubilipennella Clomene 8249

nubilis Hubner 3122

nubilofasciata Packard 4009

nucella Walsingham 6553

nuchalis Grote (Canthylidia)

1210

nuchalis Grote (Noctuelia) $\mathbf{5 1 8 2}$

nucicolora Guenee 2604

nugatis Smith 1663

nuicola Smith 2937

numa Streeker 1276

Numata

numerosana Zeller 6933

numitor Fabricius 544

nummulalis $H u b$ ner $\mathbf{5 4 2 0}$

nummulalis Lederer 4981

nummulalis Zeller 5419

nundina Drury 1163

nundinella Zeller 6284

nupera Lintner 2169

nuptialis Walker 3090

nuptula Walker 3106

nurus Walker 3083

nutrix Cramer 3397

nuttalli Strecker 794

nyetaginella Gibson 7569

nycteis Donbleday \& Hewitson 243

Nycterophata 56

Nyctiphanta

Nyctobia 104

nympha Edwards 241

nymphaella Hulat 5761

Nymphalida

Nymphalina

Nymphula

nymphulalis Haimbach 4967

Nymphuline

nysa Edwards 628

nyssrecolella Dyar 5606 nyssafoliella Clemene 7583

nyssefoliella Chambers 8379

nyssaris Guenee 4678

oaklandis B. \& MCD. 2407

Oarisma

оахасала Schaus 1386

obatra Morrieon 2933

obaurata Morrison 841

obductata Moeschler 4077

obeliscoides Guence 1356

oberon Worthington 467

oberthuralis Fernald 5037

oberthuriella Ragonot 5643

obesalis Stephens 3578

obesula Smith (Euxoa) 1236

obesula Smith (Polia) 1689

obfirmaria Hubner 4692

obfusaria Walker 3899

obfuscata Riley 7016

obispalis Dyar 5244

obispoana Kearfott $\mathbf{7 5 1 7}$

obiterella Busck 6403

objurgata Smith 1329

oblata Morrison 1447

oblectalis Hulst 5043

oblentaris Grote $\mathbf{4 7 7 1}$

oblinita Smith \& Abbot 2508

obliqua Hy. Edwards 4822

obliqua Guenee 3192

obliqua Harvey 2648

obliqua Hulst 3775

obliqus Packard 3634

obliqua Smith (Amolita) 2804

obliqua Smith (Feltia) 1411

obliqua Smith (Phuphena) 2398

obliqua Smith (Schinia) 1166

obliqua Walker 2784

obliqualis Fabricius 2874

obliqualis Grote 4993

obliqualis $\boldsymbol{H y}$. Edwards 3456

obliquana Kearfott (Phalonia)

7522

obliquana Kearfott (Pandemis) 7335 
obliquaria Grote 4549

obliquata $B$. \& McD. 849

obliquata $H y$. Edwards 5281

obliquata Grote 2784

obliquata Grote\&Robinson 3715

obliquata Smith (Heliophana) 1056

obliquata Smith (Pleroma) 2184

obliquella Dietz 8206

obliquella Strand 2923

obliquella Walker 5448

obliquifasciella Chambers 6071

obliquifera Walker 2600

obliquilinea Walker 3683

obliquistrigella Chambers 6139

oblita Grote 2077

obliteralis Walker (Loxostege) 5020

obliteralis Walker (Nymphula) 5204

obliterana Walsingham 7101

obliterata Stretch 978

obliterata Grote 2418

obliterata Warren 4598

obliterella Dietz 8030

obliviosa Hy. Edwards 3705

obliviosa Walker 2347

oblonga Grote 2791

oblongata Walsingham 6605

oblongistigma Smith 1251

oblunalis Lederer 5088

obluridata Hulst 3870

obnigra Smith 1755

obnigralis Hulst 5140

obnupsella Hulst 5505

obruptata Walker 4054

Obrussa

obrussata Grote 4782

obrutella Zeller 8422

obscura B. \& McD. 1605

obscura Cockerell 281

obscura Hy. Edwards 2491

obscura Fabricius 718

obseura Grossbeck 4528 obscura Grote 3416

obscura Riley 2574

obscura Smith (Anytus) 1537

obscura Smith (Epidemas) 2114

obscura Smith (Polia) 1722

obscura Strecker 3035

obscura Stretch 937

obscuralis $B . \& M c D: 4999$

obscuralis Grote 5200

obscuralis Lederer 4963

obscuralis Moeschler 5204

obscurata B. \& McD. 2067

obscurata Hulst 4113

obscurata McLachlan 193

obscurata Strecker 1120

obscurata Verity 69

obscurella $B$. \& $M c D .2549,1$

obscurella Beutenmuller 6628

obscurella Braun 8409

obscurella Chambers 6334

obscurella Dietz (Homosetia). 8310

obscurella Dietz (Pigritia) 6589

obscurella Kearfott 6138

obscuricostella Clemens 7890

obscurior Hulst 4167

obscurior Smith (Polia) 1755

obscurior Smith (Nelucania) 1959

obscurior Smith (Cucullia) 1999 obscuripennella Frey \& Boll 8050

obscuripennis Grote 3505

obscurofasciella Chambers (Tinagma) 7848

obscurofasciella Chambers

(Bucculatrix) 8139

obscuromaculella Chambers 6418 obscuroocelella Chambers 6262 obscurostrigella Chambers 8248 obscurosuffusella Chambers 6286 obscurusella Chambers (Mompha) 6017 
obscurusella Chombers (Gelechia) 6835

observabilis Grote 1572

obsipella Hulat 5655

obsolescens Dyar 4864

obsoleta Edwards 309

obsoleta Hy. Edwards 283

obsoleta Ehrmann (Papilio) 1

obsoleta Fabricius 1090

obsoleta Frey \& Boll 7895

obsoleta Grote (Argyrostrotis) 3162

obsoleta Grote (Tarachidia) 2950

obsoleta Reiff 785

obsoleta Seudder 410

obsoleta Smith 3503

obsoleta Swett 4115, 1

obsoleta Worthington 3051

obsoletana Walker 7345

obsoletella Zeller 6426

obstipata Fabricius 4054

obstrictella Clemens 7962

obtusa Herrich-Schaeffer 2763

obtusa Smith (Cucullia) 1990

obtusa Smith (Luperina) 2379

obtusa Smith (Trichopolia) 1867

obtusa Speyer 1428

obtusa Walker 3405

obtusangulella Ragonot 5795

obtusaria Hubner 4700

obtusilobse Frey \& Boll 7945

obtusula Zeller 2763

obumbrata Taylor $\mathbf{4 1 4 8}$

obumbratalis Lederer 5130

obversa Walker 3144

obvertens Walker 3358

obvia Walker 1464

Ocala 148

occantaria Hulst 4703

occata Grote 2038

occidens Busck 8165

occidens Grote 2265

occidens Hampson 1507

occidens Hulst $\mathbf{4 1 0 2}$

occidenta Grote 1606

occidentalis $B$. \& $M c D .4823$

occidentalis $\boldsymbol{H}$. Edwardo 711

occidentalis French (Aemilia) 930

occidentalis French (Leptaretia) 941

occidentalis Grote 5335

occidentalis Grote \& Robinson

2474

occidentalis Haimbach 4955

occidentalis Hulot (Herculia) 5260

occidentalis Hulst (Pero) $\mathbf{4 7 4 8}$

occidentalis Hulat (Rachela) 3967

occidentalis Lintner 3670

occidentalis Packard (Eupithecia) 4224

occidentalis Packard (Loxostege) 5025

occidentalis Reakirt 33

occidentalis Scudder 61

occidentalis Skinner 495

occidentalis Smith 3496

occidentalis Walsingham 5924

occidentaria Packard 3891

occidentata Taylor 4000

occidentella Chambers (Gele-

chia) 6277

occidentella Chambers (Tinea)

8261

occidentella Dietz (Dryoperia)

6591

occidentella Dietz (Amydria)

8205

occidentella Dyar 7621

occidentis Zeller 7785

occidentis Walker 3754

occiduaria Walker 4641

occiduaria Guenee 4604

occiduaria Packard 4401 
occipitana Z eller 6927 occitanica Frey \& Boll 7901 occluna Smith 1777 occulta Linnaeus 1489 occulta Hy. Edwards 3325 occultana Walker 7144 oceanus Edwards 500 ocellana Clemens 7203 ocellana Schiffermuller 7170 ocellaria Grossbeck 4682 ocellata B. \& McD. 2553 ocellata Edwards 313 ocellata Grote 4846 ocellata Stoll 3778 ocellea Haworth 5417 ocellella Chambers 6243 ocellifera Grote 345 ocellinata Guenee 4367 ocherfuscella Chambers 6336 Ochlodes

ochoa Barnes 2715

ochosalis Dyar 5155

ochracea Behr 3335

ochracea Edwards 105

ochracea Neumoegen 985

ochracea Riley 1656

ochracea Smith (Andropolia) 2521

ochracea Smith (Emarginea) 2721

ochracea Stretch (Apantesis) 978

ochracea Stretch (Platyprepia) 1028

ochracealis Walker 5291

ochraceana Fernald 6925

ochraceus Hy. Edwards 2842

ochralis Haimbach 4912

ochrea B. \& McD. 2811

ochrea Grote 3136

ochreana Clemens 6924

ochreata $B . \&$ McD. 3608

ochreata Butler 995

ochreata Grossbeck 4098 ochreata Hulst 4767

ochreata Packard 4398

ochreatus Hulst 4665

ochreella Clemens 6599

ochrefasciella Chambers 8427

ochreicostana Walsingham 7057

ochreifascia Smith 1169

ochreipennis Grote 3510

ochreipennis Harvey 3308

ochrella $B . \& M c D .5758$

ochrella Chambers (Cerostoma) 7662

ochrella Chambers (Haploptilia) 7784

ochreoalbana Walker 7490

ochreocostella Chambers 6189

ochreomaculella Chambers 7847

ochreostrigella Chambers (Gnorimoschema) 6114

ochreostrigella Chambers (Gelechia) 6239

ochreosuffusella Chamber 6258

ochrifrontella Zeller 5721

ochrimacula $B . \& M c D .2644$

ochrimaculella Ragonot 5745

ochripalpella Zeller 6361

ochripunctella Dyar 5628

ochristriata Walsingham 8082

ochristrigella Braun 8142

ochrocephala Dietz 6537

ochrocephala Walsingham 7078

ochrocomella Clemens 6590

ochrodactylus Fish 5854

ochrofuscaria Grote $\mathbf{4 5 2 6}$

ochrogaster Guenee 1363

ochromediana Kearfott 6851

ochroptena Dyar 2672

ochroscapus Grote \& Robinson 837

ochrostriata Walsingham 7786

ochroterminana Kearfott 7063

ochsenheimeri Staudinger 36

ocola Edwards 653 
octagonella Wraturingham $7787^{\text {Puno }}$ octo Guenee 2889

octolineata Hulet 4396

octomaculalis Feruald 5226

octomaculata Fabricius 1042

octomaculata Linnaeus 5176

octomaculella Chambers 6113

octonalis Zeller 5179

octopunetana Walsingham 7102

octoscripta Grote 3239

octosignalis Hulst 5137

oculana Harris 7170

oculata Smith 1147

oculatana Clemens 4891

oculatissima A. \& S. 964

oculatrix Guenee 3002

oculea Neumoegen 774

oculiferalis Ragonot 5464

ocypete Linnaeus 727

odiosella Hulat 5646

odius Fabricius 315

odontata Hulst 3939

Odontosia

odora Linnaeus 3391

odyneripennis Walker 6649

ocogenes Dyar 2785

Oecophora .161

Oecophorida

adipodalis Guenee 5105

Oedonia

125

œeneiformis Harvey 3920

Oeneis

ceno Boisduval 134

cenochroa Meyrick 7213

Oenochromina

Oenoe

anone Scudder 243

oenotherzella Chambers 6009

cenotherseseminella Chambers 6006

wenotherana Riley $\mathbf{7 4 8 7}$

cenotherivorella Chambers 6006

cenotrus Stoll 716

cetus Boisduval 119 ofellusalis Walker 4955

offectalis Hulst 7095

officioan Meyrick $\mathbf{7 4 5 5}$

offumalis Hulst $\mathbf{5 0 3 3}$

Ogdoconta

ohioensis Zeller 7618

Oiketicus

oithona Strecker 975

okakaris Packard 3862

okakensis Packard 1481

okeechobee Worthington 456

okius Oberthur 118

olancha Wright 204

olbiella $\mathrm{Hulst} 5741$

oleagina Morrison 1153

olealis Ragonot 5279

olealis Felder 4978

Olene 96

olenusaria Walker 4460

oleracea Harris 35

oleraceana Gibson 7407,1

Olethreutes

.168

olga Smith 2526

Oligia

olinalis Guenee 5268

olivacea $B$. \& McD. 1205

olivacea Cockerell 774

olivacea Grote 3579

olivacea Morrison 1755

olivacea Smith (Cerma) 2414

olivacea Smith (Schinia) 1194

olivacea Taylor 4198

olivaceana Riley 7061

oliveaceanum Fernald 6799

olivacearia Morrison 4620

olivacella $D$ yar 5739

oliveformis Braun 7889

olivalis $B$. \& $M c D .2762,1$

olivalis Grote (Arta) 5287

olivalis Grote (Euxoa) 1250

olivalis Hulst (Itame) $\mathbf{4 4 0 6}$

olivalis Hulst (Epipaschia) 5467

olivata $B . \& M c D .4646,1$ 


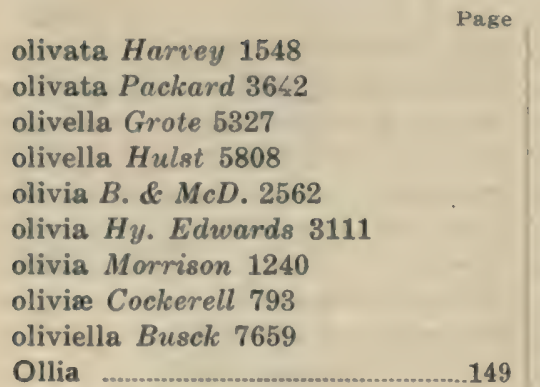

olliusalis Walker 4955

olorina Grote 2523

olvello Barnes 2716

Olyca .146

olympia Butler 3585

olympia Edwards 41

olympiadella Zeller 6203

olympus Edwards 111

olynthus Boisduval \& Le Conte 647

olyzonaria Walker 4754

omega Hubner 3248

omissa Dod 2001

omissa Walsingham 7678

Ommatochila

Ommatopteryx 140

Ommatospila 130

Ommatostola

omphacitis Meyrick 7475

Omphalocera

ona Smith 2370

onagella Dietz 8205

onagrus Guenee 2929

Oncocnemis

ondo Barnes 3466

Oneida

oneko Scudder 634

ononis Schiffermuller 1088

ontariella Bethune 6478

ontaria Smith 1313

ontario Edwards 355

onusta Grote 2365

onustana Walker 7618

Cnychagrotis

46 onychina Guenee 2121

onythesalis Walker 5147

oo Cramer 3260

Oospila 100

opaca Harvey 1385

opacalis Grote 3548

opacaria Hulst $\mathbf{4 5 8 6}$

opacella Hulst 5811

opacifrons Grote 1420

opalescalis Hulst 5329

opalescellum Hulst 5776

opalescens $\boldsymbol{H} y$. Edwards 6656

opella Grote 937

opelloides Gruef 936

opercularis Smith \& Abbot 4858

operculella Zeller 6169

operosella Zeller 8215

Opharus 31

ophelia $H y$. Edwards 3100

Ophideres 86

ophionalis Walker 5045

ophir B. \& McD. 988

ophis Edwards 650

ophrionella Dietz 8271

ophthalmica Guenee 3016

ophthalmicus Boisduval 706

opina Grote 2128

opinata Pearsall 4135

opipara Morrison 1349

opipara Hy. Edwards 2910

opis Edwards 185

oponearia Walker 4706

oporaria Zeller 3816

oporedestella Dyar 5507

Oporinia 107

Opostega 196

oppilalis Grote 5100

oppositaria Guenee 4343

ops Druce 317

optimana Dyar 6891

optimata Strecker 3973

opulenta $\boldsymbol{H}$. Edwards 1025

opulifoliella Braun 8417

Page

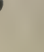


opuntiella Busck 7980

opuscularia Hulst 4504

oraculella Kenrfott 5320

orasusalis Walker 5132

oratalis Hulat 5061

orba Grote 3464

orba Grote Kusnezov 3102

orba Smith 2970

orbica Morrison 2889

orbicularis B. \& MCD. 2538

orbicularis Smith 1266

orbiculata Smith 1811

orbifere Walker 2242

orbimaculella Chambers 7724

orbipuneta $B$. d $M C D .1588,1$

orbis Grote 1594

ore Strecker 8353

oreiferalis Walker $\mathbf{3 5 1 5}$

orciferaria Walker 4636

orcus Edwards 434

ordinalis Meyrick 7652

ordinaria Walker 2367

ordinata Walker (Acidalia) 3869

ordinata Walker (Euxoв) 1304

ordinata ..W Walker (Phasiane) 4390

ordinata Wralker (Apsecasia) 4458

ordinatellus Walker $\mathbf{7 7 2 3}$

oreadella $\mathrm{H}$ ulat $\mathbf{5 3 1 6}$

oreas Eduards 276

oreasella Clemens 7695

oregona Smith 1944

oregonana Walsingham $\mathbf{7 1 1 4}$

oregonella Busck 8276

oregonella Walsingham 8428

oregonensis Butler 3620

oregonensis Harvey $2155^{\circ}$

oregonensis Strotch 1018

oregonensis Wrisingham 7926

oregonia Eduards (Papilio) 5

oreconia Eduards (Pamphila)

562 oregonica Grote 1617

oregonica Hy. Edwards 1080

oregonicus Grote 5882

Orenaia 133

oreodaphne Hy. Edwards 687

orestella Busck 7827

orestes Lintner 481

Oreta

orgyia Grote 3462

orichalcella Clemens 7836

oricus Edwards 509

orida Smith 1613

orientalides Verity 39

orientalis Grote 2798

orillata Walker 4387

orilliana Grote 1474

orina Guenee 2735

orion Fabricius 315

orites Walsingham 6509

oriunda Grote 2135

orizaba Westwood 764

orleansella Chambers 8251

ornata $B$. \& MCD. 4711

ornata Grote \& Robinson 3589

ornata Hulst 4160

ornata Neumoegen 2395

ornata Ottolengui 2751

ornata Packard 978

ornatalis Duponchel 4924

ornatella Chambers 7923

ornatella Dietz (Homosetia)

8317

ornatella Dietz (Pigritia) 6584

ornatifimbriella Clemens 6254

ornatrix Linnaeus 1030

ornatus Smith 1562

Orneodes 152

Orneodida 152

ornithogalli Guenee 2569

Ornix 189

oro Scudder 449

orobanchella Duar 5708

orobia Harvey 1823

oronella Walsingham 6083 
orono Scudder 610

oropeso Barnes 4834

orophila Hampson 3226

Orosagotis

orphisalis Walker 5153

orphnealis Walker 4959 .

orphnina Dyar 2799

orsa Boisduval 251

orseis Edwards 252

orta $B . \& M c D .2328$

orthocarpi Walsingham 5873

orthocarpi Hy. Edwards 6706

Orthofidonia

orthogonia Morrison 1233

Ortholepis 144

Orthonama 107

Orthosia 54

orthosioides Guenee 3349

orthozona Hampson 2909

ortruda Smith 1689

orythia Smith \& Abbot 286

oryzæellus Riley 5430

osceola Lintner 604

oscitalis Grote 5101

osculata Hulst (Catocala) 3051

osculata Hulst (Perizoma) 4105

oslarellum Dyar 5774

oslarellus Haimbach 5345

oslari Barnes 3702

oslari Dyar (Strymon) 357

oslari Dyar (Afilia) 3679

oslari $R$. \& J. (Sphinx) 694

oslari R. \& J. (Proserpinus) 751

oslari Rothschild (Anisota) 801

oslari Rothschild (Basilona) 813

oslari Rothschild (Halisidota) 923

oslari Rothschild (Ecpantheria) 966

oslari Skinner (Pygarctia) 1006

oslari Skinner (Mastor) 637

oslari Skinner (Oeneis) 126

Oslaria osmundana Fernald 6850

osseana Scopoli 7401

osseella Walsingham 6616

ossularia Hubner 3835

ostensackenella Fitch 7923

ostenta Hy. Edwards 933

ostentaria Walker 3896

ostreella Ragonot 5825

ostreonalis Grote 4980

ostricolorella Hulst 5722

ostrinella Clemens 5795

ostryæ Clemens 7788

ostryæella Chambers (Chrysopeli\&, 5979

ostryæella Chambers (Gracilaria) 8063

ostryæfoliella Clemens (Coptodisca) 7593

ostryæfoliella Clemens (Lithocollectis) 7891

ostryæfoliella Clemens (Nepticula) 8380

ostryarella Chambers 7965

ostryella Ely 5525

osyka Edwards 604

otero Barnes 998

othello Smith 2504

otho Barnes 926

otho Smith \& Abbot 590

otiosa Neumoegen \& Dyar 982

otiosana Clemens 7026

otisi Dyar 3990

ottawana Kearfott 7555

ottoe Edwards 568

ottolenguii D yar 3241

ou Guenee 3267

outis Skinner 477

ouwah Poling 3117

ovalis Grote 3147

ovalis Guenne 3286

ovalis Packard 5596

ovata Grote 2436

ovidius Scudder \& Burgess 529

oviduca Guenee 1821 
ovilla Grote 846

ovina Sepp 4855

oviplagalis Walker 5277

ovulalis Guenee 4944

oweni Bames 3734

oweni Eduards 183

oweni Suett 4350

oxalina Hubner 1550

oxilus Hubner 11

oxybaphi Clemens 761

Oxycilla 89

Oxycnemis 66

oxycoccana Pacliard $\mathbf{7 4 1 5}$

cxydalis Guenee 5118

oxygala Grote 1969

oxygramma Geyer 3254

oxygramma Hulat 4107

oxyleuca Meyrick 7107

Oxylos

oxymorus Grote 1793

Oxyptilus

Oramia 146

Ozarba

Ozodania 30

pacalis Grote 5208

pacalis Walker 3155

Pachylia

Pachypolia

Pachysphinx

pachystimella Busck 8097

Pachyrancla

pacifica Behr 3608

pacifica $\boldsymbol{H y}$. Edwards (Acopa) 2584

pacifica $\boldsymbol{H} y$. Edwards (Aegeris) 6653

pacifica Harvey 1916

pacifica Riley 6656

pacifiea Smith (Acronyeta) 2477

pacifiea Smith (Apamea) 2638 pacificana Walsingham 7200 pacificaris Packard (Holaretias) 3880

pacifiearia Puckard (Cabera) 4310

packardaria Grote $\mathbf{3 7 8 4}$

packardaria Hulst 4539

packardaria Moeschler 1422

packardata Grossbeck 3970

packardata Lintner 3978

packardata Taylor (Eupithecia) 4169

packardata Taylor (Enypia) 4483

packardella Chambers (Gelechis) 6224

packardella Chambers (Gracilaria) $\mathbf{8 0 5 9}$

packardella Chambera (Bucculatrix) 8134

packardella Clemens 6484

packardella Ragonot 5707

packardi Grote (Illice) 885

packardi Grote (Lygranthocia) 1113

packardi Grote (Scepsis) 825

packardi Morrison 3623

packardi Saunders 247

packardi Schaupp 960

packardi Zeller $\mathbf{7 2 3 8}$

Packardia 126

packardiana Clemens 6972

packardiana Fernald 7372

pacuvius Lintner 530

padella Linnaeus 7728

Pactes 77

psenulata Clemens 4816

panulataria Grote 4615

pagenstecheri Moeschler 3464

paginata Morrison 2604

Palada

Palacacrita

palmeno Cramer 60

palano Linnaevs 68 
palæmon Pallas 536

palæogama Guenee 3045

Palzoplusia

palamedes Drury 19

palata Grote 3212

Palatka

palatka Edwards 607

paleaceus Z Zeller 5909

pales $D . \& S .194$

palilis Harvey 1745

Palindia

palindialis Guenee 5007

palla Boisduval 226

palla French 3591

pallens $B . \& M c D .3437$

pallens $H y$. Edwards 824

pallens Strecker 710

pallens Tepper 2772

pallenscens Grossbeck 4412

pallescens Grote \& Robinson 3370

pallescens $R . \& J .687$

pallescens Smith 2646

pallialis Z eller 3562

palliatricula Guenee 2400

pallicauda Smith 1795

pallicineta Smith 1104

pallicornella Ragonot 5500

pallida Boisduval 720

pallida Bowles 802

pallida Braun 8403

pallida Cockerell 59

pallida Cockerell 64

pallida Comstock 5730

pallida Edwards 254

pallida Grote 718

pallida Herrich-Schaeffer 4853

pallida Hulst 3897

pallida Ottolengui 3239

pallida Packard (Hyphantria) 959

pallida Packard (Crambidia)

856 pallida Poling 3083

pallida Scudder 3651

pallida Skinner (Polites) 581

pallida Skinner \& Mengel (Eurymus) 57

pallida Smith 2722

pallida Strecker 3651

pallidactyla Haworth 5881

pallidata Packard 4356

pallidegrisseella Chambers 6337

pallidella Busck 6461

pallidella Chambers 6033

pallidella Dyar 5689

palliderosacella Chambers 6077

pallidice Scudder 60

pallidicollis Grote 1455

pallidicoma Grote 2499

pallidicostana Walsingham 7068

pallidior Dyar 894

pallidior Smith 3882

pallidipalpana Kearfott 6920

pallidipennella Hulst 5704

pallidissima $B . \& M c D .35$

pallidistrigella Chamber 6023

pallidochrella Chambers (Helice) 6061

pallidochrella Chambers (Gno-

rimoschema) 6103

pallidotinctella Dietz 6590

pallidula Hulst 4436

pallidulus $\boldsymbol{H y}$. Edwards 706

pallifera Grote 2519

palligera Grote 3282

palligera Smith 3543

pallimedia Grossbeck 3882

pallimedia Smith 2551

palliolella Ragonot 5523

pallipalpis Walker 6364

pallipennata $B . \& M c D .4432$

pallipennis Smith 1260 .

palliseca Smith 1946

pallistriga $B . \& M c D .5952$

palloralis Dyar 5244 
pallorana Robinsen 7366

pallula $H y$. Edwarde 3292

pallulata Hulst 4717

pallulella Hulst 5720

pallulellus B. \& MeD. 6313

palmata B. \& McD. 8338

palmeri Edwardo 323

palmeri Packard 827

palmi Beutenmuller (Datana) 3599

palmi Beutenmuller (Synanthedon) 6703

palmi Hy. Eduards 6754

palmi Neumoegen 6735

Palmia

palmillo Barnes 1797

palousana Kearfott 6940

palpalis Haworth 3578

palpalis Grote 735

palpana Walsingham 6947

palparia Walker 3563

palpata Packard $\mathbf{4 1 5 6}$

palpialbella Chambers 6338

palpiannulella Chambers 6052

Palpidia

palpilineella Chambers 6182

Palthis

Paltodora

153

paltodoriella Busck 6196

paludana Robinson 7348

paludata Thunberg 3945

palustriella Braun 8044

Palyas

pamina Neumoegen 778

Pammene 175

Pamphila

Pamphiline

pamphilus Linnaeus 106

pamphiloides Reakirt 106

pampina Guenee 2230

pampinaria Guenee 4570

pampinatrix Smith \& Abbot 744

pamponerella Dyar 5814 pan Harris 852

panatela Smith 8469

pandana Kearfott 6943

pandaria Walker $\mathbf{4 7 5 8}$

Pandemis 176

pandora Blake 781

pandoralis B. \& McD. 5186

pandorua Hubner 737

pandrosa Cramer $\mathbf{8 3 5 6}$

pandurella Dietz 8202

panella Busek 6288

Pangrapta 89

panicifoliella Clemens 7558

panisaria Walker 4689

pannaria Guenee 3840

Panopoda 86

panoquin Seudder 650

panoquinoides Skinner 651

Panthea 83

Pantheinæ _.......... 83

Pantographa. ................. 130

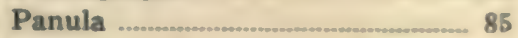

Paonias ...__ 24

Paota 103

papago Barnes 2738

Papaipema 69

paphis Linnaeus 774

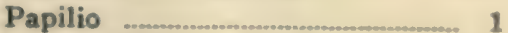

Papilionidx …n............. 1

Papilionoidea .................... 1

Parachma _...sonsonaran 138

Paracretonia … 75

Paraclemensia ........._. 196

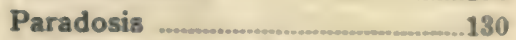

paradoxa Behr 3671

paradoxa Frey ó Boll 8012

paradoxa Giole 16 cit

paradors Riley 847.1

paradoxia Watson 790

paradoxella Dietz 8218

paradoxica Chambers 8460

paraglypta Meyrick 6903

Paralechia 156 


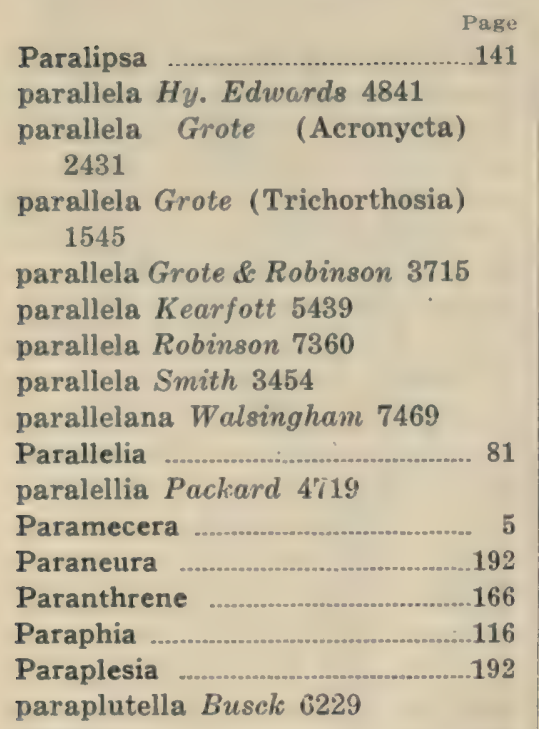

Paraptera 104

Parasa 125

Parasemia 35

Parastichtis 60

Paratrytone 21

parcata Grossbeck 4355

parcata Smith 2279

parce Fabricius 725

pardalis Behr 451

pardalis Grote 2931

pardalis Smith 3385

pardella Walsingham $\$ 480$

Parectopa .188

parentalis Grote 1542

paresa Smith 3376

Parexcelsa

Parharmonia .166

parilis Hubner 3220

parinotata Zeller 4078

parisiana Guenee $\mathbf{7 4 4 4}$

parmatana Clemens 7239

parmeliana $H y$. Edwards 1162

Parnassius

parthenos Harris 942 participialis Grote 5297

Parora

parta Guenee 3055

parthenice Kirby 973

particolor Hulst 4437

particornella Busck 5988

partita Guenee 2592

partitalis Smith 3496

partitaria Grote 4285

parva Rothschild 1025

parva $B$. \& $M c D .878$

parvana Walsingham 7087

parvella Dyar 5823

parvicristatella Chambers 6017

parvimacula Grote (Samia) 769

parvimacula Grote (Lithacodia)

2896

parvimacula Smith 2402

parvimaculana Walsingham

7492

parvipulvella Chambers (Gelechia) 6339

parvipulvella Chambers (Elachista) 7837

parvipuncta $B . \& M c D, 1201$

parvula $B$. \& $M c D .3287,1$

parvula Edwards 3116

parvula $H y$. Edwards 7736

parvula Neumoegen \& Dyar 932

parvula Walker 2948

parvulalis $B$. \& $M c D$. (Epizeuxis) 3497

parvulalis $B$. \& $M c D$. (Alpheioides) 5465

parvularia Hulst 3884

parvulella $B$. \& $M C D$. 5756

parvulella Ely 5787

parvus Walsingham 5938

pasadenana Kearfott 6771

pasadenensis Grinnell 5868

pascoensis Wright 247

pascurellas Linnaeus 5338

pasiphæia Grote 3270 


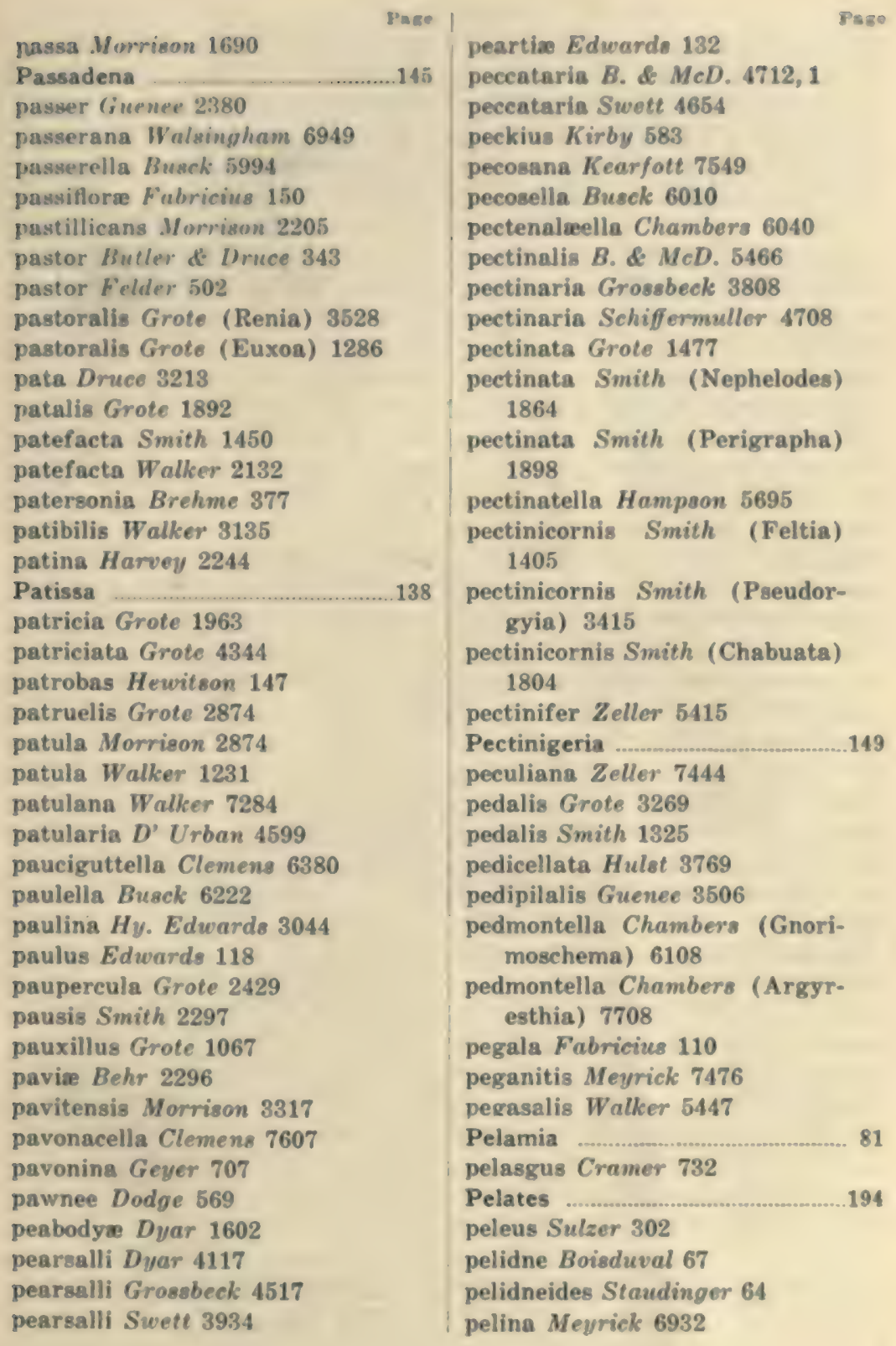


pellenis Godart 303

pellionella Linnaeu\& 8258

pellucida Herrich-Schaeffer 800

pellucidalis Grote 1494

pellucidaria Packard 4488

pellucidaria Grote \& Robinson 4651

pelviculella Hulst $\mathbf{5 7 9 5}$

pembina Edwards 433

pendens Smith 1943

pendina Smith 2352

pendula Ottolengui 2756

pendulinaria Guenee 3918

peninsularia Grote 3768

penita Morrison 2343

penitalis Grote 5129

penna Morrison 3186

pennaria Linnaeus 4663

pennella Busck 6026

pennsylvaniana Kearfott 7141

pennsylvanica Dietz 6282

pennsylvanica Smith 3693

pennsylvaniella Engel 8006

pensilis Grote 1736

Penthesilea

penumbralis Dyar 5238

penumbralis Grote 4961

penumbrata Hulst 2875

penumbrata Pearsall 4277

Peoria

Peosina

pepita Guenee 2740

peplarioides Hulst 4746

pepsidiformis Hubner 6655

peracutata Walker 4054

peralbata Packard 3839

peralto Barnes 2095

peramans Hulst 3024

perangulalis Harvey 3565

perangulata Hulst (Enypia)

4481

perangulata Hulst (Selenia) 4711

perangulata $H y$. Edwards 3653

perangustana Walsingham 7072

Perania 72

perarcuata Walker 4408

perattenta Grote 1598

perbellis Grote 2364

perbrunnea Grote 1856

perbrunneata Taylor 4233

percara Morrison 2719

percnodactyla Walsingham 5871

Percnoptilota 107

perconflua Grote 1443

perdiceas Edwards 207

perdita Dyar 8483

perdita Grote 2506

perdita Strecker 3061

perditalis Walker 3473

perdricana Walsingham 6934

perdubiella Dyar 5710

perductana Walker 6877

peredia Grote 1858

perelegans $\boldsymbol{H} y$. Edwards 690

peremptalis Grote 4905

perexcellens Grote 1364

perfecta $H y$. Edwards 3345

perfida Dod 1379

perflava Harvey 2764

perfluana Zeller 7231

perforata Grote 1818

perfracta Swett 4014

perfundis Smith 2547

perfusalis Walker 4913

perfusca Grote 1282

perfusea Hulst 4196

perfuscalis Hulst (Sarata) 5685

perfuscalis Hulst (Pilocrosis) 4937

perfuscaria Grote 3442

pergamus $\boldsymbol{H y}$. Eawards 7

pergandeana Fernald 6917

pergandeella Bisek (Agnopteryx) 6462 
pergandeella Busek (Triclon-

ella) 6500

pergentilis Grote 2559

pergilvalis Hulat 5017

pergracilidactylus Paeleard 5942

pergracilis Hulst 4577

pergratialis Hulst 5577

perichalca Meyrick 7570

periclata Su'elt 4007

Pericopida

periculella Busck 6212

periculosa Guenee 2385

periculosalis Dyar 4908

Perigea

Perigonica

Perigrapha

perillata Pearsall 4175

Perimede

perirrorata Packard 3837

periscelidactylus Fitch 5857

Perispasta 133

peristicta Meyriek 6994

perita Grote 2963

peritalis Smith 1883

peritana Clemens 7374

periusalis Walker 5013

Perizoma 108

perizomaria Hulst 4753

perleta $H y$. Edwards 3320

perlata Guenee 4659

perlata Walker 3138

perlentans Walker 1329

perlepidella Hulst 5853

perlevis Grote 1013

perlineata Packard 4116

perlubens Grote 1891

perlucida Busek 6758

perlucidula Clemens 4867

perlutea Neumoegen \& Dyar

3746

perluteella Dyar 5739

permaculata Packard 1021

permagna Grote 2791

permagnaria Grossbeek 4536 permagnarin Packand 4495

perminuta Hy. Edwards 1072

permoleatella Bueck 5974

permunda Morrison 1812

permundanum Clemens 6798

permutana Duponchel $\mathbf{7 4 2 4}$

pernana Grote 2744

pernigra Grixnell 521

pernotata Hulst 4068

pernotata Grote 2035

Pero .123

perobliqua Hampson 2651

perobsoleta Lyman 2695

perolivalis $B$. \& $M c D .2620$

perolivalis Smith 1377

perolivata Hulat 4461

perolivata Prekard 3634

Peronea 178

perophoroides Streeker 3664

peronata $B$. \& McD. 4408, 1

perpallida Dyar 3569

perpallida Grote 2586

perpallida $\boldsymbol{H} y$. Edwards 3323

perpallidaria Grote 4313

perpendiculata Warren 3810

perpenoa Grote 2289

perpicta Dyar 978

perpictaria $B$. \& $M C D .4595,1$

perplexa Hy. Edwards (Syneda)

3332

perplexa Hy. Edwards (Synan-

thedon) 6661

perplexa Grote 1777

perplexa Guenee 3148

perplexa Neumoegen \& Dyar

8344

perplexa Smith 1650

perplexa Strecker 3055

perplexalis Fernald 5027

perplexana Fernald 6983

perplexata Pearsall 4333

perpolita Morrison 1287

perpura Morrison (Anarta)

1636 
perpura Morrison (Euxoa) 1371

perquiritata Morrison $\mathbf{1 5 0 5}$ perrosea Dyar 883

perrubralis Packard 5144

perscripta Guenee 2014

perse Edwards 230

perseella B. \& McD. 5472

persephone Grote 979

persica Thomas 6655

persicæella Murtfeldt 6209

persicana Fitch 7342

persimilata Grote 3914

persimilis Grote 1069

persimilis Hulst 3856

persimplex Dyar 8155

persinuaria Guenee 4678

persius Scudder 521

personaria $\boldsymbol{H y}$. Edwards 4475

personata Morrison 1272

personata Walker 3289

perspectalis Hubner 4919

perspicelella Walsingham 8083

perspicua Grote \& Robinson

3603

perspicua Walker (Phurys)

3148

perspicua Walker (Thyris) 4888

perspicuana Robinson 7439

perstrialis Hubner 5305

perstrigata Hampson 2007

perstructana Walker 7096

persuasa Harvey 2446

pertextalis Lederer $\mathbf{5 1 1 1}$

pertracta Morrison 1973

perturbata Smith 1262

perumbraria Hulst 4534

perumbrosa Dyar 1437

pervaria Packard 4638

pervelata Walker 4506

perversa Neumoegen \& Dyar 3752

pervertipennis Hulst 4284

perviridaria Packard 3831 pervolata Hulst 4365

pestula Smith 1337

petasitis Pfaffenzeller 6287

petita Smith 1755

petræa Walker 2564

petrealis Grote 3520

petreius Edwards 489

petrella Busck 6134

petrellus Zeller 5655

petreus Cramer 302

petricola Walker 3331

petrodactyla Walker 5889

petronius Lintner 527

petrosa Walker 1024

pettitana Robinson 7288

pettiti Grote 2217

petulans Hulst 3055

petulans $\boldsymbol{H}$. Edwards 1182

petulca Grote 2138

pexata Grote 2163

pexata Swett 4564

pexellus Zeller 5403

phæa Hampson 1637

phralis Guenee 3496

Phacasiophora 169

Phadinus

phædon Herrich-Schaeffer 203

Phædrotes

phæopteralis Guenee $\mathbf{5 0 1 5}$

phrosoma Butler 1025

Phæoura

phærusalis Walker 4956

phaetana Hubner 203

phaethusa Hulst 203

phaeton Drury 203

phaeton Grote \& Robinson 755

phaetoneta Godart 203

phalænalis Grote 3449

Phalænophana 91

Phalænostola 89

phalanga Grote 3045

phalerata Harris 996

phalerosalis Walker 3528

Phalonia 


Phaloniida Paro
phantasmalis Guenee 4972
phantasmaria Strocker 4487
phantoma B. \& McD. 4648,1
phaon Edwards 246

Pharmacis .180

phasianaris Guenee $\mathbf{4 7 5 8}$

Phasiane

phasma Harvey 951

phecolisea Druee 2954

Phengommatza

Pheosia

pherecydes Cramer 816

pheres Boisduval 434

Pherne

Phigalia

phigaliaria Guenee 4615

phila Smith 1994

Philagraula

philea Linnaeus 48

Philedia

philemon Boisduval 428

philenor Linnaeus 1

phileros Boisduval 433

phileta Fabricius 30

philetas Edwards 494

Philobia

philodice Godart 60

Philometra

Philonome

philomene Hubner 68

Philosamia

Philotes

Philtraa

Phiprosopus

phleas Boisduval \& Le Conte 410

phleophaga Busck 8425

phlogophagus Grote \& Robinson 1089

Phlogophora

phlogosaria Guenee $\mathbf{4 6 7 6}$

phloxiphaga $G . \&$ \& .1089

Phlyctania

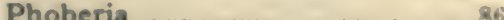

Phobetron 126

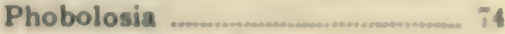

phoea Moeschler 1647

phocata Moeschler 4058

Phocides

phocion Fabricius 94

phocus Edwards 119

phoebe Hy. Edwards 3027

phoenicealis Hubner $\mathbf{5 1 4 5}$

Phoenicophanta 75

phoenix Prout 3830

phœezalis Dyar 5262

pholas Meyrick 6946

Pholisora

Pholus 26

pholus Drury 831

pholus Cramer $\mathbf{7 4 5}$

phrada Druce 871

phragmatidicola Guenee 1936

Phragmatobia 38

phragmitella Stainton 5989

Phrudocentra

Phryganeopsis 193

Phryganidia 92

Phrygionis 124

phrynia $\boldsymbol{H}$. Edwards 3053

Phthinolophus 173

Phthorimaea 156

Phuphena 64

Phurys 81

Phycanassa _.......... 21

Phyciodes ...0 10

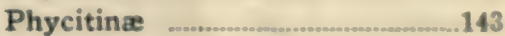

Phycitopsis 144

phylace Edwards 639

phylæus Drury 571

phylla Dyar 851

phyllinaria Zeller 3819

phyllira Drury 989

phyllisalis Walker 4985

Phyllocnistis .190

phyllophora Grote 1444

physaliella Chambers 6054 
physalivorella Chambers $6116^{\text {Page }}$

Physostegania 111

phytolacex Smith \& Abbot 2573

Phytometra 89

piasus Boisduval 448

piatrix Grote 3021

pica Walsingham 5866

pica Walker 795

piccata Pearsall 4252

picez Busck 6864, 1

piceæ Dyar 6724

picezlla Kearfott 6138

piceafoliana Kearfott 7163

picicolana Dyar 7008

picina Grote 2079

picta Edwards 253

picta Felder 3632

picta Harris 1930

picta Packard 968

picta Sepp 716

pictipennalis $B . \& M c D .5085$

pictipennata Hulst 4363

pictipennis Grinnell 5919

pictipennis Grote 1057

pictipes Grote \& Robinson 6679

picturatella Braun 7958

Pierida

Pieris

piger Dyar 8189

pigrata Walker 4054

Pigritia .163

pilacho Barnes 2775

pilalis Hulst 5121

pilatealis $B$. \& $M c D .5107$

pilatella Braun 7716

Piletocera

pilipalpis Grote 1932

pilleriana Schiffermuller 7314

Pilocrocis

pilosaria Packard 4688

pilosella Zeller 8084

pilumnus Boisduval 17

pima Edwards 45 pima Smith 2975

pimana Busck 7471

pimella Dyar 5818

pimensis $B$. \& McD. 1367

pimpinella Murtfeldt 8092

pinastri Linnueus 699

pinatubana Kearfott 7394

pindar Smith 1365

pinella Busck 6153

pineum Lintner 701

pini Dyar 3720

pini Kellicott 6723

piniaria Packard 4486

piniata Packard (Caripeta) 4466

piniata Packard (Paraphia)

4492

pinicola Dyar 3718

pinicolana Zeller 7144

pinifoliella Chambers 6166

Pinipestis

pinivorana Zeller 6103

pinorum Behrens 6728

piperana Busck 7261

piperana Kearfott 7274

piperatella Ragonot 5831

piperatella Zeller 6402

piperella Busck (Choreutis)

7614

piperella Busck (Greya) 8442

piperella Dyar 5675

piperis Grote \& Robinson 716

pirus Edwards 537

piscipellis Grote 1454

piscipellis Zeller 6031

pisoniæe Busck 6091

pisoniella Busck 7559

pistaciaria Packard 3785

pistasciaria Guenee 3813

pithecium Smith \& Abbot 4832

pittacus Edwards 619

pitychrous Grote 1272

pityochromus Grote 2767

placata Grote 3160 
placendiella Busek 7824 placentia Smith \& Abbot 994 placerana Kearfott 7221 placeraria Guenee 4718 placida B. \& McD. 1302 placida Dyar 8671 placida Grote 1585 placidana Robinaon $\mathbf{7 4 4 4}$ placidaria Guenee 3877 placidata Taylor 4234 placidata Walker 4068 placidella Dyar 5717 placidella Haimbach 5401 placidella Zeller 6059 plagalis Haimbach 5152 plagiata Smith 2770 plagiata Walker (Agrotis) 1425 plagiata Walker (Hemerocampa) 8707

plagiata Walker (Olene) 3718 plagiatella Dietz 6544

plagiatus Walker $\mathbf{8 3 5 4}$ plagifasciata Walker 4641 plagigera Morrison 1250 Plagiomimicus

Plagodis 120

plagosana Clemene 7199 plana Grote (Anytus) 1533 plana Grote (Prothymia) 3461 planalis Grote (Eriopyga) 1830 planalis Grote (Hereulia) 5260 planata Taylor 4050

planifrons Smith 1455

planiloqua Meyrick 7266

plantagenaria Hulst 3873 plantaginis Linnaeus 1024 plantaginisella Chambers 8000 Platzea 115

plantanana Clemens 7191 platanella Chambers 6253 platanella Clemens (Tetralopha) $\mathbf{5 4 8 9}$ platanella Clemens (Nepticula) 8381 platanoidielle Braun 7952 plates Clemens 8382

Plathypena 92

platina Skinner 178

Platceceticus 12:

Platyperigea 67

Platyprepia .......... 36

Platyptilia 150

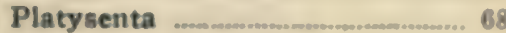

Platytes

plausipennella Chambers 8086

plautus Scudder \& Burgess 528

plebeculata Guenee 4090

plebeis Smith 1442

Plebeius 16

plebeja Fabricius 679

pleciseformis Walker 6649

plecta Linnaeus 1434

plectilis Grote \& Robinson 5102

plejadellus Zincken $\mathbf{5 4 3 0}$

plena Walker 4891

plenilinealis Grote (Renia) 3523

plenilinealis Grote (Nymphula) 5199

plenipennis Walker 3180

plenoseripta Hulst $\mathbf{4 2 1 3}$

pleonectusn Grote 2724

Pleonectyptera 88

Pleroma

Plestia

pleuritica Grote 1836

Pleuroprucha 103

Pleurota 160

plicata Smith 1681

plicatus Grote 1522

plicipunctella Walsingham 7706

Pliniaca ………………… 182

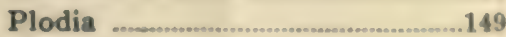

Ploiophora

plota Strecker 691

plumbaria Hulat $\mathbf{4 1 5 1}$

piumbea $\boldsymbol{H} y$. Edwards 900

plumbea Stretch 887

plumbeella Beutenmuller 6637 
plumbicostalis Grote 4939
plumbifimbriata Grote 2933
plumbifimbriellus Dyar 5369
plumbolineana Kearfott 7162
plumbosignalis Fernald 5051
plumbifrontellus Clemens 8170
plumigerella Hulst 5557
plummerella Busck 6456
plummerella Dietz 6513
plummeriana Busck
mene) 7259
plummeriana Busck (Phalonia)
7502
plumogeraria Hulst 4618
plumosa Hy. Edwards 3639
plumosaria Packard 4576
pluralis Grote 1297
Plusia

plusiæformis $H y$. Edwards 1871

Plusiinæ ............................................ 83

Plusiodonta .................................... 87

Plutella ...............................................183

plutella Chambers (Megacraspedas) 6039

plutella Chambers (Evippe) 6063

plutella Chambers (Trichotaphe) 6357

Plutellida

pluto Fabricius 757

plutonia Grote 2264

pluvialis Dyar 3750

pluviata Guenee 4014

pluviosa Walker 2268

pneumatella Hulst 5734

pnosmodiella Busck 7988

poana Zeller 6862

Poanes

pocahontas Scudder 592

Podagra

podarce Felder 429

Podiasa 166 pocilana Guenee 6999

Pcecilopsis

poetica Grote 2754

poeyi Butler 28

pohono Smith 2058

pola Boisduval 240

polaris Boisduval 196

polaris Hulst 4057

polata Duponchel 4059

Polenta 72

Polia 49

polingella Dyar 5713

polingi Barnes (Dalla) 539

polingi Barnes (Oncocnemis) 2036

polingi Kearfott 5367

polingi Skinner 661

Polingia 133

polichroa Hampson 2069

polios Cook \& Watson 385

polistiformis Harris 6751

polita Walsingham 7635

politella Walsingham 8432

Polites

politia Cramer 4778

pollinalis Schiffermuller 5075

Polloccia 138

pollostella Busck 6064

Polopeustis 144

polyactinellus Zeller 5373

Polychrosis 167

polydactyla Hubner 5954

polydamas Linnaeus 3

polygama Guenee 3106

polygoni $H y$. Edwards 6709

Polygonia

polygrammaria Packard 4572

polygrammata Hulst 4101

Polygrammate 64

Polygrammodes 131

Polyhymno .156

polyommata Tepper 780

Polyommatus 16

polyphemella Ragonot 5676 
polyphemus Boisduval 450

polyphemus Cramer 774

polyxenes Fabricius 4

Pomeria 194

pometaria Harris 3767

pometella Harris 6380

pomifoliella Clemens 8135

pomiliella Clemens 8300

pomivorella Packard 8383

pomona Smith 2143

pomonella Busele 7982

pomonella Linnaens 7270

pomonella Packard 8135

ponceana Schaus 12

ponchs Smith 1301

ponda Dyar 5459

ponderosa Grote 3046

ponderosze Dyar 5566

pontiac Edwards 610

pontiaria Taylor 4048

popana Kearfott 6929

popeanellus Clemens 8184

popofensis Smith 2373

populana Busck 7236, 1

populella Busek 8423

populetorum Frey \& Boll 8385

populi Riley 2483

populi Strecker 2122

populi Walker 8352

populicola Boisduval 711

populiella Chambers (Lithocolletis) 7903

populiella Chambers (Gracilaria) 8046

populiella Chambers (Phyllocnistis) 8110

porcellaria Guenee 4560

porcus Hubner 758

Porosagrotis

porphyria Herrich-Schaefer 915

porrectella Linnaers 7675

porrigens Walker 3349

porsenna Scudder 396

Porthetria portia Fabricius 318

portia Hy. Edwards 8079

portiandia Fabricius 90

portlandia Grote 3208

portlandia $\boldsymbol{H} y$. Edwards 3616

portulace Cockerell 7789

postera Guenes 2002

posterior Walker 3172

postica Smith 1787

posticata Grote \& Robinson 5025

posticata Harvey 2372

posticella Walsingham 6469

postrema Walker 4336

postremella Dyar 5805

postrubralis Hampgon 5144

potentalis B. \& McD. 5127

potentillø Hy. Edwarde 6734

potrillo Lucas 487

pottawattomie Worthington 613

Poujadia 149

poulella Busek 7677

puweschiek Parker 549

prrecedens $H y$. Edwards 6729

prreacuta Smith 2626

preangusta Haworth 7820

præatomata Haworth 4341

precedens $H y$. Edwards 6729

prreclara Grote \& Robinson 3107

præfectellus Zineken 5344

præfica Grote 2571

prefixa Morrison 1418

pregracilis Grote 1966

preelineata Braun 7845

præmaturella Clemens 7838

præpilata Grote 3005

præseripta Meyrick 7120

præses Grote 1904

prsestans $H_{y y}$. Edwards 6705

prasina Fabricius 1560

prasinospila Meyriele 7134

pratella Hubner 5350

pratensis Behr 249

pratensis Scudder 251

pratincola Boisduval 573 
prattiella Busck 8228

pravella Grote 5614

pravinominella Chambers 6250

praxialis Druce 4974

precationis Guenee 3261

preciosa Nixon 990

preciosella Dietz 8015

Prenes

pressus Grote 1509

pretansata Grossbeck 4258

pretiosa Lintner 3104

pretiosa Morrison 2736

prima Slosson 962

prima Smith (Perigrapha) 1894

prima Smith (Caradrina) 2581

primariana Walker 6814

primella Busck 6036

primordalis Dyar 5225

primulana Walsingham 7024

princeps Busck 6127

princeps Walker 711

princetonia Poling 27

principalis Lederer 4969

principalis Walker 5447

prini Smith \& Abbot 671

Priocycla

Prionapteryx

Prionoxystus

privata Walker 1533

proba $\mathrm{Hy}$. Edwards 955

proba Smith 1919

probata B. \& McD. 2293

proceralis Lederer 5148

Prochalia

Prochloridea

procincta Grote 1778

proclivis Smith 1386

procris Edwards 542

Prodenia

prodenialis Walker 5693

prodeniformis Smith 1706

prodeuns Walker 1842

Prodoxida 197

Prodoxus 197 producta Walker 3007

productata Packard 3902

profana Meyrick $\mathbf{7 0 7 7}$

profecta Grote 3566

profundalis Packard 5089

profunda Smith 1537

progne Cramer 277

Progona 194

prolatella Grote 5430

Proleucoptera

Prolimacodes 126

promethia Drury 771

promptana Robinson 7463

promptella Walker 3152

promulgata Pearsall 4265

promulsa Morrison 1648

Promylea 144

Pronoctua

Pronuba 197

pronuba $B . \& M c D .2565,1$

propera Grote 2892

propertius Lintner 526

prophra Schaus 927

propinqua Smith 1976

propinqualis Moeschler 4922

propinqualis Guenee 3434

propinquilinea Grote 3206

propinquinella Braun 7898

propinquus Walsingham 8181

proprialis Fernald 5204

propriaria Walker (Trichodesia) 3942

propriaria Walker (Euchlæna) 4710

propriella Walker 5743

proprius Hy. Edwards 2011

propulsa Walker 1780

propulsata Walker 3978

Prorachia 67

Proroblemma 74 prorupta Grote 1077

prosenes Dyar 5433

proserpina Edwards 305

proserpina Fabricius 784 
Pace

proserpina Seudder 318

proserpinella Frey \& Boll 6399

Proserpinus 26

Prosoparia

prosopis Hy Edwarde 6696

prosperana Kearfolt 7247

Prostomeus 166

Protagrotis

Protambulyx

proten Smith $\mathbf{3 3 7 5}$

proteella Dyar 3011

Proteides

Proteopteryx

Proteoteras

proterpis Fabricius 73

proteus Linnaeus 458

Prothrinax

protillus Herreh-Sehat ffer 459

protodice Boisduval 34

Protoparce

Protophana

protractaria Herrich-Schaeffer 3809

protumnusalis Walker 3507

proutaris Pearsall 3805

provana $K$ corfott 6875

Provia

proxantinata Walker 4343

Proxenus 68

proxima Guerin 983

proxima Hy. Edwards 6681

proxima Morrison 2331

proximalis Fernald 5079

prudens Clemens 6157

pruena Dyar 2147

pruni Harris 2438

pruniella Clemene (Anarsia) 6353

pruniella Clemens (Coptodisea) 7595

pruniella Clemens (Haploptilia) 7785 prunifoliella Chambers

(Evippe) 6062

prunifoliella Clemeno 8886

pruniramiella Clemena 8234

prunivors Walsh 7208

prunivorella Chambers 8025

pryeri Butler 782

Peacaphora

163

psammitis Zeller 7783

Psaphida 57

Psectraglea 60

pseudacaciella Fitch 7915

Pseudacontia 74

Pseudalypia 87

Pseudanarta 57

Pseudanthcecia 56

pseudargiolus Boisduval \& Le Conte 452

pseudargyria Guenee 1933

Pseudcraspedia .4

pseuderminea Peck 960

Pseudeva 84

Pseudhapigia 96

pseudoacaciella Chambers 6273

pseudoausonides Verity 39

pseudobryonise Verity 35

pseudocarye Rothschild 916

pseudocellus C. \& C. 474

pseudocorybas Verity 25

pseudodorippus Strecker 308

pseudofen Morrison 418

pseudofondella Busck 6296

Pseudogalleria 169

pseudogamma Grote 3266

Pseudohazis

pseudoleracea Verity 35

Pseudomya

pseudoneustria Boisduval 3748

Pscudopigritia 163

pseudoptiletes Boisduval \& Le Conte 420

pseudoranalis $B$. \& $M c D .5126$

Pseudorgyia 


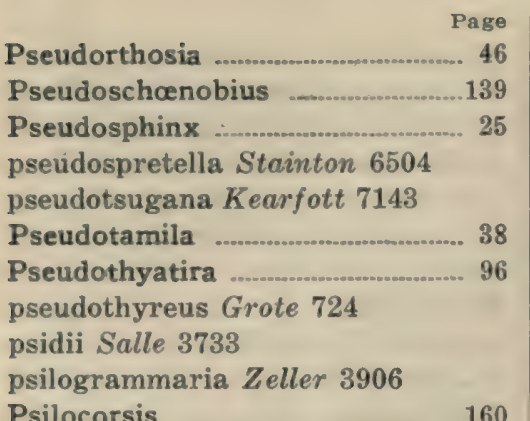

psoraliella Walsingham 6442

Psorosina

psyche Boisduval \& Le Conte 351

psychialis Hulst 4902

Psychida 124

Psychomorpha

Psychophora 107

psychotriella Busck 8119

ptelearia Riley 3885

pteliæella Chambers 8387

Ptenopoda 102

pteridis Hy. Edwards 955

pterisii Bird 2685

pterodactyla Hubner 5942

pterodactyla Linnaeus 5949

Pterophorida -.....150

Pterophorus

Pterospoda

Pterotæa

ptilodactyla Hubner 5949

ptilodonta Grote 1624

Ptycerata 153

Ptychoglene

ptychogrammos Zeller 7439

ptychophora Grote 2550

Ptychopoda 102

puber Grote \& Robinson 4755

pudefacta Dyar 3727

pudens Guenee 3693

pudens $H y$. Edwards 1016

pudibundella Ragonot 5635 pudibundella $Z$ eller 6042 pudica $H y$. Edwards 352 pudorata Morrison 2469 pudorata Smith 2024 puella Smith 2148 puellaria Dyar 4515 puer Hubner 544 puera Cramer 3583 puerilis Grote 1850 puertalis $B . \& M c D .5185$ puertata Grossbeck 4381 pugionis Smith 1247 pulchella Boisduval 249 pulchella Chambers 8048 pulchella Dietz 6528 pulchella Grossbeck 4562 pulchella Harvey 1896 pulchella Smith (Xanthia) 2234 pulchella Smith (Xylomyges) 1890

pulchella Walker 5421 pulchellana Clemens 7181 pulchellus Zeller 5342 pulcher Grote 8487 pulcherrima $B . \& M c D .3806,1$ pulcherrima Grote 2297 pulcherrima Stretch 996 pulcherrimana Walsingham 7292

pulcherrimella Chambers 5956 pulchra $B . \& M c D .2966$ pulchra Dyar 827 pulchra Strecker 84 pulchraria Minot 4486 pulchraria Taylor 3966 pulchripennis Grote 1061 pulchripictalis Hampson 5108 pulla $H y$. Edwards 101 pulla Grote 2103 pullata Braun 7976 pullata Grote 2103 pullatella Ragonot 5677 pullifimbriella Clemens 6272 
pullusella Chambers 6841

pulmonaria Grote 4579

pulsatillana Dyar 6988

pultaria Guenee 4656

pulvella Chambere (Batrachodra) 7822

pulvella Chamber (Tischeria) 7864

pulveralis Warren 5181

pulveraria Linnaeus 4641

pulveraria Hulot 4321

pulveratana Walsingham 6958

pulverea Grote \& Robinson 3640

pulverea Smith 2814

pulverilinea Grote 3201

pulverina Neumoegen 896

pulverosa Walker 3371

pulverosalis Smith 3525

pulverosalis Walker 3420,1

pulverosana Walker 7428

pulverulenta Felder 504

pulverulenta Smith (Polia) 1723

pulverulenta Smith (Byromima) 2196

pulverulenta Smith (Andropolia) 2518

pulverulentus Riley 8466

pulvipennella Clemens 6449

pumila Boisduval \& Le Conte 328

pumilella Ragonot 5619

pumilio Zeller 5945

punctadiscana Kearfott 7.181

punctalis $B$. \& $M C D$. 3498, 1

punctanum Walsingham 6801

punctata Edwards 259

punctata Fiteh 959

punctata Hulst $\mathbf{4 6 4 8}$

punctata Packard 955

punctata Walker 3625

punctatissima Neumoegen $\mathbf{8 1 3}$ punctatissima Smith \& Abbot 959
Pare

punctella Grote \& Robinuon 642

punctellus Busek 8157

puncticosta Smith $\mathbf{3 4 7 6}$

puncticostana Walker 6844

punctidactyla Haworth 5863

punctidiscanum Dyar 7252

punctidiacella Clemens 6878

punctiferella Busel 6020

punctiferella Clemene 6568

punctiferella Walsingham (Ade-

la) 8456

punctiferella Walsingham

. (Greya) $\mathbf{8 4 4 2}$

punctiformis Smith 3520

punetigera Walker 1286

punctilimbella Ragonot 5849

punctilinea Hampson 2056

punctilinea Smith 1922

punctilineella $B$. \& MCD. 5424

punctipennella Clemens 6351

punctipennis Grote 3430

punctipes Curtis $\mathbf{4 0 5 9}$

punctirena Smith 2757

punctistriga Walker $\mathbf{3 7 3 7}$

punctivena Smith (Hypocoena)

2802

punctivena Smith (Capnodes)

3380

punctofimbriata Packard 3899

punctolineata Packard $\mathbf{4 3 4 2}$

punctomacularia Hulst 4484

punctulata Braun 8402

pungens Eschscholtz 662

punitalis Smith 3446

puntagorda Slosson 3625

pupillaris Grote 3515

pupula Hubner (Eustixia) 5178

pupula Hubner (Lactura) 7732

pura B. M M D. (Crambidia)

861

pura B. \& McD. (Oslaria) 2780

pura Butler (Utetheisa) 1030

pura Hulet 3070 
pura Neumoegen 917 puraria Walker 3869 purata Guenee 3853 purcellata Taylor 4349 purgata Walker 3131 puricostella Ragonot 5850 purinosella Chambers 7859 puritana Robinson 7307 puritellus Kearfott 5429 purpurana Clemens 7358 purpuraria $B$. \& $M c D .4558$ purpuraria Pearsall 4671 purpurascens $H y$. Edwards 169 purpurascens Hulst 3842 purpurascens Packard 8483 purpurascens Walker 3398 purpurea $B . \&$ McD. 1684 purpurea Grote 2219 purpurea Walker 8454 purpurella Dietz 6580 purpurella Hulst 5651 purpureofusca Walsingham 6362

purpuriciliana Walsingham 7151

purpuriella Busck 5995

purpuriella Chambers (Chrysopeleia) 5980

purpuriella Chambers (Gracilaria) 8061

purpurifascia Grote \& Robinson 2683

purpurigera Walker 3281 purpuripennis Grote 2397 purpurissata $B$. \& $M c D .2254$ purpurissata Grossbeck 4259 purpurissata Grote (Polia) 1665

purpurissata Grote (Scelolophia) 3840

purpurocomella 6536

pusilla Dietz 6572 pusilla Frey \& Boll 7839 .
Page

pusillifoliella Frey \& Boll 7931 pusionellus Zeller 5363 pussillalis Hubner 5422 pustula Geyer 590 pustularia Guenee 4303 pustularia Hubner 4425 pustularia $H y$. Edwards 3969 pustulata $\boldsymbol{H} y$. Edwards 2847 pustulata Walker 841 pustuliferalis Lederer 5006 puta Grote \& Robinson 2225 putnami Grote 3255 putnami Hy. Edwards 362 putrescens Guevin 3175

Pygarctia 35

pygmæa $B$. \& $M c D .4808$

pygmæa Grote 3557

pygmæa Grote \& Robinson 4826 pygmæa Hubner 3005 pygmaella Hubner 7694 pygmæella Ragonot 5572

Pygmana 116

pygmæus Walsingham 5855

pygmeolaria Grote 4514

Pygoctenucha 30

Pyla 146

pylades Scudder 483

Pyralida 128

pyralidiformis Walker 6715

Pyralina 137

Pyralis 137

pyralis Hubner 3445

pyralis Smith 1517

pyraloides Strecker 1156

pyramellus $B . \& M c D .8163$

pyramidalis Walker 6744

pyramidoides Guenee 2239

pyramus Boisduval 732

pyramusalis Walker 3546

pyrana Meyrick 7334

Pyrausta 133

Pyraustina 128 


$\begin{array}{lr}\text { Pyrgus } & \text { Pase } \\ \text { pyri Harris } 6688 & \text { Is } \\ \text { pyricolana Murtfoldt } 7158 & \\ \text { pyrifoliana Clemens } 7170 \\ \text { pyrina Linnacus } 8899\end{array}$

Pyrocleptria 37

Pyroderces 152

pyrolaria Guenes 4510

pyrophiloides Harvey 1602

pyrrha Druee 3685

Pyrrhia 69

Pyrrhopyga 17

Pyrrhopygina 17

pyrrhoura Hulat 875

pyrusana Kearfott 7334

pythion Druee 3219

python Edwarde 620

pyxidifera Smith \& Abbot 4863 quadragintapunetats $D$ yar 7628 guadrangula Zetterstedt 1484 quadrannulata Morrison 1951 quadrannulata Walker 3918 quadranotata Strecker 993 quadraquina Seudder 592 quadraria Grote 4326 quadrata Grote 2457 quadrata Smith 1711 quadrate Smith 2720 quadrellus $B$. \& McD. 8194 quadricolorella Dyar 5819 quadricornis Harris 674 quadricristatella Chambers 5981 quadridentata Grote \& Robinson 1247

qquadrifasciana Fernald 7398 quadrifasciaris Packard 4306 quadrifasciata Taylor 4392 quadrifera Zeller 2886 quadrifidum Zeller 6807 quadrifilaris Hubner 3163 quadriguttalis Hubner 1042 quadriguttatus Grote 8483 quadrilinearia Packard 4410
Quadrilineata Peckard 8856

guadrilineata Grote 1762

quadrilineata Grote \& Robinson 806

quadrilineella Chambers (Cosmopteryx) 5962

quadrilineella Chambers (Haploptilia) 7790

quadrilunata Grote 1630

quadrimaculella Chambers (Anacampsis) 6189

quadrimaculella Chambers (Gelechia) 6250

quadrimaculella Chambers (Epicallima) 6494

Quadrina 97

quadrinotaria Herrich-Schaeffer 4352

quadrinotata Walker 3961

quadriplaga Smith 2976

quadriplaga Walker 3220

quadripunctats Morrison 4289

quadripunctata Packard 4068

quadripunctella Clemens 8026

quadripunctellus Dyar $\mathbf{8 1 6 9}$

quadrisignata Walker (Metalestra) 3479

quadrisignata Walker (Maearia) 4945

quadristigma Smith 1852

quadristigmalis Guenee 4978 quadristigalis Forna'd 5065

quadristrigella Busck 7812 quadristrigella Chambers 8421 quadristrigella Zeller 7684 quasita Grote 2253 quasitata Hulst 3867 quastoralis B. \& McD. 5086 quaintancella Dietz 6522 quakerata Pearsall 4241 quantulella Hulat 5786 quarta Grote 1559 quebecata Taylor 4273 


\begin{tabular}{|c|c|}
\hline $\begin{array}{l}\text { quebecensis Smith } 1320 \\
\text { quenseli Paykull } 980 \\
\text { quercana Fernald } 7296 \\
\text { querceti Herrich-Schaeffer } 4816 \\
\text { quercetorum Frey \& Boll } 7877 \\
\text { querci Hy. Edwards } 6695 \\
\text { quercialbella Fitch } 7883 \\
\text { quercicastanella Chambers } 8388 \\
\text { quercicella Busck } 6614 \\
\text { quercicella Chambers } 6389 \\
\text { quercicella Clemens } 6425 \\
\text { quercicola Herrich-Schaeffer } \\
\text { 4816 } \\
\text { quercicolella Chambers } 7690 \\
\text { quercicolella Ragonot } 5625 \\
\text { querciella B. \& McD. } 5488 \\
\text { querciella Busck } 7643 \\
\text { querciella Chambers } 6078 \\
\text { querciella Clemens } 7791 \\
\text { quercifoliana Fitch } 7364 \\
\text { quercifoliella Chambers (Gele- } \\
\text { chia) 6265 } \\
\text { quercifoliella Chambers (Acro- } \\
\text { cercops) } 7997 \\
\text { quercifoliella Fitch } 7877 \\
\text { quercinigracella Chambers } 6075 \\
\text { quercinigrella Ely } 8072 \\
\text { querciperda Fitch } 8355 \\
\text { quercipomonella Chambers } 6380 \\
\text { quercipulchella Chambers } 7883 \\
\text { quercipulchrella Chambers } 8389 \\
\text { quercitella Clemens } 7858 \\
\text { quercivororia Guenee } 4726 \\
\text { quercivorella Chambers (Recur- } \\
\text { varia) 6145 } \\
\text { quercus Boisduval } 912 \\
\text { quernaria Smith \& Abbot } 4615 \\
\text { querquera Grote } 2149\end{array}$ & 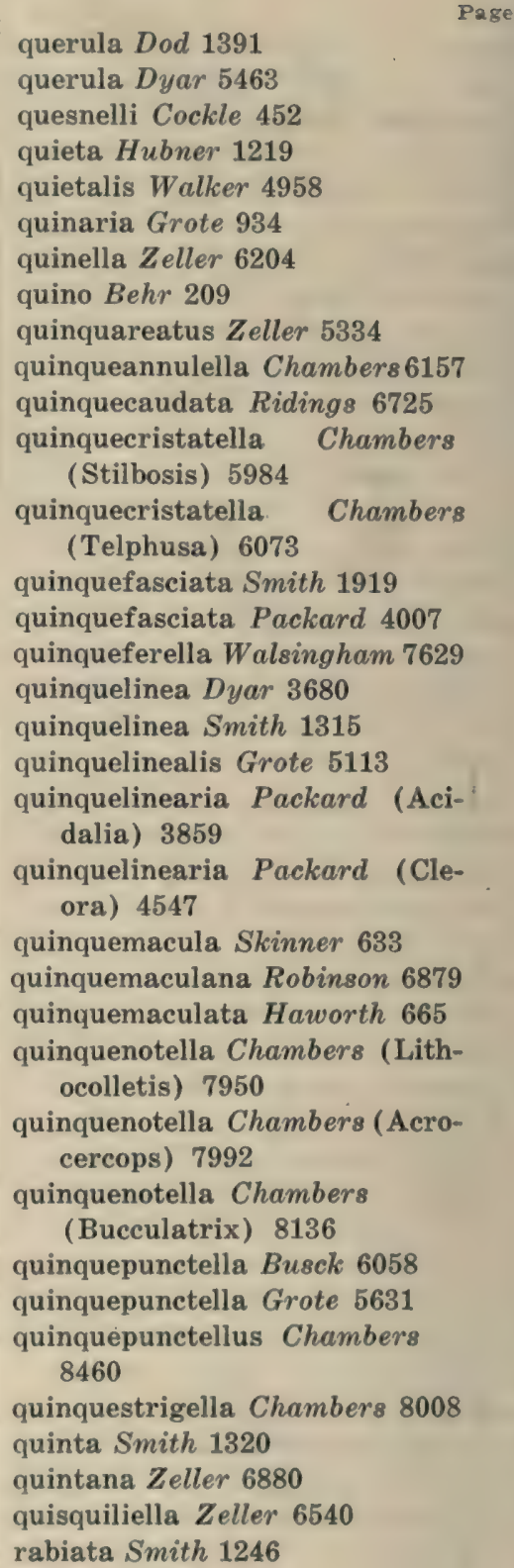 \\
\hline
\end{tabular}


rachel Bruce 75:

Rachela 104

rachelso Hulat 4617

Racheospila

radcliffei Harvey 2470

radians Walker 996

radiata Harris 1402

radiatana Waleingham 7048

radiatella Busck 6112

radiatella Donovan 7662

radiatus Snith (Lycophotia) 1485

radiatus Smith (Lythrodes) 2745

radiatus Strecker 18

radicana Walsingham 6948

radicicolana Walsingham 7257

radiola Hampson 1485

radiosalis Moeschler 5095

radiosaria Hulst $\mathbf{4 7 3 4}$

radix Walker 1712

ragonoti Walsingham 6887

Ragnotia

rainierella Dyar 5668

raineri $B$. \& $\mathbf{M c D} .193$

raineri Swith 1651

ralla Grote \& Robinson 2224

ramapoella Kearfott 8089

ramburialis Duponchel 5048

ramentalis Lederer 4937

ramosula Guenee 2515

ramuscula Dyar 8340

rans Busck 7510

ranalis Guenee 4955

Rancora

randana Kearfott 6903

randiella Busck 8007

rantalis Guenee 5025

ranunculi $\boldsymbol{H}$. Edwards 2841

rapre Linnaeus 37

rapahoe Reakirt 453

Raphia 84

Raphiptera 139 raptor Meyrick 5861

$8 \times 50$ rara Packard 1350 raracana Kearfott $7075^{\circ}$

rarata Walker 3668

rasilis Morrison 2611

raspe Boiaduval 808

rata $H_{y}$. Edwards 4869

ratifera Meyrick 7234

ratzeburgiana Saxesen $\mathbf{7 1 5 7}$

rauterbergi Skinner 459

rava Herrich-Schueffer 1484

ravocostaliata Packard 4228

rayata $B$. \& $M c D .543$

rayata $S$ mith 2080

Reabotis 88

reaghi Reiff 247

reakirti Edwards 46

reciprocata Walker 3942

reclivis Dyar 1384

reconditalis Walker 5226

reconditaria Walker 3857

rectalis Smith 3450

rectangula Kirby 3236

rectangulatn Ottolengui 3689

rectaria Grote 3811

rectarius Dyar 4878

recticincta Smith 1327

rectifascia Grote 2730

rectifascia Hulst 4312

rectifascia Smith 2120

rectiflava Smith 1854

rectilinea French 974

rectilinea Grote \& Robinson

4840

rectilinea Smith 1755

rectilinea Zeller (Chlorochla-

mys) 3818

rectilinea Zeller (Scoparia)

5232

rectilineata Taylor 4282

rectiplicana Walsingham 6964

rectistrigella Dyar 5629

rectistrigella Ragonot 5512

rectus $\boldsymbol{H y}$. Edwards 8493 


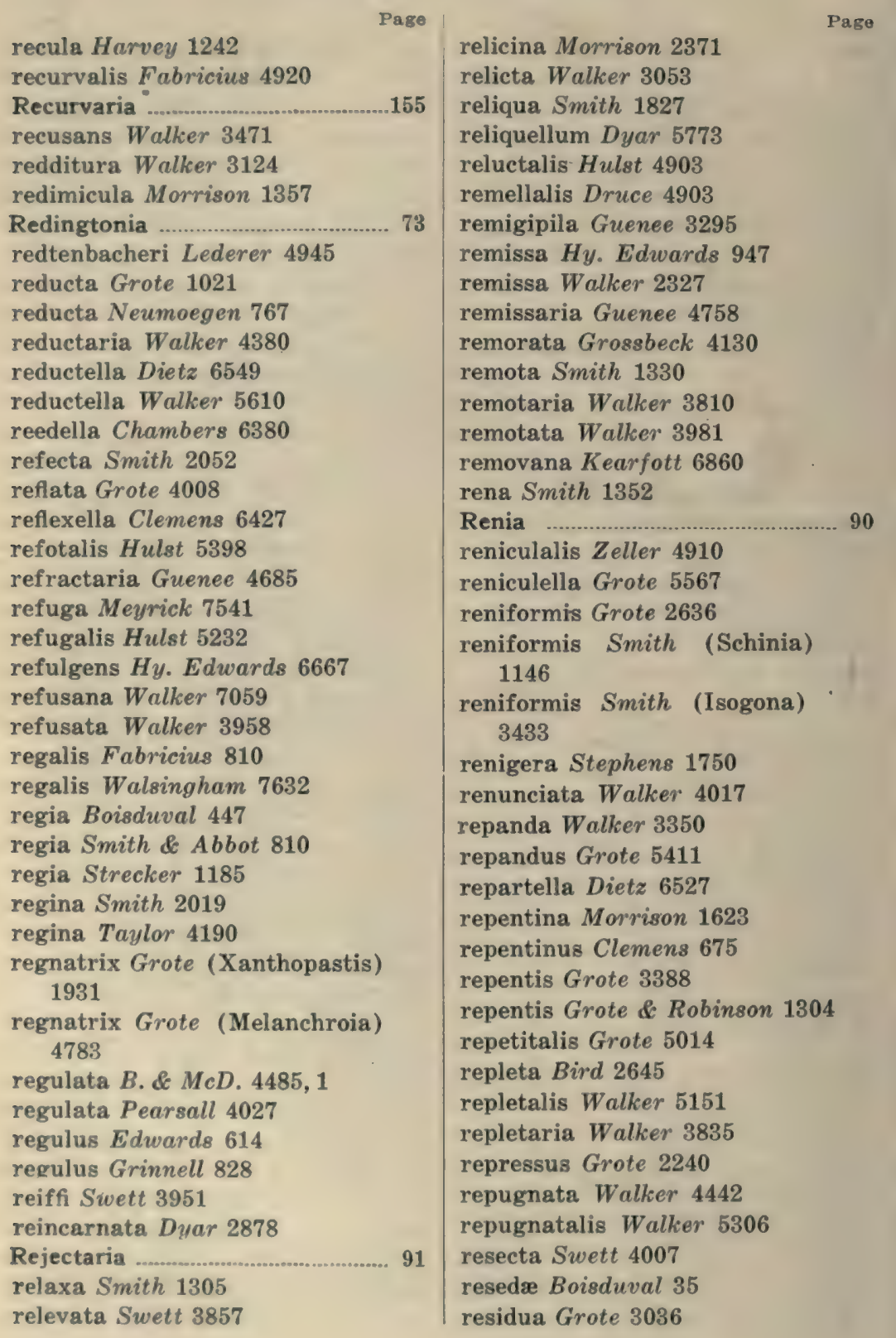


resistaris Herrich-Schneffer 4680

resoluta Meyrick 7117

resoluta Smith 2517

resoluta Zeller 3766

respersata Hulat $\mathbf{4 8 6 0}$

resplendens $H y$. Edwards 6658

resplendensella Chembers 8390

restionalis Lederer 4909

restituens Walker 3767

restora Swith 1760

restrictalis Grote 3533

restrictalis Smith (Microhelia) 2834

restrictata Walker 3857

resumens Walker (Pachylia) 723

resumens Walker (Psaphida) 2104

resumptana Walker 7122

retann Walsingham 7351

retardata Walker 2453

retecta Grote 3039

retectata Walker 4389

retectella Zeller 6524

retentata Walker 4389

reticens Walker 1304

reticulata Braun 8043

reticulatana Clemens 7294

reticulatus Lintner 8354

reticulatus Riley 8464

reticulina Beutenmuller 4895

retiniana Walsingham 7352

retinotata Walker 4345

retis Grote 2635

retractaria Walker (Ptychopoda) 3904

retractaria Walker (Macaria) 4345

retroversa Morrison 2081

reuda Strecker 1273

revayana Scopoli 3012

revellata Smith 2469

reversa Stretch 1032 reversalis Guenee 5064

reversalis Smith 3448

reversana Kearfotl 6942

reveranta Dyar 8503

revicts Morrison 1917

revocata Wriker 4622

revoluta $W$ alker 3165

Rhabdoides 18

rhas Boisduval 448

rhabus Boisduval 718

rhamnella Braun 8410

rhamnicola Braun 8410

rhena Edwards 578

rhesus Edwards 550

Rhescipha 87

rheumapterella Dietz 8465

rhexia Smith \& Abbot 1091

Rhiodinidx 13

Rhizagrotis 41

rhoda Butler 996

rhodarialis Walker 3459

rhodia Edwards 142

rhodites Meyrick 7496

rhodocentra Meyrick 7734

rhododendri Beutenmuller 6689

Rhododipsa ............................. 38

Rhodocia ................ 69

rhodope Edwarde 169

Rhodophra 143

rhodophren Meyrick 7001

Rhodophora 38

rhoiella Dyar 5683

rhoifoliella Braun 8408

rhoifoliella Chamber 8042

rhoifructans Kearfott 6786

rhoifructella Clemens $\mathbf{6 1 8 9}$

rhombiferella Frey \& Boll 8009

Rhynchagrotis 48

rhypodella Hulst 5597

ribearia Fitch 4399

ribesella Busck 7718

ribesella Chambers (Gelechia) 6213 

ribesella Chambers (Gracilaria)
8062

ribesii $H y . E d w a r d s 781$

ricara $E$ dwards 489

richardsoni Curtis 1628

richi Grote 2765

Richia 47

rickseckeri Behr 960

rickseckeri Pearsall 3923

rickseckeri Watson 788

ridenda $H y . E d w a r d s 3680$

ridingsana Robinson 6894

ridingsella Clemens 8449

ridingsiana Grote 1252

ridingsi Edwards 108

ridingsi Grote 1047

ridingsi Reakirt 556

ridingsi Riley 2919

rigida Grote 2689

rigida Smith (Orosagrotis) 1227

rigida Smith (Renia) 3527

rigida Smith (Siavana) 3351

rigida Walker 1219

rigidalis $B . \& M c D .5234$

rigidana Fernald 6761

rigidiaria $B . \& M c D .4609,1$

rigidata Walker 4109

rileiella Busck 7707

rilevaria Packard 4300

rileyana $H y$. Edwards 6660

rileyana Grote 7283

rileyana Harvey 3753

rileyana Morrison 1467

rileyana Smith 1874

rileyella Chambers (Gelechia) 6256

rileyella Chambers (Lithocolletis) 7892

rileyella Ragonot 5803

rileyi Dietz 8277

rileyi Fernald 5922

rileyi Frey \& Boll 8137

rileyi Heylaerts 4807

rileyi Walsingham 5983 rimosa Grote 1954

rimosa Packard 3615

rimosalis Guenee 5000

riparia Morrison 2046

riscana Kearfott 7548

rita B. \& McD. 445

ritaria Grossbeck 4538

riversi Dyar 1239

Rivula

rivulalis Hampson 4967

rivulana Scopoli 6841

rivularia Grote 3769

rivulosa Guènee 1130

rivulosa Smith 2771

rivulosa Stretch 978

robiginosus Morrison 4506

robiniæ Hy. Edwards 6749

robiniæ Peck 8354

robiniæfoliella Chambers 6144

robiniella Clemens (Lithocolletis) 7915

robiniella Clemens (Parectopa) 8003

robiniella Fitch 6144

robiniella Packard 6471

robinsonana Grote 6880

robinsoni Boisduval 895

robinsoni Butler 710

robinsoni Grote (Catocala) 3031

robinsoni Grote (Dicymolomia) 5447

roburella Dietz 8275

robusta Strecker 3602

robustella Dyar 5637

robustella Zeller 5480

robustior Smith 1396

Røeselia $3 n$

roessleri Z eller 7089

rogatalis Hulst 4982

rogationis Guenee 3260

rogenhoferi Moeschler 1697

rolandi Grote 2110

rolandiana Grote 2917

romonana Kearfott 7455 
roosta Smith 1482

rorana Kearfott 6931

rorulenta Smith 2269

rosa Druce 5161

rosa Eduerde 41

rosa French 934

rosa $\mathrm{MeN}$ eill 55

rosaceana Harria 7361

rosacearum Boisduval 708

rosacella Clemens 7792

rosaciliella Busck 6448

rosue Behr 3174

rosefoliella Clomen (Haploptilia) 7793

rosafoliella Clemens (Nepticula) 8391

rosalba Grote 3460

rosalinda $\boldsymbol{H}$. Edwarde 3067

rosana Linnaeus 7340

rosana Hubner 7410

rosaochreana Kearfott 6852

rosaria Grote (Agrotis) 1435

rosaria Grote (Plagodis) 4667

rosario Barnes 1212

rosea Beutenmuller 3646

rosea Harvey 1926

rosea Smith (Oncocnemis) 2053

rosea Smith (Thyreion) 1100

rosea Walker (Hyparpax) 3662

rosea Waller (Oreta) 3757

roseata Stretch 3754

roseata Walker 929

roseatella Packard 5835

roseicapitis Neumoegen \& Dyar 1008

roseicaput Neumoegen \& Dyar 8488

roseicosta B. \& McD. 3849

roseicosta Guence 3352

roseicrinella Busck 6037

roseitacta Prout 3806

roseiterminalis $\boldsymbol{B}$. \& $\boldsymbol{M}$ CD. 5029

roseitincta Harvey 1190

roseocostella Walsingham 6384 roseola Smith (Cirphis) 1937

roseola Smith (Amolita) 2805

roseomaculana Herrich-Schaeffer 6855

roseopennalis $\boldsymbol{H}$ ulat 5053

roseopennella $\mathrm{Hulat} 5810$

roseosuffusa Smith 1770

roseosuffusella Clemens 6041

roseoterminana Kearfott 7051

roseotincta $\mathrm{Hulat} 3876$

roseotinctella Ragonot 5847

roseticola Frey de Boll 7875

rossi Curtis (Erebia) 139

rossi Curtis (Gynaphora) 3699

rossi Guénes 69

rossi Ross 773

rostrana Walker 7327

rostrella Ragonot 5827

Rothschildia 28

rotundalis Walker 3490

rotundata Packard 3946

rotundata Rothschild 670

rotundopennata Packard 3900

rotundopuncta Packard 4164

Ruacodes 68

rubefacta Morrison 1929

rubefactalis Grote 1275

rubens $\boldsymbol{H}$ y. Edwards 735

rubensella Chamber 6044

ruberata Freyer 4024

rubescens Walsingham 6197

rubescentella Hulst 5605

rubi $\boldsymbol{H}$. Edwards 3194

rubi Riley 6649

rubiata Smith 3193

rubicunda Fabricius 802

rubicunda $H y$. Edwards 210

rubicunda Walker 2391

rubicundaria Hubner 934

rubida $B . \&$ MCD. 2619

rubida Ottolengui 3252

rubidella Clemens 6044

rubidus Behr $\mathbf{4 1 3}$

rubifera Grote 1438 
rubiferaria Swett 4631

rubifoliella Clemens 8392

rubigalis Guenee 5088

rubigera Hampson 3283

rubiginella Walker 5655

rubiginosa Bird 2676

rubiginosa Strecker 1189

rubiginosa Walker 2843,1

rubipunctana Kearfott 6823

rubivora Riley 3800

rubra Behr 769

rubra Neumoegen 956

rubralis $B . \& M c D .5276$

rubralis Warren 5066

rubrella Dyar 7657

rubrescens Hulst 6744

rubrescens Walker 1910

rubribasella Hulst 5690

rubrica Harvey 1888

rubricalis Hubner 5149

rubricata Edwards 98

rubricincta Beutenmuller 6676

rubricoma Guenee 2498

rubricosa Harris 948

rubricosta Ehrman 937

rubricosta Guenee 3352

rubrifasciella Packard 5543

rubrifrontaria Dyar 3799

rubrifrontaria Packard (Nemoria) 3784

rubrifrontaria Packard (Synchlora) 3802

rubrifusa Hampson 1675

rubrigutta Skinner 815

rubripallens Smith 1968

rubripalpis Felder 3737

rubripennis Grote \& Robinson 1958

rubripennis Neumoegen \& Dyar 3620

rubrisparsella Ragonot 5601

rubristigma Kellicott 6694

rubrithoracella $B$. \& $M c D .5681$

rubrofascia $H y$. Edwards 6674
Page

rubrofasciata $B . \& M C D .305$

rubrolinearia Packard (Racheospila) 3775

rubrolinearia Packard (Acidalia) 3879

rubromarginaria Packard

(Racheospila) 3773

rubromarginaria Packard

(Xystrota) 3848

rubromarginata Davis $\mathbf{7 7 3}$

rubropicta Packard 931

rubroscapus Menetries 837

rubrosuffusa Cockerell 773

rubrosuffusa Grossbeck 3937

rubrosuffusa Grote 3202

rubrosuffata Packard 4090

rubrotincta Hulst (Scelolophia) 3843

rubrotincta Hulst (Slossonia) 4660

rudana Walsingham 7310

rude $H y . E d \dot{w} a r d s 4830$

rudens Harvey 1493

ruderella Ragonot 5669

ruderella Z $Z$ eller 8215

rufa Grote 3559

rufago Hubner 2231

rufaria Grote 4549

rufescens $B . \& M c D .8327$

rufescens Boisduval 430

rufescens Hulst 3838

rufescens Wright 263

rufibasella Ragonot 5601

ruficaudis Kirby 732

ruficillata Guenee 4086

ruficollis Druce 4876

ruficornis Dyar 3636

ruficornis $H y$. Edwards 6717

rufifimbrialis Grote 5148

rufilineata Walker 3868

rufimargo Hubner 3352

rufimedia Grote 1117

rufinalis Walker (Crambus)

5380 
rufinalis Wolker (Elasmopat pus) 5655

rufinans Guense 8380

rufipectus Morrison 1565

rufipennis Grote 1303

rufisignella Zeller [.22

rufistrigalis B. \& MCD. 5196

rufocristatella Chambers 6019

rufofrontaria Gumppenberg $380^{\circ}$

rufoluteella Chambers 7751

rufopunctella Dietz 6573

rufostrigata Packard 2802

rufula Boisduval 955

rufula Grote 1815

rufula Morrison 1694

rufula Smith 1274

rufusella Chamber 6197

rugifrons Grote 2759

rugosa Morrison 1763

ruisa Forbes 1792

rumatana Smith 1245

runata Swith 2288

runcinaria Strecker 4637

rupestris Behr 180

rupta $H y$. Edwards 3680

ruptalis Walker 4926

ruptifasciatus Ploets 508

ruptilinea Walker 4824

ruptostrigella Beutenmuller 8048

ruralis Boisduval 489

rurea Edwards 604

ruricola Boisduval 564

ruricolellus Zeller 5371

rurigena Grote 3546

russata Hulst 3888

russatella Clemene 8430

russea $H_{y}$. Edwards 3266

russeliata Swett 4142

russula Grote 3415

rustica B. \& $M / c D .2084$

rustica Edwards 429

rustica Fabricius 666 rustica Sepp 712

rustica Walsingham 6393

rusticalis B. B MCD. 5092

rusticana Kearfott 6945

rusticella Hubner 8237

rusticus Edwards 271

rutile Guence 2670

rutila Wulker 3248

rutilans Hubner $\mathbf{7 4 6 1}$

rutilans $H y$. Edwards 6661

rutulus Lucas 14

ryensis Bird 2641

saawichata Swett 4094

sabalella Chambers 5992

sabina Wright 223

sabinalis Dyar 5058

sabini Kirby 4057

sabiniana Kearfott 6768

sabularia Guenee 4393

sabulella Walsingham (Paltodora) 6030

sabulella Walsingham (Agnopteryx) 6465

sabuleti Boisduval 584

sabulifera Walker 5430

Sabulodes 123

sabulosa Boisduval 1037

sabulosa $\boldsymbol{H y}$. Edwards 3299

sabulosa Smith 1063

sabura Druce 5247

saccatella Packard 7595

saccharalis Fabricius 5436

saccharella Braun (Lithocol-

letis) 7939

saccharella Braun (Nepticula)

8411

sacculalis Ragonot 5298

sackeni Grote 3228

sacramenta Heinrich 7794

sacramenti Grote \& Robinson

1046

saengeri Nermoegen 810

sepiolus Boisduval 480

sepium Boisduval 870 


Safia .................................. 92
saga Fabricius 3583
saga Staudinger 197
saganella Hulst 5329
saginella Clemens 8393
sagitella Dietz 6514
sagittalba Ottolengui 2754
sagittana Walker 7054
sagittarius Grote 1518
sagittata B.\& McD. (Catabena)
$\quad 2565$
sagittata B. \& McD. (Oncocne-
$\quad$ mis) 2070, 1
sagittigera Felder 448
sakuntala Skinner 169
salamisalis Druce 4943
Salebria ......................................145
saleppa Smith 1904
Salia

salicarum Walker 1474

saliceti Boisduval 706

saliciana Clemens 7160

salicicolana Clemens 7159

saliciella Busck 8108

saliciella Chambers 7594

salicifoliella Chambers (Lithocolletis) 7932

salicifoliella Chambers (Parectopa) 8011

salicifungiella Clemens 6045

salicipomonella Clemens 7821

salicis Behr 3174

salicis Boisduval 918

salicis $\boldsymbol{H} y$. Edwards 2843

salicis Harris 2508

salicivorella Braun 7909

salictella Clemens 7978

salienta Pearsall 4116

saligna Z Zeller 3559

saligneana Clemens 7015

salina Barnes 1381

salinaris Busck 6131

Salobrena
$\$ 2$

salona Hewitson 345

saltitans Westwood 7271

saltuellus Zeller 5355

saltusaria Hulst 3828

Saluria .149

salusalis Walker 3522

salutalis Hulst 4912

salvata Pearsall 4053

sambo Smith 1587

sambuci Behr 2813

Samea

.129

Sameodes

131

Samia

27

samoset Scudder 635

sampita Barnes 1199

sanbornana Robinson 7345

sanborni Hy. Edwards 6716

sanborni Packard 4876

sancta $H y$. Edwards 2486

sancta Hulst 3119

sancta Neumoegen \& Dyar 4879

sanctæ-monicæ Dyar 1217

sandana Kearfott 6904

sandiego Kearfott 6898

sandix Guenee 2642

sangamonia $B$. \& $M c D .3539$

sangaris Strecker 8495

sanguinaria Strecker 834

sanguinea Geyer 1188

sanguinella Beutenmuller 8048

sanguinella Busck 6458

sanguineola Boisduval 872

sanguivenosa Neumoegen 929

saniaria Hubner 3912

sanifica Meyrick 7127

sanina Smith 2031

saniptri Strecker 699

Sannina 166

sansar Strecker 2520

sansoni Dod 3271

santarita Dyar 2909

santaritella Dyar 5822

santatalis $B$. \& McD. 4953 
santella Kearfott 5317 saphirinella Chamber 6105 saporis Grote 2825 sappho Strecker 3037 sapygwoformis Walker 6713 sara Boieduval 46 sara Freveh 3080 sara Smith 1137 saracanu Kearfott 7299

Sarasota 143

Sarata 146

sardiopa Meyrick 6943

sarepta Barnes 2409

sareta Smith 1738

sarita Skinner 342

Sarrothripina

Sarrothripus

sartana Hubner 7524

sartoralis B.\& MCD. 5106

sassacus Harris 570

sassafrasella Chambers 8041

satanaria Guenec 4616

satellitia Drury 787

satiens Smith 1261

satina Strecker 2348

satis Harvey 1278

Satole

satrapellus Zincken 5332.

saturata Barnes 3625

saturata Grote 1189

Saturniida 27

Saturnioidea

saturnus Strecker 1820

satyricus Grote 1523

Satyridae 5

satyriniformis Hubner 6739

Satyrium 15

Satyrodes 6

satyrus Edwards 270

sauberi Hedemann 5446

saucia Hubner 1490

saundersana Kearfott 7215 saundersella Chambers (Epithectis) 6164

saundersella Chamber (Leucanthiza) 7986

saundersiana Grote 2034 saundersi Bethune $\mathbf{3 1 7 5}$ saundersi Grote 973 saurodonta Meyrick 7627 sauzalitre Grote 2657 sauzalitæella Chambers 8045 savignyi Latreille 457 saxea $\boldsymbol{H y}$. Edwardo 3333 saxicolana Walsingham 7533 saxifrage Hy. Edwards 6678 saxigena Morrison 1506 sayi Edwards 25 scabella Zeller 6439 scabra Fabricius 3578 scabrella Busck 6281 scabriuscula Linnaers 2243 scabrogata Pearsall 4223 scalana Walsingham 7083 scalata Warren 4009 seandens Riley 1486 scapha Harris 4837 scapularis $H_{y}$. Eduards 3272 scapularis Stretch 914

Scardia 192

seardina Zeller 8184

scarletina Smith 2839

scelestata Taylor $\mathbf{4 1 8 5}$

sceletopa Meyrick 6931

Scelidacantha .104

Scelolophia 101

Scelorthus .180

scepsiformis Grasf 1012

scepsiformis $\boldsymbol{H y}$. Edwards 6752

Scepsis 39

schaefferalis Dyar 5220

schaefferana Dyar. 4829

schaefferi Ploetz 480

schalleriana Linnneus 7420

schausaria Hy. Edwards 3768 


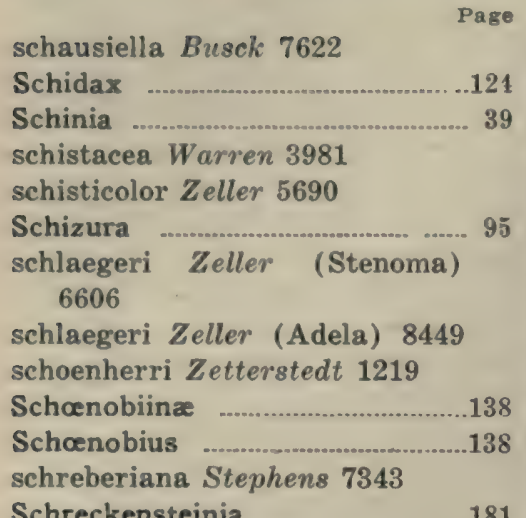

schulziana Fabricius 6874

schuylkillensis Grossbeck 4673

schwarzalis Dyar 5240

schwarzi Dyar 5943

schwarzi Smith 2993

schwarziana Busck 7451

schwarziella Busck (Euclemensia) 7575

schwarziella Busck (Cerostoma) 7664

schwarziorum Dyar 879

sciaphilella Zeller 6543

sciata Bird 2694

scintillana Clemens 6901

scintillans Grote \& Robinson 3020

scintillans Grote 5660

scintillularia Hulst 3887

scirpicola Guenee 1939

Scirpophaga

scissa Grote 1059

scissa Walker 3352

scissalis Walker 3577

scissana Walker 7453

Scissolia

seitana Walker 7210

scitata Walker 4656

scitipennis Walker 3608

scitiscripta Walker 3666 scitula Harris 6690

scitularia Walker 4114

scitulella Hulst 5547

sciurella Ragonot 5747

sciurus Boisduval 905

scobialis Grote 3487

scobiella Grote 5594

Scolecocampa 88

Scoliopteryx 87

scolopacinarius Guenee 4506

scolopendrina Boisduval 3673

Scoparia ................................136

Scopariinæ $\ldots \ldots . . . . \ldots \ldots \ldots \ldots \ldots \ldots . . .136$

scopeops Dyar 1595

scortealis Lederer 5481

Scotogramma

scribonia Stoll 964

scripta Gosse 3687

scriptaria Herrich-Schaeffer 4202

scriptipennis Walker 3485

scriptura Boisduval 492

seropulana Morrison 1480

scrupulosa $\boldsymbol{H} y$. Edwards 3300

scudderella Frey \& Boll 7911

scudderi Edwards 426

scudderi Packard 1024

scudderi Reakirt 66

scudderi Skinner (Atrytonopsis) 611

scudderi Skinner (Thanaos) 531

scudderiana Clemens 7014

sculptilis Harvey 1370

sculpta Grote 1533

scurralis Hulst 5144

scutellariæella Chambers 6120

scutellaris Grote 3563

scutosa Schiffermuller 1210

Scybalista .128

Scythridida 189

Scythris 18.? sebastianiæ Riley 7241

sebastianiella Busck 7989

secedens Walker 1772 
secta Grole 2914

sectilis Guenee 1874

sectilis Smith 2545

sectomaculata Morrison 4331

secunda Pearsall 4115

gecundalis Smith 3447

gecundaria $B$. \& $M C D .4816,1$

secundella Ely 5524

securells Walsingham 7638

sedata $H y$. Edurards 2980

sedatana Busek 7260

sedella Busck 8439

sedilis Smith 1653

seductaria Strecker 4466

sedulitella Busek 6302

segregata Pearsall 4253

segregata Smith (Polia) 1741

segregata Smith (Euxoa) 1240

segura Barnes 3481

seiferti Nenmoegen 3932

Seirarctia

Selagia

selama Strecker 1679

selecta Walker (Autographa) 3234

selects Walker (Catocala) 3083

Selenia

Selenis

selenis Smith 1284

Selicanis ie

gella Chambers 6084

selwyni Hy. Edwands 1024

semalea Meyrick 6954

semialba Ehrman 4

semialba Meyrick 7726

semiannula Robinson $\mathbf{7 4 1 3}$

cemiaperta Morrison 1798

semiatra Smith 2987

semiatrata Hulst 3975

semiauratus Neumoegen \& Dyar 8483

semicana Walker 2340

semichalcea Walker 2898

semicirculana Fernold 7383 semicircularis Grole 3695

semiclara Stretch 970

semiclarata Grote 1408

semiclarata Walker 4292

semiclusaria Walker $\mathbf{4 4 8 8}$

semicollaris Smith 2068

semicostata Zeller 5947

semicrocea Guenee 2920

semicyclionella Busek 6100

semidea Say 131

semidiaphana Harris 824

semifascia Smith 2416

semifascia Walker 4821

semiferana Walker 7354

semiflava Guenee 2965

semifuneralis Walker 5720

semifuscana Clemens 7357

semifusellus Walker 5358

semilineata Walker 3473

semilugens Zeller 6637

semiluna Smith 5071

semilunata Grote 2274

semimaculata $B$. \& $M c D .4036$

seminealis Walker 5201

seminivealis Hulst 2946

seminivella Walker 5198

seminolana Kearfott 7346

seminole Beutenmuller 6702

seminole Neumoegen 6756

seminole Seudder 565

seminole Skinner 258

seminolella Beutenmuller 8270

seminudata Walker 4652

seminobscurella Hulst 5638

semiombra Dyar 6638

semiopaca Grote (Ethmia) 6637

semiopaca Grote (Conochares) 2945

Semioscopis 161

semiovana Zeller $\mathbf{7 1 7 8}$

semipectinata Hulst 3974

semiplaga Walker 3642

semiprsestans Cockerell 6706

semipurpurana Kearfott $\mathbf{7 4 0 9}$ 
semipurpurea Walker 3460

semiradiellus Walker 5305

semiramis Edwards 176

semirelicta Grote 3069

semirubralis Packard 5143

semirufa B. \& McD. 2967

semirufescens Walker 3652

semisigna Walker 2127

semitenebrella Dyar 6632

semiusta Grote 2130

semiustana Walsingham 7325

semnitis Meyrick 6941

senatoria Smith \& Abbot 798

senatoria Smith 1806

senecioides Hy. Edwards 6658

senecionana Walsingham 7311

senescens Z Z eller 7435

senescens Grote 2127 .

senex Walsingham 7653

senicionella Busck 6464

senilis Smith 2639

sennæ Linnaeus 47

sennetti Lintner 965

senrabii Barnes 231

sensilis Grote 2401

Senta

senta Strecker 734

sentana Clemens 7332

sentinaria Geyer 3878

separans Grote 2288

separata Grote 1160

separatana Kearfott 6825

separataria Grote 4557

separatus Neumoegen 684

seposita Hy. Edwards 3338

septemberana Kearfott 7136

septemfluaria Grote 4352

septemlinearia Grote 4352

septempunctata Harvey 1198

septemstrigella Chambers 8319

septentrionalis $B . \& M c D .866$

septentrionalis $H y$. Edwards 1088

septentrionalis Moeschler 1231
Page

septentrionalis Walker (Euxoa)

1305

septentrionalis Walker (Glu-

phisia) 3680

septentrionalis Walker (Ly-

granthœeia) 1124

septentrionana Curtis 6814

septentrionella Busck 6406

septentrionella Fyles 6129

septentrionella Walsingham

8452

septentrionis Walker 1628

Septis 60

septicola Meyrick 7249

sepulchralis Boisduval 4889

sepulchralis Grote \& Robinson

811

sepulchrella Dietz 8302

sequoiæ Boisduval 698

sequoiæ Hy. Edwards 6728

sequoiolus Behrens 8492

sera Grote \& Robinson 2637

serangias Meyrick 6997

serano Smith 1491

seraphica Dyar 3728

serena Edwards 3030

serena Smith 3452

serenus Meyrick 5940

seriata Druce 3627

seriatana Zeller 7464

sericea Morrison 2203

sericearia Packard 3834

sericeata Hulst $\mathbf{4 4 2 0}$

sericeiferata Walker 4624

sericidactylus Murtfeldt 5909

sericinellus /.eller 5364

Sericoptera

120

sericoranum Walsingham 6796

Sericosema 115

serinalis Walker 5009

serinaria Herrich-Schaeffer 4667

serotinzella Chambers 8394

serotinella Ely 8010 
Paso

serotinella Busele (Gelechia) 6274

serotinella Bueck (Marmara) 7983

gerpentana Walsingham 6888

gerpentella Kearfott 5325

serrata $B$. \& MCD. $\mathbf{3 4 0 8}$

eerrata Dnury 4699

serrata Grote 2644

serrata Smilh 1825

serrataris $B$. \& $M C D .4500,2$

serraticornis Lintuer 1985

serraticornis Grote 3517

serratilineella Ragonot 5727

serratipalpella Chambers 6106

serratissimalis Zeller 5005

serrativittella Zeller 6357

serricornis Smith 1299

serrulata Packard 3919

servita Smith 1357

servulella Clemens 8301

Seryda .127

sescuplana Zeller 7377

sesquialteralis Zeller 5046

sesquilines Grote \& Robinson 4755

sesquilinearia Grote 4694

sesquistrialis Hubner 4900

sesquistriaris Hubner 3164

sessile Smith 1294

Setiostoma

181

Setomorpha

132

setosella Clemens 6367

setosella Walsingham 6379

severa $\boldsymbol{H}$. Edwards 3681

sevorsa Grote 2830

sexata Smith 1067

sexatilis Grote 1356

gexfasciata Hy. Edwards 6668

sexguttata Harrig 2404

sexmaculalis Grote 5179

sexmaculata Grote 844

sexmaculata Packard 4345 sexnotella Chambers (Starmatophora) 6001

sexnotella Chambera (Lithocol. letia) 7897

sexplagiata Smilh 1156

sexplagiata Walker 8173

sexpunctata $B$. \& McD. 2765, 1

sexpunctata Bales 4851

sexpunctata Grote 3472

sexpunctella Walsingham $\mathbf{7 5 6 4}$

sexseriata Grote 2821

sexsignata Harvey 3346

sexstrigella Chambers 6180

gexta $B . \&$ M D. 2708

sexta Johanssen 664

shaleriella Chambers $\mathbf{7 7 9 5}$

shalleriella Chambers 6498

shasta Edwards 435

shastre Walsingham 5877

shastaensis Behr 794

shastaensis French 978

shastana Walsingham 7045

shastella Beutenmuller 8048

shawiana Kearfott 7209

sheridani Edwards 391

shurtleffi Packard 4847

Siavana

sibillalis Walker 4979

Sibine 125

sicalis Walker 4913

siccaria Walker 4652

siccata Smith 1237

siculifer Packard 3760

siculodaria Schaus 3852

Sicya 120

Sidemia 63

sideraria Guence 3861

Sideridis

sideroxylonella Busck 7998

sidus Guenee 2213

sierra Wright 215

sierre B. \& MCD. 1639, 1

sierra Beutenmuller 3079

sierre Harvey 1457 


sierræHulst $4165 \quad$ Page

Sigela 74

sigmaria Guenee 4399

sigmoidella Dietz 8216

sigmoides Grote 1597

sigmoidea Beutenmuller 6699

signaria Walker 4604

signata French 2211

signata Walker (Chabuata)

1798

signata Walker (Dasylophia)

3625

signatalis Walker 5162

signatana Clemens 7084

signataria Walker 4599

signifera Meyrick 6784

signifera Walker 2574

significans $\boldsymbol{H y}$. Edwards 929

significata Walker 3656

signosa Walker 2139

silaceata Hubner 3987

silens Grote 1366

silenus Edwards 276

silphiella Grote 7616

silphii Bird 2702

silvestris Edwards 118

silvius Edwards 274

Simathis

Bimæethis Drury 342

simalis Grote 5180

similalis Guenee 5025

similalis Lederer $\mathbf{5 1 4 9}$

similana Clemens 7029

similana Hubner 7028

similana Smith 2478

similaria Walker 4309

similaris Barnes 1794

similaris Hulst 4021

similaris Smith 2004

similiella Chambers 6031

similis Edwards 3115

similis Guenee 3125

similis Strecker 628

similis Stretch 1039 similis Walker 3982

similisana Walker 6870

simius Edwards 552

simplaria Graef 3614

simplaria Morrison 1496

simplex Dyar 4489

simplex Guenee 3230

simplex Smith (Chlorocleptria)

1093

simplex Smith (Oncocnemis) 2032

simplex Stretch 971

simplex Walker (Comacla) 867

simplex Walker (Xylomyges) 1883

simplex Warren 5044

simpliciella Dietz 6513

simplicia Morrison 1496

simplicia Smith 2054

simplicialis $B . \&$ McD. 5230

simplicialis Kearfott 4911

simpliciana Walsingham 7442

simpliciaria Grote 4723

simpliciella Busck 6359

simpliciella Chambers 6332

simpliciella Walsingham 8459

simpliciellus Kearfott 5395

simplicima Ottolengui 3231

simplicior Butler 978

simplicissima Bird 2643

simplicius Stoll 460

simpsoni Busck 7270

simulana Clemens 7255

simulans Grote 6753

simularia Taylor 4463

simulata Hulst 4342

simulatalis Grote 4996

simulatilis Grote 3035

simulatricella Clemens 8446

simulatus Walsingham 8175

simulella B. \& McD. 5757

simulella Dietz (Holococera)

6558 
$\underset{\substack{\text { simulella Diels (Paraneura) } \\ 8212}}{ }$

Simyra 66

sincera Zeller 5282

sincera Herrieh-Schaeffer 1538

sinefaseia Edwards 306

sinefascin Williams 237

sineocellata Skinner 142

singula Grote 2092

singularis B. \& McD. (Neodavisia) 5499

singularis $B$. \& $M C D$. (Oncoenemis) 2042

singularis Lederer 5136

singularis Neumoegen 5498

singulella Walsingham 8455

sinualis Harvey 3816

sinuaria $B . \& M c D .4609,2$

sinuata Peckerd 4372

sinuata Warren 4374

sinuosa Grote 3097

siren Strecker 1118

sirenaria Strecker 4710

siris Edwards 580

sirius Edwards 413

siriusalis Walker 5025

siskiyousna Kearfott 6766

sissona Wright 424

sistrella Busek 6220

sisymbrii Boiaduval 32

Sisyrosea

sitellaria Guenee 3779

Sitotroga 154

siva Edwarde 375

skada Edvards 536

skinnerata Grossbeck 3881

skinnerella Dietz 8303

skinneri Barnes 67

skinneri Biederman 801

skinneri Mengel 295

slingerlandana Kearfott 6782

slingerlandella Kearfott 8384

slocanata Taylor 4235

elossonae Skinner 565 glossonalis D yar 5215

Slossonella

I'D gao

slossonella Hulst 5545

slossoni Hulat 5482

slossoni $\boldsymbol{H}$ y. Edwards 8325

slossoni Packard 3681

Siossonia

slossonia Fernald 7601

slossoniae Hulat (Myctero-

phora) $\mathbf{3 4 4 1}$

slossoniae Hulst (Philagraula)

4787

slossoniae Packard 4835

smaragdina Neumoegen 2272

smeathmanniana Fabricius 7454

smerdis Heuitson 258

Smerinthus

smilaciella Busek 8100

smilacis Boisduval \& Le Conte 377

smilacisella Chambers 7981

smintheus Dorbleday 25

smithi Butler 2438

smithi Druee (Megathymus)

658

smithi Druce (Neumoegenia) 2754

smithi Dyar (Eriopyga) 1861

smithi Dyar (Haploa) 1033

smithi Grossbeck 4739

smithi Guenee 3125

smithi Moeschler 3013

smithi Pearsall 4619

smithi Snellen 1430

smithiana Walsingham 7047

smithsonianus Clemens 4874

Smyrna

snellenella Walsingham 6174

snoviaria Hulst 4645

snoviaria Packard 4300

snoviata Packard 4377

snowi Edwards (Atrytonopsis)

612

snowi Edurards (Heodes) 412 
snowi Hy. Edwards (Auto- ${ }^{\text {Page }}$ grapha) 3229

snowi $\boldsymbol{H} y$. Edwards (Mellitia) 6740

snowi Grote (Apantesis) 990

snowi Grote (Thyreion) 1101

snowi Skinner 3405

snowi Smith 3429

Snowia .122

snowiana Grote 3048

snyderana Kearfott 7034

snyderi Skinner 175

sobria Walker (Cænurgia) $\mathbf{3 1 3 6}$

sobria Walker (Misogada) 3645

sobria Walker (Ptychopoda)

3901

sobria Walker (Calpe) 3398

sobrialis Walker 3532

sobrina Stretch 913

socia Behr 3334

socialis Grote 5142

socialis Feisthamel 781

sociata B. \& McD. 993

sociata Borkhausen 4092

sociella Linnaeus 5449

socius Butler \& Druce 454

socorro Barnes 1380

socors Walker 3131

socus Hubner 469

sodalis Walker 5261

sofia Strecker 146

solamata Hulst 4305

solanalis $B . \& M c D .5195$

solandriana Linnaeus 7030

solanella Boisduval 6169

solaniella Chambers (Phthorimæa) 6171

solaniella Chambers (Paltodora) 6031

solatus Cook \& Watson 383

Solenobia .125

solenobiella Walsingham 8441

solfataria Guenee 4644

solicitana Walker 6972 solida Ottolengui 3258

solidaginifoliella Clemens 7863

solidaginis $B$ ehr 1983

solidaginis Frey \& Boll 7972

solidaginis Hubner 2129

solidaginis Walsingham 6449

solita Walker 1934

solitaria Smith 1362

solitaria Walker 4442

solitariella Dietz 8013

solitudá $H y$. Edwards 6731

Somatolophia .118

sombreana Kearfott 7036

sommeri Lefebre 2186

somniaria Hulst 4657

somnus Dodge 3056

somnus Lintner 518

somnulentella Zeller 8120

sonomana Kearfott 7002

sonora Scudder 580

sonoræ Boisduval 223

sonorensis Felder (Apodemia) 322

sonorensis Felder (Philotes)

447

Sophronia 153

sora Smith 2252

sordescens Meyrick 7267

sordida Grote (Catocala) 3094

sordida Grote (Eucoptocnemis)

1464

sordida Grote (Heliocontia)

2924

sordida Harris 681

sordida Smith (Chorizagrotis) 1379

sordida Smith (Schinia) 1181

sordida Wright 169

sordidalis $B . \& M c D$. (Herculia) 5266

sordidalis $B$. \& $M C D$. (Patissa) 5302

sordidata Fabricius 4007

sordidellus Zincken 5308 
gordidula Grote 3567 sordidus Riley 8467 gorghiella Riley 843 soror B. \& McD. 1208 soror Smith 1373 sororeulella Dyar 7615 sororius Hy. Edwards 792 sosana Kearfoll 6932 sosybius Fabricius 96 sotnia Smith 1267 souhegan Whitney 359 spadaria Guenee 8152 spadicis Grossbeek 4819 spadix Cramer 3294 spadix $H y . E$ Edwards 368 spaldingalis $B$. \& $M c D .5243$ spaldingana Kearfott 6893 spaldingaria Grossbeck 4052 spaldingella Dyar 5792 spaldingi $B$. \& $M C D .1209$ spaldingi Smith 2281 spaldingi Taylor 4182

Spargaloma

Spargania

Sparganothis .175

sparsaria Walker 3906 sparsiciliella Clemens 6483 sparsimacula Hulat 4009 sparsipulvella Chambers 7796 sparsipunctella Walsingham 8262

sparsisquamella Busek 7637 spartinana $B$. \& McD. 7448 spatiosaria Walker 4604 specialis Grote 1238 specifica Dyar 3593 speciosa $H y$. Edwards 446 speciosa Hulet (Lygris) 3985 speciosa Hulat (Meris) 4498 speciosa Hulot (Palwerita) 4626 speciosa Hubner 1505 speciosa Moeschler 982 speciosa Morrison 2367 speciosn Ottolengui 3232 89 107

Pare

speciosn Walker 1029

speciosata Packard 1029

speciosella Hulat 5486

speciosissima Grote \& Robinson $266^{\circ}$

spectanda Strecker 1091

spectanda Smith 1340

spectans Wulker 2440

speculella Clemen 8107

speculifera Sepp 4893

speculigera Meyrick 7074

sperata Grote 2501

sperataris Walker 4615

speratella Busck 6410

sperata Grote 2501

speyeri Lintner 1992

Sphacelodea 124

spheromacaria Harvey $\mathbf{4 6 1}$

Sphecodina 26

sphilophora Meyriek 7003

Sphingidse 23

sphingiformis $B$. \& $M C D .3730$

Sphingoidea 23

Sphinx 84

spiculana Zeller 7064

spiculosa Grote 1753

spilomela Walker 2593

spilosaria Walker 4109

spinataria Packard $\mathbf{4 4 4 6}$

spinea Grote 2512

spinetorum Boisduval 372

spinigera Guenee 2475

spinosa $B$. \& MCD. 1546

spinosse Guenee 1195

spinuloides Herrich-Schaeffer 4824

spirexfoliana Clemens $\mathbf{7 1 8 3}$

spissa Guenee 1304

splendens B. \& McD. (Hemihyalea) 909

splendens B. \& McD. (Lycomorpha) 830

splendida Clemens 765

splendidaria Grossbeck 3795 
splendorata Pearsall 3929

splendoriferella Busck 6104

splendoriferella Clemens 6928

spododea Hulst 4591

Spodolepsis

spodopterata Hulst 4388

spoliana Clemens 7121

spoliata Grossbeck 4472

spoliata Walker 1873

spoliatella Dietz (Holcocera)

6559

spoliatella Dietz (Pigritia) 6588

sponsa Smith 1279

sponsata Grote 3930

sponsella Busck 8075

sporobolella Busck 8147

spraguei Grote (Pygarctia)

1003

spraguei Grote (Schinia) 1192

Spragueia

spreta Smith 1502

spretella Dietz 6566

spumosum Grote 2711

spuraria Christoph 3878

spurcata Walker 2220

sputator Grote 2344

squamealis Grote 5261

squamularis Drury 3191

squamulella Z $Z$ eller 5426

squara Smith 1641

s-signata Packard 4383

stabiliata Walker 4442

stabilis Smith 2086

Stagmatophora

staintonella Chambers (Elachista) 7840

staintonella Chambers (Bucculatrix) 8138

staintonella Clemens 8120

stalachtaria Strecker $\mathbf{4 2 8 6}$

Stamnodes 103

Staphylus

statalis Grote 5285

Statina statira Cramer 51 staudingeri Aurivillius 1627 staudingeri Moeschler 1221

Staudingeria 147

stella Edwards 46

stellaris Grote 1557

stellata Guenee 4085

stellata Hulst 4218

stellata Walker 2758

stellella Busck 6007

stellidaria Guenee 3769

stena Smith 1100

Stenaspilates 122

steneles Linaeus 290

stenialis Guenee 4956

Steniodes 136

stenocelis Dyar 2660

Stenocharis 117

Stenoma 163

Stenomidæ 163

Stenophyes 131

stenopteralis Grote 5174

Stenoptilia 151

Stenoptycha 137

stenotis Hampson 1747

Stenotrachelys 116

stephensi Skinner 659

stephensi Wright 112

stercoralis Moeschler 5229

stercoreana Z Zeller 7054

Stergamatæa 118

sterope $E d w a r d s 221$

stewarti Grote 2516

sthenele Boisduval 117

stheno Hubner 718

Sthenopis 197

Stibadium 70

sticticalis Linnaeus 5031

stictigramma Hampson 4968

stictophorella Ragonot 5582

stigma Fabricius 797

stigma Smith 2112

stigma Skinner 588

stigmata Grote 2270 
stigmata Smith 1608

stigmatalis Smith 1289

stigmaticus Mabille 507

stigmella Dyar (Acrobasis) 5.52:0

stigmella Dyar (Zophodia) 5716

stigmosa Morrison 1409

Stilbia

Stilbosis

stilla Grote 2655

stimulea Clemens 4812

stipata .Morriam 2368

stipatalis Walker 4899

stipularia $B$. \& McD. 4368

Stiria

Stiriodes

stolata Smith 1962

stollaria Guenee 3778

Storteria

stragula Grote 3606

stramentosa Guenee 2652

straminalis Hubner 5004

straminea Smith 2222

straminea Warren 5064, 1

stramineana Walsingham 7076

stramineella Chambers 6378

gtramineus Walsingham 5896

straminiella Chambers 8252

straminoides Grote 7449

straton Edu'ards 567

strattonata Packard 4173

streckeri Ellsworth 203

streckeri Grum-Grshimailo 69

streckeri Skinner (Euphyes) 608

streckeri Skinner (Megathymus) 657

strens Grote 2390

Strenoloma

strenuana Walker 6981

Stretchia

stretchi Behr (Catocala) $\mathbf{3 0 7 9}$

stretchi Behr (Syneda) 3341 stretchi Buller (Leptaretia)

941

stretchi Butler (Uthetheisa)

1030

stretchi Edwards 108

stretchi Hy. Edwards 1726

stretchi Grote 972

striana Fernald 7363

striata Druce 2321

striata Edwarde 415

striata Ottolengui 886

striata Walsingham 7320

striatalis Dyar 5210

striatana Clemene 7065

striatella Busck (Cerostoma)

7668

striatella Busek (Gelechia) 6237

striatella Grote 3284

striatella Hubner 6022

striatella Murtfeldt 6172

striatellus Fernald 5413

striatellum Dyar 5768

stricta Walker 1751

strictalis Walker 4955

strictella Walker 8036

strigalis $B . \& M C D .5697$

strigalis Dyar 5241

strigalis Smith 3386

strigata Boisduval 4816

strigata Packard 4041

strigata Schaves 2007

strigata Smith (Calophasia) 2012

strigata Smith (Gabara) 3422

strigata Smith (Rancora) 1982

strigataria Grossbeck 3787

strigataria Minot 4622

strigicollis Wallengren 1758

strigifinitella Clemens 7997

strigilis Grote 1273

strigilis Linnaeus 708

strigosa Bates 85

strigosa Brauท 7996

strigosa Fabricius 980 
strigosa Grote 3592

strigosa Harris 367

strigosa Stretch 3751

strigosa Walker 1022

strigularia Minot 3920

strigularia Walker 4385

strigulata Smith 2458

strigulataria Smith 3166

striolata Zeller 7822

Strobisia 160

strobivorella Dietz 8028

Strymon 13

stygialis $B . \&$ McD. 1290

stygialis Grote 3307

stygialis Smith 3418

stygiana Dyar 7090

stygiarius Walker 4744

stygna Walsingham 6551

stylata Smith 2078

stylobata Harvey 3167

stypticellum Grote 5772

styracis Guenee 1914

styx $B . \&$ McD. 3721

suædæ Busck 7815

suædicola Cockerell 7797

suasa Boisduval 395 .

suavata Hulst 3850

suavis Barnes 3622

suavis $B . \& M c D .1970$

suavis $H y$. Edwards 2656

subacuta Hulst 4360

subænescens Walker 5420

subrequalis Walker 4959

subsequana Zeller 7175

subæquaria Walker 4457

subserea $H y$. Edwards 6718

subalbaria Hulst 4429

subalbaria Packard 4428

subalbicans Grote 3641

subalbusella Chambers 6343

subalpina French 913

subapicalis Smith 1891

subapicana Walker 2924

subapicaria Walker 4345 subapicata Guenee 4224

subaquila Harvey 2593

subatomaria Wood 4492

subatra Smith 1068

subauratana Walker 7294

subaureola Frey \& Boll 7887

subaustralis Hulst 3769

subcærulea Walsingham 6630

subcresiella Clemens 5631

subcanalis Walker 5486

subcarnea Kellicott 2791

subcedens Walker 2308

subcervinana Walsingham 6763

subcessaria Walker 4406

subcinctaria Walker 4389

subcineraria Grossbeck 4757

subcitrina Walker 4358

subcitrinalis Hulst 2963

subcolorata Hulst 4201

subcostalis Hampson 4984

subcostella Ragonot $\mathbf{5 6 7 2}$

subcroceata Walker 3815

subdentalis Grote 5005

subdentosa Dyar 4820

subdita Moeschler 1645

subdivisalis Grote 4921

subdolens Walker 1866

subfalcata Hulst 4402

subfascianus Stephens 7389

subfasciella Walsingham 7640

subfervens Walker 7733

subflava Grote 2792

subflavana Walsingham 6906

subflavaria Pearsall $\mathbf{4 7 5 6}$

subflavidalis Grote 3451

subflavidens Grote 1213

subflexa Guenee 1091

subfoveata Dyar 4354

subfuscata Taylor 3863

subfuscella Ragonot 5639

subfuscula Grote 1652

subgenericata Dyar 4600

subgothica Haworth 1403

subhastata Nolcken 4088 
subhyalina Curtis 183

subinvicta Kearfott 7019

subiridescens Walsingham 5990

subjecta Walker 885

subjectalis Lederer (Terastia) 4989

subjectalis Lederer (Pyrausta)

5125

subjugata $D$ yar 1487

subjuneta Smith 2342

subjuncta Grote \& Robinson 1686

subjunctella Walker 8241

sublacteolata Hulst 4353

sublapidana Walsingham 7086

sublataria Guenee 3835

sublatis Grote 1351

sublepidana Kearfott 7516

sublimaria Guenee 4644

sublineata Taylor $\mathbf{4 1 4 7}$

sublucella Walsingham 7642

sublunaria Grenee 4567

subluteella Ragonot 5844

submarins Grote 1626

submarmorata Walker 4345

submedialis Grote 5121

submedianella Dyar 5581

subminiata Packard (Phasiane) 4376

subminiata Packard (Phasiane) 4377

submissa Smith 2517

submuraria Walker 4601

subnata Grote 3047

subnebulosa Dyar 944

subnicalis Warren 5167

subnisans Zeller 7084

subnivana Walker 7443

subnivosella Walker 3419

subnotata Walker 1866

subochraceus Walsingham 5913

subochrea Grote 2448

subochrearia Grote 4469

subochrearia Hulst 4758 subochreata Hulat 3907

subochreata Packard 4005

subolivacen Harvey 3462

subolivalis Packard 5140

subornata Neumoegen \& Dyar 900

subpallida B. \& McD. 313

subpallida Cockerell 280

subpartita Guenee 2592

subplicann Walsingham 6955

subporphyren Walker 1451

subprivata Walker 4667

subpunctata Harvey 1948

subpunctata Hulot $\mathbf{4 7 2 0}$

subreticulata Walsingham 7700

subrotata Harvey 3637

subruberella Chamber 6197

subrufa B. \& McD. 880

subrufalis Grote 3578

subrufella Hulst 5558

subsciurella Ragonot 5748

subsenella Zeller 6568

subsequalis Guenee 5152

subsignarius Hubner 4664

subsignata Walker 1329

subsimella Clemens 6163

subsimplex $D$ yar 2540

subsinuaria Guenee 4678

substriataria Hulat $\mathbf{4 4 9 3}$

substrigata Smith 1449

subsylvella Walsingham 7641

subtermina Smith 3303

subterminalis $B$. $M c D$.

(Renia) 3534

subterminalis $B$. \& $M c D$. (Evergestis) 4994

subterminata $B$. \& McD. 4370

subterminata Smith 1917

subterranea Busck 6132

subtetricella Ragonot 5504

subtilis $B$. \& MCD. 2546

subtinctella Ragonot 5607

subtractella Walker 6350

subusta Druce 2611 
subusta Hubner 2729

subversana Zeller 6981

subvexa Grote 2726

subvirens Dietz 4269

subviridis Harvey 3038

succandidalis Hulst 5044

succedana Schiffermuller 7088

successaria Walker 3912

succosata Z eller 4346

sudena Smith 3216

sueta Grote 1073

Sufetula 129

suffusa $B . \& M c D$. (Crambidia) 863

suffusa B. \& McD. (Namangana) 2619

suffusa Beutenmuller 3120

suffusa Cockerell 793

suffusa Laurent 591

suffusa Schiffermuller 1422

suffusa Smith (Haploa) 1032

suffusa Smith (Polia) 1657

suffusa Strecker $\mathbf{9 5 9}$

suffusa Stretch 26

suffusa Walsingham 8087

suffusa Wolcott 159

suffusalis Smith 3489

suffusana Zeller 6957

suffusana Strand 2835

suffusca Morrison 2269

suffusella Chambers (Gelechia) 6344

suffusella Chambers (Anarsia) 6354

sulfurea Grote 4821

sulfureana Clemens 7316

sulmula Strecker 1143

sulphuraria Packard (Itame) 4400

sulphuraria Packard (Hesperumia) 4398

sulphuraria Packard (Sabulodes) 4763
Page

sulphurea Frey \& Boll 7853

sulphurea Neumoegen 2760

sulphurea Packard 4400

sulphureodactylus Packard5915

sulphurica Neumoegen 985

sumptuosalis Walker 5148

superans Guenee 2447

superaria Guenee 4758

superata Walker 3813

superatalis Clemens 5467

superba Barnes 3691

superba Hy Edwards (Annaphila) 2856

superba Hy. Edwards (Heterocampa) 3637

superba Streeker 203

superba Stretch 987

superbella Chambers 6049

superbifrontella Clemens 8054

suppressaria Walker 3901

suppuraria Swett 4042

suprema Neumoegen 803

Surattha 139

surena Grote 3246

surrectalis Walker 3496

suspectata Moeschler 3992

suspiciosața Dietz 4268

susquesa Smith 2306

sutor Grenee 2330

sutor Hampson 2935

sutrina Grote 1713

sutrix Grote 2936

swainsonia Swainson 52

Swammerdamia 184

swetti Grossbeck 4260

Sylectra 87

Sylepta 130

sylla Perty 300

sylvæcolella Chambers 6210

sylvanoides Boisduval 572

sylvarum Guenee 3158

sylvatica Harris 3752 
gylvaticoides Neumoegen \& Ivar

3752

sylvia Dyur 2101

slyvia Hy. Edururds 3081

sylvicolella Buack 6162

sylviella Ely 5ร.ร5

sylvinus Buiducul 362

Symmerista 93

Symmoca

symphoricarpana hearfolt $7: 385$

symphoricarpella Chambers 7922

symphoricarpella Frey \& Boll 7922

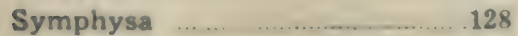

Sympistis ...... _ _ _..... 54

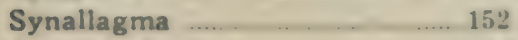

Synanthedon ... $\quad$........ 164

Synaxis ….... 12:

Synchlora ......................... 100

Synclera ……................. 129

Syneda ………................ 85

Synglochis ........__ 117

Syngrapha …....................... 83

Synnoma ……...................... 175

synochitis Grote \& Rubinson 2894

Synomila

synthetica Riley 8474

Syntomeida 29

Syntomida 29

syracosia Druce 928

syrichtus Fabricius 493

syringre Harris 6726

syringicola Packard 5102

syrissa Strecker 1799

syrtodes Meyrick 7005

Syseaura .123

Systasea

syzygiaria Hulst 4754

tabacella Ragonot 6169

tabulata Hulst 3956

tabulata Grote 1893
Page 14

Tacoma

tacoma Bentenmuller 6700

tacoma Sirecker 1709

tacomae $H_{y}$. Eiducarda 8492

tacturata Wulker 3898

tactus (jote 3479

tredata Frote 1548

tredialis Wulker 4985

taeniolalis Guenee 5146

Treniosea 63.3

tages Cramer 4922

tahoensis $B$. \& $M c D$. 4101, 1

takenaria Peargall 4584

takuata Tuylor 4062

taleana Grote 7071

taleolalis $\mathrm{Hulst} 5488$

talidiformis Guenee 2595

talis Grote 4936

Tallula

tamenund Eduards 505

Tampa 150)

tana Kenrfott 7248

tanaceti Hy. Edu'ards 6744

tandana Kearfott 7032

tanena Streeker 1184

tantalus Linnaeus 729

tantillus Grote 3481

tantiva Smith 1885

tapeta Smith 2618

tapetiella Linnaeus 8244

taracana Kearfott 7318

Tarache .................................. .77

Tarachidia .............................

tarandana Moeschler 7093

tarda Guenee 2581

tardigrada Clemens 4816

tarmitalis Hulst $\mathbf{5 6 4 9}$

tarquinius Curtis 195

tarquinius Fabricius 396

tartarea Smith 2468

tartarella Dietz 6571 
tartarellus Zeller 5657

tatalis Grote $\mathbf{5 1 5 0}$

tatila Herrich-Schaeffer 293

tatius Edwards 532

tau Scudder 26

taumas Fabricius 576

taura Smith 1293

taxifoliella Busck 6775

taxiles Edwards 594

taygete Hubner 129

taylorata Hulst $\mathbf{4 4 4 7}$

taylorata Swett 4263

taylorella Kearfott 8429

taylori Butler 4636

taylori Edwards 213

taylori Swett 4029

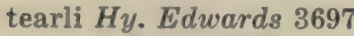

tecomæ Riley 5283

tecta Grote 2889

tecta $H y$. Edwards 6697

tecta Hubner 1482

tecta Walker 1839

tectalis $B . \& M c D .5187$

tecumseh Grinnell 584

tedyuscongalis Clemens 5201

Tegeticula

tehama Reakirt 429

tejonica Behr 3343

tela Smith 2614

telamonides Felder 20

Telea

telea Hewitson 338

teleboa Smith 1326

Telegonus

telifer Grote 3654

telifera Harris 1422

telifera Meyrick 7486

teligera Morrison 1734

telligii Barnes 4838

Telphusa

teltowa Smith 1534

telum Guenee 2461

tema Strecker 3695 temecula Barnes 2596

temerana Busck 7504

temnaria Guenee 3835

Tenaga 193

tenalis Smith 3452

tenebrella Dietz 6592

tenebrescens Hulst $\mathbf{4 1 7 7}$

tenebricosa Moeschler 2200

tenebrifera Walker 1553

tenebrosa Dyar 4849

tenebrosata Hulst 4395

tenebrosella Hulst 5625

tenella Hy. Edwards 3310

tener Druce 4857

tenera Hubner 905

tenera Smith 2361

teneralis Lederer 5060

tenisca Smith 1751

tensaria Walker $\mathbf{4 5 1 0}$

tentoriferella Clemens 6424

tenuata Hulst 4275

tenuella $B . \& M c D .5545$

tenuescens Grote 1145

tenuescens Smith (Lycophotia) 1497

tenuescens Smith (Tarachidia) 2960

tenuiana Walsingham 7066

tenuicola Morrison 1431

tenuicula Morrison 2954

tenuidactylus Fitch 5860

tenuifascia Harvey 881

tenuifascia Smith 2025

tenuilinea Smith 1536

tenuimacula $B . \& M c D, 1911$

tenuimargo $B . \& M c D .1081$

tenuimargo Holland 829

tenuipunctellum Ragonot 5776

tenuis Grote (Hæmorrhagia) 735

tenuis Grote (Isogona) 3427

tenuis Walsingham Haploptilia) 7798 
tenuis Waloingham (Aerolophus) 8186

tenuistrigata Frey \& Boll 7892 tephrella Ragonot 5684 tephriasella Chambers 6294 tephrinana Zeller 6972 tephrosiella Dyor 5555 tepida Grote 2160 teplia Smith 1242 tepperaria Hulat 9827 tepperi $H y$. Edwards 6684 tepperi Morrison 2765 tepperi Smith 1499

Terastia teratophora Herrich-Schneffer 2424

terentius Seudder \& Burgess 529

terrissa Lucas 58

terlineata Dyar $\mathbf{1 5 4 5}$

terlooti Behr 27

termessa Smith 1294

termina Grote 3377

terminalis Neumoegen \& Dyar 1029

terminalis Smith (Oncocnemis) 2027

terminalis Smith (Yrias) 3387

terminalis Walker 875

terminana Busek 7546

terminata Smith 1897

terminata Taylor 4199

terminatissima Dyar 1545

terminella Braun 8418

terminella Grote 2566

terminella Westwood 5996

terminimaculata Grote 2989

terminimaculata Kearfott 6292

terminipunctata $B$. \& $M c D$. 4021, 1

ternaria Smith 1310

ternariella Zeller 6210

terracoctana Walsingham 6965 terracottella Busck 6118

terranea Butler 476

terraria Guenes 8769

terrealis Grote 1378

terrealis Treitschke 5099

terrebralis B. \& McD. 3492

terrellus Zeller 5372

terrena Smith 1308

terrenella Zeller 5450

terrifica Smith 1483

territan $H y . E$ dwards 2750

territorialis Smith 1304

tersa Linnaeus 760

tertis Dyar 1921

tertia Grote 1173

tertialis Guenee 5102

tertialis Smith 1865

tertiella Dyar 5495

tesella Packard 3674

tesquella Clemens 5984

tesselaris Smith \& Abbot 921

tessellana Packard 6813

tessellata Harris 1329

tessellata Packard 3940

tessellata Scudder 495

tesselloides Grote 1329

tesseradactyla Linnaeus 5870

tessulata Packard 4067

testa B. \& McD. 2871

testacea Packard 4854

testata Linnaeus 3979

testula Smith 1292

testulana Zeller 7297

Tetanolita 91

tetera Smith 1960

teterrellus Zincken 5372

tethys Fabricius 302

tetra Behr 368

tetra Walker 1371

Tetracis

Tetraclonia 127

tetradactylus Walsh 4832

tetradella Zeller 5826 
tetradymiella Busck 6096
tetragona Walker 2976

Tetralopha

tetraspilaris Walker 4839

tetrella Braun 8144

tetrica Hy. Edwards 3319

tetrica Smith 1300

tetrio Linnaeus 712

tetrops Dyar 2072

teucaria Strecker 4360

teuthras Walker $\mathbf{8 1 5}$

texana B. \& McD. (Aleptina) 2557

texana B. \& McD. (Megathymus) 657

texana Edwards (Anthanassa) 258

texana Edwards (Cercyonis) 111

texana $H y$. Edwards 6701

texana French 867

texana Grote 821

texana Hampson 3154

texana Hulst 3774

texana Morrison (Borolia) 1953

texana Morrison (Pelamia) 3138

texana Riley 3409

texana Robinson 5417

texana Rothschild 918

texana Scudder $\mathbf{4 5 5}$

texana Skinner 313

texana Smith (Isogona) 3428

texana Smith (Namangana) 2623

texana Stretch 4881

texanæ French 3072

texanana Walsingham 7268

texanaria $\mathrm{Hulst} 4603$

texanella Busck 7999

texanella Chambers (Elachista) 7841

texanella Chambers (Ethmia) 6644 texanella Chambers (Gelechia) 6233

142 texanella Chambers (Haploptilia) $\mathbf{7 7 9 9}$

texanella Hulst (Hulstia) 5744

texanella Hulst (Tacoma) 5579

texanella Ragonot 5484

texanella Walsingham 6568

texanella Zeller 7917

texanellum Ragonot $\mathbf{5 7 7 5}$

texanellus Chambers 8172

texanica Frey \& Boll 7842

texanus Ehrman 18

textor Hubner 634

textor Harris 958

textrinaria Grote \& Robinson 4726

textula Herrich-Schaeffer 4828

thalialis Walker 5182

thaliastis Meyrick 6822

thalictri Lyman 2695

thallophilalis $\mathbf{H u l s t} 5034$

Thanaos

thanataria Swett 4042

thanatologia Dyar 1379

tharos Drury 247

Tharsalea 15

Thauma 27

Thaumatopsis 140

thaxteri Grotè 2168

thaxteriana Grote 2106

thecata Morrison 1851

Theclinæ 13

Theisoa 152

thekla Edwards 238

Thelethia 181

theobromæ D yar $\mathbf{5 4 5 5}$

theodora Schaus 2426

theodori Dyar 8328

theodori Grote 2525

theona Menetries 238

theonus Lucas 415

Thera

105 
theralis

theralis Walker 3500

thermopsella Chamber 8004

Theroa

thesealis Zeller 5111

theseualis Wrilker 5116

Thespieus

22

thestealis Wralker 5114

thetis Boisduval 735

thiodamus Scudder 273

thoas L.tmnurue 10

thoasalis Wulker 4940

thoe Berisducal 404

Tholera

Tholeria

thona Strecker 1027

thoosa Seudder 46

thor $\boldsymbol{H y}$. Edwards 22

thoracealbella Chambers (Gelechia) 6259

thoracealbella Chembers (Nepticula) 8395

thoracefasciella Chambers 6451

thoracella Busck 7686

thoracella Walsingham 6161

thoracenigraeella Chambers 6435

thoraceochrella Chambers 6346

thoracestrigella Chambers

(Gelechia) 6345

thoracestrigella Chambers

(Tinea) 8265

thoracica Grote 2459

thoracica $\boldsymbol{H}$ y. Edwards 2823

thoracica Putnam-Cramer 2174

thoracica Stretch 3752

thoracicoides Neumoegen \&

$$
\text { Dyar } 3752
$$

thorates Hubner 757

thoreaui Grote \& Robinson 1127

Thorybes

thraso Hubner 505

thraxalis Walker 3524

thuiella Packard 7713

thujaella Kearfott 6141 thula Sirveleer 2118

Prasen

thule Sirecker 8484

thulea Dulman 943

thurberiella Busck 8151

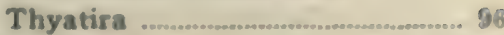

Thyatirida

thyatiroides Guenee 3280

thyatiroides Walker 3626

thycesalis Walker 5022

thymetusalis Walker 5264

Thyreion ......n........................ 88

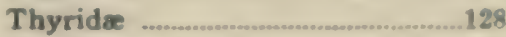

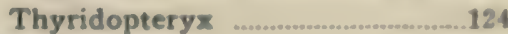

Thyridopyralis ........................... 128

Thyris _.n....... 128

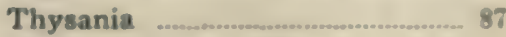

thysbe Fabricius 732

tibialis Harris 6654

tibullus Scudder \& Burgess 526

tigrinaria Guenee $\mathbf{4 7 1 0}$

tigris Guenee 2916

tigris $D$ yar $\mathbf{3 7 4 7}$

tiliæfoliella Clemens 7800

tiliaria Harris $\mathbf{4 6 2 8}$

tiliacella Chambers 7921

tiliella Braun 8407

tillialis Dyar 5093

tilosalis Smith 3523

tiltella Hulst 5493

timais Cramer 1931

Timandra 103

timandra $H y$. Edwards 3117

timandrata Walker 3868

Timetes

timidella Clemens 7280

Timochares

Timora

Tinagma 186

tinctana Walker 7324

tinctaria Walker 4290

tinctoriella Chambers 7861

Tinea 193

tineana Hubver 7203 


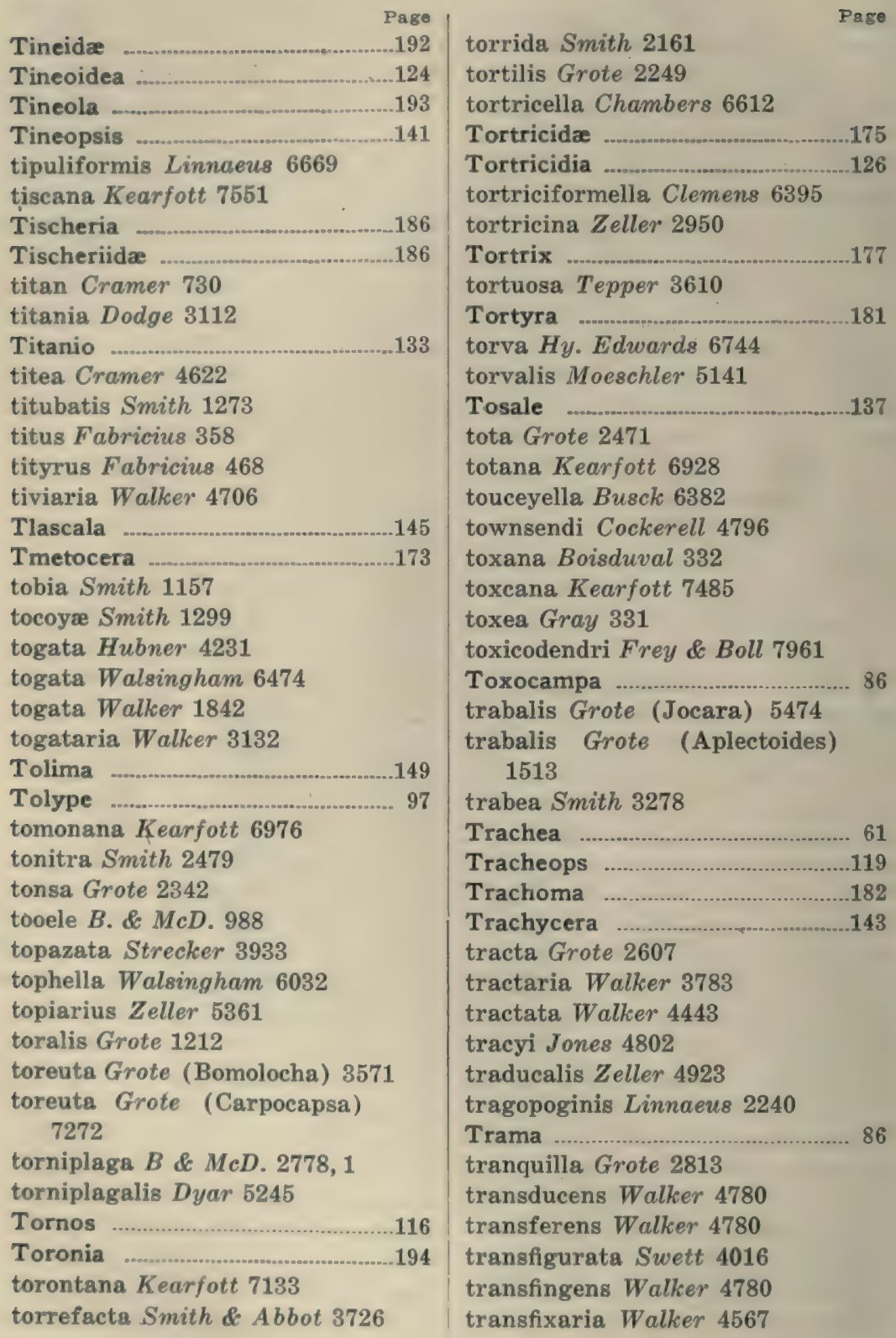


transfrons Neumoegen 2334 transitaria Walker 43.43 transitella Walken 5501 transiturana Wulker 7345 translucida Walker 912 transmissana Walker 6974 transmontana Neumoegen \& l)yar 1025

transmutans Wulker 4777 transmutata Walker 2931 transparens Grote 1903 transposita Walker 4777 transversa Walsingham 7103 transversana Walsingham 7470 transversata Braun 8146 transversata Drury 4777 transversata Smith 2484 transversata Walker 3682 transversestrigella Dietz 8215 transvertens Walker 4777 trapezalis Guenee 4927 trapezitis Meyrick 7032 traversata Kellicott 3992 treati Grote (Eubaphe) 931 treati Grote (Agrotis) 1426 tremula Harvey 2204 tremularia B.\& Me.D. 4500, 1 tremularia Walker 3840 tremuloidella Braun 7933 trentonalis Lederer 5268 trepidella Clemens 8017 trialbamaculella Chambers 6208 triangulalis $B$. \& $\mathbf{M e D} .4995$ triangulalis Moeschler $\mathbf{5 1 9 4}$ triangulana Kearfott (Eucosma) 7104

triangulana Kearfott (Tortrix) 7371

triangularia $B$. \& $M C D .4479,1$ triangularis Prout 3826

triangularis Smith 1034 triangularisella Chamberg 6543 triangulata $B$. \& $M C D .4765,1$ triangulata Packard 3981

triangulella Busck 6861

triangulifer Grote 2736

trianguliferate Packard 4679

tribunella Sohiffermuller $\mathbf{5 4 4 9}$

trichella Busck $\mathbf{7 8 2 3}$

Trichoclea . 51

Trichocosmia 71

Trichodesia ...esos..... 103

Trichophaga ......... 193

Trichoplexia ...s.............. 63

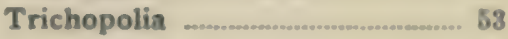

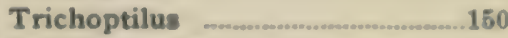

Trichorthosia ........ 47

Trichostibas 184

trichostomus Christoph 5381

Trichotaphe 159

Trichotarache _..._. 77

trichusalis Hulst 5351

tricincta Harris 6747

tricingulatella Clemens 8316

triclaris Hubner 189

Triclonella .161

tricolor Barnes 1207

tricolor Fitch 900

tricolor Packard 791

tricolor Smith 2915

tricolor Stretch 997

tricoloralis Dyar 5248

tricoloraria Packard 3799

tricolorella Grote 5548

tricosa Lintner 1403

tricristatella Chamber 5990

trientiplaga Walker 2605

trifariana Walker 2931

trifascia Smith 1829

trifascia Hubner 1152

trifascise Braun 7685

trifasciata Smith 1344

trifasciata Packard 4372

trifasciatella Chambers (Gelechia) 6218 
trifasciella Chambers (Adela) ${ }^{\text {Page }}$ 8450

trifasciella Clemens 8139

triferana Walker 7390

trifolii Rottemburg 1615

trifurcella Chamber 86635

trifurculana Zeller 7364

trigeminana Stephens 6999

trigona Grote 904

trigona Hy. Edwards 4838

trigona Smith (Rhynchagrotis) 1586

trigona Smith (Luperina) 2375 trigonana Stephens 7423

trigonana Walsingham 7634

trigrapha Zeller 8451

triguttaria Herrich-Schaeffer 3961

trilinearia Grossbeck 4414

trilinearia Packard (Platæa) 4478

trilinearia Packard (Metanema) 4726

trilineata Packard 3680

trilineata Warren 4053

trilineella Chambers (Gelechia) 6241

trilineella Chambers 7801

trimaculalis Grote 4986

trimaculata Boisduval 1049

trimaculata Smith 952

trimaculella Chambers (Trichotaphe) 6377

trimaculella Chambers (Tinea) 8254

trimaculella Chambers (Dichomeris) 6382

trimaculella Fitch 6414

trimaculella Packard 6211

trimaculosa Reakirt 938

trinotata Braun 8413

trinotata Walker 844

trinotella Busck 6365 trinotella Braun 7882

trinotella Coquillett 6386

triocellella Chambers 6110

Triocnemis 73

triolata Smith 1070

triorthia Dyar 2685

tripars Walker 1466

tripartita Smith 2934

tripartita Walker 3626

tripartitana Zeller 7025

tripartitus Grote 705

Triphosa 104

tripla Grote 3465

triplagiatus Smith 2768

triplagiella Dyar 5653

triplex Walker 2593

triplipunctaria Fitch 4492

Triprocris 127

tripuneta $B . \& M c D .2747$

tripunctaria Packard 4451

tripunctata Fabricius 4938

tripunctella Walsingham (Heliodines) 7565

tripunctella Walsingham (Incurvaria) 8430

tripunctellus Robinson 5310

triquetra Grote 2576

triquetrana Fitch 844

trisectus Walker 5392

triseriata Packard (Scelidacantha) 3949

triseriata Packard (Hydrelia) 4120

trisignana Robinson 7422

trisignata Walker 2404

tristana Hubner 7423

tristatá Linnaeus 4089

tristella Dietz 6586

tristicta Hampson 2733

tristicula Morrison 1368

tristigma Treitschke 1429

tristigmalis Hampson 4941

tristigmata Grote 2212 
tristis B. \& MCD. (Abagrotis) 1564

tristis B. \& McD. (Polia) 1683

tristis Boisduval 534

tristis Edwarde 3095

tristis Menetries 723

tristis Smich 2443

tristriata Kearfolt 7322

tristrigana Clemens 7220

tristrigella Walsingham 6200

tristrigella Walker 2601

Tristyla

tritenianella Chambere 7924

tritealis Walker 5061

tritona Hubner 2465

tritonia Edwards 147

trituberalis Zeller $\mathbf{3 5 7 4}$

triumphalis Grote 5080

trivialis $B . \& M c D .5074$

trivinctella Zeller $\mathbf{8 0 8 8}$

trivittana Zeller 7065

troilus Linnaeus 18

tronellus Smith 1258

Tropara

trophella Busck 6214

Trosia

trossulana Walaingham 7237

trouveloti Packard 3634

trumani Smith 1440

truncata Hufnagel 3992

truncataria Guenee 4644

truncataria Walker 4287

truncatella Wright 5692

truncatellus Zetterstedt 5380

truncatula Zeller 2923

truva Smith 1301

truxaliata Guence 4770

tryonana Kearfott 6880

Trypanisma

156

Tryptodema 194

tryxus Cramer 506

tuana Smith 1725

tuberculalis Lederer 5008 tuberculum Hubner 1119

tubiferella Clemens 7968

tufa Smith 1663

tulcis Bates 259

tumida Grote 2009

tumidicosta Hampson 3013

tumidulella Ragonot 5724

tunicana Walsingham 7313

turbans Christoph 980

turbata Hubner 4055

turbata Walker 1086

turbatella Grote 5705

turbatellus Walker 5365

turbida Walker (Heterocampa) 3644

turbida Walker (Fentonia) 3674

turbulenta Hubner 2283

turfosana Herrich-Schaeffer 6863

turmalis Grote 5093

turnus Linnaeus 13

turpidella Ragonot 5622

turpis Smith 2476

turris Grote 1868

Turuptiana

tusa Grote 2284

tuscanella Dietz 8263

tybo Barnes 2421

tyndarus Esper 145

typhon Klug 739

typhonalis B. \& McD. 5026

typica Smith 1601

typicodes Meyrick 6961

tyralis Guenee 5158

tyria Sloseon 3664

Tyrissa

u-album Guenee 2397

u-aureum Guenee 3238

u-brevis Guenee 3264

Ufeus 47

uhlerella Fiteh 7916

uhleri Reakirt 124 


Uinta ..................................
uintara Smith 2193
ulæ Cockerell 5399
ulalume Strecker (Proserpinus)
$\quad 753$
ulalume Strecker (Catocala)
$\quad 3044$
ulamora Smith 1801
uliginosa Smith 1767
ulmella Braun 8405
ulmella Chambers 7946
ulmella Ragonot 5734
ulmi Harris (Acronycta) 2473
ulmi Harris (Heterocampa)
$\quad 3644$ ulmiarrosorella Clemens 5734
ulodactyla Zetterstedt 5863

Ulophora .143

Ulosyneda 85

ulrica Edwards 229

ulsterata Pearsall 4332

ultima Strecker 1128

ultraria Pearsall 4540

ultrix Meyrick 7119

ultronia Hubner 3103

ululata Pearsall 3926

umatillaria Strecker 4602

umbellulariæ Walsingham 7943

umbilicata Guenee 3871

umbonata Smith 3423

umbra Hufnagel 2655

umbrabasana Kearfott 7536

umbralis Smith 3573

umbrastriana Kearfott $\mathbf{7 0 5 0}$

umbrata Packard 892

umbrata Walker 3640

umbratica Braun 7818

umbraticostella Walsingham

6434

umbratus Packard 1484

umbrella Dyar 5686

umbrifacta Hampson 2281

umbrifascia Grote 3473 umbrifascia Smith 2060

umbrifasciata Hulst 4417

umbriferata $\mathrm{Hulst} 4417$

umbrimarginella Busck 6619

umbrina Grote 3375

umbripennis Grote 3181

umbripennis Hulst (Somatolophia) 4597

umbripennis Hulst (Tlascala) 5609

umbrosa Dyar 1437

umbrosa Grote 1090

umbrosa Lintner 268

umbrosa Smith 1676

umbrosa Worthington 3051

umbrosaria Packard 4485

umbrosiarium Hubner $\mathbf{4 5 6 0}$

una Skinner 695

una Strecker 2335

Unadilla 148

uncana Hubner 7196

uncanale $\mathrm{Hulst} 5765$

uncanaria Guenee 4477

uncas Edwards 553

unctulella Zeller 6254

undalis Fabricius 4982

undatus Grote 5390

undifera Walker 4837

undinaria Strecker 3807

undosus Lintner 8353

undularis Drury 3181

undularia $B . \& M c D .4308$

undulata Linnaeus 3971

undulatella Busck 7658

undulatella Chambers 7711

undulatella Clemens 5743

undulifera Walker 2901

undulosa Walker 675

unica Neumoegen 3395

unicalcararia Guenee 4327

unicincta Smith 2360

unicipunctella Busck 7661

unicipunctella Clemens 6389 
unicolor B. \& MCD. (Pterophorus) 5917

unicolor B. ACD. (Storteria) 5307

unicolor Grote 1524

unicolor G. \& S. 53

unicolor Hulat 4163

unicolor Packard $\mathbf{3 6 4 5}$

unicolor Robinson $\mathbf{4 1 2 5}$

unicolor Walker 1461

unicoloralis B. \& MCD. (Maroa) 5070

unicoloralis B. A MCD. (Heliothela) 5190

unicoloralis B. \& MCD. (Macrotheca) $\mathbf{5 4 5 7}$

unicoloraria Hulst (Ixala) 4501

unicoloraria Hulst (Hesper- umia) 4398

unicolorella Chambers 7802

unicolorella Hulat 5615

unicolorella Walsingham 5961

unicornis Smith d Abbot 3657

unicristatella Chambers 6012

unidentaria Haworth 4039

unifascia Grote \& Robinson 882

unifascialis Packard 5140

unifasciana Clemens 7307

unifasciata Walsingham 7630

unifasciella Busck 6221

unifasciella Chambers (Lithocolletis) 7959

unifasciella Chambers (Mompha) 6018

unifasciella Chamber (Elachista) 7843

unifasciella Chambers (Neptricula) 8396

uniformis Cockerell $\mathbf{7 9 4}$

uniformis Dyar 858

uniformis Grote \& Robinson 732

uniformis Morrison $\mathbf{3 1 7 7}$ uniformis Smith (Eriopyga) 1857

uniformis Smith (Lasiestra) 1649

unijuga Walker 3068

unilinearin Taylor 3794

unilineata Grote 3176

unimacula Grote \& Robinson 5171

unimacula Morrieon 1458

unimacula Smith 1179

unimoda Lintner 2150

unimoda Smith 2690

unimodaria Morrison 4347

unio Hubner 2829

unipecta Pearsall 4115

unipuncta Dyar 5458

unipuncta Haworth (Paraphia) 4492

unipuncta Haworth (Cirphis) 1950

unipunctella Clemeno 6612

unipunctella Walsingham 756 3

unipunctellus Robinson 5309

unisignata Walker 2604

unistriatellus Packard 5343

unistriganus Dyar 8178

unistrigata Gumppenberg 3785

unistrigella Chambers 6347

unita Smith 2280

unitaria Packard 3803

unitaria Herrich-Schaeffer 4496

unna Ploetz 587

unomaculella Chambers (Perimede) 5987

unomaculella Chambers (Tinea) 8267

Untomia 160

untomiella Busck 6400

ura Smith 1264

uramia Smith 1264

urania Westwood \& Hevitson 455 


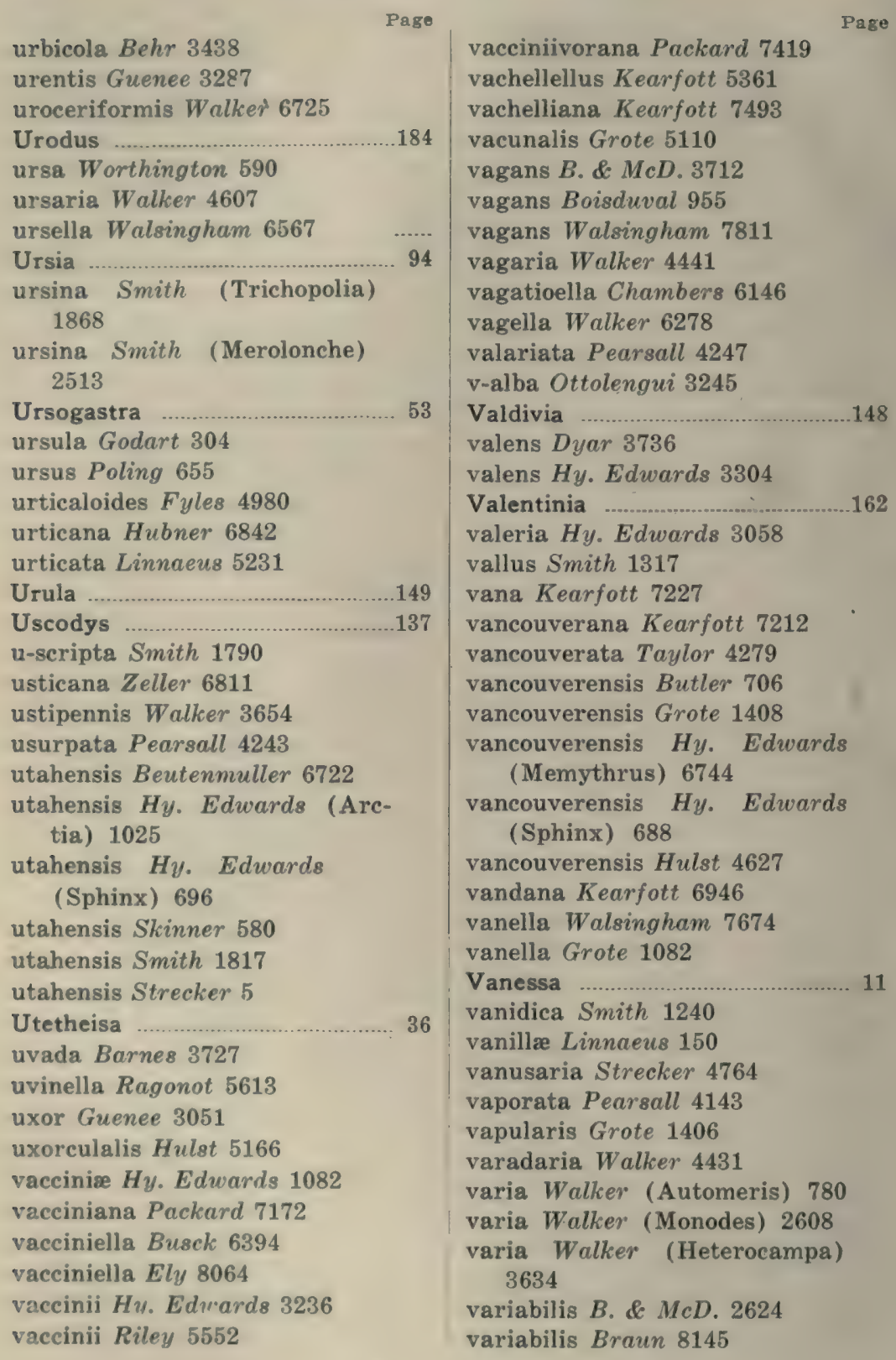


variabilis Busek 6234 variabilis Grote 1468 variabilis Smith 1872 variabilis Walsingham 8188 variana Clemens 7084 variana Fernald 7426 variana Morrison 2800 variana Ottolengui 3239 variaria Grote 4632 variata Schiffermuller 3985 variata Grole 1594 variatella Clemens 8289 variegata Smith 2852 variegata Walker 5093 variella Braun 8399 variella Chamber 6136 variella Ragonot 5497 variolana Zeller $\mathbf{7 4 1 9}$ variolaria Guenee 4307 variolata Smith 1702 varix Grote 1594

\section{Varneria} .149

varuna Edwards 124

varus Grote \& Robinson 4755 vasaliata Guenee 4109 vashti Strecker 688 vau Fitch 3589 vau-media Smith 1755 vau-orbicularis Smith 1691 v-brunneum Grote 2385 vecors Guenee (Eriopyga) 1842 vecors Guenee (Perigea) 2327 vega $D$ yar 754

vega Smith 3570

vegeta Morrison 3294

velaris Grote 1169

velata Strecker 2933

velate Walker (Rhynchagrotis) 1589

velata Walker (Eriopyga) 1842 velata Walker (Apamea) 2637 velatella Busck 6081 velatella Clemens 8306 velleda Stoll 3738 velleripennis Grote 1291

vellifera Grote 3572

vellivolata Hulat 4575

velutina $B . \& M C D .1141$

velutina $S$ mith 1805

velutinana Walker 7390

venalalis Hulst 5128

venalis Grote 5098

venata Grote 4480

venata Smith 1910

venatus Smith 2746

venerabilis Walker 1397

veneratrix Meyrick 7370

venosa Seudder 35

venosa Smith 2374

venosa Walker 833

ventilator Grote 2999

ventralis Grote \& Robinson 4933

ventrella Fitch 6389

venus Neumoegen 3663

Venusia 108

venusta Dalman 1029

venusta $H y$. Edwards 1077

venusta Walker (Hyparpax) 3662

venusta Walker (Palroplusia) 3284

venustella Clemens 7991

venustella Chambers 6398

venustula Grote 2203

venustula Walker 2963

venustus Walsh 3783

verbascoides Guenee 2246

verberata Smith 2220

verbesinella Busck 7975

verdiaria Grossbeck 4322

verecunda Hulst 3057 .

verecunda $H y$. Edwards 6720

veritata Pearsall 3954

vermiculata Grote \& Robinson 3586

verna Edwards 613

vernalis Edwards 34

vernalis Morrison 2110 


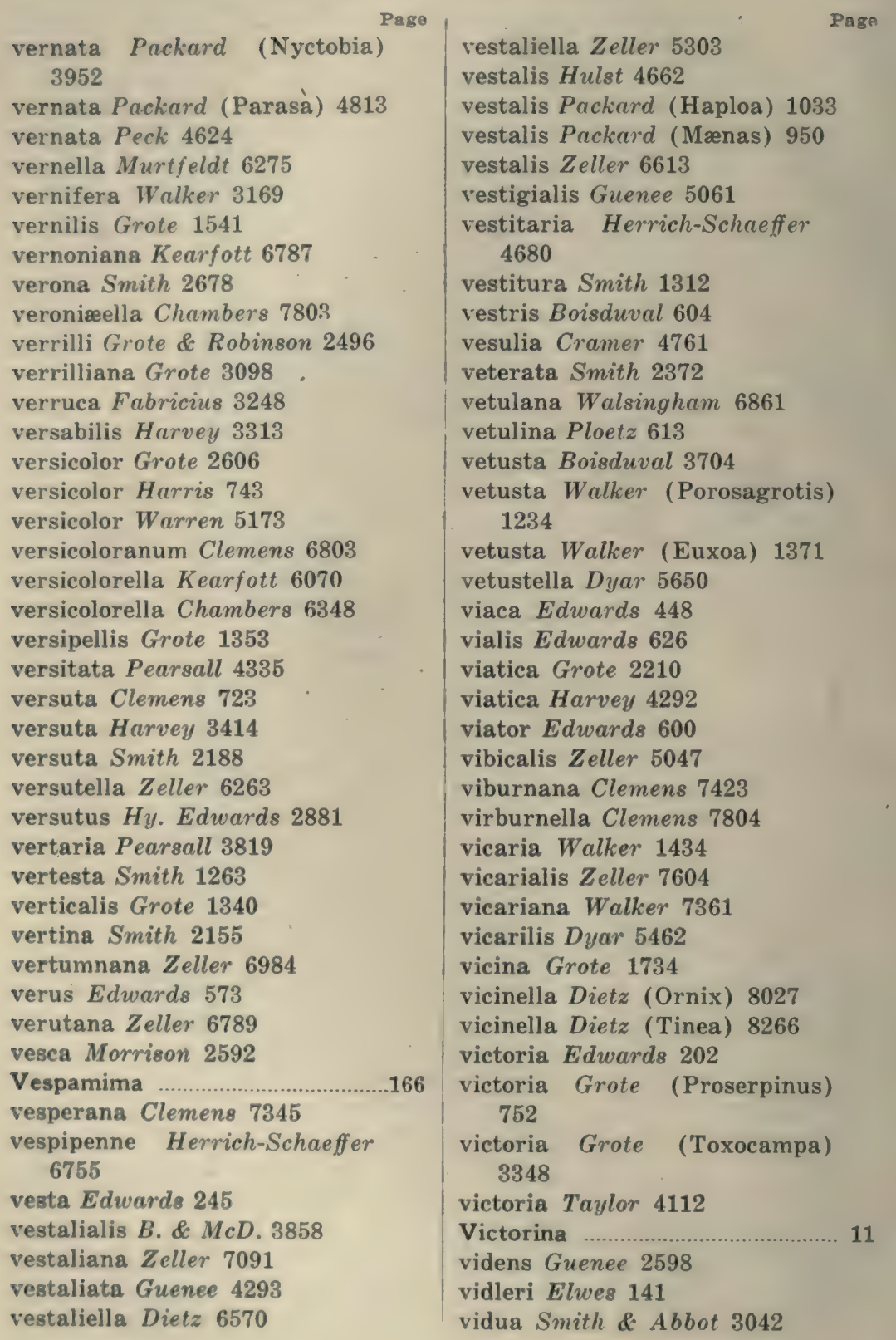


viduella Fabricius 6217

viduata Guenee $304: 3$

vidularia Grote $\mathbf{4 1 4}$

vierecki Skinner 618

vigilacella Clemens 7675

vigilans Grote 2127

vigilans Meyrick 7561

vilisana Walker $\mathbf{7 1 5 3}$

villana Busck $\mathbf{7 5 4 4}$

villella Busck (Chrysoclista) 5969,1

villella Busck (Blastobasis) 6516

villiersi Boisduval \& LeConte 2 villosa Grote 1067

villosalis Zeller 4972

villosella Clemens 8397

villosella Hulst 5690

vincta Meyriele 7548

vincta Walker 2607

vinctalis $B$. \& McD. 4997

vinculum Guenes 3143

vindemialis Grote 1929

vinela Smith 1526

vinnula Grote 2428

vinosaris Grote \& Robinson 4706

vintrella Busek 7669

vinulenta Grote 2213

vinulenta Grote \& Robinson 5162

vinulentaria Grote \& Robinson 4705

viola Maynard 97

violacea Edwards 452

violacea Grote 2332

violaceana Robinson 7306

violacearia Walker 3894

violaceellus Beutenmuller 8186

violacella Clemens 8053

violaceofusea Zeller 6245

violans Guenee 1866

violaris Grote de Robinson 1419

violascens Schau 2599

violenta $\boldsymbol{H y}$. Edwards 3099

viralis Crote 1603 virens French 3119

virescana Clemens 7377

virescens Fabricius 1091

virescens $H$ ulet 5418

virgata Packard 3649

virgata Taylor 4640

virgatella Busck 8220

virgatella Clemens 5631

virgilia $\boldsymbol{H}$ y. Edwards 3058

virgilius Seudder \& Burgess 527

virginalis Boisduval 1028

virginalis Grote 2958

virginalis $\mathrm{Hulat} \mathbf{4 4 3 0}$

virginaris Cramer 4607

virginaria Grote 3209

virginaria Hulst 4662

virginata Graef 3949

virginella $D$ yar 5842

virginiana Busele 6774

virginiana Clemene (Enarmon-

ia) $\mathbf{7 1 3 8}$

virginiana Clemens (Sparganothis) 7316

virginica Charpentier 838

virginica Fabricius 954

virginiella Chambers 7890

virginiella Clemens (Choreutis) 7608

virginiella Clemens (Nepticula) 8398

virginiensis Drury (Anisota) 800

virginiensis Drury (Vanessa) 283

virginiensis Edwards (Pieris) 35

virginiensis Edwards (Tharsalea) 398

virginiensis Gray 328

virgo Butler 4856

virgo Linnaeus 971

virgula Grote 1845

virgulti Behr 322

virguncule $K$ irby 982 
virbuncula Smith 2382

virida $B . \&$ \& $M c D .2764,1$

virida Strecker 60

viridalis $B$. \& $M c D .5280$

viridans Guenee 3170

viridata Harvey 2422

viridata Packard (Acasis) 3950

viridata Packard (Hydriomena) 4007

Viridemas

viridescens Grossbeck 4066

viridescens Walker 2104

viridicaria Hulst 3796

viridiclava Walker 4816

viridicuprella Walsingham 7805

viridifera Grote 2779

viridimedia Smith 2723

viridimusca Smith 2399

viridipallens Grote 2146

viridipallens Hulst 3821

viridipallida $B . \& M c D .2726,1$

viridipennata Hulst 3817

viridipurpurea Hulst 3809

viridirufaria Neumoegen 4464

viridis Edwards (Callophrys) 389

viridis Edwards (Pamphila) 563

viridisigma Grote 3234

viridisignata Grote 3234

viridisparsa $\operatorname{Dod} 1214$

viridisquama Walker 3170

viriditincta Smith 2039

viridoperlata Packard 4659

viridula Grote 2813

viridus Reakirt 4814

visaliella Chambers (Argyresthia) 7699

visaliella Chambers (Cyane) 8246

viscana Kearfott $\mathbf{7 4 7 6}$

viscidiflorella Walsingham 7806

vitalbata Schiffermuller 4281 viteana Clemens 6779

vitellinana Zeller 7529

vitellius $A$. \& $S .599$

viticordifoliella Clemens 7584

vitifoliella Chambers 8111

vitigenella Clemens 8112

vitis French 1670

vitis Linnaeus 740

vitivorana Packard 6779

vitralis Hubner 4974

vitrana Walsingham 7230

vitraria Grote 4647

vitrea Grote 3556

vitrina Boisduval 4890

vitrina Neumoegen 6659

vitrina Walker 2566

vittata Fabricius 996

vittata Harris 899

vittatalis Dyar 5203

vittifrons Grote 1580

vittula Grote 1768

Vitula

vivida Dyar 2145

vivida Grote 1002

vividana Dyar 7353

vocalis Grote 1455

vocaridorsana Kearfott 7308

volantaria Pearsall 3822

volubilis Harvey 1409

volucer Hulst 3936

volucrata Hulst 3848

volucris Grote 3387

volumnia $\boldsymbol{H} y$. Edwards 3058

voluntaria Meyrick 7501

volupia Fitch 1105

volupialis Grote 5163

voluta Clemens 4824

vomerina Grote 1877

vomonana Kearfott 6997

vorax Behr 2079

votiva Hulst 3098

votiva $H y$. Edwards 875

vovana Kearfott 6961 
Page

voxcana Kearfott 7475

wamsutta Harri 583

v-signatana Packard 7853

vulgalis Guenee 4949

vulgaris Grote Robinson 2251

vulgivaga Morrison 2335

vulgivagellus Clemene 5368

vulneraris Hubner 4786

vulnerata Swell 4009

vulneres Grote 2628

vulnifica Grote 2788

vulpina Grote 2486

vulpina $\boldsymbol{H y}$. Edwards 3918

vulpina Smith 1819

vultuosa Grote 2260

waco Edwards 542

wacoella Chambers 6349

wakulla Edu'ards 634

walkerana Kearfott 6979

walkerata Pearsall 3995

walkeri Andrews 2432

walkeri Felder 763

walkeri Grote 2213

walkeri Neumoegen 6650

walshella Clemens 4809

Walshia 162

walshi Edwards (Papilio) 20

walshi Edwards (Catocala) 3073

walsinghamella Beutenmuller 6642

walsinghami Butler 956

walsinghami Dietz 6280

walsinghami Hy. Edwards (Ctenucha) 837

walsinghami Hy. Edwards (Schinia) 1178

walsinghami Fernald 5891

walsinghami Kearfott $\mathbf{7 2 4 5}$

walsinghami Ragonot 5588

walsinghamiella Busck (Agnopteryx) 6443

walsinghamiella Busck (Trachoma) 7651

wana Kearfott 7226

wandana Kearjott 6950

waracana Kearfott 7543

wardi Oberthur 226

warneri Harvey 4687

washingtonalis Grote 5094

washingtonia $B . \& M c D .185$

washingtonia $H y$. Edwardo 6661

washingtonia Grote 2163

washingtoniella Busck (Gnorimoschema) 6098

washingtoniella Busck (Trichotaphe) 6369

washingtoniensis Grote 1556

wasmuthi Weeks 1

watchungana Kearfott $\mathbf{7 1 6 7}$

weidemeyeri Edwards 306

wellingtoniana Kearfott 6820

wenzelana Haimbach 7329

werdandi Herrich-Schaeffor 68

werneri Biederman 3098

westcotti Grote 3092

westermanni Staudinger 1359

westwoodi Boisduval 77

wheeleri Edwards 113

wheeleri $H_{y}$. Edwards 216

whitneyi Behr 226

whitneyi Dodge 3091

williamsi Dodge 988

williamsi Grinnell 5876

williamsi Swett 4070

willingana Kearfott 7125

willingi $B$. \& $M C D .3712$

wilsoni Grote (Xerociris) 2826

wilsoni Grote (Euxoa) 1238

wingina Scudder 587

winnata Taylor 4188

winniana Kearfott 7509

winnipeg Smith 2152

winonah Brodie 768

wiscana Kearfott 7518

wittfeldi Eduards 364 
wittfeldi $H y . E d w a r d s$ (Alypia) 1044

wittfeldi $\boldsymbol{H} y$. Edwards (Synanthedon) 6701

wockei Moeschler 1480 womonana Kearfott 6941 woodgatei Williams 563 woodi Grote 3197 worthingtoni Grote 1466 worthingtoniana Fernald 7022 wosnesenski Menetries 54 wrightiaria Hulst 4542 wrighti Edwards (Adopæa) 541 wrighti Edwards (Melitæa) 234 wrighti $\boldsymbol{H y}$. Edwards 3680 wrighti French 941 wrighti Grinnell 5856 wrighti Stretch 826 wyandot Edwards 488 wymola Barnes 3630 wythiæ Walsingham 7807 xami Reakirt 376 xandana Kearfott 6995 Xanthia

xanthobasis Zeller 7597 xanthocrypta Dyar 5177

Xanthœeia

xanthœenobares Dyar 5563

xanthoides Boisduval 401

xanthoides Guenee 2323

xanthoides Walker 7304

xantholeucalis Guenee 5299

xanthometata Walker 4300

Xanthopastis

xanthophila $R o ̈ b e r ~ 53$

Xanthoptera 75

Xanthorhoe 106

xanthospora Meyrick 7228

xanthostictella Dietz 8272

$\mathrm{X}$ anthothrix 73

Xanthotype 120

xanthus Edwards 490

Xantippides xasta $B . \& M c D .1257$

xeniolalis Hulst 4952

Xenophanes 19

xerces Boisduval 450

Xerociris 73

xicaque Reakirt 99

xiphiæformis Boisduval 6655

xithon Dyar 2893

Xylena 59

Xylestia .192

xylina Say 3406

xylina Hulst 3985

xyliniformis Guenee 2499

xyliniformis Smith 1370

xylinoides Guenee (Нyppa) 2531

xylinoides Guenee (Acronycta) 2499

Xylomyges 53

Xylomcea 68

Xylophanes 26

Xylophasia 60

xylopsamma Meyrick 5886

xylostella Packard 7683

Xylotype 59

Xyrosaria 184

xysteraria Hulst 3778

Xystrota 101 yamana Reakirt 47 yampai Barnes 4875 yandana Kearfott 6993 yaracana Kearfott 6784 yarrowi Stretch 947 yavapaella Dyar 5793 yavapai Grossbeck 4375 yavapai Kearfott 5328 yavapai Neumoegen 787 yavapai Smith 3195 ydatodes Dyar 878 yehl Skinner 603 y-inversa Packard 4841 y-inversus Riley 8463 
yosemits Grote 1528

yosemite $\boldsymbol{H}_{\text {y. }}$ Eduards 905

yosemitensis Strand 2854

youngana Kearfott 7232

youngata Taylor 4191

youngella Kearfott 6067

youngellus Kearfott 5346

youngi Holland (Erebia) 143

youngi Holland (Brenthis) 198

youngi Smith 1551

Yponomeuta

Xponomeutida

ypsilon Forbes 4614

ypsilon Rottemburg 1422

yreka Edwarde 574

yreka Realirt 37

Yrias

yucce Boisduval \& LeConte 654

yuccuecolella Dietz 6512

yuccasella Busck 6176

yuccasella Riley 8471

yuccatana Busck $\mathbf{7 5 1 2}$

yukona Holland 432

yukonensis Holland 102

yuma Edwards 611

yuma Smith 2541

yumaella Dyar 5644

yumaella Kearfott 8233

yumana Kearfott 7319

zabolicus Strecker 8354

zabulon Boisduval \& LeConte 593

zachaina Butler \& Druce 421

zachrysa Meyrick 8056

Zale

zalissaria Walker 4732

zampa Edwards 504

zana Kearfott 7228

Zanclognatha

zavaliella Busck 6647

s'a ge

zeacolella Duor $\mathbf{6 4 3 7}$

zea Filch 5807

zea Harris 2642

zeellus Fernald 5398

Zegris

zela Butler 325

zelatella Hulst 5553

zelica French 3044

zelicaon Lucas 6

Zelicodes

zella Dyar 1019

zelleraria Packard 3820

zelleri Grote (Balsa) $\mathbf{2 6 0 1}$

zelleri Grote (Epipaschia) 5468

Zelleria

zellerianum Fernald 6797

zelleriella Chambers (Ethmia) 6644

zelleriella Chambers (Fraploptilia) 7808

zelleriella Clemens 7876

zelleriella Dietz 6569

zelmira Felder 435

zembla Smith 1241)

zenobia Cramer 3392

Zenodochium

Zenodoxus

Zenophleps

zephyralis $B$. \& $M C D .5052$

zephyria Grote 779

zephyrus Edwards 273

Zerene

zerene Boisduval 168

zeroe Boisduval 405

zerynthia Hubner 300

zegtos Hubner 467

zeta Ottolengui 3239

zethus Druce 3648

zetterstedti Staudinger 2201

Zeusera

zapulata Robinson 7347

zaracana Kearfott $\mathbf{7 4 5 0}$

riczacata Walleer 4068

zillah Strecker 3060 
zilpa Butler 463

zimmermanni Grote 5560

zinckenella Treitschke 5690

zinghalis Walker 5009

ziscana Kearfott 7515

zoe Behr 3051

zolicaon Boisduval 6

zomonana Kearfott 6973

zonalis Grote 1010

zonata Drury 729

zonata Walker 3479
Page

zonulata Hulst 4760

zonulella Ragonot 5508

Zophodia 147

Zosteropoda

zoxcana Kearfott 7486

zozana Kearfott 6769

zuni Neumoegen 969

Zygænidæe 127

zygotaria Hulst 3790 


$$
x^{3}
$$





\title{
CONTRIBUTIONS
}

TO THE

\section{NATURAL HISTORY \\ or}

\section{North American Lepidoptera}

BY

\author{
Wm. BARNES, S. B., M. D. \\ AND \\ J. McDUNNOUGH, Ph. D.
}

VOL. I. .

Price

No. 1 Revision of the Cossida of N. America-7 Plates ..........\$1.50

2 The Lasiocampid Genus Gloveria and Its Allies-4 Plates 1.00

3 Revision of the Megathymids -6 Plates.............................. 1.25

4 Illustrations of Rare and Typical Lepidoptera-27 Plates 3.50

5 Fifty New Species; Notes on the Genus Alpheias-

5 Plates 1.50

6 On the Generic Types of N. Am. Diurnal Lepidoptera...... $\quad .50$

VOL. II.

No. 1 Illustrations of Rare and Typical Lepidoptera (Cont'd) -21 Plates

2 The N. American Species of the Genus Olene-7 Plates.... 2.50

3 New N. Am. Lepidoptera with Notes on Described

Species-9 Plates 3.00

4 Some Apparently New Lepidoptera from S. Florida4 Plates 1.00

5 Synonymic Notes on N. American Lepidoptera ................. .75

6 Some New N. American Pyraustina-2 Plates. 1.00

\section{VOL. III.}

No. 1 New N. Am. Lepidoptera: Notes on Walker's Types of Geometridæ in the D'Urban Collection-3 Plates...... 1.10

2 Notes on N. Am. Diurnal Lepidoptera-8 Plates .............. 1.90

3 Synonymic Notes on N. Am. Heterocera-4 Plates .......... 1.20

4 Further Notes on Philotes battoides and Its Allies;

Remarks on Grossbeck's List of Florida Lepidoptera; New Species and Varieties of Geometridre-18 Plates

To be obtained from Dr. Wm. Barnes, Decatur, III. 
Qolunur IV 101. Revisiow of the Henus tydriomena

- 2 hotes and new species 15 platos

- 3 hates and neur apecies microlefódopotero 15 flates 


\section{Exchange}

A large number of duplicates of North American Lepidoptera which have accumulated in the Barnes Collection, including many species from the West and Southwest, are offered in exchange for series of bred specimens, even of the commoner species, or for species from North America not in the collection. Desiderata List sent on application.

Identification of $\mathrm{N}$. American material will be gladly undertaken for the privilege of retaining desired specimens.

In connection with the proposed publication of a full Catalogue of $N$. American Lepidoptera records of captures are much desired in order to establish the geographical range of each species; also data regarding the location of type specimens will be gladly received.

DR. WM. BARnes, DeCatur, ILl. 


$-2$ 



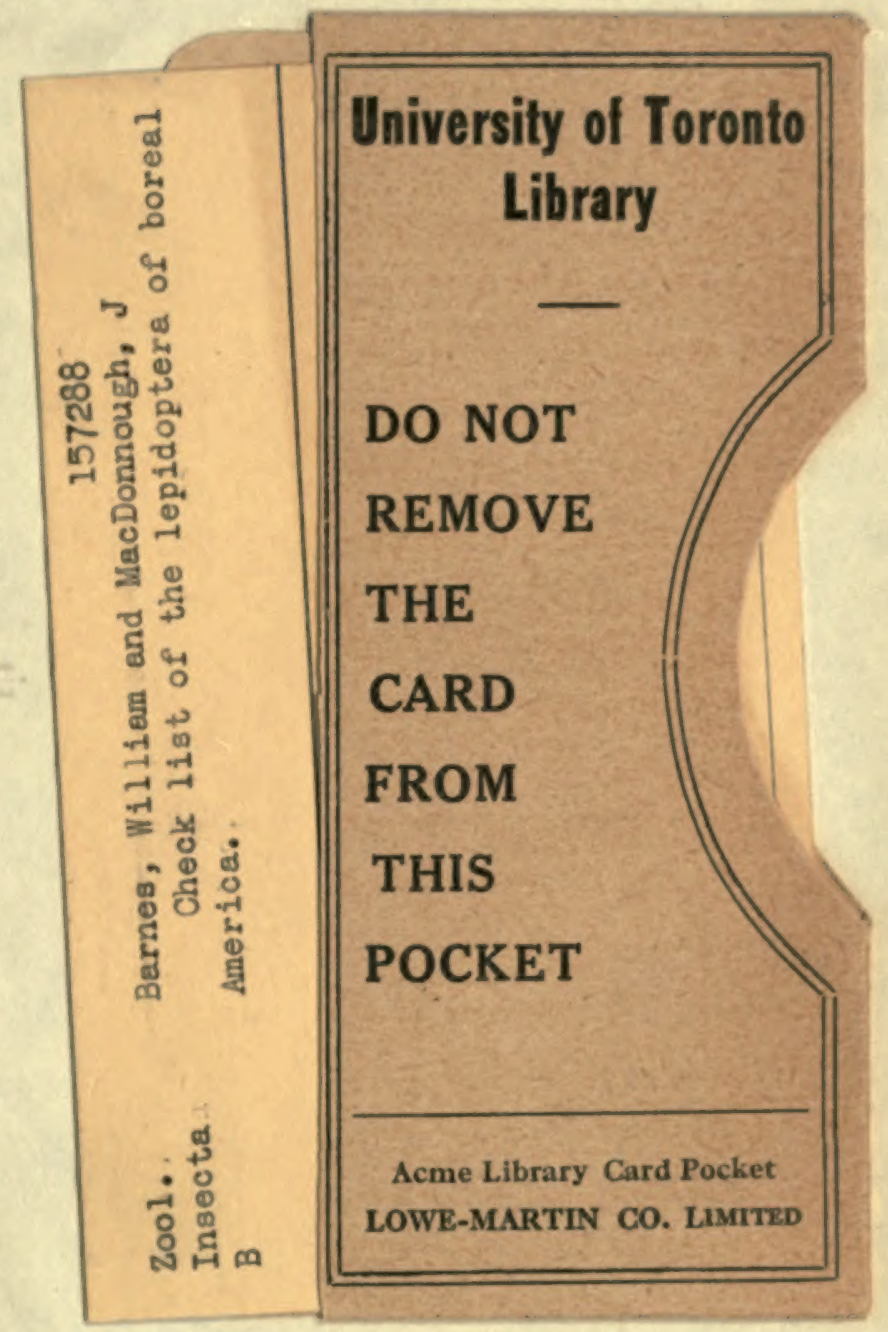


